Prepared under the auspices of the U.S. Agency for International Development

\title{
Streamflow Characteristics of Streams in the Helmand Basin, Afghanistan
}

By Tara Williams-Sether

Data Series 333

U.S. Department of the Interior

U.S. Geological Survey 


\section{Streamflow Characteristics of Streams in the Helmand Basin, Afghanistan}

By Tara Williams-Sether

Prepared under the auspices of the U.S. Agency for International Development

This study was funded by Interagency Agreement 07C442100KB between the U.S. Agency for International Development and the U.S. Geological Survey.

Data Series 333

U.S. Department of the Interior

U.S. Geological Survey 


\title{
U.S. Department of the Interior DIRK KEMPTHORNE, Secretary
}

\author{
U.S. Geological Survey \\ Mark D. Myers, Director
}

U.S. Geological Survey, Reston, Virginia: 2008

For product and ordering information:

World Wide Web: http://www.usgs.gov/pubprod

Telephone: 1-888-ASK-USGS

For more information on the USGS - the Federal source for science about the Earth, its natural and living resources, natural hazards, and the environment:

World Wide Web: http://www.usgs.gov

Telephone: 1-888-ASK-USGS

Suggested citation:

Williams-Sether, Tara, 2008, Streamflow characteristics of streams in the Helmand Basin, Afghanistan: U.S. Geological Survey Data Series 333, 341 p.

Any use of trade, product, or firm names is for descriptive purposes only and does not imply endorsement by the U.S. Government.

Although this report is in the public domain, permission must be secured from the individual copyright owners to reproduce any copyrighted material contained within this report. 


\section{Contents}

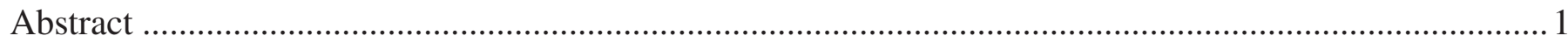

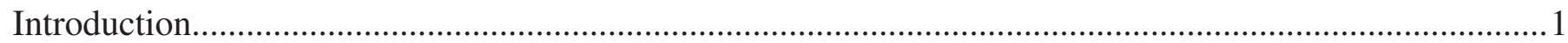

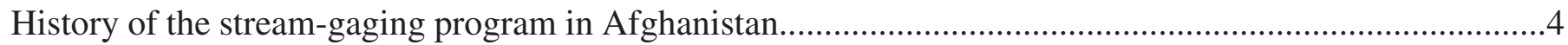

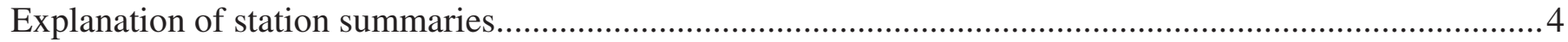

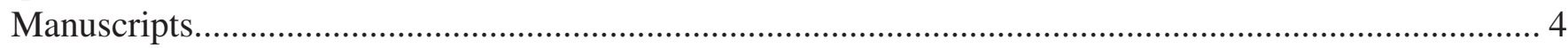

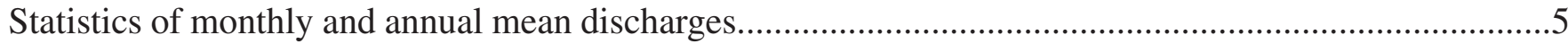

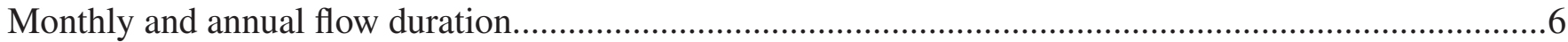

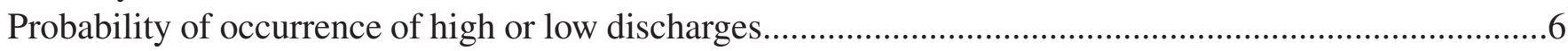

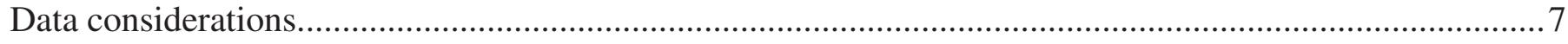

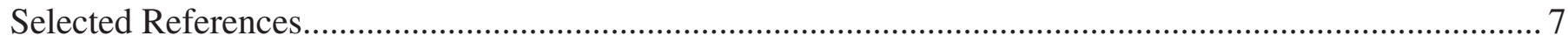

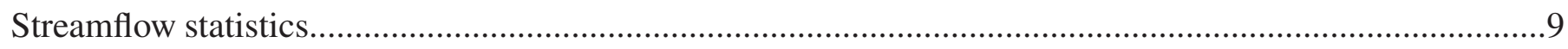

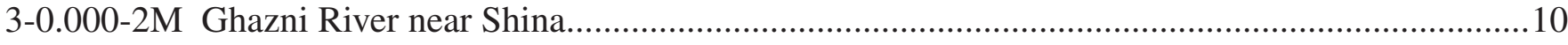

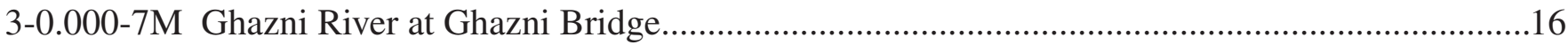

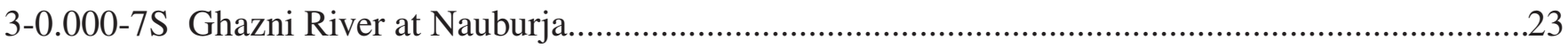

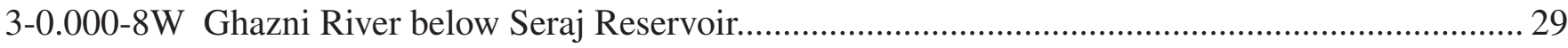

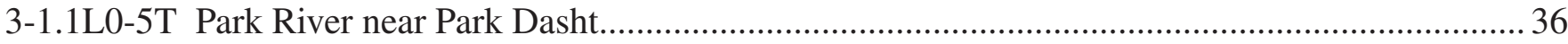

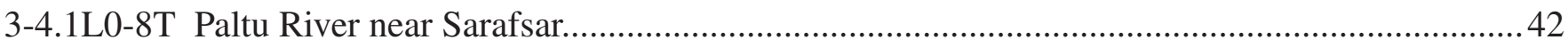

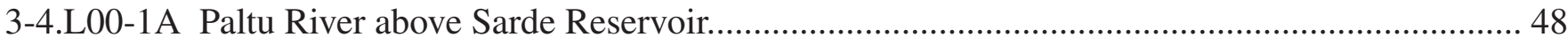

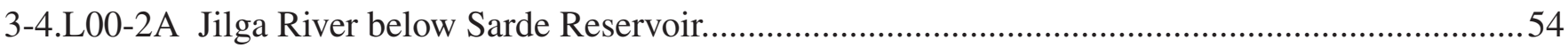

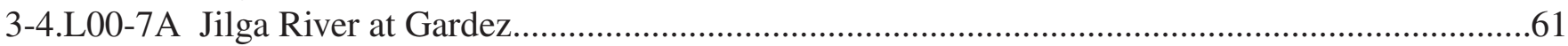

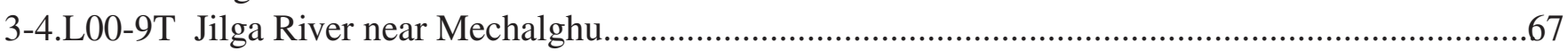

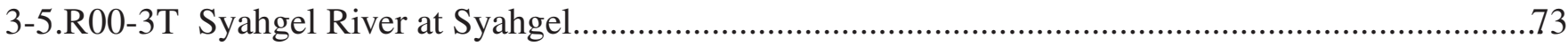

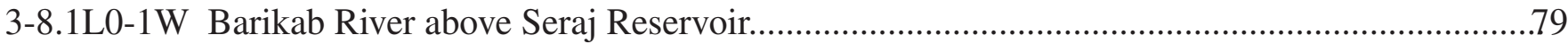

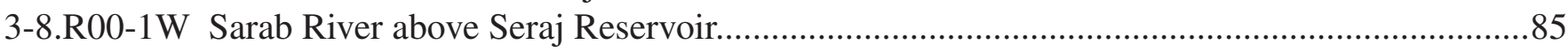

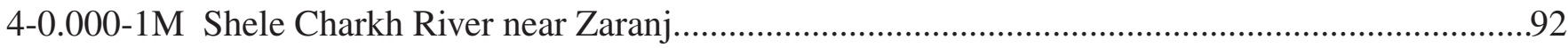

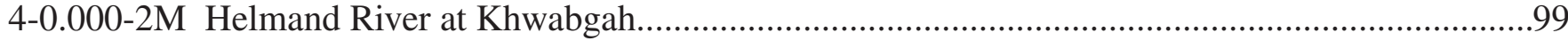

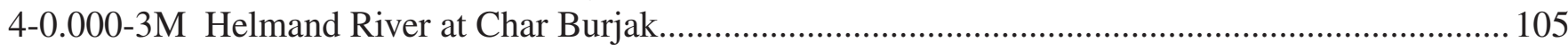

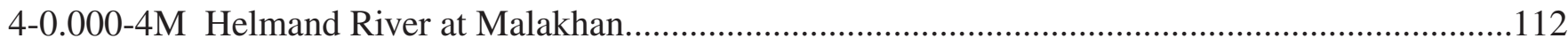

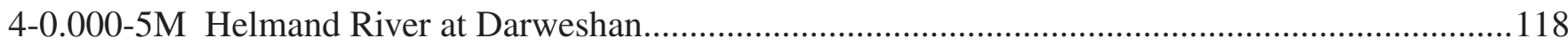

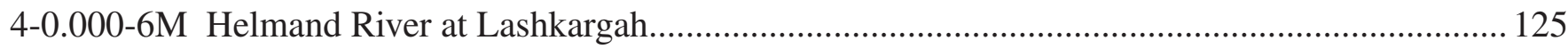

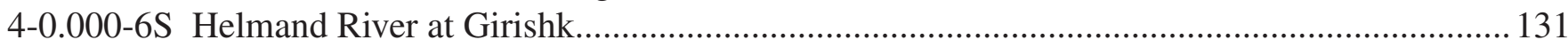

4-0.000-7M Helmand River below Kajakai Reservoir............................................................. 137

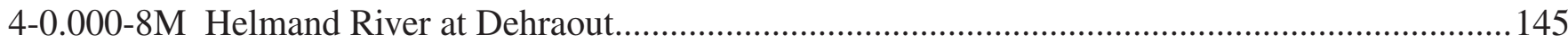

4-0.000-9M Helmand River at Gizab.....................................................................................152

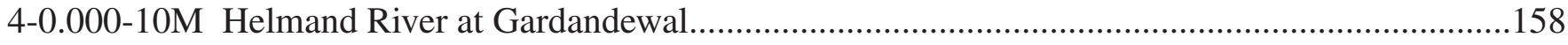

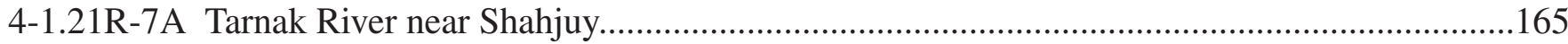

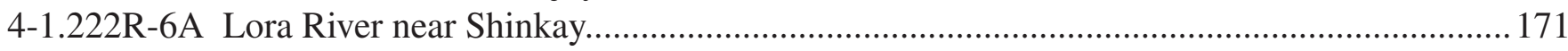

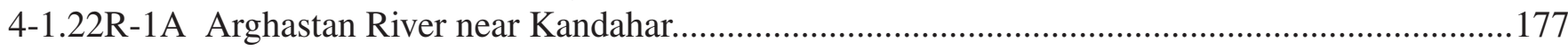

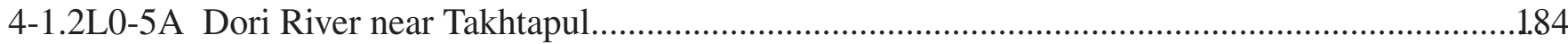

4-1.L00-1A Arghandab River at Qala-i-Bust....................................................................... 190

4-1.L00-3A Arghandab River near Kandahar........................................................................198

4-1.L00-4A Arghandab River below Arghandab Reservoir.......................................................204

4-1.L00-5A Arghandab River above Arghandab Reservoir......................................................... 212

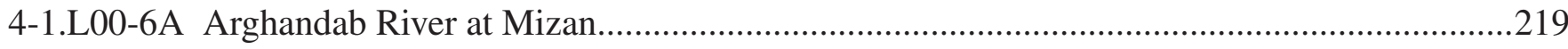


4-1.L00-9A Arghandab River at Sang-i-Masha....................................................................... 225

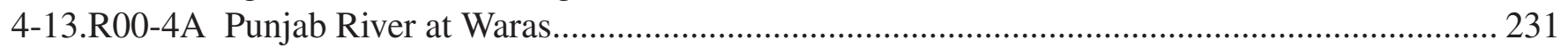

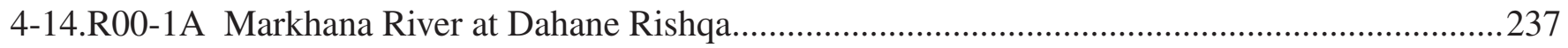

4-16.R00-3A Syahsang River near Gardandewal............................................................................243

4-3.L00-2T Sangin Wash (Seraj Canal) at Sangin........................................................................... 249

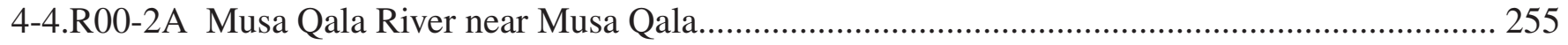

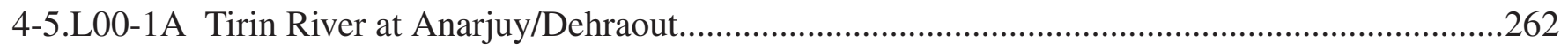

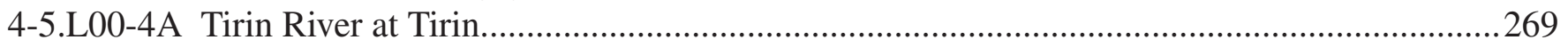

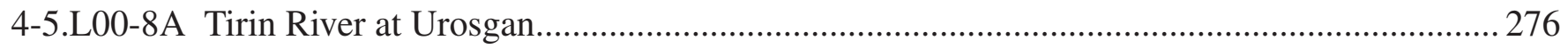

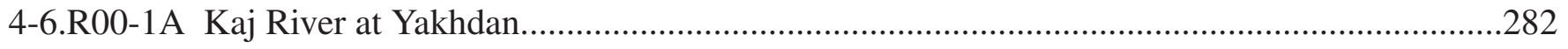

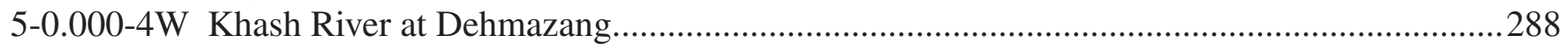

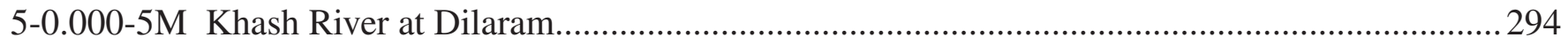

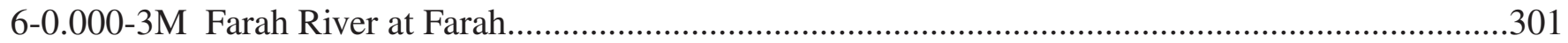

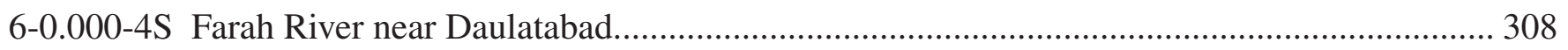

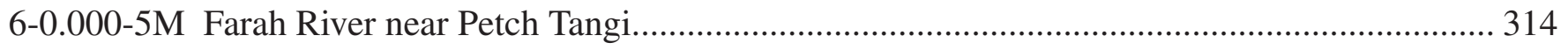

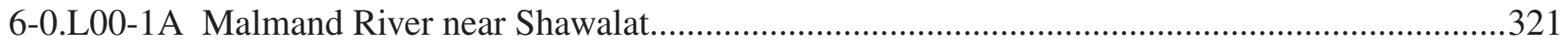

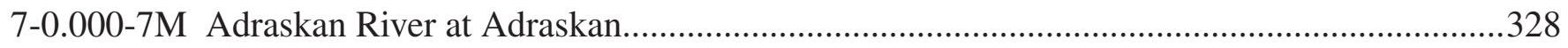

7-5.R00-1A Rud-i-Gaz River near Adraskan............................................................................... 335

\section{Figures}

1. Map showing location of streamflow-gaging stations for which streamflow statistics are published in this report.

\section{Tables}

1. List of streamflow-gaging stations for which streamflow statistics are published in this report.

\section{Conversion Factors}

\section{SI to Inch/Pound}

\begin{tabular}{|c|c|c|}
\hline Multiply & By & To obtain \\
\hline \multicolumn{3}{|c|}{ Length } \\
\hline meter $(\mathrm{m})$ & 3.281 & foot $(\mathrm{ft})$ \\
\hline kilometer $(\mathrm{km})$ & 0.6214 & mile (mi) \\
\hline \multicolumn{3}{|c|}{ Area } \\
\hline square meter $\left(\mathrm{m}^{2}\right)$ & 0.0002471 & acre \\
\hline hectare (ha) & 2.471 & acre \\
\hline square hectometer $\left(\mathrm{hm}^{2}\right)$ & 2.471 & acre \\
\hline square kilometer $\left(\mathrm{km}^{2}\right)$ & 247.1 & acre \\
\hline square meter $\left(\mathrm{m}^{2}\right)$ & 10.76 & square foot $\left(\mathrm{ft}^{2}\right)$ \\
\hline hectare (ha) & 0.003861 & square mile $\left(\mathrm{mi}^{2}\right)$ \\
\hline square kilometer $\left(\mathrm{km}^{2}\right)$ & 0.3861 & square mile $\left(\mathrm{mi}^{2}\right)$ \\
\hline \multicolumn{3}{|c|}{ Volume } \\
\hline cubic meter $\left(\mathrm{m}^{3}\right)$ & 264.2 & gallon (gal) \\
\hline
\end{tabular}




\begin{tabular}{lcl} 
cubic meter $\left(\mathrm{m}^{3}\right)$ & 0.0002642 & $\begin{array}{l}\text { million gallons }(\mathrm{Mgal}) \\
\text { cubic foot }\left(\mathrm{ft}^{3}\right)\end{array}$ \\
cubic meter $\left(\mathrm{m}^{3}\right)$ & 35.31 & cubic mile $\left(\mathrm{mi}^{3}\right)$ \\
cubic kilometer $\left(\mathrm{km}^{3}\right)$ & 0.2399 & acre-foot $(\mathrm{acre}-\mathrm{ft})$ \\
cubic meter $\left(\mathrm{m}^{3}\right)$ & 0.0008107 & Flow rate \\
\hline & 70.07 & acre-foot per day $(\mathrm{acre}-\mathrm{ft} / \mathrm{d})$ \\
\hline cubic meter per second $\left(\mathrm{m}^{3} / \mathrm{s}\right)$ & 0.000811 & acre-foot per year $(\mathrm{acre}-\mathrm{ft} / \mathrm{yr})$ \\
cubic meter per year $\left(\mathrm{m}^{3} / \mathrm{yr}\right)$ & 3.281 & foot per second $(\mathrm{ft} / \mathrm{s})$ \\
meter per second $(\mathrm{m} / \mathrm{s})$ & 35.31 & cubic foot per second $\left(\mathrm{ft} \mathrm{ft}^{3} / \mathrm{s}\right)$ \\
cubic meter per second $\left(\mathrm{m}^{3} / \mathrm{s}\right)$ &
\end{tabular}

Altitude, as used in this report, refers to distance above mean sea level at an unknown vertical datum.

\section{Definition of Terms}

Climatic year is the 12-month period April 1 through March 31. The climatic year is designated by the calendar year in which it begins.

Cubic meters per second $\left(\mathrm{m}^{3} / \mathrm{s}\right)$ is the rate of discharge representing a volume of 1 cubic meter passing a given point during 1 second and is equivalent to 1,000 liters per second or 60,000 liters per minute or 35.31 cubic feet per second.

Discharge is the volume of water in a stream at a given point and for a given period of time. Discharge is often used interchangeably with the term "streamflow."

Drainage area is the area, measured in a horizontal plane, enclosed by a topographic divide from which direct surface runoff from precipitation normally drains by gravity into the stream upstream from the station. Figures of drainage area given herein include all closed basins, or noncontributing areas, within the area unless otherwise specified.

Drainage basin is a part of the surface of the earth that is occupied by a drainage system, which consists of a surface stream or a body of impounded surface water together with all tributary surface streams and bodies of impounded surface water.

Gage heightis the water-surface elevation referred to some arbitrary gage datum. Gage height is often used interchangeably with the more general term "stage," although gage height is more appropriate when used with a reading on a gage.

Gaging station is a particular site on a stream, canal, lake, or reservoir where systematic observations of hydrologic data are obtained.

Instantaneous discharge is the discharge at a particular instant of time.

Mean is the arithmetic average of a list of values.

Mean discharge is the arithmetic mean of individual discharges during a specific period.

Period of record is the time during which a streamflow-gaging station is in operation and for which the records are published.

Probability of occurrence is the likelihood that an event will occur. Probabilities are generally expressed as a decimal number between 0 and 1 . If the probability is 0 , the event will not occur, if the probability is 1 , the event will occur absolutely. Probability also can be expressed as a percent, where 0 percent corresponds to 0 probability and 100 percent corresponds to a probability of 1 .

Recurrence interval is the average time interval between occurrences of a hydrologic event of a given or greater magnitude, usually expressed in years.

Regulation is the artificial manipulation of the flow of a stream. 
Stage (see "gage height")

Standard deviation is a measure of the variability of the values in a list of values.

Streamflow-gaging station is a particular site on a stream where a record of discharge is obtained.

Streamflow (see "discharge")

Surface water is the water on the surface of the earth.

Water year is the 12-month period 0 ctober 1 through September 30 . The water year is designated by the calendar year in which it ends and which includes 9 of the 12 months. 


\title{
Streamflow Characteristics of Streams in the Helmand Basin, Afghanistan
}

\author{
By Tara Williams-Sether
}

\section{Abstract}

Statistical summaries of streamflow data for all historical streamflow-gaging stations for the Helmand Basin upstream from the Sistan Wetlands are presented in this report. The summaries for each streamflow-gaging station include (1) manuscript (station description), (2) graph of the annual mean discharge for the period of record, (3) statistics of monthly and annual mean discharges, (4) graph of the annual flow duration, (5) monthly and annual flow duration, (6) probability of occurrence of annual high discharges, (7) probability of occurrence of annual low discharges, (8) probability of occurrence of seasonal low discharges, (9) annual peak discharge and corresponding gage height for the period of record, and (10) monthly and annual mean discharges for the period of record.

\section{Introduction}

A majority of the Afghan population lacks adequate, safe supplies of water because of contamination by a wide variety of sources, lack of water-resources management regulation, and lack of basic infrastructure, compounded by a recent 5-year drought and seasonal flooding.

The Helmand Basin is the largest in Afghanistan. It covers the southern half of the country, draining waters from the Sia Koh Mountains in Herat Province to the eastern mountains in Gardez Province (currently known as the Paktia Province) and the Parwan Mountains northwest of Kabul, and finally to the unique Sistan depression between Iran and Afghanistan (Favre and Kamal, 2004). A typical desert environment, the rivers in the Helmand Basin are fed by melting snow from the high mountains and infrequent storms. Great fluctuations in streamflow, from flood to drought, can be expected.

Knowledge of the magnitude and time distribution of streamflow is essential for all aspects of water management and environmental planning. Agencies responsible for the development and management of Afghanistan's surface-water resources can use this knowledge for making safe, economical, and environmentally sound water-resource planning decisions. To provide the Afghan managers with necessary streamflow information, the U.S. Geological Survey, in cooperation with the U.S. Agency for International Development, computed streamflow statistics for data collected at historical gaging stations within the Helmand Basin.

The purpose of this report is to provide a comprehensive publication summarizing streamflow characteristics for all historical gaging stations for the Helmand Basin upstream from the Sistan Wetlands. The historical gaging stations used are listed in table 1 and their locations are shown in figure 1. 
Table 1. List of streamflow-gaging stations for which streamflow statistics are published in this report.

\begin{tabular}{|c|c|c|}
\hline Afghan ID Number & USGS ID Number & Station Name \\
\hline $3-0.000-2 \mathrm{M}$ & 324200068060000 & Ghazni River near Shina \\
\hline $3-0.000-7 \mathrm{M}$ & 333300068250000 & Ghazni River at Ghazni Bridge \\
\hline $3-0.000-7 \mathrm{~S}$ & 333800068250000 & Ghazni River at Nauburja \\
\hline $3-0.000-8 \mathrm{~W}$ & 334500068230000 & Ghazni River below Seraj Reservoir \\
\hline $3-1.1 \mathrm{~L} 0-5 \mathrm{~T}$ & 330000068520000 & Park River near Park Dasht \\
\hline 3-4.1L0-8T & 330800069050000 & Paltu River near Sarafsar \\
\hline 3-4.L00-1A & 331600068420000 & Paltu River above Sarde Reservoir \\
\hline 3-4.L00-2A & 331800068370000 & Jilga River below Sarde Reservoir \\
\hline 3-4.L00-7A & 333500069130000 & Jilga River at Gardez \\
\hline 3-4.L00-9T & 334900069230000 & Jilga River near Mechalghu \\
\hline $3-5 . R 00-3 \mathrm{~T}$ & 333100068210000 & Syahgel River at Syahgel \\
\hline $3-8.1 \mathrm{~L} 0-1 \mathrm{~W}$ & 334600068210000 & Barikab River above Seraj Reservoir \\
\hline 3-8.R00-1W & 334601068210000 & Sarab River above Seraj Reservoir \\
\hline $4-0.000-1 \mathrm{M}$ & 310200061520000 & Shele Charkh River near Zaranj \\
\hline $4-0.000-2 \mathrm{M}$ & 304800061460000 & Helmand River at Khwabgah \\
\hline $4-0.000-3 \mathrm{M}$ & 301700062020000 & Helmand River at Char Burjak \\
\hline $4-0.000-4 \mathrm{M}$ & 302700063220000 & Helmand River at Malakhan \\
\hline $4-0.000-5 \mathrm{M}$ & 310800064110000 & Helmand River at Darweshan \\
\hline $4-0.000-6 \mathrm{M}$ & 313400064210000 & Helmand River at Lashkargah \\
\hline $4-0.000-6 \mathrm{~S}$ & 314800064350000 & Helmand River at Girishk \\
\hline $4-0.000-7 \mathrm{M}$ & 321900065060000 & Helmand River below Kajakai Reservoir \\
\hline $4-0.000-8 \mathrm{M}$ & 324200065280000 & Helmand River at Dehraout \\
\hline $4-0.000-9 \mathrm{M}$ & 332300066170000 & Helmand River at Gizab \\
\hline $4-0.000-10 \mathrm{M}$ & 343000068160000 & Helmand River at Gardandewal \\
\hline $4-1.21 \mathrm{R}-7 \mathrm{~A}$ & 323200067280000 & Tarnak River near Shahjuy \\
\hline $4-1.222 \mathrm{R}-6 \mathrm{~A}$ & 320000067180000 & Lora River near Shinkay \\
\hline $4-1.22 \mathrm{R}-1 \mathrm{~A}$ & 312600065550000 & Arghastan River near Kandahar \\
\hline $4-1.2 \mathrm{~L} 0-5 \mathrm{~A}$ & 311300065570000 & Dori River near Takhtapul \\
\hline 4-1.L00-1A & 313000064230000 & Arghandab River at Qala-i-Bust \\
\hline 4-1.L00-3A & 313700065340000 & Arghandab River near Kandahar \\
\hline 4-1.L00-4A & 315000065520000 & Arghandab River below Arghandab Reservoir \\
\hline 4-1.L00-5A & 315700066020000 & Arghandab River above Arghandab Reservoir \\
\hline 4-1.L00-6A & 321000066270000 & Arghandab River at Mizan \\
\hline 4-1.L00-9A & 330800067280000 & Arghandab River at Sang-i-Masha \\
\hline 4-13.R00-4A & 341400066550000 & Punjab River at Waras \\
\hline 4-14.R00-1A & 342100067290000 & Markhana River at Dahane Rishqa \\
\hline 4-16.R00-3A & 343400068110000 & Syahsang River near Gardandewal \\
\hline $4-3 . \mathrm{L} 00-2 \mathrm{~T}$ & 320300064500000 & Sangin Wash (Seraj Canal) at Sangin \\
\hline 4-4.R00-2A & 321700064460000 & Musa Qala River near Musa Qala \\
\hline 4-5.L00-1A & 323800065340000 & Tirin River at Anarjuy/Dehraout \\
\hline 4-5.L00-4A & 323800065560000 & Tirin River at Tirin \\
\hline 4-5.L00-8A & 325800066390000 & Tirin River at Urosgan \\
\hline 4-6.R00-1A & 325800065300000 & Kaj River at Yakhdan \\
\hline $5-0.000-4 \mathrm{~W}$ & 315500063070000 & Khash River at Dehmazang \\
\hline $5-0.000-5 \mathrm{M}$ & 320900063260000 & Khash River at Dilaram \\
\hline $6-0.000-3 \mathrm{M}$ & 322200062040000 & Farah River at Farah \\
\hline $6-0.000-4 S$ & 324500062370000 & Farah River near Daulatabad \\
\hline $6-0.000-5 \mathrm{M}$ & 325100062520000 & Farah River near Petch Tangi \\
\hline 6-0.L00-1A & 325100063180000 & Malmand River near Shawalat \\
\hline $7-0.000-7 \mathrm{M}$ & 333800062160000 & Adraskan River at Adraskan \\
\hline 7-5.R00-1A & 334200062170000 & Rud-i-Gaz River near Adraskan \\
\hline
\end{tabular}




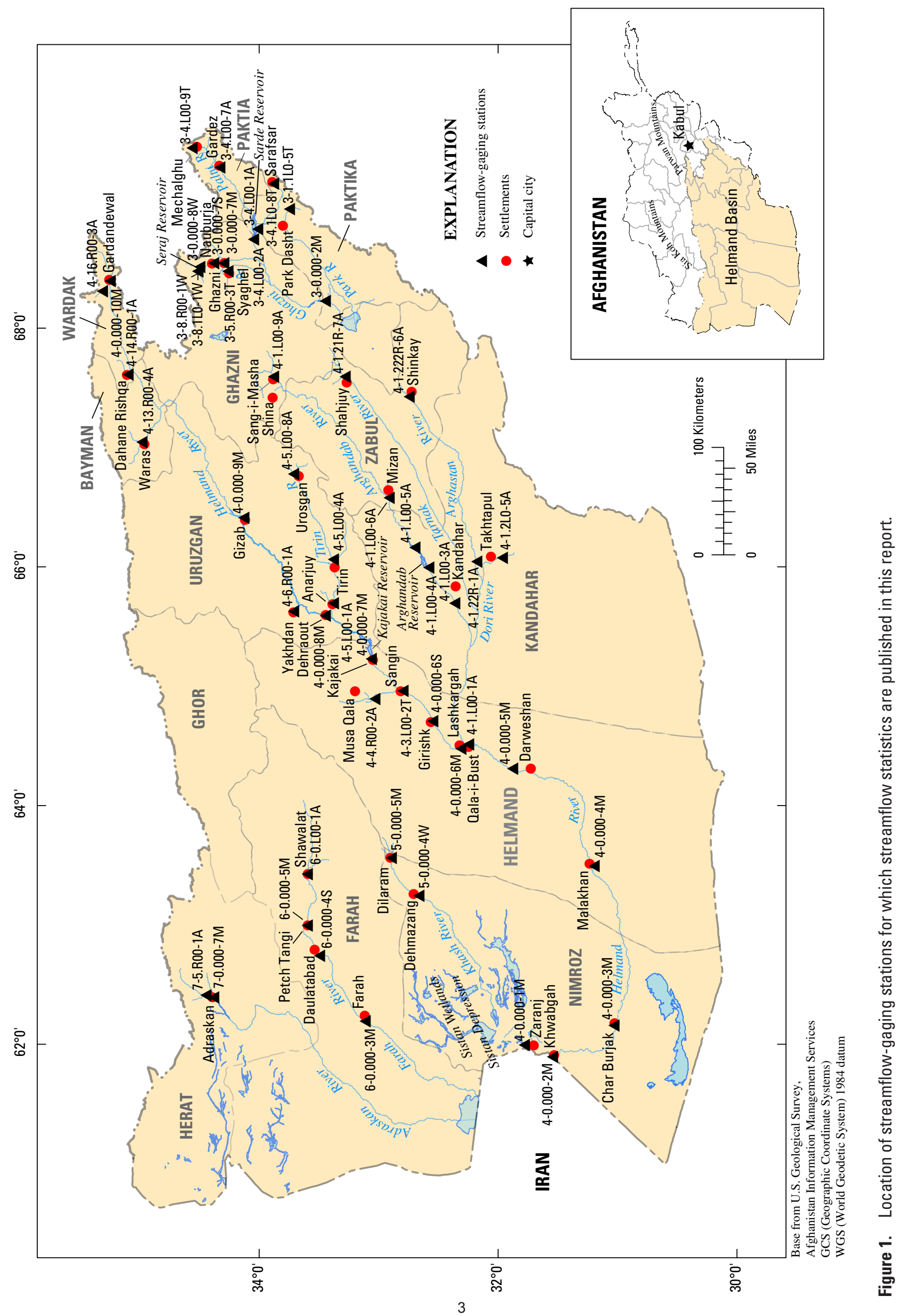




\section{History of the Streamflow-Gaging Program in Afghanistan}

River discharge measurements began in Afghanistan in the mid-1940s at a few sites. The number of sites increased over the years until the late 1970s. Measurements were discontinued soon after the Soviet invasion of Afghanistan in 1979. Until 1978, Afghanistan had a network of approximately 160 streamflow-gaging stations. No streamflow data were collected after September, 1980 until recently. In 2005, three historical streamflow-gaging stations were re-established and currently (2008) much of the historic network is in the process of being re-established.

\section{Explanation of Station Summaries}

Station summaries are presented so that each station description and tables of streamflow statistics and probabilities of occurrence are presented in the same order and format for each gaging station, including the same relative placement of the pages. Because the statistical information listed in the tables was created by "data retrievals" or from statistical program results, significant figures were not always rounded to U.S. Geological Survey standards. The order of presentation is as follows:

1. manuscript (station description),

2. graph of the annual mean discharge for the period of record,

3. table of statistics of monthly and annual mean discharges,

4. graph of the annual flow duration,

5. table of monthly and annual flow duration,

6. table of probability of occurrence of annual high discharges,

7. table of probability of occurrence of annual low discharges,

8. table of probability of occurrence of seasonal low discharges,

9. table of annual peak discharge and corresponding gage height for the period of record, and

10. table of monthly and annual mean discharges for the period of record.

\section{Manuscripts}

The location, drainage area, period of record, and other available information about each streamflow-gaging station included in this report are presented in manuscript form. This information is compiled from records maintained by the Democratic Republic of Afghanistan Ministry of Energy and Water and reports by the U.S. Geological Survey. The following comments clarify information presented under the various headings of the station manuscript.

LOCATION: Information on gaging station location is primarily limited to latitude and longitude. When available, further information is furnished with respect to cultural and physical features in the vicinity of the gaging station and the community or landmark included in the gaging station name.

DRAINAGE AREA: Drainage area is measured using Survey of India maps (where noted). The accuracy of the drainage areas is unknown.

ALTITUDE: The datum of the gage referenced to mean sea level.

PERIOD OFRECORD: The period of record is the period for which there are published records for the gaging station or for an equivalent gaging station. An equivalent gaging station is a gaging station that 
was in operation prior to the subject gaging station, and whose location is such that records from it can reasonably be considered equivalent with records from the subject gaging station. This situation arises when a gaging station is relocated upstream or downstream and given a new gaging station number and name, but the changes in drainage area and other basin characteristics are not significantly different.

GAGE: The type of gage or recorder that is or was used to collect data. A condensed history of the types, locations, and datums of previous gages are given under this heading when available.

\section{Statistics of Monthly and Annual Mean Discharges}

Statistics of monthly and annual mean discharges presented for each gaging station include (1) the maximum, minimum, and mean monthly discharges and (2) the maximum, minimum, and mean annual discharges. The water years (October 1 through September 30) in which the maximum and minimum discharges occurred are listed with the respective values, and the standard deviation and coefficient of variation of the monthly and annual mean discharges are listed with the respective values. Also, the percentage of the annual discharge that is comprised by each monthly mean discharge is listed in the table.

Each of the statistics is explained in the following paragraphs. As an aid to the readers' understanding of how the monthly mean and annual mean discharges are determined, data for the gaging station Arghandab River above Arghandab Reservoir (4-1.L00-5A) are used as an example. Each monthly mean is the mean of the daily values for the month. Months or years for which all daily values are not available are not included in the compilation of statistics.

The maximum monthly mean discharge is the maximum value of all the monthly mean values for the period of record. The maximum mean value for October is 18.7 cubic meters per second $\left(\mathrm{m}^{3} / \mathrm{s}\right)$, which occurred during water year 1966. Similarly, the minimum monthly mean discharge is the minimum value of all the monthly mean values. The minimum mean value for October is $1.08 \mathrm{~m}^{3} / \mathrm{s}$, which occurred during water year 1972. The maximum and minimum monthly mean values can be found in the statistics of monthly and annual mean discharges table or by searching the monthly and annual mean discharges table.

The mean monthly discharge is the mean of all the monthly mean discharges for a given month for the period of record, and the standard deviation is a measure of the variability of the values. The mean monthly discharge for October is $11.1 \mathrm{~m}^{3} / \mathrm{s}$, and the standard deviation is $4.1 \mathrm{~m}^{3} / \mathrm{s}$. The mean monthly discharge for October is the same as the mean of all October daily values for the period of record used. However, the standard deviation is smaller than the standard deviation obtained using all daily values. The standard deviation is smaller because the monthly values have less variability than the daily values.

The coefficient of variation is the ratio of the standard deviation to the mean. The coefficient of variation is dimensionless. Because monthly mean discharges are much greater in spring than in winter, the standard deviations also are much greater in spring than in winter. However, dividing the standard deviation by the mean monthly discharge tends to equalize the measures for all months so a more meaningful comparison among months can be made.

The percentage of the annual discharge is the percent of the annual discharge that occurred during each month. It is calculated by dividing the mean discharge for the month by the total of the 12 monthly mean discharges and multiplying by 100 . Because of rounding of the monthly percentage, the sum of the 12 percentages may not equal 100 percent

The maximum, minimum, and mean annual discharges are selected or computed from the annual mean discharges for the period of record. The water years of occurrence of the maximum and minimum values are listed with the respective values, and the standard deviation of the mean of the annual mean values is listed with the mean value. The minimum annual mean discharge of $8.61 \mathrm{~m}^{3} / \mathrm{s}$ occurred in 1971 , and the maximum annual mean discharge of $84.8 \mathrm{~m}^{3} / \mathrm{s}$ occurred in 1957 . The mean annual discharge for the period of record is $41.6 \mathrm{~m}^{3} / \mathrm{s}$. 


\section{Monthly and Annual Flow Duration}

The monthly and annual flow duration table is a magnitude and frequency analysis of daily discharge values. It is computed by tabulating the number of daily discharge values that fall within preselected class limits, computing the percentage of values within each class, and interpolating discharge values for the percentages shown in the table. Monthly values are calculated from daily values in all complete months in the record, and annual figures are calculated for all complete water years. For example, if the 90-percent flow duration value for October is $6.12 \mathrm{~m}^{3} / \mathrm{s}$, then 90 percent of all October daily discharge values for the period of record were equal to or greater than $6.12 \mathrm{~m}^{3} / \mathrm{s}$.

\section{Probability of Occurrence of High or Low Discharges}

The probabilities of occurrence of annual high discharges, annual low discharges, and seasonal low discharges are presented in three tables for each gaging station. Probability of occurrence is an estimate of the likelihood that a particular discharge in a stream will be equaled or exceeded in 1 year or, in the case of low flows, the likelihood that the discharge will not be equaled or exceeded during the year. The probability of occurrence of a high flow is called the exceedance probability, and the probability of occurrence of low flow is called the nonexceedance probability. For example, if the instantaneous discharge for the 0.20 exceedance probability is listed as $520 \mathrm{~m}^{3} / \mathrm{s}$, then a 20 percent chance exists that a discharge equal to or greater than $520 \mathrm{~m}^{3} / \mathrm{s}$ will occur once during the year.

Recurrence interval is another way of expressing annual probability and it is the reciprocal of probability of occurrence. The recurrence interval for an exceedance probability of 0.20 is 5 years ( 1 divided by 0.20 ). For a long discharge record the annual maximum discharge can be expected to equal or exceed $520 \mathrm{~m}^{3} / \mathrm{s}$ on average once every 5 years.

The table of probability of annual high discharges for each gaging station lists the maximum instantaneous discharge and the maximum mean discharge for 3, 7, 15, and 30 consecutive-day periods for selected exceedance probabilities and recurrence intervals. Values for the maximum instantaneous discharge are computed from the streamflow record according to the guidelines established by the Hydrology Subcommittee of the Interagency Advisory Committee on Water Data (1982). No adjustments are made for length of record and a station skew instead of a regional skew was used in the computations.

Values for the maximum mean discharges for 3, 7, 15, and 30 consecutive-day periods are computed from the annual high mean values of the corresponding periods. The computations are based on the log-Pearson Type III distribution using values obtained for the water year.

The table of probability of annual low discharges for each gaging station lists the minimum mean discharge for $1,3,7,14,30,60,90,120$, and 183 consecutive-day periods for selected nonexceedance probabilities and recurrence intervals. Values for the minimum mean discharges are computed from the annual low discharge values of the corresponding periods using the log-Pearson Type III distribution. Probabilities of annual low discharges are computed using values obtained for the climatic year (April 1 through March 31).

The table of probability of seasonal low discharges for each gaging station lists the minimum mean discharge for 1, 7, 14, and 30 consecutive-day periods for selected probabilities and recurrence intervals. These values are computed from the seasonal low mean values of the corresponding periods using the logPearson Type III distribution.

If the log-Pearson Type III distribution curve for the high, low or seasonal discharges failed to fit the data at the upper or lower ends, graphical interpretations were not made. Cautionary notes are provided for the user in these instances.

The annual low discharge and the seasonal low discharges that occur in any given year are sensitive to natural-channel processes, such as evapotranspiration, and human-induced hydrologic modifications, 
such as the operation of many small water-storage reservoirs; the effects of surface-water withdrawal for agricultural, municipal, and industrial use; and the effects of return flow to the river. Thus, the statistics in tables are given for recurrence intervals that generally are within twice the period of record.

\section{DATA CONSIDERATIONS}

The reliability of statistical data is related to the length of record for a stream. The Hydrology Subcommittee of the Interagency Advisory Committee on Water Data (1982) recommends that at least 10 years of record be used for computing flood frequency estimates. Therefore, the length of record criterion for inclusion of a gaging station in this report is at least 10 years. However, the record length for each gaging stations in Afghanistan can vary substantially. The 10-year requirement was relaxed so all statistics could be computed for all gaging stations. Subsequently, extreme high or low flows may be included in the streamflow record of one gaging station and not in another, resulting in inconsistencies in the streamflow statistics when comparing gaging-station data. Differences in statistical data for pre- and post-regulation periods were not addressed in this report.

\section{Selected References}

Brigham, R.H., 1964, Compilation of hydrologic data, Helmand River Valley, Afghanistan through September 1960: U.S. Geological Survey, 234 p.

Childers, Dallas, 1974, Compilation of streamflow records, Helmand River Valley and adjacent areas, Afghanistan 1961—68: U.S. Geological Survey Open File Report 74—46, 144 p.

Democratic Republic of Afghanistan, Ministry of Water and Power, date unknown, Hydrological yearbook 1961 - 1975, Part III, West flowing rivers (Khash, Farah, Adraskan and Harirud): Water and Soil Survey Department, Afghanistan Hydrologic Data Report RO 219, 77 p.

Democratic Republic of Afghanistan, Ministry of Water and Power, date unknown, Hydrological yearbook 1962 - 1975, Part II -3, Ghazni River basin: Water and Soil Survey Department, Afghanistan Hydrologic Data Report RO 217, 109 p.

Democratic Republic of Afghanistan, Ministry of Water and Power, date unknown, Hydrological yearbook 1961 — 1975, Part II — 4B, Arghandab River basin: Water and Soil Survey Department, Afghanistan Hydrologic Data Report RO 215, 121 p.

Democratic Republic of Afghanistan, Ministry of Water and Power, 1978, Hydrological yearbook 19611975, Part II-4A, Helmand River basin (excluding Arghandab River basin); Water and Soil Survey Department, Afghanistan Hydrologic Data Report RO 210, 244 p.

Democratic Republic of Afghanistan, Ministry of Water and Power, 1982, Hydrological yearbook 19761978, Part II, Rivers of Helmand Basin (Ghazni and Helmand); Water and Soil Survey Department, Afghanistan Hydrologic Data Report RO 212, 146 p.

Democratic Republic of Afghanistan, Ministry of Irrigation and Water Resources, 1985, Hydrological yearbook 1979 - 1980, Part (I and II) Rivers of Indus and Helmand Basin (Kabul, Khuram, Helmand and Ghazni): Institute of Water Resources Development, Afghanistan Hydrologic Data Report RO 211, 131 p.

Favre, R., and Kamal, G.M., 2004, Watershed atlas of Afghanistan: Kabul, Afghanistan, Afghanistan Information Management Service, 183 p. 
Hydrology Subcommittee of the Interagency Advisory Committee on Water Data, 1982, Guidelines for determining flood flow frequency: Hydrology Subcommittee Bulletin 17B, 28 p., 14 appendixes.

Republic of Afghanistan, Ministry of Water and Power, 1977, Hydrological yearbook 1961—1975, Part III-5 to 7, Khash, Farah and Adraskan River basins: Water and Soil Survey Department, Afghanistan Hydrologic Data Report RO 223, 114 p. 


\section{STREAMFLOW STATISTICS}




\section{3- 0.000-2M GHAZNI RIVER NEAR SHINA}

\section{(U.S. Geological Survey identification number: 324200068060000)}

LOCATION: Lat $32^{\circ} 42^{\prime} \mathrm{N}$., long $68^{\circ} 06^{\prime} \mathrm{E}$., about $20 \mathrm{~km}$ upstream from Lake Ab-i-Istada, and about $35 \mathrm{~km}$ southeast of Moqur.

DRAINAGE AREA: $11,680 \mathrm{~km}^{2}$.

ALTITUDE: 1,989 meters plus mean sea level.

PERIOD OF RECORD: July 18, 1967 to April 30, 1979.

GAGE: Water-stage recorder.

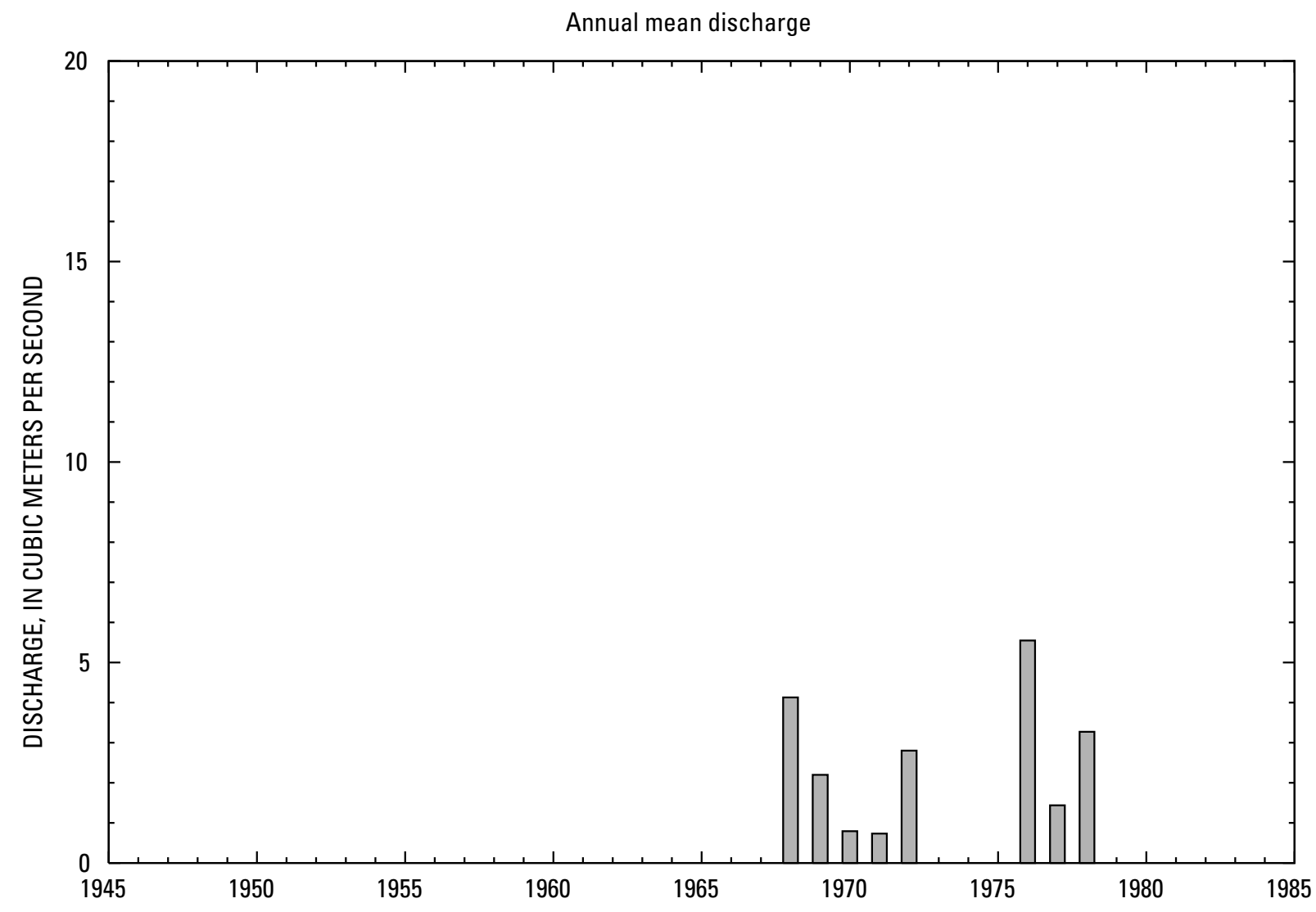




\section{3- 0.000-2M GHAZNI RIVER NEAR SHINA, Continued}

\section{Statistics of monthly and annual mean discharges}

[ $\mathrm{m}^{3} / \mathrm{s}$, meters per second]

\begin{tabular}{|c|c|c|c|c|c|c|c|c|}
\hline \multirow[b]{2}{*}{ Month } & \multicolumn{2}{|c|}{ Maximum } & \multicolumn{2}{|c|}{ Minimum } & \multicolumn{4}{|c|}{ Mean } \\
\hline & $\begin{array}{c}\text { Discharge } \\
\left(\mathrm{m}^{3} / \mathrm{s}\right)\end{array}$ & $\begin{array}{c}\text { Water year } \\
\text { of } \\
\text { occurrence }\end{array}$ & $\begin{array}{c}\text { Discharge } \\
\left(\mathrm{m}^{3} / \mathrm{s}\right)\end{array}$ & $\begin{array}{c}\text { Water year } \\
\text { of } \\
\text { occurrence }\end{array}$ & $\begin{array}{c}\text { Discharge } \\
\left(\mathrm{m}^{3} / \mathrm{s}\right)\end{array}$ & $\begin{array}{c}\text { Standard } \\
\text { deviation } \\
\left(\mathrm{m}^{3} / \mathrm{s}\right)\end{array}$ & $\begin{array}{c}\text { Coefficient } \\
\text { of } \\
\text { variation }\end{array}$ & $\begin{array}{c}\text { Percentage } \\
\text { of annual } \\
\text { discharge }\end{array}$ \\
\hline October & 0.846 & 1977 & 0.163 & 1970 & 0.42 & 0.22 & 0.52 & 1.15 \\
\hline November & 1.87 & 1977 & 0.471 & 1970 & 1.00 & 0.44 & 0.44 & 2.75 \\
\hline December & 3.53 & 1968 & 0.579 & 1970 & 2.45 & 1.37 & 0.56 & 6.75 \\
\hline January & 10.5 & 1968 & 0.989 & 1970 & 3.65 & 2.81 & 0.77 & 10.0 \\
\hline February & 9.59 & 1968 & 1.12 & 1971 & 4.57 & 3.31 & 0.73 & 12.6 \\
\hline March & 25.4 & 1973 & 2.20 & 1977 & 7.47 & 6.79 & 0.91 & 20.6 \\
\hline April & 30.8 & 1976 & 0.203 & 1971 & 8.82 & 10.5 & 1.19 & 24.3 \\
\hline May & 8.53 & 1972 & 0.096 & 1970 & 2.23 & 3.12 & 1.40 & 6.15 \\
\hline June & 1.93 & 1972 & 0.075 & 1970 & 0.69 & 0.73 & 1.07 & 1.89 \\
\hline July & 21.2 & 1978 & 0.045 & 1970 & 3.30 & 7.26 & 2.20 & 9.07 \\
\hline August & 3.92 & 1978 & 0.086 & 1971 & 1.18 & 1.33 & 1.12 & 3.26 \\
\hline September & 1.97 & 1976 & 0.131 & 1967 & 0.56 & 0.57 & 1.02 & 1.54 \\
\hline Annual & 5.55 & 1976 & 0.735 & 1971 & 2.61 & 1.68 & 0.64 & 100 \\
\hline
\end{tabular}

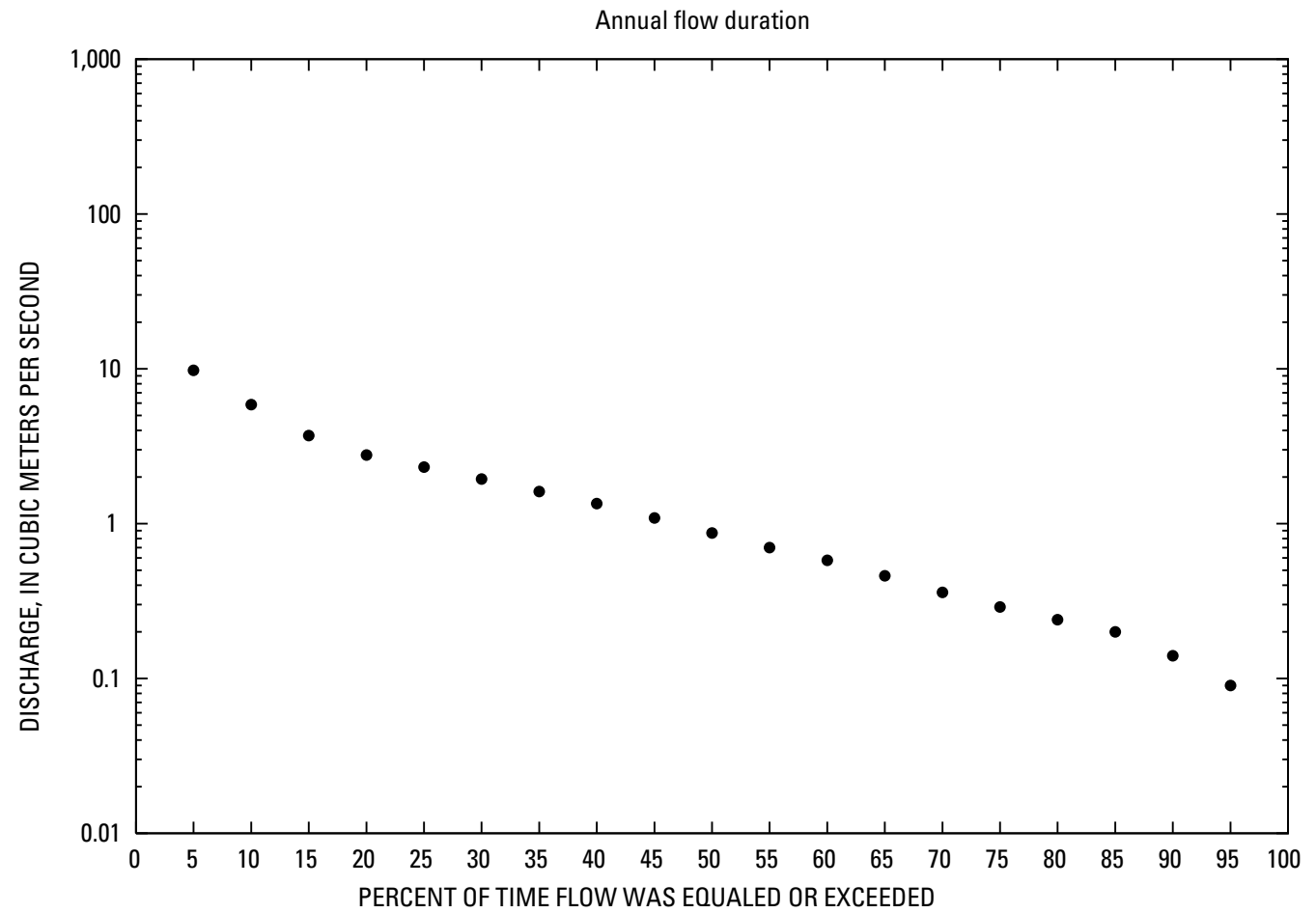




\section{3- 0.000-2M GHAZNI RIVER NEAR SHINA, Continued}

\section{Monthly and annual flow duration, in cubic meters per second}

[Data were not rounded in accordance with U.S. Geological Survey publication standards]

\begin{tabular}{|c|c|c|c|c|c|c|c|c|c|c|c|c|c|}
\hline $\begin{array}{l}\text { Percentage } \\
\text { of days } \\
\text { discharge } \\
\text { equaled or } \\
\text { exceeded }\end{array}$ & January & February & March & April & May & June & July & August & September & October & November & December & Annual \\
\hline 95 & 0.99 & 0.91 & 0.64 & 0.17 & 0.09 & 0.06 & 0.03 & 0.07 & 0.06 & 0.13 & 0.28 & 0.58 & 0.09 \\
\hline 90 & 1.05 & 1.27 & 1.13 & 0.19 & 0.14 & 0.1 & 0.05 & 0.11 & 0.07 & 0.17 & 0.31 & 0.78 & 0.14 \\
\hline 85 & 1.16 & 1.37 & 1.27 & 0.25 & 0.16 & 0.11 & 0.05 & 0.12 & 0.08 & 0.22 & 0.39 & 0.99 & 0.2 \\
\hline 80 & 1.43 & 1.48 & 1.81 & 0.29 & 0.17 & 0.11 & 0.08 & 0.18 & 0.11 & 0.23 & 0.46 & 1.12 & 0.24 \\
\hline 75 & 2.09 & 1.75 & 2.28 & 0.37 & 0.19 & 0.16 & 0.11 & 0.21 & 0.12 & 0.25 & 0.52 & 1.4 & 0.29 \\
\hline 70 & 2.4 & 1.84 & 2.64 & 0.55 & 0.2 & 0.17 & 0.18 & 0.29 & 0.15 & 0.26 & 0.62 & 1.66 & 0.36 \\
\hline 65 & 2.49 & 1.95 & 2.93 & 1.03 & 0.23 & 0.17 & 0.22 & 0.38 & 0.2 & 0.27 & 0.66 & 1.82 & 0.46 \\
\hline 60 & 2.58 & 2.44 & 3.23 & 1.17 & 0.27 & 0.18 & 0.29 & 0.42 & 0.24 & 0.29 & 0.7 & 1.96 & 0.58 \\
\hline 55 & 2.66 & 2.61 & 3.86 & 1.41 & 0.3 & 0.21 & 0.38 & 0.46 & 0.32 & 0.3 & 0.73 & 2.06 & 0.7 \\
\hline 50 & 2.75 & 2.81 & 4.17 & 1.95 & 0.39 & 0.29 & 0.44 & 0.53 & 0.39 & 0.32 & 0.78 & 2.15 & 0.87 \\
\hline 45 & 3.03 & 3.01 & 4.52 & 3.37 & 0.56 & 0.39 & 0.52 & 0.67 & 0.45 & 0.35 & 0.92 & 2.28 & 1.09 \\
\hline 40 & 3.29 & 3.17 & 5.92 & 4.29 & 0.93 & 0.48 & 0.62 & 0.75 & 0.48 & 0.45 & 1.01 & 2.41 & 1.35 \\
\hline 35 & 3.47 & 3.48 & 6.8 & 5.28 & 1.38 & 0.76 & 1.01 & 0.83 & 0.52 & 0.49 & 1.12 & 2.5 & 1.61 \\
\hline 30 & 3.65 & 3.93 & 7.49 & 6.66 & 1.65 & 0.9 & 1.23 & 0.9 & 0.55 & 0.56 & 1.2 & 2.6 & 1.94 \\
\hline 25 & 3.88 & 5.31 & 8.1 & 9.31 & 1.84 & 1.16 & 1.35 & 0.97 & 0.59 & 0.59 & 1.37 & 2.74 & 2.32 \\
\hline 20 & 4.19 & 6.95 & 9.44 & 12.2 & 2.07 & 1.5 & 1.47 & 1.12 & 0.63 & 0.64 & 1.52 & 3.11 & 2.77 \\
\hline 15 & 4.95 & 8.92 & 12.2 & 16.3 & 2.33 & 1.74 & 1.6 & 1.32 & 0.7 & 0.71 & 1.64 & 3.34 & 3.71 \\
\hline 10 & 7.07 & 12.1 & 19.2 & 24.1 & 3.12 & 2 & 2 & 1.76 & 0.81 & 0.75 & 1.88 & 3.75 & 5.87 \\
\hline 5 & 7.98 & 14.3 & 29.2 & 29.5 & 12 & 2.13 & 6.75 & 3.86 & 0.94 & 0.8 & 2.18 & 4.48 & 9.77 \\
\hline
\end{tabular}




\section{3- 0.000-2M GHAZNI RIVER NEAR SHINA, Continued}

Probability of occurrence of annual high discharges

[m³/s, meters per second; $n g$, statistic not given]

\begin{tabular}{rrrrrrr}
\hline & & & \multicolumn{5}{c}{ Maximum mean discharge } \\
$\begin{array}{c}\text { Exceedance } \\
\text { probability }\end{array}$ & $\begin{array}{c}\text { Recurrence } \\
\text { interval } \\
\text { (years) }\end{array}$ & $\begin{array}{c}\text { Maximum } \\
\text { instantaneous } \\
\left(\mathbf{m}^{\mathbf{3}} / \mathbf{s}\right)\end{array}$ & 3-day period & 7-day period & 15-day period & 30-day period \\
\cline { 5 - 7 } & 1.01 & 9.00 & 8.18 & 3.69 & 1.94 & 1.50 \\
0.99 & 1.05 & 13.6 & 9.88 & 5.28 & 3.12 & 2.42 \\
0.95 & 1.11 & 17.7 & 11.4 & 6.63 & 4.12 & 3.16 \\
0.80 & 1.25 & 25.4 & 14.3 & 9.07 & 5.88 & 4.42 \\
0.50 & 2 & 57.8 & 26.5 & 18.7 & 12.5 & 8.81 \\
0.20 & 5 & 159 & 64.3 & 46.2 & 29.3 & 18.6 \\
0.10 & 10 & 291 & 115 & 79.8 & 47.5 & 28.2 \\
0.04 & 25 & 592 & 237 & 152 & 82.2 & 44.6 \\
0.02 & 50 & 970 & 399 & 237 & 119 & 60.7 \\
0.01 & 100 & 1,550 & 661 & 363 & 168 & 80.6 \\
0.005 & 200 & 2,430 & 1,080 & 546 & 233 & 105 \\
0.002 & 500 & 4,290 & ng & ng & ng & ng \\
\hline
\end{tabular}

\section{3- 0.000-2M GHAZNI RIVER NEAR SHINA, Continued}

Probability of occurrence of annual low discharges

$\left[\mathrm{m}^{3} / \mathrm{s}\right.$, meters per second]

\begin{tabular}{|c|c|c|c|c|c|c|c|c|c|c|}
\hline \multirow{3}{*}{$\begin{array}{c}\text { Nonexceedance } \\
\text { probability }\end{array}$} & \multirow{3}{*}{$\begin{array}{c}\text { Recurrence } \\
\text { interval } \\
\text { (years) }\end{array}$} & \multicolumn{9}{|c|}{ Minimum mean discharge $\left(\mathrm{m}^{3} / \mathbf{s}\right)$} \\
\hline & & \multicolumn{9}{|c|}{ Number of consecutive days } \\
\hline & & 1 & 3 & 7 & 14 & 30 & 60 & 90 & 120 & 183 \\
\hline 0.05 & 20 & 0.004 & 0.006 & 0.013 & 0.019 & 0.035 & 0.050 & 0.055 & 0.058 & 0.118 \\
\hline 0.10 & 10 & 0.007 & 0.011 & 0.019 & 0.027 & 0.049 & 0.071 & 0.079 & 0.087 & 0.164 \\
\hline 0.20 & 5 & 0.014 & 0.021 & 0.032 & 0.043 & 0.072 & 0.107 & 0.121 & 0.140 & 0.243 \\
\hline 0.50 & 2 & 0.058 & 0.073 & 0.090 & 0.108 & 0.157 & 0.226 & 0.271 & 0.343 & 0.526 \\
\hline
\end{tabular}




\section{3- 0.000-2M GHAZNI RIVER NEAR SHINA, Continued}

\section{Probability of occurrence of seasonal low discharges}

$\left[\mathrm{m}^{3} / \mathrm{s}\right.$, meters per second]

\begin{tabular}{|c|c|c|c|c|c|c|c|c|c|}
\hline \multirow{3}{*}{$\begin{array}{l}\text { Nonexceedance } \\
\text { probability }\end{array}$} & \multirow{3}{*}{$\begin{array}{c}\text { Recurrence } \\
\text { interval } \\
\text { (years) }\end{array}$} & \multicolumn{8}{|c|}{ Minimum mean discharge $\left(\mathrm{m}^{3} / \mathrm{s}\right)$} \\
\hline & & \multicolumn{8}{|c|}{ Number of consecutive days } \\
\hline & & 1 & 7 & 14 & 30 & 1 & 7 & 14 & 30 \\
\hline & & \multicolumn{4}{|c|}{ December-January-February } & \multicolumn{4}{|c|}{ March-April-May } \\
\hline 0.05 & 20 & 0.284 & 0.548 & 0.562 & 0.658 & 0.048 & 0.054 & 0.056 & 0.066 \\
\hline 0.10 & 10 & 0.434 & 0.704 & 0.758 & 0.876 & 0.074 & 0.082 & 0.087 & 0.105 \\
\hline 0.20 & 5 & 0.674 & 0.933 & 1.05 & 1.21 & 0.125 & 0.137 & 0.149 & 0.188 \\
\hline \multirow[t]{2}{*}{0.50} & 2 & 1.27 & 1.50 & 1.74 & 2.11 & 0.339 & 0.373 & 0.425 & 0.609 \\
\hline & & \multicolumn{4}{|c|}{ June-July-August } & \multicolumn{4}{|c|}{ September-October-November } \\
\hline 0.05 & 20 & 0.005 & 0.013 & 0.020 & 0.033 & 0.010 & 0.028 & 0.035 & 0.092 \\
\hline 0.10 & 10 & 0.012 & 0.022 & 0.033 & 0.053 & 0.020 & 0.042 & 0.050 & 0.112 \\
\hline 0.20 & 5 & 0.027 & 0.042 & 0.058 & 0.093 & 0.043 & 0.068 & 0.078 & 0.143 \\
\hline 0.50 & 2 & 0.117 & 0.136 & 0.170 & 0.253 & 0.154 & 0.167 & 0.182 & 0.241 \\
\hline
\end{tabular}

3- 0.000-2M GHAZNI RIVER NEAR SHINA, Continued

Annual peak discharge and corresponding gage height

[m³/s, meters per second; --, no data]

\begin{tabular}{ccccccc}
\hline $\begin{array}{c}\text { Water } \\
\text { year }\end{array}$ Date & $\begin{array}{c}\text { Gage } \\
\text { height } \\
(\text { meters })\end{array}$ & $\begin{array}{c}\text { Peak } \\
\text { discharge } \\
\left(\mathrm{m}^{3} / \mathrm{s}\right)\end{array}$ & $\begin{array}{c}\text { Water } \\
\text { year }\end{array}$ & Date & $\begin{array}{c}\text { Gage } \\
\text { height } \\
(\mathrm{meters})\end{array}$ & $\begin{array}{c}\text { Peak } \\
\text { discharge } \\
\left(\mathrm{m}^{3} / \mathrm{s}\right)\end{array}$ \\
\hline
\end{tabular}

Annual peak discharge, by year

$\begin{array}{rrrrrrrr}1968 & \text { 30-Apr } & -- & 38.0 & 1973 & \text { 16-Mar } & -- & 35.0 \\ 1969 & \text { 10-Jan } & -- & 63.4 & 1976 & \text { 3-Sep } & -- & 278 \\ 1970 & \text { 9-Mar } & -- & 38.0 & 1977 & \text { 11-Jul } & -- & 25.0 \\ 1971 & \text { 6-Mar } & -- & 17.1 & 1978 & \text { 7-Jul } & -- & 389 \\ 1972 & \text { 18-Mar } & -- & 40.0 & 1979 & \text { 30-Mar } & -- & 263\end{array}$

Annual peak discharge, from highest to lowest

\begin{tabular}{rrrrrrrr}
1978 & 7-Jul & -- & 389 & 1970 & 9-Mar & -- & 38.0 \\
1976 & 3-Sep & -- & 278 & 1968 & 30-Apr & -- & 38.0 \\
1979 & 30-Mar & -- & 263 & 1973 & 16-Mar & -- & 35.0 \\
1969 & 10-Jan & -- & 63.4 & 1977 & 11-Jul & -- & 25.0 \\
1972 & 18-Mar & -- & 40.0 & 1971 & 6-Mar & -- & 17.1 \\
\hline
\end{tabular}




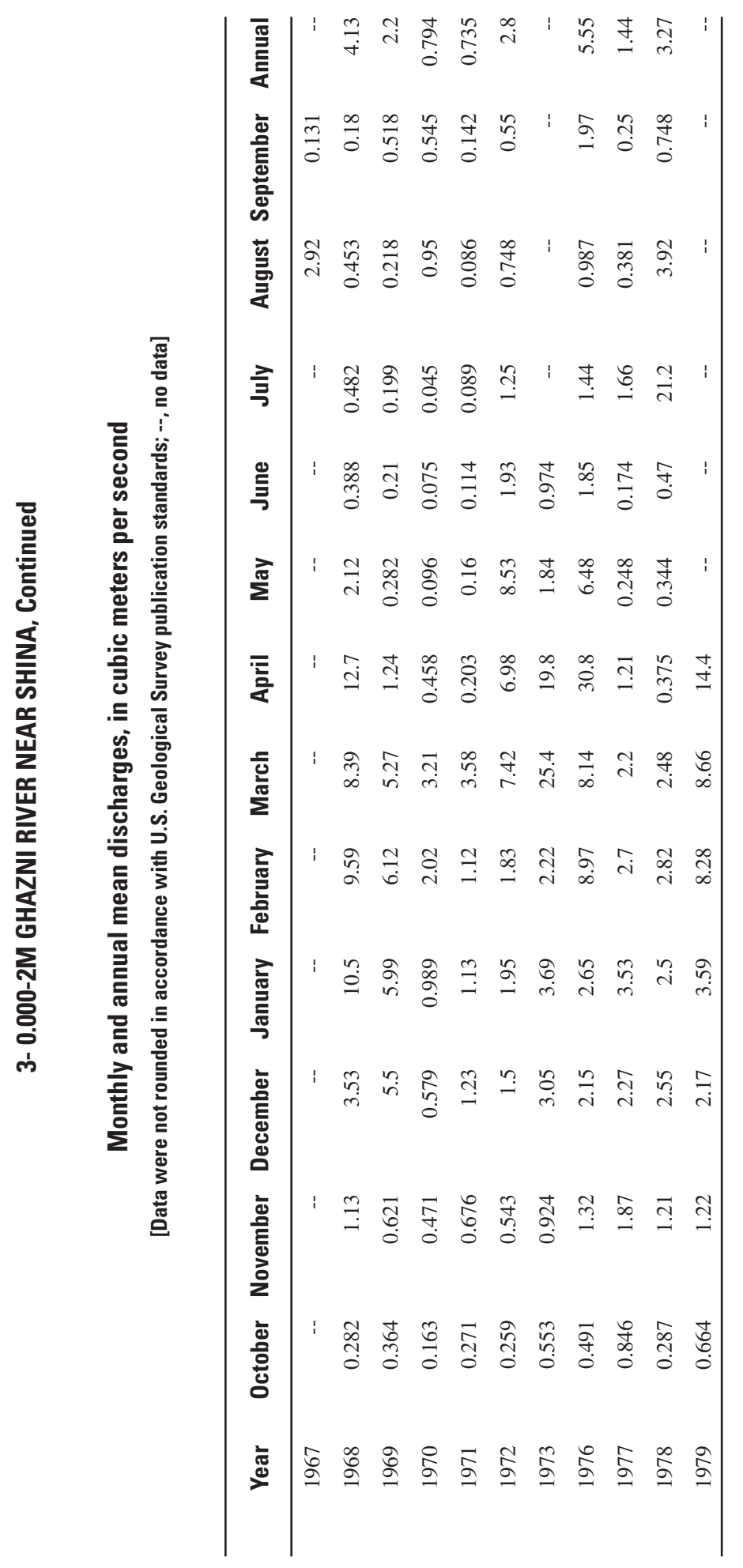




\section{3- 0.000-7M GHAZNI RIVER AT GHAZNI BRIDGE}

\section{(U.S. Geological Survey identification number: 333300068250000 )}

LOCATION: Lat $33^{\circ} 33^{\prime} N$., long 68 $25^{\prime}$ E., on downstream side of pier, near center of span of bridge on KabulKandahar Highway, at Ghazni.

DRAINAGE AREA: $1,555 \mathrm{~km}^{2}$.

ALTITUDE: 2,173 meters plus mean sea level.

PERIOD OF RECORD: April 11, 1968 to September 30, 1980.

GAGE: Water-stage recorder.

Annual mean discharge

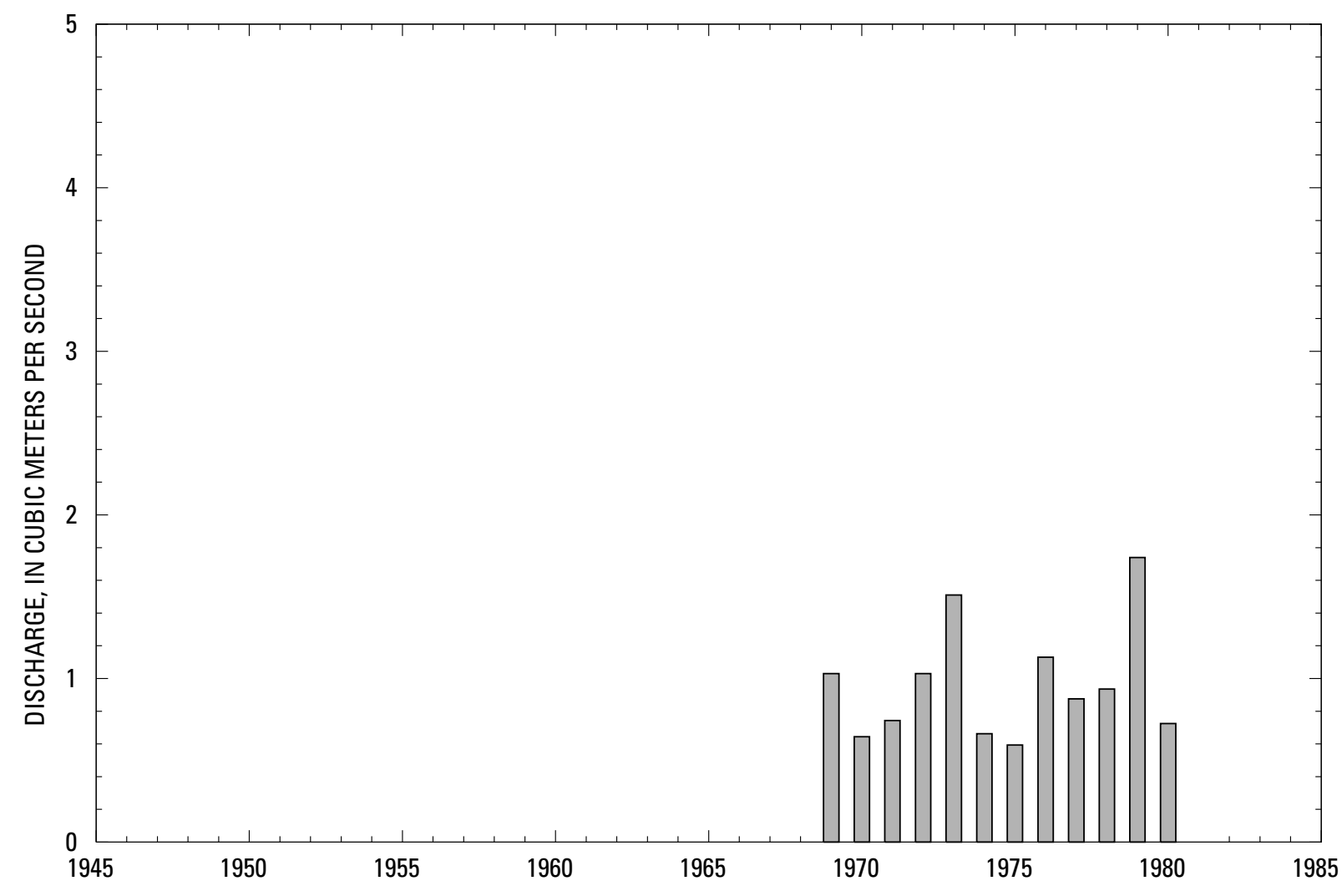




\section{3- 0.000-7M GHAZNI RIVER AT GHAZNI BRIDGE, Continued}

\section{Statistics of monthly and annual mean discharges}

[ $\mathrm{m}^{3} / \mathrm{s}$, meters per second; $\mathrm{m}$, more than 2 years of occurrence]

\begin{tabular}{|c|c|c|c|c|c|c|c|c|}
\hline \multirow[b]{2}{*}{ Month } & \multicolumn{2}{|c|}{ Maximum } & \multicolumn{2}{|c|}{ Minimum } & \multicolumn{4}{|c|}{ Mean } \\
\hline & $\begin{array}{c}\text { Discharge } \\
\left(\mathrm{m}^{3} / \mathrm{s}\right)\end{array}$ & $\begin{array}{c}\text { Water year } \\
\text { of } \\
\text { occurrence }\end{array}$ & $\begin{array}{c}\text { Discharge } \\
\left(\mathrm{m}^{3} / \mathrm{s}\right)\end{array}$ & $\begin{array}{c}\text { Water year } \\
\text { of } \\
\text { occurrence }\end{array}$ & $\begin{array}{c}\text { Discharge } \\
\left(\mathrm{m}^{3} / \mathrm{s}\right)\end{array}$ & $\begin{array}{c}\text { Standard } \\
\text { deviation } \\
\left(\mathrm{m}^{3} / \mathrm{s}\right)\end{array}$ & $\begin{array}{c}\text { Coefficient } \\
\text { of } \\
\text { variation }\end{array}$ & $\begin{array}{c}\text { Percentage } \\
\text { of annual } \\
\text { discharge }\end{array}$ \\
\hline October & 0.989 & 1979 & 0 & 1976 & 0.20 & 0.27 & 1.39 & 1.67 \\
\hline November & 1.89 & 1969 & 0.333 & 1975 & 0.99 & 0.46 & 0.47 & 8.34 \\
\hline December & 2.90 & 1969 & 0.707 & 1980 & 1.54 & 0.64 & 0.41 & 13.1 \\
\hline January & 3.57 & 1969 & 0.956 & 1972 & 2.27 & 0.85 & 0.37 & 19.2 \\
\hline February & 2.42 & 1977 & 0.739 & 1980 & 1.54 & 0.52 & 0.34 & 13.0 \\
\hline March & 3.56 & 1973 & 0.163 & 1970 & 1.04 & 1.07 & 1.02 & 8.83 \\
\hline April & 8.23 & 1979 & 0 & 1971 & 2.35 & 2.72 & 1.16 & 19.9 \\
\hline May & 2.42 & 1968 & 0 & 1975,1980 & 0.67 & 0.76 & 1.12 & 5.70 \\
\hline June & 1.50 & 1979 & 0 & 1971,1980 & 0.61 & 0.47 & 0.78 & 5.12 \\
\hline July & 1.87 & 1978 & 0 & $\mathrm{~m}$ & 0.25 & 0.58 & 2.33 & 2.11 \\
\hline August & 0.809 & 1978 & 0 & $\mathrm{~m}$ & 0.09 & 0.23 & 2.63 & 0.73 \\
\hline September & 1.20 & 1978 & 0 & $\mathrm{~m}$ & 0.27 & 0.34 & 1.24 & 2.31 \\
\hline Annual & 1.74 & 1979 & 0.593 & 1975 & 0.97 & 0.35 & 0.37 & 100 \\
\hline
\end{tabular}

Annual flow duration

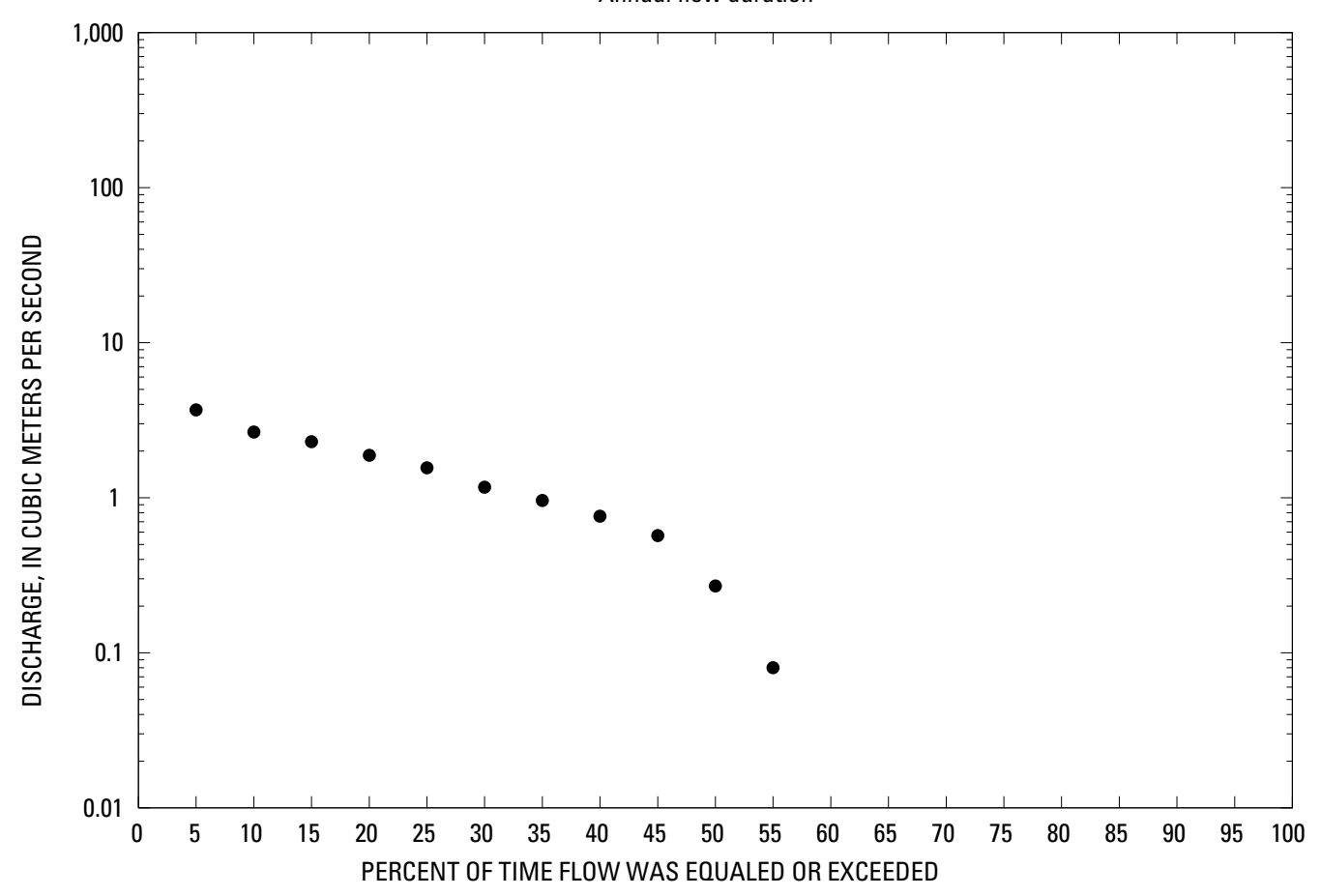




\section{3- 0.000-7M GHAZNI RIVER AT GHAZNI BRIDGE, Continued}

\section{Monthly and annual flow duration, in cubic meters per second}

[Data were not rounded in accordance with U.S. Geological Survey publication standards]

\begin{tabular}{|c|c|c|c|c|c|c|c|c|c|c|c|c|c|}
\hline $\begin{array}{l}\text { Percentage } \\
\text { of days } \\
\text { discharge } \\
\text { equaled or } \\
\text { exceeded }\end{array}$ & January & February & March & April & May & June & July & August & September & October & November & December & Annual \\
\hline 95 & 0.92 & 0.26 & 0.01 & 0 & 0 & 0 & 0 & 0 & 0 & 0 & 0.03 & 0.59 & 0 \\
\hline 90 & 0.97 & 0.66 & 0.1 & 0 & 0 & 0 & 0 & 0 & 0 & 0 & 0.2 & 0.73 & 0 \\
\hline 85 & 1.1 & 0.71 & 0.16 & 0 & 0 & 0 & 0 & 0 & 0 & 0 & 0.27 & 0.85 & 0 \\
\hline 80 & 1.56 & 0.78 & 0.19 & 0 & 0 & 0 & 0 & 0 & 0 & 0 & 0.4 & 0.93 & 0 \\
\hline 75 & 1.62 & 0.9 & 0.22 & 0.01 & 0 & 0 & 0 & 0 & 0 & 0 & 0.46 & 0.99 & 0 \\
\hline 70 & 1.77 & 0.97 & 0.26 & 0.01 & 0 & 0 & 0 & 0 & 0.01 & 0 & 0.54 & 1.03 & 0 \\
\hline 65 & 1.94 & 1.06 & 0.31 & 0.02 & 0 & 0 & 0 & 0 & 0.01 & 0 & 0.65 & 1.21 & 0 \\
\hline 60 & 2.11 & 1.3 & 0.47 & 0.02 & 0 & 0 & 0 & 0 & 0.02 & 0 & 0.72 & 1.27 & 0 \\
\hline 55 & 2.17 & 1.4 & 0.53 & 0.09 & 0 & 0 & 0 & 0 & 0.02 & 0 & 0.77 & 1.42 & 0.08 \\
\hline 50 & 2.32 & 1.49 & 0.58 & 0.28 & 0 & 0 & 0 & 0 & 0.02 & 0 & 0.85 & 1.46 & 0.27 \\
\hline 45 & 2.36 & 1.59 & 0.64 & 0.79 & 0 & 0 & 0 & 0 & 0.02 & 0 & 0.94 & 1.5 & 0.57 \\
\hline 40 & 2.4 & 1.71 & 0.76 & 1.52 & 0 & 0 & 0 & 0 & 0.02 & 0.02 & 1.01 & 1.68 & 0.76 \\
\hline 35 & 2.45 & 1.82 & 0.88 & 2.44 & 0 & 0 & 0 & 0 & 0.03 & 0.07 & 1.09 & 1.76 & 0.96 \\
\hline 30 & 2.68 & 2.11 & 1 & 3.35 & 0 & 0.29 & 0 & 0 & 0.03 & 0.13 & 1.27 & 1.89 & 1.17 \\
\hline 25 & 2.83 & 2.37 & 1.12 & 3.92 & 0.52 & 1.01 & 0 & 0 & 0.03 & 0.21 & 1.41 & 2 & 1.56 \\
\hline 20 & 3.15 & 2.43 & 1.23 & 4.91 & 0.9 & 1.44 & 0 & 0 & 0.36 & 0.28 & 1.53 & 2.1 & 1.88 \\
\hline 15 & 3.48 & 2.49 & 1.87 & 6.58 & 1.92 & 1.86 & 0 & 0 & 0.73 & 0.38 & 1.71 & 2.19 & 2.3 \\
\hline 10 & 3.57 & 2.55 & 2.63 & 7.93 & 2.99 & 2.26 & 0.03 & 0 & 1.16 & 0.57 & 1.98 & 2.4 & 2.65 \\
\hline 5 & 0 & 2.7 & 4.86 & 8.78 & 4.59 & 2.74 & 0.51 & 0.02 & 1.71 & 0.81 & 2.33 & 2.85 & 3.69 \\
\hline
\end{tabular}




\section{3- 0.000-7M GHAZNI RIVER AT GHAZNI BRIDGE, Continued}

Probability of occurrence of annual high discharges

[ $\mathrm{m}^{3} / \mathrm{s}$, meters per second; $\mathrm{ng}$, statistic not given]

\begin{tabular}{rrrrrrr}
\hline & & & \multicolumn{5}{c}{ Maximum mean discharge } \\
$\begin{array}{c}\text { Exceedance } \\
\text { probability }\end{array}$ & $\begin{array}{c}\text { Recurrence } \\
\text { interval } \\
\text { (years) }\end{array}$ & $\begin{array}{c}\text { Maximum } \\
\text { instantaneous } \\
\left(\mathbf{m}^{3} / \mathbf{s}\right)\end{array}$ & 3-day period & 7-day period & 15-day period & 30-day period \\
\cline { 5 - 7 } 0.99 & 1.01 & 1.60 & 11.83 & 11.83 & 11.74 & 11.27 \\
0.95 & 1.05 & 2.80 & 2.66 & 2.44 & 2.10 & 1.63 \\
0.90 & 1.11 & 3.90 & 3.23 & 2.86 & 2.37 & 1.89 \\
0.80 & 1.25 & 6.10 & 4.07 & 3.48 & 2.79 & 2.28 \\
0.50 & 2 & 16.3 & 6.24 & 5.13 & 4.05 & 3.38 \\
0.20 & 5 & 52.6 & 9.39 & 7.66 & 6.40 & 5.25 \\
0.10 & 10 & 105 & 11.5 & 9.51 & 8.41 & 6.74 \\
0.04 & 25 & 234 & 14.3 & 12.0 & 11.6 & 8.92 \\
0.02 & 50 & 407 & 16.4 & 14.0 & 114.5 & 10.8 \\
0.01 & 100 & 685 & 18.5 & 16.1 & 117.9 & 12.9 \\
0.005 & 200 & 1,120 & 20.6 & 18.4 & 121.9 & 15.2 \\
0.002 & 500 & 2,110 & ng & ng & ng & ng \\
\hline
\end{tabular}

'Data does not fit log-Pearson Type III curve, use with caution.

\section{3- 0.000-7M GHAZNI RIVER AT GHAZNI BRIDGE, Continued}

Probability of occurrence of annual low discharges

[m³/s, meters per second; ng, statistic not given]

\begin{tabular}{|c|c|c|c|c|c|c|c|c|c|c|}
\hline \multirow{3}{*}{$\begin{array}{c}\text { Nonexceedance } \\
\text { probability }\end{array}$} & \multirow{3}{*}{$\begin{array}{c}\text { Recurrence } \\
\text { interval } \\
\text { (years) }\end{array}$} & \multicolumn{9}{|c|}{ Minimum mean discharge $\left(\mathrm{m}^{3} / \mathrm{s}\right)$} \\
\hline & & \multicolumn{9}{|c|}{ Number of consecutive days } \\
\hline & & 1 & 3 & 7 & 14 & 30 & 60 & 90 & 120 & 183 \\
\hline 0.05 & 20 & $\mathrm{ng}$ & ng & ng & ng & ng & 0 & 0 & 0 & 0.042 \\
\hline 0.10 & 10 & ng & ng & ng & ng & ng & 0 & 0 & 0.015 & 0.071 \\
\hline 0.20 & 5 & $\mathrm{ng}$ & ng & ng & ng & ng & 0 & 0 & 0.025 & 0.122 \\
\hline 0.50 & 2 & $\mathrm{ng}$ & ng & ng & ng & ng & 0 & 0.021 & 0.054 & 0.267 \\
\hline
\end{tabular}




\section{3- 0.000-7M GHAZNI RIVER AT GHAZNI BRIDGE, Continued}

\section{Probability of occurrence of seasonal low discharges}

[ $\mathrm{m}^{3} / \mathrm{s}$, meters per second; ng, statistic not given]

\begin{tabular}{|c|c|c|c|c|c|c|c|c|c|}
\hline \multirow{3}{*}{$\begin{array}{c}\text { Nonexceedance } \\
\text { probability }\end{array}$} & \multirow{3}{*}{$\begin{array}{c}\text { Recurrence } \\
\text { interval } \\
\text { (years) }\end{array}$} & \multicolumn{8}{|c|}{ Minimum mean discharge $\left(\mathrm{m}^{3} / \mathrm{s}\right)$} \\
\hline & & \multicolumn{8}{|c|}{ Number of consecutive days } \\
\hline & & 1 & 7 & 14 & 30 & 1 & 7 & 14 & 30 \\
\hline & & \multicolumn{4}{|c|}{ December-January-February } & \multicolumn{4}{|c|}{ March-April-May } \\
\hline 0.05 & 20 & 0.096 & 0.143 & 0.459 & 0.681 & ng & ng & $\mathrm{ng}$ & 0 \\
\hline 0.10 & 10 & 0.148 & 0.226 & 0.548 & 0.814 & ng & ng & ng & 0 \\
\hline 0.20 & 5 & 0.236 & 0.366 & 0.672 & 0.988 & ng & ng & $\mathrm{ng}$ & 0 \\
\hline \multirow[t]{2}{*}{0.50} & 2 & 0.512 & 0.755 & 0.959 & 1.34 & ng & ng & $\mathrm{ng}$ & 0 \\
\hline & & \multicolumn{4}{|c|}{ June-July-August } & \multicolumn{4}{|c|}{ September-October-November } \\
\hline 0.05 & 20 & ng & ng & ng & ng & ng & ng & 0 & 0 \\
\hline 0.10 & 10 & ng & ng & ng & ng & ng & $\mathrm{ng}$ & 0 & 0 \\
\hline 0.20 & 5 & ng & ng & ng & ng & ng & ng & 0 & 0 \\
\hline 0.50 & 2 & $\mathrm{ng}$ & ng & $\mathrm{ng}$ & $\mathrm{ng}$ & ng & ng & 0 & 0.031 \\
\hline
\end{tabular}


3- 0.000-7M GHAZNI RIVER AT GHAZNI BRIDGE, Continued

Annual peak discharge and corresponding gage height

[ $\mathrm{m}^{3} / \mathrm{s}$, meters per second; --, no data]

\begin{tabular}{|c|c|c|c|c|c|c|c|}
\hline $\begin{array}{c}\text { Water } \\
\text { year }\end{array}$ & Date & $\begin{array}{c}\text { Gage } \\
\text { height } \\
\text { (meters) }\end{array}$ & $\begin{array}{c}\text { Peak } \\
\text { discharge } \\
\left(\mathrm{m}^{3} / \mathrm{s}\right)\end{array}$ & $\begin{array}{c}\text { Water } \\
\text { year }\end{array}$ & Date & $\begin{array}{c}\text { Gage } \\
\text { height } \\
\text { (meters) }\end{array}$ & $\begin{array}{c}\text { Peak } \\
\text { discharge } \\
\left(\mathrm{m}^{3} / \mathbf{s}\right)\end{array}$ \\
\hline \multicolumn{8}{|c|}{ Annual peak discharge, by year } \\
\hline 1968 & 18-May & - & 25.6 & 1975 & 18-Jan & & 3.48 \\
\hline 1969 & 23-Apr & - & 6.70 & 1976 & 25-Apr & & 9.40 \\
\hline 1970 & 12-Aug & - & 112 & 1977 & 12-Jul & & 76.4 \\
\hline 1971 & 4-May & - & 4.06 & 1978 & 13-Jul & & 237 \\
\hline 1972 & 26-Jun & - & 21.0 & 1979 & 29-Mar & & 19.0 \\
\hline 1973 & 16-Aug & - & 29.8 & 1980 & 8-Apr & & 5.80 \\
\hline 1974 & 27-Apr & - & 9.85 & & & & \\
\hline \multicolumn{8}{|c|}{ Annual peak discharge, from highest to lowest } \\
\hline 1978 & 13-Jul & - & 237 & 1974 & 27-Apr & & 9.85 \\
\hline 1970 & 12-Aug & - & 112 & 1976 & 25-Apr & & 9.40 \\
\hline 1977 & 12-Jul & - & 76.4 & 1969 & 23-Apr & & 6.70 \\
\hline 1973 & 16-Aug & - & 29.8 & 1980 & 8-Apr & & 5.80 \\
\hline 1968 & 18-May & - & 25.6 & 1971 & 4-May & & 4.06 \\
\hline 1972 & 26-Jun & - & 21.0 & 1975 & 18-Jan & & 3.48 \\
\hline 1979 & 29-Mar & - & 19.0 & & & & \\
\hline
\end{tabular}




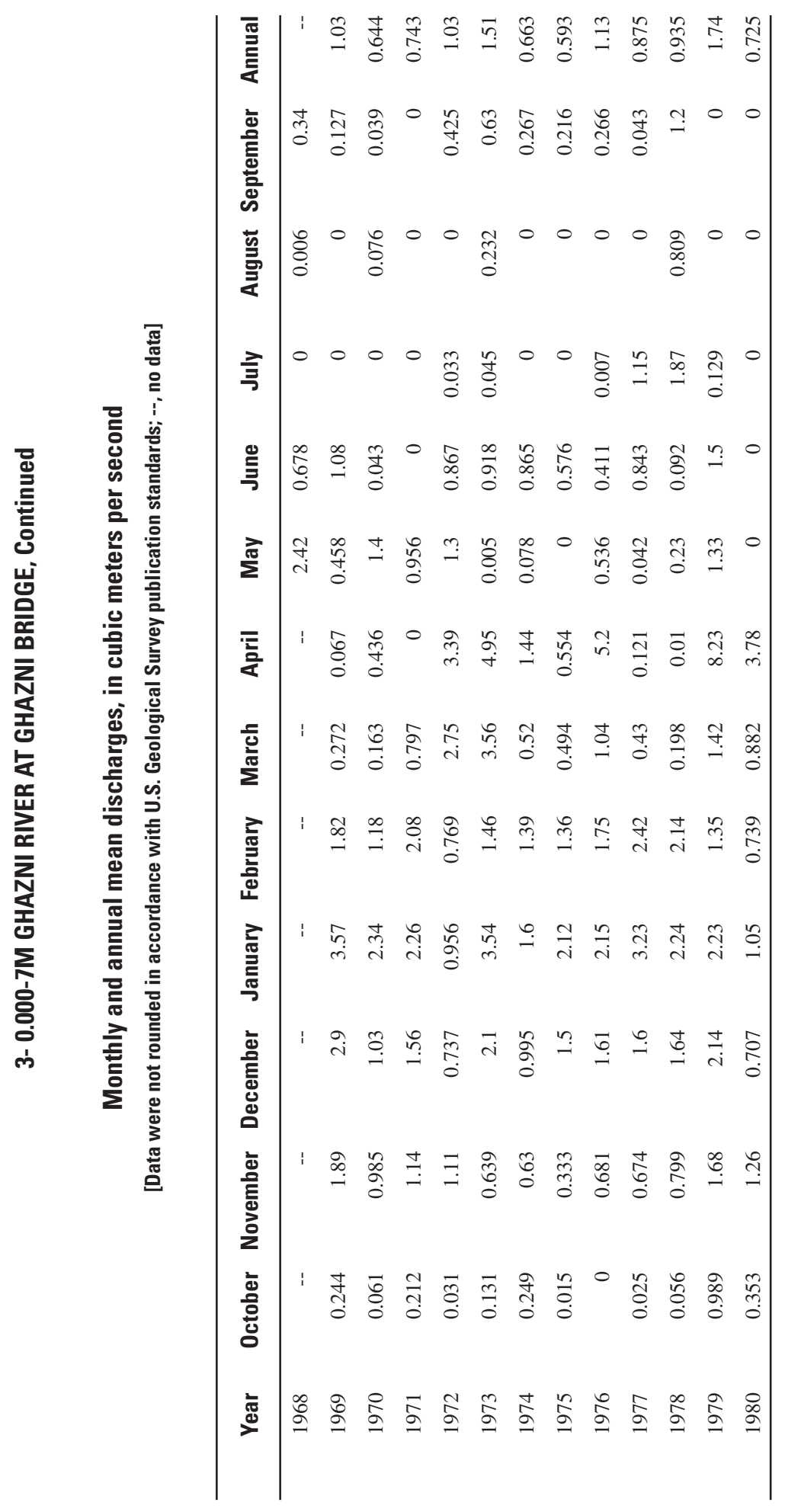




\section{3- 0.000-7S GHAZNI RIVER AT NAUBURJA}

\section{(U.S. Geological Survey identification number: 333800068250000 )}

LOCATION: Lat $33^{\circ} 38^{\prime} \mathrm{N}$., long $68^{\circ} 25^{\prime} \mathrm{E}$.

DRAINAGE AREA: $1,425 \mathrm{~km}^{2}$.

ALTITUDE: 2,235 meters plus mean sea level.

PERIOD OF RECORD: October 1, 1961 to September 30, 1967.

GAGE: --

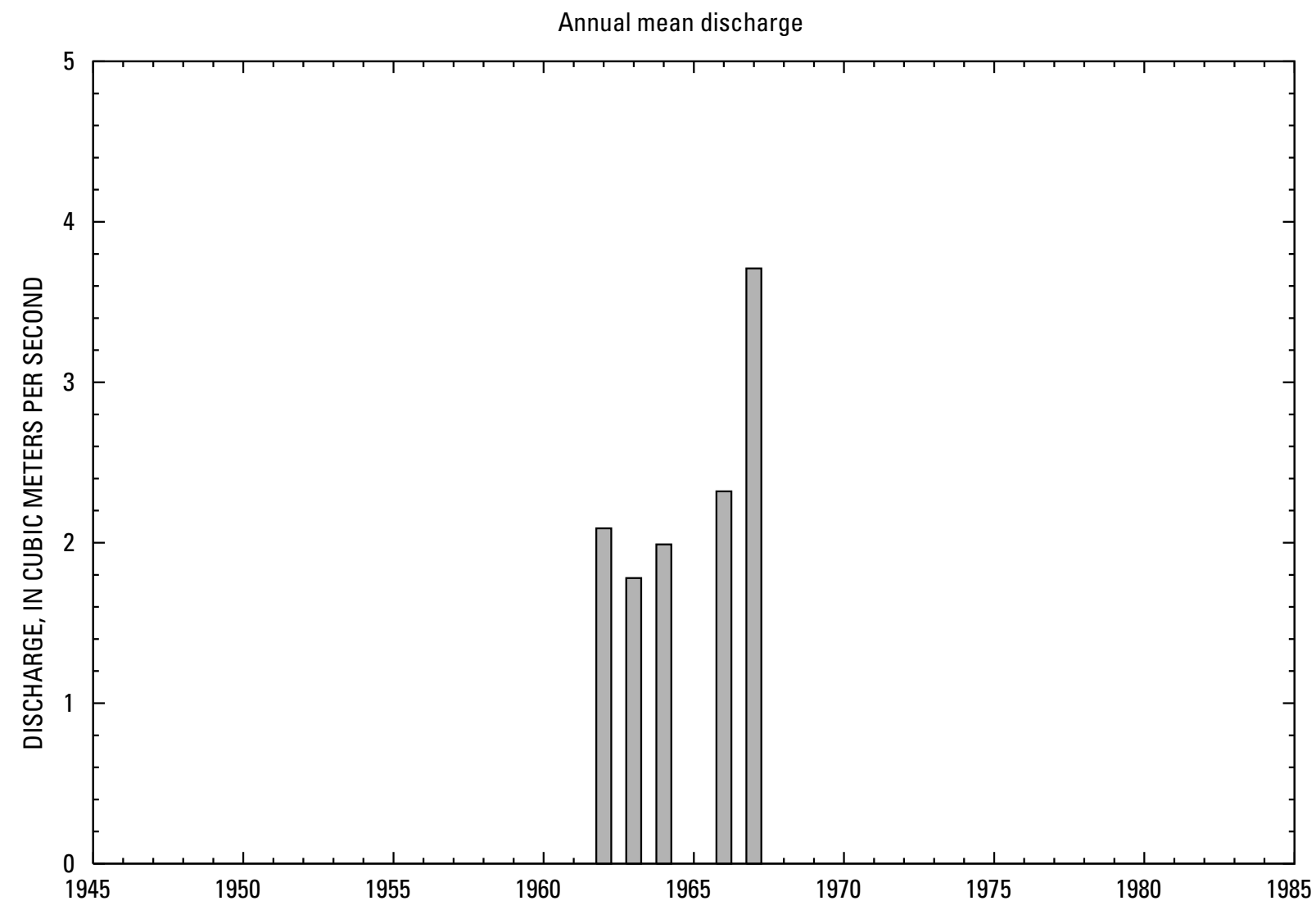


3- 0.000-7S GHAZNI RIVER AT NAUBURJA, Continued

Statistics of monthly and annual mean discharges

$\left[\mathrm{m}^{3} / \mathrm{s}\right.$, meters per second]

\begin{tabular}{|c|c|c|c|c|c|c|c|c|}
\hline \multirow[b]{2}{*}{ Month } & \multicolumn{2}{|c|}{ Maximum } & \multicolumn{2}{|c|}{ Minimum } & \multicolumn{4}{|c|}{ Mean } \\
\hline & $\begin{array}{c}\text { Discharge } \\
\left(\mathrm{m}^{3} / \mathrm{s}\right)\end{array}$ & $\begin{array}{c}\text { Water year } \\
\text { of } \\
\text { occurrence }\end{array}$ & $\begin{array}{c}\text { Discharge } \\
\left(\mathrm{m}^{3} / \mathrm{s}\right)\end{array}$ & $\begin{array}{c}\text { Water year } \\
\text { of } \\
\text { occurrence }\end{array}$ & $\begin{array}{c}\text { Discharge } \\
\left(\mathrm{m}^{3} / \mathrm{s}\right)\end{array}$ & $\begin{array}{c}\text { Standard } \\
\text { deviation } \\
\left(\mathrm{m}^{3} / \mathrm{s}\right)\end{array}$ & $\begin{array}{c}\text { Coefficient } \\
\text { of } \\
\text { variation }\end{array}$ & $\begin{array}{c}\text { Percentage } \\
\text { of annual } \\
\text { discharge }\end{array}$ \\
\hline October & 2.45 & 1962 & 0.468 & 1967 & 1.63 & 0.79 & 0.49 & 5.60 \\
\hline November & 3.09 & 1962 & 1.50 & 1967 & 2.19 & 0.60 & 0.27 & 7.51 \\
\hline December & 5.62 & 1966 & 2.79 & 1967 & 3.77 & 1.03 & 0.27 & 12.9 \\
\hline January & 5.05 & 1966 & 2.98 & 1964 & 3.95 & 0.84 & 0.21 & 13.5 \\
\hline February & 3.21 & 1966 & 0.877 & 1962 & 2.18 & 0.92 & 0.42 & 7.46 \\
\hline March & 2.86 & 1967 & 1.02 & 1963 & 1.93 & 0.76 & 0.39 & 6.60 \\
\hline April & 12.5 & 1967 & 1.22 & 1963 & 6.11 & 4.58 & 0.75 & 20.9 \\
\hline May & 11.5 & 1967 & 0.048 & 1962 & 3.24 & 4.83 & 1.49 & 11.1 \\
\hline June & 2.85 & 1962 & 0.128 & 1964 & 1.74 & 1.11 & 0.64 & 5.96 \\
\hline July & 1.86 & 1967 & 0.123 & 1964 & 0.54 & 0.74 & 1.38 & 1.85 \\
\hline August & 1.10 & 1967 & 0.068 & 1966 & 0.33 & 0.44 & 1.31 & 1.14 \\
\hline September & 4.23 & 1965 & 0.037 & 1963 & 1.58 & 1.59 & 1.01 & 5.40 \\
\hline Annual & 3.71 & 1967 & 1.78 & 1963 & 2.38 & 0.77 & 0.32 & 100 \\
\hline
\end{tabular}

Annual flow duration

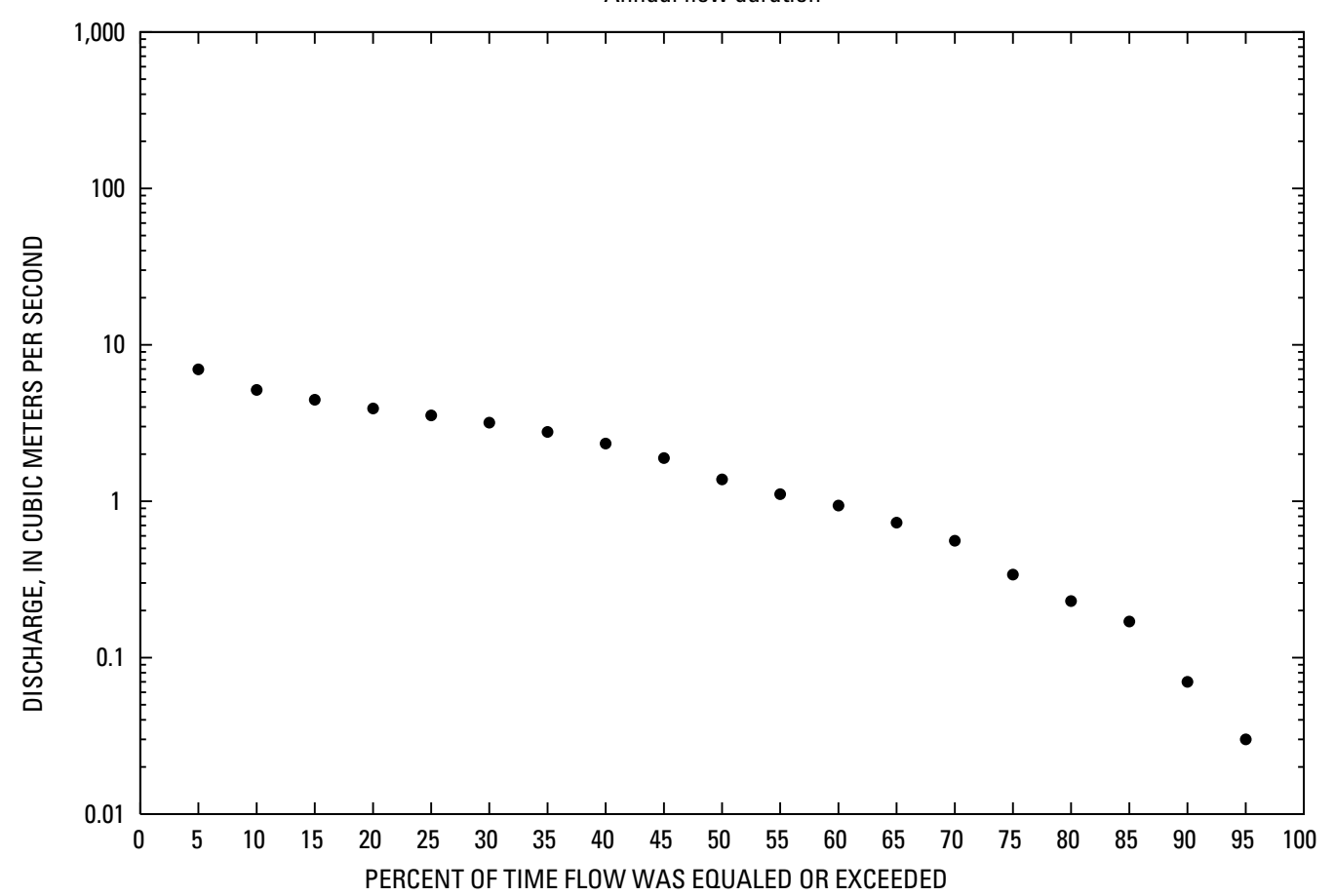




\section{3- 0.000-7S GHAZNI RIVER AT NAUBURJA, Continued}

\section{Monthly and annual flow duration, in cubic meters per second}

[Data were not rounded in accordance with U.S. Geological Survey publication standards]

\begin{tabular}{|c|c|c|c|c|c|c|c|c|c|c|c|c|c|}
\hline $\begin{array}{l}\text { Percentage } \\
\text { of days } \\
\text { discharge } \\
\text { equaled or } \\
\text { exceeded }\end{array}$ & January & February & March & April & May & June & July & August & September & October & November & December & Annual \\
\hline 95 & 2.86 & 0.66 & 0.58 & 0.77 & 0 & 0.08 & 0.07 & 0.04 & 0.02 & 0.24 & 1.13 & 2.39 & 0.03 \\
\hline 90 & 2.97 & 0.68 & 0.64 & 0.84 & 0 & 0.13 & 0.07 & 0.05 & 0.03 & 0.42 & 1.47 & 2.5 & 0.07 \\
\hline 80 & 3.14 & 0.72 & 0.74 & 1.49 & 0.01 & 0.17 & 0.13 & 0.07 & 0.07 & 0.59 & 1.6 & 2.96 & 0.23 \\
\hline 75 & 3.29 & 0.75 & 0.92 & 2.33 & 0.01 & 0.2 & 0.14 & 0.08 & 0.11 & 0.63 & 1.62 & 3.06 & 0.34 \\
\hline 70 & 3.43 & 0.79 & 0.99 & 2.54 & 0.02 & 0.31 & 0.15 & 0.08 & 0.19 & 0.67 & 1.65 & 3.24 & 0.56 \\
\hline 65 & 3.61 & 1.04 & 1.02 & 3.04 & 0.02 & 0.43 & 0.17 & 0.09 & 0.22 & 0.81 & 1.74 & 3.3 & 0.73 \\
\hline 45 & 3.87 & 2.73 & 1.14 & 5.11 & 1.04 & 0.88 & 0.25 & 0.19 & 0.64 & 1.44 & 2.34 & 3.68 & 1.89 \\
\hline 40 & 4.06 & 3.16 & 1.25 & 5.87 & 1.31 & 1.03 & 0.27 & 0.2 & 0.93 & 1.67 & 2.49 & 3.74 & 2.34 \\
\hline 35 & 4.3 & 3.29 & 1.52 & 6.69 & 2.08 & 1.21 & 0.29 & 0.24 & 1.03 & 1.86 & 2.53 & 3.82 & 2.77 \\
\hline 30 & 4.46 & 3.6 & 2.23 & 7.53 & 3.42 & 2.51 & 0.3 & 0.25 & 1.63 & 2.13 & 2.57 & 4.06 & 3.18 \\
\hline 25 & 4.52 & 3.76 & 2.41 & 8.73 & 5.28 & 3.74 & 0.33 & 0.27 & 2.72 & 2.29 & 2.62 & 4.18 & 3.54 \\
\hline 20 & 4.63 & 3.91 & 3.45 & 9.85 & 6.66 & 4.04 & 0.38 & 0.28 & 4.21 & 2.42 & 2.68 & 4.39 & 3.92 \\
\hline 15 & 4.71 & 4.05 & 4.24 & 12.1 & 8.89 & 4.35 & 0.47 & 0.61 & 4.85 & 2.7 & 2.88 & 5.02 & 4.45 \\
\hline 10 & 4.83 & 4.22 & 4.49 & 14.3 & 10.4 & 4.8 & 0.79 & 0.84 & 5.4 & 3.73 & 3.01 & 5.86 & 5.15 \\
\hline 5 & 0 & 0 & 0 & 17.9 & 15 & 5.47 & 4.76 & 1.45 & 0 & 4.8 & 3.29 & 6.23 & 6.96 \\
\hline
\end{tabular}




\section{3- 0.000-7S GHAZNI RIVER AT NAUBURJA, Continued}

Probability of occurrence of annual high discharges

[ $\mathrm{m}^{3} / \mathrm{s}$, meters per second; $\mathbf{n g}$, statistic not given]

\begin{tabular}{|c|c|c|c|c|c|c|}
\hline \multirow{2}{*}{$\begin{array}{c}\text { Exceedance } \\
\text { probability }\end{array}$} & \multirow{2}{*}{$\begin{array}{c}\text { Recurrence } \\
\text { interval } \\
\text { (years) }\end{array}$} & \multirow{2}{*}{$\begin{array}{c}\text { Maximum } \\
\text { instantaneous } \\
\left(\mathrm{m}^{3} / \mathrm{s}\right)\end{array}$} & \multicolumn{4}{|c|}{ Maximum mean discharge ${ }^{2}$} \\
\hline & & & 3-day period & 7-day period & 15-day period & 30-day period \\
\hline 0.99 & 1.01 & 3.80 & 4.11 & 4.31 & 3.38 & 2.55 \\
\hline 0.95 & 1.05 & 5.10 & 5.24 & 5.11 & 4.00 & 3.12 \\
\hline 0.90 & 1.11 & 6.10 & 6.02 & 5.70 & 4.48 & 3.55 \\
\hline 0.80 & 1.25 & 7.80 & 7.20 & 6.62 & 5.26 & 4.25 \\
\hline 0.50 & 2 & 13.1 & 10.4 & 9.34 & 7.74 & 6.45 \\
\hline 0.20 & 5 & 24.4 & 15.6 & 14.3 & 12.8 & 10.8 \\
\hline 0.10 & 10 & 35.1 & 19.7 & 18.5 & 17.4 & 14.9 \\
\hline 0.04 & 25 & 53.2 & 25.5 & 25.1 & 25.2 & 21.6 \\
\hline 0.02 & 50 & 70.9 & 30.3 & 31.0 & 32.8 & 28.0 \\
\hline 0.01 & 100 & 92.8 & 35.5 & 37.9 & 42.1 & 35.8 \\
\hline 0.005 & 200 & 120 & 41.3 & 46.0 & 53.7 & 45.5 \\
\hline 0.002 & 500 & 166 & ng & ng & ng & ng \\
\hline
\end{tabular}

'Less than 10 years of data used.

${ }^{2}$ Data does not fit log-Pearson Type III curve, use with caution.

\section{3- 0.000-7S GHAZNI RIVER AT NAUBURJA, Continued}

Probability of occurrence of annual low discharges

[m³/s, meters per second; ng, statistic not given]

\begin{tabular}{|c|c|c|c|c|c|c|c|c|c|c|}
\hline \multirow{3}{*}{$\begin{array}{c}\text { Nonexceedance } \\
\text { probability }\end{array}$} & \multirow{3}{*}{$\begin{array}{c}\text { Recurrence } \\
\text { interval } \\
\text { (years) }\end{array}$} & \multicolumn{9}{|c|}{ Minimum mean discharge $\left(\mathrm{m}^{3} / \mathrm{s}\right)$} \\
\hline & & \multicolumn{9}{|c|}{ Number of consecutive days } \\
\hline & & 1 & 3 & 7 & 14 & 30 & 60 & 90 & 120 & 183 \\
\hline 0.05 & 20 & ng & ng & 0.010 & ${ }^{1} 0.009$ & ${ }^{1} 0.006$ & 0.031 & 0.074 & 0.224 & 0.581 \\
\hline 0.10 & 10 & ng & ng & 0.010 & ${ }^{1} 0.009$ & ${ }^{1} 0.008$ & 0.036 & 0.091 & 0.257 & 0.645 \\
\hline 0.20 & 5 & ng & ng & 0.010 & 0.010 & 0.011 & 0.046 & 0.118 & 0.301 & 0.722 \\
\hline 0.50 & 2 & $\mathrm{ng}$ & ng & 0.010 & 0.011 & 0.023 & 0.084 & 0.202 & 0.397 & 0.851 \\
\hline
\end{tabular}

'Data does not fit log-Pearson Type III curve, use with caution. 


\section{3- 0.000-7S GHAZNI RIVER AT NAUBURJA, Continued}

\section{Probability of occurrence of seasonal low discharges}

$\left[\mathrm{m}^{3} / \mathrm{s}\right.$, meters per second]

\begin{tabular}{|c|c|c|c|c|c|c|c|c|c|}
\hline \multirow{3}{*}{$\begin{array}{c}\text { Nonexceedance } \\
\text { probability }\end{array}$} & \multirow{3}{*}{$\begin{array}{c}\text { Recurrence } \\
\text { interval } \\
\text { (years) }\end{array}$} & \multicolumn{8}{|c|}{ Minimum mean discharge $\left(\mathrm{m}^{3} / \mathrm{s}\right)$} \\
\hline & & \multicolumn{8}{|c|}{ Number of consecutive days } \\
\hline & & 1 & 7 & 14 & 30 & 1 & 7 & 14 & 30 \\
\hline & & \multicolumn{4}{|c|}{ December-January-February } & \multicolumn{4}{|c|}{ March-April-May } \\
\hline 0.05 & 20 & 0.515 & 0.572 & ${ }^{1} 0.544$ & 0.941 & 0 & 0 & 0 & 0.014 \\
\hline 0.10 & 10 & 0.567 & 0.616 & 0.709 & 1.19 & 0 & 0 & 0 & 0.039 \\
\hline 0.20 & 5 & 0.637 & 0.677 & 0.942 & 1.54 & 0 & 0 & 0 & 0.113 \\
\hline \multirow[t]{2}{*}{0.50} & 2 & 0.798 & 0.818 & 1.47 & 2.29 & 0.035 & 0.037 & 0.041 & 0.575 \\
\hline & & \multicolumn{4}{|c|}{ June-July-August } & \multicolumn{4}{|c|}{ September-October-November } \\
\hline 0.05 & 20 & 0.005 & 0.024 & ${ }^{1} 0.023$ & 0.036 & 0.006 & 0.006 & 0.010 & 0.019 \\
\hline 0.10 & 10 & 0.007 & 0.032 & ${ }^{1} 0.031$ & 0.043 & 0.014 & 0.014 & 0.022 & 0.045 \\
\hline 0.20 & 5 & 0.011 & 0.044 & 0.044 & 0.057 & 0.033 & 0.033 & 0.053 & 0.116 \\
\hline 0.50 & 2 & 0.028 & 0.083 & 0.096 & 0.119 & 0.135 & 0.138 & 0.199 & 0.470 \\
\hline
\end{tabular}

'Data does not fit log-Pearson Type III curve, use with caution.

\section{3- 0.000-7S GHAZNI RIVER AT NAUBURJA, Continued}

\section{Annual peak discharge and corresponding gage height}

$\left[\mathrm{m}^{3} / \mathrm{s}\right.$, meters per second; --, no data]

\begin{tabular}{|c|c|c|c|c|c|c|c|}
\hline $\begin{array}{c}\text { Water } \\
\text { year }\end{array}$ & Date & $\begin{array}{c}\text { Gage } \\
\text { height } \\
\text { (meters) }\end{array}$ & $\begin{array}{c}\text { Peak } \\
\text { discharge } \\
\left(\mathrm{m}^{3} / \mathrm{s}\right)\end{array}$ & $\begin{array}{l}\text { Water } \\
\text { year }\end{array}$ & Date & $\begin{array}{c}\text { Gage } \\
\text { height } \\
\text { (meters) }\end{array}$ & $\begin{array}{c}\text { Peak } \\
\text { discharge } \\
\left(\mathrm{m}^{3} / \mathrm{s}\right)\end{array}$ \\
\hline \multicolumn{8}{|c|}{ Annual peak discharge, by year } \\
\hline 1962 & 14-Apr & & 8.10 & 1965 & 4-Sep & & 9.90 \\
\hline 1963 & 15-May & & 41.2 & 1966 & 24-Dec & & 6.41 \\
\hline 1964 & 10-Apr & - & 17.7 & 1967 & 22-Apr & & 20.3 \\
\hline \multicolumn{8}{|c|}{ Annual peak discharge, from highest to lowest } \\
\hline 1963 & 15-May & - & 41.2 & 1965 & 4-Sep & & 9.90 \\
\hline 1967 & 22-Apr & - & 20.3 & 1962 & 14-Apr & & 8.10 \\
\hline 1964 & 10-Apr & - & 17.7 & 1966 & 24-Dec & & 6.41 \\
\hline
\end{tabular}




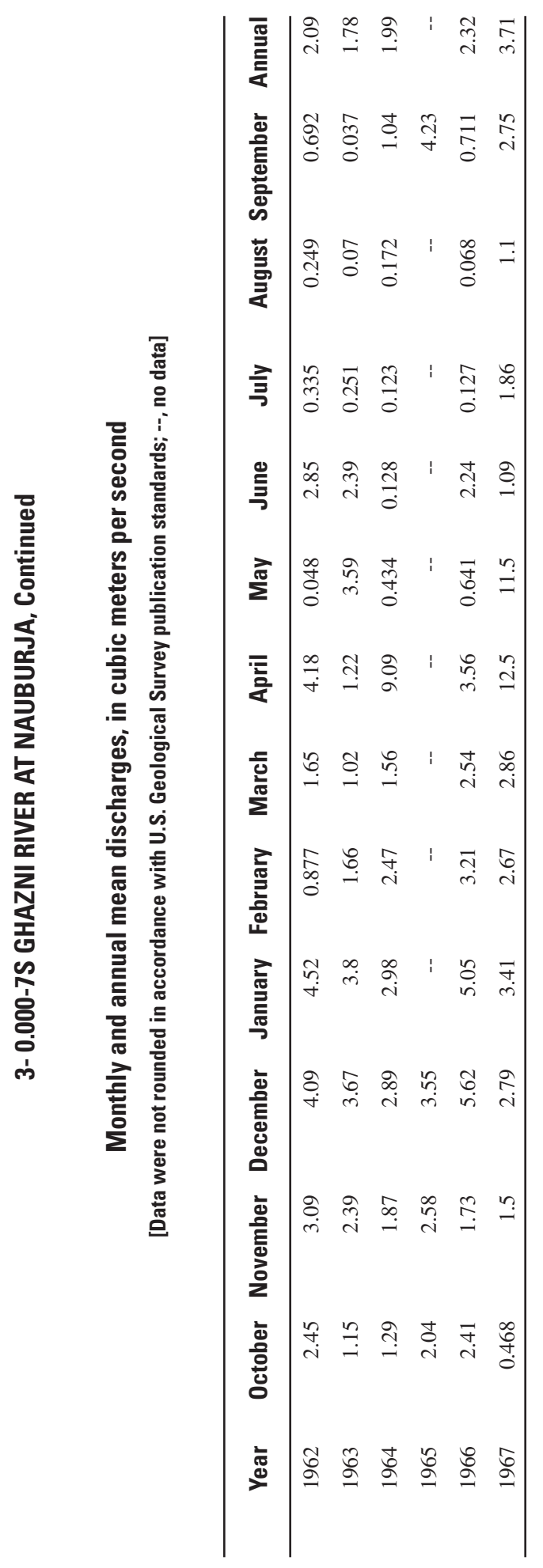




\section{3- 0.000-8W GHAZNI RIVER BELOW SERAJ RESERVOIR}

\section{(U.S. Geological Survey identification number: 334500068230000)}

LOCATION: Lat $33^{\circ} 45^{\prime} \mathrm{N}$., long 68 $23^{\prime} \mathrm{E}$., $500 \mathrm{~m}$ downstream, from Seraj Dam and about $24 \mathrm{~km}$ upstream from Ghazni.

DRAINAGE AREA: $1,165 \mathrm{~km}^{2}$.

ALTITUDE: 2,338 meters plus mean sea level.

PERIOD OF RECORD: December 7, 1947 to September 30, 1980.

GAGE: Water-stage recorder. January 11 to May 10, 1948, at site $14 \mathrm{~km}$ downstream at different datum; March 1, 1949 to April 30, 1952, at same site but different datum.

Annual mean discharge

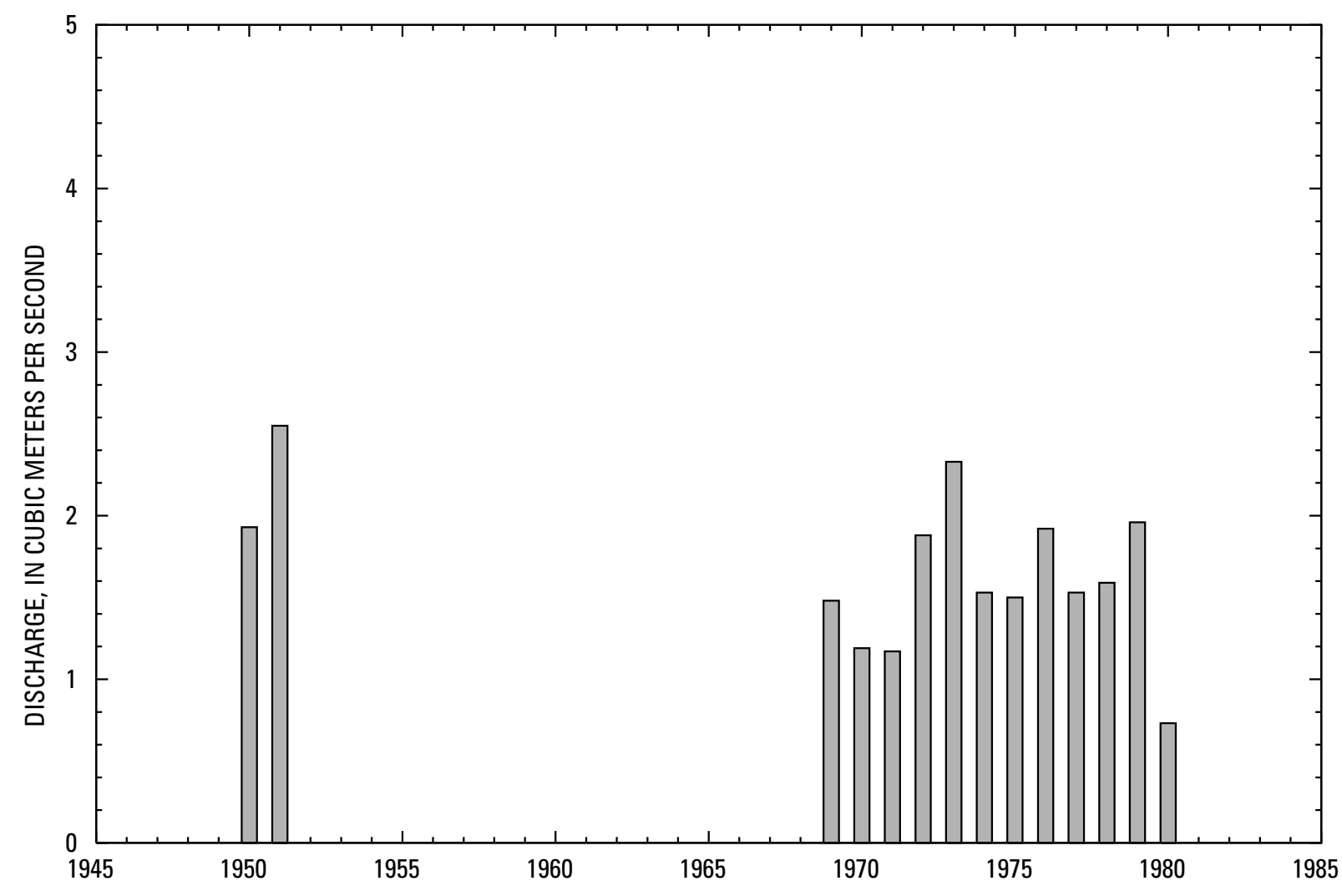


3- 0.000-8W GHAZNI RIVER BELOW SERAJ RESERVOIR, Continued

Statistics of monthly and annual mean discharges

$\left[\mathrm{m}^{3} / \mathrm{s}\right.$, meters per second]

\begin{tabular}{|c|c|c|c|c|c|c|c|c|}
\hline \multirow[b]{2}{*}{ Month } & \multicolumn{2}{|c|}{ Maximum } & \multicolumn{2}{|c|}{ Minimum } & \multicolumn{4}{|c|}{ Mean } \\
\hline & $\begin{array}{c}\text { Discharge } \\
\left(\mathrm{m}^{3} / \mathrm{s}\right)\end{array}$ & $\begin{array}{c}\text { Water year } \\
\text { of } \\
\text { occurrence }\end{array}$ & $\begin{array}{c}\text { Discharge } \\
\left(\mathrm{m}^{3} / \mathrm{s}\right)\end{array}$ & $\begin{array}{c}\text { Water year } \\
\text { of } \\
\text { occurrence }\end{array}$ & $\begin{array}{c}\text { Discharge } \\
\left(\mathrm{m}^{3} / \mathrm{s}\right)\end{array}$ & $\begin{array}{c}\text { Standard } \\
\text { deviation } \\
\left(\mathrm{m}^{3} / \mathrm{s}\right)\end{array}$ & $\begin{array}{c}\text { Coefficient } \\
\text { of } \\
\text { variation }\end{array}$ & $\begin{array}{c}\text { Percentage } \\
\text { of annual } \\
\text { discharge }\end{array}$ \\
\hline October & 3.39 & 1973 & 0.526 & 1980 & 1.30 & 0.82 & 0.63 & 6.20 \\
\hline November & 3.76 & 1969 & 1.12 & 1976 & 2.24 & 0.74 & 0.33 & 10.7 \\
\hline December & 3.37 & 1952 & 0.881 & 1980 & 2.15 & 0.66 & 0.31 & 10.2 \\
\hline January & 3.51 & 1952 & 0.897 & 1980 & 2.11 & 0.68 & 0.32 & 10.1 \\
\hline February & 3.44 & 1952 & 0.669 & 1980 & 1.76 & 0.74 & 0.42 & 8.42 \\
\hline March & 4.82 & 1952 & 0.171 & 1969 & 1.61 & 1.45 & 0.90 & 7.69 \\
\hline April & 9.44 & 1979 & 0.324 & 1971 & 3.69 & 2.66 & 0.72 & 17.6 \\
\hline May & 5.85 & 1951 & 0.090 & 1980 & 2.32 & 1.69 & 0.73 & 11.0 \\
\hline June & 3.72 & 1976 & 0.330 & 1971 & 1.83 & 1.36 & 0.74 & 8.75 \\
\hline July & 1.84 & 1978 & 0.012 & 1973 & 0.44 & 0.47 & 1.06 & 2.10 \\
\hline August & 1.37 & 1978 & 0.010 & 1973 & 0.44 & 0.41 & 0.92 & 2.10 \\
\hline September & 2.71 & 1978 & 0.064 & 1979 & 1.07 & 0.68 & 0.63 & 5.11 \\
\hline Annual & 2.55 & 1951 & 0.731 & 1980 & 1.66 & 0.48 & 0.29 & 100 \\
\hline
\end{tabular}

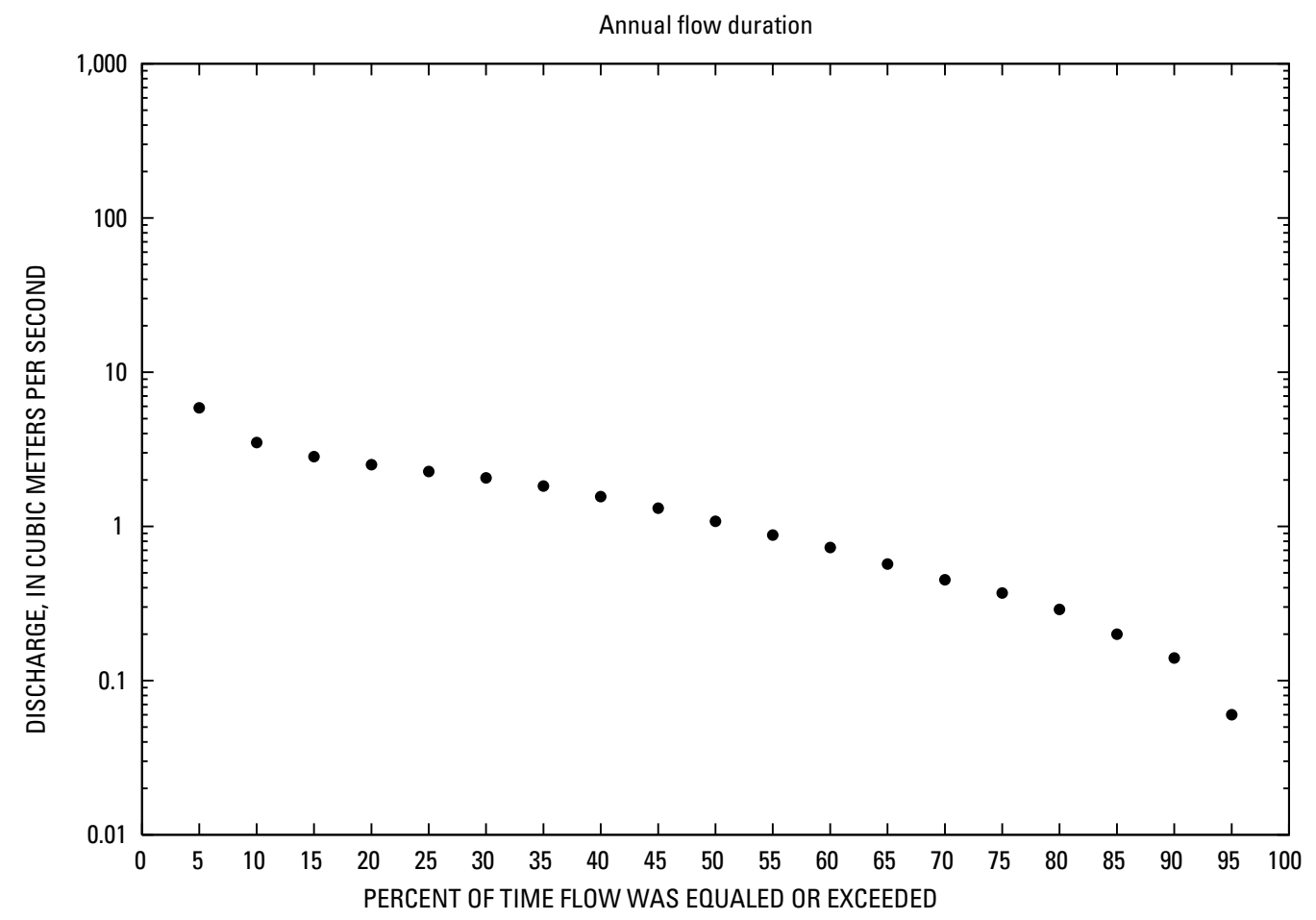




\section{3- 0.000-8W GHAZNI RIVER BELOW SERAJ RESERVOIR, Continued}

\section{Monthly and annual flow duration, in cubic meters per second}

[Data were not rounded in accordance with U.S. Geological Survey publication standards]

\begin{tabular}{|c|c|c|c|c|c|c|c|c|c|c|c|c|c|}
\hline $\begin{array}{l}\text { Percentage } \\
\text { of days } \\
\text { discharge } \\
\text { equaled or } \\
\text { exceeded }\end{array}$ & January & February & March & April & May & June & July & August & September & October & November & December & Annual \\
\hline 95 & 0.81 & 0.16 & 0.08 & 0.32 & 0.11 & 0.06 & 0.02 & 0.02 & 0.05 & 0.07 & 0.92 & 0.74 & 0.06 \\
\hline 90 & 0.98 & 0.49 & 0.15 & 0.35 & 0.35 & 0.08 & 0.05 & 0.04 & 0.07 & 0.4 & 1.16 & 1.02 & 0.14 \\
\hline 85 & 1.22 & 0.67 & 0.19 & 0.39 & 0.43 & 0.17 & 0.06 & 0.07 & 0.09 & 0.44 & 1.37 & 1.41 & 0.2 \\
\hline 80 & 1.61 & 0.82 & 0.23 & 0.47 & 0.46 & 0.21 & 0.07 & 0.11 & 0.18 & 0.48 & 1.46 & 1.56 & 0.29 \\
\hline 75 & 1.74 & 1.21 & 0.26 & 0.79 & 0.51 & 0.23 & 0.08 & 0.17 & 0.21 & 0.6 & 1.53 & 1.62 & 0.37 \\
\hline 70 & 1.9 & 1.45 & 0.31 & 1.01 & 0.63 & 0.27 & 0.16 & 0.19 & 0.25 & 0.65 & 1.59 & 1.8 & 0.45 \\
\hline 65 & 1.98 & 1.6 & 0.47 & 1.38 & 0.74 & 0.36 & 0.19 & 0.2 & 0.29 & 0.79 & 1.65 & 1.99 & 0.57 \\
\hline 60 & 2.03 & 1.75 & 0.51 & 1.82 & 0.83 & 0.38 & 0.21 & 0.21 & 0.39 & 0.84 & 1.81 & 2.07 & 0.73 \\
\hline 55 & 2.08 & 1.82 & 0.56 & 2.36 & 0.96 & 0.41 & 0.23 & 0.27 & 0.42 & 0.89 & 1.92 & 2.15 & 0.88 \\
\hline 50 & 2.16 & 1.9 & 0.77 & 2.85 & 1.12 & 0.54 & 0.31 & 0.3 & 0.46 & 1.02 & 2.01 & 2.24 & 1.08 \\
\hline 45 & 2.24 & 1.97 & 1.05 & 3.43 & 1.26 & 0.65 & 0.33 & 0.32 & 0.51 & 1.15 & 2.12 & 2.28 & 1.31 \\
\hline 40 & 2.32 & 2.04 & 1.35 & 3.95 & 1.46 & 0.79 & 0.37 & 0.46 & 0.56 & 1.27 & 2.26 & 2.32 & 1.56 \\
\hline 35 & 2.4 & 2.13 & 2.38 & 4.7 & 1.7 & 1.01 & 0.41 & 0.49 & 0.69 & 1.39 & 2.43 & 2.35 & 1.83 \\
\hline 30 & 2.49 & 2.23 & 2.55 & 5.25 & 2.35 & 1.22 & 0.45 & 0.51 & 0.82 & 1.5 & 2.6 & 2.39 & 2.06 \\
\hline 25 & 2.58 & 2.33 & 2.73 & 5.8 & 2.97 & 1.54 & 0.49 & 0.56 & 1.2 & 1.77 & 2.77 & 2.54 & 2.27 \\
\hline 20 & 2.65 & 2.43 & 2.91 & 6.65 & 4.05 & 2.47 & 0.58 & 0.62 & 1.45 & 1.93 & 2.99 & 2.72 & 2.51 \\
\hline 15 & 2.71 & 2.58 & 3.4 & 7.49 & 5.52 & 6.55 & 0.7 & 0.7 & 2.11 & 2.27 & 3.28 & 2.88 & 2.83 \\
\hline 10 & 2.78 & 2.82 & 3.99 & 8.43 & 6.89 & 7.53 & 0.94 & 1.04 & 3.34 & 2.79 & 3.67 & 3.07 & 3.5 \\
\hline 5 & 3.36 & 3.17 & 5.02 & 10 & 8.35 & 8.52 & 1.1 & 1.23 & 5.16 & 3.28 & 4.13 & 3.38 & 5.88 \\
\hline
\end{tabular}




\section{3- 0.000-8W GHAZNI RIVER BELOW SERAJ RESERVOIR, Continued}

Probability of occurrence of annual high discharges

$\left[\mathrm{m}^{3} / \mathrm{s}\right.$, meters per second; $\mathrm{ng}$, statistic not given]

\begin{tabular}{|c|c|c|c|c|c|c|}
\hline \multirow{2}{*}{$\begin{array}{c}\text { Exceedance } \\
\text { probability }\end{array}$} & \multirow{2}{*}{$\begin{array}{c}\text { Recurrence } \\
\text { interval } \\
\text { (years) }\end{array}$} & \multirow{2}{*}{$\begin{array}{c}\text { Maximum } \\
\text { instantaneous } \\
\left(\mathrm{m}^{3} / \mathrm{s}\right)\end{array}$} & \multicolumn{4}{|c|}{ Maximum mean discharge ${ }^{1}$} \\
\hline & & & 3-day period & 7-day period & 15-day period & 30-day period \\
\hline 0.99 & 1.01 & 2.60 & 2.82 & 2.50 & 2.79 & 1.99 \\
\hline 0.95 & 1.05 & 4.10 & 4.50 & 3.97 & 3.73 & 2.46 \\
\hline 0.90 & 1.11 & 5.10 & 5.53 & 4.91 & 4.31 & 2.78 \\
\hline 0.80 & 1.25 & 6.50 & 6.86 & 6.14 & 5.09 & 3.25 \\
\hline 0.50 & 2 & 9.60 & 9.33 & 8.57 & 6.83 & 4.54 \\
\hline 0.20 & 5 & 13.0 & 11.3 & 10.7 & 8.86 & 6.59 \\
\hline 0.10 & 10 & 14.8 & 12.0 & 11.6 & 10.0 & 8.15 \\
\hline 0.04 & 25 & 16.5 & 12.5 & 12.4 & 11.3 & 10.4 \\
\hline 0.02 & 50 & 17.6 & 12.7 & 12.8 & 12.2 & 12.2 \\
\hline 0.01 & 100 & 18.5 & 12.9 & 13.0 & 13.0 & 14.2 \\
\hline 0.005 & 200 & 19.2 & 13.0 & 13.2 & 13.7 & 16.3 \\
\hline 0.002 & 500 & 20.1 & ng & ng & ng & ng \\
\hline
\end{tabular}

'Data does not fit log-Pearson Type III curve, use with caution.

\section{3- 0.000-8W GHAZNI RIVER BELOW SERAJ RESERVOIR, Continued}

Probability of occurrence of annual low discharges

$\left[\mathrm{m}^{3} / \mathrm{s}\right.$, meters per second]

\begin{tabular}{|c|c|c|c|c|c|c|c|c|c|c|}
\hline \multirow{3}{*}{$\begin{array}{c}\text { Nonexceedance } \\
\text { probability }\end{array}$} & \multirow{3}{*}{$\begin{array}{c}\text { Recurrence } \\
\text { interval } \\
\text { (years) }\end{array}$} & \multicolumn{9}{|c|}{ Minimum mean discharge $\left(\mathrm{m}^{3} / \mathrm{s}\right)$} \\
\hline & & \multicolumn{9}{|c|}{ Number of consecutive days } \\
\hline & & 1 & 3 & 7 & 14 & 30 & 60 & 90 & 120 & 183 \\
\hline 0.05 & 20 & 0.010 & 0.015 & 0.017 & 0.018 & 0.020 & 0.023 & 0.031 & 0.112 & 0.427 \\
\hline 0.10 & 10 & 0.017 & 0.022 & 0.027 & 0.031 & 0.038 & 0.046 & 0.063 & 0.182 & 0.516 \\
\hline 0.20 & 5 & 0.030 & 0.037 & 0.046 & 0.056 & 0.076 & 0.098 & 0.134 & 0.302 & 0.644 \\
\hline 0.50 & 2 & 0.089 & 0.096 & 0.122 & 0.153 & 0.212 & 0.300 & 0.410 & 0.639 & 0.951 \\
\hline
\end{tabular}




\section{3- 0.000-8W GHAZNI RIVER BELOW SERAJ RESERVOIR, Continued}

\section{Probability of occurrence of seasonal low discharges}

$\left[\mathrm{m}^{3} / \mathrm{s}\right.$, meters per second]

\begin{tabular}{|c|c|c|c|c|c|c|c|c|c|}
\hline \multirow{3}{*}{$\begin{array}{c}\text { Nonexceedance } \\
\text { probability }\end{array}$} & \multirow{3}{*}{$\begin{array}{c}\text { Recurrence } \\
\text { interval } \\
\text { (years) }\end{array}$} & \multicolumn{8}{|c|}{ Minimum mean discharge $\left(\mathrm{m}^{3} / \mathrm{s}\right)$} \\
\hline & & \multicolumn{8}{|c|}{ Number of consecutive days } \\
\hline & & 1 & 7 & 14 & 30 & 1 & 7 & 14 & 30 \\
\hline & & \multicolumn{4}{|c|}{ December-January-February } & \multicolumn{4}{|c|}{ March-April-May } \\
\hline 0.05 & 20 & 0.011 & 0.035 & 0.380 & 0.637 & 0.019 & 0.021 & 0.023 & 0.101 \\
\hline 0.10 & 10 & 0.030 & 0.094 & 0.483 & 0.774 & 0.033 & 0.037 & 0.046 & 0.132 \\
\hline 0.20 & 5 & 0.091 & 0.254 & 0.644 & 0.975 & 0.062 & 0.071 & 0.096 & 0.191 \\
\hline \multirow[t]{2}{*}{0.50} & 2 & 0.524 & 1.00 & 1.12 & 1.50 & 0.198 & 0.227 & 0.327 & 0.428 \\
\hline & & \multicolumn{4}{|c|}{ June-July-August } & \multicolumn{4}{|c|}{ September-October-November } \\
\hline 0.05 & 20 & 0.016 & 0.017 & 0.018 & 0.019 & 0.019 & 0.020 & 0.055 & 0.135 \\
\hline 0.10 & 10 & 0.027 & 0.030 & 0.032 & 0.035 & 0.038 & 0.041 & 0.090 & 0.235 \\
\hline 0.20 & 5 & 0.048 & 0.056 & 0.062 & 0.071 & 0.078 & 0.089 & 0.155 & 0.414 \\
\hline 0.50 & 2 & 0.138 & 0.163 & 0.186 & 0.216 & 0.241 & 0.288 & 0.374 & 0.888 \\
\hline
\end{tabular}




\section{3- 0.000-8W GHAZNI RIVER BELOW SERAJ RESERVOIR, Continued}

Annual peak discharge and corresponding gage height

$\left[\mathrm{m}^{3} / \mathrm{s}\right.$, meters per second; --, no data]

\begin{tabular}{|c|c|c|c|c|c|c|c|}
\hline $\begin{array}{c}\text { Water } \\
\text { year }\end{array}$ & Date & $\begin{array}{c}\text { Gage } \\
\text { height } \\
\text { (meters) }\end{array}$ & $\begin{array}{c}\text { Peak } \\
\text { discharge } \\
\left(\mathrm{m}^{3} / \mathrm{s}\right)\end{array}$ & $\begin{array}{c}\text { Water } \\
\text { year }\end{array}$ & Date & $\begin{array}{c}\text { Gage } \\
\text { height } \\
\text { (meters) }\end{array}$ & $\begin{array}{c}\text { Peak } \\
\text { discharge } \\
\left(\mathrm{m}^{3} / \mathrm{s}\right)\end{array}$ \\
\hline \multicolumn{8}{|c|}{ Annual peak discharge, by year } \\
\hline 1948 & 2-Apr & 0.30 & 3.45 & 1972 & 28-Apr & -- & 9.63 \\
\hline 1949 & 10-Apr & 10.46 & 5.66 & 1973 & 15-Apr & -- & 9.63 \\
\hline 1950 & 28-Apr & 10.52 & 6.85 & 1974 & 6-Jun & -- & 9.63 \\
\hline 1951 & 6-May & 10.75 & 12.8 & 1975 & 8-Jun & -- & 8.80 \\
\hline 1952 & 7-Apr & 10.61 & 8.97 & 1976 & 22-Apr & -- & 11.1 \\
\hline 1968 & 9-Apr & -- & 11.6 & 1977 & 1-Jun & -- & 10.2 \\
\hline 1969 & 27-Sep & -- & 7.60 & 1978 & 5-Jul & -- & 18.8 \\
\hline 1970 & 21-May & -- & 10.7 & 1979 & 16-Apr & -- & 14.4 \\
\hline 1971 & 2-May & -- & 12.3 & 1980 & $29-\mathrm{Oct}$ & -- & 4.00 \\
\hline \multicolumn{8}{|c|}{ Annual peak discharge, from highest to lowest } \\
\hline 1978 & 5-Jul & -- & 18.8 & 1973 & 15-Apr & -- & 9.63 \\
\hline 1979 & 16-Apr & -- & 14.4 & 1972 & 28-Apr & -- & 9.63 \\
\hline 1951 & 6-May & 10.75 & 12.8 & 1952 & 7-Apr & 10.61 & 8.97 \\
\hline 1971 & 2-May & -- & 12.3 & 1975 & 8-Jun & -- & 8.80 \\
\hline 1968 & 9-Apr & -- & 11.6 & 1969 & 27-Sep & -- & 7.60 \\
\hline 1976 & 22-Apr & -- & 11.1 & 1950 & 28-Apr & 10.52 & 6.85 \\
\hline 1970 & 21-May & -- & 10.7 & 1949 & 10-Apr & 10.46 & 5.66 \\
\hline 1977 & 1-Jun & -- & 10.2 & 1980 & 29-Oct & -- & 4.00 \\
\hline 1974 & 6-Jun & -- & 9.63 & 1948 & 2-Apr & 0.30 & 3.45 \\
\hline
\end{tabular}




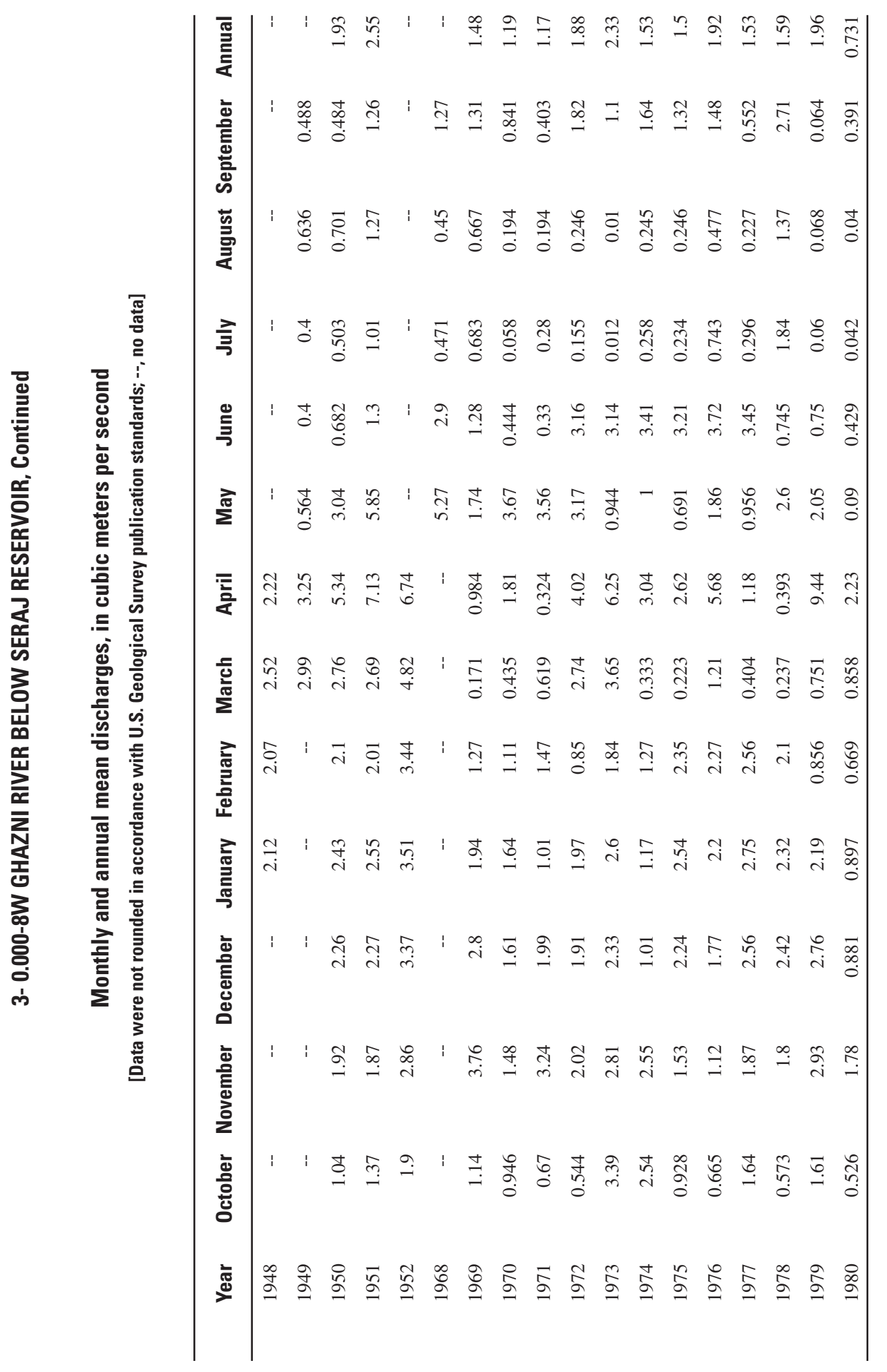




\section{3- 1.1L0-5T PARK RIVER NEAR PARK DASHT}

\section{(U.S. Geological Survey identification number: $\mathbf{3 3 0 0 0 0 0 6 8 5 2 0 0 0 0 )}$}

LOCATION: Lat $33^{\circ} 00^{\prime} N$., long 68²'․

DRAINAGE AREA: $260 \mathrm{~km}^{2}$.

ALTITUDE: 2,290 meters plus mean sea level.

PERIOD OF RECORD: January 16, 1968 to April 30, 1979.

GAGE: --

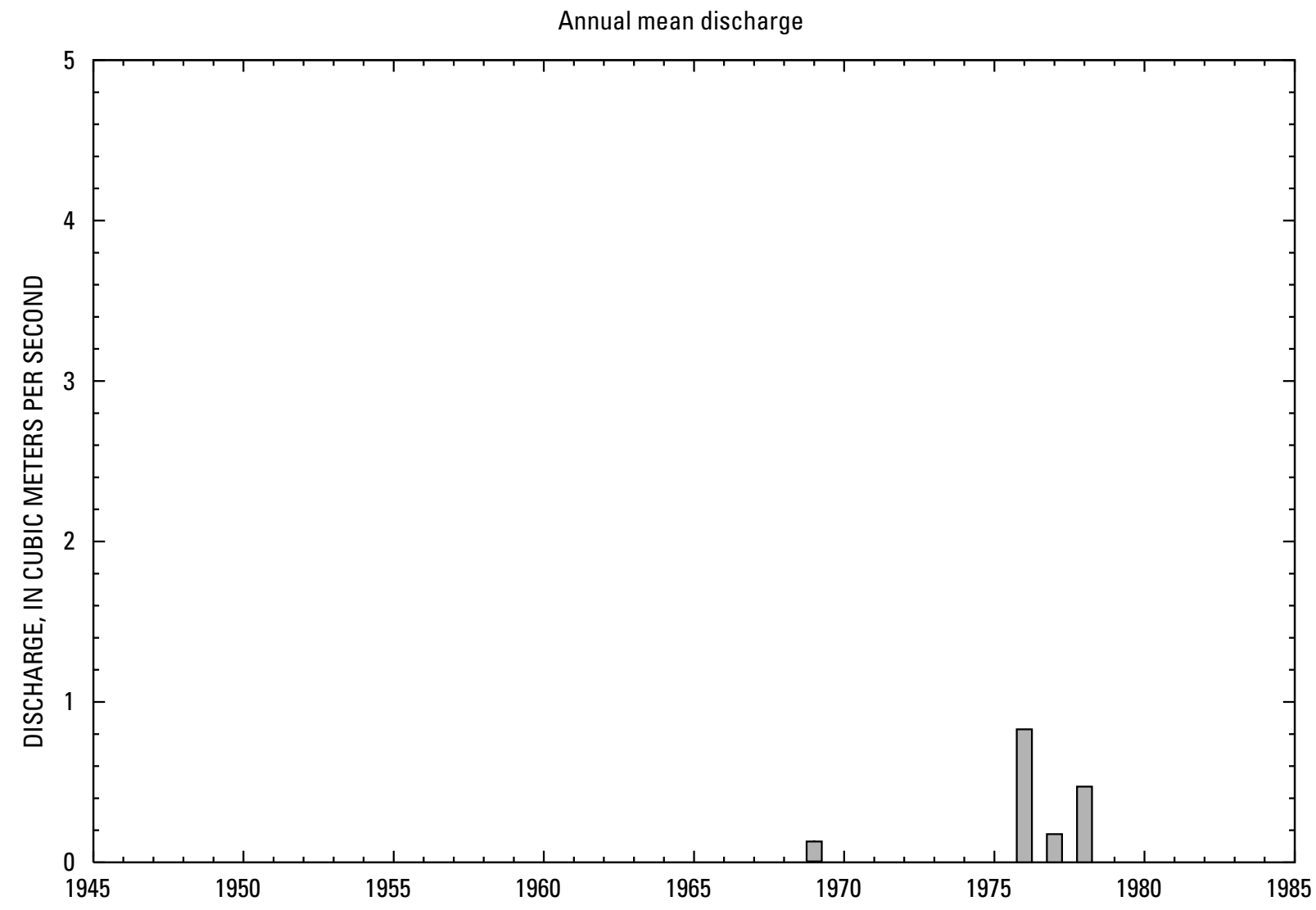




\section{3- 1.1L0-5T PARK RIVER NEAR PARK DASHT, Continued}

\section{Statistics of monthly and annual mean discharges}

$\left[\mathrm{m}^{3} / \mathrm{s}\right.$, meters per second]

\begin{tabular}{|c|c|c|c|c|c|c|c|c|}
\hline \multirow[b]{2}{*}{ Month } & \multicolumn{2}{|c|}{ Maximum } & \multicolumn{2}{|c|}{ Minimum } & \multicolumn{4}{|c|}{ Mean } \\
\hline & $\begin{array}{c}\text { Discharge } \\
\left(\mathrm{m}^{3} / \mathrm{s}\right)\end{array}$ & $\begin{array}{c}\text { Water year } \\
\text { of } \\
\text { occurrence }\end{array}$ & $\begin{array}{c}\text { Discharge } \\
\left(\mathrm{m}^{3} / \mathrm{s}\right)\end{array}$ & $\begin{array}{c}\text { Water year } \\
\text { of } \\
\text { occurrence }\end{array}$ & $\begin{array}{c}\text { Discharge } \\
\left(\mathrm{m}^{3} / \mathrm{s}\right)\end{array}$ & $\begin{array}{c}\text { Standard } \\
\text { deviation } \\
\left(\mathrm{m}^{3} / \mathrm{s}\right)\end{array}$ & $\begin{array}{c}\text { Coefficient } \\
\text { of } \\
\text { variation }\end{array}$ & $\begin{array}{c}\text { Percentage } \\
\text { of annual } \\
\text { discharge }\end{array}$ \\
\hline October & 0.091 & 1976 & 0.040 & 1978 & 0.06 & 0.02 & 0.32 & 1.12 \\
\hline November & 0.104 & 1979 & 0.048 & 1978 & 0.06 & 0.02 & 0.33 & 1.19 \\
\hline December & 0.133 & 1969 & 0.043 & 1970 & 0.09 & 0.03 & 0.35 & 1.72 \\
\hline January & 0.149 & 1977 & 0.041 & 1970 & 0.11 & 0.04 & 0.37 & 1.98 \\
\hline February & 1.13 & 1976 & 0.070 & 1969 & 0.32 & 0.37 & 1.17 & 5.85 \\
\hline March & 3.58 & 1968 & 0.144 & 1969 & 1.48 & 1.26 & 0.85 & 27.2 \\
\hline April & 3.83 & 1979 & 0.552 & 1969 & 1.89 & 1.41 & 0.75 & 34.8 \\
\hline May & 1.42 & 1976 & 0.088 & 1978 & 0.46 & 0.55 & 1.18 & 8.52 \\
\hline June & 0.272 & 1976 & 0.057 & 1978 & 0.13 & 0.09 & 0.68 & 2.33 \\
\hline July & 1.97 & 1978 & 0.042 & 1968 & 0.55 & 0.82 & 1.48 & 10.2 \\
\hline August & 0.447 & 1976 & 0.039 & 1969 & 0.20 & 0.20 & 0.97 & 3.72 \\
\hline September & 0.172 & 1976 & 0.030 & 1968 & 0.08 & 0.06 & 0.79 & 1.44 \\
\hline Annual & 0.829 & 1976 & 0.136 & 1969 & 0.40 & 0.32 & 0.80 & 100 \\
\hline
\end{tabular}

Annual flow duration

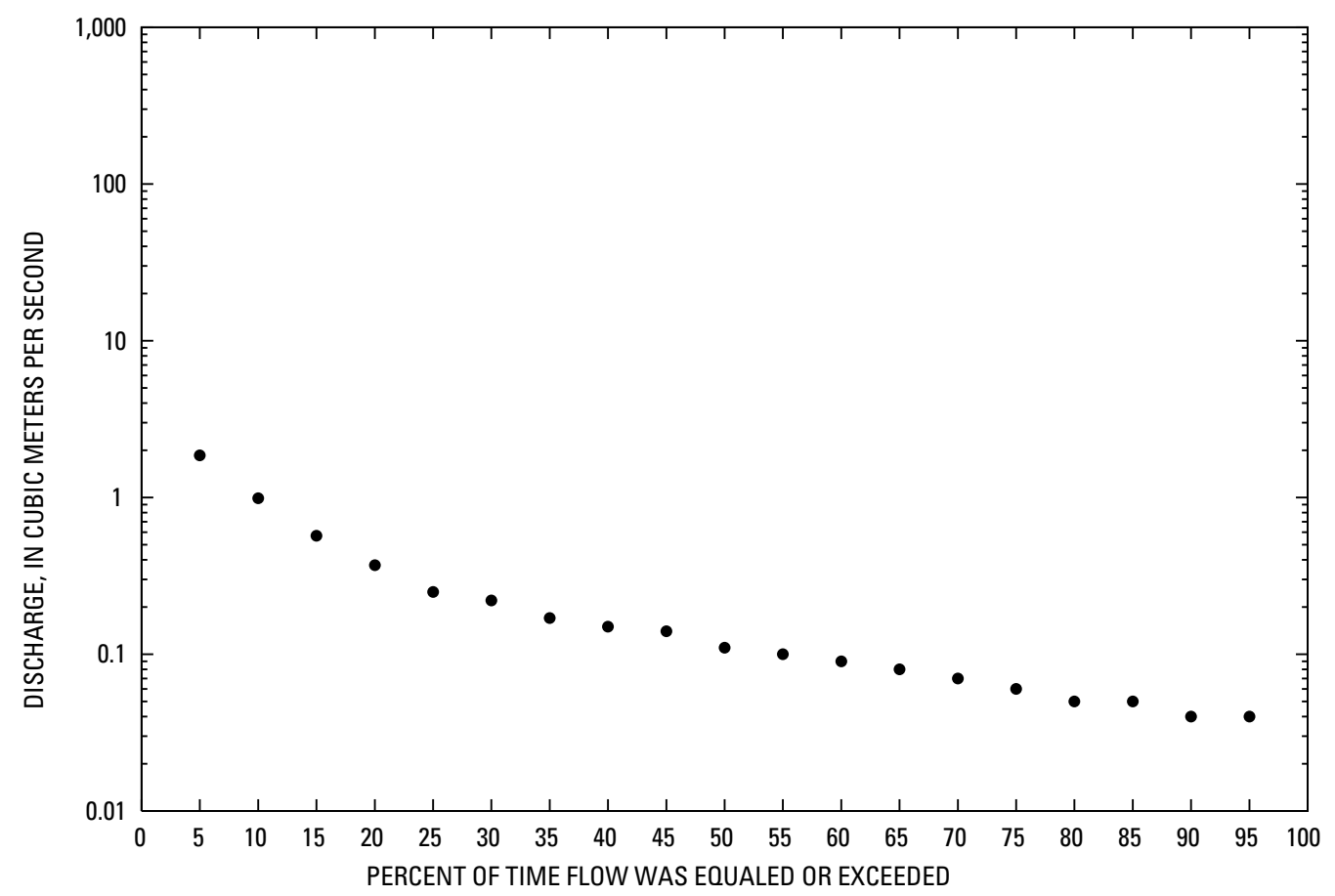




\section{3- 1.1L0-5T PARK RIVER NEAR PARK DASHT, Continued}

\section{Monthly and annual flow duration, in cubic meters per second}

[Data were not rounded in accordance with U.S. Geological Survey publication standards]

\begin{tabular}{|c|c|c|c|c|c|c|c|c|c|c|c|c|c|}
\hline $\begin{array}{l}\text { Percentage } \\
\text { of days } \\
\text { discharge } \\
\text { equaled or } \\
\text { exceeded }\end{array}$ & January & February & March & April & May & June & July & August & September & October & November & December & Annual \\
\hline 95 & 0.04 & 0.05 & 0.13 & 0.23 & 0.05 & 0.04 & 0.04 & 0.04 & 0.03 & 0.04 & 0.04 & 0.04 & 0.04 \\
\hline 90 & 0.05 & 0.06 & 0.16 & 0.3 & 0.08 & 0.04 & 0.04 & 0.04 & 0.03 & 0.04 & 0.04 & 0.05 & 0.04 \\
\hline 85 & 0.05 & 0.07 & 0.17 & 0.42 & 0.11 & 0.05 & 0.04 & 0.04 & 0.04 & 0.04 & 0.05 & 0.06 & 0.05 \\
\hline 80 & 0.08 & 0.09 & 0.18 & 0.58 & 0.12 & 0.05 & 0.04 & 0.04 & 0.04 & 0.04 & 0.05 & 0.07 & 0.05 \\
\hline 75 & 0.1 & 0.09 & 0.21 & 0.71 & 0.14 & 0.06 & 0.05 & 0.04 & 0.04 & 0.05 & 0.05 & 0.07 & 0.06 \\
\hline 70 & 0.1 & 0.1 & 0.23 & 0.75 & 0.15 & 0.06 & 0.05 & 0.05 & 0.04 & 0.05 & 0.05 & 0.07 & 0.07 \\
\hline 65 & 0.1 & 0.12 & 0.48 & 0.79 & 0.15 & 0.07 & 0.05 & 0.05 & 0.04 & 0.05 & 0.06 & 0.08 & 0.08 \\
\hline 60 & 0.11 & 0.14 & 0.54 & 0.85 & 0.16 & 0.08 & 0.05 & 0.05 & 0.04 & 0.06 & 0.06 & 0.08 & 0.09 \\
\hline 55 & 0.11 & 0.15 & 0.71 & 0.99 & 0.19 & 0.08 & 0.05 & 0.05 & 0.05 & 0.06 & 0.07 & 0.08 & 0.1 \\
\hline 50 & 0.11 & 0.16 & 0.88 & 1.04 & 0.2 & 0.1 & 0.06 & 0.05 & 0.05 & 0.06 & 0.07 & 0.09 & 0.11 \\
\hline 45 & 0.11 & 0.17 & 1.07 & 1.1 & 0.2 & 0.1 & 0.08 & 0.1 & 0.05 & 0.07 & 0.07 & 0.1 & 0.14 \\
\hline 40 & 0.12 & 0.19 & 1.73 & 1.47 & 0.27 & 0.11 & 0.18 & 0.11 & 0.05 & 0.07 & 0.07 & 0.1 & 0.15 \\
\hline 35 & 0.13 & 0.2 & 1.92 & 1.81 & 0.31 & 0.12 & 0.2 & 0.13 & 0.08 & 0.07 & 0.07 & 0.1 & 0.17 \\
\hline 30 & 0.13 & 0.25 & 2.05 & 1.9 & 0.4 & 0.15 & 0.32 & 0.2 & 0.1 & 0.08 & 0.08 & 0.11 & 0.22 \\
\hline 25 & 0.14 & 0.28 & 2.14 & 1.99 & 0.51 & 0.18 & 0.36 & 0.22 & 0.12 & 0.08 & 0.08 & 0.12 & 0.25 \\
\hline 20 & 0.15 & 0.5 & 2.23 & 2.31 & 0.56 & 0.2 & 0.4 & 0.26 & 0.13 & 0.08 & 0.08 & 0.13 & 0.37 \\
\hline 15 & 0.16 & 0.71 & 2.47 & 3.33 & 0.65 & 0.24 & 0.49 & 0.31 & 0.14 & 0.09 & 0.09 & 0.14 & 0.57 \\
\hline 10 & 0.16 & 0.84 & 3.07 & 5.16 & 0.92 & 0.28 & 0.56 & 0.43 & 0.15 & 0 & 0 & 0.15 & 0.99 \\
\hline 5 & 0.2 & 0 & 5.42 & 7.12 & 2.08 & 0.32 & 1.41 & 0.89 & 0.16 & 0 & 0 & 0.16 & 1.86 \\
\hline
\end{tabular}




\section{3- 1.1L0-5T PARK RIVER NEAR PARK DASHT, Continued}

Probability of occurrence of annual high discharges

$\left[\mathrm{m}^{3} / \mathrm{s}\right.$, meters per second; $\mathrm{ng}$, statistic not given]

\begin{tabular}{|c|c|c|c|c|c|c|}
\hline \multirow{2}{*}{$\begin{array}{c}\text { Exceedance } \\
\text { probability }\end{array}$} & \multirow{2}{*}{$\begin{array}{c}\text { Recurrence } \\
\text { interval } \\
\text { (years) }\end{array}$} & \multirow{2}{*}{$\begin{array}{c}\text { Maximum } \\
\text { instantaneous }{ }^{1} \\
\left(\mathrm{~m}^{3} / \mathrm{s}\right)\end{array}$} & \multicolumn{4}{|c|}{ Maximum mean discharge } \\
\hline & & & 3-day period & 7-day period & 15-day period & 30-day period \\
\hline 0.99 & 1.01 & 0.300 & ${ }^{2} 0.158$ & ${ }^{2} 0.170$ & ${ }^{2} 0.241$ & ${ }^{2} 0.266$ \\
\hline 0.95 & 1.05 & 1.1 & ${ }^{2} 0.421$ & ${ }^{2} 0.440$ & ${ }^{2} 0.463$ & ${ }^{2} 0.408$ \\
\hline 0.90 & 1.11 & 2.1 & ${ }^{2} 0.700$ & 0.708 & 0.652 & 0.520 \\
\hline 0.80 & 1.25 & 4.4 & 1.28 & 1.22 & 0.985 & 0.709 \\
\hline 0.50 & 2 & 17.1 & 3.94 & 3.17 & 2.13 & 1.34 \\
\hline 0.20 & 5 & 58.3 & 11.5 & 7.34 & 4.54 & 2.72 \\
\hline 0.10 & 10 & 106 & 19.9 & 10.9 & 6.68 & 4.03 \\
\hline 0.04 & 25 & 193 & 35.1 & 16.2 & 10.0 & 6.25 \\
\hline 0.02 & 50 & 279 & 50.3 & 20.5 & 13.0 & 8.40 \\
\hline 0.01 & 100 & 383 & 69.2 & 25.1 & 16.4 & 11.0 \\
\hline 0.005 & 200 & 508 & 92.3 & 29.9 & 20.3 & 14.3 \\
\hline 0.002 & 500 & 706 & ng & ng & ng & ng \\
\hline
\end{tabular}

'Less than 10 years of data used.

${ }^{2}$ Data does not fit log-Pearson Type III curve, use with caution.

3- 1.1L0-5T PARK RIVER NEAR PARK DASHT, Continued

Probability of occurrence of annual low discharges

$\left[\mathrm{m}^{3} / \mathrm{s}\right.$, meters per second]

\begin{tabular}{|c|c|c|c|c|c|c|c|c|c|c|}
\hline \multirow{3}{*}{$\begin{array}{c}\text { Nonexceedance } \\
\text { probability }\end{array}$} & \multirow{3}{*}{$\begin{array}{c}\text { Recurrence } \\
\text { interval } \\
\text { (years) }\end{array}$} & \multicolumn{9}{|c|}{ Minimum mean discharge $\left(\mathrm{m}^{3} / \mathrm{s}\right)$} \\
\hline & & \multicolumn{9}{|c|}{ Number of consecutive days } \\
\hline & & 1 & 3 & 7 & 14 & 30 & 60 & 90 & 120 & 183 \\
\hline 0.05 & 20 & 0.018 & 0.018 & 0.024 & 0.027 & 0.027 & 0.029 & 0.029 & 0.031 & 0.033 \\
\hline 0.10 & 10 & 0.021 & 0.021 & 0.026 & 0.029 & 0.030 & 0.032 & 0.032 & 0.035 & 0.039 \\
\hline 0.20 & 5 & 0.025 & 0.025 & 0.028 & 0.031 & 0.033 & 0.035 & 0.037 & 0.041 & 0.047 \\
\hline 0.50 & 2 & 0.032 & 0.032 & 0.033 & 0.036 & 0.040 & 0.045 & 0.052 & 0.057 & 0.070 \\
\hline
\end{tabular}




\section{3- 1.1L0-5T PARK RIVER NEAR PARK DASHT, Continued}

\section{Probability of occurrence of seasonal low discharges}

$\left[\mathrm{m}^{3} / \mathrm{s}\right.$, meters per second]

\begin{tabular}{|c|c|c|c|c|c|c|c|c|c|}
\hline \multirow{3}{*}{$\begin{array}{l}\text { Nonexceedance } \\
\text { probability }\end{array}$} & \multirow{3}{*}{$\begin{array}{c}\text { Recurrence } \\
\text { interval } \\
\text { (years) }\end{array}$} & \multicolumn{8}{|c|}{ Minimum mean discharge $\left(\mathrm{m}^{3} / \mathrm{s}\right)$} \\
\hline & & \multicolumn{8}{|c|}{ Number of consecutive days } \\
\hline & & 1 & 7 & 14 & 30 & 1 & 7 & 14 & 30 \\
\hline & & \multicolumn{4}{|c|}{ December-January-February } & \multicolumn{4}{|c|}{ March-April-May } \\
\hline 0.05 & 20 & 0.034 & 0.037 & 0.037 & 0.038 & 0.031 & ${ }^{1} 0.030$ & 0.046 & 0.062 \\
\hline 0.10 & 10 & 0.038 & 0.041 & 0.043 & 0.046 & 0.042 & 0.042 & 0.057 & 0.074 \\
\hline 0.20 & 5 & 0.044 & 0.048 & 0.051 & 0.057 & 0.061 & 0.063 & 0.076 & 0.097 \\
\hline 0.50 & 2 & 0.057 & 0.061 & 0.069 & 0.081 & 0.125 & 0.137 & 0.146 & 0.198 \\
\hline & & \multicolumn{4}{|c|}{ June-July-August } & \multicolumn{4}{|c|}{ September-October-November } \\
\hline 0.05 & 20 & 0.018 & 0.023 & 0.027 & 0.031 & 0.026 & 0.026 & 0.027 & 0.026 \\
\hline 0.10 & 10 & 0.020 & 0.025 & 0.028 & 0.033 & 0.028 & 0.028 & 0.029 & 0.029 \\
\hline 0.20 & 5 & 0.024 & 0.027 & 0.030 & 0.037 & 0.030 & 0.030 & 0.031 & 0.033 \\
\hline 0.50 & 2 & 0.036 & 0.037 & 0.041 & 0.053 & 0.037 & 0.037 & 0.039 & 0.044 \\
\hline
\end{tabular}

'Data does not fit log-Pearson Type III curve, use with caution.

\section{3- 1.1L0-5T PARK RIVER NEAR PARK DASHT, Continued}

\section{Annual peak discharge and corresponding gage height}

$\left[\mathrm{m}^{3} / \mathrm{s}\right.$, meters per second; --, no data]

\begin{tabular}{|c|c|c|c|c|c|c|c|}
\hline $\begin{array}{c}\text { Water } \\
\text { year }\end{array}$ & Date & $\begin{array}{c}\text { Gage } \\
\text { height } \\
\text { (meters) }\end{array}$ & $\begin{array}{c}\text { Peak } \\
\text { discharge } \\
\left(\mathrm{m}^{3} / \mathrm{s}\right)\end{array}$ & $\begin{array}{l}\text { Water } \\
\text { year }\end{array}$ & Date & $\begin{array}{c}\text { Gage } \\
\text { height } \\
\text { (meters) }\end{array}$ & $\begin{array}{c}\text { Peak } \\
\text { discharge } \\
\left(\mathrm{m}^{3} / \mathrm{s}\right)\end{array}$ \\
\hline \multicolumn{8}{|c|}{ Annual peak discharge, by year } \\
\hline 1968 & 10-Mar & - & 11.7 & 1977 & 30-Jun & & 13.0 \\
\hline 1969 & 10-Dec & - & 1.27 & 1978 & 6-Jul & & 100 \\
\hline 1970 & 25-Mar & - & 5.30 & 1979 & 1-Apr & & 25.0 \\
\hline 1976 & 13-Jul & - & 89.0 & & & & \\
\hline \multicolumn{8}{|c|}{ Annual peak discharge, from highest to lowest } \\
\hline 1978 & 6-Jul & - & 100 & 1968 & 10-Mar & & 11.7 \\
\hline 1976 & 13-Jul & - & 89.0 & 1970 & 25-Mar & & 5.30 \\
\hline 1979 & 1-Apr & - & 25.0 & 1969 & 10-Dec & & 1.27 \\
\hline 1977 & 30-Jun & - & 13.0 & & & & \\
\hline
\end{tabular}




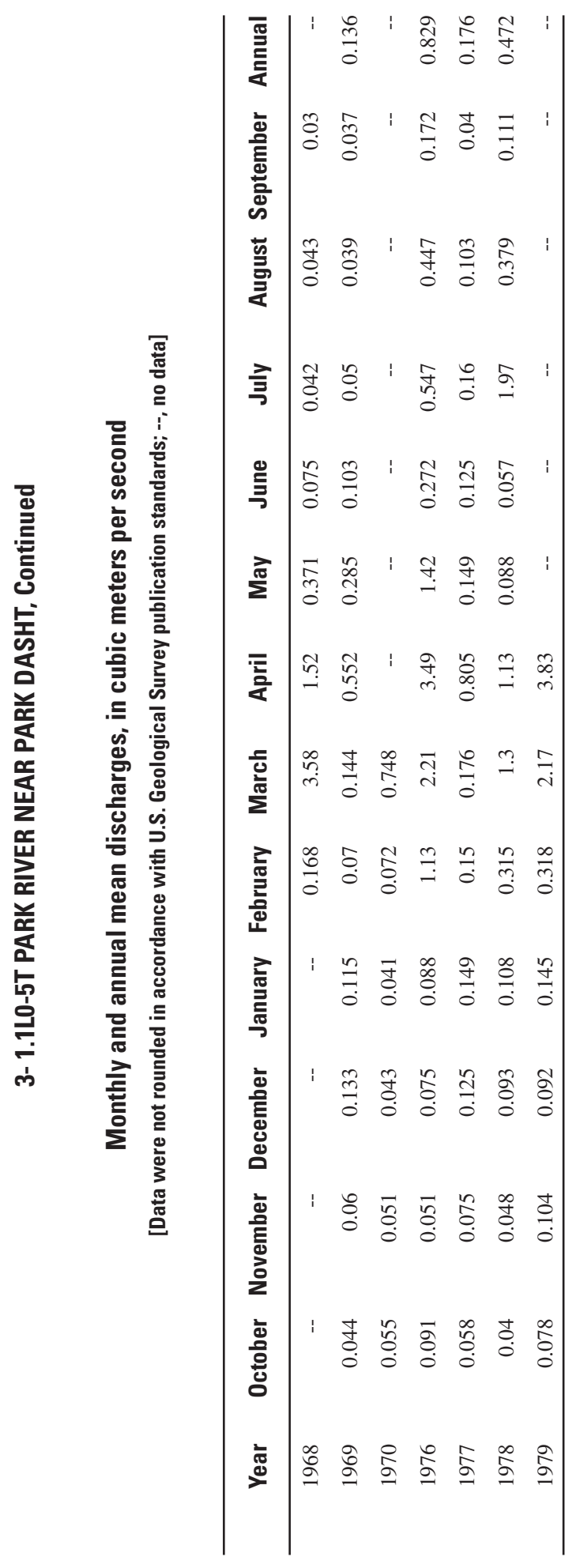




\section{3- 4.1L0-8T PALTU RIVER NEAR SARAFSAR}

\section{(U.S. Geological Survey identification number: 330800069050000 )}

LOCATION: Lat $33^{\circ} 08^{\prime} N$., long 6905'E., on right bank of Paltu River, $1 \mathrm{~km}$ downstream from the Paltu Dam, about $19 \mathrm{~km}$ east of Sarafsar, $51 \mathrm{~km}$ south of Gardez, and $72 \mathrm{~km}$ southeast of Ghazni.

DRAINAGE AREA: $105 \mathrm{~km}^{2}$.

ALTITUDE: 2,633 meters plus mean sea level.

PERIOD OF RECORD: May 1, 1949 to April 15, 1952.

GAGE: Water-stage recorder.

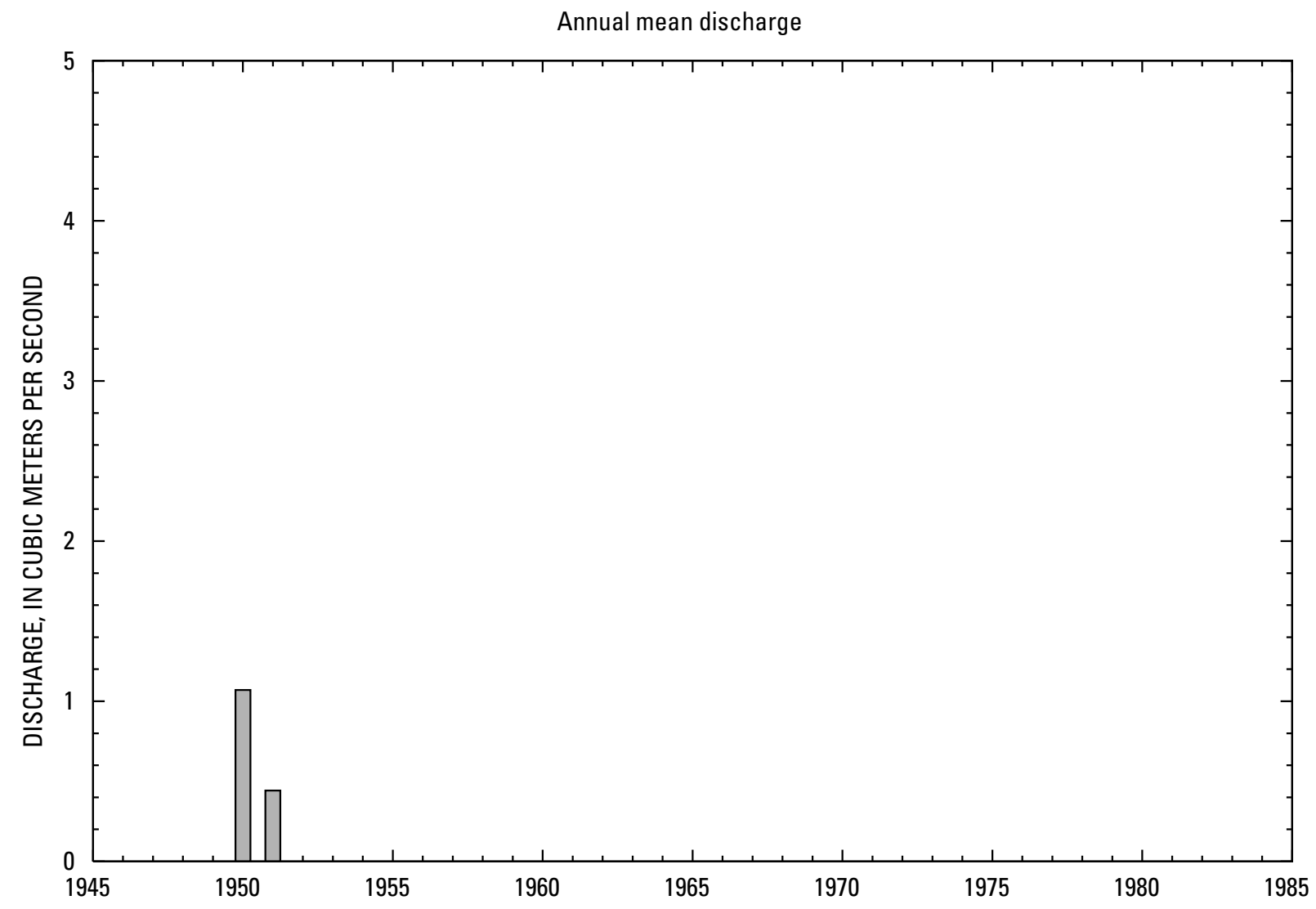




\section{3- 4.1L0-8T PALTU RIVER NEAR SARAFSAR, Continued}

\section{Statistics of monthly and annual mean discharges}

$\left[\mathrm{m}^{3} / \mathrm{s}\right.$, meters per second]

\begin{tabular}{|c|c|c|c|c|c|c|c|c|}
\hline \multirow[b]{2}{*}{ Month } & \multicolumn{2}{|c|}{ Maximum } & \multicolumn{2}{|c|}{ Minimum } & \multicolumn{4}{|c|}{ Mean } \\
\hline & $\begin{array}{c}\text { Discharge } \\
\left(\mathrm{m}^{3} / \mathrm{s}\right)\end{array}$ & $\begin{array}{c}\text { Water year } \\
\text { of } \\
\text { occurrence }\end{array}$ & $\begin{array}{c}\text { Discharge } \\
\left(\mathrm{m}^{3} / \mathrm{s}\right)\end{array}$ & $\begin{array}{c}\text { Water year } \\
\text { of } \\
\text { occurrence }\end{array}$ & $\begin{array}{c}\text { Discharge } \\
\left(\mathrm{m}^{3} / \mathrm{s}\right)\end{array}$ & $\begin{array}{c}\text { Standard } \\
\text { deviation } \\
\left(\mathrm{m}^{3} / \mathrm{s}\right)\end{array}$ & $\begin{array}{c}\text { Coefficient } \\
\text { of } \\
\text { variation }\end{array}$ & $\begin{array}{c}\text { Percentage } \\
\text { of annual } \\
\text { discharge }\end{array}$ \\
\hline October & 0.358 & 1952 & 0.070 & 1950 & 0.17 & 0.16 & 0.93 & 1.98 \\
\hline November & 0.406 & 1952 & 0.093 & 1950 & 0.22 & 0.16 & 0.74 & 2.53 \\
\hline December & 0.421 & 1952 & 0.090 & 1950 & 0.23 & 0.17 & 0.74 & 2.63 \\
\hline January & 0.429 & 1952 & 0.066 & 1950 & 0.22 & 0.19 & 0.84 & 2.54 \\
\hline February & 0.457 & 1952 & 0.126 & 1950 & 0.26 & 0.17 & 0.67 & 2.99 \\
\hline March & 1.15 & 1950 & 0.512 & 1951 & 0.74 & 0.36 & 0.48 & 8.46 \\
\hline April & 6.86 & 1950 & 1.01 & 1951 & 3.93 & 4.14 & 1.05 & 45.0 \\
\hline May & 3.55 & 1950 & 0.767 & 1949 & 1.75 & 1.56 & 0.89 & 20.0 \\
\hline June & 0.627 & 1951 & 0.344 & 1949 & 0.50 & 0.14 & 0.29 & 5.74 \\
\hline July & 0.591 & 1951 & 0.185 & 1950 & 0.33 & 0.23 & 0.69 & 3.77 \\
\hline August & 0.522 & 1951 & 0.086 & 1949 & 0.25 & 0.24 & 0.98 & 2.81 \\
\hline September & 0.290 & 1951 & 0.043 & 1950 & 0.13 & 0.14 & 1.05 & 1.50 \\
\hline Annual & 1.07 & 1950 & 0.442 & 1951 & 0.76 & 0.44 & 0.59 & 100 \\
\hline
\end{tabular}

Annual flow duration

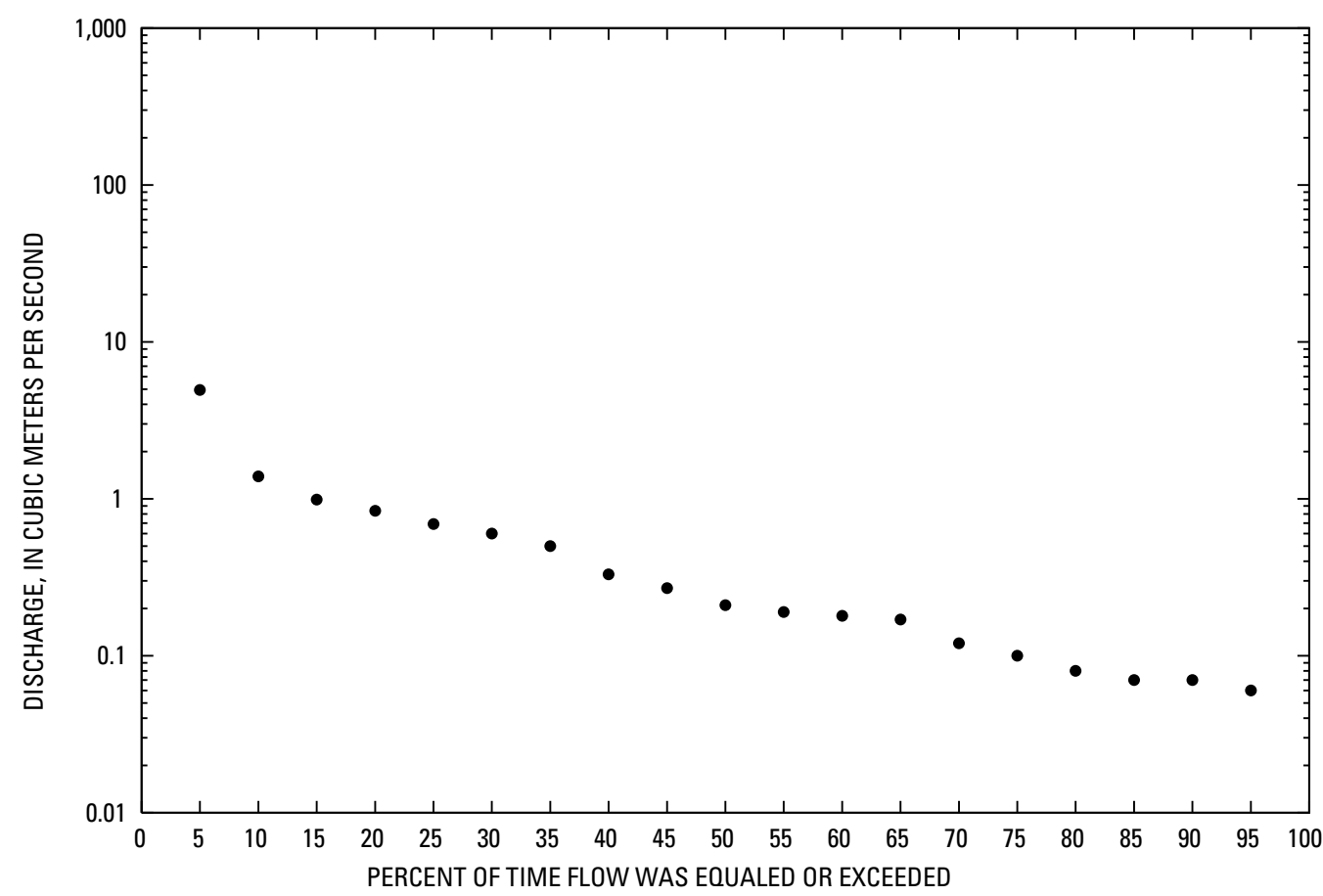




\section{3- 4.1L0-8T PALTU RIVER NEAR SARAFSAR, Continued}

Monthly and annual flow duration, in cubic meters per second

[Data were not rounded in accordance with U.S. Geological Survey publication standards]

\begin{tabular}{|c|c|c|c|c|c|c|c|c|c|c|c|c|c|}
\hline $\begin{array}{l}\text { Percentage } \\
\text { of days } \\
\text { discharge } \\
\text { equaled or } \\
\text { exceeded }\end{array}$ & January & February & March & April & May & June & July & August & September & October & November & December & Annual \\
\hline 95 & 0.06 & 0.06 & 0.24 & 0.88 & 0.59 & 0.21 & 0.11 & 0.06 & 0.03 & 0.06 & 0.08 & 0.08 & 0.06 \\
\hline 90 & 0.06 & 0.07 & 0.35 & 0.89 & 0.62 & 0.25 & 0.12 & 0.06 & 0.04 & 0.06 & 0.09 & 0.08 & 0.07 \\
\hline 85 & 0.07 & 0.11 & 0.45 & 0.91 & 0.74 & 0.27 & 0.14 & 0.06 & 0.04 & 0.06 & 0.09 & 0.09 & 0.07 \\
\hline 80 & 0.07 & 0.12 & 0.51 & 0.93 & 0.81 & 0.29 & 0.15 & 0.07 & 0.04 & 0.07 & 0.09 & 0.09 & 0.08 \\
\hline 75 & 0.08 & 0.19 & 0.53 & 0.94 & 0.83 & 0.31 & 0.15 & 0.07 & 0.06 & 0.07 & 0.11 & 0.11 & 0.1 \\
\hline 70 & 0.09 & 0.19 & 0.55 & 0.97 & 0.86 & 0.34 & 0.17 & 0.07 & 0.06 & 0.07 & 0.12 & 0.12 & 0.12 \\
\hline 65 & 0.17 & 0.2 & 0.56 & 1.04 & 0.88 & 0.38 & 0.18 & 0.08 & 0.06 & 0.07 & 0.14 & 0.17 & 0.17 \\
\hline 60 & 0.17 & 0.2 & 0.56 & 1.18 & 0.9 & 0.41 & 0.2 & 0.09 & 0.06 & 0.08 & 0.15 & 0.17 & 0.18 \\
\hline 55 & 0.17 & 0.2 & 0.57 & 1.29 & 0.92 & 0.42 & 0.21 & 0.11 & 0.07 & 0.09 & 0.17 & 0.17 & 0.19 \\
\hline 50 & 0.18 & 0.21 & 0.58 & 1.63 & 0.95 & 0.52 & 0.25 & 0.12 & 0.07 & 0.09 & 0.17 & 0.18 & 0.21 \\
\hline 45 & 0.18 & 0.21 & 0.59 & 4.87 & 1 & 0.62 & 0.26 & 0.15 & 0.07 & 0.12 & 0.18 & 0.18 & 0.27 \\
\hline 40 & 0.18 & 0.24 & 0.75 & 5.21 & 1.06 & 0.63 & 0.3 & 0.18 & 0.07 & 0.14 & 0.18 & 0.2 & 0.33 \\
\hline 35 & 0.18 & 0.25 & 0.77 & 5.45 & 1.17 & 0.63 & 0.54 & 0.29 & 0.08 & 0.15 & 0.2 & 0.21 & 0.5 \\
\hline 30 & 0 & 0.44 & 0.83 & 6.23 & 1.51 & 0.64 & 0.56 & 0.32 & 0 & 0.31 & 0.4 & 0 & 0.6 \\
\hline 25 & 0 & 0.45 & 0.87 & 6.66 & 1.98 & 0.64 & 0.57 & 0.41 & 0 & 0.33 & 0.41 & 0 & 0.69 \\
\hline 20 & 0 & 0.46 & 1.19 & 7.24 & 2.7 & 0.65 & 0.59 & 0.43 & 0 & 0 & 0.41 & 0 & 0.84 \\
\hline 15 & 0 & 0.47 & 1.25 & 7.92 & 4.18 & 0.66 & 0.6 & 0.47 & 0 & 0 & 0.41 & 0 & 0.99 \\
\hline 10 & 0 & 0.48 & 1.32 & 8.91 & 5.02 & 0.73 & 0.61 & 0.52 & 0 & 0 & 0.42 & 0 & 1.39 \\
\hline 5 & 0 & 0 & 1.47 & 0 & 5.3 & 0.93 & 0.64 & 0.54 & 0 & 0 & 0 & 0 & 4.95 \\
\hline
\end{tabular}




\section{3- 4.1L0-8T PALTU RIVER NEAR SARAFSAR, Continued}

\section{Probability of occurrence of annual high discharges}

[ $\mathrm{m}^{3} / \mathrm{s}$, meters per second; $\mathrm{ng}$, statistic not given]

\begin{tabular}{rrrrrrl}
\hline & & & \multicolumn{5}{c}{ Maximum mean discharge } \\
$\begin{array}{c}\text { Exceedance } \\
\text { probability }\end{array}$ & $\begin{array}{c}\text { Recurrence } \\
\text { interval } \\
\text { (years) }\end{array}$ & $\begin{array}{c}\text { Maximum } \\
\text { instantaneous } \\
\left(\mathbf{m}^{3} / \mathbf{s}\right)\end{array}$ & 3-day period & 7-day period & 15-day period & 30-day period \\
\cline { 5 - 7 } & 1.01 & $\mathrm{ng}$ & $\mathrm{ng}$ & $\mathrm{ng}$ & $\mathrm{ng}$ & $\mathrm{ng}$ \\
0.99 & 1.05 & $\mathrm{ng}$ & $\mathrm{ng}$ & $\mathrm{ng}$ & $\mathrm{ng}$ & $\mathrm{ng}$ \\
0.95 & 1.11 & $\mathrm{ng}$ & $\mathrm{ng}$ & $\mathrm{ng}$ & $\mathrm{ng}$ & $\mathrm{ng}$ \\
0.90 & 1.25 & $\mathrm{ng}$ & $\mathrm{ng}$ & $\mathrm{ng}$ & $\mathrm{ng}$ & $\mathrm{ng}$ \\
0.80 & 2 & $\mathrm{ng}$ & $\mathrm{ng}$ & $\mathrm{ng}$ & $\mathrm{ng}$ & $\mathrm{ng}$ \\
0.50 & 5 & $\mathrm{ng}$ & $\mathrm{ng}$ & $\mathrm{ng}$ & $\mathrm{ng}$ & $\mathrm{ng}$ \\
0.20 & 10 & $\mathrm{ng}$ & $\mathrm{ng}$ & $\mathrm{ng}$ & $\mathrm{ng}$ & $\mathrm{ng}$ \\
0.10 & 25 & $\mathrm{ng}$ & $\mathrm{ng}$ & $\mathrm{ng}$ & $\mathrm{ng}$ & $\mathrm{ng}$ \\
0.04 & 50 & $\mathrm{ng}$ & $\mathrm{ng}$ & $\mathrm{ng}$ & $\mathrm{ng}$ & $\mathrm{ng}$ \\
0.02 & 100 & $\mathrm{ng}$ & $\mathrm{ng}$ & $\mathrm{ng}$ & $\mathrm{ng}$ & $\mathrm{ng}$ \\
0.01 & 200 & $\mathrm{ng}$ & $\mathrm{ng}$ & $\mathrm{ng}$ & $\mathrm{ng}$ & $\mathrm{ng}$ \\
0.005 & 500 & $\mathrm{ng}$ & $\mathrm{ng}$ & $\mathrm{ng}$ & $\mathrm{ng}$ & $\mathrm{ng}$ \\
0.002 & & & & &
\end{tabular}

\section{3- 4.1L0-8T PALTU RIVER NEAR SARAFSAR, Continued}

Probability of occurrence of annual low discharges

[m³/s, meters per second; ng, statistic not given]

\begin{tabular}{|c|c|c|c|c|c|c|c|c|c|c|}
\hline \multirow{3}{*}{$\begin{array}{c}\text { Nonexceedance } \\
\text { probability }\end{array}$} & \multirow{3}{*}{$\begin{array}{c}\text { Recurrence } \\
\text { interval } \\
\text { (years) }\end{array}$} & \multicolumn{9}{|c|}{ Minimum mean discharge $\left(\mathrm{m}^{3} / \mathrm{s}\right)$} \\
\hline & & \multicolumn{9}{|c|}{ Number of consecutive days } \\
\hline & & 1 & 3 & 7 & 14 & 30 & 60 & 90 & 120 & 183 \\
\hline 0.05 & 20 & ng & ng & ng & ng & ng & ng & ng & ng & ng \\
\hline 0.10 & 10 & ng & ng & $\mathrm{ng}$ & ng & ng & ng & ng & $\mathrm{ng}$ & ng \\
\hline 0.20 & 5 & ng & ng & ng & ng & ng & ng & ng & ng & ng \\
\hline 0.50 & 2 & ng & ng & ng & ng & ng & ng & ng & ng & ng \\
\hline
\end{tabular}




\section{3- 4.1L0-8T PALTU RIVER NEAR SARAFSAR, Continued}

Probability of occurrence of seasonal low discharges

[ $\mathrm{m}^{3} / \mathrm{s}$, meters per second; $\mathrm{ng}$, statistic not given]

\begin{tabular}{|c|c|c|c|c|c|c|c|c|c|}
\hline \multirow{3}{*}{$\begin{array}{c}\text { Nonexceedance } \\
\text { probability }\end{array}$} & \multirow{3}{*}{$\begin{array}{c}\text { Recurrence } \\
\text { interval } \\
\text { (years) }\end{array}$} & \multicolumn{8}{|c|}{ Minimum mean discharge $\left(\mathrm{m}^{3} / \mathrm{s}\right)$} \\
\hline & & \multicolumn{8}{|c|}{ Number of consecutive days } \\
\hline & & 1 & 7 & 14 & 30 & 1 & 7 & 14 & 30 \\
\hline & & \multicolumn{4}{|c|}{ December-January-February } & \multicolumn{4}{|c|}{ March-April-May } \\
\hline 0.05 & 20 & ng & ng & ng & ng & ng & ng & ng & ng \\
\hline 0.10 & 10 & ng & ng & ng & ng & ng & ng & ng & ng \\
\hline 0.20 & 5 & ng & ng & ng & ng & ng & ng & ng & ng \\
\hline \multirow[t]{2}{*}{0.50} & 2 & ng & ng & ng & ng & ng & ng & ng & ng \\
\hline & & \multicolumn{4}{|c|}{ June-July-August } & \multicolumn{4}{|c|}{ September-October-November } \\
\hline 0.05 & 20 & ng & ng & ng & ng & ng & ng & ng & ng \\
\hline 0.10 & 10 & ng & $\mathrm{ng}$ & $\mathrm{ng}$ & $\mathrm{ng}$ & $\mathrm{ng}$ & ng & ng & ng \\
\hline 0.20 & 5 & ng & $\mathrm{ng}$ & $\mathrm{ng}$ & ng & ng & ng & ng & ng \\
\hline 0.50 & 2 & $\mathrm{ng}$ & $\mathrm{ng}$ & $\mathrm{ng}$ & $\mathrm{ng}$ & $\mathrm{ng}$ & ng & ng & ng \\
\hline
\end{tabular}

\section{3- 4.1L0-8T PALTU RIVER NEAR SARAFSAR, Continued}

Annual peak discharge and corresponding gage height

[ $\mathrm{m}^{3} / \mathrm{s}$, meters per second; --, no data]

\begin{tabular}{|c|c|c|c|c|c|c|c|}
\hline $\begin{array}{c}\text { Water } \\
\text { year }\end{array}$ & Date & $\begin{array}{c}\text { Gage } \\
\text { height } \\
\text { (meters) }\end{array}$ & $\begin{array}{c}\text { Peak } \\
\text { discharge } \\
\left(\mathrm{m}^{3} / \mathrm{s}\right)\end{array}$ & $\begin{array}{c}\text { Water } \\
\text { year }\end{array}$ & Date & $\begin{array}{c}\text { Gage } \\
\text { height } \\
\text { (meters) }\end{array}$ & $\begin{array}{c}\text { Peak } \\
\text { discharge } \\
\left(\mathrm{m}^{3} / \mathrm{s}\right)\end{array}$ \\
\hline \multicolumn{8}{|c|}{ Annual peak discharge, by year } \\
\hline 1950 & 3-Apr & 2.19 & 15.3 & & & & \\
\hline 1951 & 11-Aug & 2.24 & 22.1 & & & & \\
\hline \multicolumn{8}{|c|}{ Annual peak discharge, from highest to lowest } \\
\hline 1951 & 11-Aug & 2.24 & 22.1 & & & & \\
\hline 1950 & 3-Apr & 2.19 & 15.3 & & & & \\
\hline
\end{tabular}




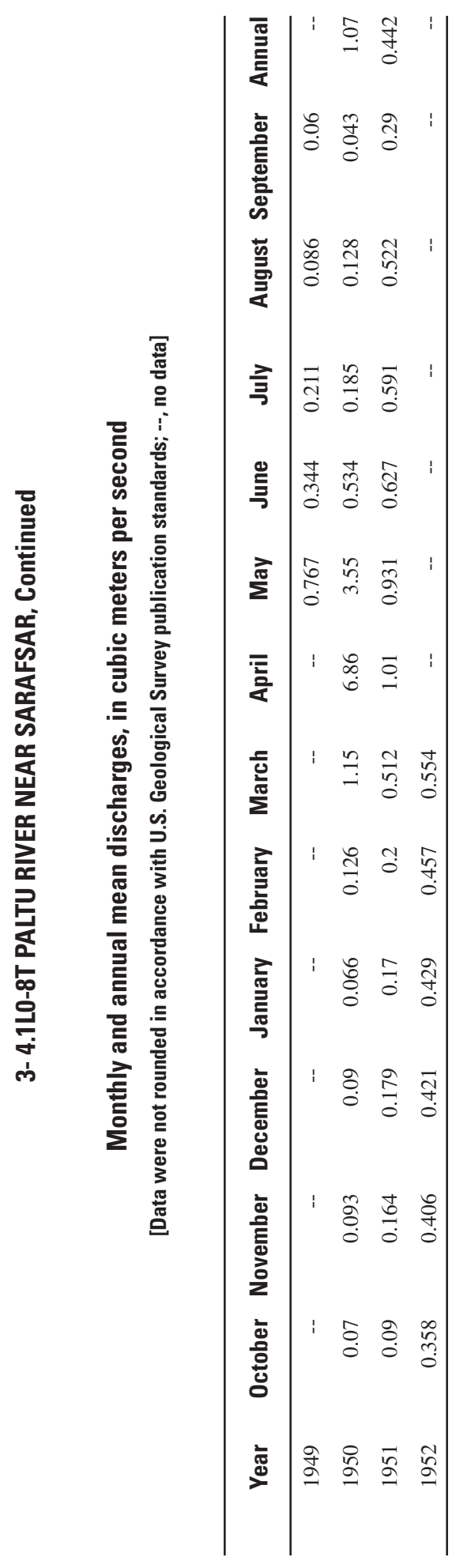




\section{3- 4.LO0-1A PALTU RIVER ABOVE SARDE RESERVOIR}

\section{(U.S. Geological Survey identification number: 331600068420000)}

LOCATION: Lat $33^{\circ} 16^{\prime}$ N., long $68^{\circ} 42^{\prime}$ E., on right bank 1 km upstream from Sarde Dam, about $51 \mathrm{~km}$ southwest of Gardez.

DRAINAGE AREA: $805 \mathrm{~km}^{2}$.

ALTITUDE: 2,115 meters plus mean sea level.

PERIOD OF RECORD: May 6, 1969 to September 30, 1980.

GAGE: Water-stage recorder.

Annual mean discharge

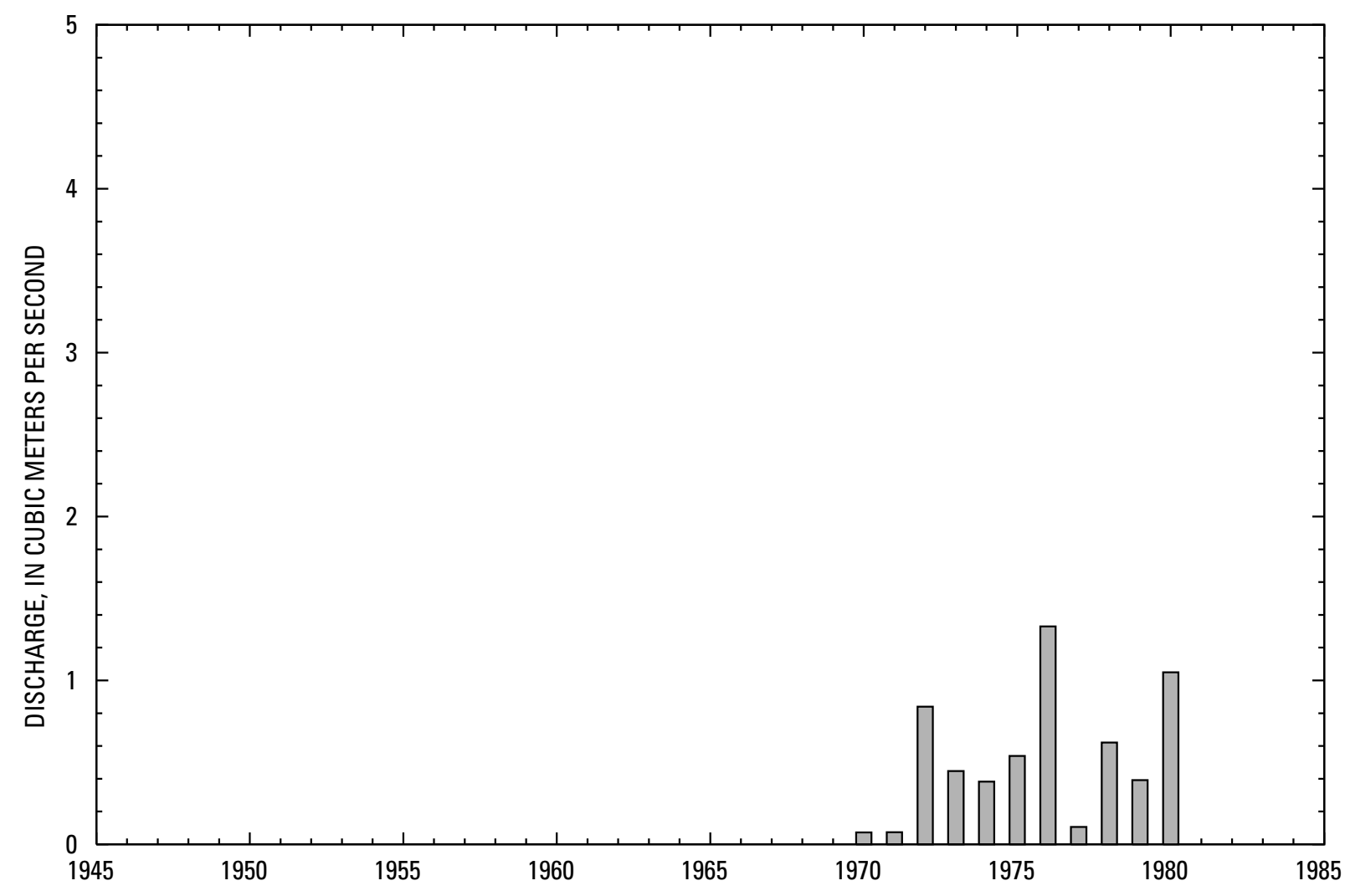




\section{3- 4.LOO-1A PALTU RIVER ABOVE SARDE RESERVOIR, Continued}

\section{Statistics of monthly and annual mean discharges}

[ $\mathrm{m}^{3} / \mathrm{s}$, meters per second; $\mathrm{m}$, more than 2 years of occurrence; $\mathrm{ng}$, no data]

\begin{tabular}{|c|c|c|c|c|c|c|c|c|}
\hline \multirow[b]{2}{*}{ Month } & \multicolumn{2}{|c|}{ Maximum } & \multicolumn{2}{|c|}{ Minimum } & \multicolumn{4}{|c|}{ Mean } \\
\hline & $\begin{array}{c}\text { Discharge } \\
\left(\mathrm{m}^{3} / \mathrm{s}\right)\end{array}$ & $\begin{array}{c}\text { Water year } \\
\text { of } \\
\text { occurrence }\end{array}$ & $\begin{array}{c}\text { Discharge } \\
\left(\mathrm{m}^{3} / \mathrm{s}\right)\end{array}$ & $\begin{array}{c}\text { Water year } \\
\text { of } \\
\text { occurrence }\end{array}$ & $\begin{array}{c}\text { Discharge } \\
\left(\mathrm{m}^{3} / \mathrm{s}\right)\end{array}$ & $\begin{array}{c}\text { Standard } \\
\text { deviation } \\
\left(\mathrm{m}^{3} / \mathrm{s}\right)\end{array}$ & $\begin{array}{c}\text { Coefficient } \\
\text { of } \\
\text { variation }\end{array}$ & $\begin{array}{c}\text { Percentage } \\
\text { of annual } \\
\text { discharge }\end{array}$ \\
\hline October & 0 & $\mathrm{~m}$ & 0 & $\mathrm{~m}$ & 0 & 0 & ng & 0 \\
\hline November & 0.404 & 1978 & 0 & $\mathrm{~m}$ & 0.05 & 0.12 & 2.63 & 0.72 \\
\hline December & 0.167 & 1979 & 0 & 1972 & 0.06 & 0.06 & 0.95 & 1.03 \\
\hline January & 0.271 & 1979 & 0.010 & $\mathrm{~m}$ & 0.13 & 0.11 & 0.89 & 2.02 \\
\hline February & 2.32 & 1973 & 0.010 & 1974,1975 & 0.50 & 0.70 & 1.40 & 7.95 \\
\hline March & 3.15 & 1980 & 0.091 & 1977 & 1.53 & 1.26 & 0.82 & 24.2 \\
\hline April & 8.14 & 1976 & 0 & 1970, 1971 & 2.45 & 2.72 & 1.11 & 38.8 \\
\hline May & 3.13 & 1976 & 0 & $\mathrm{~m}$ & 0.67 & 1.14 & 1.70 & 10.6 \\
\hline June & 0.219 & 1972 & 0 & $\mathrm{~m}$ & 0.02 & 0.06 & 3.46 & 0.29 \\
\hline July & 3.15 & 1978 & 0 & $\mathrm{~m}$ & 0.39 & 0.91 & 2.37 & 6.11 \\
\hline August & 2.54 & 1978 & 0 & $\mathrm{~m}$ & 0.48 & 0.77 & 1.60 & 7.63 \\
\hline September & 0.324 & 1976 & 0 & $\mathrm{~m}$ & 0.05 & 0.11 & 2.38 & 0.73 \\
\hline Annual & 1.33 & 1976 & 0.073 & 1970 & 0.53 & 0.41 & 0.77 & 100 \\
\hline
\end{tabular}

Annual flow duration

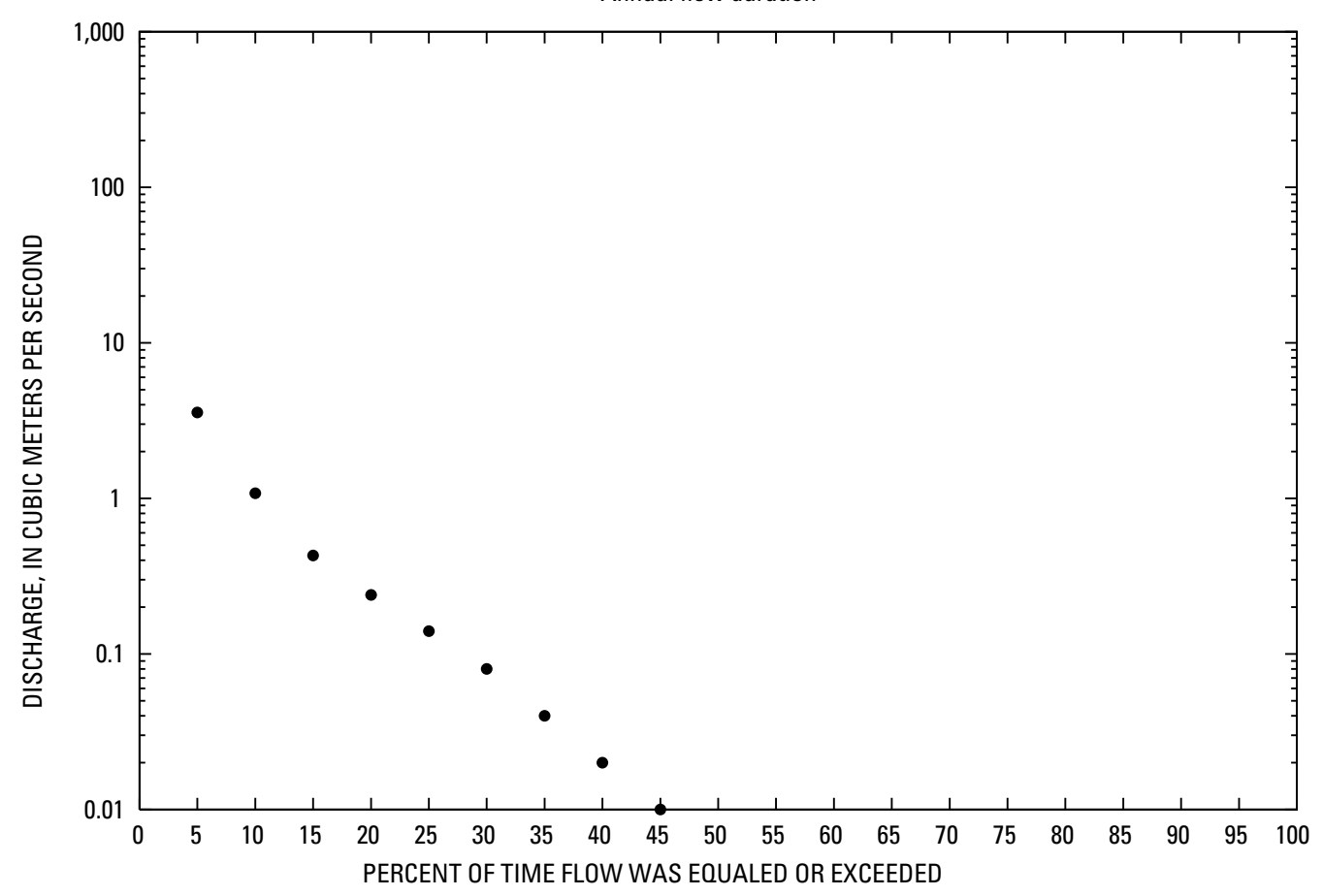




\section{3- 4.L00-1A PALTU RIVER ABOVE SARDE RESERVOIR, Continued}

Monthly and annual flow duration, in cubic meters per second

[Data were not rounded in accordance with U.S. Geological Survey publication standards]

\begin{tabular}{|c|c|c|c|c|c|c|c|c|c|c|c|c|c|}
\hline $\begin{array}{l}\text { Percentage } \\
\text { of days } \\
\text { discharge } \\
\text { equaled or } \\
\text { exceeded }\end{array}$ & January & February & March & April & May & June & July & August & September & October & November & December & Annual \\
\hline 95 & 0.01 & 0.01 & 0 & 0 & 0 & 0 & 0 & 0 & 0 & 0 & 0 & 0 & 0 \\
\hline 90 & 0.01 & 0.01 & 0.02 & 0 & 0 & 0 & 0 & 0 & 0 & 0 & 0 & 0 & 0 \\
\hline 80 & 0.02 & 0.02 & 0.13 & 0 & 0 & 0 & 0 & 0 & 0 & 0 & 0 & 0.01 & 0 \\
\hline 75 & 0.02 & 0.03 & 0.17 & 0.01 & 0 & 0 & 0 & 0 & 0 & 0 & 0 & 0.01 & 0 \\
\hline 70 & 0.04 & 0.05 & 0.24 & 0.02 & 0 & 0 & 0 & 0 & 0 & 0 & 0 & 0.01 & 0 \\
\hline 65 & 0.05 & 0.05 & 0.36 & 0.06 & 0 & 0 & 0 & 0 & 0 & 0 & 0 & 0.02 & 0 \\
\hline 60 & 0.08 & 0.06 & 0.45 & 0.33 & 0 & 0 & 0 & 0 & 0 & 0 & 0 & 0.02 & 0 \\
\hline 40 & 0.14 & 0.25 & 1.08 & 1.66 & 0 & 0 & 0 & 0 & 0 & 0 & 0 & 0.06 & 0.02 \\
\hline 35 & 0.15 & 0.28 & 1.83 & 2.58 & 0 & 0 & 0 & 0 & 0 & 0 & 0 & 0.09 & 0.04 \\
\hline 30 & 0.16 & 0.31 & 2.44 & 3.19 & 0 & 0 & 0 & 0 & 0 & 0 & 0 & 0.1 & 0.08 \\
\hline 25 & 0.2 & 0.35 & 2.75 & 3.71 & 0.01 & 0 & 0 & 0 & 0 & 0 & 0 & 0.13 & 0.14 \\
\hline 20 & 0.26 & 0.4 & 2.99 & 4.9 & 0.13 & 0 & 0 & 0.01 & 0 & 0 & 0 & 0.14 & 0.24 \\
\hline 15 & 0.3 & 0.66 & 3.3 & 5.93 & 1.02 & 0 & 0.02 & 0.02 & 0 & 0 & 0.03 & 0.15 & 0.43 \\
\hline 10 & 0.35 & 0.91 & 4.11 & 7.13 & 3.71 & 0 & 0.12 & 0.43 & 0 & 0 & 0.06 & 0.16 & 1.08 \\
\hline 5 & 0.38 & 2.46 & 4.62 & 8.75 & 5.25 & 0 & 1.55 & 2.83 & 0.02 & 0 & 0.1 & 0.22 & 3.57 \\
\hline
\end{tabular}




\section{3- 4.L00-1A PALTU RIVER ABOVE SARDE RESERVOIR, Continued}

Probability of occurrence of annual high discharges

[ $\mathrm{m}^{3} / \mathrm{s}$, meters per second; $\mathrm{ng}$, statistic not given]

\begin{tabular}{|c|c|c|c|c|c|c|}
\hline \multirow{2}{*}{$\begin{array}{c}\text { Exceedance } \\
\text { probability }\end{array}$} & \multirow{2}{*}{$\begin{array}{c}\text { Recurrence } \\
\text { interval } \\
\text { (years) }\end{array}$} & \multirow{2}{*}{$\begin{array}{c}\text { Maximum } \\
\text { instantaneous } \\
\left(\mathrm{m}^{3} / \mathrm{s}\right)\end{array}$} & \multicolumn{4}{|c|}{ Maximum mean discharge } \\
\hline & & & 3-day period & 7-day period & 15-day period & 30-day period \\
\hline 0.99 & 1.01 & 0.200 & ${ }^{1} 0.080$ & ${ }^{1} 0.073$ & ${ }^{1} 0.088$ & ${ }^{1} 0.083$ \\
\hline 0.95 & 1.05 & 1.00 & 0.468 & 0.387 & 0.350 & 0.279 \\
\hline 0.90 & 1.11 & 2.10 & 1.03 & 0.817 & 0.661 & 0.497 \\
\hline 0.80 & 1.25 & 4.60 & 2.37 & 1.79 & 1.30 & 0.935 \\
\hline 0.50 & 2 & 15.7 & 7.92 & 5.61 & 3.67 & 2.59 \\
\hline 0.20 & 5 & 38.2 & 17.2 & 11.7 & 7.58 & 5.68 \\
\hline 0.10 & 10 & 53.8 & 22.3 & 15.0 & 9.95 & 7.88 \\
\hline 0.04 & 25 & 71.6 & 27.0 & 18.0 & 12.4 & 10.5 \\
\hline 0.02 & 50 & 82.8 & 29.4 & 19.6 & 13.8 & 12.3 \\
\hline 0.01 & 100 & 92.2 & 31.1 & 20.7 & 15.0 & 14.0 \\
\hline 0.005 & 200 & 100 & 32.3 & 21.5 & 15.9 & 15.4 \\
\hline 0.002 & 500 & 108 & $\mathrm{ng}$ & ng & $\mathrm{ng}$ & $\mathrm{ng}$ \\
\hline
\end{tabular}

'Data does not fit log-Pearson Type III curve, use with caution.

\section{3- 4.L00-1A PALTU RIVER ABOVE SARDE RESERVOIR, Continued}

Probability of occurrence of annual low discharges

[m³/s, meters per second; ng, statistic not given]

\begin{tabular}{|c|c|c|c|c|c|c|c|c|c|c|}
\hline \multirow{3}{*}{$\begin{array}{c}\text { Nonexceedance } \\
\text { probability }\end{array}$} & \multirow{3}{*}{$\begin{array}{c}\text { Recurrence } \\
\text { interval } \\
\text { (years) }\end{array}$} & \multicolumn{9}{|c|}{ Minimum mean discharge $\left(\mathrm{m}^{3} / \mathrm{s}\right)$} \\
\hline & & \multicolumn{9}{|c|}{ Number of consecutive days } \\
\hline & & 1 & 3 & 7 & 14 & 30 & 60 & 90 & 120 & 183 \\
\hline 0.05 & 20 & ng & ng & ng & ng & ng & ng & ng & 0 & 0 \\
\hline 0.10 & 10 & ng & ng & ng & ng & ng & ng & ng & 0 & 0 \\
\hline 0.20 & 5 & ng & ng & ng & ng & ng & ng & ng & 0 & 0 \\
\hline 0.50 & 2 & ng & ng & ng & ng & ng & ng & ng & 0.003 & 0.019 \\
\hline
\end{tabular}


Probability of occurrence of seasonal low discharges

[ $\mathrm{m}^{3} / \mathrm{s}$, meters per second; $\mathbf{n g}$, statistic not given]

\begin{tabular}{|c|c|c|c|c|c|c|c|c|c|}
\hline \multirow{3}{*}{$\begin{array}{l}\text { Nonexceedance } \\
\text { probability }\end{array}$} & \multirow{3}{*}{$\begin{array}{c}\text { Recurrence } \\
\text { interval } \\
\text { (years) }\end{array}$} & \multicolumn{8}{|c|}{ Minimum mean discharge $\left(\mathrm{m}^{3} / \mathrm{s}\right)$} \\
\hline & & \multicolumn{8}{|c|}{ Number of consecutive days } \\
\hline & & 1 & 7 & 14 & 30 & 1 & 7 & 14 & 30 \\
\hline & & \multicolumn{4}{|c|}{ December-January-February } & \multicolumn{4}{|c|}{ March-April-May } \\
\hline 0.05 & 20 & 0 & 0 & 0 & 0 & ng & ng & ng & 0 \\
\hline 0.10 & 10 & 0 & 0 & 0.001 & 0.002 & ng & ng & $\mathrm{ng}$ & 0 \\
\hline 0.20 & 5 & 0 & 0 & 0.004 & 0.009 & ng & ng & ng & 0 \\
\hline \multirow[t]{2}{*}{0.50} & 2 & 0.008 & 0.012 & 0.017 & 0.033 & ng & ng & ng & 0 \\
\hline & & \multicolumn{4}{|c|}{ June-July-August } & \multicolumn{4}{|c|}{ September-October-November } \\
\hline 0.05 & 20 & ng & ng & ng & ng & ng & ng & ng & $\mathrm{ng}$ \\
\hline 0.10 & 10 & $\mathrm{ng}$ & $\mathrm{ng}$ & $\mathrm{ng}$ & $\mathrm{ng}$ & $\mathrm{ng}$ & $\mathrm{ng}$ & $\mathrm{ng}$ & ng \\
\hline 0.20 & 5 & $\mathrm{ng}$ & ng & ng & ng & ng & ng & $\mathrm{ng}$ & ng \\
\hline 0.50 & 2 & $\mathrm{ng}$ & $\mathrm{ng}$ & $\mathrm{ng}$ & $\mathrm{ng}$ & $\mathrm{ng}$ & ng & ng & $\mathrm{ng}$ \\
\hline
\end{tabular}

3- 4.L00-1A PALTU RIVER ABOVE SARDE RESERVOIR, Continued

Annual peak discharge and corresponding gage height

[ $\mathrm{m}^{3} / \mathrm{s}$, meters per second; --, no data]

\begin{tabular}{|c|c|c|c|c|c|c|c|}
\hline $\begin{array}{c}\text { Water } \\
\text { year }\end{array}$ & Date & $\begin{array}{c}\text { Gage } \\
\text { height } \\
\text { (meters) }\end{array}$ & $\begin{array}{c}\text { Peak } \\
\text { discharge } \\
\left(\mathrm{m}^{3} / \mathrm{s}\right)\end{array}$ & $\begin{array}{c}\text { Water } \\
\text { year }\end{array}$ & Date & $\begin{array}{c}\text { Gage } \\
\text { height } \\
\text { (meters) }\end{array}$ & $\begin{array}{c}\text { Peak } \\
\text { discharge } \\
\left(\mathrm{m}^{3} / \mathrm{s}\right)\end{array}$ \\
\hline
\end{tabular}

Annual peak discharge, by year

\begin{tabular}{|c|c|c|c|c|c|c|c|}
\hline 1970 & 18-Feb & -- & 1.04 & 1976 & 25-Jul & -- & 16.5 \\
\hline 1971 & 13-May & -- & 1.10 & 1977 & 17-Apr & -- & 12.7 \\
\hline 1972 & 27-Apr & -- & 46.4 & 1978 & 20-Aug & -- & 16.5 \\
\hline 1973 & 5-Aug & -- & 62.1 & 1979 & 30-Mar & -- & 14.4 \\
\hline 1974 & 8-Apr & -- & 28.0 & 1980 & 29-Mar & -- & 8.60 \\
\hline 1975 & 19-Aug & -- & 25.1 & & & & \\
\hline \multicolumn{8}{|c|}{ Annual peak discharge, from highest to lowest } \\
\hline 1973 & 5-Aug & -- & 62.1 & 1979 & 30-Mar & -- & 14.4 \\
\hline 1972 & 27-Apr & -- & 46.4 & 1977 & 17-Apr & -- & 12.7 \\
\hline 1974 & 8-Apr & -- & 28.0 & 1980 & 29-Mar & -- & 8.60 \\
\hline 1975 & 19-Aug & -- & 25.1 & 1971 & 13-May & -- & 1.10 \\
\hline 1978 & 20-Aug & -- & 16.5 & 1970 & $18-\mathrm{Feb}$ & -- & 1.04 \\
\hline 1976 & 25-Jul & -- & 16.5 & & & & \\
\hline
\end{tabular}




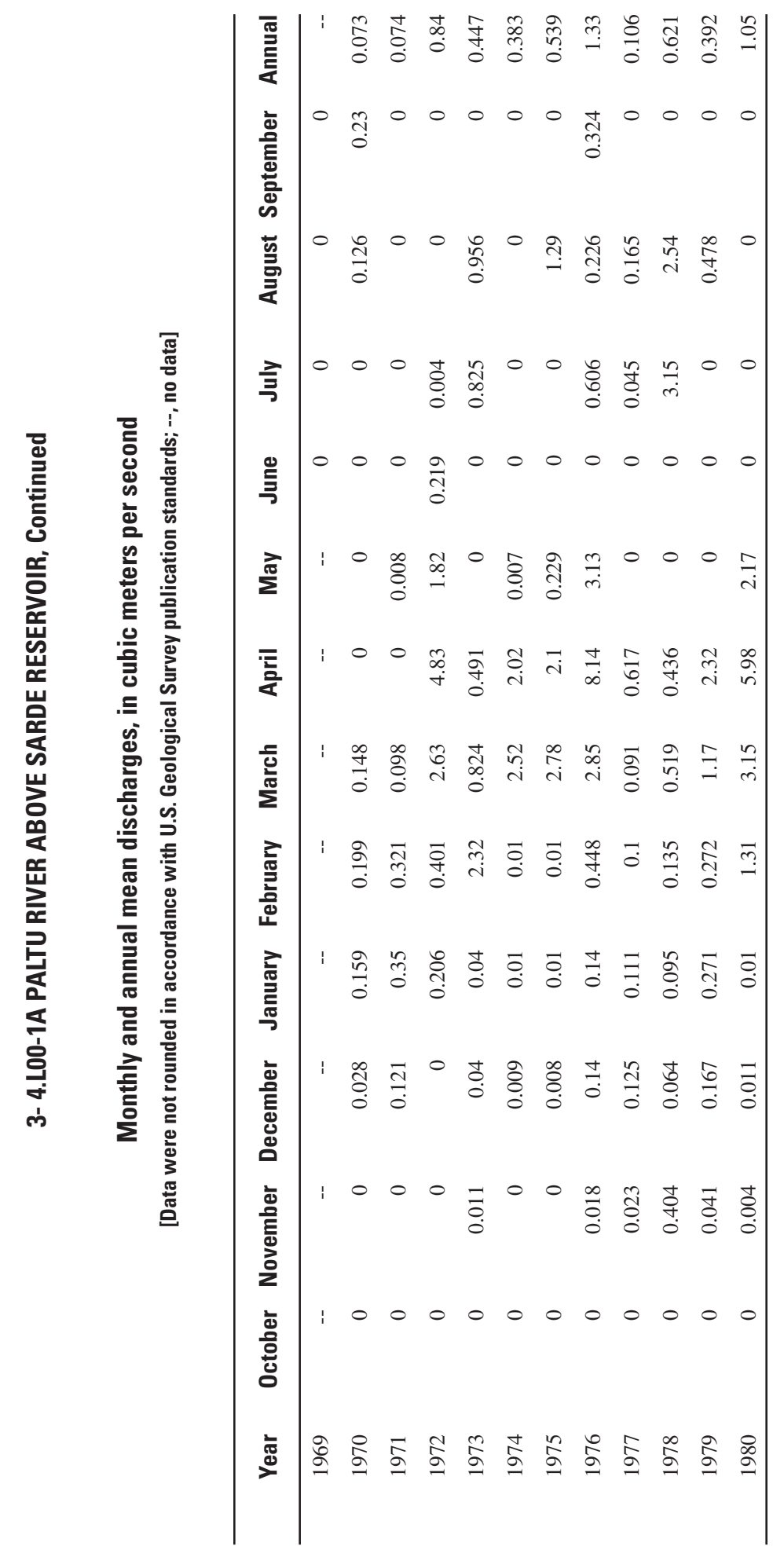




\section{3- 4.LO0-2A JILGA RIVER BELOW SARDE RESERVOIR}

\section{(U.S. Geological Survey identification number: 331800068370000 )}

LOCATION: Lat $33^{\circ} 18^{\prime} \mathrm{N}$., long 68³7'E.

DRAINAGE AREA: $4,340 \mathrm{~km}^{2}$.

ALTITUDE: 2,086 meters plus mean sea level.

PERIOD OF RECORD: April 8, 1969 to September 30, 1979.

GAGE: --

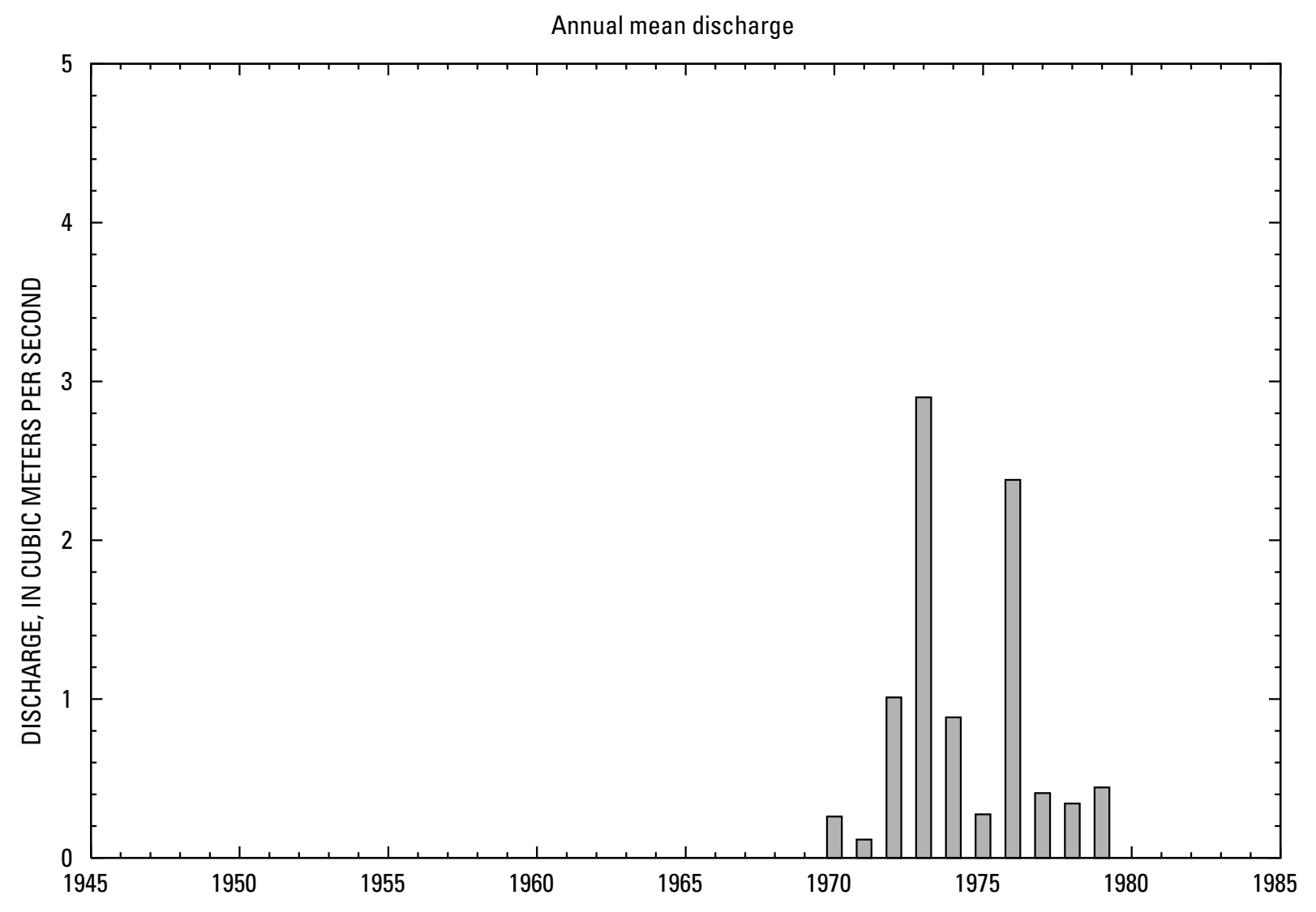




\section{3- 4.L00-2A JILGA RIVER BELOW SARDE RESERVOIR, Continued}

\section{Statistics of monthly and annual mean discharges}

$\left[\mathrm{m}^{3} / \mathrm{s}\right.$, meters per second]

\begin{tabular}{|c|c|c|c|c|c|c|c|c|}
\hline \multirow[b]{2}{*}{ Month } & \multicolumn{2}{|c|}{ Maximum } & \multicolumn{2}{|c|}{ Minimum } & \multicolumn{4}{|c|}{ Mean } \\
\hline & $\begin{array}{c}\text { Discharge } \\
\left(\mathrm{m}^{3} / \mathrm{s}\right)\end{array}$ & $\begin{array}{c}\text { Water year } \\
\text { of } \\
\text { occurrence }\end{array}$ & $\begin{array}{c}\text { Discharge } \\
\left(\mathrm{m}^{3} / \mathrm{s}\right)\end{array}$ & $\begin{array}{c}\text { Water year } \\
\text { of } \\
\text { occurrence }\end{array}$ & $\begin{array}{c}\text { Discharge } \\
\left(\mathrm{m}^{3} / \mathrm{s}\right)\end{array}$ & $\begin{array}{c}\text { Standard } \\
\text { deviation } \\
\left(\mathrm{m}^{3} / \mathrm{s}\right)\end{array}$ & $\begin{array}{c}\text { Coefficient } \\
\text { of } \\
\text { variation }\end{array}$ & $\begin{array}{c}\text { Percentage } \\
\text { of annual } \\
\text { discharge }\end{array}$ \\
\hline October & 0.393 & 1977 & 0.039 & 1972 & 0.22 & 0.11 & 0.51 & 2.05 \\
\hline November & 0.324 & 1977 & 0.070 & 1970 & 0.21 & 0.09 & 0.44 & 1.93 \\
\hline December & 0.320 & 1977 & 0.067 & 1972 & 0.21 & 0.09 & 0.42 & 1.92 \\
\hline January & 0.301 & 1979 & 0.050 & 1972 & 0.21 & 0.09 & 0.44 & 1.94 \\
\hline February & 0.786 & 1973 & 0.074 & 1972 & 0.28 & 0.21 & 0.72 & 2.67 \\
\hline March & 18.5 & 1973 & 0.191 & 1972 & 2.59 & 5.74 & 2.22 & 24.3 \\
\hline April & 21.9 & 1976 & 0.210 & 1971 & 4.04 & 7.09 & 1.76 & 37.8 \\
\hline May & 7.98 & 1972 & 0.104 & 1971 & 1.56 & 2.45 & 1.57 & 14.6 \\
\hline June & 1.47 & 1972 & 0.108 & 1971 & 0.50 & 0.39 & 0.79 & 4.65 \\
\hline July & 0.675 & 1973 & 0.108 & 1971 & 0.36 & 0.19 & 0.51 & 3.39 \\
\hline August & 0.477 & 1974 & 0.052 & 1971 & 0.27 & 0.14 & 0.49 & 2.58 \\
\hline September & 0.361 & 1976 & 0.100 & 1970 & 0.23 & 0.10 & 0.41 & 2.20 \\
\hline Annual & 2.90 & 1973 & 0.115 & 1971 & 0.90 & 0.97 & 1.07 & 100 \\
\hline
\end{tabular}

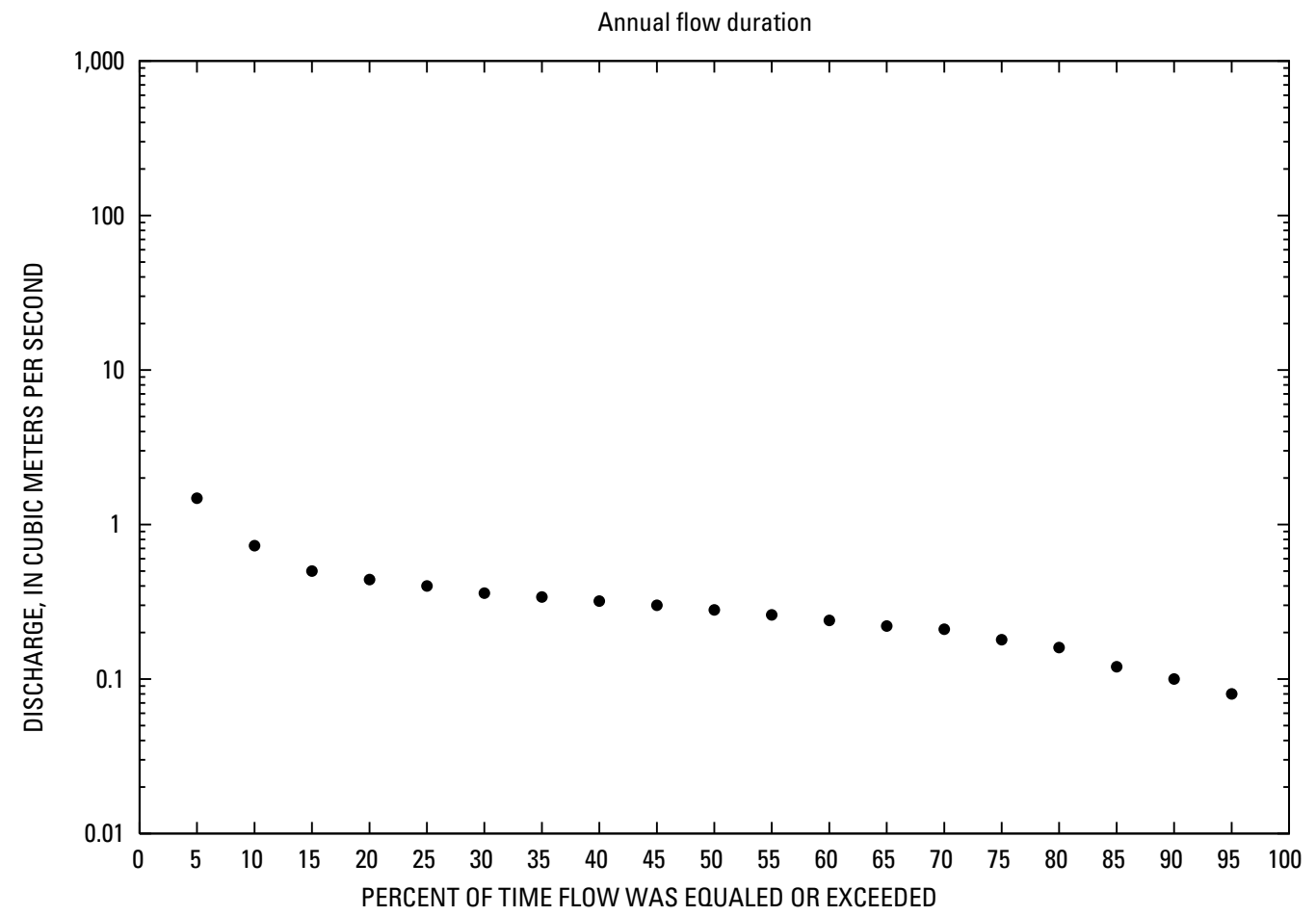




\section{3- 4.L00-2A JILGA RIVER BELOW SARDE RESERVOIR, Continued}

\section{Monthly and annual flow duration, in cubic meters per second}

[Data were not rounded in accordance with U.S. Geological Survey publication standards]

\begin{tabular}{|c|c|c|c|c|c|c|c|c|c|c|c|c|c|}
\hline $\begin{array}{l}\text { Percentage } \\
\text { of days } \\
\text { discharge } \\
\text { equaled or } \\
\text { exceeded }\end{array}$ & January & February & March & April & May & June & July & August & September & October & November & December & Annual \\
\hline 95 & 0.05 & 0.07 & 0.14 & 0.16 & 0.11 & 0.11 & 0.11 & 0.07 & 0.03 & 0.04 & 0.05 & 0.07 & 0.08 \\
\hline 90 & 0.1 & 0.1 & 0.16 & 0.27 & 0.19 & 0.13 & 0.14 & 0.1 & 0.1 & 0.07 & 0.08 & 0.08 & 0.1 \\
\hline 85 & 0.1 & 0.11 & 0.18 & 0.31 & 0.24 & 0.2 & 0.17 & 0.12 & 0.1 & 0.1 & 0.1 & 0.1 & 0.12 \\
\hline 80 & 0.11 & 0.11 & 0.19 & 0.34 & 0.3 & 0.24 & 0.19 & 0.13 & 0.11 & 0.11 & 0.11 & 0.11 & 0.16 \\
\hline 75 & 0.11 & 0.14 & 0.21 & 0.36 & 0.33 & 0.26 & 0.2 & 0.18 & 0.13 & 0.13 & 0.13 & 0.13 & 0.18 \\
\hline 70 & 0.17 & 0.17 & 0.22 & 0.38 & 0.35 & 0.3 & 0.23 & 0.22 & 0.2 & 0.14 & 0.14 & 0.14 & 0.21 \\
\hline 65 & 0.19 & 0.19 & 0.24 & 0.4 & 0.37 & 0.31 & 0.26 & 0.23 & 0.21 & 0.17 & 0.18 & 0.2 & 0.22 \\
\hline 60 & 0.2 & 0.21 & 0.26 & 0.42 & 0.39 & 0.32 & 0.3 & 0.24 & 0.22 & 0.2 & 0.2 & 0.21 & 0.24 \\
\hline 55 & 0.23 & 0.22 & 0.29 & 0.44 & 0.41 & 0.32 & 0.31 & 0.25 & 0.24 & 0.21 & 0.2 & 0.21 & 0.26 \\
\hline 50 & 0.24 & 0.23 & 0.32 & 0.49 & 0.44 & 0.36 & 0.32 & 0.29 & 0.25 & 0.24 & 0.21 & 0.21 & 0.28 \\
\hline 45 & 0.24 & 0.26 & 0.34 & 0.55 & 0.48 & 0.38 & 0.32 & 0.3 & 0.26 & 0.25 & 0.22 & 0.22 & 0.3 \\
\hline 40 & 0.25 & 0.3 & 0.37 & 0.62 & 0.53 & 0.39 & 0.36 & 0.31 & 0.27 & 0.25 & 0.25 & 0.22 & 0.32 \\
\hline 35 & 0.26 & 0.31 & 0.4 & 0.75 & 0.58 & 0.42 & 0.37 & 0.32 & 0.27 & 0.26 & 0.26 & 0.24 & 0.34 \\
\hline 30 & 0.27 & 0.32 & 0.45 & 0.89 & 0.79 & 0.48 & 0.38 & 0.33 & 0.28 & 0.3 & 0.27 & 0.25 & 0.36 \\
\hline 25 & 0.29 & 0.33 & 0.69 & 1.11 & 0.91 & 0.58 & 0.39 & 0.37 & 0.32 & 0.31 & 0.28 & 0.27 & 0.4 \\
\hline 20 & 0.3 & 0.34 & 1.31 & 2.12 & 1 & 0.73 & 0.5 & 0.38 & 0.33 & 0.32 & 0.31 & 0.28 & 0.44 \\
\hline 15 & 0.31 & 0.36 & 1.77 & 4.2 & 1.19 & 0.94 & 0.62 & 0.45 & 0.34 & 0.32 & 0 & 0.31 & 0.5 \\
\hline 10 & 0 & 0.38 & 11.4 & 11.3 & 1.7 & 0.98 & 0.65 & 0.48 & 0.36 & 0.33 & 0 & 0.32 & 0.73 \\
\hline 5 & 0 & 0.4 & 17.6 & 14.5 & 4.64 & 1.48 & 0.83 & 0.51 & 0.38 & 0 & 0 & 0.33 & 1.48 \\
\hline
\end{tabular}




\section{3- 4.L00-2A JILGA RIVER BELOW SARDE RESERVOIR, Continued}

Probability of occurrence of annual high discharges

[ $\mathrm{m}^{3} / \mathrm{s}$, meters per second; $\mathrm{ng}$, statistic not given]

\begin{tabular}{|c|c|c|c|c|c|c|}
\hline \multirow{2}{*}{$\begin{array}{l}\text { Exceedance } \\
\text { probability }\end{array}$} & \multirow{2}{*}{$\begin{array}{c}\text { Recurrence } \\
\text { interval } \\
\text { (years) }\end{array}$} & \multirow{2}{*}{$\begin{array}{c}\text { Maximum } \\
\text { instantaneous } \\
\left(\mathrm{m}^{3} / \mathrm{s}\right)\end{array}$} & \multicolumn{4}{|c|}{ Maximum mean discharge ${ }^{1}$} \\
\hline & & & 3-day period & 7-day period & 15-day period & 30-day period \\
\hline 0.99 & 1.01 & 0.100 & 0.109 & 0.089 & 0.079 & 0.075 \\
\hline 0.95 & 1.05 & 0.400 & 0.270 & 0.229 & 0.207 & 0.184 \\
\hline 0.90 & 1.11 & 0.800 & 0.458 & 0.395 & 0.356 & 0.305 \\
\hline 0.80 & 1.25 & 1.80 & 0.902 & 0.795 & 0.709 & 0.579 \\
\hline 0.50 & 2 & 7.60 & 3.74 & 0.345 & 2.91 & 2.13 \\
\hline 0.20 & 5 & 29.5 & 18.4 & 17.8 & 13.6 & 8.72 \\
\hline 0.10 & 10 & 57.9 & 45.6 & 45.0 & 31.9 & 19.0 \\
\hline 0.04 & 25 & 116 & 126 & 128 & 82.9 & 45.2 \\
\hline 0.02 & 50 & 180 & 252 & 260 & 157 & 80.7 \\
\hline 0.01 & 100 & 264 & 478 & 501 & 283 & 137 \\
\hline 0.005 & 200 & 373 & 874 & 930 & 492 & 226 \\
\hline 0.002 & 500 & 561 & ng & ng & $\mathrm{ng}$ & ng \\
\hline
\end{tabular}

'Data does not fit log-Pearson Type III curve, use with caution.

\section{3- 4.L00-2A JILGA RIVER BELOW SARDE RESERVOIR, Continued}

Probability of occurrence of annual low discharges

[ $\mathrm{m}^{3} / \mathrm{s}$, meters per second]

\begin{tabular}{|c|c|c|c|c|c|c|c|c|c|c|}
\hline \multirow{3}{*}{$\begin{array}{c}\text { Nonexceedance } \\
\text { probability }\end{array}$} & \multirow{3}{*}{$\begin{array}{c}\text { Recurrence } \\
\text { interval } \\
\text { (years) }\end{array}$} & \multicolumn{9}{|c|}{ Minimum mean discharge $\left(\mathrm{m}^{3} / \mathrm{s}\right)$} \\
\hline & & \multicolumn{9}{|c|}{ Number of consecutive days } \\
\hline & & 1 & 3 & 7 & 14 & 30 & 60 & 90 & 120 & 183 \\
\hline 0.05 & 20 & 0.015 & 0.016 & 0.017 & 0.021 & 0.032 & 0.040 & 0.046 & 0.059 & 0.061 \\
\hline 0.10 & 10 & 0.032 & 0.034 & 0.035 & 0.042 & 0.055 & 0.065 & 0.073 & 0.087 & 0.090 \\
\hline 0.20 & 5 & 0.064 & 0.069 & 0.072 & 0.080 & 0.095 & 0.106 & 0.115 & 0.130 & 0.132 \\
\hline 0.50 & 2 & 0.161 & 0.168 & 0.176 & 0.183 & 0.193 & 0.152 & 0.214 & 0.224 & 0.229 \\
\hline
\end{tabular}




\section{3- 4.L00-2A JILGA RIVER BELOW SARDE RESERVOIR, Continued}

\section{Probability of occurrence of seasonal low discharges}

$\left[\mathrm{m}^{3} / \mathrm{s}\right.$, meters per second]

\begin{tabular}{|c|c|c|c|c|c|c|c|c|c|}
\hline \multirow{3}{*}{$\begin{array}{l}\text { Nonexceedance } \\
\text { probability }\end{array}$} & \multirow{3}{*}{$\begin{array}{c}\text { Recurrence } \\
\text { interval } \\
\text { (years) }\end{array}$} & \multicolumn{8}{|c|}{ Minimum mean discharge $\left(\mathrm{m}^{3} / \mathrm{s}\right)$} \\
\hline & & \multicolumn{8}{|c|}{ Number of consecutive days } \\
\hline & & 1 & 7 & 14 & 30 & 1 & 7 & 14 & 30 \\
\hline & & \multicolumn{4}{|c|}{ December-January-February } & \multicolumn{4}{|c|}{ March-April-May } \\
\hline 0.05 & 20 & 0.017 & 0.036 & 0.041 & 0.049 & 0.066 & 0.091 & 0.102 & 0.119 \\
\hline 0.10 & 10 & 0.033 & 0.054 & 0.060 & 0.069 & 0.081 & 0.105 & 0.118 & 0.138 \\
\hline 0.20 & 5 & 0.066 & 0.082 & 0.090 & 0.100 & 0.105 & 0.129 & 0.144 & 0.168 \\
\hline \multirow[t]{2}{*}{0.50} & 2 & ${ }^{1} 0.162$ & 0.157 & 0.166 & 0.175 & 0.190 & 0.209 & 0.229 & 0.264 \\
\hline & & \multicolumn{4}{|c|}{ June-July-August } & \multicolumn{4}{|c|}{ September-October-November } \\
\hline 0.05 & 20 & 0.030 & 0.045 & 0.050 & 0.067 & 0.015 & 0.015 & 0.021 & 0.030 \\
\hline 0.10 & 10 & 0.053 & 0.070 & 0.075 & 0.096 & 0.030 & 0.030 & 0.039 & 0.050 \\
\hline 0.20 & 5 & 0.094 & 0.109 & 0.116 & 0.140 & 0.061 & 0.061 & 0.072 & 0.086 \\
\hline 0.50 & 2 & 0.208 & 0.211 & 0.219 & 0.245 & 0.160 & 0.163 & 0.171 & 0.184 \\
\hline
\end{tabular}

'Data does not fit log-Pearson Type III curve, use with caution. 


\section{3- 4.L00-2A JILGA RIVER BELOW SARDE RESERVOIR, Continued}

Annual peak discharge and corresponding gage height

[ $\mathrm{m}^{3} / \mathrm{s}$, meters per second; --, no data]

\begin{tabular}{|c|c|c|c|c|c|c|c|c|}
\hline $\begin{array}{l}\text { Water } \\
\text { year }\end{array}$ & Date & $\begin{array}{c}\text { Gage } \\
\text { height } \\
\text { (meter) }\end{array}$ & $\begin{array}{c}\text { Peak } \\
\text { discharge } \\
\left(\mathrm{m}^{3} / \mathrm{s}\right)\end{array}$ & $\begin{array}{l}\text { Water } \\
\text { year }\end{array}$ & Date & $\begin{array}{c}\text { Gage } \\
\text { height } \\
\text { (meter) }\end{array}$ & & $\begin{array}{c}\text { Peak } \\
\text { discharge } \\
\left(\mathrm{m}^{3} / \mathrm{s}\right)\end{array}$ \\
\hline \multicolumn{9}{|c|}{ Annual peak discharge, by year } \\
\hline 1969 & 25-Sep & & 2.50 & 1975 & 16-Мay & & -- & 5.82 \\
\hline 1970 & 29-Apr & & 1.37 & 1976 & 25-Apr & & -- & 96.9 \\
\hline 1971 & 7-Apr & & 0.38 & 1977 & 14-Mar & & -- & 1.65 \\
\hline 1972 & 6-May & & 28.1 & 1978 & 19-Aug & & -- & 33.0 \\
\hline 1973 & 6-Mar & & 26.9 & 1979 & 2-Apr & & -- & 7.15 \\
\hline 1974 & 24-Mar & & 11.8 & & & & & \\
\hline \multicolumn{9}{|c|}{ Annual peak discharge, from highest to lowest } \\
\hline 1976 & 25-Apr & & 96.9 & 1975 & 16-May & & -- & 5.82 \\
\hline 1978 & 19-Aug & & 33.0 & 1969 & 25-Sep & & -- & 2.50 \\
\hline 1972 & 6-May & & 28.1 & 1977 & 14-Mar & & -- & 1.65 \\
\hline 1973 & 6-Mar & & 26.9 & 1970 & 29-Apr & & -- & 1.37 \\
\hline 1974 & 24-Mar & & 11.8 & 1971 & 7-Apr & & -- & 0.38 \\
\hline 1979 & 2-Apr & & 7.15 & & & & & \\
\hline
\end{tabular}




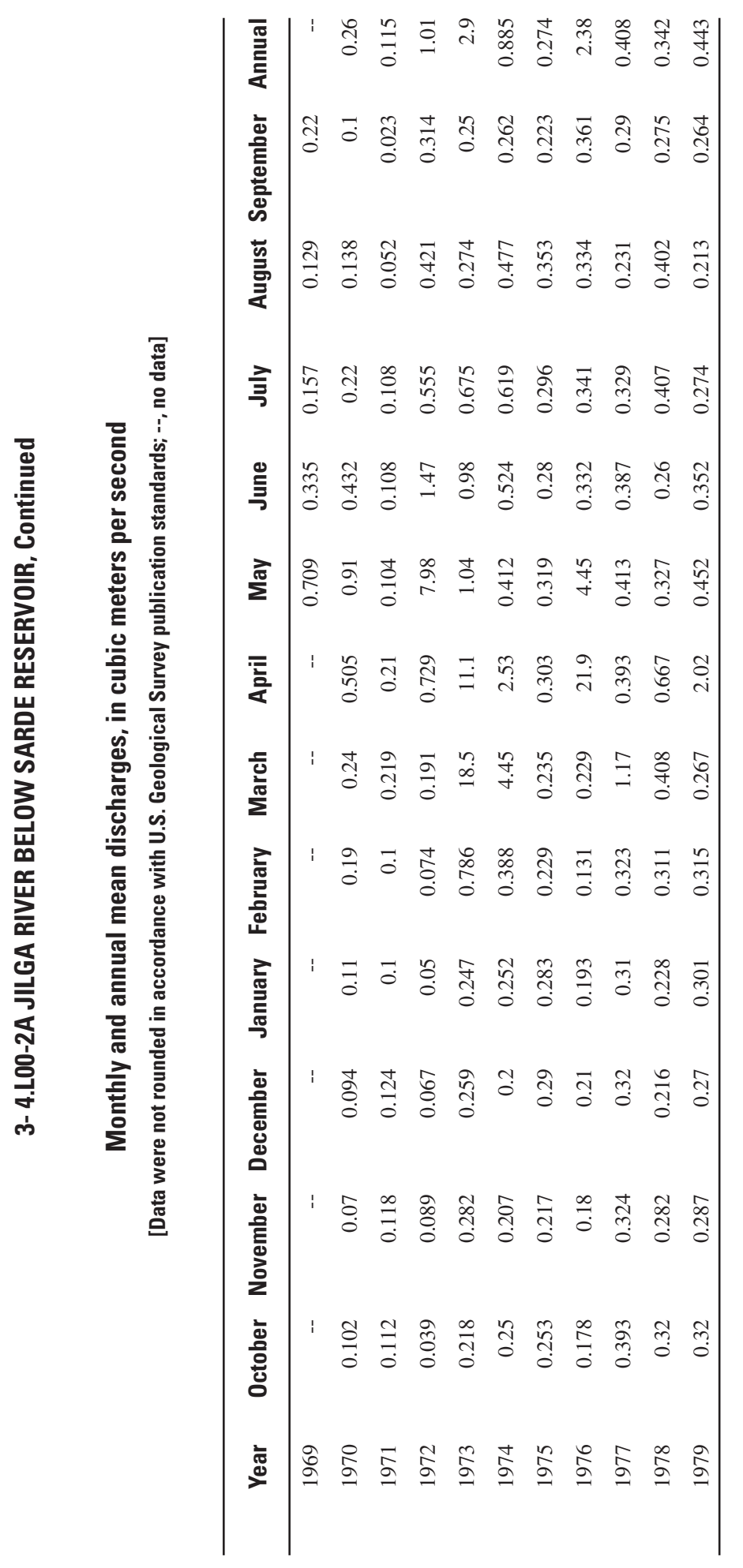




\section{3- 4.L00-7A JILGA RIVER AT GARDEZ}

\section{(U.S. Geological Survey identification number: 333500069130000)}

LOCATION: Lat $33^{\circ} 35^{\prime} \mathrm{N}$., long $69^{\circ} 13^{\prime} \mathrm{E}$.

DRAINAGE AREA: $1,065 \mathrm{~km}^{2}$.

ALTITUDE: 2,294 meters plus mean sea level.

PERIOD OF RECORD: August 6, 1970 to September 30, 1978.

GAGE: --

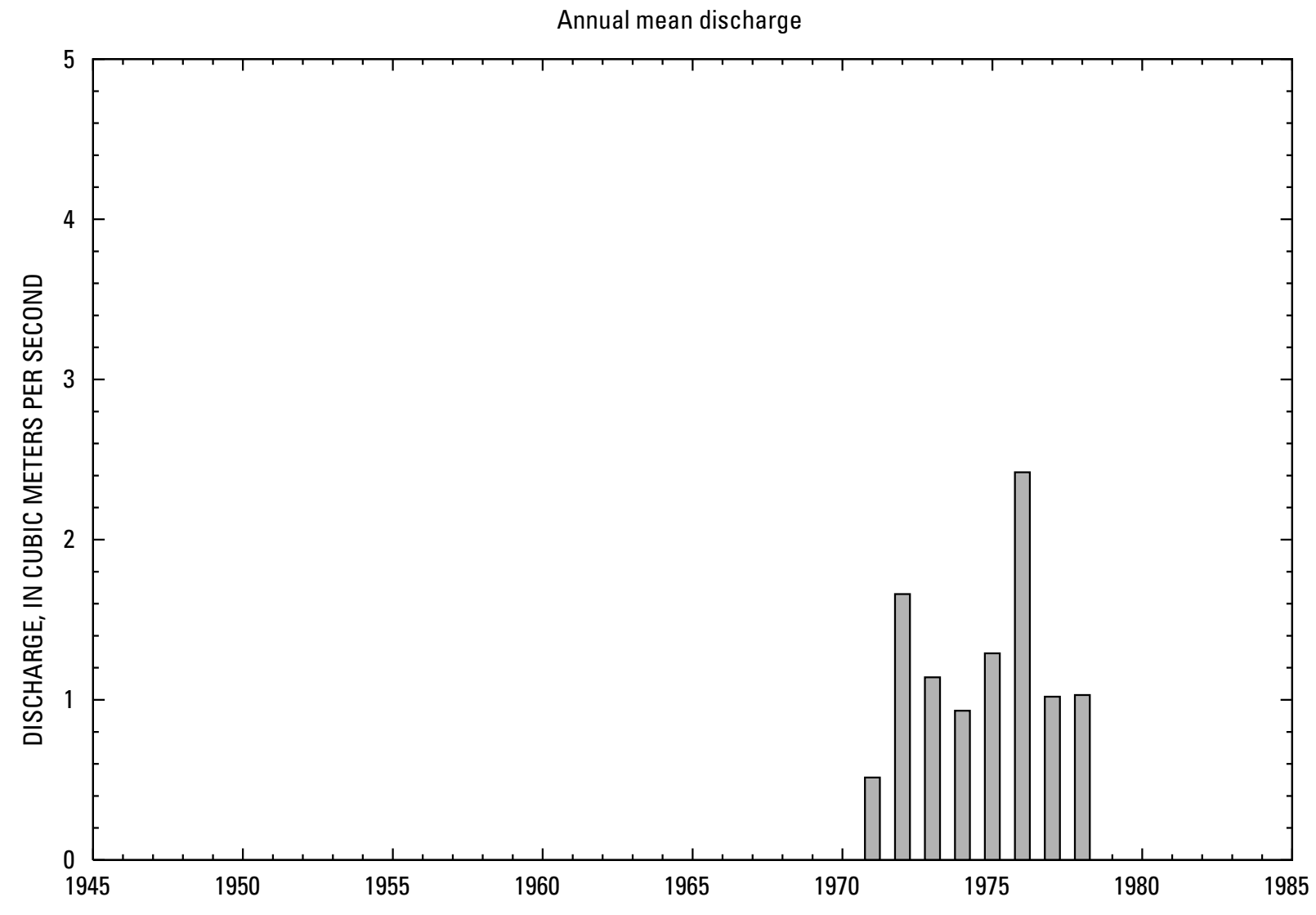




\section{3- 4.L00-7A JILGA RIVER AT GARDEZ, Continued}

\section{Statistics of monthly and annual mean discharges}

[ $\mathrm{m}^{3} / \mathrm{s}$, meters per second]

\begin{tabular}{|c|c|c|c|c|c|c|c|c|}
\hline \multirow[b]{2}{*}{ Month } & \multicolumn{2}{|c|}{ Maximum } & \multicolumn{2}{|c|}{ Minimum } & \multicolumn{4}{|c|}{ Mean } \\
\hline & $\begin{array}{c}\text { Discharge } \\
\left(\mathrm{m}^{3} / \mathrm{s}\right)\end{array}$ & $\begin{array}{c}\text { Water year } \\
\text { of } \\
\text { occurrence }\end{array}$ & $\begin{array}{c}\text { Discharge } \\
\left(\mathrm{m}^{3} / \mathrm{s}\right)\end{array}$ & $\begin{array}{c}\text { Water year } \\
\text { of } \\
\text { occurrence }\end{array}$ & $\begin{array}{c}\text { Discharge } \\
\left(\mathrm{m}^{3} / \mathrm{s}\right)\end{array}$ & $\begin{array}{c}\text { Standard } \\
\text { deviation } \\
\left(\mathrm{m}^{3} / \mathrm{s}\right)\end{array}$ & $\begin{array}{c}\text { Coefficient } \\
\text { of } \\
\text { variation }\end{array}$ & $\begin{array}{c}\text { Percentage } \\
\text { of annual } \\
\text { discharge }\end{array}$ \\
\hline October & 0.726 & 1977 & 0.029 & 1972 & 0.37 & 0.24 & 0.67 & 2.43 \\
\hline November & 1.38 & 1977 & 0.317 & 1972 & 0.89 & 0.37 & 0.41 & 5.88 \\
\hline December & 1.99 & 1977 & 0.565 & 1971 & 1.22 & 0.55 & 0.45 & 8.06 \\
\hline January & 1.94 & 1977 & 0.360 & 1972 & 1.21 & 0.52 & 0.43 & 8.00 \\
\hline February & 1.78 & 1978 & 0.582 & 1972 & 1.31 & 0.42 & 0.32 & 8.67 \\
\hline March & 3.36 & 1976 & 1.63 & 1971 & 2.60 & 0.58 & 0.22 & 17.2 \\
\hline April & 17.3 & 1976 & 0.700 & 1971 & 5.29 & 5.54 & 1.05 & 35.1 \\
\hline May & 5.53 & 1972 & 0.002 & 1977 & 1.15 & 1.84 & 1.60 & 7.65 \\
\hline June & 0.674 & 1972 & 0.004 & 1977 & 0.16 & 0.24 & 1.57 & 1.03 \\
\hline July & 2.16 & 1973 & 0 & 1971 & 0.55 & 0.73 & 1.33 & 3.64 \\
\hline August & 0.936 & 1975 & 0 & 1977 & 0.23 & 0.33 & 1.43 & 1.52 \\
\hline September & 0.440 & 1976 & 0.002 & 1977 & 0.12 & 0.14 & 1.14 & 0.83 \\
\hline Annual & 2.42 & 1976 & 0.515 & 1971 & 1.25 & 0.57 & 0.46 & 100 \\
\hline
\end{tabular}

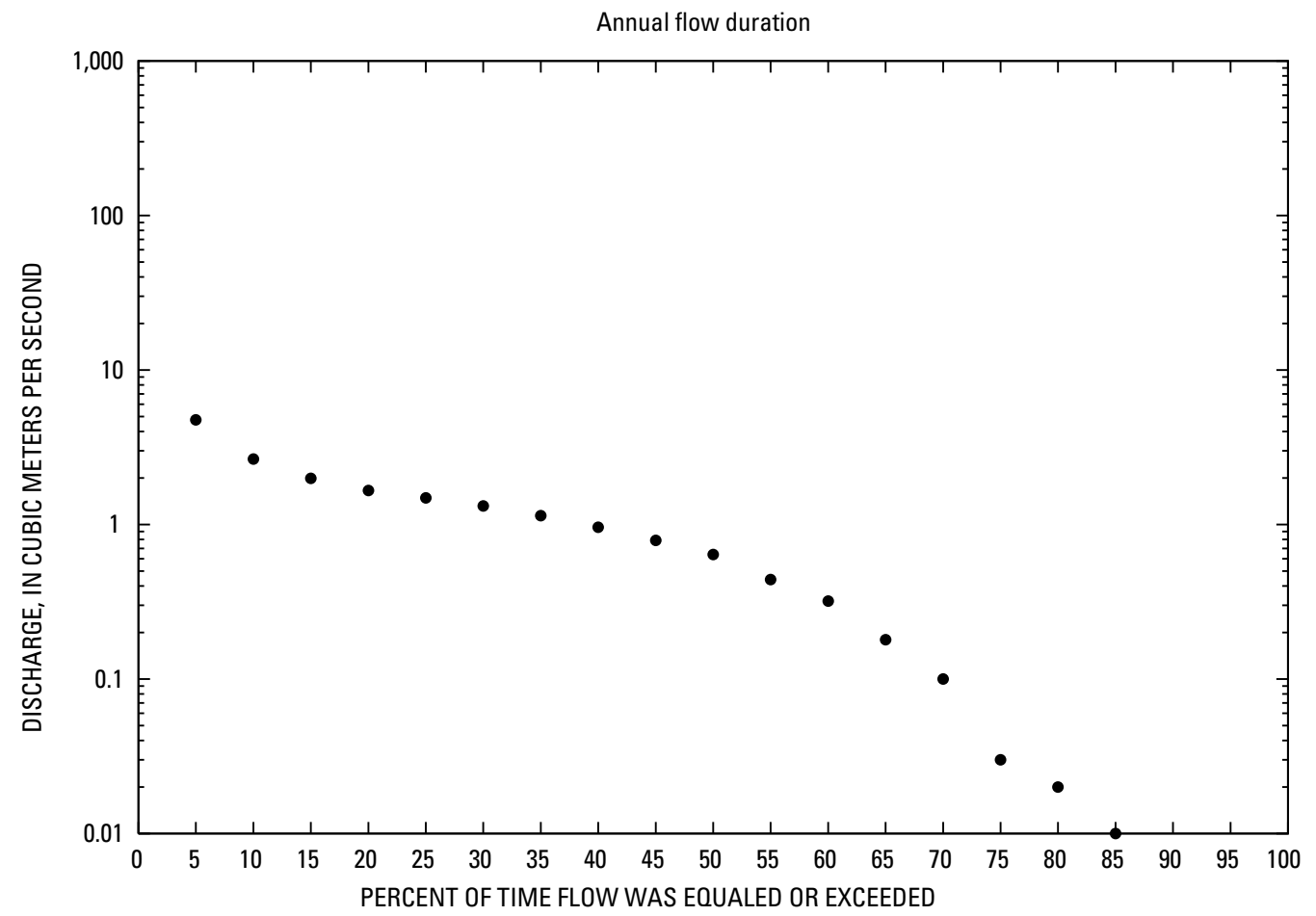


Monthly and annual flow duration, in cubic meters per second

[Data were not rounded in accordance with U.S. Geological Survey publication standards]

\begin{tabular}{|c|c|c|c|c|c|c|c|c|c|c|c|c|c|}
\hline $\begin{array}{l}\text { Percentage } \\
\text { of days } \\
\text { discharge } \\
\text { equaled or } \\
\text { exceeded }\end{array}$ & January & February & March & April & May & June & July & August & September & October & November & December & Annual \\
\hline 95 & 0.32 & 0.46 & 1.04 & 0.17 & 0 & 0 & 0 & 0 & 0 & 0 & 0.3 & 0.44 & 0 \\
\hline 90 & 0.47 & 0.64 & 1.24 & 0.56 & 0 & 0 & 0 & 0 & 0 & 0.01 & 0.33 & 0.59 & 0 \\
\hline 85 & 0.64 & 0.75 & 1.33 & 0.92 & 0.01 & 0 & 0 & 0 & 0 & 0.03 & 0.4 & 0.63 & 0.01 \\
\hline 80 & 0.69 & 0.87 & 1.47 & 1.28 & 0.01 & 0 & 0.01 & 0 & 0 & 0.1 & 0.45 & 0.66 & 0.02 \\
\hline 75 & 0.77 & 1.1 & 1.62 & 1.53 & 0.01 & 0 & 0.01 & 0 & 0.01 & 0.11 & 0.57 & 0.74 & 0.03 \\
\hline 70 & 0.91 & 1.18 & 1.72 & 1.75 & 0.02 & 0.01 & 0.01 & 0 & 0.02 & 0.11 & 0.61 & 0.77 & 0.1 \\
\hline 65 & 0.97 & 1.23 & 1.84 & 1.97 & 0.02 & 0.01 & 0.01 & 0 & 0.02 & 0.12 & 0.77 & 0.86 & 0.18 \\
\hline 60 & 1.04 & 1.3 & 2.07 & 2.18 & 0.03 & 0.01 & 0.02 & 0 & 0.05 & 0.15 & 0.86 & 0.97 & 0.32 \\
\hline 55 & 1.26 & 1.36 & 2.18 & 2.85 & 0.05 & 0.01 & 0.02 & 0.01 & 0.06 & 0.2 & 0.91 & 1.16 & 0.44 \\
\hline 50 & 1.34 & 1.4 & 2.26 & 3.51 & 0.08 & 0.02 & 0.02 & 0.01 & 0.07 & 0.27 & 1 & 1.27 & 0.64 \\
\hline 45 & 1.37 & 1.43 & 2.41 & 4.07 & 0.13 & 0.02 & 0.02 & 0.02 & 0.09 & 0.31 & 1.03 & 1.31 & 0.79 \\
\hline 40 & 1.39 & 1.47 & 2.62 & 4.59 & 0.41 & 0.02 & 0.03 & 0.02 & 0.11 & 0.37 & 1.06 & 1.36 & 0.96 \\
\hline 35 & 1.44 & 1.5 & 2.75 & 5.12 & 0.53 & 0.02 & 0.05 & 0.04 & 0.11 & 0.42 & 1.08 & 1.42 & 1.14 \\
\hline 30 & 1.52 & 1.53 & 2.93 & 5.66 & 0.72 & 0.02 & 0.12 & 0.09 & 0.12 & 0.54 & 1.12 & 1.48 & 1.32 \\
\hline 25 & 1.58 & 1.56 & 3.1 & 6.23 & 0.9 & 0.03 & 0.29 & 0.12 & 0.15 & 0.61 & 1.15 & 1.6 & 1.49 \\
\hline 20 & 1.64 & 1.6 & 3.4 & 7.17 & 1.15 & 0.09 & 0.43 & 0.14 & 0.2 & 0.73 & 1.19 & 1.78 & 1.66 \\
\hline 15 & 1.71 & 1.64 & 3.93 & 8.21 & 1.9 & 0.28 & 0.92 & 0.19 & 0.3 & 0.83 & 1.25 & 1.92 & 1.99 \\
\hline 10 & 1.93 & 1.68 & 4.47 & 9.78 & 3.49 & 0.32 & 1.89 & 0.29 & 0.34 & 0.91 & 1.34 & 2.04 & 2.65 \\
\hline 5 & 0 & 1.78 & 5.48 & 18.9 & 8.03 & 0.43 & 3.41 & 0.66 & 0.45 & 0.98 & 1.49 & 2.17 & 4.76 \\
\hline
\end{tabular}




\section{3- 4.L00-7A JILGA RIVER AT GARDEZ, Continued}

Probability of occurrence of annual high discharges

[ $\mathrm{m}^{3} / \mathrm{s}$, meters per second; ng, statistic not given]

\begin{tabular}{|c|c|c|c|c|c|c|}
\hline \multirow{2}{*}{$\begin{array}{c}\text { Exceedance } \\
\text { probability }\end{array}$} & \multirow{2}{*}{$\begin{array}{c}\text { Recurrence } \\
\text { interval } \\
\text { (years) }\end{array}$} & \multirow{2}{*}{$\begin{array}{c}\text { Maximum } \\
\text { instantaneous }{ }^{1} \\
\left(\mathrm{~m}^{3} / \mathrm{s}\right)\end{array}$} & \multicolumn{4}{|c|}{ Maximum mean discharge } \\
\hline & & & 3-day period & 7-day period & 15-day period & 30-day period \\
\hline 0.99 & 1.01 & 2.80 & 2.02 & 1.89 & 1.59 & 1.13 \\
\hline 0.95 & 1.05 & 5.00 & 2.39 & 2.22 & 1.91 & 1.56 \\
\hline 0.90 & 1.11 & 6.70 & 2.72 & 2.52 & 2.19 & 1.88 \\
\hline 0.80 & 1.25 & 9.50 & 3.34 & 3.06 & 2.70 & 2.43 \\
\hline 0.50 & 2 & 17.7 & 5.84 & 5.22 & 4.63 & 4.29 \\
\hline 0.20 & 5 & 31.7 & 13.2 & 11.3 & 9.82 & 8.40 \\
\hline 0.10 & 10 & 42.2 & 22.7 & 18.8 & 16.0 & 12.5 \\
\hline 0.04 & 25 & 56.5 & 44.4 & 35.4 & 28.9 & 19.7 \\
\hline 0.02 & 50 & 67.8 & 272.2 & 55.8 & 44.3 & 27.0 \\
\hline 0.01 & 100 & 79.5 & ${ }^{2} 116$ & ${ }^{2} 86.8$ & 66.9 & 36.3 \\
\hline 0.005 & 200 & 91.6 & ${ }^{2} 184$ & ${ }^{2} 134$ & ${ }^{2} 100$ & 48.1 \\
\hline 0.002 & 500 & 108 & ng & ng & ng & ng \\
\hline
\end{tabular}

'Less than 10 years of data used.

${ }^{2}$ Data does not fit log-Pearson Type III curve, use with caution.

\section{3- 4.L00-7A JILGA RIVER AT GARDEZ, Continued}

Probability of occurrence of annual low discharges

[m³/s, meters per second; ng, statistic not given]

\begin{tabular}{|c|c|c|c|c|c|c|c|c|c|c|}
\hline \multirow{3}{*}{$\begin{array}{c}\text { Nonexceedance } \\
\text { probability }\end{array}$} & \multirow{3}{*}{$\begin{array}{c}\text { Recurrence } \\
\text { interval } \\
\text { (years) }\end{array}$} & \multicolumn{9}{|c|}{ Minimum mean discharge $\left(\mathrm{m}^{3} / \mathrm{s}\right)$} \\
\hline & & \multicolumn{9}{|c|}{ Number of consecutive days } \\
\hline & & 1 & 3 & 7 & 14 & 30 & 60 & 90 & 120 & 183 \\
\hline 0.05 & 20 & ng & ng & ng & ng & 0 & 0 & 0.001 & 0.002 & 0.020 \\
\hline 0.10 & 10 & ng & ng & ng & ng & 0 & 0 & 0.002 & 0.004 & 0.035 \\
\hline 0.20 & 5 & ng & ng & ng & ng & 0 & 0 & 0.005 & 0.013 & 0.067 \\
\hline 0.50 & 2 & ng & ng & ng & ng & 0.002 & 0.010 & 0.030 & 0.077 & 0.201 \\
\hline
\end{tabular}


Probability of occurrence of seasonal low discharges

$\left[\mathrm{m}^{3} / \mathrm{s}\right.$, meters per second]

\begin{tabular}{|c|c|c|c|c|c|c|c|c|c|}
\hline \multirow{3}{*}{$\begin{array}{c}\text { Nonexceedance } \\
\text { probability }\end{array}$} & \multirow{3}{*}{$\begin{array}{c}\text { Recurrence } \\
\text { interval } \\
\text { (years) }\end{array}$} & \multicolumn{8}{|c|}{ Minimum mean discharge $\left(\mathrm{m}^{3} / \mathrm{s}\right)$} \\
\hline & & \multicolumn{8}{|c|}{ Number of consecutive days } \\
\hline & & 1 & 7 & 14 & 30 & 1 & 7 & 14 & 30 \\
\hline & & \multicolumn{4}{|c|}{ December-January-February } & \multicolumn{4}{|c|}{ March-April-May } \\
\hline 0.05 & 20 & 0.258 & 0.272 & 0.312 & 0.342 & 0 & 0 & 0 & 0.001 \\
\hline 0.10 & 10 & 0.342 & 0.365 & 0.411 & 0.448 & 0 & 0 & 0 & 0.003 \\
\hline 0.20 & 5 & 0.471 & 0.507 & 0.559 & 0.607 & 0 & 0 & 0.003 & 0.013 \\
\hline \multirow[t]{2}{*}{0.50} & 2 & 0.813 & 0.876 & 0.933 & 1.00 & 0.019 & 0.026 & 0.048 & 0.176 \\
\hline & & \multicolumn{4}{|c|}{ June-July-August } & \multicolumn{4}{|c|}{ September-October-November } \\
\hline 0.05 & 20 & 0 & 0 & 0 & 0 & 0 & 0 & 0 & 0 \\
\hline 0.10 & 10 & 0 & 0 & 0 & 0 & 0 & 0 & 0 & 0.001 \\
\hline 0.20 & 5 & 0 & 0 & 0 & 0 & 0 & 0 & 0 & 0.004 \\
\hline 0.50 & 2 & 0 & 0 & 0 & 0.004 & 0 & 0.012 & 0.028 & 0.029 \\
\hline
\end{tabular}

\section{3- 4.L00-7A JILGA RIVER AT GARDEZ, Continued}

Annual peak discharge and corresponding gage height

[m³/s, meters per second; --, no data]

\begin{tabular}{|c|c|c|c|c|c|c|c|}
\hline $\begin{array}{c}\text { Water } \\
\text { year }\end{array}$ & Date & $\begin{array}{c}\text { Gage } \\
\text { height } \\
\text { (meters) }\end{array}$ & $\begin{array}{c}\text { Peak } \\
\text { discharge } \\
\left(\mathrm{m}^{3} / \mathrm{s}\right)\end{array}$ & $\begin{array}{c}\text { Water } \\
\text { year }\end{array}$ & Date & $\begin{array}{c}\text { Gage } \\
\text { height } \\
\text { (meters) }\end{array}$ & $\begin{array}{c}\text { Peak } \\
\text { discharge } \\
\left(\mathrm{m}^{3} / \mathrm{s}\right)\end{array}$ \\
\hline \multicolumn{8}{|c|}{ Annual peak discharge, by year } \\
\hline 1971 & 26-Mar & & 5.11 & 1975 & 14-Aug & & 24.4 \\
\hline 1972 & 30-Jun & & 25.5 & 1976 & 19-Apr & & 50.0 \\
\hline 1973 & 16-Aug & & 28.8 & 1977 & 17-Apr & & 12.8 \\
\hline 1974 & 7-Apr & & 12.8 & 1978 & 5-Jul & & 10.0 \\
\hline \multicolumn{8}{|c|}{ Annual peak discharge, from highest to lowest } \\
\hline 1976 & 19-Apr & & 50.0 & 1977 & 17-Apr & & 12.8 \\
\hline 1973 & 16-Aug & & 28.8 & 1974 & 7-Apr & & 12.8 \\
\hline 1972 & 30-Jun & & 25.5 & 1978 & 5-Jul & & 10.0 \\
\hline 1975 & 14-Aug & & 24.4 & 1971 & 26-Mar & & 5.11 \\
\hline
\end{tabular}




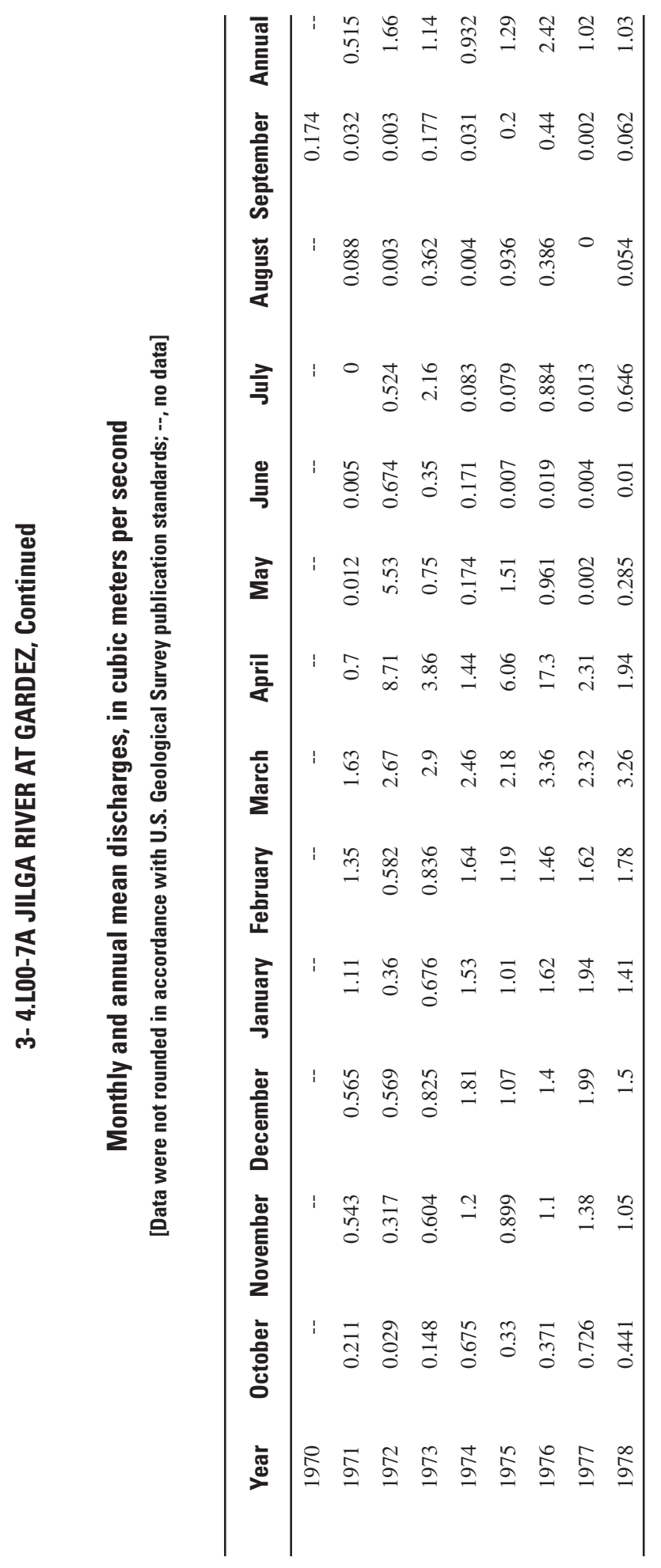




\section{3- 4.L00-9T JILGA RIVER NEAR MECHALGHU}

\section{(U.S. Geological Survey identification number: 334900069230000)}

LOCATION: Lat 334'N., long 69²3'E.

DRAINAGE AREA: $65 \mathrm{~km}^{2}$.

ALTITUDE: 2,690 meters plus mean sea level.

PERIOD OF RECORD: August 11, 1970 to September 30, 1978.

GAGE: --

Annual mean discharge

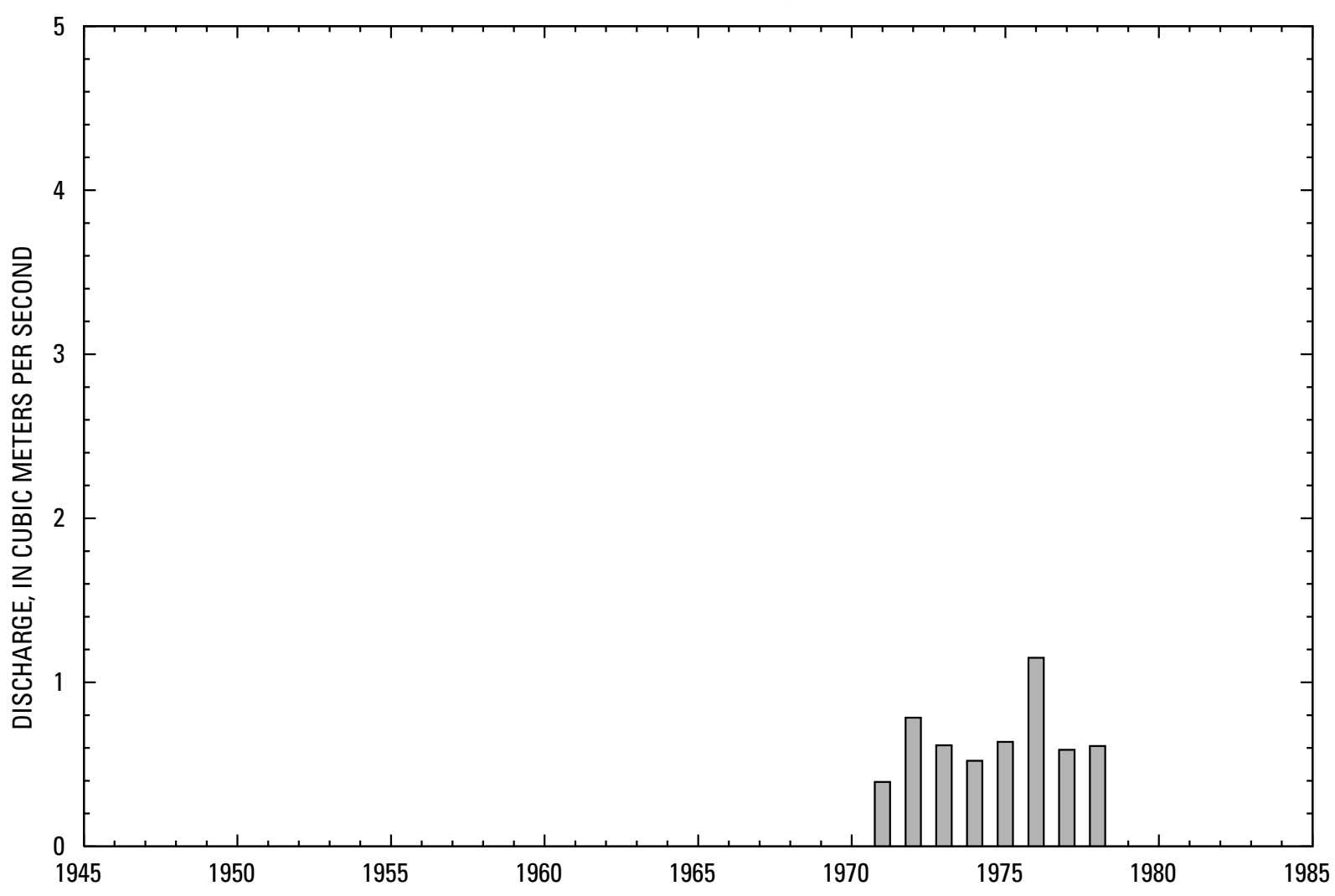




\section{3- 4.L00-9T JILGA RIVER NEAR MECHALGHU, Continued}

\section{Statistics of monthly and annual mean discharges}

[ $\mathrm{m}^{3} / \mathrm{s}$, meters per second]

\begin{tabular}{|c|c|c|c|c|c|c|c|c|}
\hline \multirow[b]{2}{*}{ Month } & \multicolumn{2}{|c|}{ Maximum } & \multicolumn{2}{|c|}{ Minimum } & \multicolumn{4}{|c|}{ Mean } \\
\hline & $\begin{array}{c}\text { Discharge } \\
\left(\mathrm{m}^{3} / \mathrm{s}\right)\end{array}$ & $\begin{array}{c}\text { Water year } \\
\text { of } \\
\text { occurrence }\end{array}$ & $\begin{array}{c}\text { Discharge } \\
\left(\mathrm{m}^{3} / \mathrm{s}\right)\end{array}$ & $\begin{array}{c}\text { Water year } \\
\text { of } \\
\text { occurrence }\end{array}$ & $\begin{array}{c}\text { Discharge } \\
\left(\mathrm{m}^{3} / \mathrm{s}\right)\end{array}$ & $\begin{array}{c}\text { Standard } \\
\text { deviation } \\
\left(\mathrm{m}^{3} / \mathrm{s}\right)\end{array}$ & $\begin{array}{c}\text { Coefficient } \\
\text { of } \\
\text { variation }\end{array}$ & $\begin{array}{c}\text { Percentage } \\
\text { of annual } \\
\text { discharge }\end{array}$ \\
\hline October & 0.626 & 1977 & 0.344 & 1975 & 0.47 & 0.10 & 0.22 & 5.86 \\
\hline November & 0.625 & 1977 & 0.177 & 1973 & 0.43 & 0.14 & 0.32 & 5.38 \\
\hline December & 0.707 & 1978 & 0.222 & 1973 & 0.46 & 0.17 & 0.37 & 5.73 \\
\hline January & 0.652 & 1978 & 0.098 & 1973 & 0.41 & 0.21 & 0.51 & 5.10 \\
\hline February & 0.691 & 1978 & 0.209 & 1973 & 0.44 & 0.18 & 0.41 & 5.52 \\
\hline March & 1.09 & 1978 & 0.518 & 1971 & 0.78 & 0.20 & 0.25 & 9.79 \\
\hline April & 4.49 & 1976 & 0.627 & 1971 & 1.86 & 1.35 & 0.73 & 23.4 \\
\hline May & 2.18 & 1976 & 0.380 & 1971 & 1.06 & 0.56 & 0.53 & 13.3 \\
\hline June & 1.09 & 1972 & 0.297 & 1978 & 0.61 & 0.29 & 0.48 & 7.69 \\
\hline July & 1.04 & 1976 & 0.334 & 1971 & 0.60 & 0.25 & 0.41 & 7.52 \\
\hline August & 0.687 & 1976 & 0.311 & 1978 & 0.45 & 0.14 & 0.32 & 5.62 \\
\hline September & 0.653 & 1976 & 0.233 & 1978 & 0.40 & 0.14 & 0.34 & 5.07 \\
\hline Annual & 1.15 & 1976 & 0.393 & 1971 & 0.66 & 0.23 & 0.34 & 100 \\
\hline
\end{tabular}

Annual flow duration

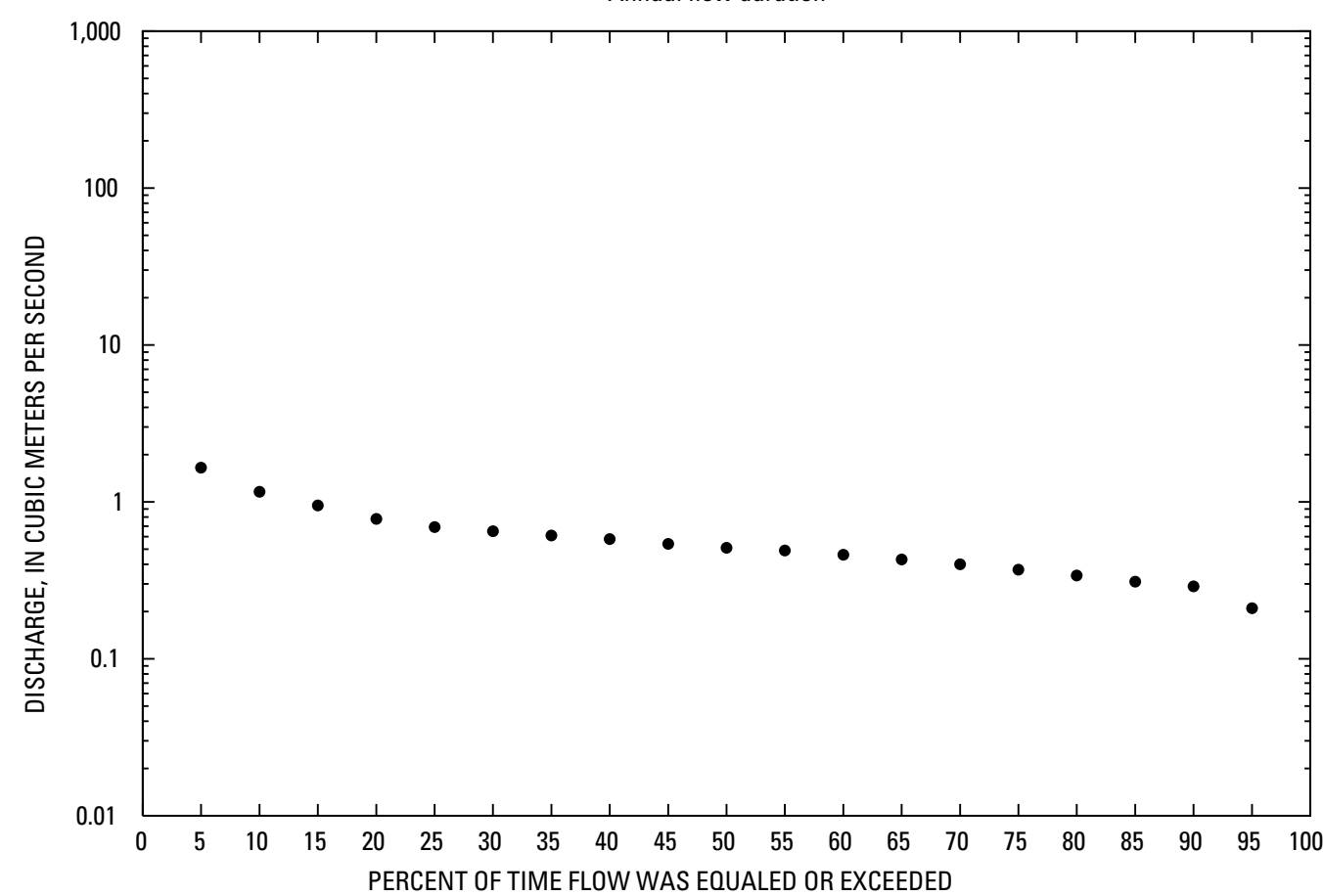




\section{3- 4.L00-9T JILGA RIVER NEAR MECHALGHU, Continued}

\section{Monthly and annual flow duration, in cubic meters per second}

[Data were not rounded in accordance with U.S. Geological Survey publication standards]

\begin{tabular}{|c|c|c|c|c|c|c|c|c|c|c|c|c|c|}
\hline $\begin{array}{l}\text { Percentage } \\
\text { of days } \\
\text { discharge } \\
\text { equaled or } \\
\text { exceeded }\end{array}$ & January & February & March & April & May & June & July & August & September & October & November & December & Annual \\
\hline 95 & 0.08 & 0.2 & 0.36 & 0.63 & 0.38 & 0.29 & 0.27 & 0.28 & 0.2 & 0.34 & 0.16 & 0.23 & 0.21 \\
\hline 90 & 0.15 & 0.21 & 0.4 & 0.67 & 0.44 & 0.29 & 0.28 & 0.29 & 0.21 & 0.35 & 0.24 & 0.24 & 0.29 \\
\hline 85 & 0.16 & 0.26 & 0.41 & 0.71 & 0.64 & 0.3 & 0.29 & 0.29 & 0.27 & 0.36 & 0.3 & 0.29 & 0.31 \\
\hline 80 & 0.17 & 0.3 & 0.46 & 0.75 & 0.65 & 0.35 & 0.3 & 0.3 & 0.28 & 0.36 & 0.33 & 0.34 & 0.34 \\
\hline 75 & 0.26 & 0.31 & 0.51 & 0.87 & 0.66 & 0.42 & 0.33 & 0.3 & 0.3 & 0.37 & 0.36 & 0.35 & 0.37 \\
\hline 70 & 0.3 & 0.31 & 0.52 & 0.92 & 0.67 & 0.44 & 0.36 & 0.32 & 0.33 & 0.37 & 0.37 & 0.36 & 0.4 \\
\hline 65 & 0.31 & 0.32 & 0.57 & 1.01 & 0.68 & 0.44 & 0.41 & 0.36 & 0.34 & 0.41 & 0.38 & 0.37 & 0.43 \\
\hline 60 & 0.34 & 0.36 & 0.59 & 1.09 & 0.81 & 0.45 & 0.43 & 0.37 & 0.35 & 0.44 & 0.4 & 0.38 & 0.46 \\
\hline 55 & 0.36 & 0.4 & 0.69 & 1.14 & 0.83 & 0.46 & 0.44 & 0.39 & 0.37 & 0.45 & 0.41 & 0.41 & 0.49 \\
\hline 50 & 0.43 & 0.46 & 0.73 & 1.23 & 0.87 & 0.48 & 0.45 & 0.41 & 0.38 & 0.46 & 0.42 & 0.44 & 0.51 \\
\hline 45 & 0.49 & 0.5 & 0.78 & 1.39 & 1.02 & 0.55 & 0.58 & 0.44 & 0.39 & 0.47 & 0.43 & 0.45 & 0.54 \\
\hline 40 & 0.55 & 0.52 & 0.81 & 1.57 & 1.16 & 0.62 & 0.62 & 0.46 & 0.4 & 0.48 & 0.44 & 0.46 & 0.58 \\
\hline 35 & 0.56 & 0.54 & 0.83 & 1.74 & 1.23 & 0.65 & 0.64 & 0.48 & 0.41 & 0.49 & 0.46 & 0.55 & 0.61 \\
\hline 30 & 0.57 & 0.56 & 0.87 & 1.91 & 1.28 & 0.7 & 0.65 & 0.49 & 0.46 & 0.56 & 0.56 & 0.56 & 0.65 \\
\hline 25 & 0.59 & 0.59 & 0.96 & 2.31 & 1.31 & 0.75 & 0.69 & 0.52 & 0.48 & 0.57 & 0.58 & 0.57 & 0.69 \\
\hline 20 & 0.6 & 0.61 & 0.99 & 2.6 & 1.42 & 0.86 & 0.75 & 0.56 & 0.57 & 0.57 & 0.6 & 0.64 & 0.78 \\
\hline 15 & 0.62 & 0.64 & 1.11 & 3.09 & 1.53 & 0.9 & 0.84 & 0.59 & 0.59 & 0.6 & 0.63 & 0.66 & 0.95 \\
\hline 10 & 0.63 & 0.68 & 1.23 & 3.69 & 1.67 & 0.93 & 1.04 & 0.6 & 0.61 & 0 & 0 & 0.68 & 1.16 \\
\hline 5 & 0.66 & 0.73 & 1.53 & 5.29 & 2.43 & 1.09 & 1.29 & 0.63 & 0.63 & 0 & 0 & 0.85 & 1.65 \\
\hline
\end{tabular}




\section{3- 4.L00-9T JILGA RIVER NEAR MECHALGHU, Continued}

Probability of occurrence of annual high discharges

[ $\mathrm{m}^{3} / \mathrm{s}$, meters per second; $\mathrm{ng}$, statistic not given]

\begin{tabular}{|c|c|c|c|c|c|c|}
\hline \multirow{2}{*}{$\begin{array}{c}\text { Exceedance } \\
\text { probability }\end{array}$} & \multirow{2}{*}{$\begin{array}{c}\text { Recurrence } \\
\text { interval } \\
\text { (years) }\end{array}$} & \multirow{2}{*}{$\begin{array}{c}\text { Maximum } \\
\text { instantaneous } \\
\left(\mathrm{m}^{3} / \mathrm{s}\right)\end{array}$} & \multicolumn{4}{|c|}{ Maximum mean discharge } \\
\hline & & & 3-day period & 7-day period & 15-day period & 30-day period \\
\hline 0.99 & 1.01 & 0.700 & 0.569 & 0.492 & 0.468 & 0.434 \\
\hline 0.95 & 1.05 & 1.20 & 0.794 & 0.667 & 0.627 & 0.583 \\
\hline 0.90 & 1.11 & 1.60 & 0.973 & 0.806 & 0.752 & 0.696 \\
\hline 0.80 & 1.25 & 2.30 & 1.28 & 1.04 & 0.960 & 0.883 \\
\hline 0.50 & 2 & 5.00 & 2.32 & 1.86 & 1.66 & 1.48 \\
\hline 0.20 & 5 & 11.3 & 4.75 & 3.74 & 3.24 & 2.74 \\
\hline 0.10 & 10 & 17.9 & 7.23 & 5.69 & 4.82 & 3.92 \\
\hline 0.04 & 25 & 30.0 & 11.8 & 9.27 & 7.65 & 5.92 \\
\hline 0.02 & 50 & 42.4 & 16.4 & 13.0 & 10.5 & 7.86 \\
\hline 0.01 & 100 & 58.3 & 22.5 & 17.9 & 14.2 & 10.3 \\
\hline 0.005 & 200 & 78.6 & 30.4 & 24.3 & 19.0 & 13.2 \\
\hline 0.002 & 500 & 114 & $\mathrm{ng}$ & $\mathrm{ng}$ & $\mathrm{ng}$ & ng \\
\hline
\end{tabular}

'Less than 10 years of data used.

3- 4.LO0-9T JILGA RIVER NEAR MECHALGHU, Continued

Probability of occurrence of annual low discharges

$\left[\mathrm{m}^{3} / \mathrm{s}\right.$, meters per second]

\begin{tabular}{|c|c|c|c|c|c|c|c|c|c|c|}
\hline \multirow{3}{*}{$\begin{array}{c}\text { Nonexceedance } \\
\text { probability }\end{array}$} & \multirow{3}{*}{$\begin{array}{c}\text { Recurrence } \\
\text { interval } \\
\text { (years) }\end{array}$} & \multicolumn{9}{|c|}{ Minimum mean discharge $\left(\mathrm{m}^{3} / \mathrm{s}\right)$} \\
\hline & & \multicolumn{9}{|c|}{ Number of consecutive days } \\
\hline & & 1 & 3 & 7 & 14 & 30 & 60 & 90 & 120 & 183 \\
\hline 0.05 & 20 & 0.060 & 0.067 & 0.069 & 0.069 & 0.074 & 0.123 & 0.145 & 0.164 & 0.230 \\
\hline 0.10 & 10 & 0.082 & 0.097 & 0.102 & 0.104 & 0.109 & 0.160 & 0.184 & 0.205 & 0.262 \\
\hline 0.20 & 5 & 0.117 & 0.145 & 0.155 & 0.159 & 0.165 & 0.213 & 0.240 & 0.261 & 0.306 \\
\hline 0.50 & 2 & 0.219 & 0.269 & 0.290 & 0.299 & 0.308 & 0.339 & 0.366 & 0.386 & 0.405 \\
\hline
\end{tabular}




\section{3- 4.L00-9T JILGA RIVER NEAR MECHALGHU, Continued}

Probability of occurrence of seasonal low discharges

$\left[\mathrm{m}^{3} / \mathrm{s}\right.$, meters per second]

\begin{tabular}{|c|c|c|c|c|c|c|c|c|c|}
\hline \multirow{3}{*}{$\begin{array}{l}\text { Nonexceedance } \\
\text { probability }\end{array}$} & \multirow{3}{*}{$\begin{array}{c}\text { Recurrence } \\
\text { interval } \\
\text { (years) }\end{array}$} & \multicolumn{8}{|c|}{ Minimum mean discharge $\left(\mathrm{m}^{3} / \mathrm{s}\right)$} \\
\hline & & \multicolumn{8}{|c|}{ Number of consecutive days } \\
\hline & & 1 & 7 & 14 & 30 & 1 & 7 & 14 & 30 \\
\hline & & \multicolumn{4}{|c|}{ December-January-February } & \multicolumn{4}{|c|}{ March-April-May } \\
\hline 0.05 & 20 & 0.073 & 0.075 & ${ }^{1} 0.074$ & 0.080 & 0.256 & 0.281 & 0.324 & 0.392 \\
\hline 0.10 & 10 & 0.108 & 0.115 & 0.115 & 0.122 & 0.279 & 0.313 & 0.355 & 0.453 \\
\hline 0.20 & 5 & 0.166 & 0.181 & 0.183 & 0.190 & 0.313 & 0.358 & 0.399 & 0.530 \\
\hline \multirow[t]{2}{*}{0.50} & 2 & 0.318 & 0.348 & 0.361 & 0.369 & 0.406 & 0.466 & 0.504 & 0.683 \\
\hline & & \multicolumn{4}{|c|}{ June-July-August } & \multicolumn{4}{|c|}{ September-October-November } \\
\hline 0.05 & 20 & 0.136 & 0.215 & 0.226 & 0.249 & 0.067 & 0.096 & 0.131 & 0.184 \\
\hline 0.10 & 10 & 0.158 & 0.230 & 0.246 & 0.272 & 0.100 & 0.133 & 0.165 & 0.215 \\
\hline 0.20 & 5 & 0.191 & 0.254 & 0.274 & 0.306 & 0.154 & 0.187 & 0.213 & 0.258 \\
\hline 0.50 & 2 & 0.277 & 0.318 & 0.349 & 0.386 & 0.300 & 0.320 & 0.325 & 0.354 \\
\hline
\end{tabular}

'Data does not fit log-Pearson Type III curve, use with caution.

\section{3- 4.L00-9T JILGA RIVER NEAR MECHALGHU, Continued}

\section{Annual peak discharge and corresponding gage height}

[m³/s, meters per second; --, no data]

\begin{tabular}{|c|c|c|c|c|c|c|c|}
\hline $\begin{array}{c}\text { Water } \\
\text { year }\end{array}$ & Date & $\begin{array}{c}\text { Gage } \\
\text { height } \\
\text { (meters) }\end{array}$ & $\begin{array}{c}\text { Peak } \\
\text { discharge } \\
\left(\mathrm{m}^{3} / \mathrm{s}\right)\end{array}$ & $\begin{array}{c}\text { Water } \\
\text { year }\end{array}$ & Date & $\begin{array}{c}\text { Gage } \\
\text { height } \\
\text { (meters) }\end{array}$ & $\begin{array}{c}\text { Peak } \\
\text { discharge } \\
\left(\mathrm{m}^{3} / \mathrm{s}\right)\end{array}$ \\
\hline \multicolumn{8}{|c|}{ Annual peak discharge, by year } \\
\hline 1971 & 30-Jul & & 20.8 & 1975 & 25-Apr & & 4.12 \\
\hline 1972 & 28-Apr & & 7.34 & 1976 & 18-Apr & & 17.4 \\
\hline 1973 & 2-Jul & & 4.40 & 1977 & 17-Apr & & 1.91 \\
\hline 1974 & 23-Mar & & 1.50 & 1978 & $5-J u l$ & & 4.00 \\
\hline \multicolumn{8}{|c|}{ Annual peak discharge, from highest to lowest } \\
\hline 1971 & 30-Jul & & 20.8 & 1975 & 25-Apr & & 4.12 \\
\hline 1976 & 18-Apr & & 17.4 & 1978 & 5-Jul & & 4.00 \\
\hline 1972 & 28-Apr & & 7.34 & 1977 & 17-Apr & & 1.91 \\
\hline 1973 & 2-Jul & & 4.40 & 1974 & 23-Mar & & 1.50 \\
\hline
\end{tabular}




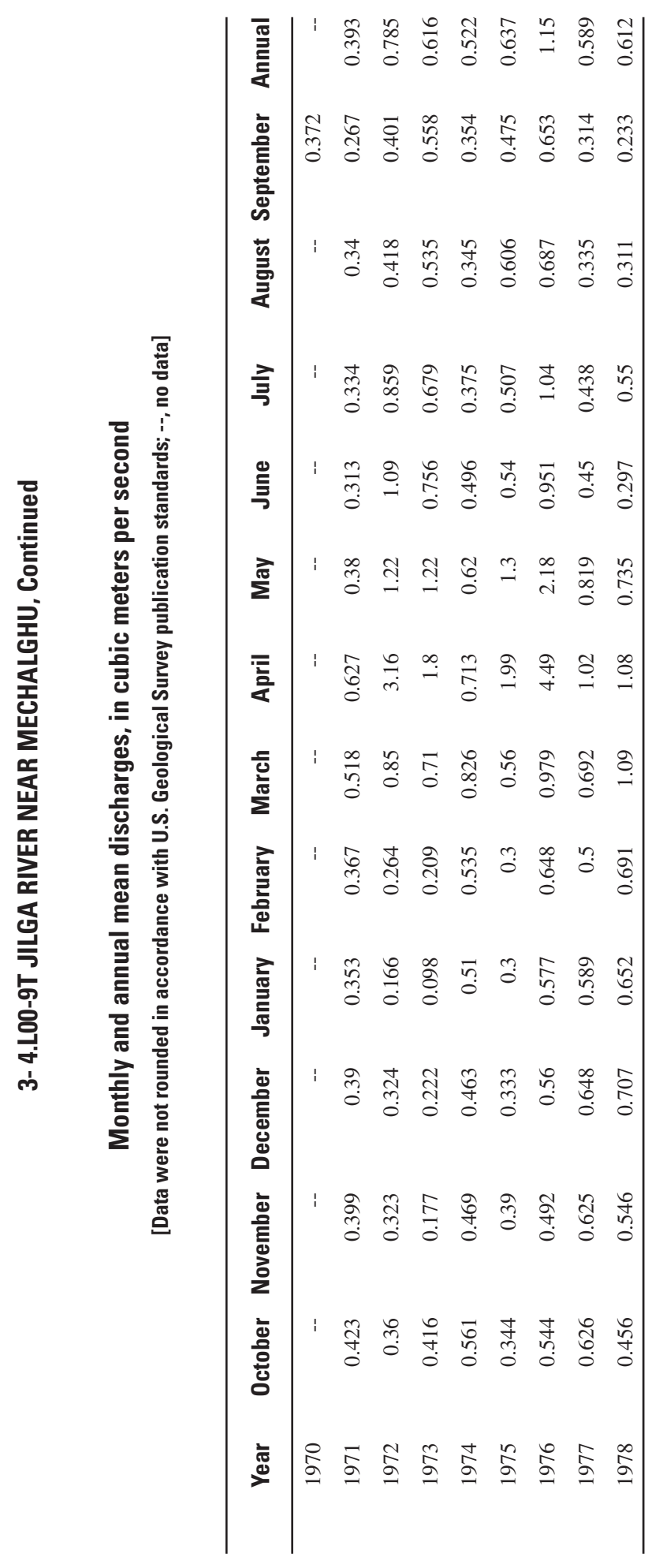




\section{3- 5.R00-3T SYAHGEL RIVER AT SYAHGEL}

\section{(U.S. Geological Survey identification number: 333100068210000)}

LOCATION: Lat $33^{\circ} 31^{\prime} \mathrm{N}$. , long $68^{\circ} 21^{\prime} \mathrm{E}$.

DRAINAGE AREA: $145 \mathrm{~km}^{2}$.

ALTITUDE: 2,241 meters plus mean sea level.

PERIOD OF RECORD: April 7, 1968 to September 22, 1976.

GAGE: --

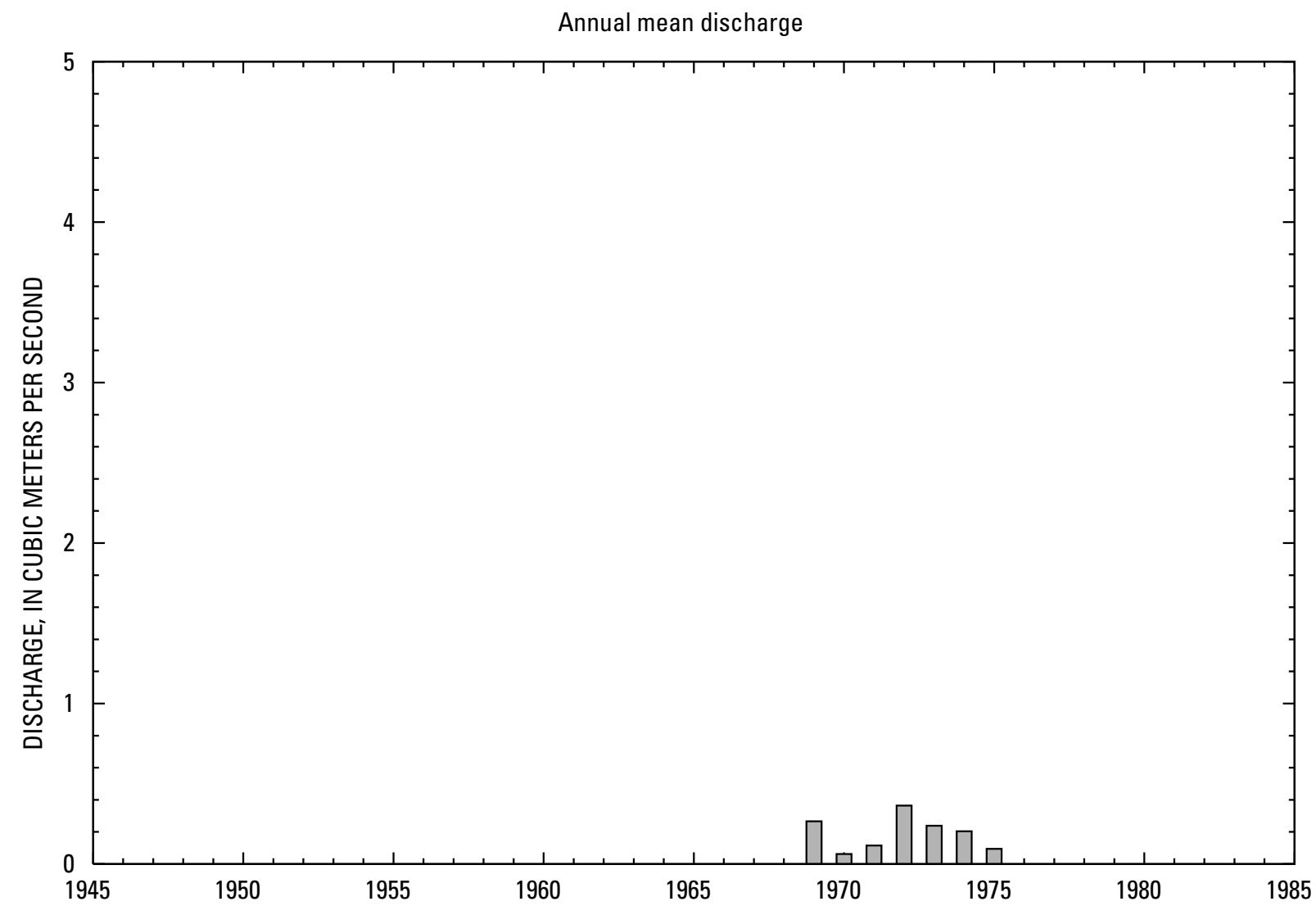




\section{3- 5.R00-3T SYAHGEL RIVER AT SYAHGEL, Continued}

\section{Statistics of monthly and annual mean discharges}

$\left[\mathrm{m}^{3} / \mathrm{s}\right.$, meters per second]

\begin{tabular}{|c|c|c|c|c|c|c|c|c|}
\hline \multirow[b]{2}{*}{ Month } & \multicolumn{2}{|c|}{ Maximum } & \multicolumn{2}{|c|}{ Minimum } & \multicolumn{4}{|c|}{ Mean } \\
\hline & $\begin{array}{c}\text { Discharge } \\
\left(\mathrm{m}^{3} / \mathrm{s}\right)\end{array}$ & $\begin{array}{c}\text { Water year } \\
\text { of } \\
\text { occurrence }\end{array}$ & $\begin{array}{c}\text { Discharge } \\
\left(\mathrm{m}^{3} / \mathrm{s}\right)\end{array}$ & $\begin{array}{c}\text { Water year } \\
\text { of } \\
\text { occurrence }\end{array}$ & $\begin{array}{c}\text { Discharge } \\
\left(\mathrm{m}^{3} / \mathrm{s}\right)\end{array}$ & $\begin{array}{c}\text { Standard } \\
\text { deviation } \\
\left(\mathrm{m}^{3} / \mathrm{s}\right)\end{array}$ & $\begin{array}{c}\text { Coefficient } \\
\text { of } \\
\text { variation }\end{array}$ & $\begin{array}{c}\text { Percentage } \\
\text { of annual } \\
\text { discharge }\end{array}$ \\
\hline October & 0.110 & 1970 & 0.010 & 1969 & 0.04 & 0.03 & 0.86 & 1.43 \\
\hline November & 0.220 & 1973 & 0.010 & 1969 & 0.05 & 0.07 & 1.29 & 2.08 \\
\hline December & 0.390 & 1973 & 0.034 & 1969 & 0.12 & 0.14 & 1.11 & 4.84 \\
\hline January & 0.796 & 1969 & 0.066 & 1975 & 0.29 & 0.26 & 0.9 & 11.2 \\
\hline February & 0.960 & 1969 & 0.078 & 1975 & 0.34 & 0.29 & 0.85 & 13.5 \\
\hline March & 0.727 & 1976 & 0.154 & 1970 & 0.42 & 0.19 & 0.45 & 16.3 \\
\hline April & 1.94 & 1972 & 0.057 & 1969 & 0.57 & 0.66 & 1.16 & 22.3 \\
\hline May & 1.07 & 1968 & 0.023 & 1971 & 0.34 & 0.38 & 1.12 & 13.5 \\
\hline June & 0.606 & 1972 & 0.012 & 1970 & 0.14 & 0.18 & 1.30 & 5.54 \\
\hline July & 0.298 & 1969 & 0.025 & 1972 & 0.10 & 0.10 & 0.96 & 4.02 \\
\hline August & 0.410 & 1969 & 0.019 & 1972 & 0.10 & 0.12 & 1.18 & 4.05 \\
\hline September & 0.060 & 1969 & 0.012 & 1970 & 0.03 & 0.02 & 0.49 & 1.29 \\
\hline Annual & 0.364 & 1972 & 0.062 & 1970 & 0.19 & 0.11 & 0.56 & 100 \\
\hline
\end{tabular}

Annual flow duration

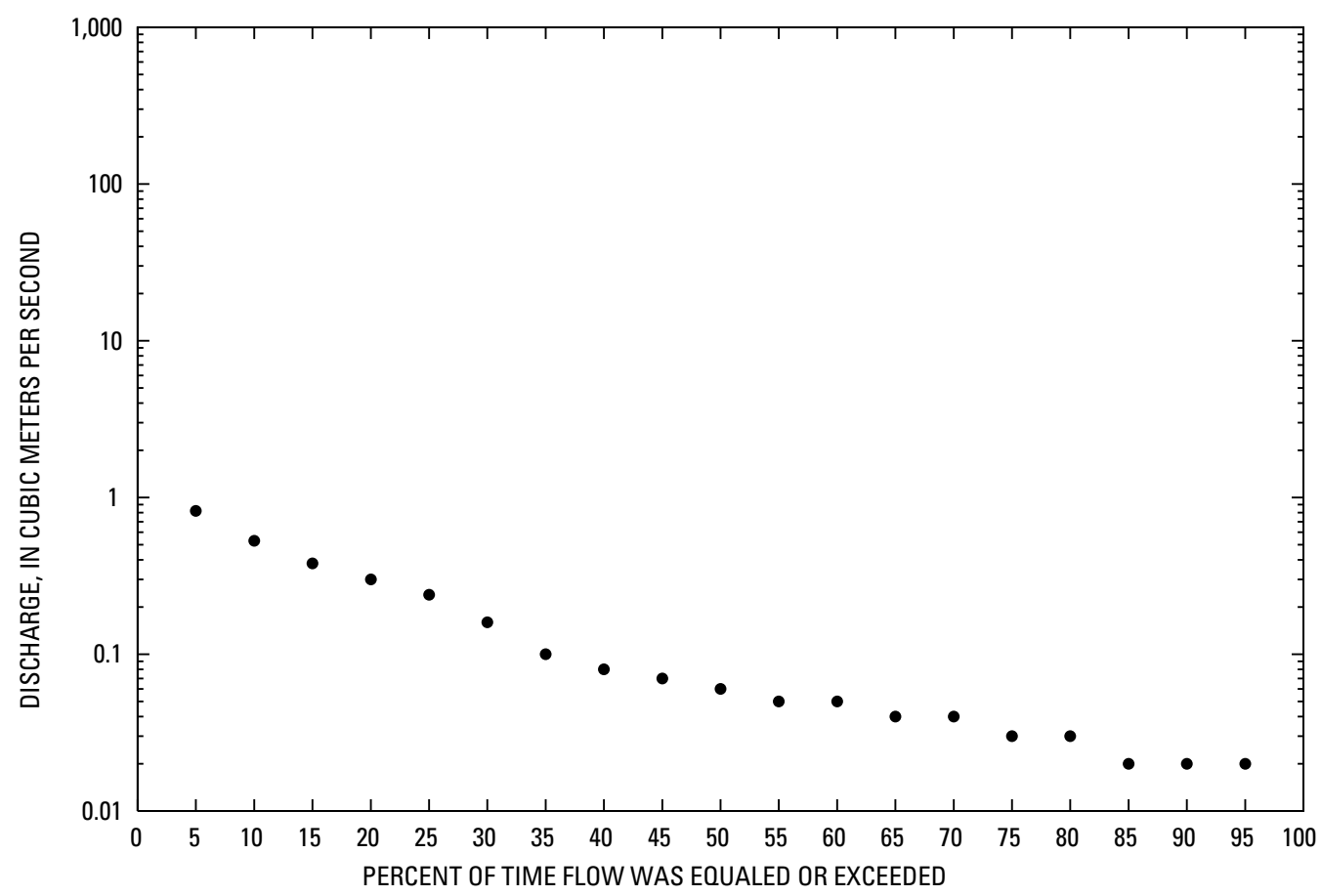




\section{3- 5.R00-3T SYAHGEL RIVER AT SYAHgEL, Continued}

\section{Monthly and annual flow duration, in cubic meters per second}

[Data were not rounded in accordance with U.S. Geological Survey publication standards]

\begin{tabular}{|c|c|c|c|c|c|c|c|c|c|c|c|c|c|}
\hline $\begin{array}{l}\text { Percentage } \\
\text { of days } \\
\text { discharge } \\
\text { equaled or } \\
\text { exceeded }\end{array}$ & January & February & March & April & May & June & July & August & September & October & November & December & Annual \\
\hline 95 & 0.04 & 0.06 & 0.07 & 0.02 & 0.02 & 0.02 & 0.01 & 0.01 & 0.01 & 0.01 & 0.01 & 0.02 & 0.02 \\
\hline 90 & 0.06 & 0.07 & 0.11 & 0.03 & 0.03 & 0.02 & 0.02 & 0.02 & 0.02 & 0.02 & 0.02 & 0.03 & 0.02 \\
\hline 80 & 0.08 & 0.1 & 0.19 & 0.05 & 0.04 & 0.03 & 0.02 & 0.02 & 0.02 & 0.02 & 0.02 & 0.04 & 0.03 \\
\hline 75 & 0.08 & 0.11 & 0.23 & 0.06 & 0.05 & 0.03 & 0.03 & 0.03 & 0.03 & 0.02 & 0.02 & 0.04 & 0.03 \\
\hline 70 & 0.09 & 0.15 & 0.25 & 0.07 & 0.05 & 0.04 & 0.03 & 0.03 & 0.03 & 0.02 & 0.03 & 0.04 & 0.04 \\
\hline 65 & 0.1 & 0.2 & 0.28 & 0.08 & 0.06 & 0.04 & 0.03 & 0.03 & 0.03 & 0.03 & 0.03 & 0.05 & 0.04 \\
\hline 60 & 0.13 & 0.24 & 0.34 & 0.14 & 0.07 & 0.05 & 0.04 & 0.04 & 0.03 & 0.03 & 0.03 & 0.05 & 0.05 \\
\hline 40 & 0.26 & 0.31 & 0.45 & 0.47 & 0.32 & 0.07 & 0.05 & 0.05 & 0.04 & 0.03 & 0.04 & 0.07 & 0.08 \\
\hline 35 & 0.27 & 0.33 & 0.47 & 0.52 & 0.36 & 0.09 & 0.06 & 0.05 & 0.04 & 0.04 & 0.04 & 0.09 & 0.1 \\
\hline 30 & 0.28 & 0.35 & 0.52 & 0.6 & 0.43 & 0.11 & 0.06 & 0.06 & 0.04 & 0.04 & 0.04 & 0.11 & 0.16 \\
\hline 25 & 0.29 & 0.42 & 0.57 & 0.89 & 0.53 & 0.12 & 0.07 & 0.08 & 0.04 & 0.04 & 0.05 & 0.13 & 0.24 \\
\hline 20 & 0.35 & 0.54 & 0.61 & 1 & 0.66 & 0.16 & 0.08 & 0.1 & 0.05 & 0.04 & 0.06 & 0.2 & 0.3 \\
\hline 15 & 0.54 & 0.69 & 0.67 & 1.29 & 0.85 & 0.22 & 0.14 & 0.13 & 0.05 & 0.06 & 0.07 & 0.34 & 0.38 \\
\hline 10 & 0.68 & 0.82 & 0.82 & 1.64 & 0.93 & 0.3 & 0.19 & 0.15 & 0.07 & 0.07 & 0.16 & 0.37 & 0.53 \\
\hline 5 & 1 & 1.09 & 0.93 & 1.91 & 1.24 & 0.44 & 0.36 & 0.66 & 0.09 & 0.14 & 0 & 0 & 0.82 \\
\hline
\end{tabular}




\section{3- 5.RO0-3T SYAHGEL RIVER AT SYAHGEL, Continued}

Probability of occurrence of annual high discharges

[ $\mathrm{m}^{3} / \mathrm{s}$, meters per second; $\mathbf{n g}$, statistic not given]

\begin{tabular}{|c|c|c|c|c|c|c|}
\hline \multirow{2}{*}{$\begin{array}{l}\text { Exceedance } \\
\text { probability }\end{array}$} & \multirow{2}{*}{$\begin{array}{c}\text { Recurrence } \\
\text { interval } \\
\text { (years) }\end{array}$} & \multirow{2}{*}{$\begin{array}{c}\text { Maximum } \\
\text { instantaneous } \\
\left(\mathrm{m}^{3} / \mathrm{s}\right)\end{array}$} & \multicolumn{4}{|c|}{ Maximum mean discharge } \\
\hline & & & 3-day period & 7-day period & 15-day period & 30-day period \\
\hline 0.99 & 1.01 & 0.800 & 0.242 & 0.135 & 0.124 & 0.095 \\
\hline 0.95 & 1.05 & 1.60 & 0.362 & 0.232 & 0.213 & 0.171 \\
\hline 0.90 & 1.11 & 2.20 & 0.458 & 0.310 & 0.283 & 0.232 \\
\hline 0.80 & 1.25 & 3.20 & 0.621 & 0.443 & 0.400 & 0.332 \\
\hline 0.50 & 2 & 5.90 & 1.18 & 0.879 & 0.770 & 0.641 \\
\hline 0.20 & 5 & 10.1 & 2.45 & 1.76 & 1.48 & 1.19 \\
\hline 0.10 & 10 & 12.8 & 3.70 & 2.55 & 2.07 & 1.63 \\
\hline 0.04 & 25 & 16.0 & 5.92 & 3.78 & 2.97 & 2.24 \\
\hline 0.02 & 50 & 18.3 & 8.14 & 4.89 & 3.74 & 2.74 \\
\hline 0.01 & 100 & 20.4 & 11.0 & 6.16 & 4.60 & 3.28 \\
\hline 0.005 & 200 & 22.4 & 14.5 & 7.62 & 5.57 & 3.85 \\
\hline 0.002 & 500 & 24.8 & ng & ng & ng & ng \\
\hline
\end{tabular}

'Less than 10 years data used.

3- 5.R00-3T SYAHGEL RIVER AT SYAHGEL, Continued

Probability of occurrence of annual low discharges

$\left[\mathrm{m}^{3} / \mathrm{s}\right.$, meters per second]

\begin{tabular}{|c|c|c|c|c|c|c|c|c|c|c|}
\hline \multirow{3}{*}{$\begin{array}{c}\text { Nonexceedance } \\
\text { probability }\end{array}$} & \multirow{3}{*}{$\begin{array}{c}\text { Recurrence } \\
\text { interval } \\
\text { (years) }\end{array}$} & \multicolumn{9}{|c|}{ Minimum mean discharge $\left(\mathrm{m}^{3} / \mathrm{s}\right)$} \\
\hline & & \multicolumn{9}{|c|}{ Number of consecutive days } \\
\hline & & 1 & 3 & 7 & 14 & 30 & 60 & 90 & 120 & 183 \\
\hline 0.05 & 20 & 0.007 & 0.007 & 0.009 & 0.010 & 0.010 & 0.015 & 0.015 & 0.017 & 0.022 \\
\hline 0.10 & 10 & 0.008 & 0.008 & 0.010 & 0.012 & 0.012 & 0.016 & 0.016 & 0.019 & 0.025 \\
\hline 0.20 & 5 & 0.010 & 0.010 & 0.011 & 0.014 & 0.015 & 0.017 & 0.018 & 0.021 & 0.031 \\
\hline 0.50 & 2 & 0.013 & 0.013 & 0.015 & 0.018 & 0.021 & 0.022 & 0.024 & 0.027 & 0.046 \\
\hline
\end{tabular}




\section{3- 5.R00-3T SYAHGEL RIVER AT SYAHGEL, Continued}

\section{Probability of occurrence of seasonal low discharges}

$\left[\mathrm{m}^{3} / \mathrm{s}\right.$, meters per second]

\begin{tabular}{|c|c|c|c|c|c|c|c|c|c|}
\hline \multirow{3}{*}{$\begin{array}{l}\text { Nonexceedance } \\
\text { probability }\end{array}$} & \multirow{3}{*}{$\begin{array}{c}\text { Recurrence } \\
\text { interval } \\
\text { (years) }\end{array}$} & \multicolumn{8}{|c|}{ Minimum mean discharge $\left(\mathrm{m}^{3} / \mathrm{s}\right)$} \\
\hline & & \multicolumn{8}{|c|}{ Number of consecutive days } \\
\hline & & 1 & 7 & 14 & 30 & 1 & 7 & 14 & 30 \\
\hline & & \multicolumn{4}{|c|}{ December-January-February } & \multicolumn{4}{|c|}{ March-April-May } \\
\hline 0.05 & 20 & 0.008 & 0.011 & ${ }^{1} 0.010$ & 0.024 & 0.006 & 0.008 & 0.008 & 0.013 \\
\hline 0.10 & 10 & 0.010 & 0.013 & 0.013 & 0.028 & 0.008 & 0.012 & 0.012 & 0.020 \\
\hline 0.20 & 5 & 0.013 & 0.017 & 0.019 & 0.034 & 0.014 & 0.019 & 0.020 & 0.031 \\
\hline \multirow[t]{2}{*}{0.50} & 2 & 0.028 & 0.033 & 0.040 & 0.059 & 0.040 & 0.051 & 0.058 & 0.084 \\
\hline & & \multicolumn{4}{|c|}{ June-July-August } & \multicolumn{4}{|c|}{ September-October-November } \\
\hline 0.05 & 20 & 0.007 & 0.008 & 0.010 & 0.010 & 0.008 & 0.009 & 0.009 & 0.009 \\
\hline 0.10 & 10 & 0.008 & 0.009 & 0.012 & 0.013 & 0.009 & 0.011 & 0.011 & 0.011 \\
\hline 0.20 & 5 & 0.010 & 0.011 & 0.015 & 0.017 & 0.011 & 0.013 & 0.013 & 0.014 \\
\hline 0.50 & 2 & 0.014 & 0.016 & 0.022 & 0.031 & 0.016 & 0.019 & 0.019 & 0.022 \\
\hline
\end{tabular}

'Data does not fit log-Pearson Type III curve, use with caution.

\section{3- 5.R00-3T SYAHGEL RIVER AT SYAHGEL, Continued}

\section{Annual peak discharge and corresponding gage height}

[m³/s, meters per second; --, no data]

\begin{tabular}{|c|c|c|c|c|c|c|c|}
\hline $\begin{array}{c}\text { Water } \\
\text { year }\end{array}$ & Date & $\begin{array}{c}\text { Gage } \\
\text { height } \\
\text { (meters) }\end{array}$ & $\begin{array}{c}\text { Peak } \\
\text { discharge } \\
\left(\mathrm{m}^{3} / \mathrm{s}\right)\end{array}$ & $\begin{array}{c}\text { Water } \\
\text { year }\end{array}$ & Date & $\begin{array}{c}\text { Gage } \\
\text { height } \\
\text { (meters) }\end{array}$ & $\begin{array}{c}\text { Peak } \\
\text { discharge } \\
\left(\mathrm{m}^{3} / \mathrm{s}\right)\end{array}$ \\
\hline \multicolumn{8}{|c|}{ Annual peak discharge, by year } \\
\hline 1968 & 19-May & & 2.58 & 1973 & 7-Aug & & 5.20 \\
\hline 1969 & 9-May & & 3.00 & 1974 & 12-May & & 10.0 \\
\hline 1970 & 7-Jul & & 6.60 & 1975 & 11-Aug & & 12.0 \\
\hline 1971 & 4-Jul & & 9.00 & 1976 & 23-Apr & & 1.69 \\
\hline 1972 & 26-Apr & & 10 & & & & \\
\hline \multicolumn{8}{|c|}{ Annual peak discharge, from highest to lowest } \\
\hline 1975 & 11-Aug & & 12.0 & 1973 & 7-Aug & & 5.20 \\
\hline 1974 & 12-May & & 10.0 & 1969 & 9-May & & 3.00 \\
\hline 1972 & 26-Apr & & 10.0 & 1968 & 19-May & & 2.58 \\
\hline 1971 & 4-Jul & & 9.00 & 1976 & 23-Apr & & 1.69 \\
\hline 1970 & 7-Jul & & 6.60 & & & & \\
\hline
\end{tabular}




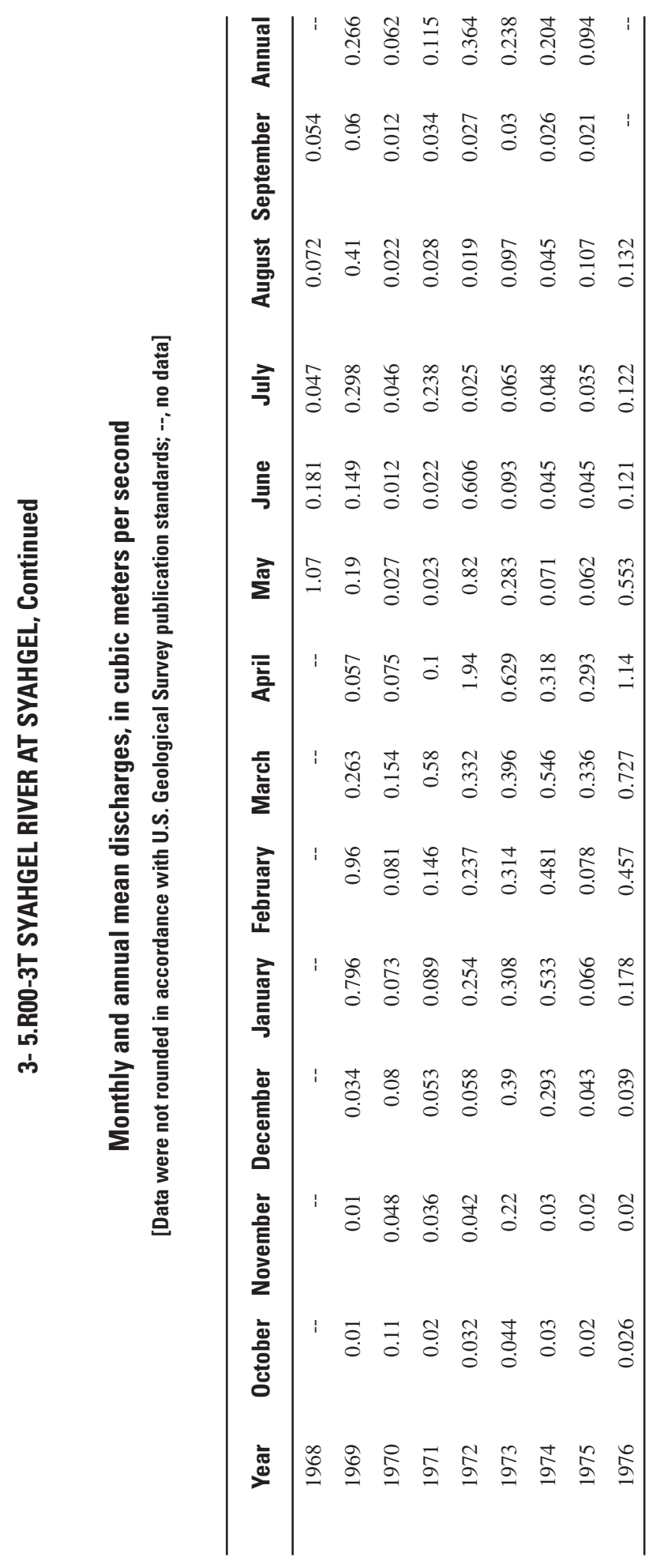




\section{3- 8.1L0-1W BARIKAB RIVER ABOVE SERAJ RESERVOIR}

\section{(U.S. Geological Survey identification number: 334600068210000 )}

LOCATION: Lat $33^{\circ} 46^{\prime}$ N., long $68^{\circ} 21^{\prime}$ E., on left bank at Khojagan.

DRAINAGE AREA: $165 \mathrm{~km}^{2}$.

ALTITUDE: 2,386 meters plus mean sea level.

PERIOD OF RECORD: April 16, 1968 to September 18, 1976.

GAGE: Staff gage read once or twice daily.

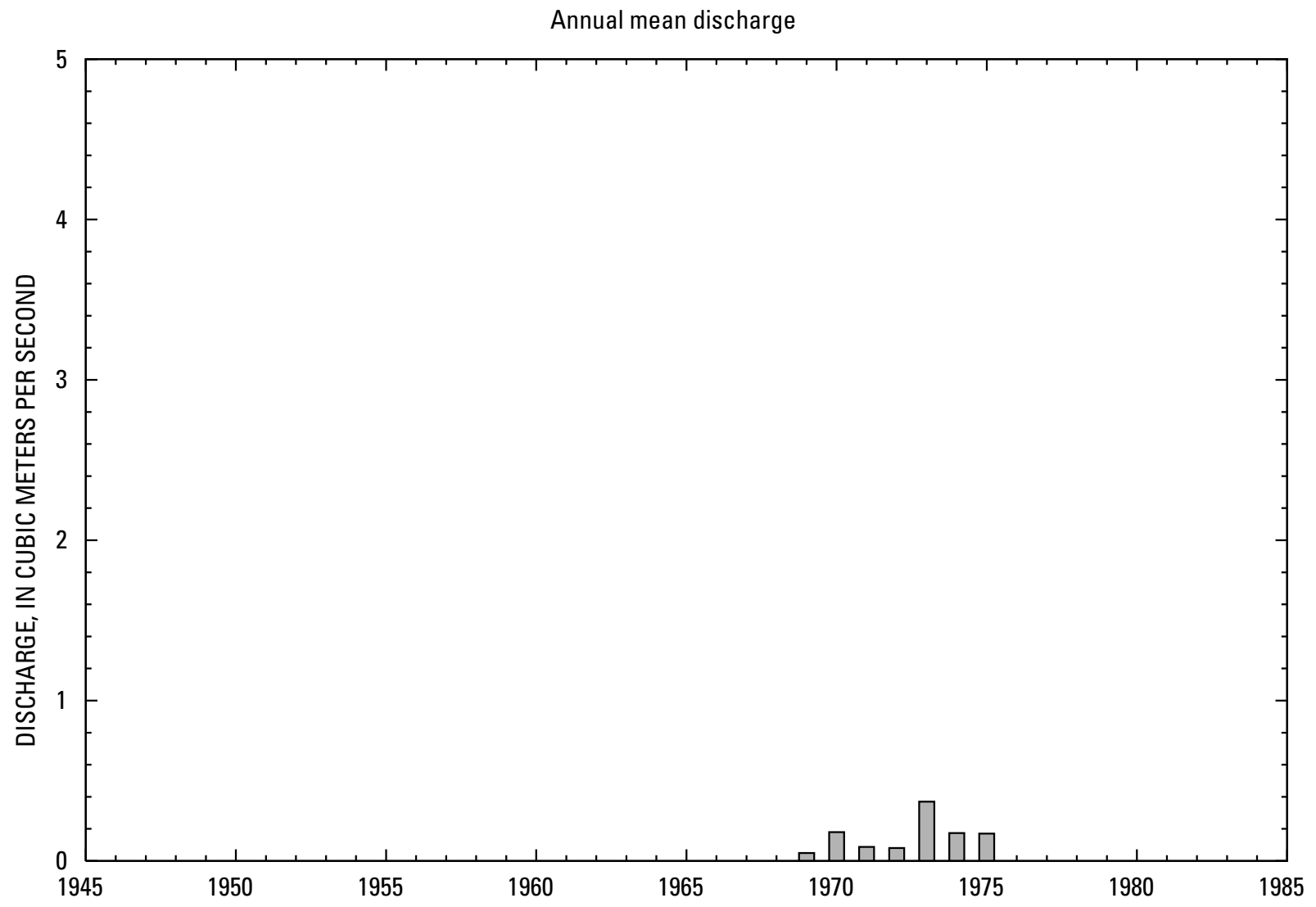




\section{3- 8.1LO-1W BARIKAB RIVER ABOVE SERAJ RESERVOIR, Continued}

\section{Statistics of monthly and annual mean discharges}

[ $\mathrm{m}^{3} / \mathrm{s}$, meters per second; $\mathrm{m}$, more than 2 years of occurrence]

\begin{tabular}{|c|c|c|c|c|c|c|c|c|}
\hline \multirow[b]{2}{*}{ Month } & \multicolumn{2}{|c|}{ Maximum } & \multicolumn{2}{|c|}{ Minimum } & \multicolumn{4}{|c|}{ Mean } \\
\hline & $\begin{array}{c}\text { Discharge } \\
\left(\mathrm{m}^{3} / \mathrm{s}\right)\end{array}$ & $\begin{array}{c}\text { Water year } \\
\text { of } \\
\text { occurrence }\end{array}$ & $\begin{array}{c}\text { Discharge } \\
\left(\mathrm{m}^{3} / \mathrm{s}\right)\end{array}$ & $\begin{array}{c}\text { Water year } \\
\text { of } \\
\text { occurrence }\end{array}$ & $\begin{array}{c}\text { Discharge } \\
\left(\mathrm{m}^{3} / \mathrm{s}\right)\end{array}$ & $\begin{array}{c}\text { Standard } \\
\text { deviation } \\
\left(\mathrm{m}^{3} / \mathrm{s}\right)\end{array}$ & $\begin{array}{c}\text { Coefficient } \\
\text { of } \\
\text { variation }\end{array}$ & $\begin{array}{c}\text { Percentage } \\
\text { of annual } \\
\text { discharge }\end{array}$ \\
\hline October & 0.215 & 1975 & 0.010 & $\mathrm{~m}$ & 0.06 & 0.07 & 1.25 & 3.12 \\
\hline November & 0.465 & 1973 & 0.011 & 1976 & 0.13 & 0.15 & 1.17 & 6.87 \\
\hline December & 0.640 & 1973 & 0.010 & 1976 & 0.17 & 0.21 & 1.24 & 8.73 \\
\hline January & 0.575 & 1973 & 0.010 & 1969 & 0.24 & 0.21 & 0.84 & 12.8 \\
\hline February & 0.496 & 1973 & 0.010 & 1969 & 0.23 & 0.18 & 0.77 & 12.3 \\
\hline March & 0.639 & 1973 & 0.014 & 1969 & 0.29 & 0.21 & 0.73 & 15.2 \\
\hline April & 1.35 & 1973 & 0.067 & 1971 & 0.51 & 0.47 & 0.93 & 26.5 \\
\hline May & 0.117 & 1969 & 0.035 & 1971 & 0.07 & 0.03 & 0.40 & 3.66 \\
\hline June & 0.106 & 1974 & 0.010 & 1971 & 0.04 & 0.03 & 0.73 & 2.30 \\
\hline July & 0.170 & 1974 & 0.019 & 1975 & 0.06 & 0.05 & 0.80 & 3.23 \\
\hline August & 0.359 & 1970 & 0.005 & 1971 & 0.08 & 0.11 & 1.35 & 4.19 \\
\hline September & 0.083 & 1968 & 0 & 1971 & 0.02 & 0.03 & 1.17 & 1.18 \\
\hline Annual & 0.370 & 1973 & 0.050 & 1969 & 0.16 & 0.11 & 0.67 & 100 \\
\hline
\end{tabular}

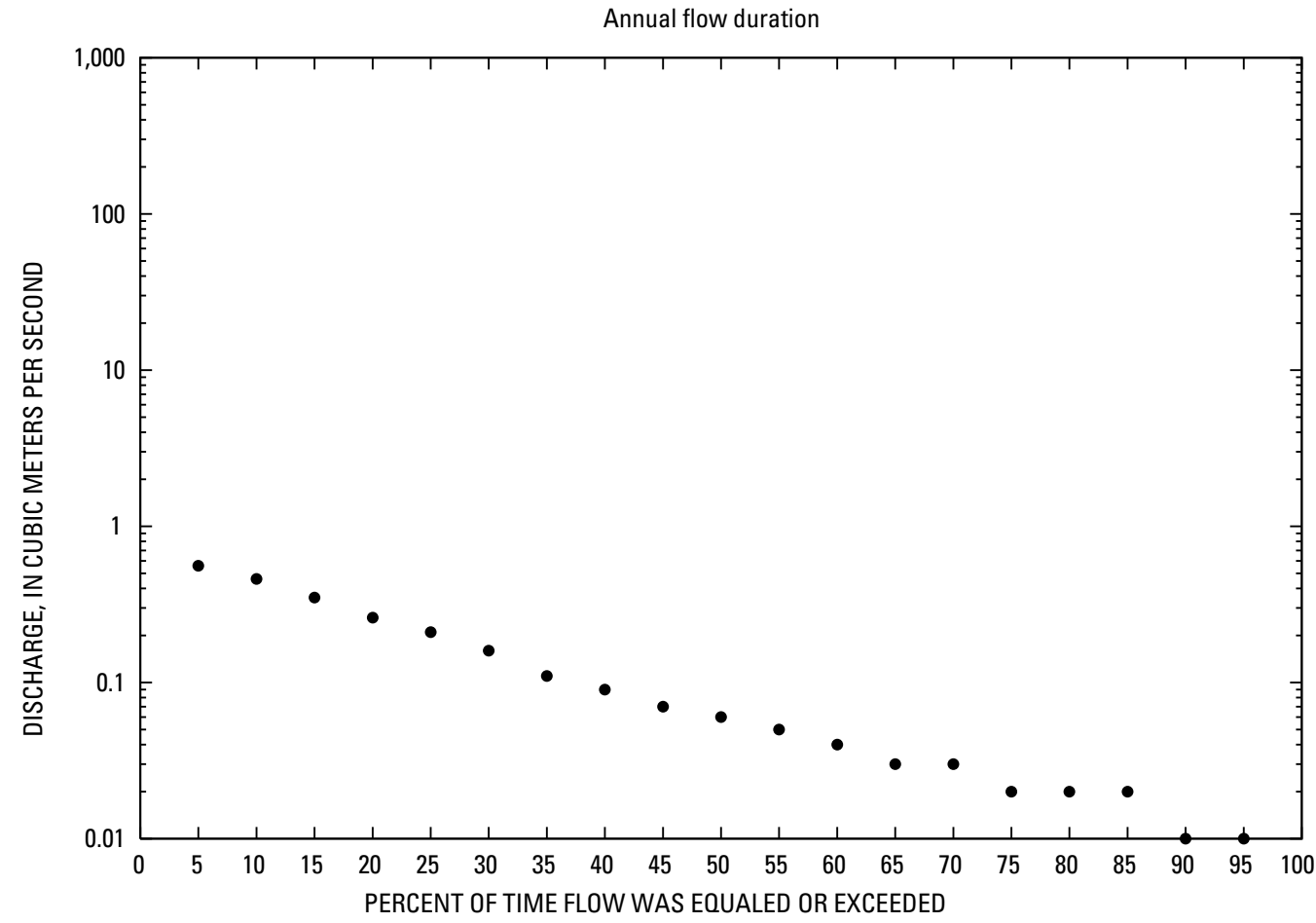


3- 8.1LO-1W BARIKAB RIVER ABOVE SERAJ RESERVOIR, Continued

Monthly and annual flow duration, in cubic meters per second

[Data were not rounded in accordance with U.S. Geological Survey publication standards]

\begin{tabular}{|c|c|c|c|c|c|c|c|c|c|c|c|c|c|}
\hline $\begin{array}{l}\text { Percentage } \\
\text { of days } \\
\text { discharge } \\
\text { equaled or } \\
\text { exceeded }\end{array}$ & January & February & March & April & May & June & July & August & September & October & November & December & Annual \\
\hline 95 & 0.01 & 0.01 & 0.02 & 0.04 & 0.02 & 0.01 & 0.01 & 0 & 0 & 0.01 & 0.01 & 0.01 & 0.01 \\
\hline 90 & 0.02 & 0.02 & 0.02 & 0.05 & 0.03 & 0.01 & 0.01 & 0.01 & 0 & 0.01 & 0.02 & 0.02 & 0.01 \\
\hline 85 & 0.02 & 0.02 & 0.04 & 0.06 & 0.03 & 0.02 & 0.02 & 0.01 & 0.01 & 0.01 & 0.02 & 0.02 & 0.02 \\
\hline 80 & 0.03 & 0.03 & 0.07 & 0.07 & 0.04 & 0.02 & 0.02 & 0.02 & 0.01 & 0.01 & 0.02 & 0.02 & 0.02 \\
\hline 75 & 0.05 & 0.05 & 0.11 & 0.09 & 0.04 & 0.02 & 0.02 & 0.02 & 0.01 & 0.01 & 0.03 & 0.03 & 0.02 \\
\hline 70 & 0.13 & 0.09 & 0.12 & 0.1 & 0.04 & 0.02 & 0.03 & 0.02 & 0.01 & 0.02 & 0.03 & 0.05 & 0.03 \\
\hline 65 & 0.16 & 0.13 & 0.18 & 0.11 & 0.05 & 0.03 & 0.03 & 0.03 & 0.01 & 0.02 & 0.03 & 0.06 & 0.03 \\
\hline 60 & 0.16 & 0.14 & 0.22 & 0.15 & 0.05 & 0.03 & 0.03 & 0.03 & 0.02 & 0.02 & 0.05 & 0.06 & 0.04 \\
\hline 55 & 0.17 & 0.18 & 0.24 & 0.23 & 0.05 & 0.03 & 0.03 & 0.04 & 0.02 & 0.02 & 0.06 & 0.07 & 0.05 \\
\hline 50 & 0.18 & 0.23 & 0.29 & 0.28 & 0.06 & 0.04 & 0.04 & 0.05 & 0.02 & 0.02 & 0.07 & 0.09 & 0.06 \\
\hline 45 & 0.2 & 0.26 & 0.34 & 0.35 & 0.06 & 0.04 & 0.04 & 0.05 & 0.02 & 0.02 & 0.08 & 0.12 & 0.07 \\
\hline 40 & 0.26 & 0.27 & 0.37 & 0.46 & 0.07 & 0.04 & 0.04 & 0.06 & 0.02 & 0.04 & 0.1 & 0.14 & 0.09 \\
\hline 35 & 0.34 & 0.29 & 0.39 & 0.53 & 0.07 & 0.05 & 0.06 & 0.07 & 0.02 & 0.06 & 0.12 & 0.15 & 0.11 \\
\hline 30 & 0.41 & 0.38 & 0.41 & 0.6 & 0.08 & 0.06 & 0.07 & 0.08 & 0.03 & 0.09 & 0.16 & 0.17 & 0.16 \\
\hline 25 & 0.42 & 0.42 & 0.43 & 0.71 & 0.09 & 0.07 & 0.08 & 0.08 & 0.03 & 0.1 & 0.19 & 0.19 & 0.21 \\
\hline 20 & 0.44 & 0.46 & 0.44 & 0.82 & 0.1 & 0.08 & 0.09 & 0.09 & 0.04 & 0.11 & 0.22 & 0.29 & 0.26 \\
\hline 15 & 0.48 & 0.49 & 0.48 & 1.06 & 0.12 & 0.09 & 0.1 & 0.09 & 0.05 & 0.13 & 0.26 & 0.34 & 0.35 \\
\hline 10 & 0.56 & 0.51 & 0.51 & 1.46 & 0.13 & 0.09 & 0.15 & 0.1 & 0 & 0.18 & 0.31 & 0.57 & 0.46 \\
\hline 5 & 0 & 0.53 & 0.64 & 2.01 & 0.18 & 0.11 & 0.24 & 0.1 & 0 & 0.23 & 0.58 & 0.66 & 0.56 \\
\hline
\end{tabular}




\section{3- 8.1LO-1W BARIKAB RIVER ABOVE SERAJ RESERVOIR, Continued}

Probability of occurrence of annual high discharges

$\left[\mathrm{m}^{3} / \mathrm{s}\right.$, meters per second; $\mathrm{ng}$, statistic not given]

\begin{tabular}{rrrrrrr}
\hline & & & \multicolumn{5}{c}{ Maximum mean discharge $^{2}$} \\
$\begin{array}{c}\text { Exceedance } \\
\text { probability }\end{array}$ & $\begin{array}{c}\text { Recurrence } \\
\text { interval } \\
\text { (years) }\end{array}$ & $\begin{array}{c}\text { Maximum } \\
\text { instantaneous } \\
\left(\mathbf{m}^{3} / \mathbf{s}\right)\end{array}$ & 3-day period & 7-day period & 15-day period & 30-day period \\
\cline { 5 - 7 } 0.99 & 1.01 & 0.100 & 0.075 & 0.069 & 0.069 & 0.062 \\
0.95 & 1.05 & 0.100 & 0.154 & 0.149 & 0.134 & 0.119 \\
0.90 & 1.11 & 0.200 & 0.224 & 0.218 & 0.188 & 0.166 \\
0.80 & 1.25 & 0.300 & 0.353 & 0.339 & 0.281 & 0.243 \\
0.50 & 2 & 0.800 & 0.823 & 0.731 & 0.581 & 0.485 \\
0.20 & 5 & 3.00 & 1.88 & 1.44 & 1.14 & 0.913 \\
0.10 & 10 & 6.10 & 2.86 & 1.99 & 1.60 & 1.24 \\
0.04 & 25 & 13.9 & 4.46 & 2.73 & 2.25 & 1.70 \\
0.02 & 50 & 24.2 & 5.92 & 3.31 & 2.79 & 2.06 \\
0.01 & 100 & 40.7 & 7.61 & 3.90 & 3.36 & 2.44 \\
0.005 & 200 & 66.5 & 9.56 & 4.50 & 3.97 & 2.84 \\
0.002 & 500 & 123 & ng & ng & ng & ng \\
\hline
\end{tabular}

'Less than 10 years of data used.

${ }^{2}$ Data does not fit log-Pearson Type III curve, use with caution.

\section{3- 8.1L0-1W BARIKAB RIVER ABOVE SERAJ RESERVOIR, Continued}

\section{Probability of occurrence of annual low discharges}

[ $\mathrm{m}^{3} / \mathrm{s}$, meters per second;ng, statistic not given]

\begin{tabular}{|c|c|c|c|c|c|c|c|c|c|c|}
\hline \multirow{3}{*}{$\begin{array}{c}\text { Nonexceedance } \\
\text { probability }\end{array}$} & \multirow{3}{*}{$\begin{array}{c}\text { Recurrence } \\
\text { interval } \\
\text { (years) }\end{array}$} & \multicolumn{9}{|c|}{ Minimum mean discharge $\left(\mathrm{m}^{3} / \mathrm{s}\right)$} \\
\hline & & \multicolumn{9}{|c|}{ Number of consecutive days } \\
\hline & & 1 & 3 & 7 & 14 & 30 & 60 & 90 & 120 & 183 \\
\hline 0.05 & 20 & ng & 0 & 0 & 0 & ng & 0.002 & 0.005 & 0.007 & 0.010 \\
\hline 0.10 & 10 & ng & 0 & 0 & 0 & ng & 0.003 & 0.006 & 0.009 & 0.014 \\
\hline 0.20 & 5 & ng & 0.006 & 0.009 & 0.009 & ng & ${ }^{1} 0.006$ & 0.009 & 0.011 & 0.020 \\
\hline 0.50 & 2 & ng & 0.009 & 0.010 & 0.010 & ng & 0.012 & 0.016 & 0.021 & 0.039 \\
\hline
\end{tabular}

'Data does not fit log-Pearson Type III curve, use with caution. 


\section{3- 8.1LO-1W BARIKAB RIVER ABOVE SERAJ RESERVOIR, Continued}

\section{Probability of occurrence of seasonal low discharges}

$\left[\mathrm{m}^{3} / \mathrm{s}\right.$, meters per second]

\begin{tabular}{|c|c|c|c|c|c|c|c|c|c|}
\hline \multirow{3}{*}{$\begin{array}{l}\text { Nonexceedance } \\
\text { probability }\end{array}$} & \multirow{3}{*}{$\begin{array}{c}\text { Recurrence } \\
\text { interval } \\
\text { (years) }\end{array}$} & \multicolumn{8}{|c|}{ Minimum mean discharge $\left(\mathrm{m}^{3} / \mathrm{s}\right)$} \\
\hline & & \multicolumn{8}{|c|}{ Number of consecutive days } \\
\hline & & 1 & 7 & 14 & 30 & 1 & 7 & 14 & 30 \\
\hline & & \multicolumn{4}{|c|}{ December-January-February } & \multicolumn{4}{|c|}{ March-April-May } \\
\hline 0.05 & 20 & 0.005 & 0.005 & 0.005 & 0.005 & 0.007 & 0.008 & 0.010 & 0.015 \\
\hline 0.10 & 10 & 0.008 & 0.009 & 0.009 & 0.010 & 0.008 & 0.011 & 0.013 & 0.021 \\
\hline 0.20 & 5 & 0.014 & 0.015 & 0.017 & 0.020 & 0.010 & 0.014 & 0.017 & 0.030 \\
\hline \multirow[t]{2}{*}{0.50} & 2 & 0.042 & 0.047 & 0.055 & 0.071 & 0.015 & 0.024 & 0.029 & 0.051 \\
\hline & & \multicolumn{4}{|c|}{ June-July-August } & \multicolumn{4}{|c|}{ September-October-November } \\
\hline 0.05 & 20 & 0 & 0 & 0 & 0.005 & 0 & 0 & 0 & 0 \\
\hline 0.10 & 10 & 0 & 0 & 0 & 0.008 & 0 & 0 & 0 & 0 \\
\hline 0.20 & 5 & 0 & 0.007 & 0.009 & 0.012 & 0 & 0.007 & 0.007 & 0.007 \\
\hline 0.50 & 2 & 0.012 & 0.012 & 0.018 & 0.024 & 0.010 & 0.011 & 0.011 & 0.012 \\
\hline
\end{tabular}

3- 8.1LO-1W BARIKAB RIVER ABOVE SERAJ RESERVOIR, Continued

Annual peak discharge and corresponding gage height

[ $\mathrm{m}^{3} / \mathrm{s}$, meters per second; --, no data]

\begin{tabular}{|c|c|c|c|c|c|c|c|}
\hline $\begin{array}{c}\text { Water } \\
\text { year }\end{array}$ & Date & $\begin{array}{c}\text { Gage } \\
\text { height } \\
\text { (meters) }\end{array}$ & $\begin{array}{c}\text { Peak } \\
\text { discharge } \\
\left(\mathrm{m}^{3} / \mathrm{s}\right)\end{array}$ & $\begin{array}{c}\text { Water } \\
\text { year }\end{array}$ & Date & $\begin{array}{c}\text { Gage } \\
\text { height } \\
\text { (meters) }\end{array}$ & $\begin{array}{c}\text { Peak } \\
\text { discharge } \\
\left(\mathrm{m}^{3} / \mathrm{s}\right)\end{array}$ \\
\hline \multicolumn{8}{|c|}{ Annual peak discharge, by year } \\
\hline 1968 & 11-Aug & & 0.09 & 1973 & 17-Apr & & 2.22 \\
\hline 1969 & 14-May & & 0.28 & 1974 & 7-Apr & & 0.58 \\
\hline 1970 & 21-Aug & & 15.1 & 1975 & 11-Apr & & 1.10 \\
\hline 1971 & 16-Jul & & 0.50 & 1976 & 20-Apr & & 1.64 \\
\hline 1972 & 13-Apr & & 1.10 & & & & \\
\hline \multicolumn{8}{|c|}{ Annual peak discharge, from highest to lowest } \\
\hline 1970 & 21-Aug & & 15.1 & 1974 & 7-Apr & & 0.58 \\
\hline 1973 & 17-Apr & & 2.22 & 1971 & 16-Jul & & 0.50 \\
\hline 1976 & 20-Apr & & 1.64 & 1969 & 14-May & & 0.28 \\
\hline 1975 & 11-Apr & & 1.10 & 1968 & 11-Aug & & 0.09 \\
\hline 1972 & 13-Apr & & 1.10 & & & & \\
\hline
\end{tabular}




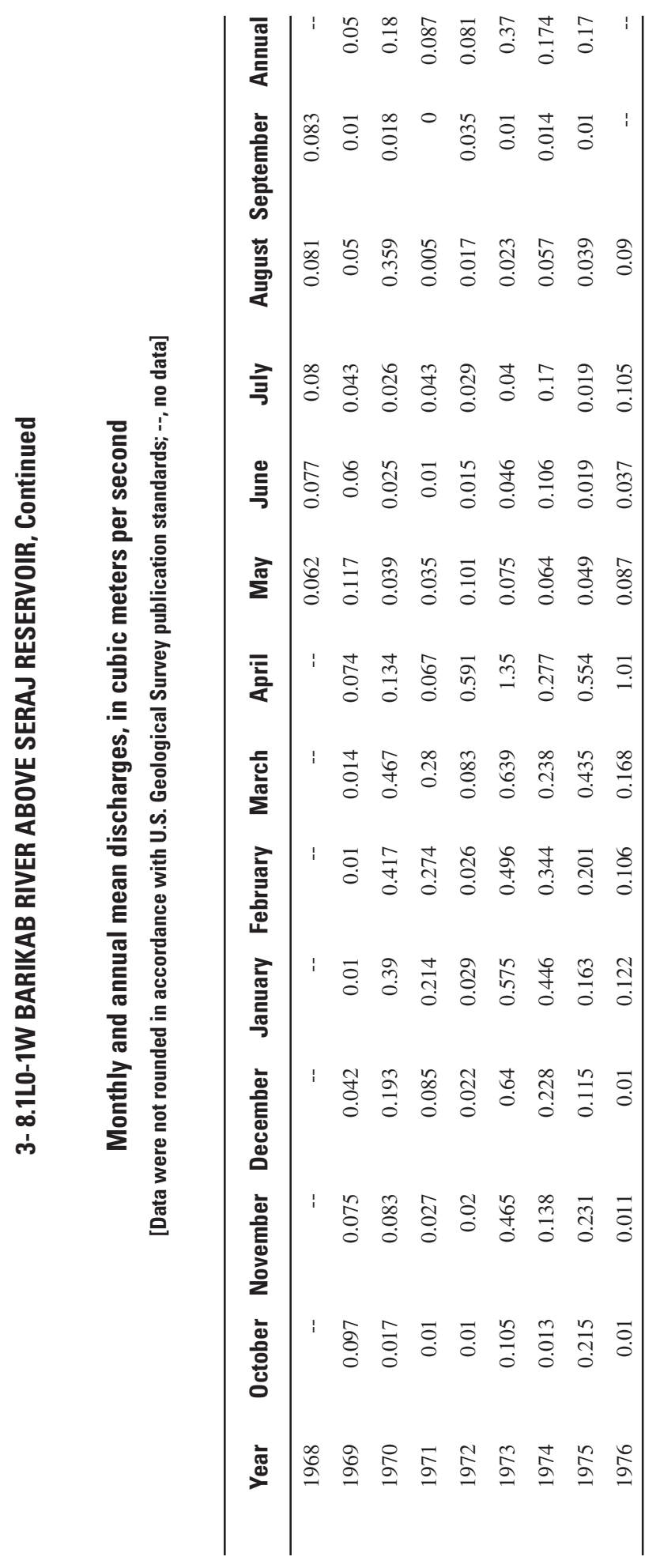




\section{3- 8.ROO-1W SARAB RIVER ABOVE SERAJ RESERVOIR}

\section{(U.S. Geological Survey identification number: 334601068210000)}

LOCATION: Lat $33^{\circ} 46^{\prime} 01^{\prime \prime}$ N., long $68^{\circ} 21^{\prime}$ E., on right bank at Khojagan.

DRAINAGE AREA: $730 \mathrm{~km}^{2}$.

ALTITUDE: 2,385 meters plus mean sea level.

PERIOD OF RECORD: April 16, 1968 to September 30, 1980.

GAGE: Staff gage read once or twice daily.

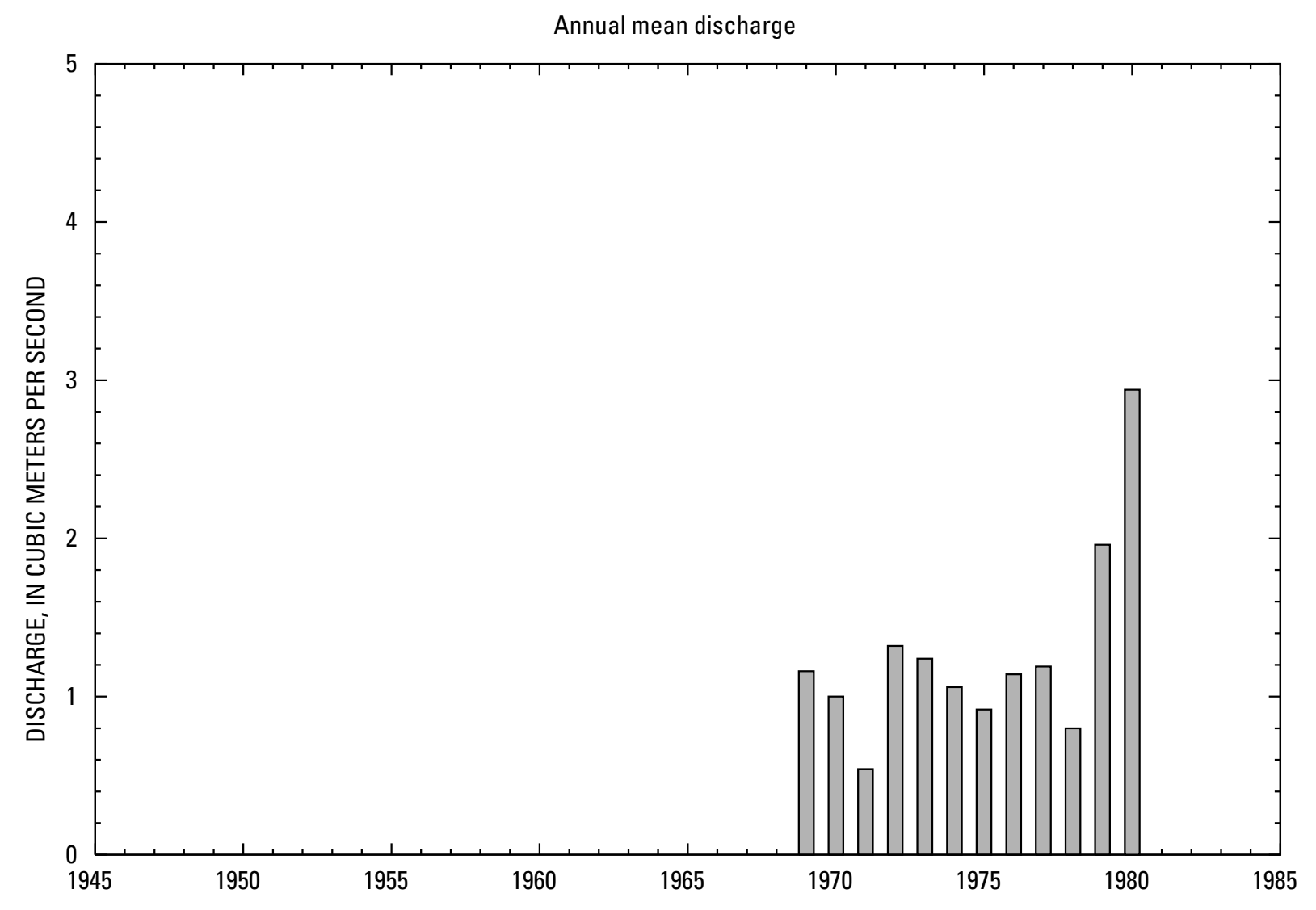




\section{3- 8.RO0-1W SARAB RIVER ABOVE SERAJ RESERVOIR, Continued}

\section{Statistics of monthly and annual mean discharges}

[ $\mathrm{m}^{3} / \mathrm{s}$, meters per second; $\mathrm{m}$, more than 2 years of occurrence]

\begin{tabular}{|c|c|c|c|c|c|c|c|c|}
\hline \multirow[b]{2}{*}{ Month } & \multicolumn{2}{|c|}{ Maximum } & \multicolumn{2}{|c|}{ Minimum } & \multicolumn{4}{|c|}{ Mean } \\
\hline & $\begin{array}{c}\text { Discharge } \\
\left(\mathrm{m}^{3} / \mathrm{s}\right)\end{array}$ & $\begin{array}{c}\text { Water year } \\
\text { of } \\
\text { occurrence }\end{array}$ & $\begin{array}{c}\text { Discharge } \\
\left(\mathrm{m}^{3} / \mathrm{s}\right)\end{array}$ & $\begin{array}{c}\text { Water year } \\
\text { of } \\
\text { occurrence }\end{array}$ & $\begin{array}{c}\text { Discharge } \\
\left(\mathrm{m}^{3} / \mathrm{s}\right)\end{array}$ & $\begin{array}{c}\text { Standard } \\
\text { deviation } \\
\left(\mathrm{m}^{3} / \mathrm{s}\right)\end{array}$ & $\begin{array}{c}\text { Coefficient } \\
\text { of } \\
\text { variation }\end{array}$ & $\begin{array}{c}\text { Percentage } \\
\text { of annual } \\
\text { discharge }\end{array}$ \\
\hline October & 1.37 & 1969 & 0.155 & 1976 & 0.55 & 0.36 & 0.66 & 3.56 \\
\hline November & 2.61 & 1980 & 0.606 & 1976 & 1.34 & 0.59 & 0.44 & 8.63 \\
\hline December & 3.20 & 1980 & 0.893 & 1976 & 1.59 & 0.66 & 0.41 & 10.2 \\
\hline January & 3.27 & 1980 & 0.908 & 1971 & 1.75 & 0.72 & 0.41 & 11.2 \\
\hline February & 3.40 & 1980 & 0.770 & 1976 & 1.72 & 0.76 & 0.44 & 11.1 \\
\hline March & 4.58 & 1980 & 0.851 & 1976 & 2.12 & 0.95 & 0.45 & 13.6 \\
\hline April & 10.4 & 1980 & 0.716 & 1969 & 3.62 & 3.31 & 0.91 & 23.3 \\
\hline May & 5.26 & 1980 & 0.122 & 1978 & 1.50 & 1.60 & 1.07 & 9.62 \\
\hline June & 1.45 & 1980 & 0.020 & 1971 & 0.42 & 0.45 & 1.07 & 2.68 \\
\hline July & 0.755 & 1978 & 0.040 & 1970 & 0.38 & 0.26 & 0.69 & 2.45 \\
\hline August & 1.68 & 1970 & 0.028 & 1975 & 0.41 & 0.46 & 1.13 & 2.63 \\
\hline September & 0.662 & 1968 & 0.010 & $\mathrm{~m}$ & 0.15 & 0.19 & 1.24 & 1.00 \\
\hline Annual & 2.94 & 1980 & 0.542 & 1971 & 1.27 & 0.62 & 0.49 & 100 \\
\hline
\end{tabular}

Annual flow duration

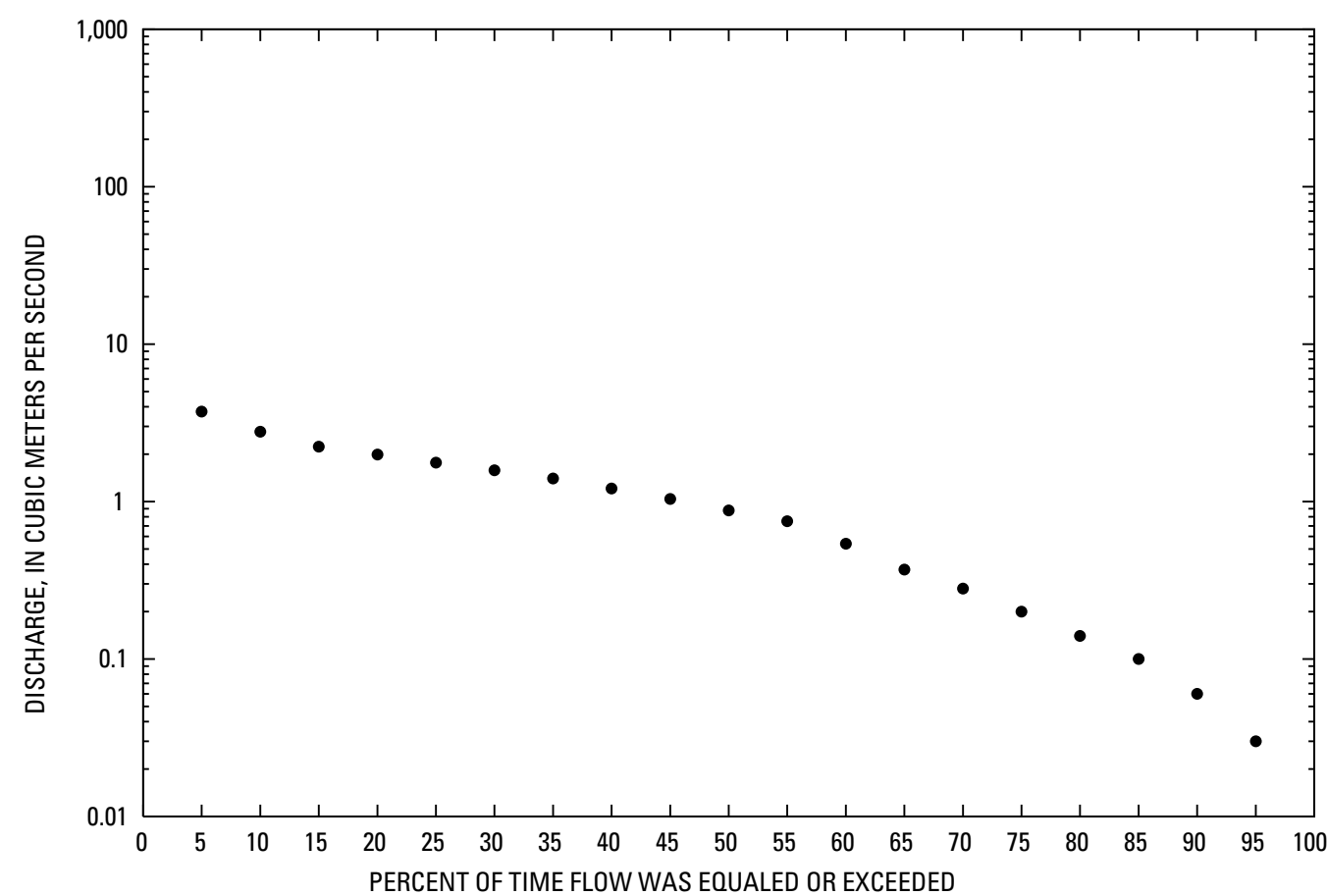




\section{3- 8.RO0-1W SARAB RIVER ABOVE SERAJ RESERVOIR, Continued}

\section{Monthly and annual flow duration, in cubic meters per second}

[Data were not rounded in accordance with U.S. Geological Survey publication standards]

\begin{tabular}{|c|c|c|c|c|c|c|c|c|c|c|c|c|c|}
\hline $\begin{array}{l}\text { Percentage } \\
\text { of days } \\
\text { discharge } \\
\text { equaled or } \\
\text { exceeded }\end{array}$ & January & February & March & April & May & June & July & August & September & October & November & December & Annual \\
\hline 95 & 0.85 & 0.79 & 0.81 & 0.24 & 0.09 & 0.03 & 0.04 & 0.02 & 0.01 & 0.06 & 0.59 & 0.91 & 0.03 \\
\hline 90 & 0.92 & 0.8 & 0.91 & 0.32 & 0.11 & 0.05 & 0.05 & 0.03 & 0.01 & 0.1 & 0.75 & 0.95 & 0.06 \\
\hline 80 & 1 & 0.89 & 1.4 & 0.74 & 0.21 & 0.09 & 0.08 & 0.05 & 0.02 & 0.14 & 0.87 & 1.06 & 0.14 \\
\hline 75 & 1.17 & 1.13 & 1.48 & 1 & 0.24 & 0.1 & 0.09 & 0.06 & 0.02 & 0.16 & 0.93 & 1.13 & 0.2 \\
\hline 70 & 1.29 & 1.39 & 1.54 & 1.37 & 0.29 & 0.12 & 0.1 & 0.07 & 0.02 & 0.21 & 0.98 & 1.16 & 0.28 \\
\hline 65 & 1.46 & 1.45 & 1.66 & 1.65 & 0.32 & 0.17 & 0.15 & 0.1 & 0.04 & 0.28 & 1.02 & 1.24 & 0.37 \\
\hline 60 & 1.51 & 1.47 & 1.8 & 1.92 & 0.35 & 0.2 & 0.17 & 0.12 & 0.05 & 0.33 & 1.05 & 1.27 & 0.54 \\
\hline 45 & 1.67 & 1.67 & 2.05 & 2.94 & 0.72 & 0.27 & 0.22 & 0.17 & 0.11 & 0.52 & 1.25 & 1.48 & 1.04 \\
\hline 40 & 1.75 & 1.74 & 2.13 & 3.21 & 0.88 & 0.3 & 0.24 & 0.18 & 0.12 & 0.61 & 1.29 & 1.52 & 1.21 \\
\hline 35 & 1.96 & 1.82 & 2.2 & 3.7 & 1.29 & 0.35 & 0.29 & 0.19 & 0.13 & 0.68 & 1.37 & 1.57 & 1.4 \\
\hline 30 & 2.09 & 2.06 & 2.29 & 4.17 & 1.61 & 0.41 & 0.4 & 0.22 & 0.15 & 0.75 & 1.46 & 1.79 & 1.58 \\
\hline 25 & 2.36 & 2.14 & 2.4 & 4.73 & 2.12 & 0.48 & 0.57 & 0.4 & 0.18 & 0.83 & 1.58 & 2.07 & 1.77 \\
\hline 20 & 2.42 & 2.23 & 2.71 & 5.78 & 2.76 & 0.59 & 0.64 & 0.5 & 0.33 & 0.92 & 1.82 & 2.13 & 1.99 \\
\hline 15 & 2.49 & 2.42 & 2.92 & 7.33 & 3.83 & 0.66 & 0.73 & 0.56 & 0.36 & 1.03 & 2.2 & 2.2 & 2.23 \\
\hline 10 & 0 & 3.12 & 3.54 & 9.9 & 4.31 & 0.99 & 0.85 & 0.72 & 0.38 & 1.24 & 2.37 & 2.36 & 2.78 \\
\hline 5 & 0 & 0 & 4.04 & 11.2 & 5.22 & 1.29 & 1.05 & 1.06 & 0.63 & 1.51 & 2.68 & 0 & 3.73 \\
\hline
\end{tabular}




\section{3- 8.R00-1W SARAB RIVER ABOVE SERAJ RESERVOIR, Continued}

Probability of occurrence of annual high discharges

$\left[\mathrm{m}^{3} / \mathrm{s}\right.$, meters per second; $\mathrm{ng}$, statistic not given]

\begin{tabular}{|c|c|c|c|c|c|c|}
\hline \multirow{2}{*}{$\begin{array}{c}\text { Exceedance } \\
\text { probability }\end{array}$} & \multirow{2}{*}{$\begin{array}{c}\text { Recurrence } \\
\text { interval } \\
\text { (years) }\end{array}$} & \multirow{2}{*}{$\begin{array}{c}\text { Maximum } \\
\text { instantaneous } \\
\left(\mathrm{m}^{3} / \mathrm{s}\right)\end{array}$} & \multicolumn{4}{|c|}{ Maximum mean discharge } \\
\hline & & & 3-day period & 7-day period & 15-day period & 30-day period \\
\hline 0.99 & 1.01 & 1.80 & 1.55 & 0.978 & ${ }^{1} 0.995$ & 0.953 \\
\hline 0.95 & 1.05 & 2.30 & 2.07 & 1.52 & 1.43 & 1.31 \\
\hline 0.90 & 1.11 & 2.70 & 2.47 & 1.92 & 1.75 & 1.58 \\
\hline 0.80 & 1.25 & 3.50 & 3.12 & 2.56 & 2.27 & 2.01 \\
\hline 0.50 & 2 & 7.10 & 5.19 & 4.48 & 3.86 & 3.34 \\
\hline 0.20 & 5 & 18.5 & 9.43 & 7.88 & 6.86 & 5.92 \\
\hline 0.10 & 10 & 34.6 & 13.4 & 10.6 & 9.44 & 8.20 \\
\hline 0.04 & 25 & 74.4 & 20.0 & 14.6 & 13.5 & 11.9 \\
\hline 0.02 & 50 & 129 & 26.3 & 18.0 & 17.1 & 15.2 \\
\hline 0.01 & 100 & 219 & 34.1 & 21.8 & 21.3 & 19.2 \\
\hline 0.005 & 200 & 368 & 43.7 & 25.9 & ${ }^{1} 26.2$ & 23.9 \\
\hline 0.002 & 500 & 720 & ng & ng & ng & ng \\
\hline
\end{tabular}

'Data does not fit log-Pearson Type III curve, use with caution.

\section{3- 8.RO0-1W SARAB RIVER ABOVE SERAJ RESERVOIR, Continued}

Probability of occurrence of annual low discharges

$\left[\mathrm{m}^{3} / \mathrm{s}\right.$, meters per second]

\begin{tabular}{|c|c|c|c|c|c|c|c|c|c|c|}
\hline \multirow{3}{*}{$\begin{array}{l}\text { Nonexceedance } \\
\text { probability }\end{array}$} & \multirow{3}{*}{$\begin{array}{c}\text { Recurrence } \\
\text { interval } \\
\text { (years) }\end{array}$} & \multicolumn{9}{|c|}{ Minimum mean discharge $\left(\mathrm{m}^{3} / \mathrm{s}\right)$} \\
\hline & & \multicolumn{9}{|c|}{ Number of consecutive days } \\
\hline & & 1 & 3 & 7 & 14 & 30 & 60 & 90 & 120 & 183 \\
\hline 0.05 & 20 & 0 & 0.005 & 0.005 & 0.005 & 0.006 & 0.017 & 0.029 & 0.053 & 0.117 \\
\hline 0.10 & 10 & 0.004 & 0.007 & 0.008 & 0.008 & 0.008 & 0.023 & 0.041 & 0.070 & 0.145 \\
\hline 0.20 & 5 & 0.009 & 0.011 & 0.012 & 0.012 & 0.014 & 0.033 & 0.061 & 0.099 & 0.189 \\
\hline 0.50 & 2 & 0.027 & 0.028 & 0.032 & 0.034 & 0.040 & 0.071 & 0.130 & 0.184 & 0.314 \\
\hline
\end{tabular}




\section{3- 8.RO0-1W SARAB RIVER ABOVE SERAJ RESERVOIR, Continued}

\section{Probability of occurrence of seasonal low discharges}

$\left[\mathrm{m}^{3} / \mathrm{s}\right.$, meters per second]

\begin{tabular}{|c|c|c|c|c|c|c|c|c|c|}
\hline \multirow{3}{*}{$\begin{array}{l}\text { Nonexceedance } \\
\text { probability }\end{array}$} & \multirow{3}{*}{$\begin{array}{c}\text { Recurrence } \\
\text { interval } \\
\text { (years) }\end{array}$} & \multicolumn{8}{|c|}{ Minimum mean discharge $\left(\mathrm{m}^{3} / \mathrm{s}\right)$} \\
\hline & & \multicolumn{8}{|c|}{ Number of consecutive days } \\
\hline & & 1 & 7 & 14 & 30 & 1 & 7 & 14 & 30 \\
\hline & & \multicolumn{4}{|c|}{ December-January-February } & \multicolumn{4}{|c|}{ March-April-May } \\
\hline 0.05 & 20 & 0.598 & 0.655 & 0.685 & 0.713 & 0.039 & 0.048 & 0.075 & 0.114 \\
\hline 0.10 & 10 & 0.694 & 0.746 & 0.776 & 0.818 & 0.051 & 0.065 & 0.100 & 0.156 \\
\hline 0.20 & 5 & 0.836 & 0.882 & 0.912 & 0.972 & 0.076 & 0.097 & 0.146 & 0.233 \\
\hline \multirow[t]{2}{*}{0.50} & 2 & 1.21 & 1.25 & 1.28 & 1.38 & 0.185 & 0.237 & 0.339 & 0.552 \\
\hline & & \multicolumn{4}{|c|}{ June-July-August } & \multicolumn{4}{|c|}{ September-October-November } \\
\hline 0.05 & 20 & 0.006 & 0.009 & 0.018 & 0.020 & 0 & 0.005 & 0.005 & 0.005 \\
\hline 0.10 & 10 & 0.010 & 0.014 & 0.024 & 0.030 & 0.003 & 0.008 & 0.008 & 0.009 \\
\hline 0.20 & 5 & 0.017 & 0.023 & 0.035 & 0.048 & 0.010 & 0.013 & 0.014 & 0.017 \\
\hline 0.50 & 2 & 0.048 & 0.062 & 0.078 & 0.113 & 0.044 & ${ }^{1} 0.043$ & 0.047 & 0.059 \\
\hline
\end{tabular}

'Data does not fit log-Pearson Type III curve, use with caution. 
3- 8.ROO-1W SARAB RIVER ABOVE SERAJ RESERVOIR, Continued

Annual peak discharge and corresponding gage height

[ $\mathrm{m}^{3} / \mathrm{s}$, meters per second; --, no data]

\begin{tabular}{|c|c|c|c|c|c|c|c|}
\hline $\begin{array}{c}\text { Water } \\
\text { year }\end{array}$ & Date & $\begin{array}{c}\text { Gage } \\
\text { height } \\
\text { (meters) }\end{array}$ & $\begin{array}{c}\text { Peak } \\
\text { discharge } \\
\left(\mathrm{m}^{3} / \mathrm{s}\right)\end{array}$ & $\begin{array}{c}\text { Water } \\
\text { year }\end{array}$ & Date & $\begin{array}{c}\text { Gage } \\
\text { height } \\
\text { (meters) }\end{array}$ & $\begin{array}{c}\text { Peak } \\
\text { discharge } \\
\left(\mathrm{m}^{3} / \mathbf{s}\right)\end{array}$ \\
\hline \multicolumn{8}{|c|}{ Annual peak discharge, by year } \\
\hline 1968 & 11-Apr & & 7.60 & 1975 & 14-Apr & & 6.22 \\
\hline 1969 & 20-Nov & & 2.71 & 1976 & 17-Jul & & 25.0 \\
\hline 1970 & 23-Aug & & 35.2 & 1977 & 13-Apr & & 2.96 \\
\hline 1971 & 16-Jul & & 5.00 & 1978 & 5-Jul & & 8.00 \\
\hline 1972 & 12-May & & 5.06 & 1979 & 17-Apr & & 11.9 \\
\hline 1973 & 18-Apr & & 5.56 & 1980 & 18-Apr & & 90.0 \\
\hline 1974 & 20-Apr & - & 3.30 & & & & \\
\hline \multicolumn{8}{|c|}{ Annual peak discharge, from highest to lowest } \\
\hline 1980 & 18-Apr & . & 90.0 & 1973 & 18-Apr & & 5.56 \\
\hline 1970 & 23-Aug & . & 35.2 & 1972 & 12-May & & 5.06 \\
\hline 1976 & 17-Jul & . & 25.0 & 1971 & 16-Jul & & 5.00 \\
\hline 1979 & 17-Apr & . & 11.9 & 1974 & 20-Apr & & 3.30 \\
\hline 1978 & 5-Jul & . & 8.00 & 1977 & 13-Apr & & 2.96 \\
\hline 1968 & 11-Apr & . & 7.60 & 1969 & $20-\mathrm{Nov}$ & & 2.71 \\
\hline 1975 & 14-Apr & . & 6.22 & & & & \\
\hline
\end{tabular}




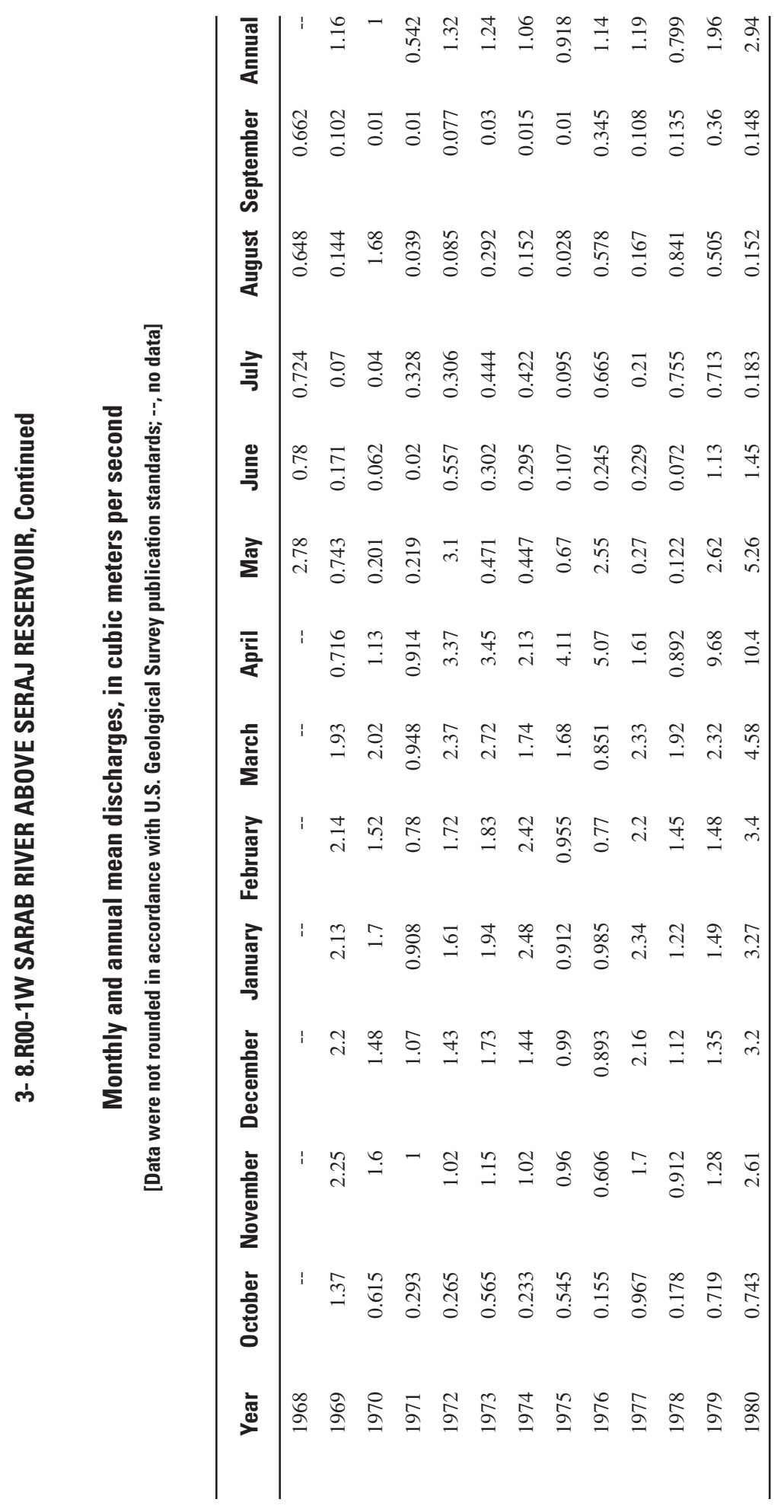




\section{4- 0.000-1M SHELE CHARKH RIVER NEAR ZARANJ}

\section{(U.S. Geological Survey identification number: 310200061520000)}

LOCATION: Lat $31^{\circ} 02^{\prime}$ N., long $61^{\circ} 52^{\prime} E$., on left bank at bridge site at Shele Charkh, $8 \mathrm{~km}$ south of Qala-iKang, $24 \mathrm{~km}$ southwest of Chakhansur, $28 \mathrm{~km}$ downstream from Sistan diversion, and about $105 \mathrm{~km}$ downstream from Char Burjak.

DRAINAGE AREA: --

ALTITUDE: 476 meters plus mean sea level.

PERIOD OF RECORD: October 1, 1955 to June 1, 1979.

GAGE: Water-stage recorder.

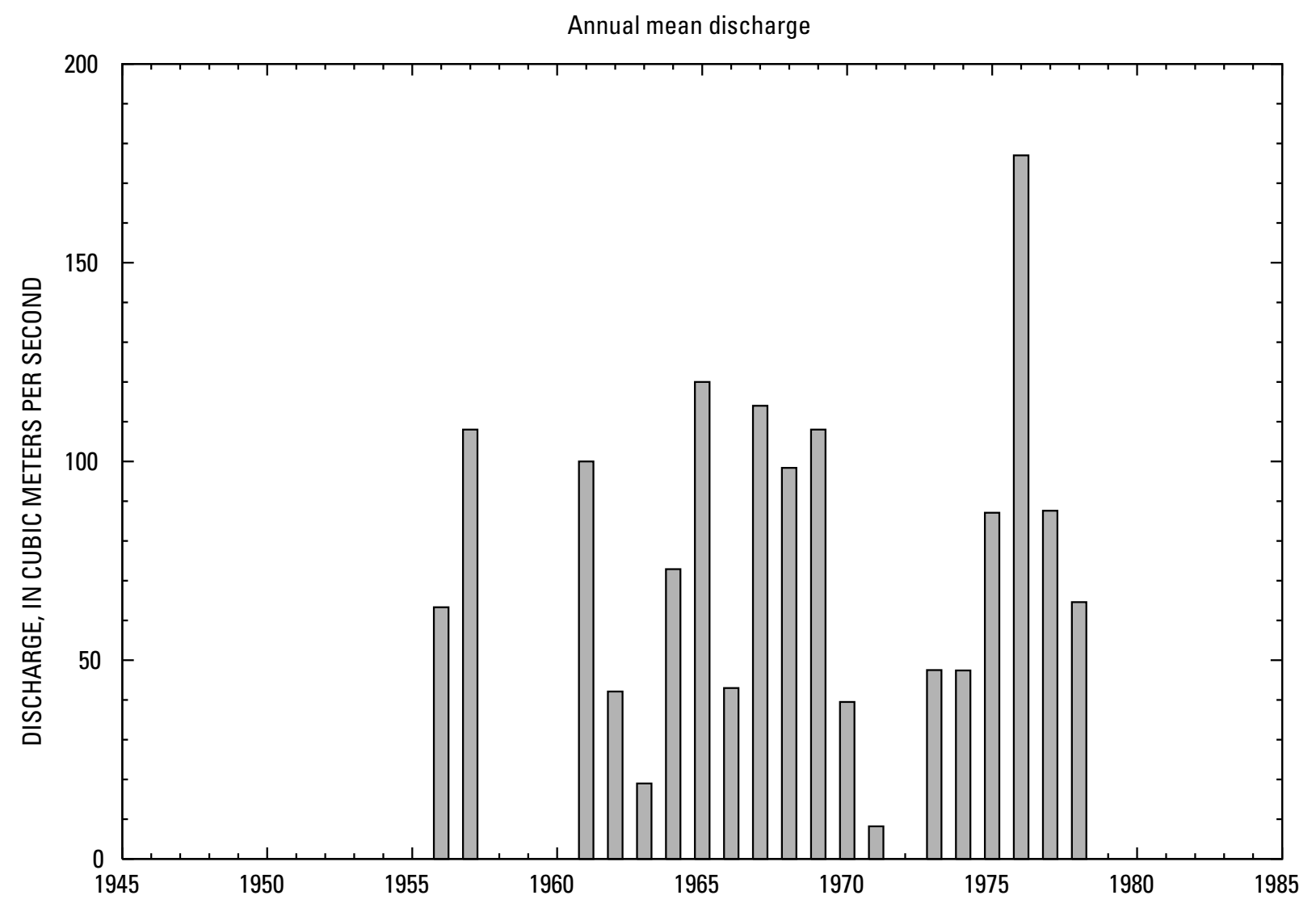




\section{4- 0.000-1M SHELE CHARKH RIVER NEAR ZARANJ, Continued}

\section{Statistics of monthly and annual mean discharges}

$\left[\mathrm{m}^{3} / \mathrm{s}\right.$, meters per second]

\begin{tabular}{|c|c|c|c|c|c|c|c|c|}
\hline \multirow[b]{2}{*}{ Month } & \multicolumn{2}{|c|}{ Maximum } & \multicolumn{2}{|c|}{ Minimum } & \multicolumn{4}{|c|}{ Mean } \\
\hline & $\begin{array}{c}\text { Discharge } \\
\left(\mathrm{m}^{3} / \mathrm{s}\right)\end{array}$ & $\begin{array}{c}\text { Water year } \\
\text { of } \\
\text { occurrence }\end{array}$ & $\begin{array}{c}\text { Discharge } \\
\left(\mathrm{m}^{3} / \mathrm{s}\right)\end{array}$ & $\begin{array}{c}\text { Water year } \\
\text { of } \\
\text { occurrence }\end{array}$ & $\begin{array}{c}\text { Discharge } \\
\left(\mathrm{m}^{3} / \mathrm{s}\right)\end{array}$ & $\begin{array}{c}\text { Standard } \\
\text { deviation } \\
\left(\mathrm{m}^{3} / \mathrm{s}\right)\end{array}$ & $\begin{array}{c}\text { Coefficient } \\
\text { of } \\
\text { variation }\end{array}$ & $\begin{array}{c}\text { Percentage } \\
\text { of annual } \\
\text { discharge }\end{array}$ \\
\hline October & 67.6 & 1977 & 2.73 & 1963 & 26.6 & 18.4 & 0.69 & 2.84 \\
\hline November & 78.4 & 1977 & 1.84 & 1963 & 30.4 & 20.2 & 0.67 & 3.26 \\
\hline December & 85.4 & 1962 & 8.19 & 1963 & 44.5 & 23.2 & 0.52 & 4.76 \\
\hline January & 124 & 1977 & 15.6 & 1967 & 64.2 & 31.9 & 0.50 & 6.87 \\
\hline February & 133 & 1968 & 13.6 & 1971 & 79.4 & 36.5 & 0.46 & 8.50 \\
\hline March & 281 & 1976 & 6.10 & 1971 & 82.8 & 61.5 & 0.74 & 8.86 \\
\hline April & 448 & 1976 & 7.21 & 1971 & 153 & 138 & 0.90 & 16.4 \\
\hline May & 737 & 1967 & 11.5 & 1971 & 255 & 216 & 0.85 & 24.3 \\
\hline June & 241 & 1965 & 2.44 & 1971 & 92.7 & 72.0 & 0.78 & 9.92 \\
\hline July & 93.5 & 1976 & 0.003 & 1971 & 43.9 & 27.4 & 0.62 & 4.70 \\
\hline August & 85.2 & 1956 & 0 & 1971 & 33.8 & 24.1 & 0.71 & 3.62 \\
\hline September & 73.1 & 1956 & 0 & 1971 & 27.6 & 19.7 & 0.71 & 2.95 \\
\hline Annual & 177 & 1976 & 8.21 & 1971 & 76.2 & 41.0 & 0.54 & 100 \\
\hline
\end{tabular}

Annual flow duration

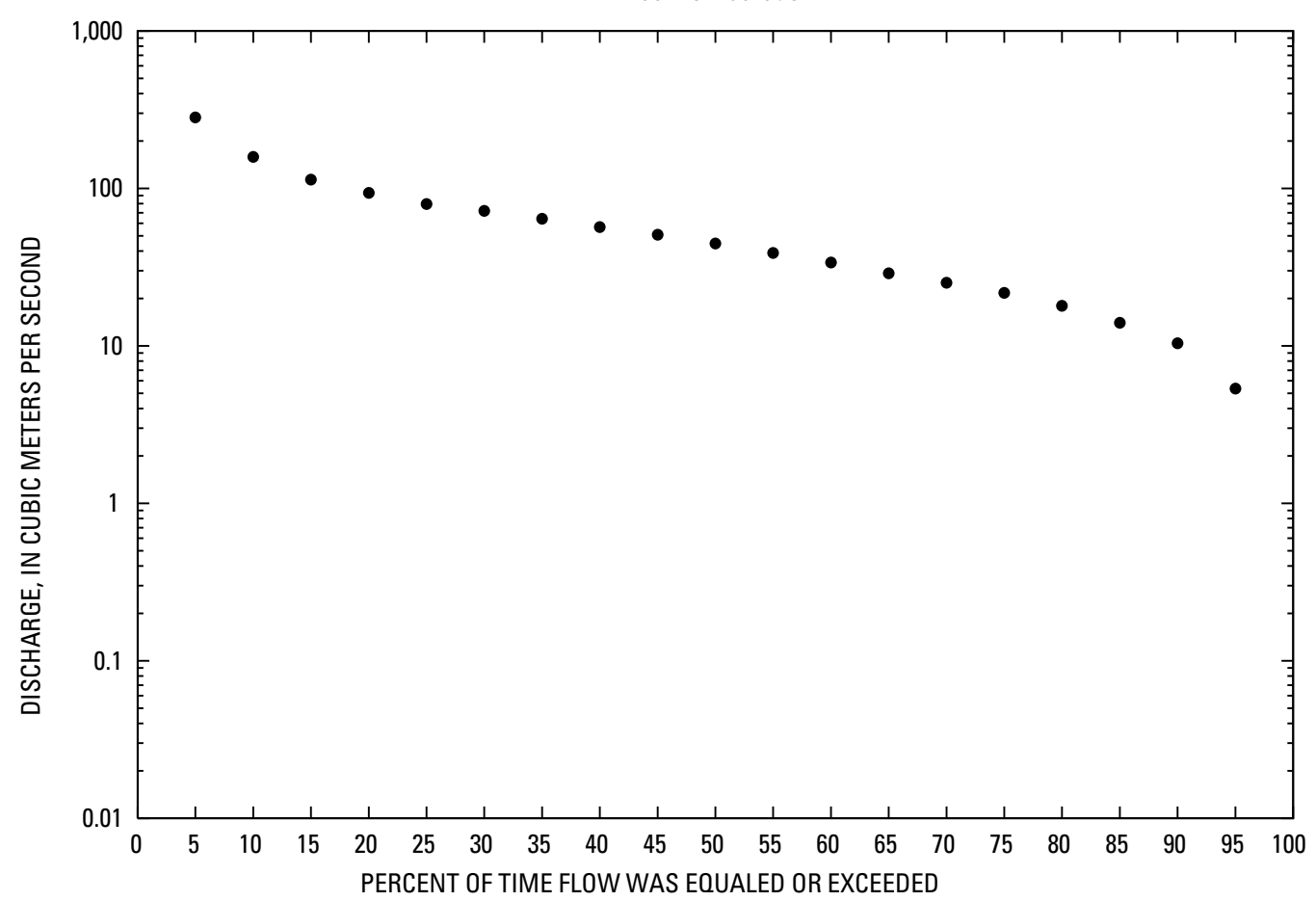




\section{4- 0.000-1M SHELE CHARKH RIVER NEAR ZARANJ, Continued}

Monthly and annual flow duration, in cubic meters per second

[Data were not rounded in accordance with U.S. Geological Survey publication standards]

\begin{tabular}{|c|c|c|c|c|c|c|c|c|c|c|c|c|c|}
\hline $\begin{array}{l}\text { Percentage } \\
\text { of days } \\
\text { discharge } \\
\text { equaled or } \\
\text { exceeded }\end{array}$ & January & February & March & April & May & June & July & August & September & October & November & December & Annual \\
\hline 95 & 12.5 & 14.7 & 11.7 & 9.97 & 12.5 & 3.88 & 0.02 & 4.75 & 1 & 3.85 & 3.19 & 12.1 & 5.36 \\
\hline 90 & 19 & 22 & 24.5 & 16.2 & 22.2 & 7.82 & 5.66 & 6.97 & 5.05 & 4.91 & 5.59 & 16.8 & 10.4 \\
\hline 85 & 25.3 & 28.9 & 28.2 & 21.7 & 30.6 & 23 & 11.2 & 9.46 & 7.99 & 6.88 & 8.77 & 19.4 & 14 \\
\hline 80 & 30.2 & 35.7 & 35.1 & 29.2 & 41.5 & 27.1 & 14 & 11.9 & 10.5 & 10.3 & 13.1 & 21.3 & 18 \\
\hline 75 & 35 & 43.7 & 43.8 & 40.7 & 68.1 & 30.1 & 18.3 & 13.5 & 11.4 & 11.5 & 15 & 23.2 & 21.7 \\
\hline 70 & 39.2 & 49.1 & 48.5 & 46.9 & 82.8 & 34.7 & 21.9 & 15.3 & 13.1 & 14 & 17.1 & 25 & 25.2 \\
\hline 65 & 43.2 & 54.1 & 53.1 & 57.4 & 101 & 41.2 & 24.3 & 17.7 & 14.7 & 16.2 & 19 & 26.7 & 28.9 \\
\hline 60 & 46.2 & 60.5 & 57.3 & 65.2 & 127.3 & 48.5 & 26.7 & 20.5 & 17 & 18.8 & 21.1 & 29 & 33.9 \\
\hline 55 & 50.1 & 65.5 & 61.5 & 71.7 & 145.1 & 56.3 & 29.9 & 22.7 & 18.5 & 21.9 & 22.5 & 32.4 & 39 \\
\hline 50 & 56.4 & 68.8 & 66 & 79.2 & 181.2 & 66.1 & 35.5 & 24.2 & 22.7 & 23 & 23.8 & 35.5 & 44.7 \\
\hline 45 & 63.2 & 72 & 70.5 & 88.9 & 223.4 & 77.6 & 41.1 & 28.3 & 25.4 & 24 & 26.6 & 39.1 & 50.8 \\
\hline 40 & 67.7 & 78.1 & 75.3 & 108.2 & 267.4 & 91.3 & 46.7 & 31.4 & 28.2 & 26.7 & 31.5 & 44.8 & 56.8 \\
\hline 35 & 76.9 & 84.8 & 80.3 & 122.1 & 299.8 & 105.8 & 52.2 & 39.3 & 31.7 & 28.9 & 35 & 51.3 & 64.2 \\
\hline 30 & 83.7 & 92.1 & 85.9 & 145.2 & 335.6 & 120.4 & 56.8 & 45.6 & 37.9 & 30.9 & 38 & 59.6 & 72 \\
\hline 25 & 88.5 & 100.1 & 95.1 & 203 & 375.2 & 138.1 & 61 & 49.8 & 42.6 & 35.9 & 41.2 & 66.3 & 79.7 \\
\hline 20 & 92.8 & 109.4 & 105.3 & 249.8 & 424.6 & 156.5 & 65.3 & 55.1 & 45.7 & 41 & 44.9 & 71.5 & 93.7 \\
\hline 15 & 98.7 & 121 & 116.5 & 293.5 & 498.2 & 184.4 & 69.5 & 63.5 & 50 & 45.7 & 56.8 & 75.8 & 113.7 \\
\hline 10 & 107.4 & 141.4 & 137.6 & 411.3 & 561 & 217.7 & 74.3 & 67.9 & 57.8 & 51.6 & 67.8 & 80.1 & 158.5 \\
\hline 5 & 118.9 & 186 & 182.5 & 558.2 & 720.5 & 261.8 & 94 & 78.9 & 67.8 & 68.1 & 75 & 91 & 282.3 \\
\hline
\end{tabular}




\section{4- 0.000-1M SHELE CHARKH RIVER NEAR ZARANJ, Continued}

\section{Probability of occurrence of annual high discharges}

[ $\mathrm{m}^{3} / \mathrm{s}$, meters per second; $\mathrm{ng}$, statistic not given]

\begin{tabular}{|c|c|c|c|c|c|c|}
\hline \multirow{2}{*}{$\begin{array}{l}\text { Exceedance } \\
\text { probability }\end{array}$} & \multirow{2}{*}{$\begin{array}{c}\text { Recurrence } \\
\text { interval } \\
\text { (years) }\end{array}$} & \multirow{2}{*}{$\begin{array}{c}\text { Maximum } \\
\text { instantaneous } \\
\left(\mathrm{m}^{3} / \mathrm{s}\right)\end{array}$} & \multicolumn{4}{|c|}{ Maximum mean discharge ${ }^{1}$} \\
\hline & & & 3-day period & 7-day period & 15-day period & 30-day period \\
\hline 0.99 & 1.01 & 29.1 & 1.38 & 4.26 & 1.35 & 2.62 \\
\hline 0.95 & 1.05 & 72.2 & 12.5 & 18.4 & 9.89 & 12.5 \\
\hline 0.90 & 1.11 & 113 & 33.1 & 36.7 & 24.2 & 25.8 \\
\hline 0.80 & 1.25 & 189 & 90.1 & 70.1 & 61.6 & 56.4 \\
\hline 0.50 & 2 & 457 & 367 & 262 & 241 & 189 \\
\hline 0.20 & 5 & 979 & 840 & 660 & 575 & 451 \\
\hline 0.10 & 10 & 1,390 & 1,080 & 960 & 769 & 632 \\
\hline 0.04 & 25 & 1,970 & 1,280 & 1,350 & 953 & 836 \\
\hline 0.02 & 50 & 2,410 & 1,360 & 1,620 & 1,050 & 965 \\
\hline 0.01 & 100 & 2,870 & 1,420 & 1,860 & 1,120 & 1,070 \\
\hline 0.005 & 200 & 3,330 & 1,450 & 2,090 & 1,160 & 1,160 \\
\hline 0.002 & 500 & 3,940 & ng & ng & ng & ng \\
\hline
\end{tabular}

'Data does not fit log-Pearson Type III curve, use with caution.

\section{4- 0.000-1M SHELE CHARKH RIVER NEAR ZARANJ, Continued}

Probability of occurrence of annual low discharges

$\left[\mathrm{m}^{3} / \mathrm{s}\right.$, meters per second]

\begin{tabular}{|c|c|c|c|c|c|c|c|c|c|c|}
\hline \multirow{3}{*}{$\begin{array}{l}\text { Nonexceedance } \\
\text { probability }\end{array}$} & \multirow{3}{*}{$\begin{array}{c}\text { Recurrence } \\
\text { interval } \\
\text { (years) }\end{array}$} & \multicolumn{9}{|c|}{ Minimum mean discharge $\left(\mathrm{m}^{3} / \mathrm{s}\right)$} \\
\hline & & \multicolumn{9}{|c|}{ Number of consecutive days } \\
\hline & & 1 & 13 & 17 & 14 & 30 & 60 & 90 & 120 & 183 \\
\hline 0.05 & 20 & 1.18 & 0 & 0 & 2.05 & 2.63 & 3.39 & 4.46 & 5.31 & 7.08 \\
\hline 0.10 & 10 & 2.07 & 1.48 & 1.76 & 3.15 & 4.08 & 5.15 & 6.52 & 7.75 & 10.3 \\
\hline 0.20 & 5 & 3.88 & 3.25 & 3.85 & 5.12 & 6.63 & 8.19 & 10.0 & 11.8 & 15.6 \\
\hline 0.50 & 2 & 11.1 & 9.35 & 10.6 & 11.6 & 14.6 & 17.6 & 20.5 & 23.8 & 30.3 \\
\hline
\end{tabular}

'Data does not fit log-Pearson Type III curve, use with caution. 


\section{4- 0.000-1M SHELE CHARKH RIVER NEAR ZARANJ, Continued}

\section{Probability of occurrence of seasonal low discharges}

$\left[\mathrm{m}^{3} / \mathrm{s}\right.$, meters per second]

\begin{tabular}{|c|c|c|c|c|c|c|c|c|c|}
\hline \multirow{3}{*}{$\begin{array}{c}\text { Nonexceedance } \\
\text { probability }\end{array}$} & \multirow{3}{*}{$\begin{array}{c}\text { Recurrence } \\
\text { interval } \\
\text { (years) }\end{array}$} & \multicolumn{8}{|c|}{ Minimum mean discharge $\left(\mathrm{m}^{3} / \mathrm{s}\right)$} \\
\hline & & \multicolumn{8}{|c|}{ Number of consecutive days } \\
\hline & & 1 & 7 & 14 & 30 & 1 & 7 & 14 & 30 \\
\hline & & \multicolumn{4}{|c|}{ December-January-February } & \multicolumn{4}{|c|}{ March-April-May } \\
\hline 0.05 & 20 & 4.56 & 6.58 & 7.68 & 10.4 & 5.56 & 6.57 & ${ }^{1} 4.11$ & 10.3 \\
\hline 0.10 & 10 & 6.80 & 9.27 & 11.1 & 14.2 & 8.34 & 9.95 & ${ }^{17} .21$ & 15.1 \\
\hline 0.20 & 5 & 10.6 & 13.7 & 16.5 & 20.1 & 13.2 & 15.8 & ${ }^{1} 13.2$ & 23.6 \\
\hline \multirow[t]{2}{*}{0.50} & 2 & 22.5 & 26.5 & 31.5 & 36.0 & 28.7 & 34.2 & 133.9 & 51.3 \\
\hline & & \multicolumn{4}{|c|}{ June-July-August } & \multicolumn{4}{|c|}{ September-October-November } \\
\hline 0.05 & 20 & 0 & 0 & 0 & 0 & 0 & 0 & 2.29 & 2.78 \\
\hline 0.10 & 10 & 3.16 & 3.58 & 4.14 & 5.29 & 1.29 & 2.05 & 3.53 & 4.39 \\
\hline 0.20 & 5 & 6.13 & 6.97 & 7.93 & 9.99 & 3.12 & 4.42 & 5.78 & 7.31 \\
\hline 0.50 & 2 & 15.9 & 17.7 & 19.4 & 23.5 & 10.1 & 12.2 & 13.5 & 16.9 \\
\hline
\end{tabular}

'Data does not fit log-Pearson Type III curve, use with caution. 


\section{4- 0.000-1M SHELE CHARKH RIVER NEAR ZARANJ, Continued}

\section{Annual peak discharge and corresponding gage height}

[m³/s, meters per second; --, no data]

\begin{tabular}{|c|c|c|c|c|c|c|c|}
\hline $\begin{array}{l}\text { Water } \\
\text { year }\end{array}$ & Date & $\begin{array}{c}\text { Gage } \\
\text { height } \\
\text { (meters) }\end{array}$ & $\begin{array}{c}\text { Peak } \\
\text { discharge } \\
\left(\mathrm{m}^{3} / \mathrm{s}\right)\end{array}$ & $\begin{array}{c}\text { Water } \\
\text { year }\end{array}$ & Date & $\begin{array}{c}\text { Gage } \\
\text { height } \\
\text { (meters) }\end{array}$ & $\begin{array}{c}\text { Peak } \\
\text { discharge } \\
\left(\mathrm{m}^{3} / \mathrm{s}\right)\end{array}$ \\
\hline \multicolumn{8}{|c|}{ Annual peak discharge, by year } \\
\hline 1956 & 24-Jul & -- & 345 & 1969 & 20-Apr & -- & 1,000 \\
\hline 1957 & 13-May & -- & 1,019 & 1970 & $1-\mathrm{Feb}$ & -- & 380 \\
\hline 1961 & 15-Apr & 4.99 & 1,044 & 1971 & 11-Jan & -- & 58.8 \\
\hline 1962 & 3-Jan & 2.64 & 142 & 1973 & 11-May & -- & 147 \\
\hline 1963 & 15-May & -- & 115 & 1974 & 27-Feb & -- & 185 \\
\hline 1964 & 4-May & -- & 828 & 1975 & 23-May & -- & 645 \\
\hline 1965 & 30-Apr & -- & 1,040 & 1976 & 2-May & -- & 1,304 \\
\hline 1966 & 3-Mar & -- & 91.6 & 1977 & 13-Jan & -- & 500 \\
\hline 1967 & 7-May & -- & 1,582 & 1978 & 23-Apr & -- & 329 \\
\hline 1968 & 4-May & -- & 450 & 1979 & 16-Feb & -- & 1,006 \\
\hline \multicolumn{8}{|c|}{ Annual peak discharge, from highest to lowest } \\
\hline 1967 & 7-May & -- & 1,582 & 1968 & 4-May & -- & 450 \\
\hline 1976 & 2-May & -- & 1,304 & 1970 & $1-\mathrm{Feb}$ & -- & 380 \\
\hline 1961 & 15-Apr & 4.99 & 1,044 & 1956 & 24-Jul & -- & 345 \\
\hline 1965 & 30-Apr & -- & 1,040 & 1978 & 23-Apr & -- & 329 \\
\hline 1957 & 13-May & -- & 1,019 & 1974 & 27-Feb & -- & 185 \\
\hline 1979 & 16-Feb & -- & 1,006 & 1973 & 11-May & -- & 147 \\
\hline 1969 & 20-Apr & -- & 1,000 & 1962 & 3-Jan & 2.64 & 142 \\
\hline 1964 & 4-May & -- & 828 & 1963 & 15-May & -- & 115 \\
\hline 1975 & 23-May & -- & 645 & 1966 & 3-Mar & -- & 91.6 \\
\hline 1977 & 13-Jan & -- & 500 & 1971 & 11-Jan & -- & 58.8 \\
\hline
\end{tabular}




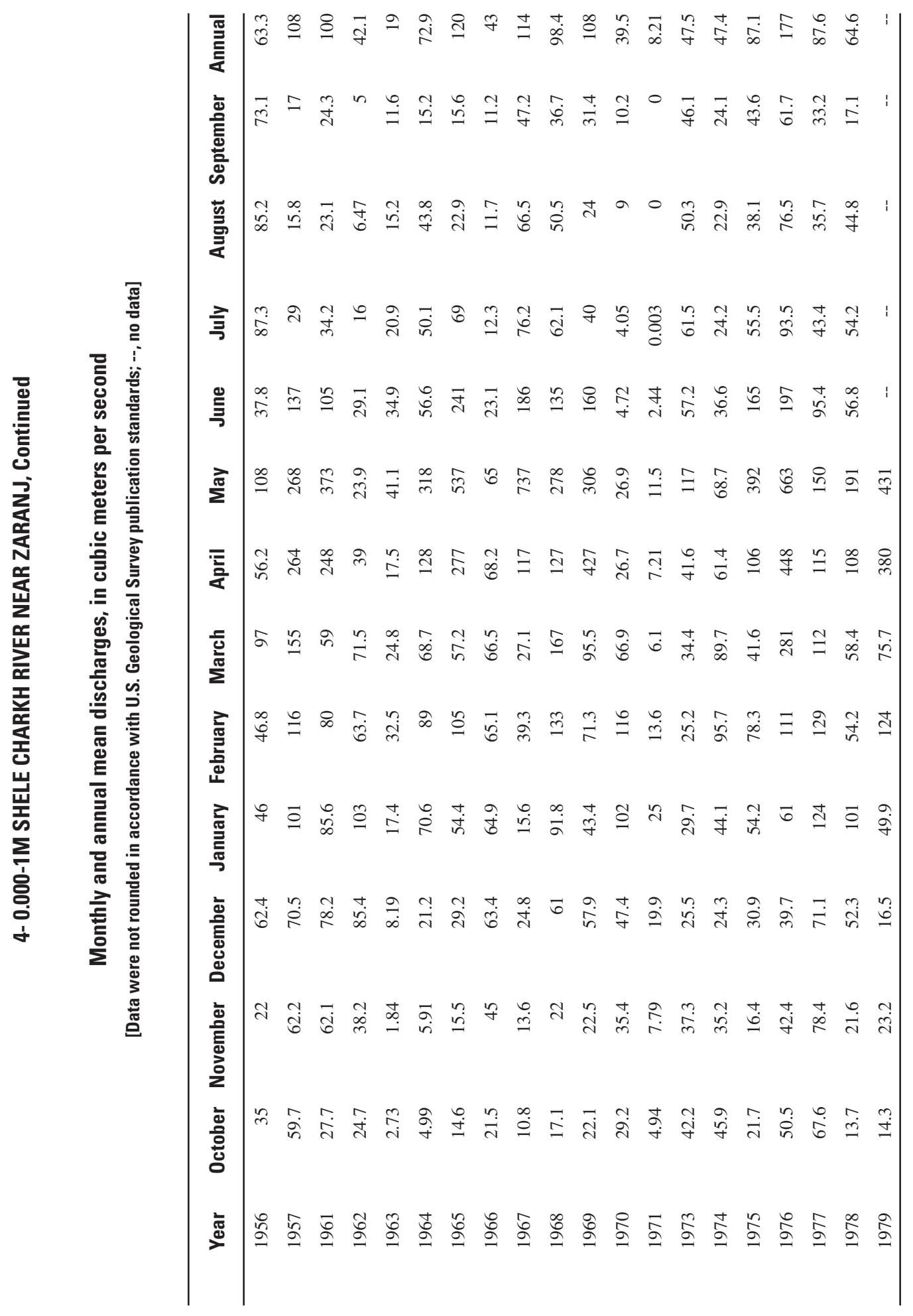




\section{4- 0.000-2M HELMAND RIVER AT KHWABGAH}

\section{(U.S. Geological Survey identification number: 304800061460000)}

LOCATION: Lat $30^{\circ} 48^{\prime} \mathrm{N}$., long $61^{\circ} 46^{\prime} \mathrm{E}$.

DRAINAGE AREA: $137,765 \mathrm{~km}^{2}$, of which approximately $19,400 \mathrm{~km}^{2}$ is considered noncontributing.

ALTITUDE: 491 meters plus mean sea level.

PERIOD OF RECORD: January 24, 1969 to May 31, 1979.

GAGE: --

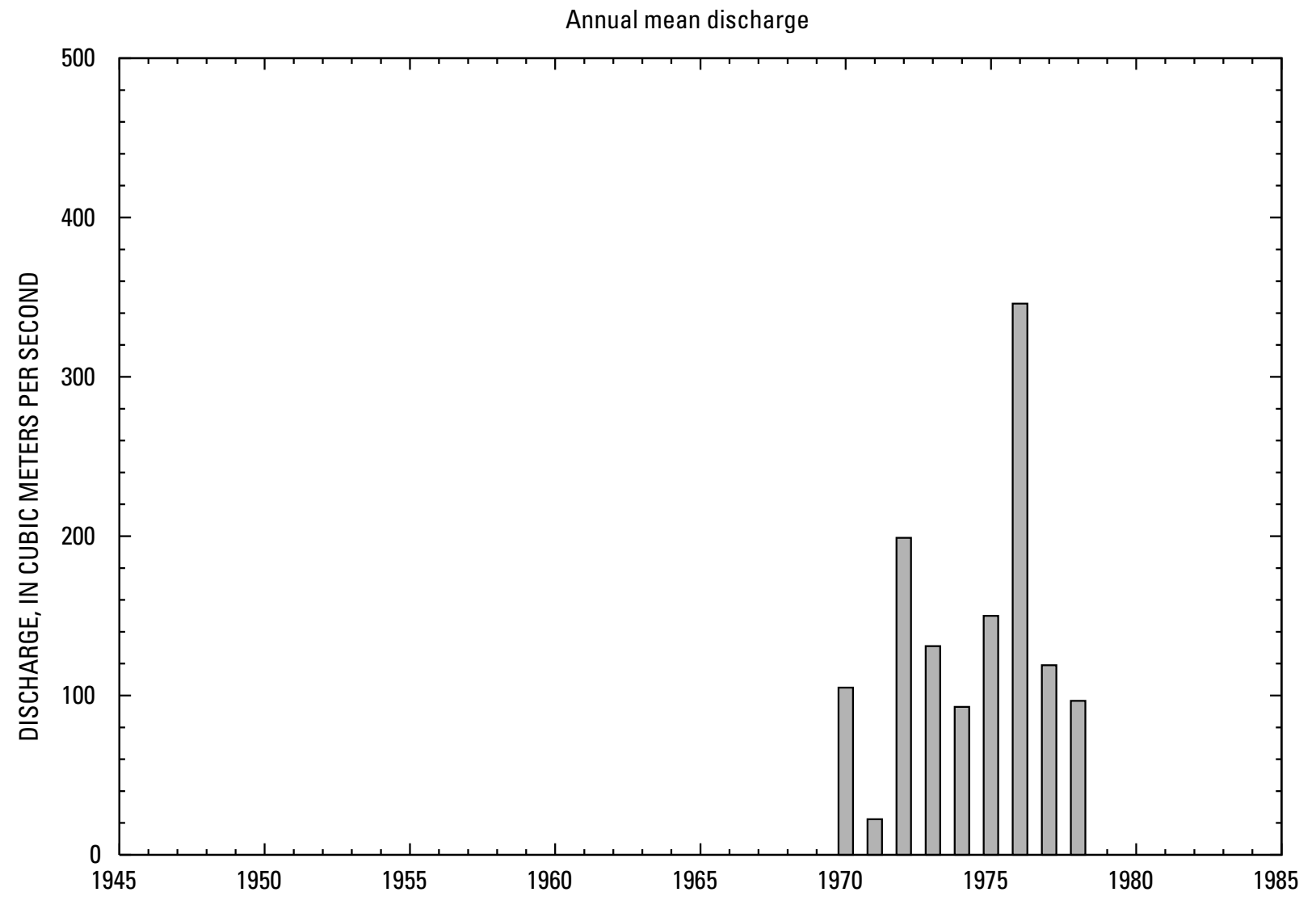




\section{4- 0.000-2M HELMAND RIVER AT KHWABGAH, Continued}

\section{Statistics of monthly and annual mean discharges}

$\left[\mathrm{m}^{3} / \mathrm{s}\right.$, meters per second]

\begin{tabular}{|c|c|c|c|c|c|c|c|c|}
\hline \multirow[b]{2}{*}{ Month } & \multicolumn{2}{|c|}{ Maximum } & \multicolumn{2}{|c|}{ Minimum } & \multicolumn{4}{|c|}{ Mean } \\
\hline & $\begin{array}{c}\text { Discharge } \\
\left(\mathrm{m}^{3} / \mathrm{s}\right)\end{array}$ & $\begin{array}{c}\text { Water year } \\
\text { of } \\
\text { occurrence }\end{array}$ & $\begin{array}{c}\text { Discharge } \\
\left(\mathrm{m}^{3} / \mathrm{s}\right)\end{array}$ & $\begin{array}{c}\text { Water year } \\
\text { of } \\
\text { occurrence }\end{array}$ & $\begin{array}{c}\text { Discharge } \\
\left(\mathrm{m}^{3} / \mathrm{s}\right)\end{array}$ & $\begin{array}{c}\text { Standard } \\
\text { deviation } \\
\left(\mathrm{m}^{3} / \mathrm{s}\right)\end{array}$ & $\begin{array}{c}\text { Coefficient } \\
\text { of } \\
\text { variation }\end{array}$ & $\begin{array}{c}\text { Percentage } \\
\text { of annual } \\
\text { discharge }\end{array}$ \\
\hline October & 118 & 1973 & 5.52 & 1972 & 66.5 & 39.5 & 0.59 & 3.74 \\
\hline November & 132 & 1977 & 10.6 & 1972 & 67.9 & 37.7 & 0.56 & 3.82 \\
\hline December & 113 & 1977 & 11.5 & 1972 & 68.7 & 29.0 & 0.42 & 3.86 \\
\hline January & 185 & 1970 & 44.5 & 1971 & 111 & 40.7 & 0.37 & 6.22 \\
\hline February & 224 & 1970 & 34.3 & 1971 & 136 & 53.4 & 0.39 & 7.66 \\
\hline March & 520 & 1976 & 11.8 & 1971 & 177 & 135 & 0.76 & 9.94 \\
\hline April & 1,110 & 1976 & 24.6 & 1971 & 350 & 328 & 0.94 & 19.7 \\
\hline May & 1,240 & 1976 & 33.7 & 1971 & 425 & 351 & 0.83 & 23.9 \\
\hline June & 398 & 1976 & 12.1 & 1971 & 166 & 126 & 0.76 & 9.31 \\
\hline July & 165 & 1976 & 10.2 & 1971 & 84.5 & 52.6 & 0.62 & 4.75 \\
\hline August & 126 & 1976 & 5.59 & 1971 & 64.4 & 42.0 & 0.65 & 3.62 \\
\hline September & 112 & 1972 & 4.97 & 1971 & 62.9 & 37.8 & 0.60 & 3.54 \\
\hline Annual & 346 & 1976 & 22.4 & 1971 & 140 & 90.6 & 0.65 & 100 \\
\hline
\end{tabular}

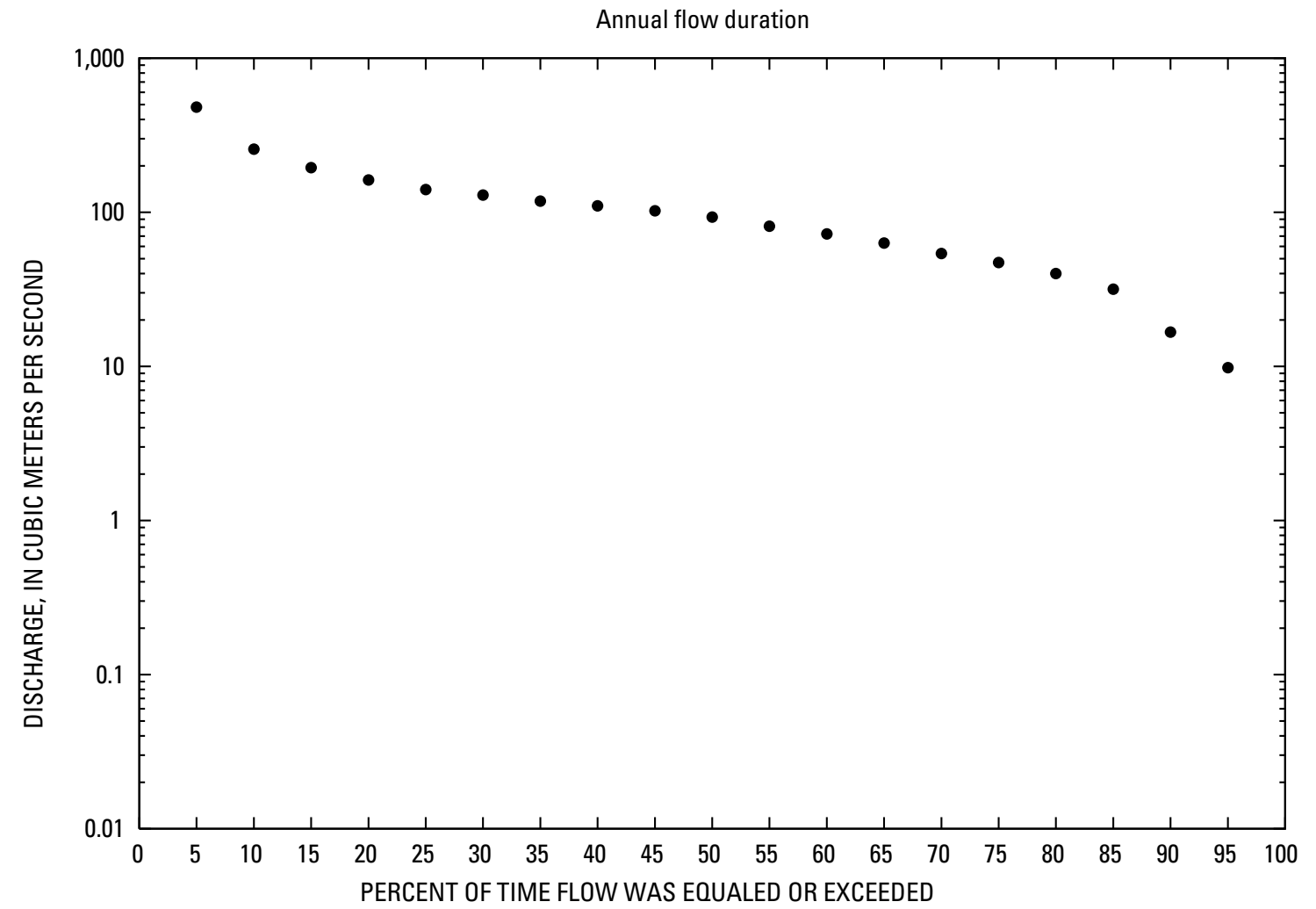




\section{4- 0.000-2M HELMAND RIVER AT KHWABGAH, Continued}

\section{Monthly and annual flow duration, in cubic meters per second}

[Data were not rounded in accordance with U.S. Geological Survey publication standards]

\begin{tabular}{|c|c|c|c|c|c|c|c|c|c|c|c|c|c|}
\hline $\begin{array}{l}\text { Percentage } \\
\text { of days } \\
\text { discharge } \\
\text { equaled or } \\
\text { exceeded }\end{array}$ & January & February & March & April & May & June & July & August & September & October & November & December & Annual \\
\hline 95 & 31.7 & 34.7 & 12.7 & 22.8 & 34.6 & 9.12 & 11 & 4.5 & 4.78 & 5.3 & 8.23 & 11.3 & 9.82 \\
\hline 90 & 43.3 & 41.4 & 53.3 & 32.7 & 67.8 & 26.2 & 12.7 & 9.12 & 6.8 & 8.22 & 14.3 & 14.7 & 16.7 \\
\hline 85 & 49.5 & 58.7 & 71.4 & 69.1 & 91.4 & 43.3 & 27.1 & 13.8 & 19.2 & 15.5 & 17.4 & 45.4 & 31.7 \\
\hline 80 & 55.1 & 71 & 80.1 & 95.7 & 129.9 & 50.1 & 31 & 15.5 & 27.6 & 19.3 & 34.3 & 48.4 & 40 \\
\hline 75 & 63.5 & 81.6 & 90.5 & 117.1 & 147.5 & 57 & 39.2 & 18.1 & 32.7 & 42.6 & 39.1 & 50.3 & 47.2 \\
\hline 70 & 68.8 & 87.7 & 99.9 & 128.4 & 159.9 & 68.4 & 43.9 & 33.9 & 40.5 & 45.5 & 41.1 & 52.1 & 54 \\
\hline 65 & 73 & 93.9 & 108.6 & 138.3 & 194.3 & 85.8 & 48.1 & 37.3 & 45.1 & 50.1 & 45.6 & 54.4 & 63.1 \\
\hline 60 & 84.8 & 101 & 116.5 & 147.2 & 235.9 & 100.1 & 53.3 & 42.2 & 51.2 & 54.5 & 61.3 & 57 & 72.3 \\
\hline 55 & 92.8 & 115.9 & 123.9 & 156 & 275.8 & 118.1 & 63.3 & 46.3 & 55.2 & 58.5 & 66.7 & 59.8 & 81.3 \\
\hline 50 & 97.2 & 127.6 & 131.3 & 175.5 & 371.5 & 130.2 & 72.4 & 51 & 58.6 & 62.6 & 69.9 & 63 & 93.1 \\
\hline 45 & 101.5 & 137.9 & 143.3 & 216 & 423.4 & 140 & 79 & 61.6 & 61.4 & 68.9 & 74.4 & 65.9 & 102.2 \\
\hline 40 & 106.6 & 143.9 & 155.7 & 270 & 474.4 & 159.6 & 86.5 & 72.1 & 71.9 & 77.7 & 79.8 & 68.5 & 110 \\
\hline 35 & 114.7 & 151.7 & 165.9 & 341.8 & 503.3 & 182 & 106.2 & 78.4 & 81.4 & 81.9 & 84.6 & 71 & 118.1 \\
\hline 30 & 123.6 & 172 & 175.9 & 401.1 & 532.1 & 205.7 & 115.5 & 95.8 & 90.6 & 95.7 & 90 & 75.1 & 129.3 \\
\hline 25 & 134 & 189.5 & 186.6 & 496.2 & 571.7 & 231.7 & 123 & 104.1 & 102.7 & 102.9 & 95.3 & 80.8 & 140.6 \\
\hline 20 & 163.8 & 201.4 & 202.7 & 662.4 & 614 & 265.8 & 130.6 & 108.8 & 109.2 & 109.6 & 100.1 & 95.7 & 162 \\
\hline 15 & 188.2 & 210.8 & 219.7 & 735.9 & 678.5 & 312.6 & 139.9 & 113.5 & 112.1 & 115.8 & 107.4 & 103.9 & 194.4 \\
\hline 10 & 201 & 221.7 & 253.8 & 839.2 & 878.6 & 365.6 & 150 & 121 & 115.1 & 121.4 & 120.9 & 109.6 & 257 \\
\hline 5 & 224.6 & 245 & 503.5 & 1165.5 & 1156.1 & 461 & 165.5 & 139.6 & 118 & 126.7 & 0 & 129.7 & 481.6 \\
\hline
\end{tabular}




\section{4- 0.000-2M HELMAND RIVER AT KHWABGAH, Continued}

Probability of occurrence of annual high discharges

[m³/s, meters per second; $n g$, statistic not given]

\begin{tabular}{|c|c|c|c|c|c|c|}
\hline \multirow{2}{*}{$\begin{array}{c}\text { Exceedance } \\
\text { probability }\end{array}$} & \multirow{2}{*}{$\begin{array}{c}\text { Recurrence } \\
\text { interval } \\
\text { (years) }\end{array}$} & \multirow{2}{*}{$\begin{array}{c}\text { Maximum } \\
\text { instantaneous } \\
\left(\mathrm{m}^{3} / \mathrm{s}\right)\end{array}$} & \multicolumn{4}{|c|}{ Maximum mean discharge } \\
\hline & & & 3-day period & 7-day period & 15-day period & 30-day period \\
\hline 0.99 & 1.01 & ng & 33.7 & 31.7 & 27.6 & 27.4 \\
\hline 0.95 & 1.05 & ng & 84.6 & 73.3 & 65.2 & 61.2 \\
\hline 0.90 & 1.11 & 345 & 133 & 112 & 100 & 92.0 \\
\hline 0.80 & 1.25 & 457 & 221 & 183 & 164 & 147 \\
\hline 0.50 & 2 & 773 & 529 & 437 & 391 & 341 \\
\hline 0.20 & 5 & 1,280 & 1,110 & 962 & 844 & 732 \\
\hline 0.10 & 10 & 1,660 & 1,550 & 1,410 & 1,210 & 1,060 \\
\hline 0.04 & 25 & 2,170 & 2,140 & 2,060 & 1,740 & 1,530 \\
\hline 0.02 & 50 & 2,580 & ${ }^{12,590}$ & ${ }^{12,610}$ & 2,170 & 1,920 \\
\hline 0.01 & 100 & 3,000 & ${ }^{1} 3,030$ & ${ }^{13}, 190$ & 2,620 & 2,340 \\
\hline 0.005 & 200 & 3,440 & ${ }^{1} 3,470$ & ${ }^{1} 3,820$ & 3,080 & 2,790 \\
\hline 0.002 & 500 & 4,040 & $\mathrm{ng}$ & $\mathrm{ng}$ & $\mathrm{ng}$ & $\mathrm{ng}$ \\
\hline
\end{tabular}

'Data does not fit log-Pearson Type III curve, use with caution.

\section{4- 0.000-2M HELMAND RIVER AT KHWABGAH, Continued}

\section{Probability of occurrence of annual low discharges}

$\left[\mathrm{m}^{3} / \mathrm{s}\right.$, meters per second]

\begin{tabular}{|c|c|c|c|c|c|c|c|c|c|c|}
\hline \multirow{3}{*}{$\begin{array}{c}\text { Nonexceedance } \\
\text { probability }\end{array}$} & \multirow{3}{*}{$\begin{array}{c}\text { Recurrence } \\
\text { interval } \\
\text { (years) }\end{array}$} & \multicolumn{9}{|c|}{ Minimum mean discharge $\left(\mathrm{m}^{3} / \mathrm{s}\right)$} \\
\hline & & \multicolumn{9}{|c|}{ Number of consecutive days } \\
\hline & & 1 & 3 & 7 & 14 & 30 & 60 & 90 & 120 & 183 \\
\hline 0.05 & 20 & 3.54 & 3.66 & 3.77 & 4.00 & 5.26 & 6.72 & 7.32 & 8.12 & 10.5 \\
\hline 0.10 & 10 & 6.05 & 6.24 & 6.49 & 7.02 & 9.36 & 12.2 & 13.1 & 14.2 & 17.7 \\
\hline 0.20 & 5 & 10.8 & 11.1 & 11.7 & 12.8 & 17.2 & 22.3 & 23.9 & 25.2 & 30.2 \\
\hline 0.50 & 2 & 27.0 & 28.0 & 29.8 & 33.0 & 41.7 & 52.1 & 55.2 & 57.3 & 64.7 \\
\hline
\end{tabular}




\section{4- 0.000-2M HELMAND RIVER AT KHWABGAH, Continued}

\section{Probability of occurrence of seasonal low discharges}

$\left[\mathrm{m}^{3} / \mathrm{s}\right.$, meters per second]

\begin{tabular}{|c|c|c|c|c|c|c|c|c|c|}
\hline \multirow{3}{*}{$\begin{array}{c}\text { Nonexceedance } \\
\text { probability }\end{array}$} & \multirow{3}{*}{$\begin{array}{c}\text { Recurrence } \\
\text { interval } \\
\text { (years) }\end{array}$} & \multicolumn{8}{|c|}{ Minimum mean discharge $\left(\mathrm{m}^{3} / \mathrm{s}\right)$} \\
\hline & & \multicolumn{8}{|c|}{ Number of consecutive days } \\
\hline & & 1 & 7 & 14 & 30 & 1 & 7 & 14 & 30 \\
\hline & & \multicolumn{4}{|c|}{ December-January-February } & \multicolumn{4}{|c|}{ March-April-May } \\
\hline 0.05 & 20 & 11.9 & 13.2 & 14.3 & 15.2 & 9.85 & 10.8 & 11.8 & 17.8 \\
\hline 0.10 & 10 & 16.6 & 19.7 & 21.6 & 23.7 & 18.5 & 20.5 & 22.4 & 29.4 \\
\hline 0.20 & 5 & 23.9 & 29.6 & 32.8 & 37.0 & 34.4 & 38.5 & 42.1 & 51.0 \\
\hline \multirow[t]{2}{*}{0.50} & 2 & 42.3 & 52.6 & 57.7 & 67.0 & 76.2 & 85.3 & 94.8 & 124 \\
\hline & & \multicolumn{4}{|c|}{ June-July-August } & \multicolumn{4}{|c|}{ September-October-November } \\
\hline 0.05 & 20 & 4.26 & 4.50 & 4.70 & 6.56 & 5.05 & 5.34 & 5.62 & 6.53 \\
\hline 0.10 & 10 & 7.51 & 7.96 & 8.33 & 11.0 & 9.13 & 9.64 & 10.3 & 11.7 \\
\hline 0.20 & 5 & 14.0 & 14.9 & 15.6 & 19.5 & 17.1 & 18.0 & 19.5 & 21.5 \\
\hline 0.50 & 2 & 38.8 & 41.0 & 42.5 & 48.8 & 43.4 & 45.3 & 49.1 & 52.5 \\
\hline
\end{tabular}

\section{4- 0.000-2M HELMAND RIVER AT KHWABGAH, Continued}

Annual peak discharge and corresponding gage height

[m³/s, meters per second; --, no data]

\begin{tabular}{cccccccc}
\hline $\begin{array}{c}\text { Water } \\
\text { year }\end{array}$ Date & $\begin{array}{c}\text { Gage } \\
\text { height } \\
(\text { meters })\end{array}$ & $\begin{array}{c}\text { Peak } \\
\text { discharge } \\
\left(\mathrm{m}^{3} / \mathrm{s}\right)\end{array}$ & $\begin{array}{c}\text { Water } \\
\text { year }\end{array}$ & Date & $\begin{array}{c}\text { Gage } \\
\text { height } \\
(\text { meters })\end{array}$ & $\begin{array}{c}\text { Peak } \\
\text { discharge } \\
\left(\mathrm{m}^{3} / \mathrm{s}\right)\end{array}$ \\
\hline
\end{tabular}

Annual peak discharge, by year

\begin{tabular}{rrrrrcrr}
1969 & 21-Apr & -- & 1,244 & 1975 & 22-May & -- & 1,078 \\
1970 & 1-Feb & -- & 680 & 1976 & 3-May & -- & 2,071 \\
1971 & 11-Jan & -- & 76.6 & 1977 & 13-Jan & -- & 772 \\
1972 & 2-May & -- & 1,431 & 1978 & 30-Dec & -- & 470 \\
1973 & 4-May & -- & 436 & 1979 & 16-Feb & -- & 1,006 \\
1974 & 24-Jan & -- & 348 & & & & \\
& & Annual peak discharge, from highest to lowest & & \\
1976 & 3-May & -- & 2,071 & 1970 & 1-Feb & -- & 680 \\
1972 & 2-May & -- & 1,431 & 1978 & 30-Dec & -- & 470 \\
1969 & 21-Apr & -- & 1,244 & 1973 & 4-May & -- & 436 \\
1975 & 22-May & -- & 1,078 & 1974 & 24-Jan & -- & 348 \\
1979 & 16-Feb & -- & 1,006 & 1971 & 11-Jan & -- & 76.6 \\
1977 & 13-Jan & -- & 772 & & & & \\
\hline
\end{tabular}




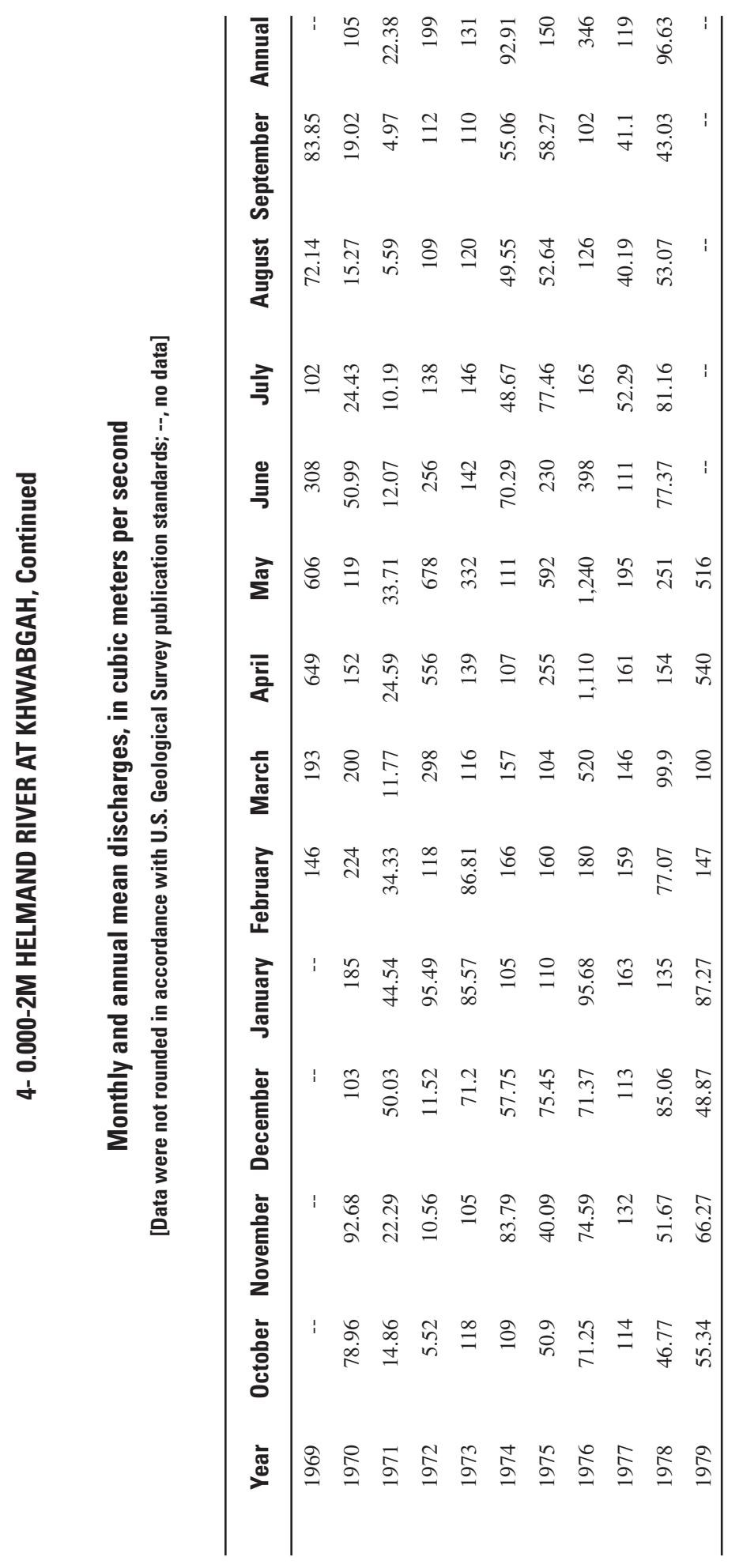




\section{4- 0.000-3M HELMAND RIVER AT CHAR BURJAK}

\section{(U.S. Geological Survey identification number: 301700062020000)}

LOCATION: Lat $30^{\circ} 17^{\prime} \mathrm{N}$. , long $62^{\circ} 02^{\prime} \mathrm{E}$., on right bank $1.2 \mathrm{~km}$ downstream from Char Burjak, about $80 \mathrm{~km}$ upstream from international boundary, and about $445 \mathrm{~km}$ downstream from Kajakai Dam.

DRAINAGE AREA: $136,735 \mathrm{~km}^{2}$, of which approximately $19,100 \mathrm{~km}^{2}$ is considered noncontributing.

ALTITUDE: 524 meters plus mean sea level.

PERIOD OF RECORD: October 1, 1948 to May 21, 1979.

GAGE: Water-stage recorder.

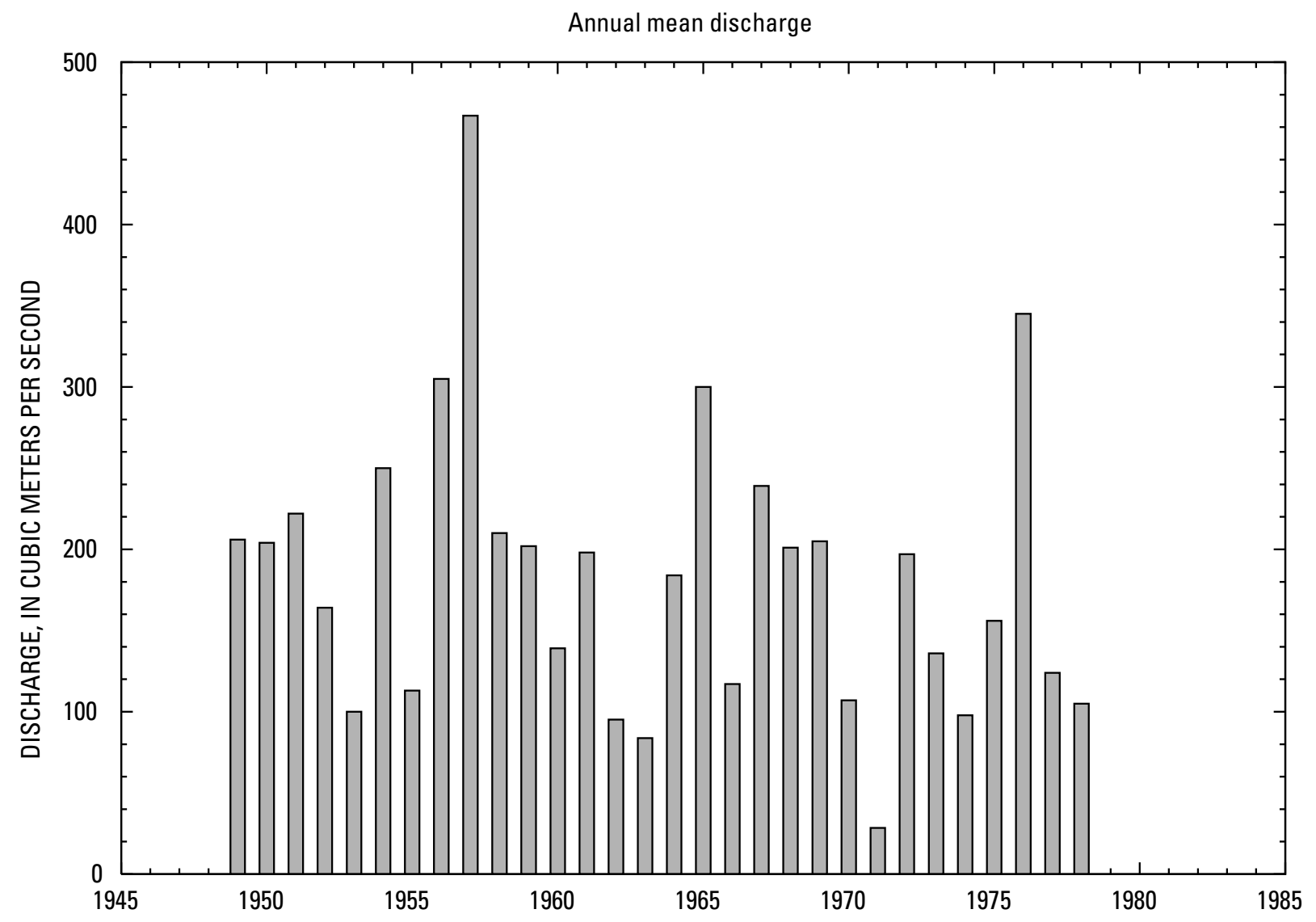




\section{4- 0.000-3M HELMAND RIVER AT CHAR BURJAK, Continued}

\section{Statistics of monthly and annual mean discharges}

[ $\mathrm{m}^{3} / \mathrm{s}$, meters per second]

\begin{tabular}{|c|c|c|c|c|c|c|c|c|}
\hline \multirow[b]{2}{*}{ Month } & \multicolumn{2}{|c|}{ Maximum } & \multicolumn{2}{|c|}{ Minimum } & \multicolumn{4}{|c|}{ Mean } \\
\hline & $\begin{array}{c}\text { Discharge } \\
\left(\mathrm{m}^{3} / \mathrm{s}\right)\end{array}$ & $\begin{array}{c}\text { Water year } \\
\text { of } \\
\text { occurrence }\end{array}$ & $\begin{array}{c}\text { Discharge } \\
\left(\mathrm{m}^{3} / \mathrm{s}\right)\end{array}$ & $\begin{array}{c}\text { Water year } \\
\text { of } \\
\text { occurrence }\end{array}$ & $\begin{array}{c}\text { Discharge } \\
\left(\mathrm{m}^{3} / \mathrm{s}\right)\end{array}$ & $\begin{array}{c}\text { Standard } \\
\text { deviation } \\
\left(\mathrm{m}^{3} / \mathrm{s}\right)\end{array}$ & $\begin{array}{c}\text { Coefficient } \\
\text { of } \\
\text { variation }\end{array}$ & $\begin{array}{c}\text { Percentage } \\
\text { of annual } \\
\text { discharge }\end{array}$ \\
\hline October & 172 & 1958 & 13.0 & 1972 & 66.3 & 39.2 & 0.59 & 3.02 \\
\hline November & 150 & 1966 & 18.5 & 1972 & 72.4 & 36.3 & 0.50 & 3.29 \\
\hline December & 295 & 1958 & 16.5 & 1972 & 96.2 & 57.1 & 0.59 & 4.38 \\
\hline January & 257 & 1957 & 41.6 & 1950 & 127 & 60.3 & 0.48 & 5.77 \\
\hline February & 536 & 1954 & 41.8 & 1971 & 171 & 88.4 & 0.52 & 7.78 \\
\hline March & 519 & 1976 & 17.3 & 1971 & 218 & 128 & 0.59 & 9.93 \\
\hline April & 1,600 & 1957 & 33.9 & 1971 & 438 & 376 & 0.86 & 19.9 \\
\hline May & 1,500 & 1957 & 46.3 & 1971 & 517 & 382 & 0.74 & 23.5 \\
\hline June & 622 & 1957 & 17.2 & 1971 & 226 & 145 & 0.64 & 10.3 \\
\hline July & 410 & 1956 & 15.2 & 1971 & 111 & 76.7 & 0.69 & 5.07 \\
\hline August & 299 & 1956 & 11.2 & 1971 & 83.9 & 57.5 & 0.69 & 3.82 \\
\hline September & 172 & 1957 & 11.5 & 1971 & 71.3 & 42.0 & 0.59 & 3.25 \\
\hline Annual & 467 & 1957 & 28.5 & 1971 & 183 & 89.7 & 0.49 & 100 \\
\hline
\end{tabular}

Annual flow duration

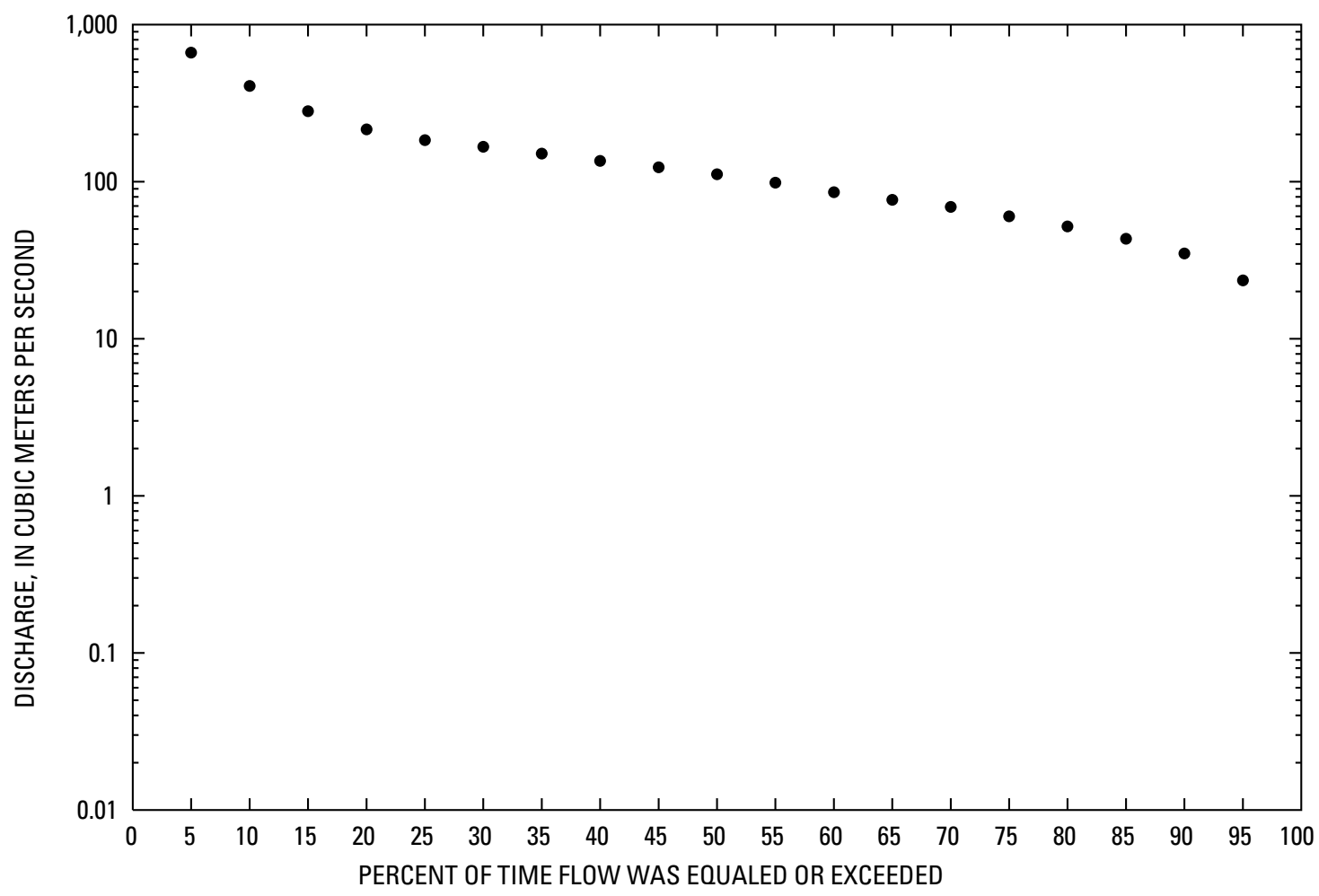




\section{4- 0.000-3M HELMAND RIVER AT CHAR BURJAK, Continued}

Monthly and annual flow duration, in cubic meters per second

[Data were not rounded in accordance with U.S. Geological Survey publication standards]

\begin{tabular}{|c|c|c|c|c|c|c|c|c|c|c|c|c|c|}
\hline $\begin{array}{l}\text { Percentage } \\
\text { of days } \\
\text { discharge } \\
\text { equaled or } \\
\text { exceeded }\end{array}$ & January & February & March & April & May & June & July & August & September & October & November & December & Annual \\
\hline 95 & 40.5 & 57.5 & 63.5 & 61.4 & 72.9 & 45.6 & 26.3 & 17.4 & 14.2 & 14.3 & 21.4 & 32.4 & 23.5 \\
\hline 90 & 45.1 & 69.2 & 85.2 & 78.3 & 91.1 & 58.1 & 39.1 & 25.2 & 20.9 & 18.9 & 28.5 & 37.7 & 34.9 \\
\hline 80 & 59.2 & 88.6 & 107.6 & 116.3 & 151.4 & 92.4 & 51.2 & 31.6 & 27.3 & 29.9 & 37.2 & 45.4 & 52 \\
\hline 75 & 62.6 & 96.3 & 114.1 & 132.8 & 167 & 112.5 & 55.2 & 37.4 & 33 & 32.9 & 39.4 & 50 & 60.2 \\
\hline 70 & 69.2 & 109.6 & 120.5 & 150.8 & 204.2 & 133.5 & 59.7 & 45.1 & 44.6 & 38.4 & 41.1 & 54.8 & 69.1 \\
\hline 65 & 89.6 & 125.5 & 128.4 & 165.9 & 273.5 & 148.2 & 65.3 & 50.7 & 51.2 & 45.9 & 46.2 & 59 & 76.6 \\
\hline 60 & 98.8 & 134.5 & 137.7 & 204 & 324.6 & 158.2 & 73.5 & 56.3 & 54.9 & 52.8 & 57.8 & 64.3 & 85.7 \\
\hline 45 & 122.5 & 161.3 & 172.7 & 339.7 & 483.8 & 200.2 & 101.3 & 76.5 & 68.6 & 68.4 & 74 & 83.3 & 123.6 \\
\hline 40 & 132.9 & 168.9 & 185 & 379.5 & 533.3 & 216.6 & 126 & 85.4 & 74.2 & 72.1 & 77.2 & 91.2 & 135.5 \\
\hline 35 & 153.9 & 176.6 & 197.3 & 454.3 & 583.9 & 235.2 & 133 & 103.8 & 79.4 & 75.4 & 80 & 105.1 & 150.8 \\
\hline 30 & 167.8 & 187.6 & 222.1 & 539.2 & 644.3 & 260.2 & 138.3 & 114.4 & 88 & 78.7 & 82.8 & 114 & 166.9 \\
\hline 25 & 178 & 199.7 & 259.5 & 608.2 & 728.6 & 290.1 & 143.6 & 120.8 & 105.8 & 82.3 & 91.6 & 125.1 & 183.9 \\
\hline 20 & 185.2 & 213.4 & 296.3 & 684.7 & 845.7 & 327.3 & 150.9 & 127.2 & 114.5 & 86.3 & 101.2 & 144.4 & 215.6 \\
\hline 15 & 192.4 & 233.8 & 328.6 & 808.3 & 965.6 & 378.7 & 159.9 & 137.3 & 121.5 & 114.3 & 109.1 & 159.9 & 281.4 \\
\hline 10 & 208.8 & 272.2 & 372.9 & 1036.5 & 1106.7 & 445.8 & 173.2 & 149.3 & 127.7 & 127.6 & 132.6 & 173.7 & 406.7 \\
\hline 5 & 254.4 & 385.8 & 511 & 1388 & 1303.3 & 572 & 215.9 & 166.4 & 169.4 & 143 & 160.4 & 187.7 & 662.8 \\
\hline
\end{tabular}




\section{4- 0.000-3M HELMAND RIVER AT CHAR BURJAK, Continued}

\section{Probability of occurrence of annual high discharges}

[ $\mathrm{m}^{3} / \mathrm{s}$, meters per second; $\mathrm{ng}$, statistic not given]

\begin{tabular}{|c|c|c|c|c|c|c|}
\hline \multirow{2}{*}{$\begin{array}{l}\text { Exceedance } \\
\text { probability }\end{array}$} & \multirow{2}{*}{$\begin{array}{c}\text { Recurrence } \\
\text { interval } \\
\text { (years) }\end{array}$} & \multirow{2}{*}{$\begin{array}{c}\text { Maximum } \\
\text { instantaneous } \\
\left(\mathrm{m}^{3} / \mathrm{s}\right)\end{array}$} & \multicolumn{4}{|c|}{ Maximum mean discharge } \\
\hline & & & 3-day period & 7-day period & 15-day period & 30-day period \\
\hline 0.99 & 1.01 & ng & 70.2 & 63.1 & 53.8 & 50.5 \\
\hline 0.95 & 1.05 & 266 & 163 & 143 & 122 & 109 \\
\hline 0.90 & 1.11 & 352 & 244 & 211 & 180 & 160 \\
\hline 0.80 & 1.25 & 489 & 379 & 327 & 281 & 246 \\
\hline 0.50 & 2 & 883 & 773 & 676 & 594 & 513 \\
\hline 0.20 & 5 & 1,520 & 1,340 & 1,220 & 1,100 & 959 \\
\hline 0.10 & 10 & 1,970 & 1,700 & 1,570 & 1,450 & 1,280 \\
\hline 0.04 & 25 & 2,570 & 2,090 & 1,990 & 1,880 & 1,680 \\
\hline 0.02 & 50 & 3,030 & 2,340 & 2,280 & 2,190 & 1,980 \\
\hline 0.01 & 100 & 3,500 & 2,560 & 2,550 & 2,470 & 2,260 \\
\hline 0.005 & 200 & 3,970 & 2,750 & ${ }^{2} 2,790$ & 2,750 & 2,540 \\
\hline 0.002 & 500 & 4,600 & ng & ng & ng & ng \\
\hline
\end{tabular}

'Historic peaks of 1885 and 1903 not used.

${ }^{2}$ Data does not fit log-Pearson Type III curve, use with caution.

\section{4- 0.000-3M HELMAND RIVER AT CHAR BURJAK, Continued}

Probability of occurrence of annual low discharges

$\left[\mathrm{m}^{3} / \mathrm{s}\right.$, meters per second]

\begin{tabular}{|c|c|c|c|c|c|c|c|c|c|c|}
\hline \multirow{3}{*}{$\begin{array}{c}\text { Nonexceedance } \\
\text { probability }\end{array}$} & \multirow{3}{*}{$\begin{array}{c}\text { Recurrence } \\
\text { interval } \\
\text { (years) }\end{array}$} & \multicolumn{9}{|c|}{ Minimum mean discharge $\left(\mathrm{m}^{3} / \mathrm{s}\right)$} \\
\hline & & \multicolumn{9}{|c|}{ Number of consecutive days } \\
\hline & & 1 & 3 & 7 & 14 & 30 & 60 & 90 & 120 & 183 \\
\hline 0.05 & 20 & 12.4 & 12.7 & 13.1 & 13.8 & 15.1 & 16.4 & 17.6 & 19.2 & 23.6 \\
\hline 0.10 & 10 & 16.0 & 16.4 & 17.0 & 18.3 & 20.1 & 22.1 & 23.8 & 25.9 & 31.5 \\
\hline 0.20 & 5 & 21.3 & 21.7 & 22.8 & 24.9 & 27.7 & 30.7 & 33.2 & 36.1 & 43.4 \\
\hline 0.50 & 2 & 34.4 & 35.2 & 37.2 & 41.1 & 47.0 & 52.8 & 57.2 & 62.1 & 73.8 \\
\hline
\end{tabular}




\section{4- 0.000-3M HELMAND RIVER AT CHAR BURJAK, Continued}

\section{Probability of occurrence of seasonal low discharges}

$\left[\mathrm{m}^{3} / \mathrm{s}\right.$, meters per second]

\begin{tabular}{|c|c|c|c|c|c|c|c|c|c|}
\hline \multirow{3}{*}{$\begin{array}{c}\text { Nonexceedance } \\
\text { probability }\end{array}$} & \multirow{3}{*}{$\begin{array}{c}\text { Recurrence } \\
\text { interval } \\
\text { (years) }\end{array}$} & \multicolumn{8}{|c|}{ Minimum mean discharge $\left(\mathrm{m}^{3} / \mathrm{s}\right)$} \\
\hline & & \multicolumn{8}{|c|}{ Number of consecutive days } \\
\hline & & 1 & 7 & 14 & 30 & 1 & 7 & 14 & 30 \\
\hline & & \multicolumn{4}{|c|}{ December-January-February } & \multicolumn{4}{|c|}{ March-April-May } \\
\hline 0.05 & 20 & ${ }^{1} 15.1$ & 11.1 & 11.7 & 13.9 & 28.8 & 30.8 & 33.6 & 41.9 \\
\hline 0.10 & 10 & ${ }^{1} 20.5$ & 17.8 & 19.0 & 24.2 & 39.9 & 44.0 & 49.2 & 60.1 \\
\hline 0.20 & 5 & 29.0 & 29.2 & 31.7 & 42.0 & 57.1 & 64.8 & 74.0 & 89.9 \\
\hline \multirow[t]{2}{*}{0.50} & 2 & 53.0 & 61.2 & 67.7 & 86.7 & 103 & 119 & 137 & 175 \\
\hline & & \multicolumn{4}{|c|}{ June-July-August } & \multicolumn{4}{|c|}{ September-October-November } \\
\hline 0.05 & 20 & 14.4 & 14.9 & 15.5 & 17.6 & 13.2 & 14.0 & 14.8 & 15.7 \\
\hline 0.10 & 10 & 19.3 & 20.4 & 21.7 & 24.4 & 17.0 & 18.2 & 19.6 & 21.0 \\
\hline 0.20 & 5 & 27.0 & 29.4 & 31.7 & 35.3 & 22.8 & 24.6 & 26.8 & 29.2 \\
\hline 0.50 & 2 & 49.3 & 55.4 & 60.2 & 66.2 & 38.7 & 41.7 & 45.6 & 51.1 \\
\hline
\end{tabular}

'Data does not fit log-Pearson Type III curve, use with caution. 


\section{4- 0.000-3M HELMAND RIVER AT CHAR BURJAK, Continued}

Annual peak discharge and corresponding gage height

[m³/s, meters per second; --, no data]

\begin{tabular}{|c|c|c|c|c|c|c|c|}
\hline $\begin{array}{l}\text { Water } \\
\text { year }\end{array}$ & Date & $\begin{array}{c}\text { Gage } \\
\text { height } \\
\text { (meters) }\end{array}$ & $\begin{array}{c}\text { Peak } \\
\text { discharge } \\
\left(\mathrm{m}^{3} / \mathrm{s}\right)\end{array}$ & $\begin{array}{l}\text { Water } \\
\text { year }\end{array}$ & Date & $\begin{array}{c}\text { Gage } \\
\text { height } \\
\text { (meters) }\end{array}$ & $\begin{array}{c}\text { Peak } \\
\text { discharge } \\
\left(\mathrm{m}^{3} / \mathrm{s}\right)\end{array}$ \\
\hline \multicolumn{8}{|c|}{ Annual peak discharge, by year } \\
\hline 1885 & -- & -- & 16,980 & 1965 & 29-Apr & -- & 1,660 \\
\hline 1903 & -- & -- & 2,590 & 1966 & 26-Dec & -- & 275 \\
\hline 1949 & 15-Mar & 12.23 & 2,266 & 1967 & 3-May & -- & 1,638 \\
\hline 1950 & 2-Feb & 11.30 & 1,623 & 1968 & 5-May & -- & 830 \\
\hline 1951 & 12-May & -- & 1,585 & 1969 & 21-Apr & -- & 1,154 \\
\hline 1953 & 18-Feb & -- & 283 & 1970 & 31-Jan & -- & 826 \\
\hline 1955 & 18-Mar & 10.02 & 861 & 1971 & 8-Jan & -- & 73 \\
\hline 1956 & 10-Apr & 11.25 & 1,620 & 1972 & 2-May & -- & 1,038 \\
\hline 1957 & 21-Mar & 12.98 & 3,311 & 1973 & 9-May & -- & 451 \\
\hline 1958 & 14-Dec & 10.20 & 903 & 1974 & 23-Jan & -- & 388 \\
\hline 1959 & 4-Mar & 9.81 & 915 & 1975 & 25-Apr & -- & 790 \\
\hline 1960 & 20-May & 9.55 & 719 & 1976 & 27-Mar & -- & 1,900 \\
\hline 1961 & 14-Apr & -- & 1,288 & 1977 & 14-Jan & -- & 700 \\
\hline 1962 & 2-Jan & -- & 215 & 1978 & 29-Dec & -- & 577 \\
\hline 1963 & 16-May & -- & 532 & 1979 & 16-Feb & -- & 810 \\
\hline 1964 & 1-May & -- & 810 & & & & \\
\hline \multicolumn{8}{|c|}{ Annual peak discharge, from highest to lowest } \\
\hline 1885 & -- & -- & 16,980 & 1968 & 5-May & -- & 830 \\
\hline 1957 & 21-Mar & 12.98 & 3,311 & 1970 & 31-Jan & -- & 826 \\
\hline 1903 & -- & -- & 2,590 & 1979 & 16-Feb & -- & 810 \\
\hline 1949 & 15-Mar & 12.23 & 2,266 & 1964 & 1-May & -- & 810 \\
\hline 1976 & 27-Mar & -- & 1,900 & 1975 & 25-Apr & -- & 790 \\
\hline 1965 & 29-Apr & -- & 1,660 & 1960 & 20-May & 9.55 & 719 \\
\hline 1950 & 2-Feb & 11.30 & 1,623 & 1978 & 29-Dec & -- & 577 \\
\hline 1956 & 10-Apr & 11.25 & 1,620 & 1963 & 16-May & -- & 532 \\
\hline 1951 & 12-May & -- & 1,585 & 1973 & 9-May & -- & 451 \\
\hline 1961 & 14-Apr & -- & 1,288 & 1974 & 23-Jan & -- & 388 \\
\hline 1969 & 21-Apr & -- & 1,154 & 1953 & 18-Feb & -- & 283 \\
\hline 1972 & 2-May & -- & 1,038 & 1966 & 26-Dec & -- & 275 \\
\hline 1959 & 4-Mar & 9.81 & 915 & 1962 & 2-Jan & -- & 215 \\
\hline 1958 & 14-Dec & 10.20 & 903 & 1971 & 8-Jan & -- & 73 \\
\hline 1955 & 18-Mar & 10.02 & 861 & & & & \\
\hline
\end{tabular}




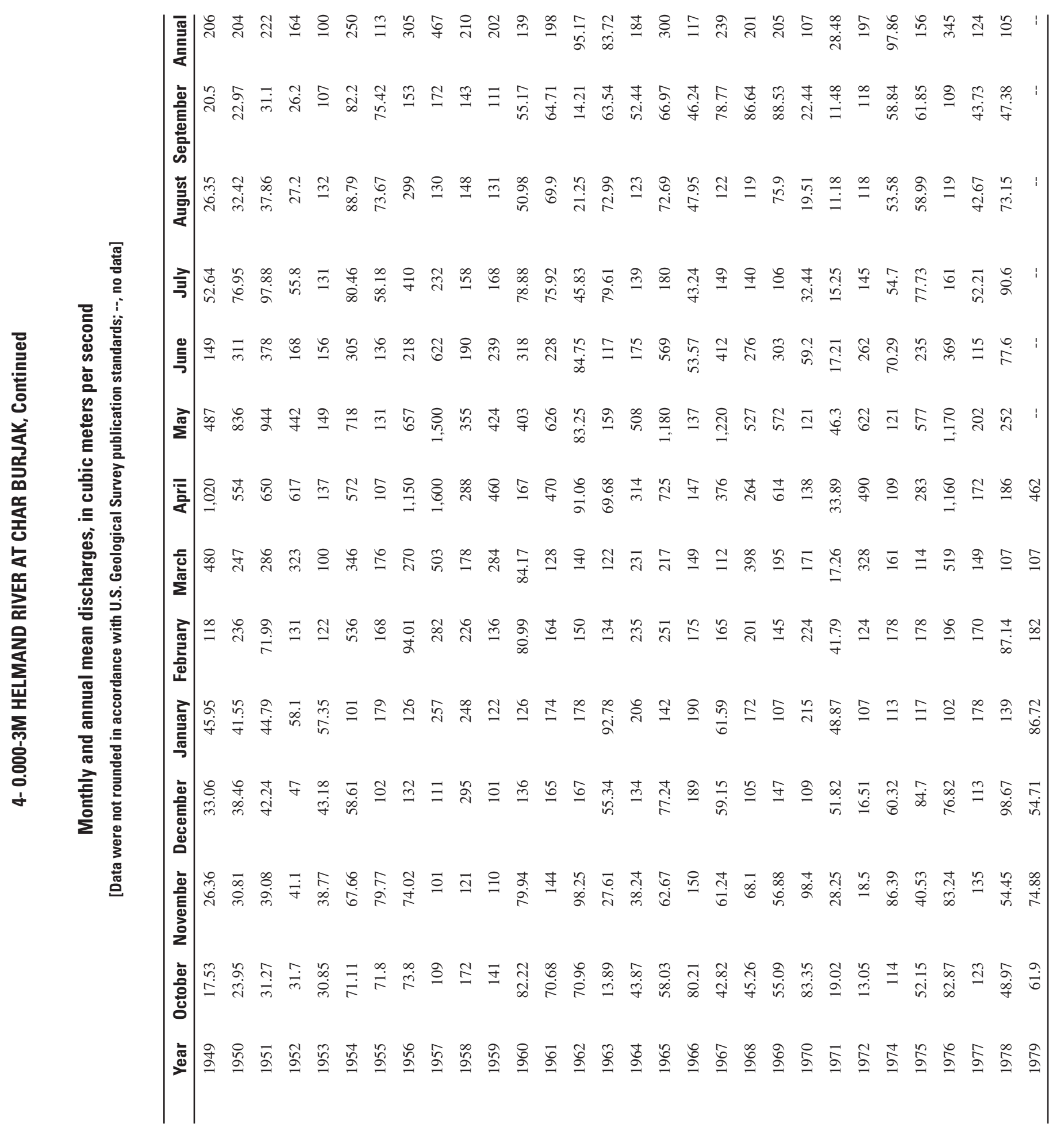




\section{4- 0.000-4M HELMAND RIVER AT MALAKHAN}

\section{(U.S. Geological Survey identification number: 302700063220000)}

LOCATION: Lat $30^{\circ} 27^{\prime} \mathrm{N}$., long $63^{\circ} 22^{\prime} \mathrm{E}$.

DRAINAGE AREA: $132,880 \mathrm{~km}^{2}$, of which approximately $17400 \mathrm{~km}^{2}$ is considered noncontributing.

ALTITUDE: 591 meters plus mean sea level.

PERIOD OF RECORD: January 19, 1970 to September 30, 1978.

GAGE: --

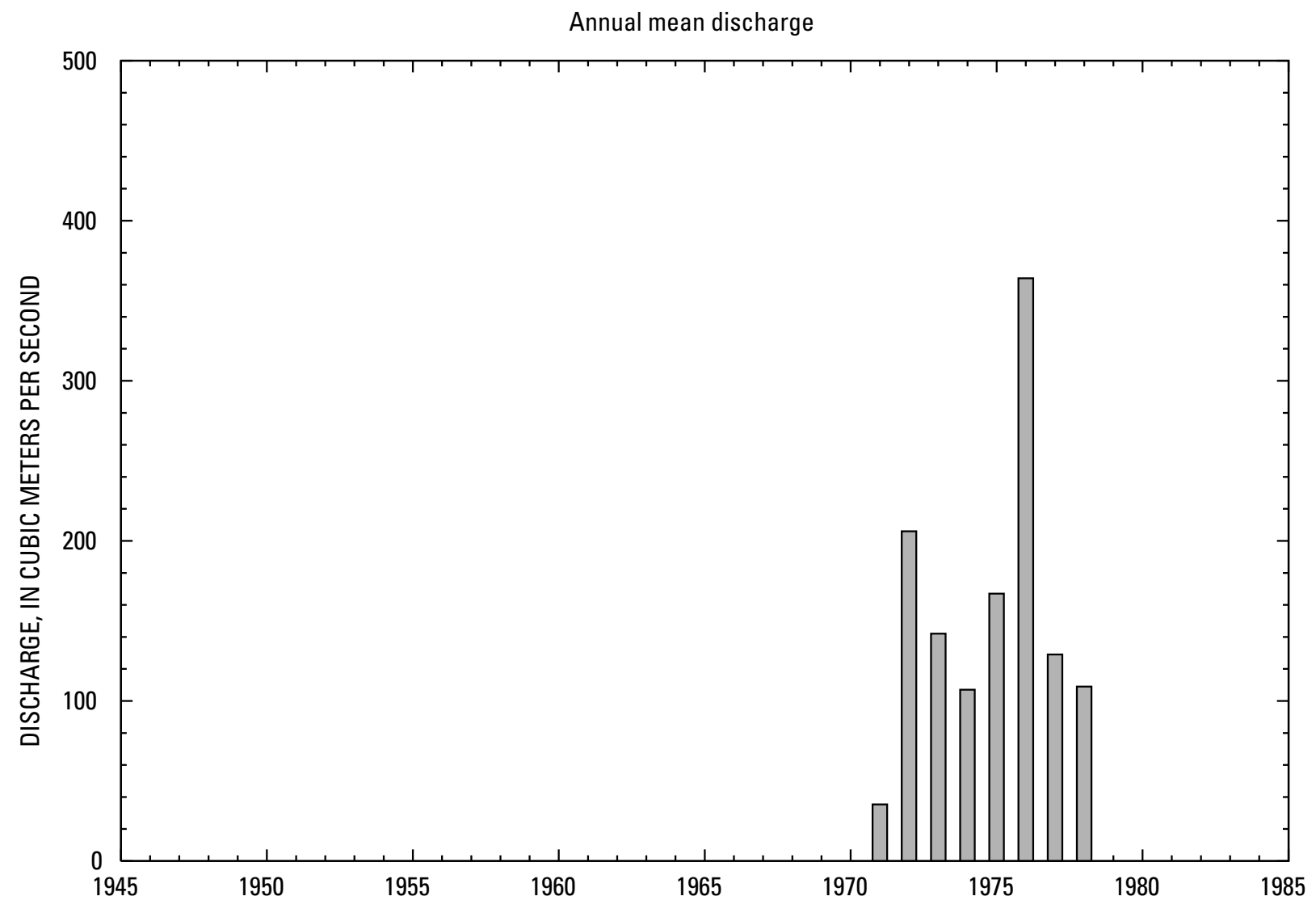




\section{4- 0.000-4M HELMAND RIVER AT MALAKAN, Continued}

\section{Statistics of monthly and annual mean discharges}

$\left[\mathrm{m}^{3} / \mathrm{s}\right.$, meters per second]

\begin{tabular}{|c|c|c|c|c|c|c|c|c|}
\hline \multirow[b]{2}{*}{ Month } & \multicolumn{2}{|c|}{ Maximum } & \multicolumn{2}{|c|}{ Minimum } & \multicolumn{4}{|c|}{ Mean } \\
\hline & $\begin{array}{c}\text { Discharge } \\
\left(\mathrm{m}^{3} / \mathrm{s}\right)\end{array}$ & $\begin{array}{c}\text { Water year } \\
\text { of } \\
\text { occurrence }\end{array}$ & $\begin{array}{c}\text { Discharge } \\
\left(\mathrm{m}^{3} / \mathrm{s}\right)\end{array}$ & $\begin{array}{c}\text { Water year } \\
\text { of } \\
\text { occurrence }\end{array}$ & $\begin{array}{c}\text { Discharge } \\
\left(\mathrm{m}^{3} / \mathrm{s}\right)\end{array}$ & $\begin{array}{c}\text { Standard } \\
\text { deviation } \\
\left(\mathrm{m}^{3} / \mathrm{s}\right)\end{array}$ & $\begin{array}{c}\text { Coefficient } \\
\text { of } \\
\text { variation }\end{array}$ & $\begin{array}{c}\text { Percentage } \\
\text { of annual } \\
\text { discharge }\end{array}$ \\
\hline October & 130 & 1977 & 13.6 & 1972 & 75.0 & 45.6 & 0.61 & 4.13 \\
\hline November & 142 & 1977 & 34.3 & 1972 & 77.6 & 38.6 & 0.50 & 4.27 \\
\hline December & 129 & 1977 & 30.8 & 1972 & 86.6 & 30.3 & 0.35 & 4.77 \\
\hline January & 174 & 1977 & 65.4 & 1971 & 120 & 32.4 & 0.27 & 6.62 \\
\hline February & 226 & 1970 & 46.1 & 1971 & 147 & 60.2 & 0.41 & 8.08 \\
\hline March & 576 & 1976 & 24.3 & 1971 & 199 & 165 & 0.83 & 11.0 \\
\hline April & 1,270 & 1976 & 41.6 & 1971 & 333 & 378 & 1.13 & 18.4 \\
\hline May & 1,200 & 1976 & 53.5 & 1971 & 394 & 368 & 0.93 & 21.7 \\
\hline June & 357 & 1976 & 20.7 & 1971 & 148 & 110 & 0.74 & 8.16 \\
\hline July & 175 & 1976 & 15.5 & 1971 & 90.9 & 54.9 & 0.60 & 5.01 \\
\hline August & 133 & 1972 & 16.0 & 1971 & 75.0 & 44.5 & 0.59 & 4.14 \\
\hline September & 124 & 1972 & 14.8 & 1971 & 68.5 & 40.6 & 0.59 & 3.78 \\
\hline Annual & 364 & 1976 & 35.4 & 1971 & 157 & 97.1 & 0.62 & 100 \\
\hline
\end{tabular}

Annual flow duration

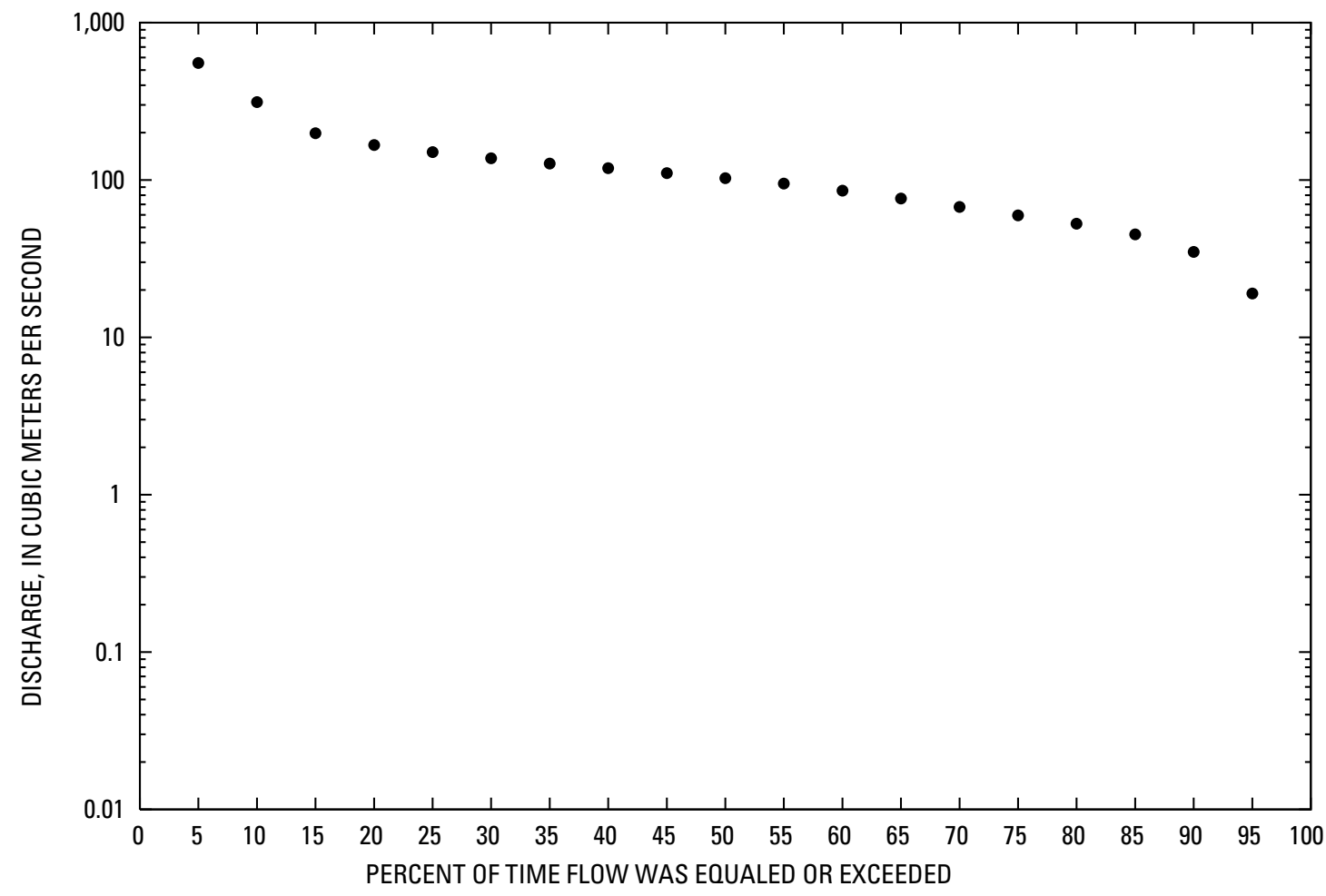




\section{4- 0.000-4M HELMAND RIVER AT MALAKAN, Continued}

Monthly and annual flow duration, in cubic meters per second

[Data were not rounded in accordance with U.S. Geological Survey publication standards]

\begin{tabular}{|c|c|c|c|c|c|c|c|c|c|c|c|c|c|}
\hline $\begin{array}{l}\text { Percentage } \\
\text { of days } \\
\text { discharge } \\
\text { equaled or } \\
\text { exceeded }\end{array}$ & January & February & March & April & May & June & July & August & September & October & November & December & Annual \\
\hline 95 & 49.9 & 49.4 & 19.7 & 36.8 & 54 & 18.2 & 15.8 & 16 & 13.7 & 12.4 & 23.3 & 31.2 & 19 \\
\hline 90 & 60.2 & 52.6 & 35.5 & 57.9 & 61.5 & 31.2 & 16.6 & 17.9 & 19.4 & 15.8 & 31.4 & 33.2 & 34.9 \\
\hline 80 & 75.3 & 88.3 & 91.7 & 98.3 & 119.4 & 57.1 & 43.2 & 27.7 & 25.7 & 24.5 & 40.3 & 67.8 & 52.8 \\
\hline 75 & 82.4 & 92.1 & 97.5 & 122.9 & 140.5 & 61.5 & 47 & 38.7 & 40.2 & 26 & 42.1 & 70.3 & 59.6 \\
\hline 70 & 90.7 & 95.9 & 104.9 & 134.5 & 154.4 & 65.2 & 49.6 & 40.2 & 45.2 & 49.4 & 43.5 & 71.8 & 67.3 \\
\hline 65 & 96.9 & 102.7 & 114.4 & 142.7 & 163.4 & 72 & 53.1 & 42.1 & 49.7 & 51.8 & 51.3 & 73.4 & 76.2 \\
\hline 60 & 101.4 & 117.9 & 123 & 150.4 & 173.4 & 95 & 58.2 & 55.3 & 54 & 55.2 & 57 & 74.9 & 85.6 \\
\hline 45 & 109.9 & 149.5 & 147.9 & 175 & 290.5 & 127.1 & 78 & 59.9 & 63.8 & 81.8 & 85.4 & 80.7 & 110.7 \\
\hline 40 & 113.2 & 154.9 & 156.4 & 184.7 & 361.7 & 136.8 & 100.4 & 65.3 & 66.8 & 87.5 & 90.7 & 83 & 118.9 \\
\hline 35 & 116.5 & 163.2 & 163.7 & 235.8 & 433.7 & 153.4 & 104.5 & 105.9 & 71.7 & 110.6 & 94.3 & 85.4 & 127.4 \\
\hline 30 & 119.8 & 172.1 & 170.6 & 320.6 & 469.2 & 170.1 & 108.6 & 111 & 110.1 & 113.8 & 100.2 & 87.9 & 137.4 \\
\hline 25 & 126.9 & 188 & 177.6 & 373 & 546.3 & 191.9 & 115.2 & 116.8 & 113.3 & 116.9 & 105.7 & 92.6 & 150.3 \\
\hline 20 & 135.9 & 202.4 & 187.7 & 458 & 628.6 & 224.5 & 134 & 124.4 & 116.4 & 120.1 & 109.7 & 100.1 & 167.1 \\
\hline 15 & 144.9 & 215.6 & 208.3 & 662 & 742.6 & 255.5 & 147.4 & 133.1 & 119.4 & 123.2 & 120.5 & 112.2 & 198.4 \\
\hline 10 & 173.5 & 227.8 & 440.8 & 760 & 931.6 & 317.8 & 160.4 & 142.3 & 122.4 & 126.3 & 139.4 & 129.1 & 313.1 \\
\hline 5 & 246.9 & 271 & 686.7 & 1345 & 1210.6 & 359.4 & 196.3 & 151.4 & 126 & 135.7 & 143.6 & 140.8 & 554.4 \\
\hline
\end{tabular}




\section{4- 0.000-4M HELMAND RIVER AT MALAKAN, Continued}

Probability of occurrence of annual high discharges

[ $\mathrm{m}^{3} / \mathrm{s}$, meters per second; $\mathrm{ng}$, statistic not given]

\begin{tabular}{|c|c|c|c|c|c|c|}
\hline \multirow{2}{*}{$\begin{array}{c}\text { Exceedance } \\
\text { probability }\end{array}$} & \multirow{2}{*}{$\begin{array}{c}\text { Recurrence } \\
\text { interval } \\
\text { (years) }\end{array}$} & \multirow{2}{*}{$\begin{array}{c}\text { Maximum } \\
\text { instantaneous }{ }^{1} \\
\left(\mathrm{~m}^{3} / \mathrm{s}\right)\end{array}$} & \multicolumn{4}{|c|}{ Maximum mean discharge } \\
\hline & & & 3-day period & 7-day period & 15-day period & 30-day period \\
\hline 0.99 & 1.01 & ng & 51.0 & 44.8 & 38.3 & 33.7 \\
\hline 0.95 & 1.05 & ng & 111 & 94.4 & 83.4 & 72.9 \\
\hline 0.90 & 1.11 & ng & 165 & 138 & 124 & 108 \\
\hline 0.80 & 1.25 & 564 & 258 & 216 & 195 & 170 \\
\hline 0.50 & 2 & 805 & 569 & 486 & 441 & 386 \\
\hline 0.20 & 5 & 1,230 & 1,150 & 1,030 & 923 & 814 \\
\hline 0.10 & 10 & 1,580 & ${ }^{2} 1,600$ & 1,500 & 1,320 & 1,170 \\
\hline 0.04 & 25 & 2,110 & 22,220 & ${ }^{2} 2,190$ & 1,890 & 1,690 \\
\hline 0.02 & 50 & 2,580 & ${ }^{2} 2,720$ & 22,770 & 2,360 & 2,120 \\
\hline 0.01 & 100 & 3,110 & ${ }^{2} 3,220$ & ${ }^{2} 3,400$ & 2,860 & 2,580 \\
\hline 0.005 & 200 & 3,720 & ${ }^{23}, 740$ & ${ }^{2} 4,100$ & 3,380 & 3,070 \\
\hline 0.002 & 500 & 4,670 & ng & ng & ng & ng \\
\hline
\end{tabular}

'Less than 10 years of data used.

${ }^{2}$ Data does not fit log-Pearson Type III curve, use with caution.

\section{4- 0.000-4M HELMAND RIVER AT MALAKAN, Continued}

Probability of occurrence of annual low discharges

$\left[\mathrm{m}^{3} / \mathrm{s}\right.$, meters per second]

\begin{tabular}{|c|c|c|c|c|c|c|c|c|c|c|}
\hline \multirow{3}{*}{$\begin{array}{c}\text { Nonexceedance } \\
\text { probability }\end{array}$} & \multirow{3}{*}{$\begin{array}{l}\text { Recurrence } \\
\text { interval } \\
\text { (years) }\end{array}$} & \multicolumn{9}{|c|}{ Minimum mean discharge $\left(\mathrm{m}^{3} / \mathrm{s}\right)$} \\
\hline & & \multicolumn{9}{|c|}{ Number of consecutive days } \\
\hline & & 1 & 3 & 7 & 14 & 30 & 60 & 90 & 120 & 183 \\
\hline 0.05 & 20 & 8.54 & 8.74 & 9.65 & 10.4 & 12.8 & 13.6 & 14.0 & 14.1 & 18.9 \\
\hline 0.10 & 10 & 12.8 & 13.1 & 14.3 & 15.2 & 18.3 & 19.4 & 20.2 & 20.6 & 26.4 \\
\hline 0.20 & 5 & 20.1 & 20.6 & 22.1 & 23.3 & 27.0 & 28.9 & 30.2 & 31.3 & 38.2 \\
\hline 0.50 & 2 & 42.0 & 43.1 & 45.5 & 47.3 & 51.8 & 55.4 & 58.5 & 61.4 & 69.7 \\
\hline
\end{tabular}




\section{4- 0.000-4M HELMAND RIVER AT MALAKAN, Continued}

\section{Probability of occurrence of seasonal low discharges}

$\left[\mathrm{m}^{3} / \mathrm{s}\right.$, meters per second]

\begin{tabular}{|c|c|c|c|c|c|c|c|c|c|}
\hline \multirow{3}{*}{$\begin{array}{l}\text { Nonexceedance } \\
\text { probability }\end{array}$} & \multirow{3}{*}{$\begin{array}{c}\text { Recurrence } \\
\text { interval } \\
\text { (years) }\end{array}$} & \multicolumn{8}{|c|}{ Minimum mean discharge $\left(\mathrm{m}^{3} / \mathrm{s}\right)$} \\
\hline & & \multicolumn{8}{|c|}{ Number of consecutive days } \\
\hline & & 1 & 7 & 14 & 30 & 1 & 7 & 14 & 30 \\
\hline & & \multicolumn{4}{|c|}{ December-January-February } & \multicolumn{4}{|c|}{ March-April-May } \\
\hline 0.05 & 20 & 21.1 & 26.2 & 29.8 & 31.0 & 18.4 & 20.8 & 22.9 & 28.4 \\
\hline 0.10 & 10 & 27.5 & 33.4 & 37.4 & 40.2 & 30.3 & 33.4 & 36.3 & 39.9 \\
\hline 0.20 & 5 & 37.0 & 43.4 & 47.8 & 52.9 & 49.0 & 52.7 & 57.1 & 59.9 \\
\hline \multirow[t]{2}{*}{0.50} & 2 & 59.5 & 66.3 & 70.5 & 80.1 & 87.2 & 91.5 & 102 & 128 \\
\hline & & \multicolumn{4}{|c|}{ June-July-August } & \multicolumn{4}{|c|}{ September-October-November } \\
\hline 0.05 & 20 & 13.6 & 14.2 & 14.2 & 15.0 & 10.1 & 10.9 & 11.2 & 12.5 \\
\hline 0.10 & 10 & 18.6 & 19.3 & 19.5 & 20.7 & 15.1 & 16.2 & 16.8 & 18.4 \\
\hline 0.20 & 5 & 26.8 & 27.7 & 28.0 & 30.0 & 23.4 & 25.0 & 26.2 & 28.1 \\
\hline 0.50 & 2 & 51.2 & 52.4 & 53.5 & 57.9 & 48.3 & 51.1 & 54.0 & 56.8 \\
\hline
\end{tabular}

\section{4- 0.000-4M HELMAND RIVER AT MALAKAN, Continued}

Annual peak discharge and corresponding gage height

[m³/s, meters per second; --, no data]

\begin{tabular}{|c|c|c|c|c|c|c|c|}
\hline $\begin{array}{c}\text { Water } \\
\text { year }\end{array}$ & Date & $\begin{array}{c}\text { Gage } \\
\text { height } \\
\text { (meters) }\end{array}$ & $\begin{array}{c}\text { Peak } \\
\text { discharge } \\
\left(\mathrm{m}^{3} / \mathrm{s}\right)\end{array}$ & $\begin{array}{c}\text { Water } \\
\text { year }\end{array}$ & Date & $\begin{array}{c}\text { Gage } \\
\text { height } \\
\text { (meters) }\end{array}$ & $\begin{array}{c}\text { Peak } \\
\text { discharge } \\
\left(\mathrm{m}^{3} / \mathrm{s}\right)\end{array}$ \\
\hline \multicolumn{8}{|c|}{ Annual peak discharge, by year } \\
\hline 1970 & 30-Jan & - & 982 & 1975 & 21-May & & 898 \\
\hline 1971 & 8-Jan & - & 105 & 1976 & 29-Apr & & 2,143 \\
\hline 1972 & 1-May & - & 1,166 & 1977 & 13-Jan & & 900 \\
\hline 1973 & 8-May & - & 481 & 1978 & 27-Dec & & 778 \\
\hline 1974 & 21-Jan & - & 620 & & & & \\
\hline \multicolumn{8}{|c|}{ Annual peak discharge, from highest to lowest } \\
\hline 1976 & 29-Apr & - & 2,143 & 1978 & 27-Dec & & 778 \\
\hline 1972 & 1-May & - & 1,166 & 1974 & 21-Jan & & 620 \\
\hline 1970 & 30-Jan & - & 982 & 1973 & 8-May & & 481 \\
\hline 1977 & 13-Jan & - & 900 & 1971 & 8-Jan & & 105 \\
\hline 1975 & 21-May & - & 898 & & & & \\
\hline
\end{tabular}




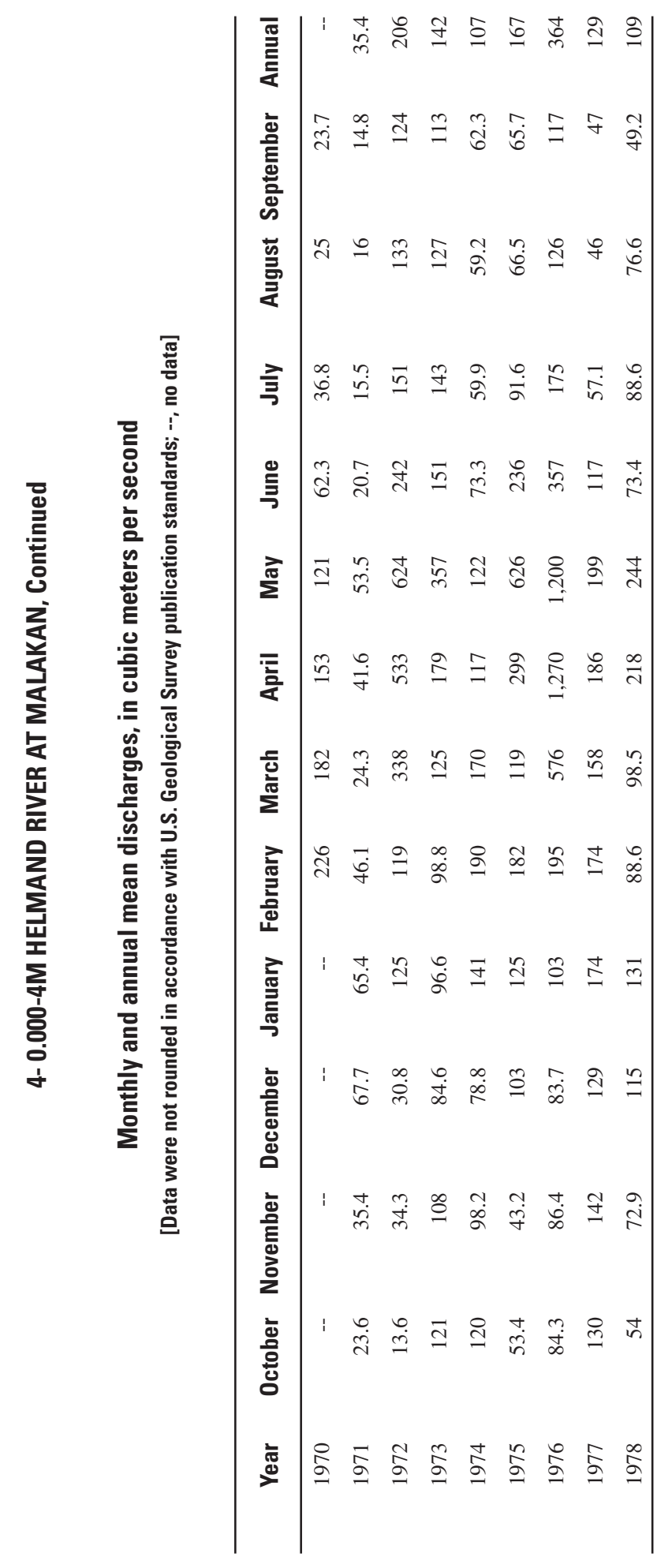




\section{4- 0.000-5M HELMAND RIVER AT DARWESHAN}

\section{(U.S. Geological Survey identification number: 310800064110000)}

LOCATION: Lat $31^{\circ} 08^{\prime}$ N., long $64^{\circ} 11^{\prime}$ E., at road bridge in Darweshan, $55 \mathrm{~km}$ downstream from Arghandab River, and $180 \mathrm{~km}$ downstream from Kajakai Dam.

DRAINAGE AREA: $118,335 \mathrm{~km}^{2}$, of which approximately $4,400 \mathrm{~km}^{2}$ is considered noncontributing.

ALTITUDE: 713 meters plus mean sea level.

PERIOD OF RECORD: October 1, 1956 to April 7, 1980.

GAGE: Water-stage recorder.

Annual mean discharge

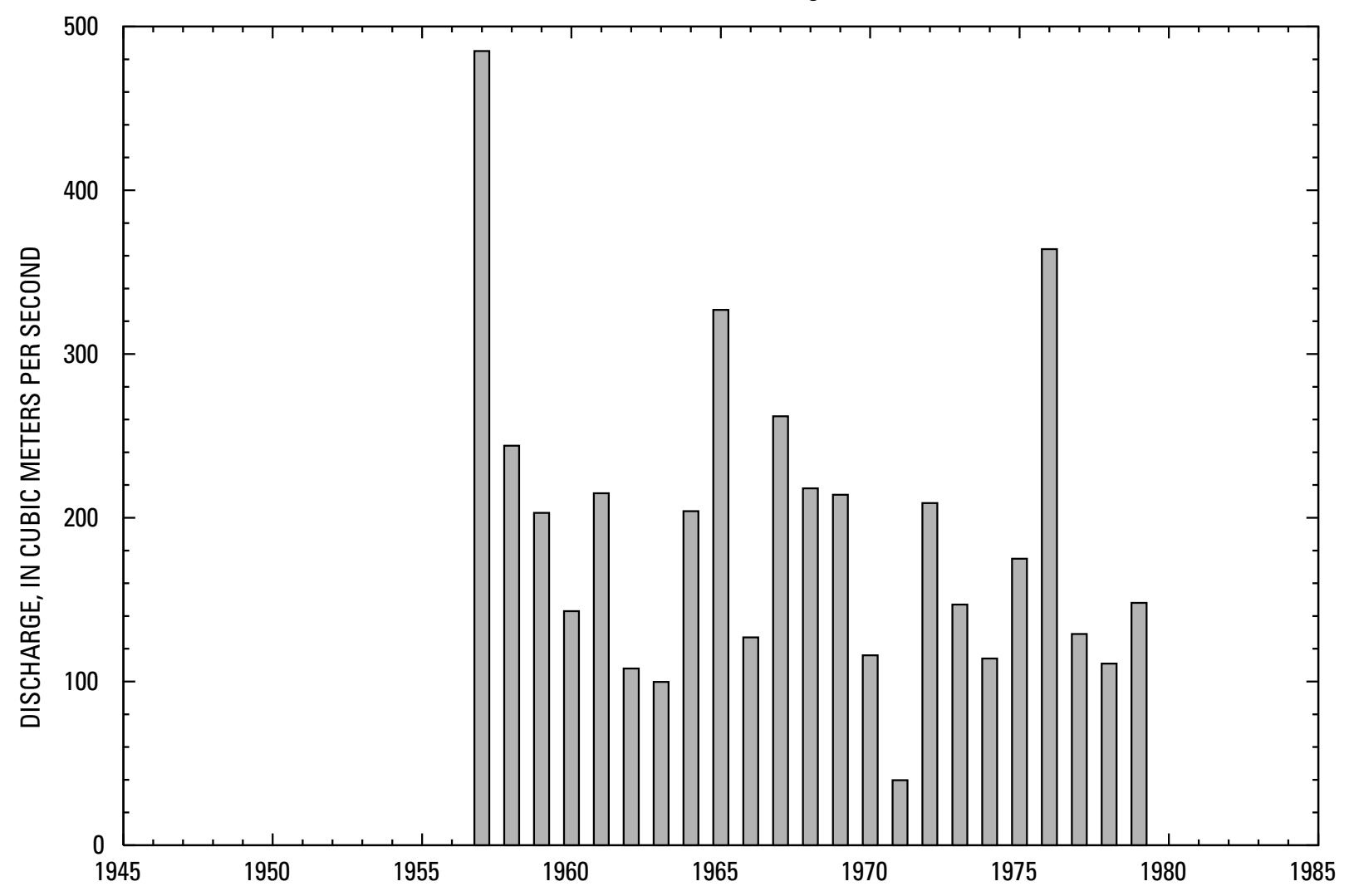




\section{4- 0.000-5M HELMAND RIVER AT DARWESHAN, Continued}

\section{Statistics of monthly and annual mean discharges}

$\left[\mathrm{m}^{3} / \mathrm{s}\right.$, meters per second]

\begin{tabular}{|c|c|c|c|c|c|c|c|c|}
\hline \multirow[b]{2}{*}{ Month } & \multicolumn{2}{|c|}{ Maximum } & \multicolumn{2}{|c|}{ Minimum } & \multicolumn{4}{|c|}{ Mean } \\
\hline & $\begin{array}{c}\text { Discharge } \\
\left(\mathrm{m}^{3} / \mathrm{s}\right)\end{array}$ & $\begin{array}{c}\text { Water year } \\
\text { of } \\
\text { occurrence }\end{array}$ & $\begin{array}{c}\text { Discharge } \\
\left(\mathrm{m}^{3} / \mathrm{s}\right)\end{array}$ & $\begin{array}{c}\text { Water year } \\
\text { of } \\
\text { occurrence }\end{array}$ & $\begin{array}{c}\text { Discharge } \\
\left(\mathrm{m}^{3} / \mathrm{s}\right)\end{array}$ & $\begin{array}{c}\text { Standard } \\
\text { deviation } \\
\left(\mathrm{m}^{3} / \mathrm{s}\right)\end{array}$ & $\begin{array}{c}\text { Coefficient } \\
\text { of } \\
\text { variation }\end{array}$ & $\begin{array}{c}\text { Percentage } \\
\text { of annual } \\
\text { discharge }\end{array}$ \\
\hline October & 192 & 1958 & 15.7 & 1972 & 82.1 & 41.4 & 0.50 & 3.54 \\
\hline November & 178 & 1961 & 34.4 & 1972 & 93.5 & 38.9 & 0.42 & 4.03 \\
\hline December & 350 & 1958 & 30.8 & 1972 & 128 & 67.0 & 0.52 & 5.53 \\
\hline January & 312 & 1957 & 70.2 & 1971 & 161 & 62.6 & 0.39 & 6.97 \\
\hline February & 311 & 1957 & 49.8 & 1971 & 178 & 64.2 & 0.36 & 7.68 \\
\hline March & 662 & 1980 & 30.8 & 1971 & 232 & 168 & 0.72 & 10.0 \\
\hline April & 1,590 & 1957 & 47.8 & 1971 & 436 & 391 & 0.90 & 18.8 \\
\hline May & 1,450 & 1957 & 51.4 & 1971 & 507 & 399 & 0.79 & 21.9 \\
\hline June & 616 & 1957 & 22.1 & 1971 & 212 & 147 & 0.69 & 9.17 \\
\hline July & 235 & 1957 & 17.8 & 1971 & 115 & 57.4 & 0.50 & 4.96 \\
\hline August & 169 & 1958 & 20.5 & 1971 & 90.3 & 44.5 & 0.49 & 3.90 \\
\hline September & 190 & 1957 & 17.5 & 1971 & 81.1 & 44.0 & 0.54 & 3.50 \\
\hline Annual & 485 & 1957 & 39.8 & 1971 & 191 & 99.0 & 0.52 & 100 \\
\hline
\end{tabular}

Annual flow duration

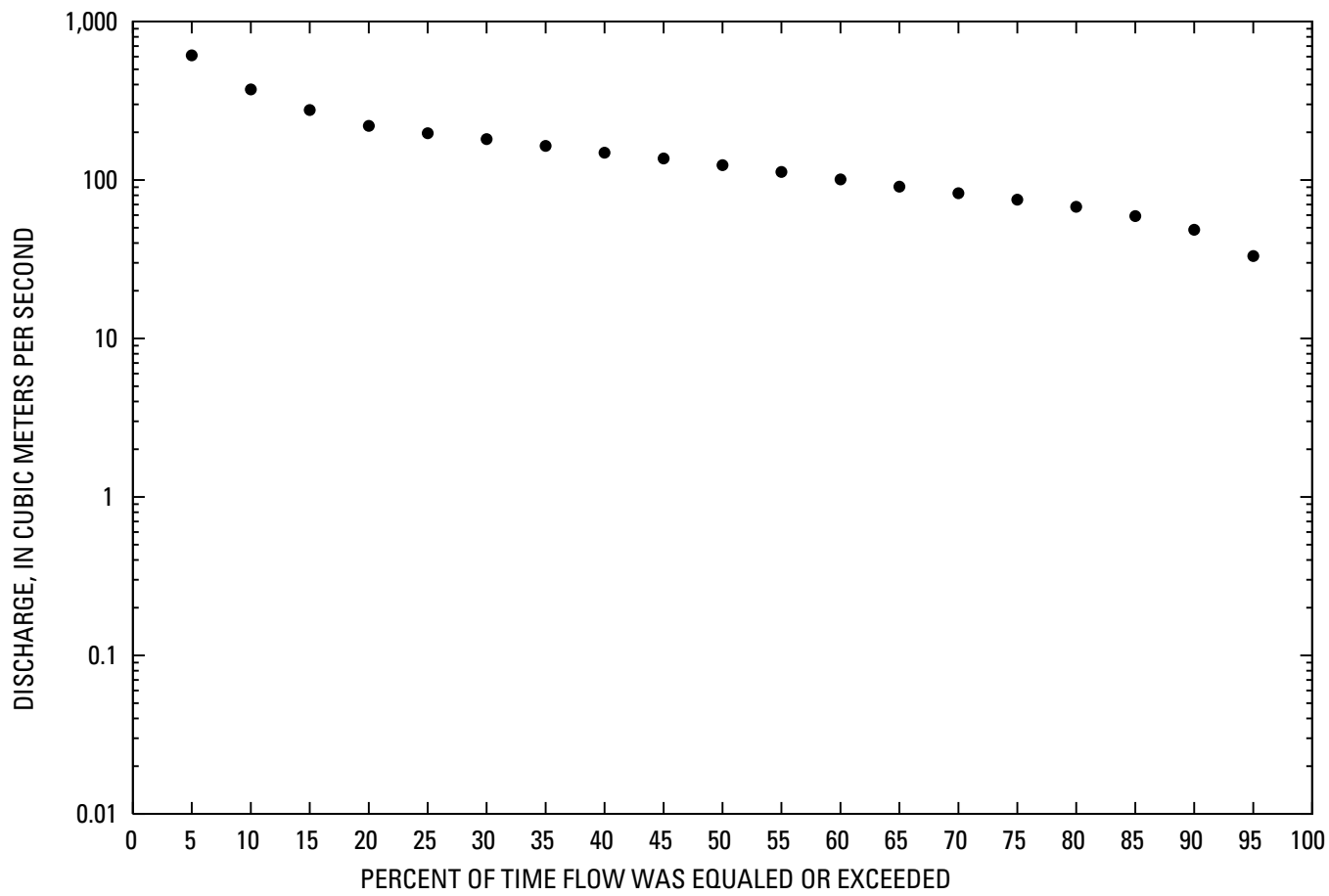




\section{4- 0.000-5M HELMAND RIVER AT DARWESHAN, Continued}

Monthly and annual flow duration, in cubic meters per second

[Data were not rounded in accordance with U.S. Geological Survey publication standards]

\begin{tabular}{|c|c|c|c|c|c|c|c|c|c|c|c|c|c|}
\hline $\begin{array}{l}\text { Percentage } \\
\text { of days } \\
\text { discharge } \\
\text { equaled or } \\
\text { exceeded }\end{array}$ & January & February & March & April & May & June & July & August & September & October & November & December & Annual \\
\hline 95 & 68 & 64.3 & 58.6 & 67.7 & 68.4 & 47.7 & 29.5 & 22.7 & 21.2 & 24.3 & 33.2 & 56.2 & 33.1 \\
\hline 90 & 79 & 77.2 & 84.7 & 81.9 & 95.4 & 58.8 & 44.7 & 32.3 & 28.8 & 28.3 & 45 & 64 & 48.5 \\
\hline 80 & 97.7 & 96.6 & 106.9 & 124.8 & 156.1 & 88.3 & 54.7 & 42.9 & 40.3 & 46.1 & 56.4 & 74.8 & 67.7 \\
\hline 75 & 103.7 & 110 & 116.4 & 143.9 & 173.2 & 104.2 & 59.5 & 50.4 & 46.9 & 51.2 & 66.5 & 78.2 & 75.1 \\
\hline 70 & 107.8 & 126.8 & 126.5 & 159.3 & 207.2 & 115.7 & 66.5 & 56.8 & 52.2 & 55.9 & 69.9 & 81.6 & 82.6 \\
\hline 65 & 111.4 & 138.4 & 136.1 & 175 & 266 & 132.6 & 78.5 & 61.7 & 57.7 & 60.9 & 73.9 & 84.9 & 90.9 \\
\hline 60 & 115 & 148.2 & 144.3 & 199.7 & 311.3 & 147.3 & 86.5 & 67.7 & 62.5 & 67 & 77 & 88.4 & 100.9 \\
\hline 45 & 144.1 & 176.9 & 178.4 & 296.1 & 443.6 & 184.6 & 114.7 & 87.5 & 73.1 & 79.2 & 85.1 & 111.9 & 136.5 \\
\hline 40 & 180.2 & 185.3 & 190.6 & 338 & 485.8 & 198.8 & 137.1 & 92.8 & 80.5 & 84.4 & 91.5 & 120.2 & 148.8 \\
\hline 35 & 192.3 & 193.7 & 202.3 & 374.3 & 529.2 & 213.6 & 146.7 & 120.8 & 90.1 & 90.2 & 101.5 & 127.9 & 164.4 \\
\hline 30 & 198.5 & 205.4 & 218.6 & 439.7 & 589 & 237.9 & 153 & 131 & 102.2 & 96 & 109.3 & 149.1 & 181.3 \\
\hline 25 & 204.7 & 217.3 & 248.2 & 537.4 & 668.6 & 269.7 & 160.1 & 136.3 & 119.1 & 102.1 & 116.3 & 168.6 & 197.2 \\
\hline 20 & 214.2 & 234.9 & 287.8 & 634.1 & 783.2 & 308.5 & 169.8 & 140.6 & 128.2 & 117.6 & 129.2 & 184.6 & 219.4 \\
\hline 15 & 227.6 & 257 & 318.4 & 736.2 & 952.4 & 353.9 & 180 & 144.8 & 134.4 & 129.4 & 139.9 & 198.2 & 276.2 \\
\hline 10 & 260.9 & 289.5 & 446.4 & 1152.2 & 1160.9 & 422.6 & 190.9 & 156.1 & 142 & 144.5 & 173 & 211.2 & 372.8 \\
\hline 5 & 292.1 & 331.6 & 681.5 & 1615.5 & 1383.5 & 556.5 & 214.6 & 172.3 & 176.8 & 182.5 & 185.5 & 257.2 & 612.6 \\
\hline
\end{tabular}




\section{4- 0.000-5M HELMAND RIVER AT DARWESHAN, Continued}

\section{Probability of occurrence of annual high discharges}

[ $\mathrm{m}^{3} / \mathrm{s}$, meters per second; $\mathbf{n g}$, statistic not given]

\begin{tabular}{|c|c|c|c|c|c|c|}
\hline \multirow{2}{*}{$\begin{array}{c}\text { Exceedance } \\
\text { probability }\end{array}$} & \multirow{2}{*}{$\begin{array}{c}\text { Recurrence } \\
\text { interval } \\
\text { (years) }\end{array}$} & \multirow{2}{*}{$\begin{array}{c}\text { Maximum } \\
\text { instantaneous } \\
\left(\mathrm{m}^{3} / \mathrm{s}\right)\end{array}$} & \multicolumn{4}{|c|}{ Maximum mean discharge } \\
\hline & & & 3-day period & 7-day period & 15-day period & 30-day period \\
\hline 0.99 & 1.01 & ng & 87.3 & 78.6 & 71.3 & 69.5 \\
\hline 0.95 & 1.05 & 329 & 180 & 156 & 140 & 127 \\
\hline 0.90 & 1.11 & 456 & 258 & 220 & 196 & 174 \\
\hline 0.80 & 1.25 & 657 & 387 & 328 & 290 & 251 \\
\hline 0.50 & 2 & 1,220 & 777 & 661 & 581 & 491 \\
\hline 0.20 & 5 & 2,030 & 1,410 & 1,230 & 1,080 & 921 \\
\hline 0.10 & 10 & 2,560 & 1,860 & 1,660 & 1,460 & 1,260 \\
\hline 0.04 & 25 & 3,180 & 2,420 & 2,240 & 1,970 & 1,740 \\
\hline 0.02 & 50 & 3,600 & 2,840 & 2,680 & 2,370 & 2,120 \\
\hline 0.01 & 100 & 4,000 & 3,240 & 3,130 & 2,780 & 2,530 \\
\hline 0.005 & 200 & 4,370 & 3,630 & 3,580 & 3,190 & 2,970 \\
\hline 0.002 & 500 & 4,820 & ng & ng & ng & ng \\
\hline
\end{tabular}

\section{4- 0.000-5M HELMAND RIVER AT DARWESHAN, Continued}

Probability of occurrence of annual low discharges

[m³/s, meters per second]

\begin{tabular}{|c|c|c|c|c|c|c|c|c|c|c|}
\hline \multirow{3}{*}{$\begin{array}{c}\text { Nonexceedance } \\
\text { probability }\end{array}$} & \multirow{3}{*}{$\begin{array}{c}\text { Recurrence } \\
\text { interval } \\
\text { (years) }\end{array}$} & \multicolumn{9}{|c|}{ Minimum mean discharge $\left(\mathrm{m}^{3} / \mathrm{s}\right)$} \\
\hline & & \multicolumn{9}{|c|}{ Number of consecutive days } \\
\hline & & 1 & 3 & 7 & 14 & 30 & 60 & 90 & 120 & 183 \\
\hline 0.05 & 20 & 14.5 & 15.6 & 17.4 & 19.0 & 21.8 & 23.6 & 25.5 & 26.7 & 33.3 \\
\hline 0.10 & 10 & 19.2 & 20.5 & 22.4 & 24.9 & 28.0 & 30.7 & 33.1 & 35.2 & 43.1 \\
\hline 0.20 & 5 & 26.2 & 27.7 & 29.8 & 33.1 & 37.1 & 40.9 & 44.0 & 47.5 & 57.1 \\
\hline 0.50 & 2 & 43.4 & 45.3 & 47.3 & 52.1 & 58.5 & 65.4 & 70.1 & 76.3 & 89.9 \\
\hline
\end{tabular}




\section{4- 0.000-5M HELMAND RIVER AT DARWESHAN, Continued}

\section{Probability of occurrence of seasonal low discharges}

[ $\mathrm{m}^{3} / \mathrm{s}$, meters per second]

\begin{tabular}{|c|c|c|c|c|c|c|c|c|c|}
\hline \multirow{3}{*}{$\begin{array}{c}\text { Nonexceedance } \\
\text { probability }\end{array}$} & \multirow{3}{*}{$\begin{array}{c}\text { Recurrence } \\
\text { interval } \\
\text { (years) }\end{array}$} & \multicolumn{8}{|c|}{ Minimum mean discharge $\left(\mathrm{m}^{3} / \mathrm{s}\right)$} \\
\hline & & \multicolumn{8}{|c|}{ Number of consecutive days } \\
\hline & & 1 & 7 & 14 & 30 & 1 & 7 & 14 & 30 \\
\hline & & \multicolumn{4}{|c|}{ December-January-February } & \multicolumn{4}{|c|}{ March-April-May } \\
\hline 0.05 & 20 & 32.2 & 36.7 & 40.4 & 45.7 & 28.9 & 34.9 & 38.5 & 51.8 \\
\hline 0.10 & 10 & 40.4 & 44.5 & 48.1 & 55.3 & 39.8 & 46.4 & 51.5 & 65.3 \\
\hline 0.20 & 5 & 52.2 & 55.9 & 59.5 & 69.0 & 56.5 & 64.0 & 71.6 & 86.7 \\
\hline \multirow[t]{2}{*}{0.50} & 2 & 80.4 & 84.2 & 88.9 & 103 & 98.6 & 110 & 124 & 151 \\
\hline & & \multicolumn{4}{|c|}{ June-July-August } & \multicolumn{4}{|c|}{ September-October-November } \\
\hline 0.05 & 20 & 16.6 & 20.8 & 22.4 & 24.6 & 17.1 & 18.1 & 19.8 & 21.9 \\
\hline 0.10 & 10 & 22.7 & 27.8 & 30.3 & 33.0 & 22.2 & 23.3 & 25.8 & 28.5 \\
\hline 0.20 & 5 & 32.4 & 38.6 & 42.4 & 45.9 & 29.6 & 30.9 & 34.5 & 38.3 \\
\hline 0.50 & 2 & 59.7 & 68.5 & 74.9 & 80.0 & 48.3 & 50.5 & 55.5 & 62.7 \\
\hline
\end{tabular}




\section{4- 0.000-5M HELMAND RIVER AT DARWESHAN, Continued}

Annual peak discharge and corresponding gage height

$\left[\mathrm{m}^{3} / \mathrm{s}\right.$, meters per second; --, no data]

\begin{tabular}{|c|c|c|c|c|c|c|c|}
\hline $\begin{array}{c}\text { Water } \\
\text { year }\end{array}$ & Date & $\begin{array}{c}\text { Gage } \\
\text { height } \\
\text { (meters) }\end{array}$ & $\begin{array}{c}\text { Peak } \\
\text { discharge } \\
\left(\mathrm{m}^{3} / \mathrm{s}\right)\end{array}$ & $\begin{array}{c}\text { Water } \\
\text { year }\end{array}$ & Date & $\begin{array}{c}\text { Gage } \\
\text { height } \\
\text { (meters) }\end{array}$ & $\begin{array}{c}\text { Peak } \\
\text { discharge } \\
\left(\mathrm{m}^{3} / \mathrm{s}\right)\end{array}$ \\
\hline \multicolumn{8}{|c|}{ Annual peak discharge, by year } \\
\hline 1957 & 18-Mar & 709.80 & 3,764 & 1969 & 18-Apr & -- & 1,364 \\
\hline 1958 & 13-Dec & 707.44 & 1,319 & 1970 & 29-Jan & -- & 1,444 \\
\hline 1959 & 3-Mar & 707.49 & 1,354 & 1971 & 6-Jan & -- & 107 \\
\hline 1960 & 19-May & 706.34 & 680 & 1972 & 21-Jan & -- & 1,418 \\
\hline 1961 & 11-Apr & -- & 2,717 & 1973 & 6-May & -- & 478 \\
\hline 1962 & 3-Mar & -- & 260 & 1974 & 20-Jan & -- & 800 \\
\hline 1963 & 14-May & -- & 710 & 1975 & 18-May & -- & 945 \\
\hline 1964 & 19-Feb & 707.44 & 1,237 & 1976 & 24-Mar & -- & 2,235 \\
\hline 1965 & 24-Apr & 709.16 & 2,122 & 1977 & 11-Jan & -- & 1,451 \\
\hline 1966 & 23-Dec & -- & 322 & 1978 & 26-Dec & -- & 985 \\
\hline 1967 & 1-May & -- & 2,334 & 1979 & 14-Feb & -- & 1,400 \\
\hline 1968 & 3-May & -- & 955 & 1980 & 22-Mar & -- & 2,263 \\
\hline \multicolumn{8}{|c|}{ Annual peak discharge, from highest to lowest } \\
\hline 1957 & 18-Mar & 709.80 & 3,764 & 1958 & 13-Dec & 707.44 & 1,319 \\
\hline 1961 & 11-Apr & -- & 2,717 & 1964 & 19-Feb & 707.44 & 1,237 \\
\hline 1967 & 1-May & -- & 2,334 & 1978 & 26-Dec & -- & 985 \\
\hline 1980 & 22-Mar & -- & 2,263 & 1968 & 3-May & -- & 955 \\
\hline 1976 & 24-Mar & -- & 2,235 & 1975 & 18-May & -- & 945 \\
\hline 1965 & 24-Apr & 709.16 & 2,122 & 1974 & 20-Jan & -- & 800 \\
\hline 1977 & 11-Jan & -- & 1,451 & 1963 & 14-May & -- & 710 \\
\hline 1970 & 29-Jan & -- & 1,444 & 1960 & 19-May & 706.34 & 680 \\
\hline 1972 & 21-Jan & -- & 1,418 & 1973 & 6-May & -- & 478 \\
\hline 1979 & $14-F e b$ & -- & 1,400 & 1966 & 23-Dec & -- & 322 \\
\hline 1969 & 18-Apr & -- & 1,364 & 1962 & 3-Mar & -- & 260 \\
\hline 1959 & 3-Mar & 707.49 & 1,354 & 1971 & 6-Jan & -- & 107 \\
\hline
\end{tabular}




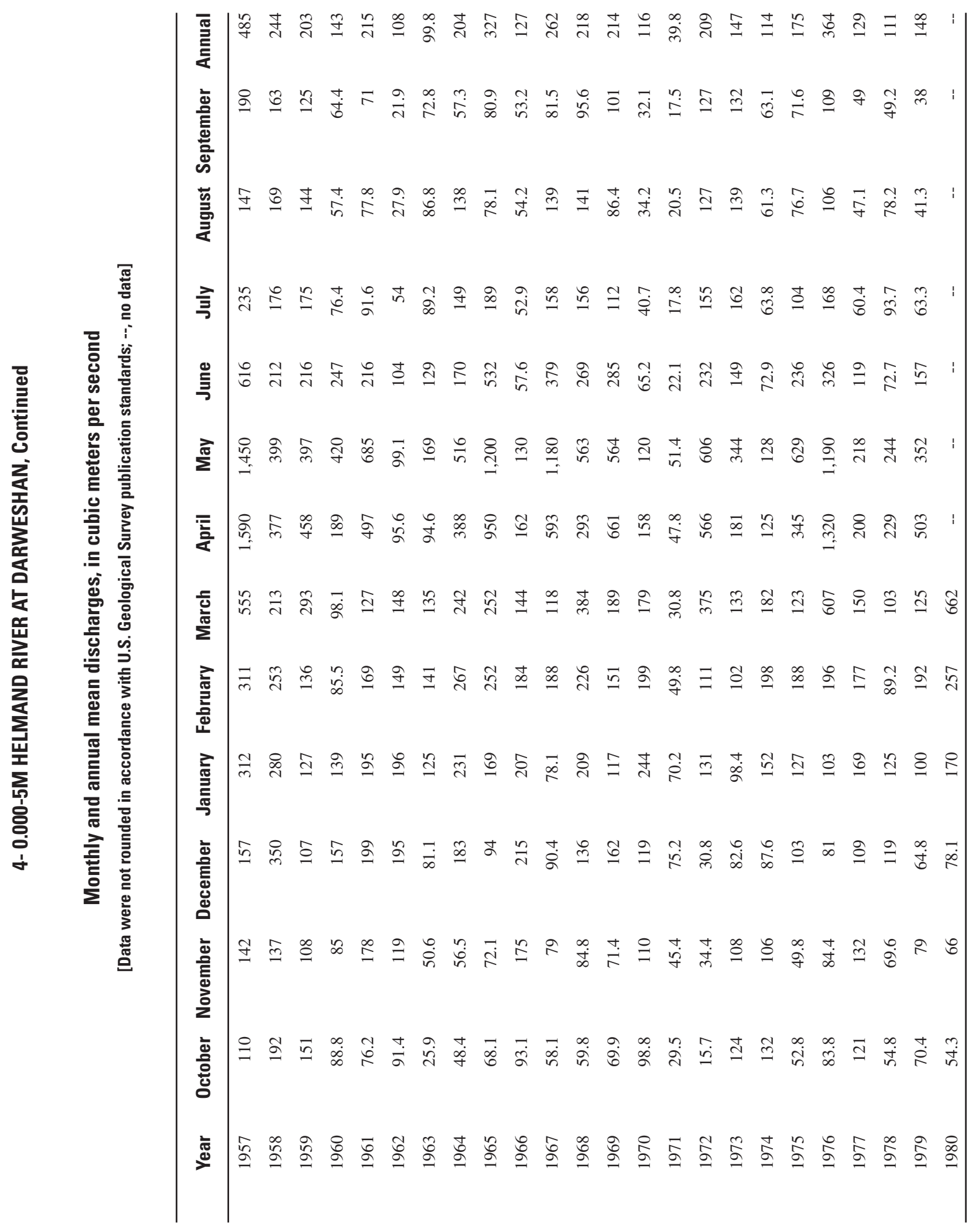




\section{4- 0.000-6M HELMAND RIVER AT LASHKARGAH}

\section{(U.S. Geological Survey identification number: 313400064210000)}

LOCATION: Lat $31^{\circ} 34^{\prime} N$., long 64² $21^{\prime} E$., on bridge pier at Lashkargah, 8 km upstream from Arghandab River, and $117 \mathrm{~km}$ downstream from Kajakai Dam.

DRAINAGE AREA: $61,720 \mathrm{~km}^{2}$.

ALTITUDE: 775 meters plus mean sea level.

PERIOD OF RECORD: October 1, 1953 to September 30, 1980.

GAGE: Staff gage read once or twice daily.

Annual mean discharge

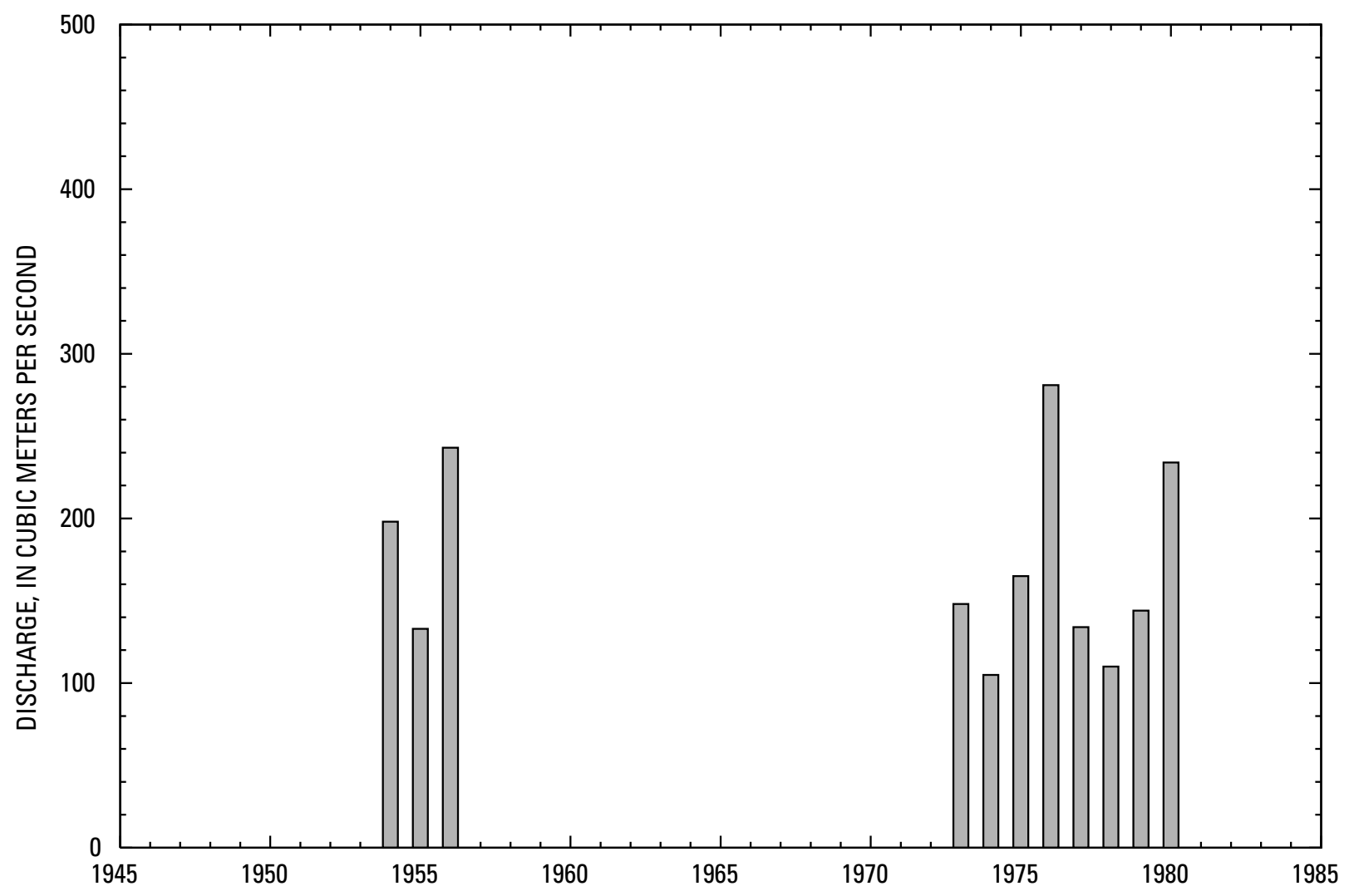




\section{4- 0.000-6M HELMAND RIVER AT LASHKARGAH, Continued}

\section{Statistics of monthly and annual mean discharges}

$\left[\mathrm{m}^{3} / \mathrm{s}\right.$, meters per second]

\begin{tabular}{|c|c|c|c|c|c|c|c|c|}
\hline \multirow[b]{2}{*}{ Month } & \multicolumn{2}{|c|}{ Maximum } & \multicolumn{2}{|c|}{ Minimum } & \multicolumn{4}{|c|}{ Mean } \\
\hline & $\begin{array}{c}\text { Discharge } \\
\left(\mathrm{m}^{3} / \mathrm{s}\right)\end{array}$ & $\begin{array}{c}\text { Water year } \\
\text { of } \\
\text { occurrence }\end{array}$ & $\begin{array}{c}\text { Discharge } \\
\left(\mathrm{m}^{3} / \mathrm{s}\right)\end{array}$ & $\begin{array}{c}\text { Water year } \\
\text { of } \\
\text { occurrence }\end{array}$ & $\begin{array}{c}\text { Discharge } \\
\left(\mathrm{m}^{3} / \mathrm{s}\right)\end{array}$ & $\begin{array}{c}\text { Standard } \\
\text { deviation } \\
\left(\mathrm{m}^{3} / \mathrm{s}\right)\end{array}$ & $\begin{array}{c}\text { Coefficient } \\
\text { of } \\
\text { variation }\end{array}$ & $\begin{array}{c}\text { Percentage } \\
\text { of annual } \\
\text { discharge }\end{array}$ \\
\hline October & 141 & 1977 & 57.9 & 1980 & 96.4 & 27.4 & 0.28 & 4.32 \\
\hline November & 163 & 1957 & 62.5 & 1975 & 99.4 & 30.2 & 0.30 & 4.46 \\
\hline December & 174 & 1957 & 68.1 & 1954 & 105 & 32.4 & 0.31 & 4.71 \\
\hline January & 287 & 1957 & 90.2 & 1979 & 136 & 56.3 & 0.41 & 6.09 \\
\hline February & 271 & 1957 & 100 & 1978 & 162 & 54.3 & 0.34 & 7.27 \\
\hline March & 432 & 1957 & 86.3 & 1975 & 201 & 111 & 0.55 & 9.04 \\
\hline April & 1,080 & 1957 & 92.3 & 1974 & 472 & 356 & 0.75 & 21.2 \\
\hline May & 984 & 1957 & 143 & 1974 & 475 & 284 & 0.60 & 21.3 \\
\hline June & 276 & 1976 & 66.0 & 1978 & 175 & 65.0 & 0.37 & 7.86 \\
\hline July & 185 & 1956 & 58.8 & 1978 & 111 & 42.1 & 0.38 & 4.99 \\
\hline August & 210 & 1956 & 54.1 & 1979 & 101 & 44.3 & 0.44 & 4.55 \\
\hline September & 162 & 1956 & 43.5 & 1979 & 94.4 & 33.6 & 0.36 & 4.23 \\
\hline Annual & 281 & 1976 & 105 & 1974 & 172 & 58.4 & 0.34 & 100 \\
\hline
\end{tabular}

Annual flow duration

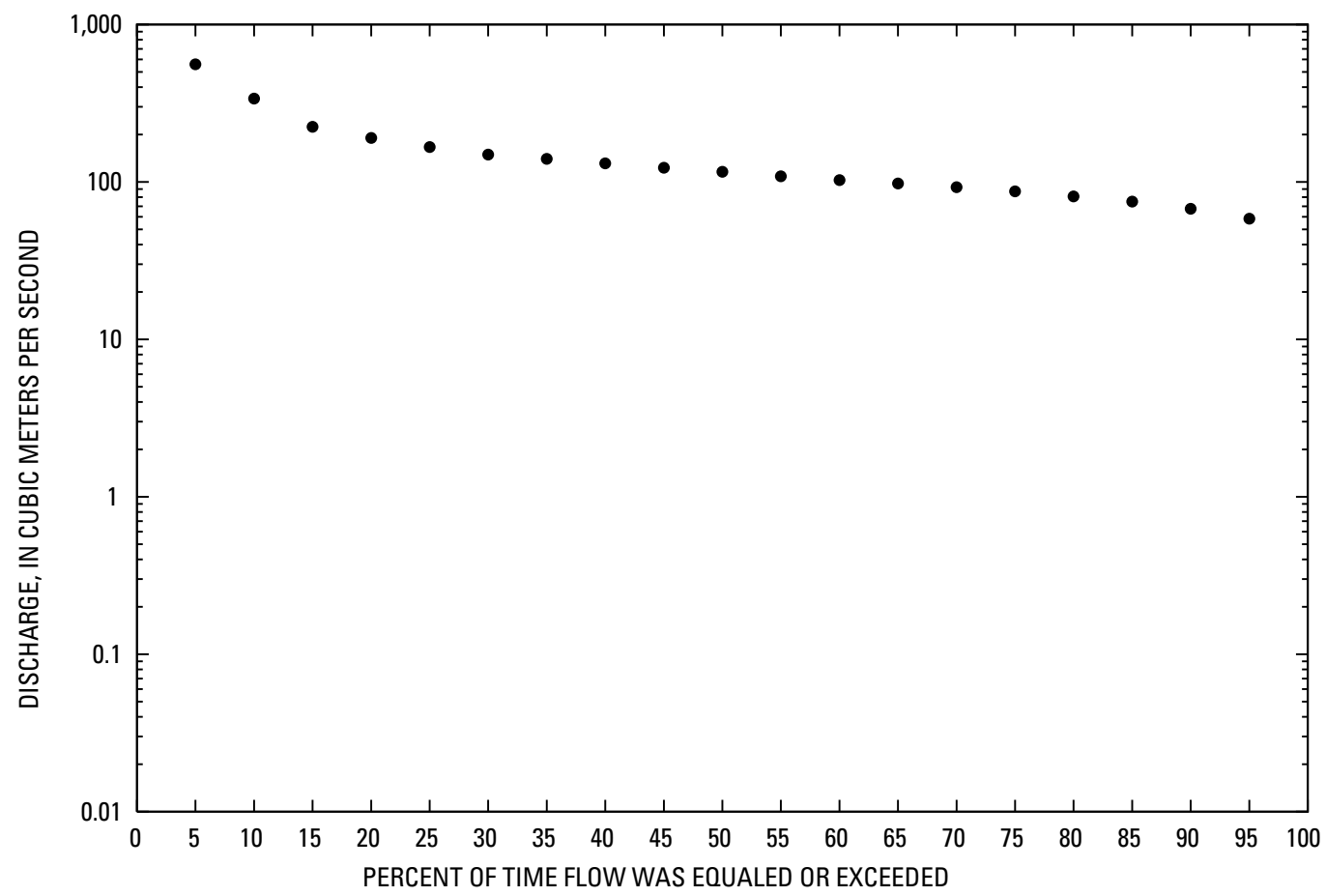




\section{4- 0.000-6M HELMAND RIVER AT LASHKARGAH, Continued}

Monthly and annual flow duration, in cubic meters per second

[Data were not rounded in accordance with U.S. Geological Survey publication standards]

\begin{tabular}{|c|c|c|c|c|c|c|c|c|c|c|c|c|c|}
\hline $\begin{array}{l}\text { Percentage } \\
\text { of days } \\
\text { discharge } \\
\text { equaled or } \\
\text { exceeded }\end{array}$ & January & February & March & April & May & June & July & August & September & October & November & December & Annual \\
\hline 95 & 71.7 & 87.2 & 77.5 & 90.1 & 141.2 & 64.3 & 58.4 & 52 & 42.6 & 49.4 & 58.5 & 54.5 & 58.5 \\
\hline 90 & 83.1 & 100.1 & 84.9 & 101.3 & 148.7 & 79.4 & 62 & 60.9 & 48.5 & 57.2 & 62.6 & 62 & 67.5 \\
\hline 80 & 95.8 & 109.4 & 106.7 & 127.4 & 196.4 & 108 & 73.6 & 66.9 & 65.2 & 62 & 67.5 & 72.3 & 81 \\
\hline 75 & 98 & 115 & 114 & 133.9 & 213.6 & 121.8 & 75.8 & 70.7 & 69.1 & 66.7 & 73.7 & 79.9 & 87.1 \\
\hline 70 & 100.1 & 120.9 & 119.8 & 140.8 & 260.1 & 135.2 & 78.1 & 74.3 & 74.4 & 73.5 & 85.3 & 83.7 & 92.6 \\
\hline 65 & 102.2 & 126.1 & 125.1 & 159.3 & 304.1 & 140.6 & 80.8 & 76.6 & 76.2 & 81.1 & 88.3 & 87.4 & 97.8 \\
\hline 45 & 121.8 & 149.9 & 153 & 364.5 & 462.3 & 164.9 & 96.5 & 93.5 & 96.4 & 100.1 & 98.5 & 99.8 & 123.3 \\
\hline 40 & 130.6 & 156.8 & 165.2 & 448.4 & 491.2 & 172.6 & 104.3 & 97.9 & 99.4 & 102.7 & 100.8 & 103.9 & 131.2 \\
\hline 35 & 137.8 & 165.7 & 179.9 & 525.8 & 537 & 181.7 & 119.5 & 117.3 & 103.4 & 105.3 & 104.7 & 108.2 & 140.2 \\
\hline 30 & 144.3 & 176.1 & 190.2 & 661.5 & 588.2 & 194.5 & 128.6 & 121 & 111 & 107.9 & 110.2 & 112.5 & 149.2 \\
\hline 25 & 152.2 & 185.9 & 214.1 & 808.5 & 650.5 & 207.6 & 134.4 & 124.6 & 118.1 & 123.5 & 116.1 & 130.5 & 166.7 \\
\hline 20 & 173.8 & 196.5 & 253.4 & 903.9 & 708.9 & 228.3 & 140.2 & 128 & 120.8 & 130 & 122.3 & 139 & 190.2 \\
\hline 15 & 202.9 & 214.2 & 284.1 & 983.8 & 775.9 & 266.2 & 148.9 & 133 & 123.6 & 136.8 & 141.5 & 147.6 & 223.8 \\
\hline 10 & 219.4 & 267.3 & 418.2 & 1090 & 913.2 & 302.8 & 157.9 & 142.7 & 128.1 & 144.2 & 150.5 & 158.2 & 338.5 \\
\hline 5 & 245.2 & 296.9 & 557.8 & 1200 & 1125.5 & 348.5 & 211.7 & 199.3 & 187.6 & 157.4 & 160.2 & 170.7 & 559.6 \\
\hline
\end{tabular}




\section{4- 0.000-6M HELMAND RIVER AT LASHKARGAH, Continued}

\section{Probability of occurrence of annual high discharges}

[ $\mathrm{m}^{3} / \mathrm{s}$, meters per second; ng, statistic not given]

\begin{tabular}{rrrrrrr}
\hline & & & & \multicolumn{5}{c}{ Maximum mean discharge } \\
\cline { 6 - 7 } $\begin{array}{c}\text { Exceedance } \\
\text { probability }\end{array}$ & $\begin{array}{c}\text { Recurrence } \\
\text { interval } \\
\text { (years) }\end{array}$ & $\begin{array}{c}\text { Maximum } \\
\text { instantaneous } \\
\left(\mathbf{m}^{3} / \mathbf{s}\right)\end{array}$ & 3-day period & 7-day period & 15-day period & 30-day period \\
\cline { 5 - 7 } & 1.01 & 261 & 55.1 & 156.6 & 52.8 & 48.3 \\
0.99 & 1.05 & 387 & 124 & 120 & 107 & 94.7 \\
0.95 & 1.11 & 472 & 184 & 173 & 153 & 133 \\
0.80 & 1.25 & 596 & 284 & 261 & 229 & 198 \\
0.50 & 2 & 903 & 584 & 528 & 463 & 400 \\
0.20 & 5 & 1,320 & 1,040 & 953 & 862 & 758 \\
0.10 & 10 & 1,590 & 1,340 & 1,240 & 1,150 & 1,030 \\
0.04 & 25 & 1,920 & 1,700 & 1,610 & 1,540 & 1,410 \\
0.02 & 50 & 2,160 & 1,940 & 1,870 & 1,830 & 1,710 \\
0.01 & 100 & 2,380 & 2,160 & 12,110 & 2,120 & 2,020 \\
0.005 & 200 & 2,610 & 2,360 & 2,350 & 12,410 & 2,330 \\
0.002 & 500 & 2,890 & $n g$ & $n g$ & $n g$ & $n g$ \\
\hline
\end{tabular}

'Data does not fit log-Pearson Type III curve, use with caution.

\section{4- 0.000-6M HELMAND RIVER AT LASHKARGAH, Continued}

\section{Probability of occurrence of annual low discharges}

$\left[\mathrm{m}^{3} / \mathrm{s}\right.$, meters per second]

\begin{tabular}{|c|c|c|c|c|c|c|c|c|c|c|}
\hline \multirow{3}{*}{$\begin{array}{c}\text { Nonexceedance } \\
\text { probability }\end{array}$} & \multirow{3}{*}{$\begin{array}{c}\text { Recurrence } \\
\text { interval } \\
\text { (years) }\end{array}$} & \multicolumn{9}{|c|}{ Minimum mean discharge $\left(\mathrm{m}^{3} / \mathrm{s}\right)$} \\
\hline & & \multicolumn{9}{|c|}{ Number of consecutive days } \\
\hline & & 1 & 3 & 7 & 14 & 30 & 60 & 90 & 120 & 183 \\
\hline 0.05 & 20 & 3.91 & 5.88 & 13.5 & 23.6 & 45.2 & 47.1 & 50.7 & 53.9 & 60.6 \\
\hline 0.10 & 10 & 7.29 & 10.6 & 21.1 & 31.5 & 50.6 & 52.7 & 56.1 & 59.6 & 66.1 \\
\hline 0.20 & 5 & 14.3 & 19.6 & 33.1 & 42.6 & 57.7 & 60.4 & 63.8 & 67.7 & 73.8 \\
\hline 0.50 & 2 & 41.5 & 49.2 & 61.2 & 65.6 & 72.7 & 78.4 & 82.5 & 87.3 & 92.9 \\
\hline
\end{tabular}




\section{4- 0.000-6M HELMAND RIVER AT LASHKARGAH, Continued}

Probability of occurrence of seasonal low discharges

$\left[\mathrm{m}^{3} / \mathrm{s}\right.$, meters per second]

\begin{tabular}{|c|c|c|c|c|c|c|c|c|c|}
\hline \multirow{3}{*}{$\begin{array}{c}\text { Nonexceedance } \\
\text { probability }\end{array}$} & \multirow{3}{*}{$\begin{array}{c}\text { Recurrence } \\
\text { interval } \\
\text { (years) }\end{array}$} & \multicolumn{8}{|c|}{ Minimum mean discharge $\left(\mathrm{m}^{3} / \mathrm{s}\right)$} \\
\hline & & \multicolumn{8}{|c|}{ Number of consecutive days } \\
\hline & & 1 & 7 & 14 & 30 & 1 & 7 & 14 & 30 \\
\hline & & \multicolumn{4}{|c|}{ December-January-February } & \multicolumn{4}{|c|}{ March-April-May } \\
\hline 0.05 & 20 & 6.52 & 13.0 & ${ }^{1} 11.8$ & 55.7 & 23.1 & 27.2 & 31.1 & 45.4 \\
\hline 0.10 & 10 & 12.8 & 23.1 & ${ }^{1} 22.3$ & 62.8 & 34.1 & 40.7 & 45.5 & 58.2 \\
\hline 0.20 & 5 & 25.3 & 40.6 & 41.3 & 72.4 & 51.2 & 61.8 & 67.9 & 78.4 \\
\hline \multirow[t]{2}{*}{0.50} & 2 & 64.7 & 84.1 & 89.1 & 94.4 & 91.6 & 113 & 122 & 136 \\
\hline & & \multicolumn{4}{|c|}{ June-July-August } & \multicolumn{4}{|c|}{ September-October-November } \\
\hline 0.05 & 20 & 12.3 & 47.1 & 50.0 & 53.0 & 9.86 & 14.7 & 23.4 & 45.4 \\
\hline 0.10 & 10 & 19.7 & 50.4 & 53.5 & 57.7 & 19.0 & 24.4 & 32.4 & 52.5 \\
\hline 0.20 & 5 & 32.1 & 55.4 & 58.8 & 64.2 & 36.0 & 40.4 & 45.5 & 61.9 \\
\hline 0.50 & 2 & 65.5 & 69.1 & 72.9 & 80.4 & 78.0 & 177.3 & ${ }^{1} 75.0$ & 82.0 \\
\hline
\end{tabular}

'Data does not fit log-Pearson Type III curve, use with caution.

\section{4- 0.000-6M HELMAND RIVER AT LASHKARGAH, Continued}

\section{Annual peak discharge and corresponding gage height}

[m³/s, meters per second; --, no data]

\begin{tabular}{|c|c|c|c|c|c|c|c|}
\hline $\begin{array}{c}\text { Water } \\
\text { year }\end{array}$ & Date & $\begin{array}{c}\text { Gage } \\
\text { height } \\
\text { (meters) }\end{array}$ & $\begin{array}{c}\text { Peak } \\
\text { discharge } \\
\left(\mathrm{m}^{3} / \mathrm{s}\right)\end{array}$ & $\begin{array}{c}\text { Water } \\
\text { year }\end{array}$ & Date & $\begin{array}{c}\text { Gage } \\
\text { height } \\
\text { (meters) }\end{array}$ & $\begin{array}{c}\text { Peak } \\
\text { discharge } \\
\left(\mathrm{m}^{3} / \mathrm{s}\right)\end{array}$ \\
\hline \multicolumn{8}{|c|}{ Annual peak discharge, by year } \\
\hline 1954 & $10-\mathrm{Feb}$ & 3.70 & 835 & 1975 & 19-May & -- & 918 \\
\hline 1955 & 15-Mar & 4.58 & 1,234 & 1976 & 27-Apr & -- & 1,862 \\
\hline 1956 & 9-Apr & 4.54 & 1,214 & 1977 & 28-Apr & -- & 361 \\
\hline 1957 & 5-May & 4.78 & 1,406 & 1978 & 25-Dec & -- & 738 \\
\hline 1973 & 5-May & -- & 500 & 1979 & 13-Feb & -- & 690 \\
\hline 1974 & 26-Jan & -- & 610 & 1980 & 21-Mar & -- & 1,295 \\
\hline \multicolumn{8}{|c|}{ Annual peak discharge, from highest to lowest } \\
\hline 1976 & 27-Apr & -- & 1,862 & 1954 & $10-\mathrm{Feb}$ & 3.70 & 835 \\
\hline 1957 & 5-May & 4.78 & 1,406 & 1978 & 25-Dec & -- & 738 \\
\hline 1980 & 21-Mar & -- & 1,295 & 1979 & 13-Feb & -- & 690 \\
\hline 1955 & 15-Mar & 4.58 & 1,234 & 1974 & 26-Jan & -- & 610 \\
\hline 1956 & 9-Apr & 4.54 & 1,214 & 1973 & 5-May & -- & 500 \\
\hline 1975 & 19-May & -- & 918 & 1977 & 28-Apr & -- & 361 \\
\hline
\end{tabular}




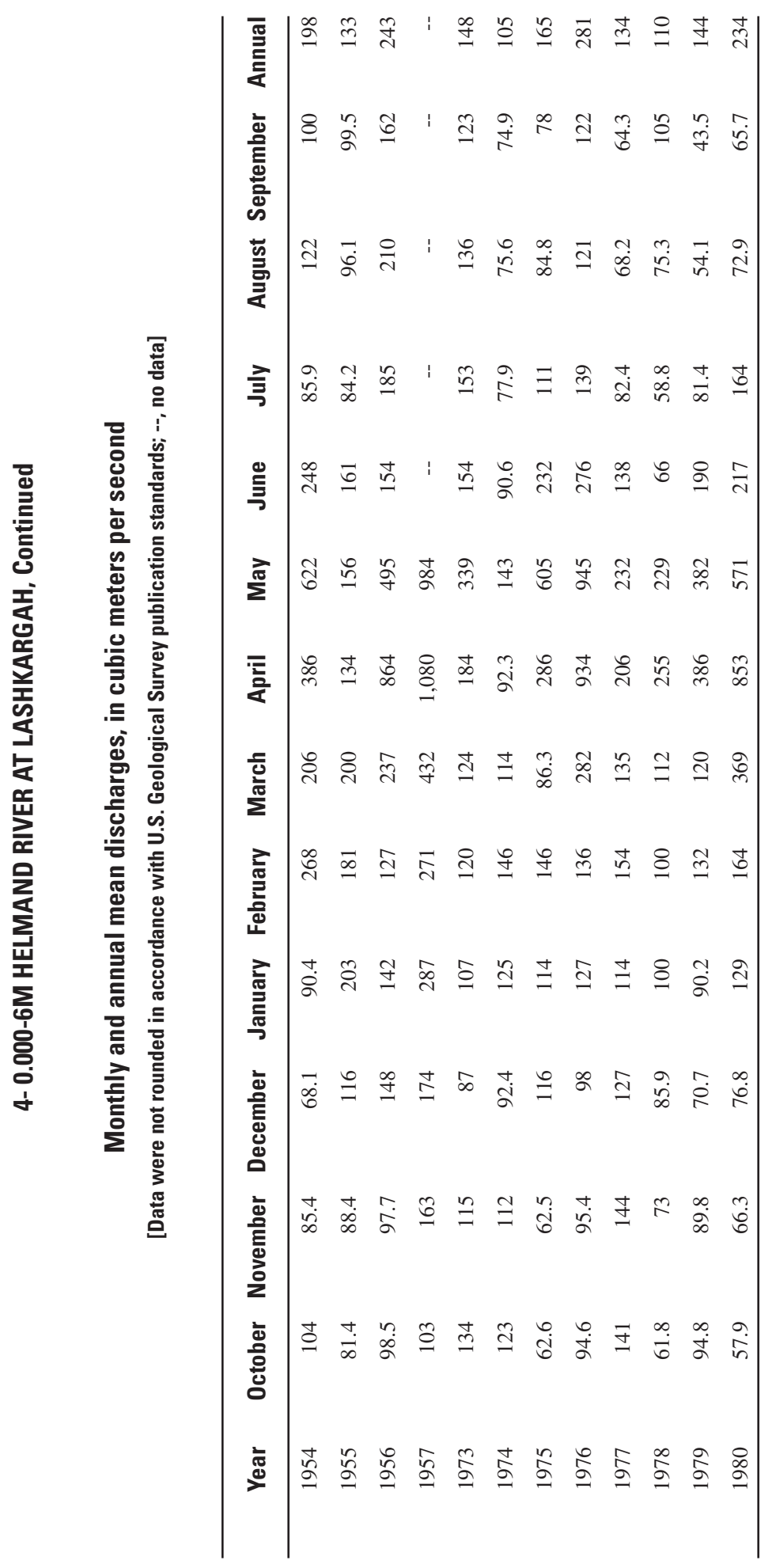




\section{4- 0.000-6S HELMAND RIVER AT GIRISHK}

\section{(U.S. Geological Survey identification number: 314800064350000)}

LOCATION: Lat $31^{\circ} 48^{\prime} \mathrm{N}$., long 64³5'E., on left bank under highway bridge, $2.5 \mathrm{~km}$ east of Girishk, $51 \mathrm{~km}$ upstream from Arghandab River and 73 km downstream from Kajakai Dam.

DRAINAGE AREA: $58,070 \mathrm{~km}^{2}$ (from Survey of India maps).

ALTITUDE: 821 meters plus mean sea level.

PERIOD OF RECORD: July 1, 1946 to March 11, 1949.

GAGE: Water-stage recorder. February 16 to April 30, 1947, at site $5 \mathrm{~km}$ upstream at different datum.

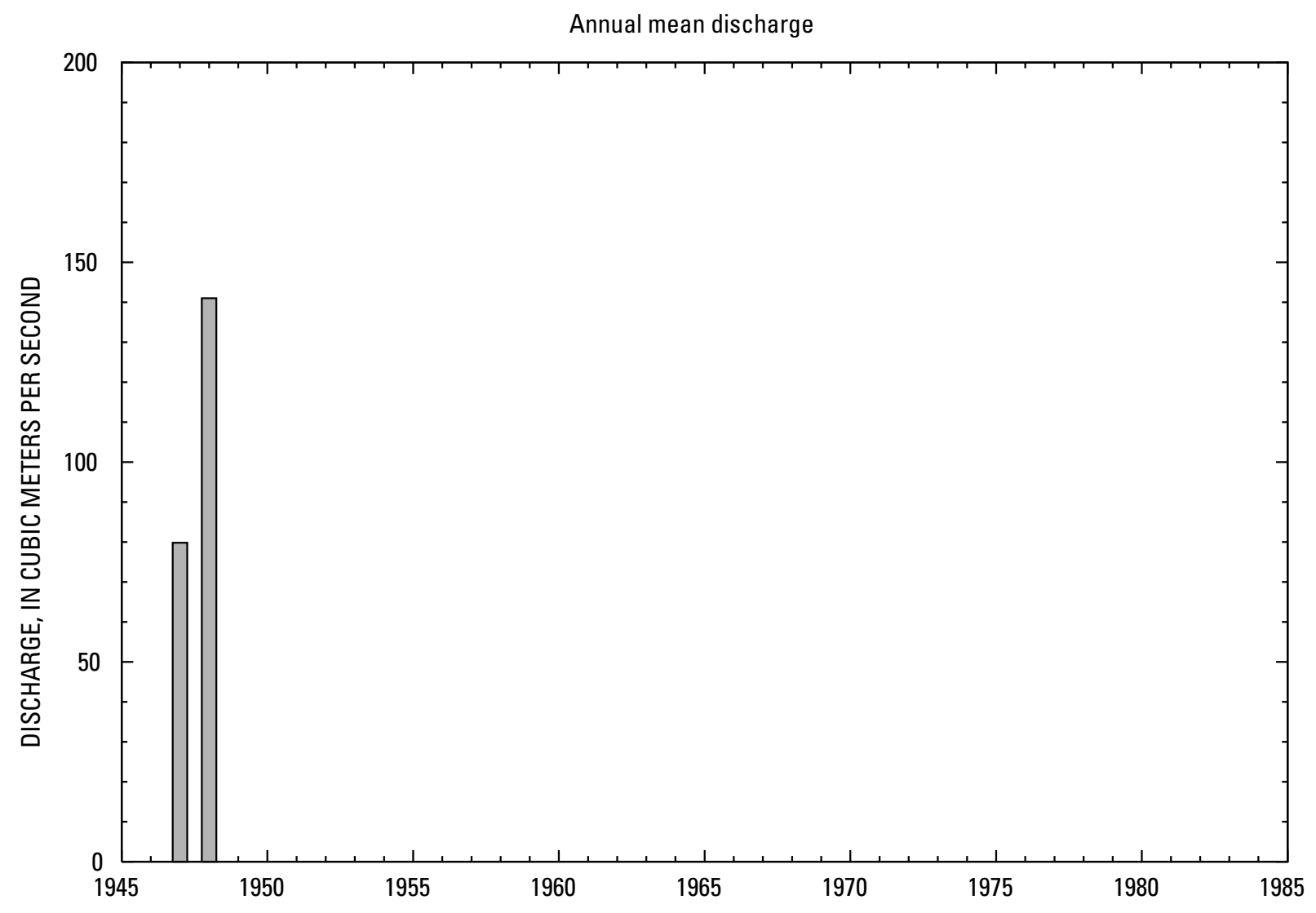




\section{4- 0.000-6S HELMAND RIVER AT GIRISHK, Continued}

\section{Statistics of monthly and annual mean discharges}

$\left[\mathrm{m}^{3} / \mathrm{s}\right.$, meters per second]

\begin{tabular}{|c|c|c|c|c|c|c|c|c|}
\hline \multirow[b]{2}{*}{ Month } & \multicolumn{2}{|c|}{ Maximum } & \multicolumn{2}{|c|}{ Minimum } & \multicolumn{4}{|c|}{ Mean } \\
\hline & $\begin{array}{c}\text { Discharge } \\
\left(\mathrm{m}^{3} / \mathrm{s}\right)\end{array}$ & $\begin{array}{c}\text { Water year } \\
\text { of } \\
\text { occurrence }\end{array}$ & $\begin{array}{c}\text { Discharge } \\
\left(\mathrm{m}^{3} / \mathrm{s}\right)\end{array}$ & $\begin{array}{c}\text { Water year } \\
\text { of } \\
\text { occurrence }\end{array}$ & $\begin{array}{c}\text { Discharge } \\
\left(\mathrm{m}^{3} / \mathrm{s}\right)\end{array}$ & $\begin{array}{c}\text { Standard } \\
\text { deviation } \\
\left(\mathrm{m}^{3} / \mathrm{s}\right)\end{array}$ & $\begin{array}{c}\text { Coefficient } \\
\text { of } \\
\text { variation }\end{array}$ & $\begin{array}{c}\text { Percentage } \\
\text { of annual } \\
\text { discharge }\end{array}$ \\
\hline October & 50.1 & 1947 & 33.0 & 1948 & 42.9 & 8.85 & 0.21 & 3.18 \\
\hline November & 61.6 & 1949 & 49.0 & 1948 & 57.0 & 6.93 & 0.12 & 4.23 \\
\hline December & 67.5 & 1947 & 62.6 & 1948 & 65.6 & 2.63 & 0.04 & 4.87 \\
\hline January & 74.0 & 1947 & 67.5 & 1948 & 70.0 & 3.48 & 0.05 & 5.20 \\
\hline February & 114 & 1949 & 77.9 & 1948 & 93.9 & 18.4 & 0.20 & 6.97 \\
\hline March & 279 & 1948 & 161 & 1947 & 220 & 83.4 & 0.38 & 16.3 \\
\hline April & 565 & 1948 & 211 & 1947 & 388 & 250 & 0.65 & 28.8 \\
\hline May & 332 & 1948 & 131 & 1947 & 232 & 142 & 0.61 & 17.2 \\
\hline June & 111 & 1948 & 67.6 & 1947 & 89.3 & 30.7 & 0.34 & 6.62 \\
\hline July & 51.9 & 1948 & 23.0 & 1947 & 37.9 & 14.5 & 0.38 & 2.81 \\
\hline August & 34.4 & 1948 & 10.2 & 1947 & 24.5 & 12.7 & 0.52 & 1.82 \\
\hline September & 38.6 & 1946 & 13.7 & 1947 & 27.4 & 12.6 & 0.46 & 2.04 \\
\hline Annual & 141 & 1948 & 79.8 & 1947 & 110 & 43.3 & 0.39 & 100 \\
\hline
\end{tabular}

Annual flow duration

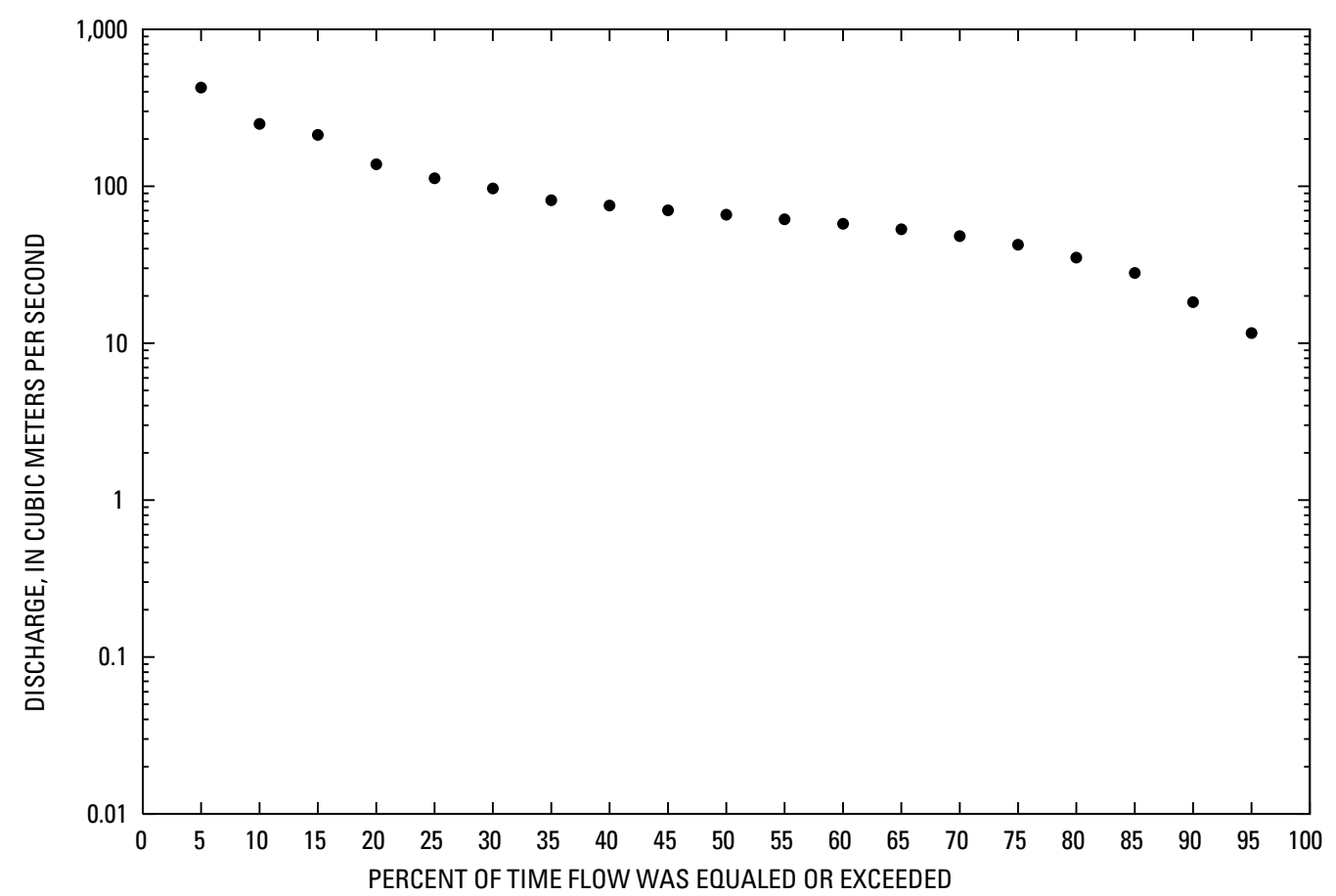




\section{4- 0.000-6S HELMAND RIVER AT GIRISHK, Continued}

\section{Monthly and annual flow duration, in cubic meters per second}

[Data were not rounded in accordance with U.S. Geological Survey publication standards]

\begin{tabular}{|c|c|c|c|c|c|c|c|c|c|c|c|c|c|}
\hline $\begin{array}{l}\text { Percentage } \\
\text { of days } \\
\text { discharge } \\
\text { equaled or } \\
\text { exceeded }\end{array}$ & January & February & March & April & May & June & July & August & September & October & November & December & Annual \\
\hline 95 & 60.7 & 71.1 & 106.1 & 169 & 102.5 & 47 & 13 & 9.69 & 11.5 & 24.4 & 44 & 57.9 & 11.6 \\
\hline 90 & 62.1 & 73.8 & 110.5 & 197 & 111.4 & 50.5 & 14.4 & 9.87 & 11.9 & 27 & 45.3 & 60.9 & 18.3 \\
\hline 80 & 64.1 & 76.9 & 118 & 213.1 & 127.8 & 62.9 & 24.3 & 10.3 & 13.6 & 35.5 & 52.3 & 62.3 & 35.1 \\
\hline 75 & 64.7 & 78.3 & 121.8 & 216.8 & 131.4 & 67.6 & 29.8 & 10.7 & 15.2 & 36.9 & 52.8 & 63.8 & 42.4 \\
\hline 70 & 65.1 & 79.3 & 126.8 & 220.8 & 135 & 69.5 & 32.8 & 11.2 & 18.2 & 38.2 & 53.8 & 64.2 & 48.1 \\
\hline 65 & 66.6 & 80.5 & 132.8 & 224.9 & 138.6 & 75.4 & 34.3 & 24.1 & 24.4 & 40.4 & 56.8 & 64.6 & 53.2 \\
\hline 60 & 67.1 & 82 & 155 & 229 & 150.8 & 81.1 & 34.8 & 26.5 & 25.8 & 42.5 & 58.1 & 64.9 & 57.6 \\
\hline 45 & 70.4 & 87 & 229.2 & 400 & 171.9 & 94.8 & 38.8 & 28.1 & 30.4 & 45 & 59.6 & 65.8 & 70.3 \\
\hline 40 & 71.7 & 88 & 240.4 & 443 & 212.4 & 96.2 & 42 & 28.7 & 34 & 45.6 & 59.9 & 66.4 & 75.4 \\
\hline 35 & 72.5 & 89.7 & 257.4 & 478 & 230.4 & 98.2 & 45.4 & 29.5 & 35.2 & 47.6 & 60.2 & 66.6 & 81.4 \\
\hline 30 & 73.2 & 93 & 275.4 & 498.7 & 255.1 & 101 & 47.7 & 30.7 & 36.2 & 48.8 & 61.1 & 66.7 & 96.7 \\
\hline 25 & 75 & 97.1 & 288.6 & 542 & 294.5 & 106.5 & 49.4 & 32.2 & 37.1 & 50 & 62.3 & 66.9 & 112.4 \\
\hline 20 & 75.5 & 99.2 & 295.9 & 631 & 345.8 & 110 & 51.4 & 34.4 & 37.9 & 51.1 & 62.7 & 68 & 138.1 \\
\hline 15 & 76.5 & 102.4 & 303.3 & 655.8 & 428.4 & 117 & 54.3 & 36.8 & 39.2 & 53.2 & 63.1 & 69.5 & 212.5 \\
\hline 10 & 78.3 & 108 & 318.7 & 698 & 479.6 & 127.5 & 56.8 & 38.8 & 40.7 & 54.7 & 64.2 & 70.9 & 249.6 \\
\hline 5 & 79.7 & 123 & 393.5 & 735 & 527.6 & 140 & 59.8 & 0 & 42.2 & 55.5 & 0.05 & 74.3 & 425.7 \\
\hline
\end{tabular}




\section{4- 0.000-6S HELMAND RIVER AT GIRISHK, Continued}

\section{Probability of occurrence of annual high discharges}

[ $\mathrm{m}^{3} / \mathrm{s}$, meters per second; ng, statistic not given]

\begin{tabular}{|c|c|c|c|c|c|c|}
\hline \multirow{2}{*}{$\begin{array}{l}\text { Exceedance } \\
\text { probability }\end{array}$} & \multirow{2}{*}{$\begin{array}{c}\text { Recurrence } \\
\text { interval } \\
\text { (years) }\end{array}$} & \multirow{2}{*}{$\begin{array}{c}\text { Maximum } \\
\text { instantaneous } \\
\left(\mathrm{m}^{3} / \mathrm{s}\right)\end{array}$} & \multicolumn{4}{|c|}{ Maximum mean discharge } \\
\hline & & & 3-day period & 7-day period & 15-day period & 30-day period \\
\hline 0.99 & 1.01 & ng & ng & ng & ng & ng \\
\hline 0.95 & 1.05 & ng & ng & ng & ng & ng \\
\hline 0.90 & 1.11 & ng & ng & ng & ng & ng \\
\hline 0.80 & 1.25 & $\mathrm{ng}$ & ng & ng & ng & ng \\
\hline 0.50 & 2 & ng & ng & ng & ng & ng \\
\hline 0.20 & 5 & ng & ng & ng & ng & ng \\
\hline 0.10 & 10 & ng & ng & ng & ng & ng \\
\hline 0.04 & 25 & ng & ng & ng & ng & ng \\
\hline 0.02 & 50 & ng & ng & ng & ng & ng \\
\hline 0.01 & 100 & $\mathrm{ng}$ & ng & ng & ng & $\mathrm{ng}$ \\
\hline 0.005 & 200 & ng & ng & ng & $\mathrm{ng}$ & $\mathrm{ng}$ \\
\hline 0.002 & 500 & ng & ng & ng & ng & $\mathrm{ng}$ \\
\hline
\end{tabular}

\section{4- 0.000-6S HELMAND RIVER AT GIRISHK, Continued}

Probability of occurrence of annual low discharges

[ $\mathrm{m}^{3} / \mathrm{s}$, meters per second; ng, statistic not given]

\begin{tabular}{|c|c|c|c|c|c|c|c|c|c|c|}
\hline \multirow{3}{*}{$\begin{array}{l}\text { Nonexceedance } \\
\text { probability }\end{array}$} & \multirow{3}{*}{$\begin{array}{c}\text { Recurrence } \\
\text { interval } \\
\text { (years) }\end{array}$} & \multicolumn{9}{|c|}{ Minimum mean discharge $\left(\mathrm{m}^{3} / \mathrm{s}\right)$} \\
\hline & & \multicolumn{9}{|c|}{ Number of consecutive days } \\
\hline & & 1 & 3 & 7 & 14 & 30 & 60 & 90 & 120 & 183 \\
\hline 0.05 & 20 & ng & ng & ng & ng & ng & ng & ng & ng & ng \\
\hline 0.10 & 10 & ng & ng & ng & ng & ng & ng & ng & ng & ng \\
\hline 0.20 & 5 & ng & ng & ng & ng & ng & ng & ng & ng & ng \\
\hline 0.50 & 2 & ng & ng & $\mathrm{ng}$ & ng & ng & ng & ng & $\mathrm{ng}$ & ng \\
\hline
\end{tabular}




\section{4- 0.000-6S HELMAND RIVER AT GIRISHK, Continued}

\section{Probability of occurrence of seasonal low discharges}

[m³/s, meters per second; ng, statistic not given]

\begin{tabular}{|c|c|c|c|c|c|c|c|c|c|}
\hline \multirow{3}{*}{$\begin{array}{c}\text { Nonexceedance } \\
\text { probability }\end{array}$} & \multirow{3}{*}{$\begin{array}{c}\text { Recurrence } \\
\text { interval } \\
\text { (years) }\end{array}$} & \multicolumn{8}{|c|}{ Minimum mean discharge $\left(\mathrm{m}^{3} / \mathrm{s}\right)$} \\
\hline & & \multicolumn{8}{|c|}{ Number of consecutive days } \\
\hline & & 1 & 7 & 14 & 30 & 1 & 7 & 14 & 30 \\
\hline & & \multicolumn{4}{|c|}{ December-January-February } & \multicolumn{4}{|c|}{ March-April-May } \\
\hline 0.05 & 20 & ng & ng & ng & ng & $\mathrm{ng}$ & $\mathrm{ng}$ & ng & $\mathrm{ng}$ \\
\hline 0.10 & 10 & ng & $\mathrm{ng}$ & ng & ng & ng & ng & ng & $\mathrm{ng}$ \\
\hline 0.20 & 5 & ng & ng & ng & $\mathrm{ng}$ & ng & $\mathrm{ng}$ & ng & $\mathrm{ng}$ \\
\hline \multirow[t]{2}{*}{0.50} & 2 & ng & ng & ng & ng & ng & ng & ng & ng \\
\hline & & \multicolumn{4}{|c|}{ June-July-August } & \multicolumn{4}{|c|}{ September-October-November } \\
\hline 0.05 & 20 & ng & ng & ng & ng & $\mathrm{ng}$ & ng & ng & $\mathrm{ng}$ \\
\hline 0.10 & 10 & $\mathrm{ng}$ & ng & $\mathrm{ng}$ & $\mathrm{ng}$ & $\mathrm{ng}$ & ng & ng & $\mathrm{ng}$ \\
\hline 0.20 & 5 & ng & ng & ng & ng & $\mathrm{ng}$ & ng & ng & $\mathrm{ng}$ \\
\hline 0.50 & 2 & $\mathrm{ng}$ & $\mathrm{ng}$ & $\mathrm{ng}$ & $\mathrm{ng}$ & $\mathrm{ng}$ & $\mathrm{ng}$ & $\mathrm{ng}$ & $\mathrm{ng}$ \\
\hline
\end{tabular}

\section{4- 0.000-6S HELMAND RIVER AT GIRISHK, Continued}

Annual peak discharge and corresponding gage height

[m³/s, meters per second; --, no data]

\begin{tabular}{|c|c|c|c|c|c|c|c|}
\hline $\begin{array}{c}\text { Water } \\
\text { year }\end{array}$ & Date & $\begin{array}{c}\text { Gage } \\
\text { height } \\
\text { (meters) }\end{array}$ & $\begin{array}{c}\text { Peak } \\
\text { discharge } \\
\left(\mathrm{m}^{3} / \mathrm{s}\right)\end{array}$ & $\begin{array}{c}\text { Water } \\
\text { year }\end{array}$ & Date & $\begin{array}{c}\text { Gage } \\
\text { height } \\
\text { (meters) }\end{array}$ & $\begin{array}{c}\text { Peak } \\
\text { discharge } \\
\left(\mathrm{m}^{3} / \mathrm{s}\right)\end{array}$ \\
\hline
\end{tabular}

Annual peak discharge, by year

$\begin{array}{rrrr}1947 & \text { 22-Mar } & 28.90 & 317 \\ 1948 & \text { 8-Mar } & 18.08 & 1,019 \\ 1949 & \text { 10-Mar } & -- & 912\end{array}$

Annual peak discharge, from highest to lowest

$1948 \quad$ 8-Mar $\quad 18.08 \quad 1,019$

1949 10-Mar $\quad$-- $\quad 912$

$1947 \quad 22-M a r \quad 28.90 \quad 317$ 


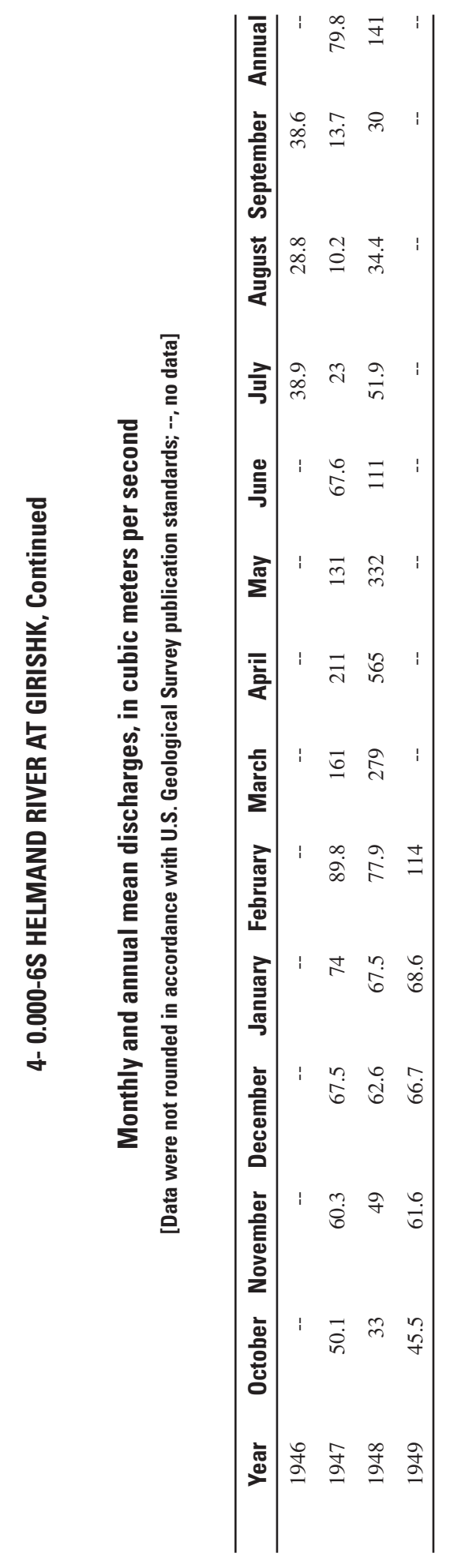




\section{4- 0.000-7M HELMAND RIVER BELOW KAJAKAI RESERVOIR}

\section{(U.S. Geological Survey identification number: 321900065060000)}

LOCATION: Lat $32^{\circ} 19^{\prime}$ N., long 6506'E., on left bank $1 \mathrm{~km}$ east of Kajakai, $2 \mathrm{~km}$ downstream from Kajakai Dam, and $123 \mathrm{~km}$ upstream from Arghandab River.

DRAINAGE AREA: $46,600 \mathrm{~km}^{2}$ (from Survey of India maps).

ALTITUDE: 955 meters plus mean sea level.

PERIOD OF RECORD: October 1, 1947 to April 6, 1980.

GAGE: Water-stage recorder. Datum of gage is $960.169 \mathrm{~m}$ above mean sea level (Survey of India datum).

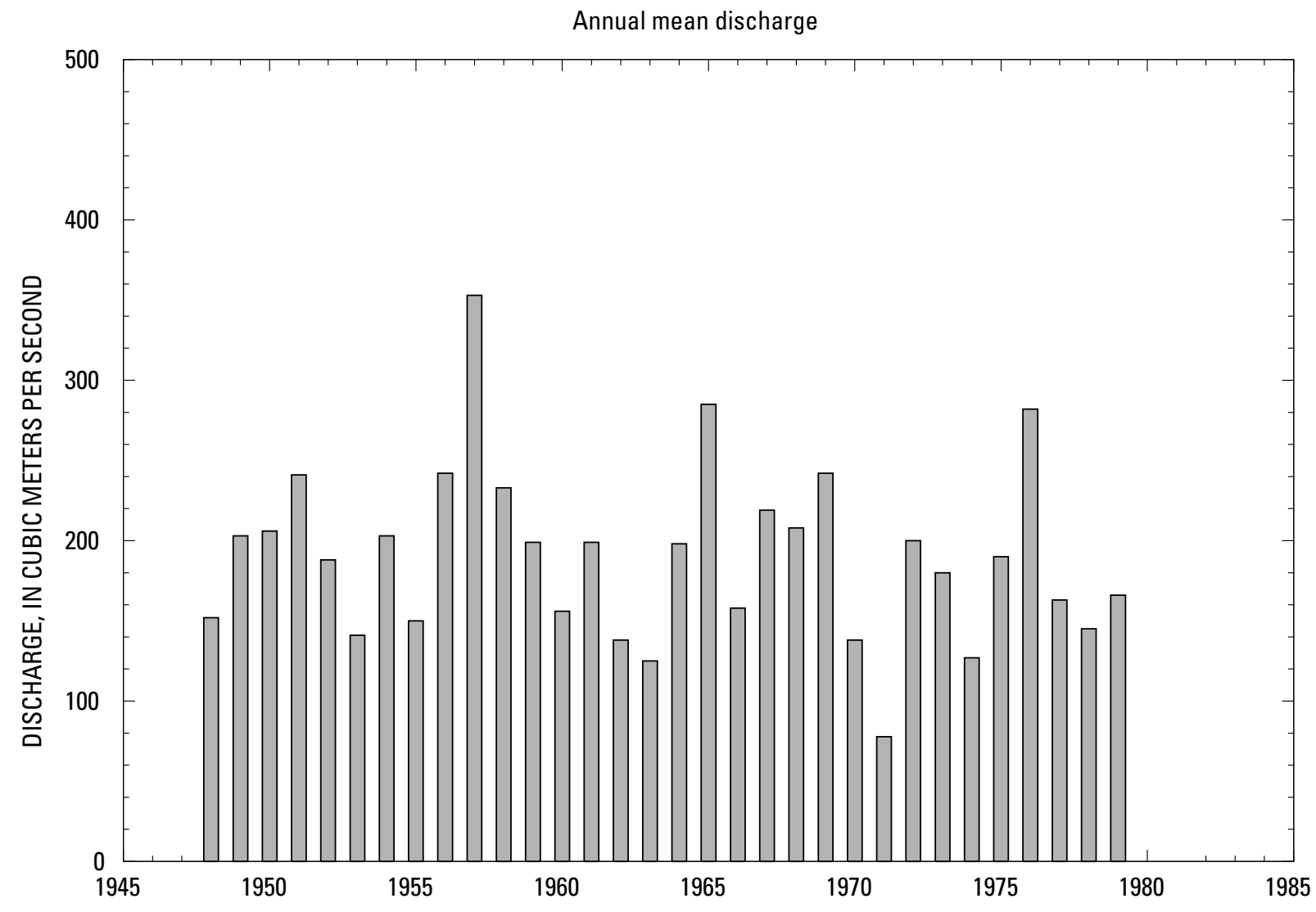




\section{4- 0.000-7M HELMAND RIVER BELOW KAJAKAI RESERVOIR, Continued}

\section{Statistics of monthly and annual mean discharges}

$\left[\mathrm{m}^{3} / \mathrm{s}\right.$, meters per second]

\begin{tabular}{|c|c|c|c|c|c|c|c|c|}
\hline \multirow[b]{2}{*}{ Month } & \multicolumn{2}{|c|}{ Maximum } & \multicolumn{2}{|c|}{ Minimum } & \multicolumn{4}{|c|}{ Mean } \\
\hline & $\begin{array}{c}\text { Discharge } \\
\left(\mathrm{m}^{3} / \mathrm{s}\right)\end{array}$ & $\begin{array}{c}\text { Water year } \\
\text { of } \\
\text { occurrence }\end{array}$ & $\begin{array}{c}\text { Discharge } \\
\left(\mathrm{m}^{3} / \mathrm{s}\right)\end{array}$ & $\begin{array}{c}\text { Water year } \\
\text { of } \\
\text { occurrence }\end{array}$ & $\begin{array}{c}\text { Discharge } \\
\left(\mathrm{m}^{3} / \mathrm{s}\right)\end{array}$ & $\begin{array}{c}\text { Standard } \\
\text { deviation } \\
\left(\mathrm{m}^{3} / \mathrm{s}\right)\end{array}$ & $\begin{array}{c}\text { Coefficient } \\
\text { of } \\
\text { variation }\end{array}$ & $\begin{array}{c}\text { Percentage } \\
\text { of annual } \\
\text { discharge }\end{array}$ \\
\hline October & 207 & 1958 & 41.0 & 1948 & 108 & 40.2 & 0.37 & 4.71 \\
\hline November & 219 & 1966 & 54.2 & 1948 & 110 & 37.2 & 0.34 & 4.79 \\
\hline December & 209 & 1966 & 60.8 & 1972 & 117 & 41.0 & 0.35 & 5.10 \\
\hline January & 206 & 1958 & 52.9 & 1972 & 118 & 47.2 & 0.40 & 5.14 \\
\hline February & 204 & 1969 & 42.7 & 1971 & 129 & 46.5 & 0.36 & 5.65 \\
\hline March & 346 & 1952 & 61.7 & 1972 & 172 & 71.6 & 0.42 & 7.51 \\
\hline April & 985 & 1957 & 100 & 1960 & 407 & 262 & 0.64 & 17.8 \\
\hline May & 1,180 & 1957 & 112 & 1971 & 479 & 276 & 0.58 & 20.9 \\
\hline June & 536 & 1957 & 76.0 & 1971 & 235 & 99.7 & 0.42 & 10.3 \\
\hline July & 237 & 1957 & 62.0 & 1948 & 155 & 51.7 & 0.33 & 6.77 \\
\hline August & 216 & 1958 & 38.9 & 1948 & 139 & 53.7 & 0.39 & 6.08 \\
\hline September & 209 & 1957 & 42.4 & 1948 & 121 & 45.6 & 0.38 & 5.28 \\
\hline Annual & 353 & 1957 & 77.8 & 1971 & 191 & 54.8 & 0.29 & 100 \\
\hline
\end{tabular}

Annual flow duration

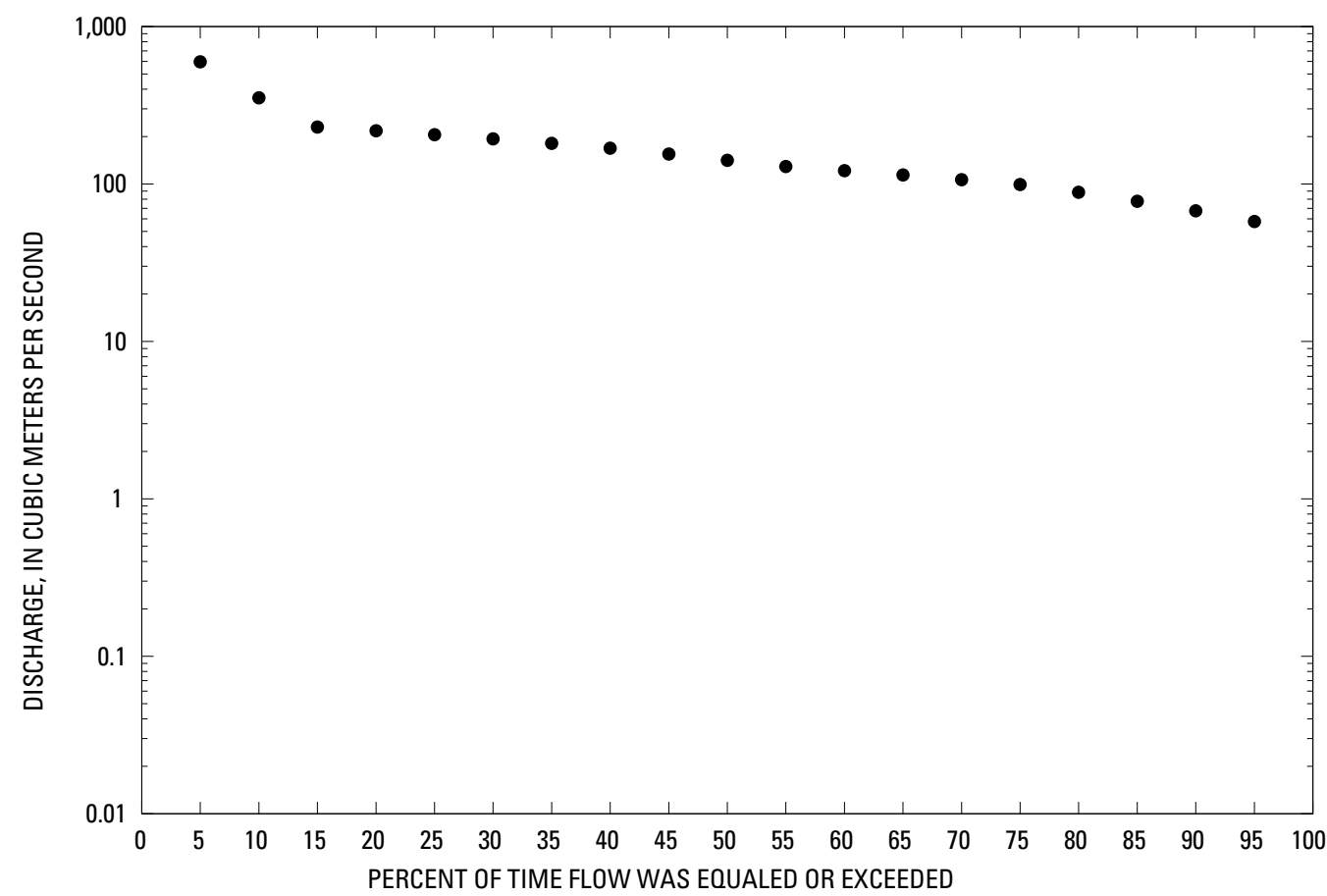




\section{4-0.000-7M HELMAND RIVER BELOW KAJAKAI RESERVOIR, Continued}

Monthly and annual flow duration, in cubic meters per second

[Data were not rounded in accordance with U.S. Geological Survey publication standards]

\begin{tabular}{|c|c|c|c|c|c|c|c|c|c|c|c|c|c|}
\hline $\begin{array}{l}\text { Percentage } \\
\text { of days } \\
\text { discharge } \\
\text { equaled or } \\
\text { exceeded }\end{array}$ & January & February & March & April & May & June & July & August & September & October & November & December & Annual \\
\hline 95 & 53 & 48 & 66.8 & 101.5 & 122.7 & 104.6 & 70 & 52.7 & 51.7 & 50.9 & 57.6 & 62.4 & 57.8 \\
\hline 90 & 60.5 & 60.9 & 88.9 & 108.8 & 148.1 & 114.9 & 82.4 & 58.9 & 56 & 55.4 & 65.4 & 71 & 67.6 \\
\hline 85 & 69.7 & 72.5 & 98.1 & 122 & 174.3 & 132 & 89.1 & 66.1 & 60.3 & 63.2 & 72 & 76.4 & 77.8 \\
\hline 80 & 74.3 & 80.4 & 107.1 & 137.6 & 200.3 & 141.7 & 98.4 & 70.9 & 64.8 & 68.1 & 77.4 & 81.8 & 88.6 \\
\hline 75 & 78 & 88 & 115.5 & 163.2 & 217.8 & 152.3 & 112.7 & 90.1 & 69.3 & 71.3 & 81.7 & 87.3 & 99.1 \\
\hline 70 & 81.3 & 94.2 & 123.9 & 176.9 & 267.3 & 164 & 121.1 & 121.8 & 93.1 & 76.8 & 86.1 & 91.2 & 106.6 \\
\hline 65 & 84.3 & 100.3 & 134.1 & 190.8 & 309.4 & 176.9 & 129.5 & 127.6 & 103.8 & 84.9 & 90.5 & 95.1 & 114.1 \\
\hline 60 & 89.3 & 106.5 & 147 & 203 & 343.4 & 191 & 139.1 & 132 & 112.7 & 102.4 & 94.9 & 99 & 121.6 \\
\hline 55 & 99.8 & 113.9 & 158.9 & 211.6 & 379.3 & 204.5 & 144.2 & 136 & 117.1 & 105.9 & 99.4 & 102.9 & 129.2 \\
\hline 50 & 103.8 & 123.1 & 165.1 & 220.4 & 418.9 & 212.5 & 149.3 & 139.5 & 121.6 & 109.3 & 104 & 106.8 & 141.4 \\
\hline 45 & 107.7 & 132.3 & 171.2 & 239.1 & 461.7 & 220.5 & 155.7 & 142.9 & 126.1 & 112.6 & 108.5 & 111.2 & 155.1 \\
\hline 40 & 116.6 & 144.6 & 177.4 & 383.7 & 504.7 & 229.7 & 162.2 & 147 & 130.6 & 115.8 & 113.1 & 116.4 & 168.8 \\
\hline 35 & 122.8 & 161.9 & 183.6 & 467.9 & 550.6 & 241.6 & 185.9 & 152.4 & 137.9 & 119.8 & 117.9 & 121.6 & 181.5 \\
\hline 30 & 158.4 & 176.2 & 189.7 & 532.2 & 602.4 & 254.8 & 201.9 & 188.7 & 145.5 & 124.1 & 124.5 & 126.8 & 193.6 \\
\hline 25 & 177.6 & 183.5 & 199.7 & 612.5 & 657 & 277.3 & 212.6 & 196.2 & 153.1 & 129.3 & 131.1 & 132 & 205.7 \\
\hline 20 & 184.6 & 190.8 & 211.9 & 686.9 & 723.6 & 307.7 & 216.9 & 200.6 & 171.6 & 135.1 & 137.7 & 146.8 & 217.8 \\
\hline 15 & 188.6 & 198.1 & 224.1 & 774 & 819.1 & 348 & 221.3 & 205 & 185.4 & 150.7 & 144.3 & 176.8 & 229.9 \\
\hline 10 & 192.7 & 205.4 & 253.8 & 928.9 & 916.5 & 390.2 & 225.7 & 210 & 194.5 & 172.3 & 164 & 189.9 & 352.4 \\
\hline 5 & 196.7 & 212.6 & 346.7 & 1121.4 & 1033.3 & 455 & 230.3 & 215.4 & 203.7 & 192.9 & 196.7 & 203 & 596.8 \\
\hline
\end{tabular}




\section{4- 0.000-7M HELMAND RIVER BELOW KAJAKAI RESERVOIR, Continued}

Probability of occurrence of annual high discharges

[ $\mathrm{m}^{3} / \mathrm{s}$, meters per second; $\mathrm{ng}$, statistic not given]

\begin{tabular}{|c|c|c|c|c|c|c|}
\hline \multirow{2}{*}{$\begin{array}{c}\text { Exceedance } \\
\text { probability }\end{array}$} & \multirow{2}{*}{$\begin{array}{c}\text { Recurrence } \\
\text { interval } \\
\text { (years) }\end{array}$} & \multirow{2}{*}{$\begin{array}{c}\text { Maximum } \\
\text { instantaneous } \\
\left(\mathrm{m}^{3} / \mathrm{s}\right)\end{array}$} & \multicolumn{4}{|c|}{ Maximum mean discharge } \\
\hline & & & 3-day period & 7-day period & 15-day period & 30-day period \\
\hline 0.99 & 1.01 & 87.8 & ${ }^{1} 89.3$ & ${ }^{1} 89.6$ & ${ }^{1} 89.8$ & 191.2 \\
\hline 0.95 & 1.05 & 174 & 174 & 173 & 169 & 162 \\
\hline 0.90 & 1.11 & 243 & 242 & 238 & 230 & 215 \\
\hline 0.80 & 1.25 & 356 & 351 & 342 & 326 & 298 \\
\hline 0.50 & 2 & 686 & 665 & 636 & 592 & 523 \\
\hline 0.20 & 5 & 1,210 & 1,150 & 1,080 & 979 & 857 \\
\hline 0.10 & 10 & 1,560 & 1,480 & 1,370 & 1,230 & 1,080 \\
\hline 0.04 & 25 & 2,010 & 1,880 & 1,720 & 1,530 & 1,350 \\
\hline 0.02 & 50 & 2,340 & 2,170 & 1,970 & 1,740 & 1,550 \\
\hline 0.01 & 100 & 2,650 & 2,450 & 2,200 & 1,940 & 1,730 \\
\hline 0.005 & 200 & 2,950 & 2,710 & 2,430 & 2,130 & 1,910 \\
\hline 0.002 & 500 & 3,330 & $\mathrm{ng}$ & ng & ng & ng \\
\hline
\end{tabular}

'Data does not fit log-Pearson Type III curve, use with caution.

\section{4- 0.000-7M HELMAND RIVER BELOW KAJAKAI RESERVOIR, Continued}

Probability of occurrence of annual low discharges

[m³/s, meters per second; ng, statistic not given]

\begin{tabular}{|c|c|c|c|c|c|c|c|c|c|c|}
\hline \multirow{3}{*}{$\begin{array}{c}\text { Nonexceedance } \\
\text { probability }\end{array}$} & \multirow{3}{*}{$\begin{array}{c}\text { Recurrence } \\
\text { interval } \\
\text { (years) }\end{array}$} & \multicolumn{9}{|c|}{ Minimum mean discharge $\left(\mathrm{m}^{3} / \mathrm{s}\right)$} \\
\hline & & \multicolumn{9}{|c|}{ Number of consecutive days } \\
\hline & & 1 & 3 & 7 & 14 & 30 & 60 & 90 & 120 & 183 \\
\hline 0.05 & 20 & 0.431 & 1.20 & ng & 33.6 & 42.5 & 47.8 & 52.8 & 57.2 & 61.4 \\
\hline 0.10 & 10 & 1.82 & 4.72 & ng & 40.1 & 49.3 & 55.3 & 60.6 & 65.1 & 70.7 \\
\hline 0.20 & 5 & 7.69 & 17.1 & ng & 49.1 & 58.4 & 65.4 & 70.8 & 75.5 & 83.1 \\
\hline 0.50 & 2 & 51.9 & 73.8 & ng & 96.4 & 78.3 & 87.2 & 93.0 & 98.2 & 110 \\
\hline
\end{tabular}




\section{4- 0.000-7M HELMAND RIVER BELOW KAJAKAI RESERVOIR, Continued}

\section{Probability of occurrence of seasonal low discharges}

[ $\mathrm{m}^{3} / \mathrm{s}$, meters per second; ng, statistic not given]

\begin{tabular}{|c|c|c|c|c|c|c|c|c|c|}
\hline \multirow{3}{*}{$\begin{array}{c}\text { Nonexceedance } \\
\text { probability }\end{array}$} & \multirow{3}{*}{$\begin{array}{c}\text { Recurrence } \\
\text { interval } \\
\text { (years) }\end{array}$} & \multicolumn{8}{|c|}{ Minimum mean discharge $\left(\mathrm{m}^{3} / \mathrm{s}\right)$} \\
\hline & & \multicolumn{8}{|c|}{ Number of consecutive days } \\
\hline & & 1 & 7 & 14 & 30 & 1 & 7 & 14 & 30 \\
\hline & & \multicolumn{4}{|c|}{ December-January-February } & \multicolumn{4}{|c|}{ March-April-May } \\
\hline 0.05 & 20 & 1.35 & ng & 39.4 & 47.8 & ng & 59.9 & 62.4 & 74.5 \\
\hline 0.10 & 10 & 5.56 & ng & 48.8 & 55.5 & ng & 71.4 & 74.9 & 85.6 \\
\hline 0.20 & 5 & 21.0 & ng & 62.0 & 66.5 & ng & 87.2 & 92.6 & 103 \\
\hline \multirow[t]{2}{*}{0.50} & 2 & 194.6 & ng & 92.3 & 93.5 & ng & 123 & 135 & 148 \\
\hline & & \multicolumn{4}{|c|}{ June-July-August } & \multicolumn{4}{|c|}{ September-October-November } \\
\hline 0.05 & 20 & 31.1 & 47.1 & 49.7 & 52.2 & ng & ng & 41.2 & 49.5 \\
\hline 0.10 & 10 & 41.0 & 58.6 & 62.6 & 65.8 & ng & ng & 48.1 & 57.1 \\
\hline 0.20 & 5 & 56.0 & 75.1 & 81.0 & 84.9 & ng & ng & 57.8 & 67.4 \\
\hline 0.50 & 2 & 94.7 & 115 & 124 & 129 & ng & $\mathrm{ng}$ & 80.8 & 90.8 \\
\hline
\end{tabular}

'Data does not fit log-Pearson Type III curve, use with caution. 


\section{4- 0.000-7M HELMAND RIVER BELOW KAJAKAI RESERVOIR, Continued}

\section{Annual peak discharge and corresponding gage height}

[m³/s, meters per second; --, no data]

\begin{tabular}{|c|c|c|c|c|c|c|c|}
\hline $\begin{array}{l}\text { Water } \\
\text { year }\end{array}$ & Date & $\begin{array}{c}\text { Gage } \\
\text { height } \\
\text { (meters) }\end{array}$ & $\begin{array}{c}\text { Peak } \\
\text { discharge } \\
\left(\mathrm{m}^{3} / \mathrm{s}\right)\end{array}$ & $\begin{array}{l}\text { Water } \\
\text { year }\end{array}$ & Date & $\begin{array}{c}\text { Gage } \\
\text { height } \\
\text { (meters) }\end{array}$ & $\begin{array}{c}\text { Peak } \\
\text { discharge } \\
\left(\mathrm{m}^{3} / \mathrm{s}\right)\end{array}$ \\
\hline
\end{tabular}

Annual peak discharge, by year

\begin{tabular}{|c|c|c|c|c|c|c|c|}
\hline 1948 & 8-Mar & 2.76 & 877 & 1964 & 1-May & -- & 693 \\
\hline 1949 & 26-Apr & 3.79 & 1,418 & 1965 & 6-May & -- & 1,275 \\
\hline 1950 & 11-May & 3.10 & 1,056 & 1966 & 4-Nov & 1.16 & 232 \\
\hline 1951 & 7-May & 3.70 & 1,370 & 1967 & 29-Apr & -- & 1,820 \\
\hline 1952 & 20-Apr & 2.65 & 821 & 1968 & 1-May & -- & 785 \\
\hline 1953 & 28-May & 1.32 & 221 & 1969 & 17-Apr & -- & 1,214 \\
\hline 1954 & 3-May & 2.84 & 911 & 1970 & 3-Jan & -- & 195 \\
\hline 1955 & 30-May & 1.47 & 263 & 1971 & 21-Apr & -- & 153 \\
\hline 1956 & 21-Apr & -- & 1,259 & 1972 & 29-Apr & -- & 1,100 \\
\hline 1957 & 5-May & -- & 1,692 & 1973 & 5-May & -- & 547 \\
\hline 1958 & 27-Apr & 2.26 & 705 & 1974 & 15-May & -- & 245 \\
\hline 1959 & 23-Apr & 2.11 & 623 & 1975 & 18-May & -- & 851 \\
\hline 1960 & 18-May & 2.15 & 662 & 1976 & 25-Apr & -- & 1,724 \\
\hline 1961 & 7-May & -- & 911 & 1977 & 25-Apr & -- & 402 \\
\hline 1962 & 30-Nov & 1.08 & 209 & 1978 & 19-Apr & -- & 582 \\
\hline 1963 & 10-Jun & -- & 213 & 1979 & 18-Apr & -- & 685 \\
\hline \multicolumn{8}{|c|}{ Annual peak discharge, from highest to lowest } \\
\hline 1967 & 29-Apr & -- & 1,820 & 1958 & 27-Apr & 2.26 & 705 \\
\hline 1976 & 25-Apr & -- & 1,724 & 1964 & 1-May & -- & 693 \\
\hline 1957 & 5-May & -- & 1,692 & 1979 & 18-Apr & -- & 685 \\
\hline 1949 & 26-Apr & 3.79 & 1,418 & 1960 & 18-May & 2.15 & 662 \\
\hline 1951 & 7-May & 3.70 & 1,370 & 1959 & 23-Apr & 2.11 & 623 \\
\hline 1965 & 6-May & -- & 1,275 & 1978 & 19-Apr & -- & 582 \\
\hline 1956 & 21-Apr & -- & 1,259 & 1973 & 5-May & -- & 547 \\
\hline 1969 & 17-Apr & -- & 1,214 & 1977 & 25-Apr & -- & 402 \\
\hline 1972 & 29-Apr & -- & 1,100 & 1955 & 30-May & 1.47 & 263 \\
\hline 1950 & 11-May & 3.10 & 1,056 & 1974 & 15-May & -- & 245 \\
\hline 1961 & 7-May & -- & 911 & 1966 & 4-Nov & 1.16 & 232 \\
\hline 1954 & 3-May & 2.84 & 911 & 1953 & 28-May & 1.32 & 221 \\
\hline 1948 & 8-Mar & 2.76 & 877 & 1963 & 10-Jun & -- & 213 \\
\hline 1975 & 18-May & -- & 851 & 1962 & 30-Nov & 1.08 & 209 \\
\hline 1952 & 20-Apr & 2.65 & 821 & 1970 & 3-Jan & -- & 195 \\
\hline 1968 & 1-May & -- & 785 & 1971 & 21-Apr & -- & 153 \\
\hline
\end{tabular}




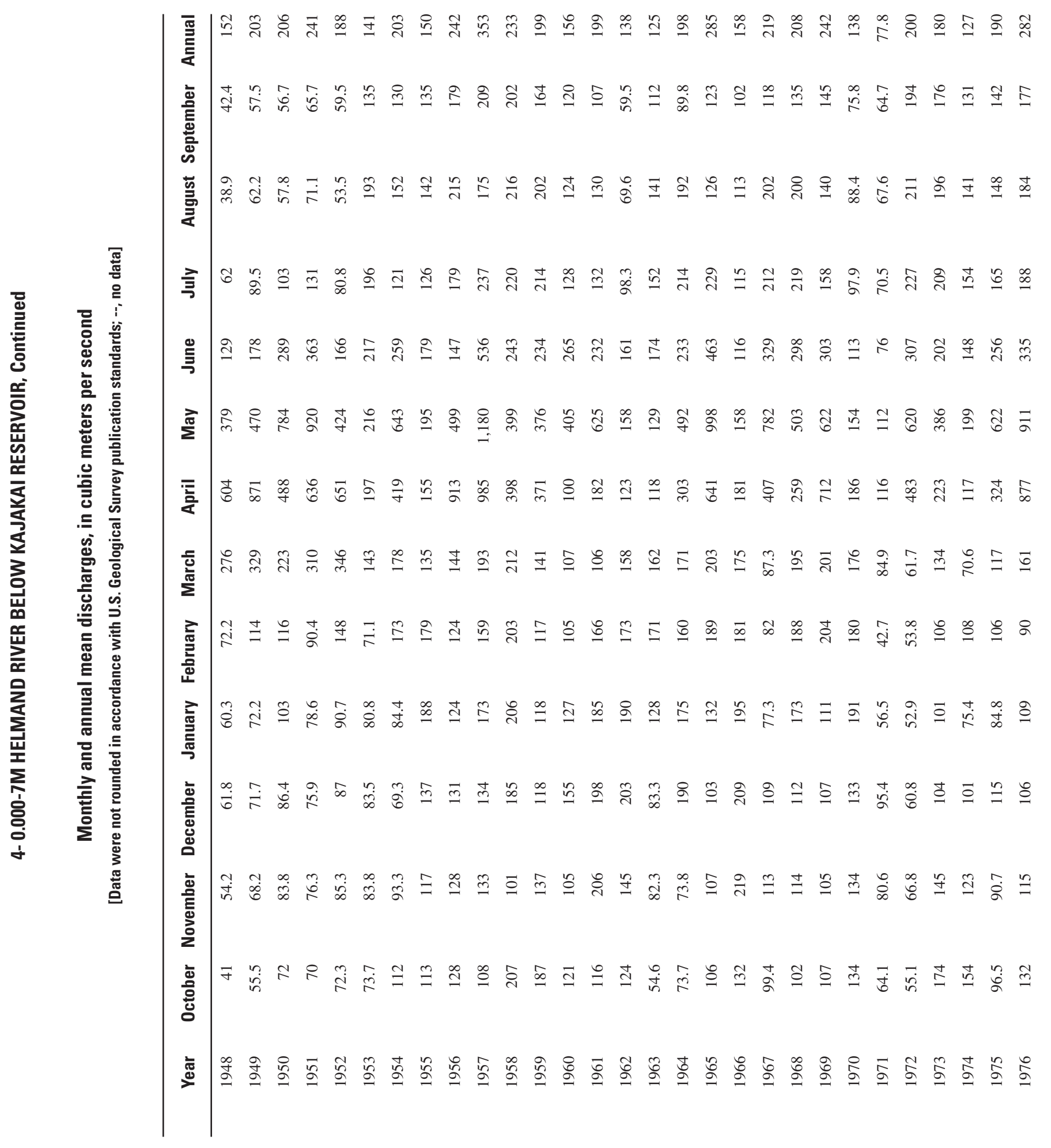




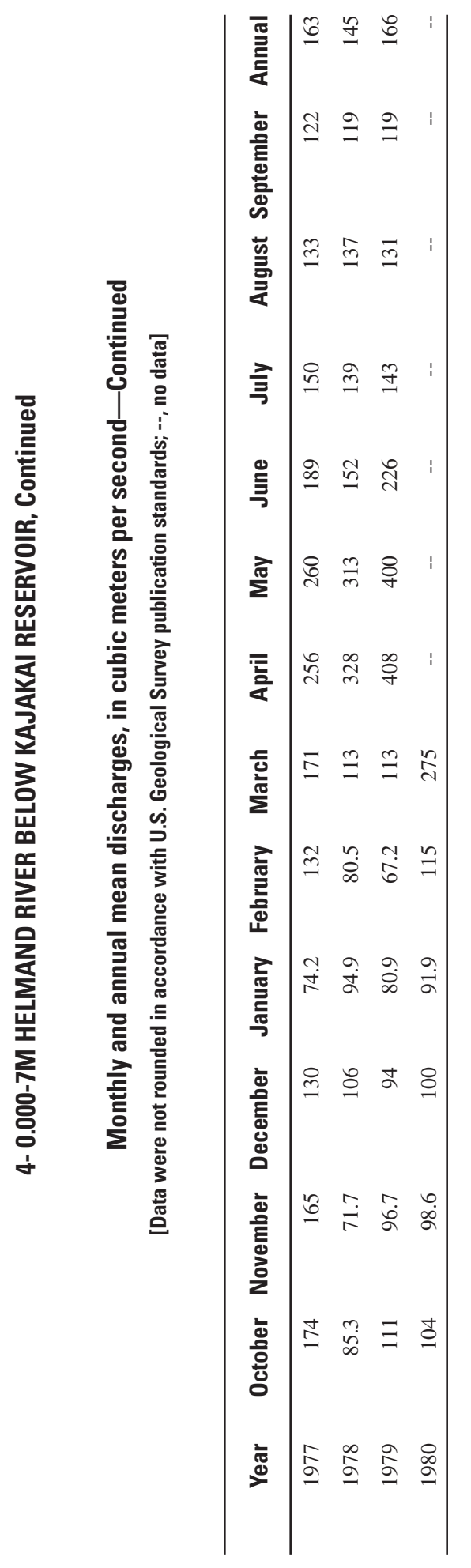




\section{4- 0.000-8M HELMAND RIVER AT DEHRAOUT}

\section{(U.S. Geological Survey identification number: 324200065280000)}

LOCATION: Lat $32^{\circ} 42^{\prime} \mathrm{N}$., long $65^{\circ} 28^{\prime} \mathrm{E}$., on right bank $10 \mathrm{~km}$ upstream from Dehraout, $15 \mathrm{~km}$ upstream from Tirin River, $70 \mathrm{~km}$ upstream from Kajakai Dam, and $120 \mathrm{~km}$ north of Kandahar.

DRAINAGE AREA: $36,990 \mathrm{~km}^{2}$ (Survey of India maps).

ALTITUDE: 1,065 meters plus mean sea level.

PERIOD OF RECORD: October 1, 1952 to September 30, 1979.

GAGE: Water-stage recorder. Prior to November 18, 1952, staff gage at site $2 \mathrm{~km}$ upstream at different datum.

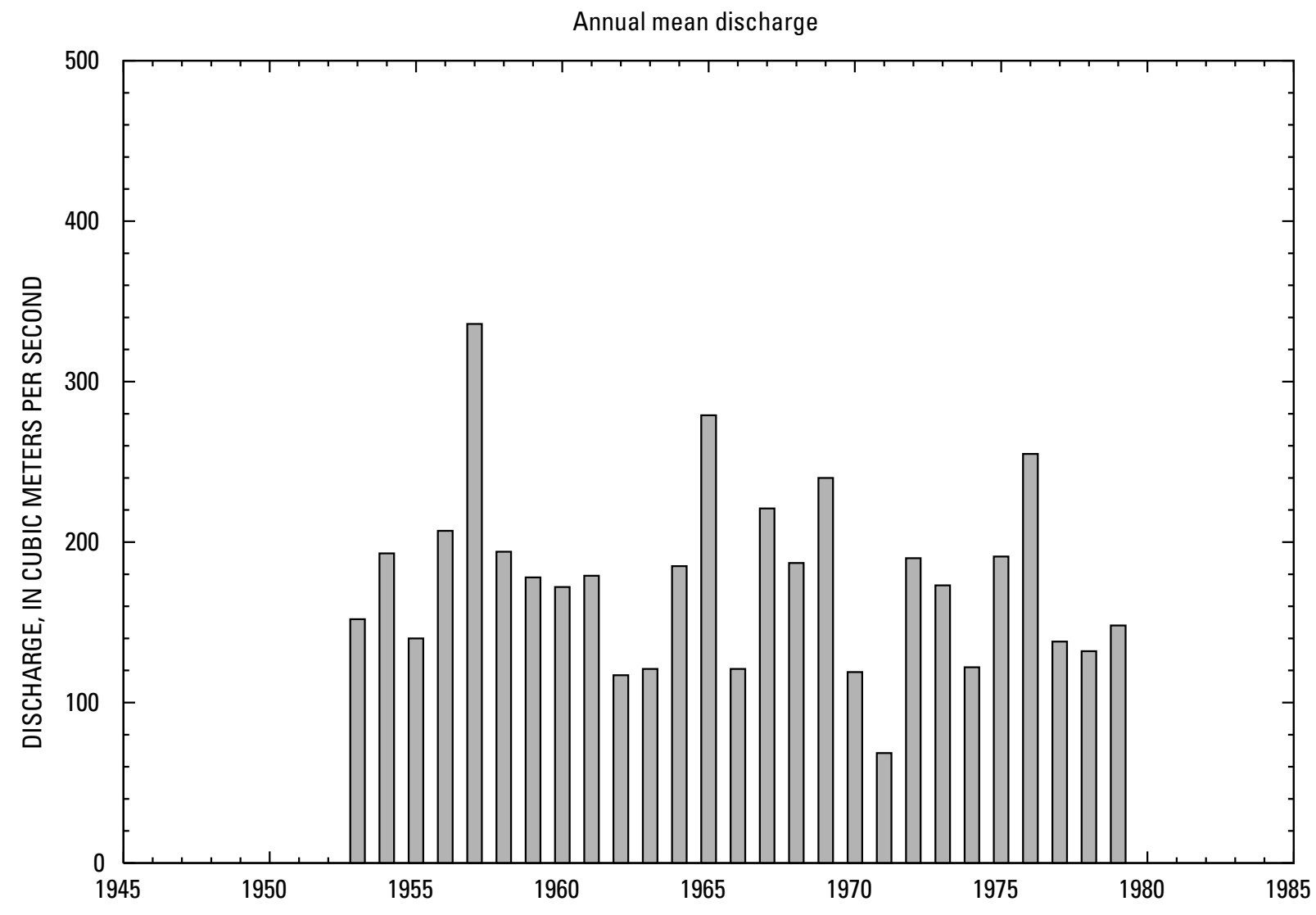




\section{4- 0.000-8M HELMAND RIVER AT DEHRAOUT, Continued}

\section{Statistics of monthly and annual mean discharges}

[ $\mathrm{m}^{3} / \mathrm{s}$, meters per second]

\begin{tabular}{|c|c|c|c|c|c|c|c|c|}
\hline \multirow[b]{2}{*}{ Month } & \multicolumn{2}{|c|}{ Maximum } & \multicolumn{2}{|c|}{ Minimum } & \multicolumn{4}{|c|}{ Mean } \\
\hline & $\begin{array}{c}\text { Discharge } \\
\left(\mathrm{m}^{3} / \mathrm{s}\right)\end{array}$ & $\begin{array}{c}\text { Water year } \\
\text { of } \\
\text { occurrence }\end{array}$ & $\begin{array}{c}\text { Discharge } \\
\left(\mathrm{m}^{3} / \mathrm{s}\right)\end{array}$ & $\begin{array}{c}\text { Water year } \\
\text { of } \\
\text { occurrence }\end{array}$ & $\begin{array}{c}\text { Discharge } \\
\left(\mathrm{m}^{3} / \mathrm{s}\right)\end{array}$ & $\begin{array}{c}\text { Standard } \\
\text { deviation } \\
\left(\mathrm{m}^{3} / \mathrm{s}\right)\end{array}$ & $\begin{array}{c}\text { Coefficient } \\
\text { of } \\
\text { variation }\end{array}$ & $\begin{array}{c}\text { Percentage } \\
\text { of annual } \\
\text { discharge }\end{array}$ \\
\hline October & 98.3 & 1958 & 37.0 & 1972 & 67.7 & 13.2 & 0.19 & 3.20 \\
\hline November & 141 & 1970 & 46.3 & 1972 & 79.0 & 19.5 & 0.25 & 3.73 \\
\hline December & 142 & 1958 & 47.6 & 1972 & 78.6 & 18.2 & 0.23 & 3.71 \\
\hline January & 137 & 1958 & 54.8 & 1967 & 80.1 & 17.8 & 0.22 & 3.79 \\
\hline February & 200 & 1965 & 68.8 & 1971 & 106 & 31.5 & 0.30 & 5.00 \\
\hline March & 492 & 1957 & 111 & 1971 & 280 & 106 & 0.38 & 13.2 \\
\hline April & 1,130 & 1957 & 199 & 1971 & 557 & 227 & 0.41 & 26.4 \\
\hline May & 1,160 & 1957 & 100 & 1971 & 461 & 235 & 0.51 & 21.8 \\
\hline June & 473 & 1957 & 36.8 & 1971 & 198 & 100 & 0.51 & 9.37 \\
\hline July & 176 & 1957 & 23.7 & 1971 & 91.8 & 39.7 & 0.43 & 4.34 \\
\hline August & 96.0 & 1965 & 21.1 & 1971 & 59.2 & 17.0 & 0.29 & 2.80 \\
\hline September & 77.6 & 1969 & 26.1 & 1971 & 56.8 & 12.8 & 0.23 & 2.68 \\
\hline Annual & 336 & 1957 & 68.6 & 1971 & 176 & 56.9 & 0.32 & 100 \\
\hline
\end{tabular}

Annual flow duration

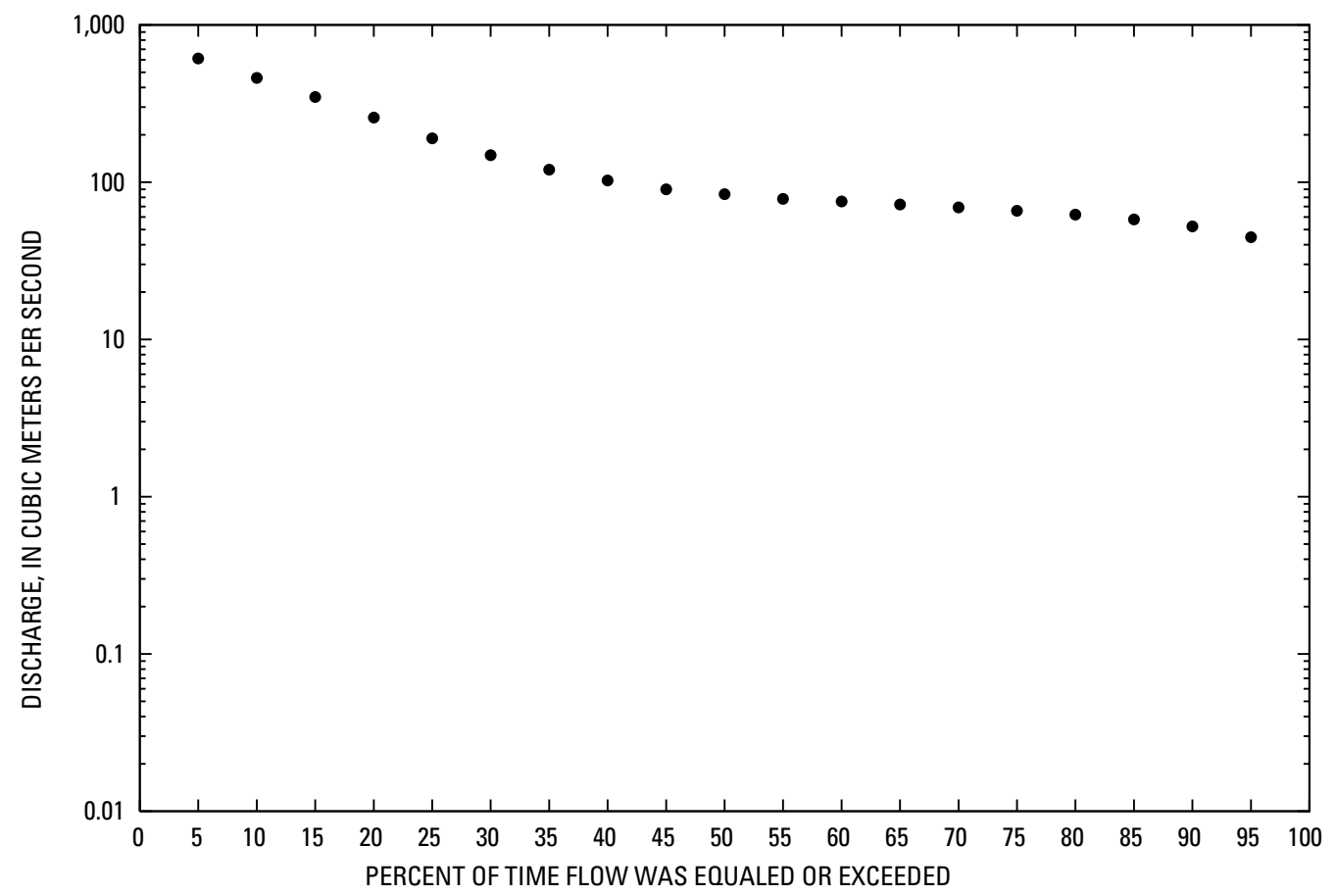




\section{4- 0.000-8M HELMAND RIVER AT DEHRAOUT, Continued}

Monthly and annual flow duration, in cubic meters per second

[Data were not rounded in accordance with U.S. Geological Survey publication standards]

\begin{tabular}{|c|c|c|c|c|c|c|c|c|c|c|c|c|c|}
\hline $\begin{array}{l}\text { Percentage } \\
\text { of days } \\
\text { discharge } \\
\text { equaled or } \\
\text { exceeded }\end{array}$ & January & February & March & April & May & June & July & August & September & October & November & December & Annual \\
\hline 95 & 56.6 & 63.0 & 91.8 & 195.8 & 150.4 & 61.4 & 40.9 & 35.4 & 38.6 & 48.6 & 58.5 & 56.8 & 44.7 \\
\hline 90 & 59.9 & 67.2 & 109.6 & 251.1 & 195.0 & 78.2 & 46.8 & 39.1 & 42.4 & 52.0 & 60.7 & 60.9 & 52.4 \\
\hline 80 & 65.8 & 73.4 & 134.6 & 342.5 & 245.7 & 107.9 & 54.1 & 44.2 & 44.8 & 57.1 & 64.6 & 65.5 & 62.2 \\
\hline 75 & 68.2 & 75.6 & 148.8 & 374.5 & 274.2 & 119.9 & 58.1 & 46.1 & 46.2 & 58.8 & 66.6 & 67.3 & 65.7 \\
\hline 70 & 69.7 & 77.9 & 165.4 & 405.1 & 302.7 & 129.7 & 62.9 & 48.0 & 47.8 & 60.5 & 68.8 & 69.0 & 69.1 \\
\hline 65 & 71.2 & 80.8 & 179.7 & 436.3 & 327.1 & 139.6 & 68.5 & 50.5 & 50.5 & 62.1 & 71.2 & 70.6 & 72.2 \\
\hline 45 & 76.8 & 100.4 & 261.8 & 549.8 & 430.0 & 181.9 & 86.1 & 61.8 & 59.3 & 67.9 & 76.5 & 75.4 & 90.0 \\
\hline 40 & 78.7 & 106.2 & 287.6 & 577.6 & 460.4 & 193.6 & 91.8 & 63.9 & 62.2 & 69.5 & 77.8 & 77.2 & 102.6 \\
\hline 35 & 80.6 & 111.1 & 310.1 & 599.3 & 501.3 & 208.3 & 98.2 & 66.1 & 64.0 & 71.5 & 79.8 & 80.4 & 120.1 \\
\hline 30 & 82.9 & 115.6 & 334.6 & 621.0 & 539.9 & 225.6 & 104.7 & 68.2 & 65.5 & 73.4 & 82.2 & 83.1 & 148.6 \\
\hline 25 & 85.7 & 119.7 & 370.2 & 663.3 & 582.7 & 246.2 & 111.2 & 70.4 & 66.8 & 74.8 & 84.9 & 85.7 & 190.1 \\
\hline 20 & 89.3 & 129.4 & 405.6 & 716.6 & 642.1 & 272.6 & 120.9 & 72.5 & 68.9 & 76.9 & 87.9 & 88.6 & 257.2 \\
\hline 15 & 96.6 & 141.9 & 466.0 & 791.3 & 722.1 & 309.2 & 133.1 & 75.6 & 71.5 & 81.4 & 91.8 & 93.1 & 348.4 \\
\hline 10 & 106.0 & 159.4 & 517.6 & 946.0 & 846.1 & 352.0 & 151.1 & 80.4 & 74.3 & 86.0 & 99.1 & 100.9 & 459.9 \\
\hline 5 & 124.2 & 187.5 & 582.8 & 1106.0 & 952.4 & 439.3 & 178.3 & 91.7 & 77.2 & 92.3 & 121.2 & 110.8 & 613.0 \\
\hline
\end{tabular}




\section{4- 0.000-8M HELMAND RIVER AT DEHRAOUT, Continued}

Probability of occurrence of annual high discharges

[ $\mathrm{m}^{3} / \mathrm{s}$, meters per second; ng, statistic not given]

\begin{tabular}{rrrrrrr}
\hline & & & \multicolumn{5}{c}{ Maximum mean discharge } \\
\cline { 5 - 7 } $\begin{array}{c}\text { Exceedance } \\
\text { probability }\end{array}$ & $\begin{array}{c}\text { Recurrence } \\
\text { interval } \\
\text { (years) }\end{array}$ & $\begin{array}{c}\text { Maximum } \\
\text { instantaneous } \\
\left(\mathbf{m}^{3} / \mathbf{s}\right)\end{array}$ & 3-day period & 7-day period & 15-day period & 30-day period \\
\cline { 5 - 7 } & 1.01 & 278 & 268 & 260 & 234 & 206 \\
0.99 & 1.05 & 396 & 366 & 347 & 318 & 285 \\
0.95 & 1.11 & 475 & 432 & 405 & 372 & 337 \\
0.90 & 1.25 & 590 & 526 & 488 & 450 & 409 \\
0.80 & 2 & 880 & 763 & 696 & 638 & 584 \\
0.50 & 5 & 1,280 & 1,100 & 988 & 892 & 816 \\
0.20 & 10 & 1,550 & 1,320 & 1,190 & 1,060 & 963 \\
0.10 & 25 & 1,890 & 1,620 & 1,440 & 1,260 & 1,140 \\
0.04 & 50 & 2,140 & 1,840 & 1,630 & 1,410 & 1,270 \\
0.02 & 100 & 2,390 & 2,060 & 1,830 & 1,560 & 1,390 \\
0.01 & 200 & 2,640 & 2,280 & 2,020 & 1,710 & 1,510 \\
0.005 & 500 & 2,970 & ng & ng & ng & ng \\
\hline 0.002 & & & & &
\end{tabular}

\section{4- 0.000-8M HELMAND RIVER AT DEHRAOUT, Continued}

Probability of occurrence of annual low discharges

$\left[\mathrm{m}^{3} / \mathrm{s}\right.$, meters per second]

\begin{tabular}{|c|c|c|c|c|c|c|c|c|c|c|}
\hline \multirow{3}{*}{$\begin{array}{c}\text { Nonexceedance } \\
\text { probability }\end{array}$} & \multirow{3}{*}{$\begin{array}{c}\text { Recurrence } \\
\text { interval } \\
\text { (years) }\end{array}$} & \multicolumn{9}{|c|}{ Minimum mean discharge $\left(\mathrm{m}^{3} / \mathrm{s}\right)$} \\
\hline & & \multicolumn{9}{|c|}{ Number of consecutive days } \\
\hline & & 1 & 3 & 7 & 14 & 30 & 60 & 90 & 120 & 183 \\
\hline 0.05 & 20 & 28.9 & 29.1 & 29.4 & 29.8 & 30.4 & 31.8 & 33.7 & 36.4 & 42.7 \\
\hline 0.10 & 10 & 34.2 & 34.5 & 34.8 & 35.2 & 36.0 & 37.7 & 39.7 & 42.6 & 48.2 \\
\hline 0.20 & 5 & 41.0 & 41.3 & 41.6 & 42.1 & 43.0 & 45.1 & 47.3 & 50.2 & 55.1 \\
\hline 0.50 & 2 & 53.9 & 54.3 & 54.7 & 55.2 & 56.3 & 58.9 & 61.6 & 64.9 & 68.9 \\
\hline
\end{tabular}




\section{4- 0.000-8M HELMAND RIVER AT DEHRAOUT, Continued}

\section{Probability of occurrence of seasonal low discharges}

[ $\mathrm{m}^{3} / \mathrm{s}$, meters per second]

\begin{tabular}{|c|c|c|c|c|c|c|c|c|c|}
\hline \multirow{3}{*}{$\begin{array}{c}\text { Nonexceedance } \\
\text { probability }\end{array}$} & \multirow{3}{*}{$\begin{array}{c}\text { Recurrence } \\
\text { interval } \\
\text { (years) }\end{array}$} & \multicolumn{8}{|c|}{ Minimum mean discharge $\left(\mathrm{m}^{3} / \mathrm{s}\right)$} \\
\hline & & \multicolumn{8}{|c|}{ Number of consecutive days } \\
\hline & & 1 & 7 & 14 & 30 & 1 & 7 & 14 & 30 \\
\hline & & \multicolumn{4}{|c|}{ December-January-February } & \multicolumn{4}{|c|}{ March-April-May } \\
\hline 0.05 & 20 & 49.7 & 52.9 & 53.8 & 55.6 & 65.9 & 74.9 & 86.8 & 114 \\
\hline 0.10 & 10 & 51.9 & 55.2 & 56.4 & 58.2 & 76.5 & 87.6 & 102 & 139 \\
\hline 0.20 & 5 & 55.2 & 58.7 & 60.1 & 62.0 & 91.0 & 106 & 124 & 174 \\
\hline \multirow[t]{2}{*}{0.50} & 2 & 63.6 & 67.4 & 69.4 & 71.4 & 124 & 150 & 178 & 256 \\
\hline & & \multicolumn{4}{|c|}{ June-July-August } & \multicolumn{4}{|c|}{ September-October-November } \\
\hline 0.05 & 20 & 29.0 & 29.5 & 30.0 & 30.8 & 30.4 & 31.0 & 31.9 & 34.8 \\
\hline 0.10 & 10 & 34.2 & 34.8 & 35.3 & 36.4 & 35.8 & 36.3 & 37.2 & 39.8 \\
\hline 0.20 & 5 & 40.8 & 41.6 & 42.2 & 43.7 & 42.5 & 43.0 & 43.8 & 46.1 \\
\hline 0.50 & 2 & 54.0 & 55.0 & 55.9 & 58.6 & 55.1 & 55.6 & 56.3 & 58.0 \\
\hline
\end{tabular}




\section{4- 0.000-8M HELMAND RIVER AT DEHRAOUT, Continued}

\section{Annual peak discharge and corresponding gage height}

$\left[\mathrm{m}^{3} / \mathrm{s}\right.$, meters per second; --, no data]

\begin{tabular}{|c|c|c|c|c|c|c|c|}
\hline $\begin{array}{c}\text { Water } \\
\text { year }\end{array}$ & Date & $\begin{array}{c}\text { Gage } \\
\text { height } \\
\text { (meters) }\end{array}$ & $\begin{array}{c}\text { Peak } \\
\text { discharge } \\
\left(\mathrm{m}^{3} / \mathrm{s}\right)\end{array}$ & $\begin{array}{c}\text { Water } \\
\text { year }\end{array}$ & Date & $\begin{array}{c}\text { Gage } \\
\text { height } \\
\text { (meters) }\end{array}$ & $\begin{array}{c}\text { Peak } \\
\text { discharge } \\
\left(\mathrm{m}^{3} / \mathrm{s}\right)\end{array}$ \\
\hline \multicolumn{8}{|c|}{ Annual peak discharge, by year } \\
\hline 1953 & 7-Mar & & 693 & 1967 & 28-Apr & & 1,750 \\
\hline 1954 & 30-Mar & & 860 & 1968 & 30-Apr & & 1,106 \\
\hline 1955 & 15-Mar & . & 1,152 & 1969 & 15-Apr & & 1,969 \\
\hline 1956 & 2-Apr & & 1,361 & 1970 & 12-Apr & & 399 \\
\hline 1957 & 3-May & . & 1,873 & 1971 & 14-Apr & & 290 \\
\hline 1958 & 27-Apr & & 736 & 1972 & 28-Apr & & 1,030 \\
\hline 1959 & 11-Apr & & 691 & 1973 & 25-Apr & & 630 \\
\hline 1960 & 3-May & . & 951 & 1974 & 23-Mar & & 555 \\
\hline 1961 & 3-Apr & . & 903 & 1975 & 4-Apr & & 1,264 \\
\hline 1962 & 1-May & & 540 & 1976 & 25-Apr & & 1,529 \\
\hline 1963 & 13-May & . & 722 & 1977 & 14-Apr & & 573 \\
\hline 1964 & 24-Apr & . & 1,105 & 1978 & 17-Apr & & 714 \\
\hline 1965 & 16-Apr & . & 1,079 & 1979 & 30-Mar & & 818 \\
\hline 1966 & 13-Apr & . & 588 & & & & \\
\hline \multicolumn{8}{|c|}{ Annual peak discharge, from highest to lowest } \\
\hline 1969 & 15-Apr & & 1,969 & 1979 & 30-Mar & & 818 \\
\hline 1957 & 3-May & & 1,873 & 1958 & 27-Apr & & 736 \\
\hline 1967 & 28-Apr & . & 1,750 & 1963 & 13-May & & 722 \\
\hline 1976 & 25-Apr & & 1,529 & 1978 & 17-Apr & & 714 \\
\hline 1956 & 2-Apr & . & 1,361 & 1953 & 7-Mar & & 693 \\
\hline 1975 & 4-Apr & . & 1,264 & 1959 & 11-Apr & & 691 \\
\hline 1955 & 15-Mar & . & 1,152 & 1973 & 25-Apr & & 630 \\
\hline 1968 & 30-Apr & & 1,106 & 1966 & 13-Apr & & 588 \\
\hline 1964 & 24-Apr & . & 1,105 & 1977 & 14-Apr & & 573 \\
\hline 1965 & 16-Apr & . & 1,079 & 1974 & 23-Mar & & 555 \\
\hline 1972 & 28-Apr & & 1,030 & 1962 & 1-May & & 540 \\
\hline 1960 & 3-May & . & 951 & 1970 & 12-Apr & & 399 \\
\hline 1961 & 3-Apr & . & 903 & 1971 & 14-Apr & & 290 \\
\hline 1954 & 30-Mar & . & 860 & & & & \\
\hline
\end{tabular}




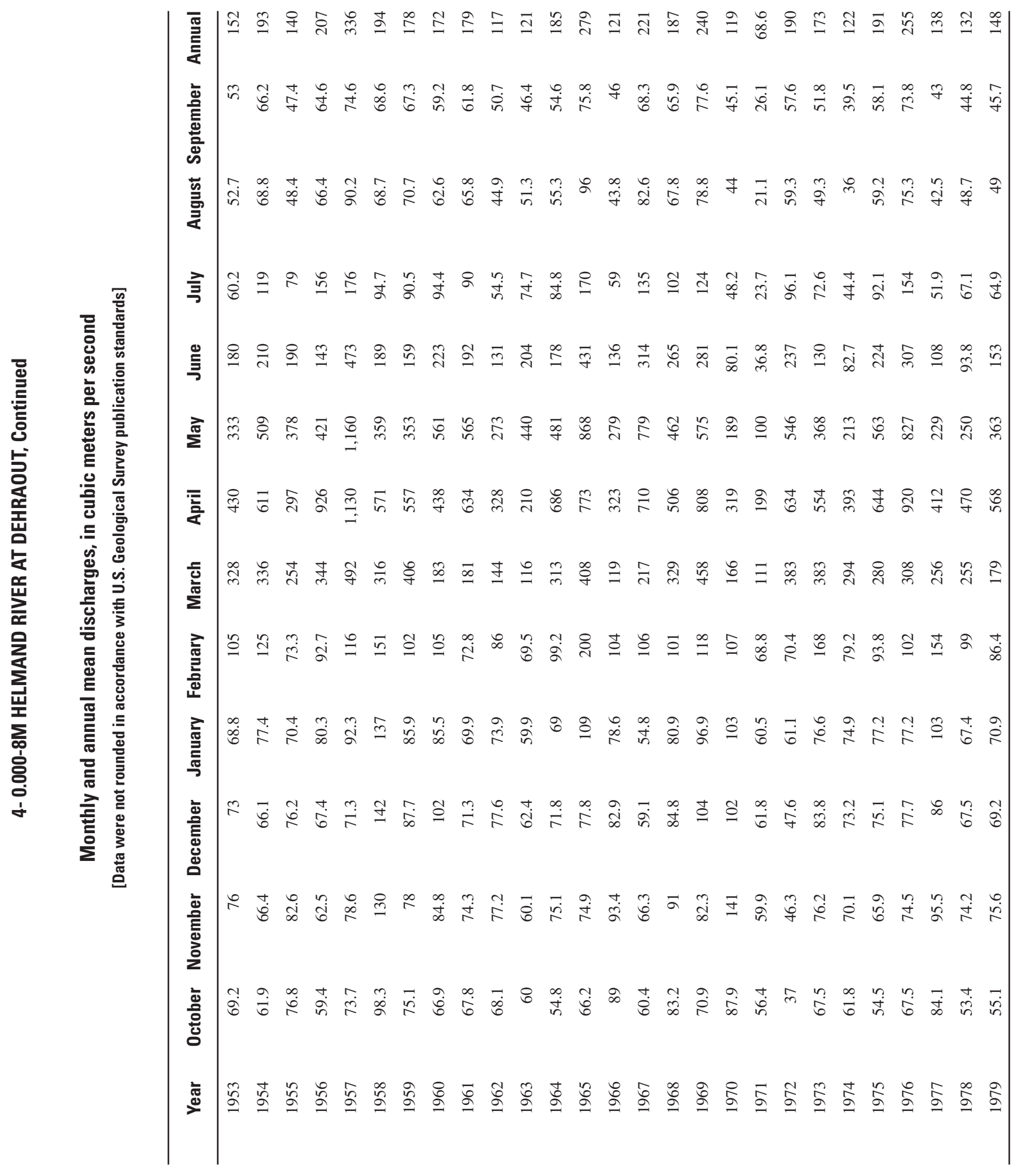




\section{4- 0.000-9M HELMAND RIVER AT GIZAB}

\section{(U.S. Geological Survey identification number: 332300066170000 )}

LOCATION: Lat $33^{\circ} 23^{\prime} \mathrm{N}$., long $66^{\circ} 17^{\prime} \mathrm{E}$.

DRAINAGE AREA: $20,750 \mathrm{~km}^{2}$.

ALTITUDE: 1,325 meters plus mean sea level.

PERIOD OF RECORD: July 19, 1971 to March 11, 1979.

GAGE: --

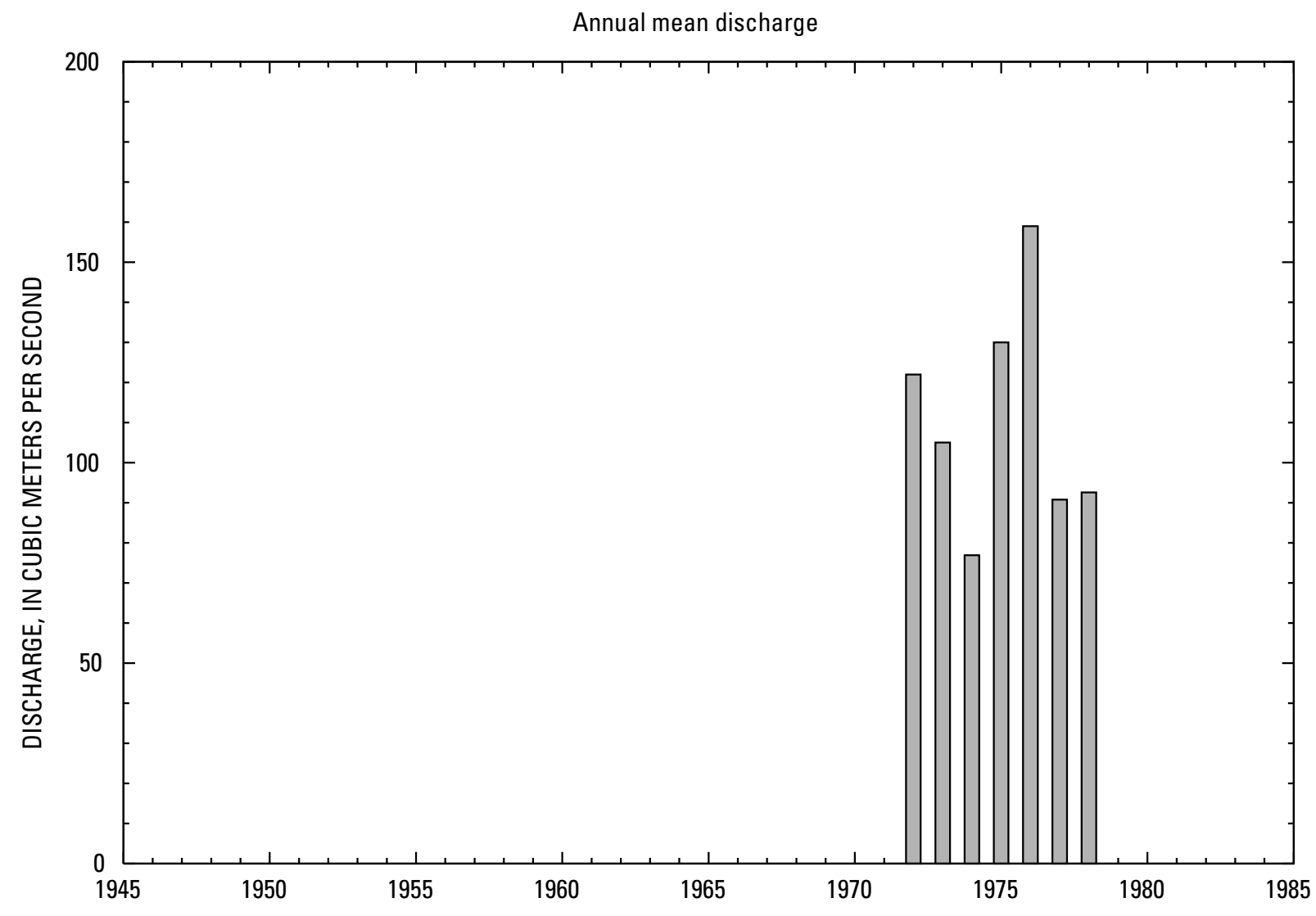




\section{4- 0.000-9M HELMAND RIVER AT GIZAB, Continued}

\section{Statistics of monthly and annual mean discharges}

[ $\mathrm{m}^{3} / \mathrm{s}$, meters per second]

\begin{tabular}{|c|c|c|c|c|c|c|c|c|}
\hline \multirow[b]{2}{*}{ Month } & \multicolumn{2}{|c|}{ Maximum } & \multicolumn{2}{|c|}{ Minimum } & \multicolumn{4}{|c|}{ Mean } \\
\hline & $\begin{array}{c}\text { Discharge } \\
\left(\mathrm{m}^{3} / \mathrm{s}\right)\end{array}$ & $\begin{array}{c}\text { Water year } \\
\text { of } \\
\text { occurrence }\end{array}$ & $\begin{array}{c}\text { Discharge } \\
\left(\mathrm{m}^{3} / \mathrm{s}\right)\end{array}$ & $\begin{array}{c}\text { Water year } \\
\text { of } \\
\text { occurrence }\end{array}$ & $\begin{array}{c}\text { Discharge } \\
\left(\mathrm{m}^{3} / \mathrm{s}\right)\end{array}$ & $\begin{array}{c}\text { Standard } \\
\text { deviation } \\
\left(\mathrm{m}^{3} / \mathrm{s}\right)\end{array}$ & $\begin{array}{c}\text { Coefficient } \\
\text { of } \\
\text { variation }\end{array}$ & $\begin{array}{c}\text { Percentage } \\
\text { of annual } \\
\text { discharge }\end{array}$ \\
\hline October & 50.0 & 1977 & 28.2 & 1972 & 41.3 & 6.56 & 0.16 & 3.11 \\
\hline November & 61.2 & 1977 & 32.2 & 1972 & 47.4 & 8.58 & 0.18 & 3.57 \\
\hline December & 57.3 & 1977 & 30.5 & 1972 & 46.8 & 8.09 & 0.17 & 3.53 \\
\hline January & 60.8 & 1977 & 32.7 & 1972 & 48.0 & 8.42 & 0.18 & 3.61 \\
\hline February & 102 & 1973 & 35.7 & 1972 & 66.8 & 22.4 & 0.33 & 5.03 \\
\hline March & 259 & 1972 & 143 & 1978 & 186 & 39.4 & 0.21 & 14.0 \\
\hline April & 563 & 1976 & 230 & 1974 & 360 & 112 & 0.31 & 27.1 \\
\hline May & 543 & 1976 & 137 & 1974 & 286 & 142 & 0.50 & 21.5 \\
\hline June & 189 & 1976 & 71.5 & 1974 & 119 & 52.7 & 0.44 & 8.99 \\
\hline July & 95.3 & 1976 & 30.2 & 1974 & 57.8 & 22.0 & 0.38 & 4.35 \\
\hline August & 45.1 & 1975 & 18.3 & 1971 & 34.1 & 8.87 & 0.26 & 2.57 \\
\hline September & 46.3 & 1976 & 22.0 & 1971 & 35.0 & 8.47 & 0.24 & 2.64 \\
\hline Annual & 159 & 1976 & 76.9 & 1974 & 111 & 28.1 & 0.25 & 100 \\
\hline
\end{tabular}

Annual flow duration

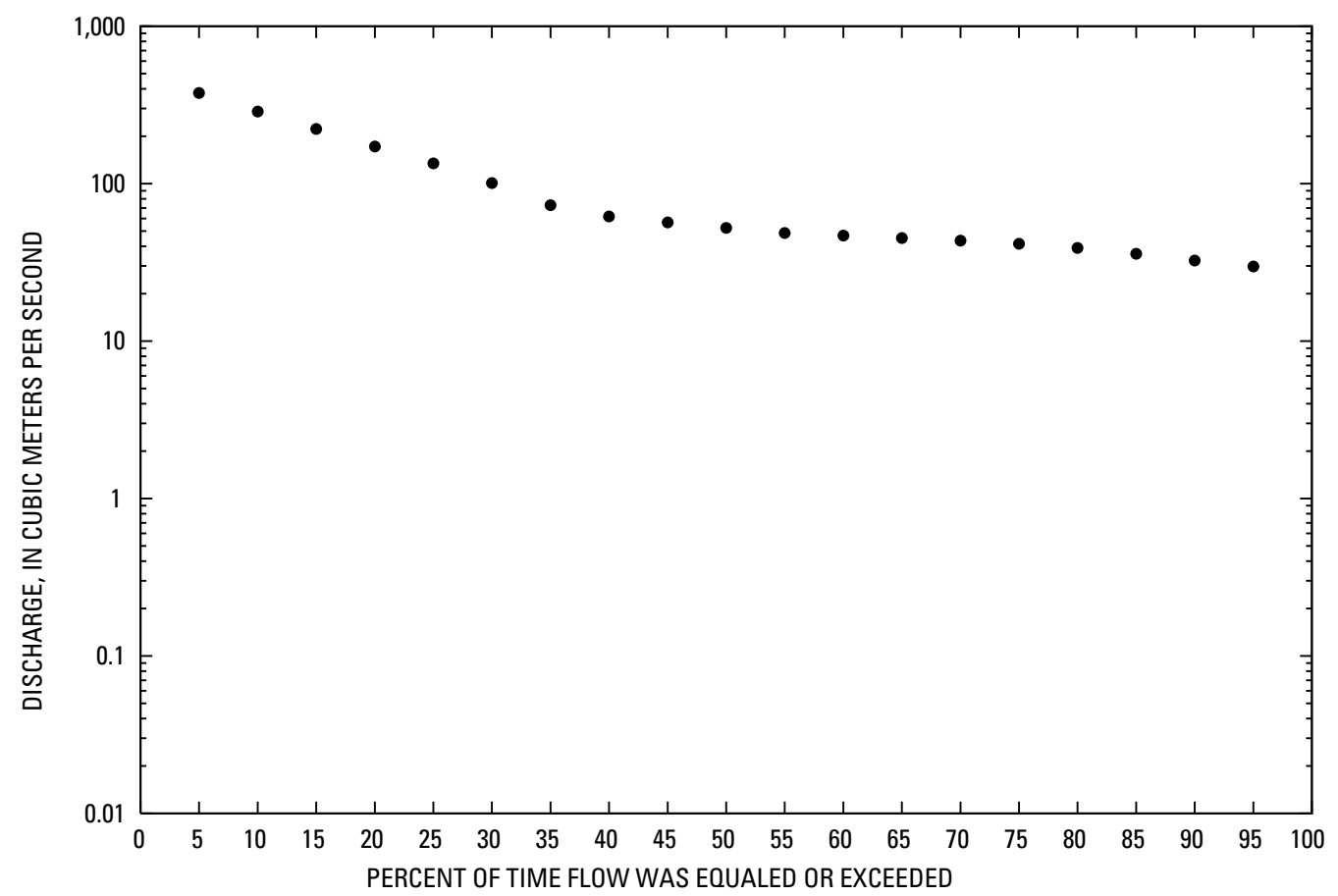




\section{4- 0.000-9M HELMAND RIVER AT GIZAB, Continued}

\section{Monthly and annual flow duration, in cubic meters per second}

[Data were not rounded in accordance with U.S. Geological Survey publication standards]

\begin{tabular}{|c|c|c|c|c|c|c|c|c|c|c|c|c|c|}
\hline $\begin{array}{l}\text { Percentage } \\
\text { of days } \\
\text { discharge } \\
\text { equaled or } \\
\text { exceeded }\end{array}$ & January & February & March & April & May & June & July & August & September & October & November & December & Annual \\
\hline 95 & 29.6 & 34.2 & 74.5 & 210.1 & 114.7 & 54.2 & 26.9 & 18.4 & 21.1 & 27.8 & 31.9 & 30.5 & 29.8 \\
\hline 90 & 36.5 & 38.2 & 106.9 & 231.5 & 142.9 & 58.9 & 32.5 & 19 & 23.8 & 29.7 & 33.1 & 31.2 & 32.5 \\
\hline 80 & 41.9 & 47 & 117.9 & 248.9 & 163.6 & 65.8 & 36.4 & 26.6 & 26.2 & 36.6 & 40.2 & 42.5 & 39.1 \\
\hline 75 & 42.9 & 49 & 125.3 & 257.7 & 171.7 & 69 & 38.2 & 27.7 & 27 & 38 & 43.9 & 43.4 & 41.5 \\
\hline 70 & 44.6 & 51 & 135.7 & 271 & 180.2 & 74.9 & 40.2 & 30.3 & 31.5 & 40.4 & 45.1 & 43.9 & 43.5 \\
\hline 65 & 45.2 & 53.1 & 143.3 & 288.5 & 189.9 & 80.8 & 42 & 30.9 & 32.5 & 41.2 & 45.8 & 44.5 & 45.2 \\
\hline 60 & 46 & 55.3 & 148.5 & 301 & 207.7 & 87 & 44.3 & 31.8 & 33.2 & 41.9 & 46.8 & 45.5 & 46.8 \\
\hline 40 & 50.9 & 64.1 & 172 & 355.3 & 286.5 & 119.9 & 58 & 36.1 & 37.4 & 43.3 & 48.1 & 48.9 & 61.9 \\
\hline 35 & 51.8 & 67.3 & 180.7 & 366.9 & 322.2 & 127.9 & 60.6 & 39.1 & 38.8 & 43.6 & 48.7 & 50 & 72.9 \\
\hline 30 & 52.5 & 70 & 195.8 & 379.6 & 344 & 139 & 65.5 & 41.1 & 40.8 & 44.5 & 49.5 & 51.8 & 100.8 \\
\hline 25 & 53.1 & 73.5 & 220.3 & 394.5 & 360.3 & 155.3 & 70.7 & 41.9 & 42.3 & 46 & 51 & 52.8 & 134.4 \\
\hline 20 & 55.5 & 78.8 & 244.6 & 411.3 & 384.1 & 173.6 & 76.8 & 42.6 & 43 & 46.4 & 53.7 & 54.2 & 171.9 \\
\hline 15 & 57 & 91.4 & 273.1 & 431.6 & 414.5 & 188.9 & 82.9 & 43.4 & 44.2 & 47 & 58.6 & 56.1 & 222.5 \\
\hline 10 & 58.2 & 103.9 & 311.9 & 499 & 540.9 & 206.9 & 90.3 & 44.2 & 45.4 & 47.8 & 59.7 & 57.4 & 287.5 \\
\hline 5 & 62.6 & 124.9 & 387.3 & 725 & 618.5 & 250.2 & 99.9 & 46.4 & 46.1 & 50.4 & 62.1 & 58.7 & 376.7 \\
\hline
\end{tabular}




\section{4- 0.000-9M HELMAND RIVER AT GIZAB, Continued}

Probability of occurrence of annual high discharges

$\left[\mathrm{m}^{3} / \mathrm{s}\right.$, meters per second; $\mathrm{ng}$, statistic not given]

\begin{tabular}{rrrrrrr}
\hline & & & \multicolumn{5}{c}{ Maximum mean discharge } \\
$\begin{array}{c}\text { Exceedance } \\
\text { probability }\end{array}$ & $\begin{array}{c}\text { Recurrence } \\
\text { interval } \\
\text { (years) }\end{array}$ & $\begin{array}{c}\text { Maximum } \\
\text { instantaneous } \\
\left(\mathbf{m}^{\mathbf{3}} / \mathbf{s}\right)\end{array}$ & 3-day period & 7-day period & 15-day period & 30-day period \\
\cline { 5 - 7 } & 1.01 & 212 & 211 & 2223 & 2230 & 2215 \\
0.99 & 1.05 & 258 & 251 & 251 & 248 & 233 \\
0.95 & 1.11 & 292 & 279 & 273 & 264 & 247 \\
0.90 & 1.25 & 344 & 324 & 307 & 289 & 271 \\
0.50 & 2 & 496 & 451 & 412 & 369 & 343 \\
0.20 & 5 & 768 & 678 & 609 & 525 & 479 \\
0.10 & 10 & 994 & 864 & 778 & 661 & 595 \\
0.04 & 25 & 1,340 & 1,150 & 1,050 & 877 & 776 \\
0.02 & 50 & 1,650 & 1,400 & 1,290 & 1,080 & 939 \\
0.01 & 100 & 2,000 & 1,680 & 1,580 & 1,310 & 1,130 \\
0.005 & 200 & 2,410 & 2,010 & 1,920 & 1,590 & 1,350 \\
0.002 & 500 & 3,040 & ng & ng & ng & ng \\
\hline
\end{tabular}

'Less than 10 years data used.

${ }^{2}$ Data does not fit log-Pearson Type III curve, use with caution.

\section{4- 0.000-9M HELMAND RIVER AT GIZAB, Continued}

Probability of occurrence of annual low discharges

$\left[\mathrm{m}^{3} / \mathrm{s}\right.$, meters per second]

\begin{tabular}{|c|c|c|c|c|c|c|c|c|c|c|}
\hline \multirow{3}{*}{$\begin{array}{c}\text { Nonexceedance } \\
\text { probability }\end{array}$} & \multirow{3}{*}{$\begin{array}{c}\text { Recurrence } \\
\text { interval } \\
\text { (years) }\end{array}$} & \multicolumn{9}{|c|}{ Minimum mean discharge $\left(\mathrm{m}^{3} / \mathrm{s}\right)$} \\
\hline & & \multicolumn{9}{|c|}{ Number of consecutive days } \\
\hline & & 1 & 3 & 7 & 14 & 30 & 60 & 90 & 120 & 183 \\
\hline 0.05 & 20 & 23.0 & 23.0 & 122.9 & 23.4 & 24.3 & 24.6 & 25.6 & 28.5 & 33.5 \\
\hline 0.10 & 10 & 25.1 & 25.3 & 125.2 & 25.7 & 26.6 & 27.2 & 28.5 & 31.1 & 35.3 \\
\hline 0.20 & 5 & 27.9 & 28.1 & 28.2 & 28.5 & 29.4 & 30.4 & 32.0 & 34.3 & 37.7 \\
\hline 0.50 & 2 & 33.3 & 33.8 & 34.0 & 34.2 & 35.1 & 36.8 & 38.6 & 40.6 & 42.7 \\
\hline
\end{tabular}

'Data does not fit log-Pearson Type III curve, use with caution. 


\section{4- 0.000-9M HELMAND RIVER AT GIZAB, Continued}

\section{Probability of occurrence of seasonal low discharges}

$\left[\mathrm{m}^{3} / \mathrm{s}\right.$, meters per second]

\begin{tabular}{|c|c|c|c|c|c|c|c|c|c|}
\hline \multirow{3}{*}{$\begin{array}{c}\text { Nonexceedance } \\
\text { probability }\end{array}$} & \multirow{3}{*}{$\begin{array}{c}\text { Recurrence } \\
\text { interval } \\
\text { (years) }\end{array}$} & \multicolumn{8}{|c|}{ Minimum mean discharge $\left(\mathrm{m}^{3} / \mathrm{s}\right)$} \\
\hline & & \multicolumn{8}{|c|}{ Number of consecutive days } \\
\hline & & 1 & 7 & 14 & 30 & 1 & 7 & 14 & 30 \\
\hline & & \multicolumn{4}{|c|}{ December-January-February } & \multicolumn{4}{|c|}{ March-April-May } \\
\hline 0.05 & 20 & 25.4 & 27.5 & 28.8 & 30.2 & 38.0 & 61.1 & 97.2 & 124 \\
\hline 0.10 & 10 & 28.1 & 30.8 & 32.3 & 33.9 & 46.0 & 71.5 & 102 & 132 \\
\hline 0.20 & 5 & 31.6 & 34.8 & 36.6 & 38.3 & 57.3 & 85.1 & 108 & 144 \\
\hline \multirow[t]{2}{*}{0.50} & 2 & 38.7 & 42.6 & 44.4 & 46.2 & 84.1 & 113 & 124 & 172 \\
\hline & & \multicolumn{4}{|c|}{ June-July-August } & \multicolumn{4}{|c|}{ September-October-November } \\
\hline 0.05 & 20 & 24.2 & 24.3 & 24.7 & 25.2 & 18.2 & 18.9 & 19.8 & 21.5 \\
\hline 0.10 & 10 & 25.8 & 26.0 & 26.5 & 27.3 & 21.1 & 21.8 & 22.6 & 24.3 \\
\hline 0.20 & 5 & 28.1 & 28.2 & 28.8 & 30.0 & 24.9 & 25.5 & 26.2 & 27.8 \\
\hline 0.50 & 2 & 33.0 & 33.1 & 33.8 & 35.6 & 32.4 & 32.9 & 33.6 & 34.9 \\
\hline
\end{tabular}

\section{4- 0.000-9M HELMAND RIVER AT GIZAB, Continued}

\section{Annual peak discharge and corresponding gage height}

$\left[\mathrm{m}^{3} / \mathrm{s}\right.$, meters per second; --, no data]

\begin{tabular}{cccccccc}
\hline $\begin{array}{c}\text { Water } \\
\text { year }\end{array}$ & Date & $\begin{array}{c}\text { Gage } \\
\text { height } \\
(\text { meters })\end{array}$ & $\begin{array}{c}\text { Peak } \\
\text { discharge } \\
\left(\mathrm{m}^{3} / \mathrm{s}\right)\end{array}$ & $\begin{array}{c}\text { Water } \\
\text { year }\end{array}$ & Date & $\begin{array}{c}\text { Gage } \\
\text { height } \\
(\text { meters })\end{array}$ & $\begin{array}{c}\text { Peak } \\
\text { discharge } \\
\left(\mathrm{m}^{3} / \mathrm{s}\right)\end{array}$ \\
\hline
\end{tabular}

Annual peak discharge, by year

\begin{tabular}{|c|c|c|c|c|c|c|c|}
\hline 1972 & 28-Apr & -- & 646 & 1976 & 24-Apr & -- & 1,196 \\
\hline 1973 & 11-Apr & -- & 410 & 1977 & 14-Apr & -- & 342 \\
\hline 1974 & 30-Mar & -- & 288 & 1978 & 16-Apr & -- & 485 \\
\hline 1975 & 4-Apr & -- & 695 & & & & \\
\hline \multicolumn{8}{|c|}{ Annual peak discharge, from highest to lowest } \\
\hline 1976 & 24-Apr & -- & 1,196 & 1973 & 11-Apr & -- & 410 \\
\hline 1975 & 4-Apr & -- & 695 & 1977 & 14-Apr & -- & 342 \\
\hline 1972 & 28-Apr & -- & 646 & 1974 & 30-Mar & -- & 288 \\
\hline 1978 & 16-Apr & -- & 485 & & & & \\
\hline
\end{tabular}




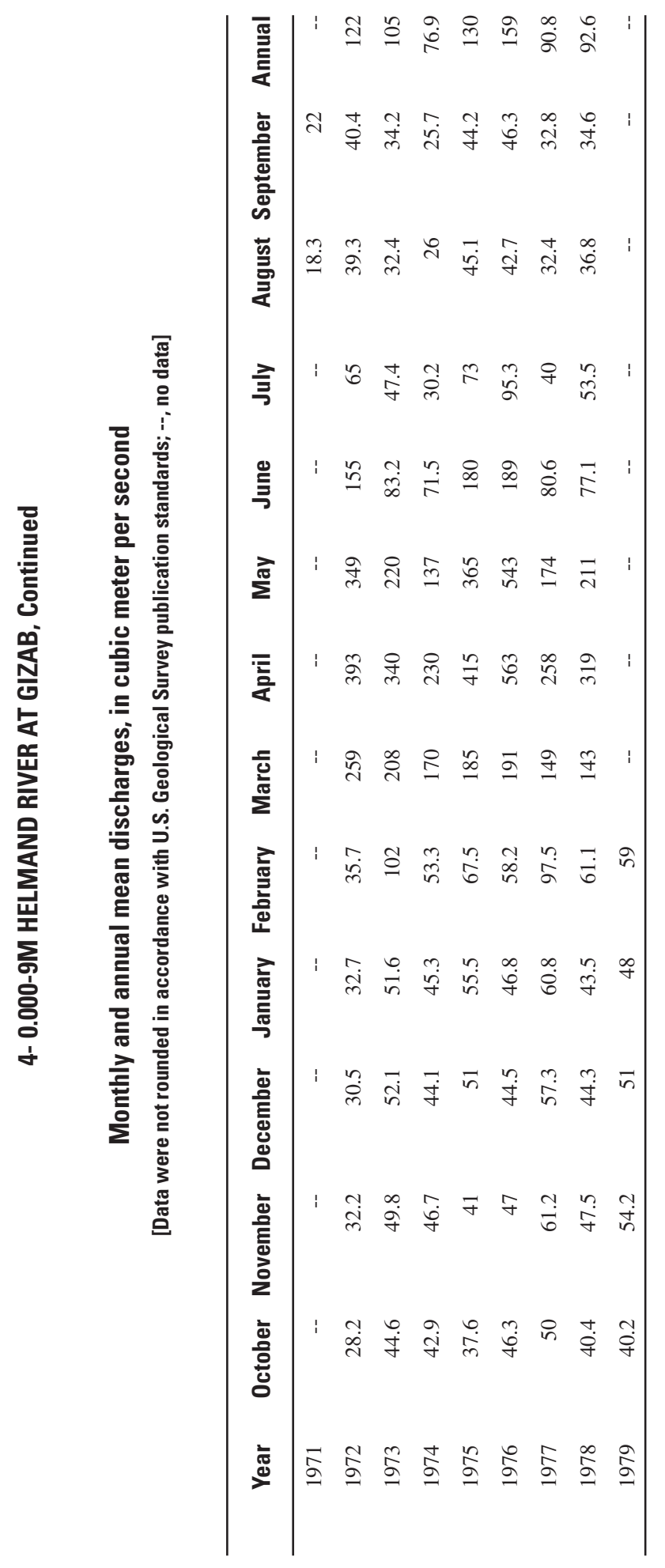




\section{4- 0.000-10M HELMAND RIVER AT GARDANDEWAL \\ (U.S. Geological Survey identification number: 343000068160000)}

LOCATION: Lat $34^{\circ} 30^{\prime} \mathrm{N}$. , long $68^{\circ} 16^{\prime} \mathrm{E}$.

DRAINAGE AREA: $605 \mathrm{~km}^{2}$.

ALTITUDE: 2,955 meters plus mean sea level.

PERIOD OF RECORD: August 8, 1969 to September 30, 1980.

GAGE: --

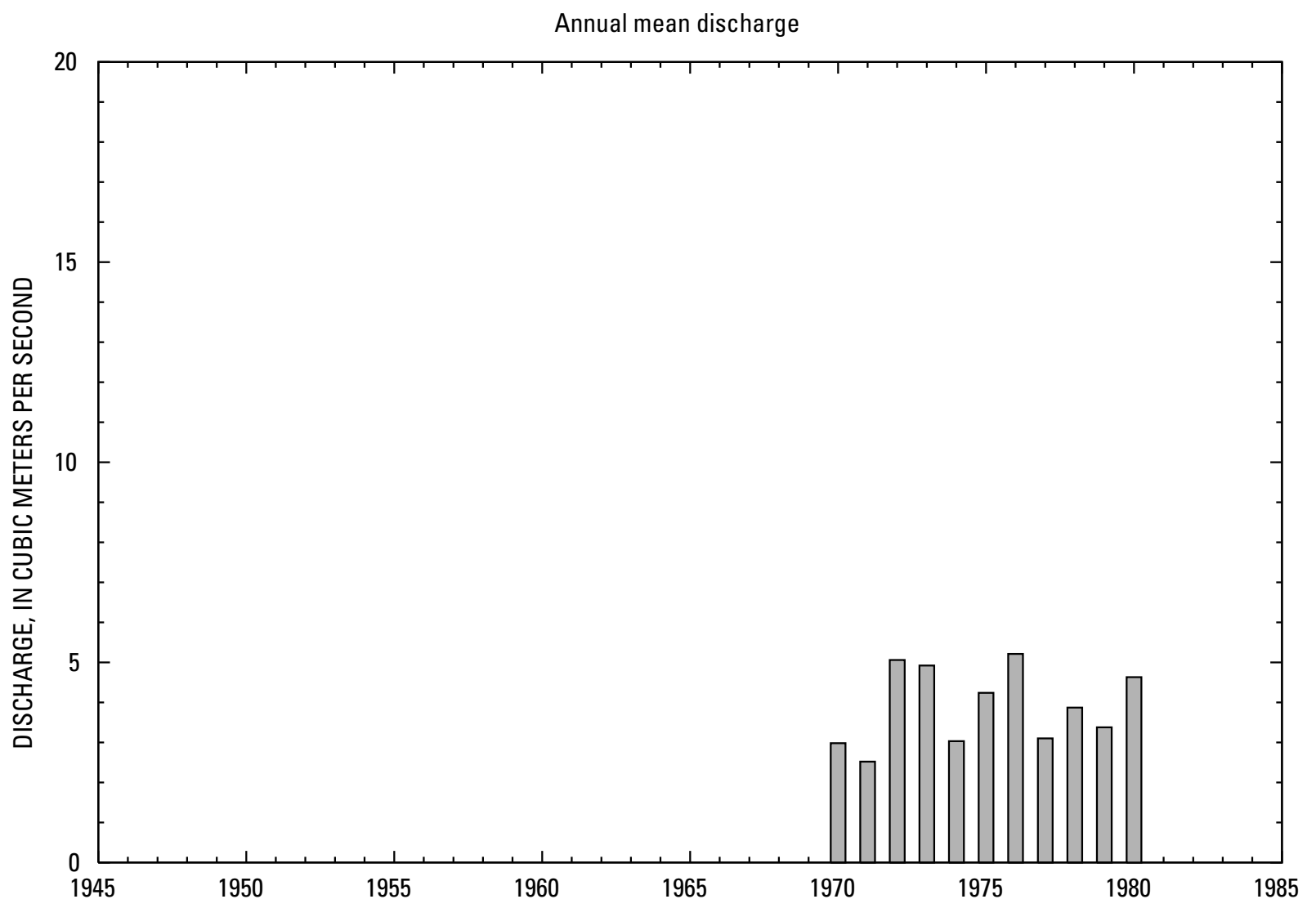




\section{4- 0.000-10M HELMAND RIVER AT GARDANDEWAL, Continued}

\section{Statistics of monthly and annual mean discharges}

$\left[\mathrm{m}^{3} / \mathrm{s}\right.$, meters per second]

\begin{tabular}{|c|c|c|c|c|c|c|c|c|}
\hline \multirow[b]{2}{*}{ Month } & \multicolumn{2}{|c|}{ Maximum } & \multicolumn{2}{|c|}{ Minimum } & \multicolumn{4}{|c|}{ Mean } \\
\hline & $\begin{array}{c}\text { Discharge } \\
\qquad\left(\mathrm{m}^{3} / \mathrm{s}\right)\end{array}$ & $\begin{array}{c}\text { Water year } \\
\text { of } \\
\text { occurrence }\end{array}$ & $\begin{array}{c}\text { Discharge } \\
\left(\mathrm{m}^{3} / \mathrm{s}\right)\end{array}$ & $\begin{array}{c}\text { Water year } \\
\text { of } \\
\text { occurrence }\end{array}$ & $\begin{array}{c}\text { Discharge } \\
\left(\mathrm{m}^{3} / \mathrm{s}\right)\end{array}$ & $\begin{array}{c}\text { Standard } \\
\text { deviation } \\
\left(\mathrm{m}^{3} / \mathrm{s}\right)\end{array}$ & $\begin{array}{c}\text { Coefficient } \\
\text { of } \\
\text { variation }\end{array}$ & $\begin{array}{c}\text { Percentage } \\
\text { of annual } \\
\text { discharge }\end{array}$ \\
\hline October & 1.54 & 1970 & 0.811 & 1978 & 1.19 & 0.24 & 0.20 & 2.55 \\
\hline November & 1.62 & 1973 & 1.00 & 1972 & 1.24 & 0.21 & 0.17 & 2.65 \\
\hline December & 1.87 & 1973 & 0.935 & 1980 & 1.22 & 0.27 & 0.22 & 2.60 \\
\hline January & 1.85 & 1973 & 0.889 & 1976 & 1.24 & 0.30 & 0.25 & 2.64 \\
\hline February & 1.77 & 1970 & 0.960 & 1976 & 1.31 & 0.33 & 0.25 & 2.80 \\
\hline March & 2.53 & 1978 & 1.58 & 1980 & 2.05 & 0.38 & 0.18 & 4.37 \\
\hline April & 13.5 & 1976 & 5.52 & 1971 & 8.93 & 2.74 & 0.31 & 19.1 \\
\hline May & 24.2 & 1976 & 8.78 & 1971 & 15.3 & 5.91 & 0.39 & 32.7 \\
\hline June & 17.1 & 1972 & 3.03 & 1971 & 8.47 & 4.38 & 0.52 & 18.1 \\
\hline July & 5.68 & 1980 & 1.68 & 1971 & 2.91 & 1.09 & 0.37 & 6.22 \\
\hline August & 2.08 & 1979 & 0.945 & 1971 & 1.58 & 0.32 & 0.21 & 3.37 \\
\hline September & 1.79 & 1969 & 0.910 & 1977 & 1.39 & 0.32 & 0.23 & 2.97 \\
\hline Annual & 5.21 & 1976 & 2.52 & 1971 & 3.90 & 0.96 & 0.25 & 100 \\
\hline
\end{tabular}

Annual flow duration

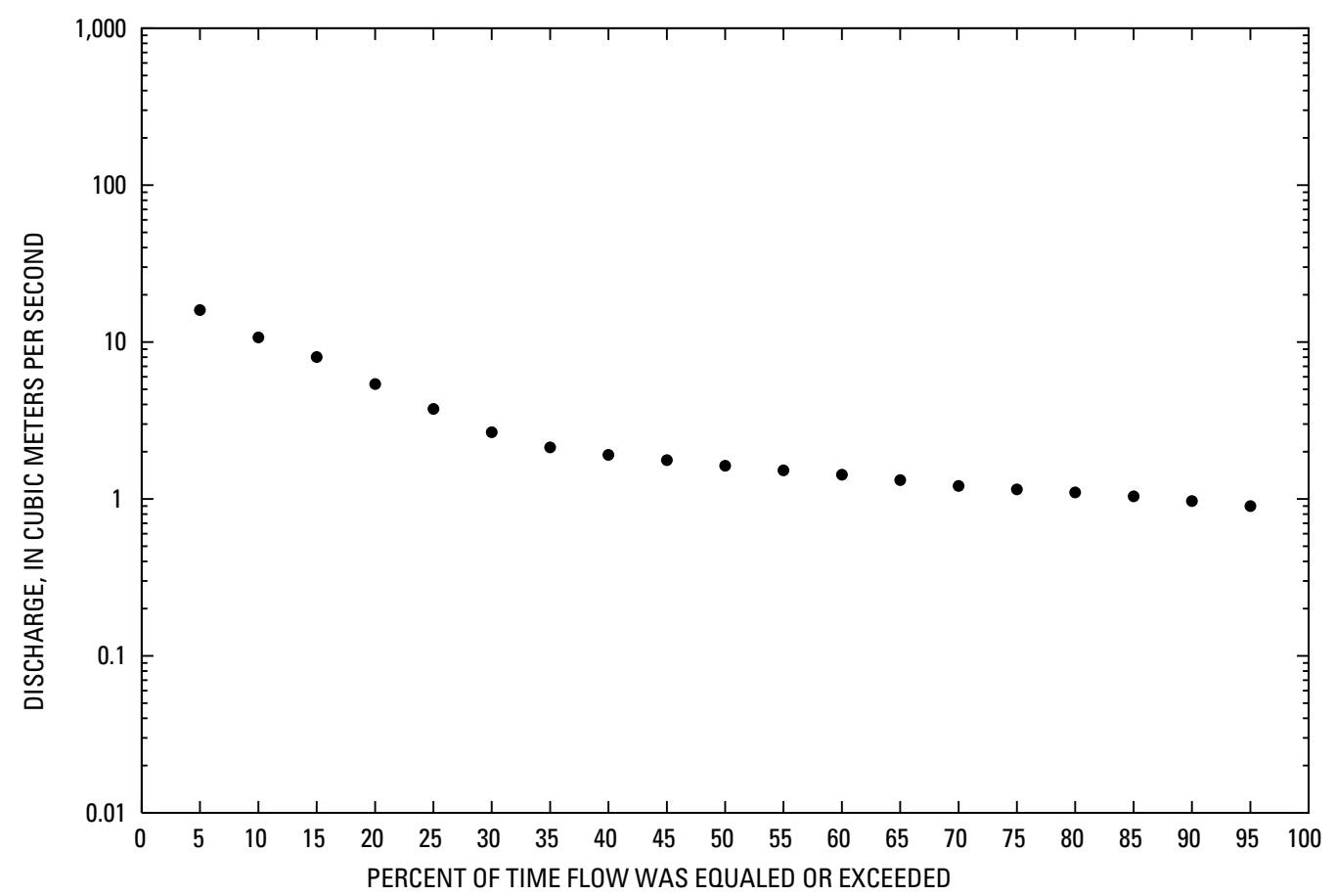




\section{4- 0.000-10M HELMAND RIVER AT GARDANDEWAL, Continued}

Monthly and annual flow duration, in cubic meters per second

[Data were not rounded in accordance with U.S. Geological Survey publication standards]

\begin{tabular}{|c|c|c|c|c|c|c|c|c|c|c|c|c|c|}
\hline $\begin{array}{l}\text { Percentage } \\
\text { of days } \\
\text { discharge } \\
\text { equaled or } \\
\text { exceeded }\end{array}$ & January & February & March & April & May & June & July & August & September & October & November & December & Annual \\
\hline 95 & 0.87 & 0.94 & 1.12 & 3.23 & 7.75 & 2.63 & 1.56 & 0.95 & 0.94 & 0.86 & 0.88 & 0.94 & 0.9 \\
\hline 90 & 0.94 & 0.95 & 1.23 & 4.09 & 8.18 & 3.33 & 1.71 & 1.04 & 0.96 & 0.93 & 0.94 & 0.95 & 0.97 \\
\hline 80 & 0.96 & 1.02 & 1.32 & 5.02 & 8.89 & 3.99 & 2.03 & 1.24 & 1.04 & 1.02 & 1.02 & 0.96 & 1.1 \\
\hline 75 & 1.03 & 1.04 & 1.45 & 5.53 & 9.29 & 4.28 & 2.09 & 1.36 & 1.13 & 1.03 & 1.03 & 1.03 & 1.15 \\
\hline 70 & 1.03 & 1.05 & 1.5 & 5.88 & 10.3 & 4.53 & 2.17 & 1.39 & 1.14 & 1.03 & 1.03 & 1.04 & 1.21 \\
\hline 65 & 1.04 & 1.06 & 1.57 & 6.23 & 10.8 & 5.01 & 2.29 & 1.47 & 1.2 & 1.04 & 1.1 & 1.12 & 1.32 \\
\hline 60 & 1.05 & 1.12 & 1.66 & 6.72 & 11.4 & 5.46 & 2.39 & 1.52 & 1.22 & 1.04 & 1.12 & 1.13 & 1.43 \\
\hline 40 & 1.21 & 1.33 & 1.98 & 8.8 & 16 & 7.86 & 2.76 & 1.74 & 1.49 & 1.22 & 1.33 & 1.21 & 1.91 \\
\hline 35 & 1.22 & 1.46 & 2.01 & 9.56 & 17 & 8.82 & 2.91 & 1.77 & 1.54 & 1.3 & 1.38 & 1.21 & 2.13 \\
\hline 30 & 1.4 & 1.58 & 2.2 & 10.3 & 18.1 & 10.6 & 3.16 & 1.79 & 1.63 & 1.31 & 1.39 & 1.22 & 2.66 \\
\hline 25 & 1.48 & 1.6 & 2.34 & 11 & 19.6 & 11.5 & 3.37 & 1.81 & 1.65 & 1.4 & 1.41 & 1.33 & 3.75 \\
\hline 20 & 1.56 & 1.67 & 2.53 & 12.2 & 21.2 & 13.5 & 3.51 & 1.93 & 1.67 & 1.5 & 1.46 & 1.41 & 5.4 \\
\hline 15 & 1.6 & 1.77 & 2.77 & 13.1 & 23 & 14.2 & 3.82 & 1.97 & 1.74 & 1.52 & 1.48 & 1.5 & 8.04 \\
\hline 10 & 1.66 & 1.8 & 3.47 & 15.2 & 25.5 & 16.4 & 4.28 & 2.01 & 1.77 & 1.54 & 1.55 & 1.57 & 10.7 \\
\hline 5 & 0 & 1.89 & 4.18 & 20 & 29.3 & 20.1 & 5.44 & 2.05 & 1.97 & 1.56 & 1.78 & 0 & 16 \\
\hline
\end{tabular}




\section{4- 0.000-10M HELMAND RIVER AT GARDANDEWAL, Continued}

Probability of occurrence of annual high discharges

[ $\mathrm{m}^{3} / \mathrm{s}$, meters per second; ng, statistic not given]

\begin{tabular}{|c|c|c|c|c|c|c|}
\hline \multirow{2}{*}{$\begin{array}{c}\text { Exceedance } \\
\text { probability }\end{array}$} & \multirow{2}{*}{$\begin{array}{c}\text { Recurrence } \\
\text { interval } \\
\text { (years) }\end{array}$} & \multirow{2}{*}{$\begin{array}{c}\text { Maximum } \\
\text { instantaneous } \\
\left(\mathrm{m}^{3} / \mathrm{s}\right)\end{array}$} & \multicolumn{4}{|c|}{ Maximum mean discharge } \\
\hline & & & 3-day period & 7-day period & 15-day period & 30-day period \\
\hline 0.99 & 1.01 & 8.30 & 6.51 & 6.46 & 6.12 & 5.88 \\
\hline 0.95 & 1.05 & 11.2 & 9.23 & 8.96 & 8.33 & 7.84 \\
\hline 0.90 & 1.11 & 13.2 & 11.0 & 10.6 & 9.80 & 9.12 \\
\hline 0.80 & 1.25 & 16.0 & 13.7 & 13.0 & 11.9 & 10.9 \\
\hline 0.50 & 2 & 22.9 & 20.1 & 18.9 & 17.2 & 15.4 \\
\hline 0.20 & 5 & 32.5 & 29.0 & 27.2 & 24.5 & 21.6 \\
\hline 0.10 & 10 & 38.9 & 34.7 & 32.7 & 29.5 & 25.8 \\
\hline 0.04 & 25 & 46.9 & 41.9 & 39.6 & 35.8 & 31.0 \\
\hline 0.02 & 50 & 52.9 & 47.1 & 44.8 & 40.4 & 34.9 \\
\hline 0.01 & 100 & 58.8 & 52.2 & 49.9 & 45.2 & 38.7 \\
\hline 0.005 & 200 & 64.7 & 57.2 & 55.0 & 49.9 & 42.7 \\
\hline 0.002 & 500 & 72.6 & ng & ng & ng & ng \\
\hline
\end{tabular}

\section{4- 0.000-10M HELMAND RIVER AT GARDANDEWAL, Continued}

Probability of occurrence of annual low discharges

$\left[\mathrm{m}^{3} / \mathrm{s}\right.$, meters per second]

\begin{tabular}{|c|c|c|c|c|c|c|c|c|c|c|}
\hline \multirow{3}{*}{$\begin{array}{c}\text { Nonexceedance } \\
\text { probability }\end{array}$} & \multirow{3}{*}{$\begin{array}{c}\text { Recurrence } \\
\text { interval } \\
\text { (years) }\end{array}$} & \multicolumn{9}{|c|}{ Minimum mean discharge $\left(\mathrm{m}^{3} / \mathrm{s}\right)$} \\
\hline & & \multicolumn{9}{|c|}{ Number of consecutive days } \\
\hline & & 1 & 3 & 7 & 14 & 30 & 60 & 90 & 120 & 183 \\
\hline 0.05 & 20 & 0.774 & 0.778 & 0.780 & 0.782 & 0.815 & 0.835 & 0.866 & 0.910 & 0.983 \\
\hline 0.10 & 10 & 0.796 & 0.800 & 0.806 & 0.811 & 0.840 & 0.863 & 0.897 & 0.936 & 1.01 \\
\hline 0.20 & 5 & 0.830 & 0.835 & 0.844 & 0.852 & 0.877 & 0.905 & 0.943 & 0.976 & 1.04 \\
\hline 0.50 & 2 & 0.925 & 0.929 & 0.945 & 0.957 & 0.978 & 1.02 & 1.06 & 1.09 & 1.15 \\
\hline
\end{tabular}




\section{4- 0.000-10M HELMAND RIVER AT GARDANDEWAL, Continued}

\section{Probability of occurrence of seasonal low discharges}

$\left[\mathrm{m}^{3} / \mathrm{s}\right.$, meters per second]

\begin{tabular}{|c|c|c|c|c|c|c|c|c|c|}
\hline \multirow{3}{*}{$\begin{array}{c}\text { Nonexceedance } \\
\text { probability }\end{array}$} & \multirow{3}{*}{$\begin{array}{c}\text { Recurrence } \\
\text { interval } \\
\text { (years) }\end{array}$} & \multicolumn{8}{|c|}{ Minimum mean discharge $\left(\mathrm{m}^{3} / \mathrm{s}\right)$} \\
\hline & & \multicolumn{8}{|c|}{ Number of consecutive days } \\
\hline & & 1 & 7 & 14 & 30 & 1 & 7 & 14 & 30 \\
\hline & & \multicolumn{4}{|c|}{ December-January-February } & \multicolumn{4}{|c|}{ March-April-May } \\
\hline 0.05 & 20 & 0.807 & 0.829 & ${ }^{1} 0.825$ & 0.847 & 0.985 & 0.992 & 1.06 & 1.43 \\
\hline 0.10 & 10 & 0.842 & 0.867 & 0.868 & 0.890 & 1.04 & 1.06 & 1.14 & 1.53 \\
\hline 0.20 & 5 & 0.894 & 0.924 & 0.931 & 0.953 & 1.12 & 1.16 & 1.24 & 1.67 \\
\hline \multirow[t]{2}{*}{0.50} & 2 & 1.03 & 1.07 & 1.09 & 1.12 & 1.31 & 1.38 & 1.48 & 1.96 \\
\hline & & \multicolumn{4}{|c|}{ June-July-August } & \multicolumn{4}{|c|}{ September-October-November } \\
\hline 0.05 & 20 & 0.846 & 0.901 & 0.941 & 1.00 & 0.779 & 0.792 & 0.800 & 0.837 \\
\hline 0.10 & 10 & 0.944 & 0.995 & 1.04 & 1.13 & 0.813 & 0.829 & 0.841 & 0.882 \\
\hline 0.20 & 5 & 1.07 & 1.12 & 1.18 & 1.29 & 0.860 & 0.881 & 0.896 & 0.942 \\
\hline 0.50 & 2 & 1.35 & 1.38 & 1.44 & 1.59 & 0.977 & 1.00 & 1.03 & 1.08 \\
\hline
\end{tabular}

'Data does not fit log-Pearson Type III curve, use with caution. 


\section{4- 0.000-10M HELMAND RIVER AT GARDANDEWAL, Continued}

Annual peak discharge and corresponding gage height

$\left[\mathrm{m}^{3} / \mathrm{s}\right.$, meters per second; --, no data]

\begin{tabular}{|c|c|c|c|c|c|c|c|c|}
\hline $\begin{array}{c}\text { Water } \\
\text { year }\end{array}$ & Date & $\begin{array}{c}\text { Gage } \\
\text { height } \\
\text { (meter) }\end{array}$ & $\begin{array}{c}\text { Peak } \\
\text { discharge } \\
\left(\mathrm{m}^{3} / \mathrm{s}\right)\end{array}$ & $\begin{array}{c}\text { Water } \\
\text { year }\end{array}$ & Date & $\begin{array}{c}\text { Gage } \\
\text { height } \\
\text { (meter) }\end{array}$ & & $\begin{array}{c}\text { Peak } \\
\text { discharge } \\
\left(\mathrm{m}^{3} / \mathrm{s}\right)\end{array}$ \\
\hline \multicolumn{9}{|c|}{ Annual peak discharge, by year } \\
\hline 1970 & 21-May & & 15.6 & 1976 & 15-May & & -- & 39.5 \\
\hline 1971 & 12-May & & 13.1 & 1977 & 27-May & & -- & 13.9 \\
\hline 1972 & 21-May & & 39.5 & 1978 & 16-Apr & & -- & 25.8 \\
\hline 1973 & 9-May & & 30.5 & 1979 & 26-Apr & & -- & 20.8 \\
\hline 1974 & 31-May & & 13.9 & 1980 & 20-May & & -- & 27.0 \\
\hline 1975 & 16-May & & 31.2 & & & & & \\
\hline \multicolumn{9}{|c|}{ Annual peak discharge, from highest to lowest } \\
\hline 1976 & 15-May & & 39.5 & 1979 & 26-Apr & & -- & 20.8 \\
\hline 1972 & 21-May & & 39.5 & 1970 & 21-May & & -- & 15.6 \\
\hline 1975 & 16-May & & 31.2 & 1977 & 27-May & & -- & 13.9 \\
\hline 1973 & 9-May & & 30.5 & 1974 & 31-May & & -- & 13.9 \\
\hline 1980 & 20-May & & 27.0 & 1971 & 12-May & & -- & 13.1 \\
\hline 1978 & 16-Apr & & 25.8 & & & & & \\
\hline
\end{tabular}




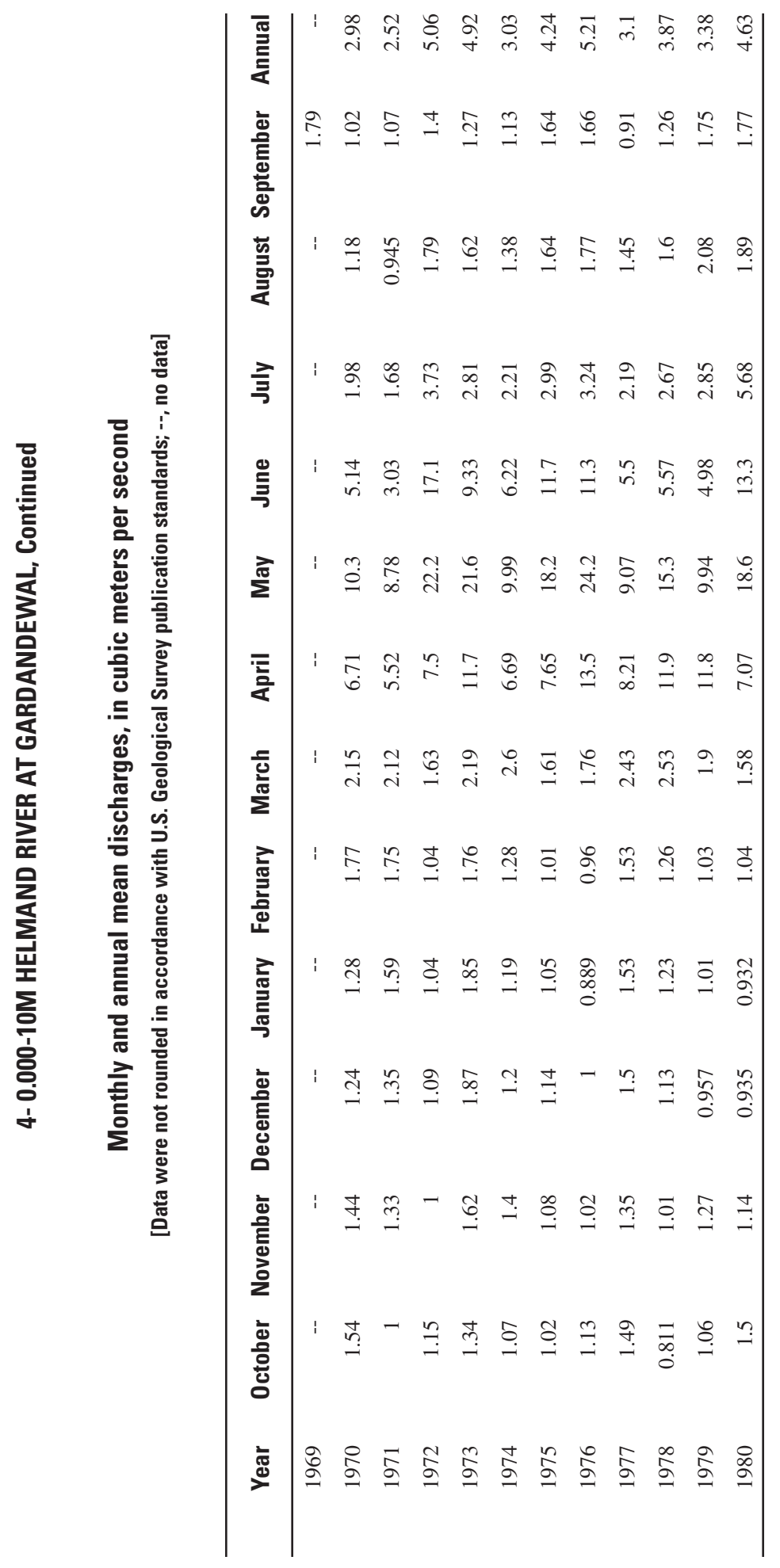




\section{4- 1.21R-7A TARNAK RIVER NEAR SHAHJUY}

\section{(U.S. Geological Survey identification number: 323200067280000)}

LOCATION: Lat $32^{\circ} 32^{\prime} \mathrm{N}$., long $67^{\circ} 28^{\prime} \mathrm{E}$.

DRAINAGE AREA: $8,120 \mathrm{~km}^{2}$.

ALTITUDE: 1,043 meters plus mean sea level.

PERIOD OF RECORD: February 19, 1969 to September 30, 1980.

GAGE: --

Annual mean discharge

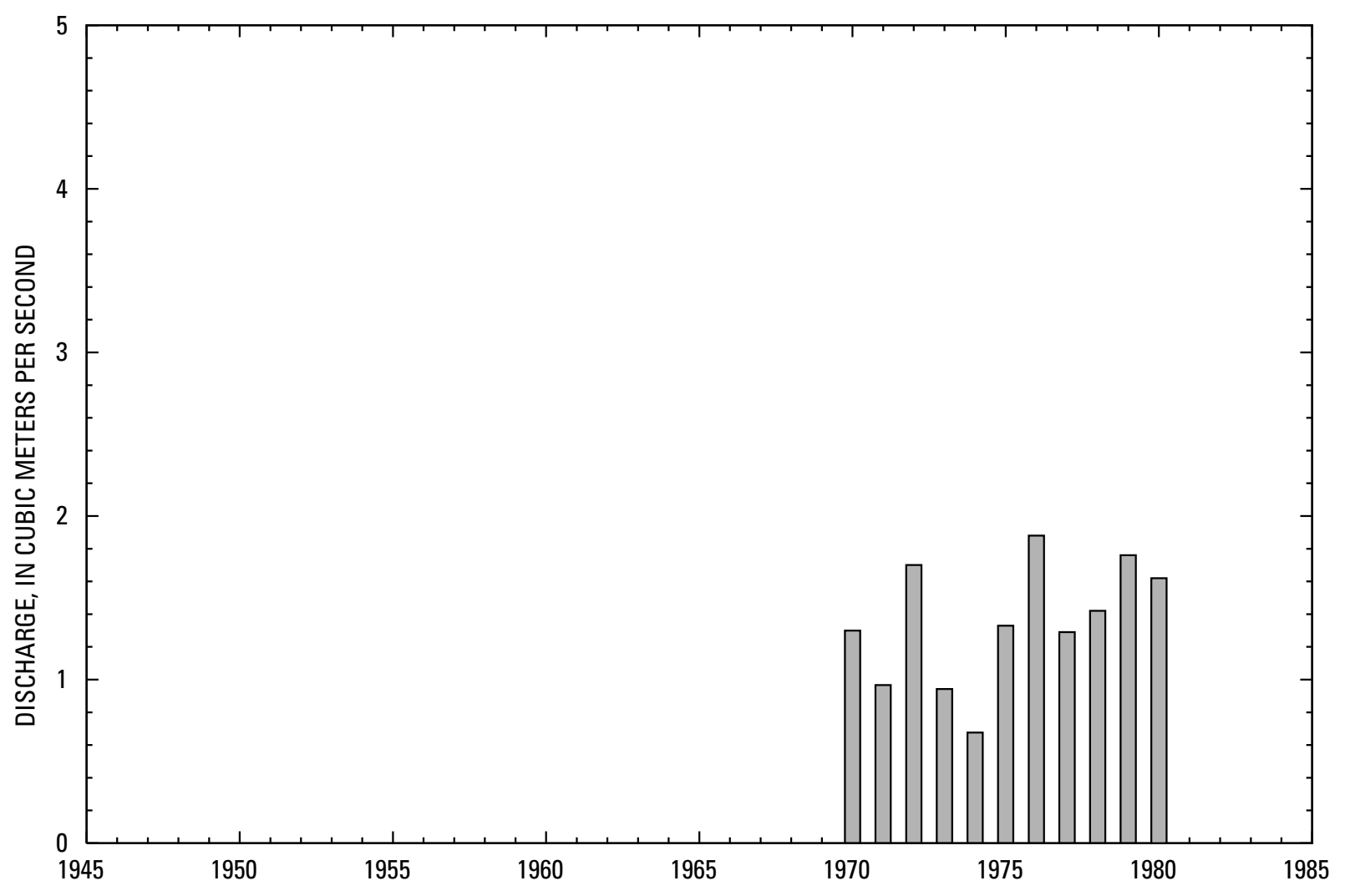




\section{4- 1.21R-7A TARNAK RIVER NEAR SHAHJUY, Continued}

\section{Statistics of monthly and annual mean discharges}

$\left[\mathrm{m}^{3} / \mathrm{s}\right.$, meters per second]

\begin{tabular}{|c|c|c|c|c|c|c|c|c|}
\hline \multirow[b]{2}{*}{ Month } & \multicolumn{2}{|c|}{ Maximum } & \multicolumn{2}{|c|}{ Minimum } & \multicolumn{4}{|c|}{ Mean } \\
\hline & $\begin{array}{c}\text { Discharge } \\
\left(\mathrm{m}^{3} / \mathrm{s}\right)\end{array}$ & $\begin{array}{c}\text { Water year } \\
\text { of } \\
\text { occurrence }\end{array}$ & $\begin{array}{c}\text { Discharge } \\
\left(\mathrm{m}^{3} / \mathrm{s}\right)\end{array}$ & $\begin{array}{c}\text { Water year } \\
\text { of } \\
\text { occurrence }\end{array}$ & $\begin{array}{c}\text { Discharge } \\
\left(\mathrm{m}^{3} / \mathrm{s}\right)\end{array}$ & $\begin{array}{c}\text { Standard } \\
\text { deviation } \\
\left(\mathrm{m}^{3} / \mathrm{s}\right)\end{array}$ & $\begin{array}{c}\text { Coefficient } \\
\text { of } \\
\text { variation }\end{array}$ & $\begin{array}{c}\text { Percentage } \\
\text { of annual } \\
\text { discharge }\end{array}$ \\
\hline October & 1.24 & 1980 & 0.500 & 1974 & 0.79 & 0.20 & 0.25 & 4.94 \\
\hline November & 2.20 & 1970 & 0.484 & 1974 & 1.16 & 0.42 & 0.36 & 7.24 \\
\hline December & 2.14 & 1970 & 0.407 & 1974 & 1.36 & 0.50 & 0.37 & 8.48 \\
\hline January & 2.81 & 1972 & 0.413 & 1974 & 1.50 & 0.67 & 0.44 & 9.39 \\
\hline February & 3.61 & 1979 & 0.486 & 1974 & 1.90 & 0.94 & 0.50 & 11.9 \\
\hline March & 5.90 & 1980 & 1.08 & 1969 & 2.36 & 1.32 & 0.56 & 14.7 \\
\hline April & 3.76 & 1972 & 0.883 & 1978 & 1.90 & 0.98 & 0.52 & 11.8 \\
\hline May & 2.69 & 1976 & 0.660 & 1978 & 1.22 & 0.67 & 0.55 & 7.58 \\
\hline June & 1.75 & 1976 & 0.527 & 1975 & 0.82 & 0.38 & 0.46 & 5.13 \\
\hline July & 3.75 & 1978 & 0.522 & 1971 & 1.07 & 0.92 & 0.86 & 6.71 \\
\hline August & 2.60 & 1978 & 0.573 & 1972 & 1.19 & 0.73 & 0.61 & 7.45 \\
\hline September & 1.26 & 1976 & 0.471 & 1977 & 0.75 & 0.26 & 0.34 & 4.68 \\
\hline Annual & 1.88 & 1976 & 0.676 & 1974 & 1.35 & 0.38 & 0.28 & 100 \\
\hline
\end{tabular}

Annual flow duration

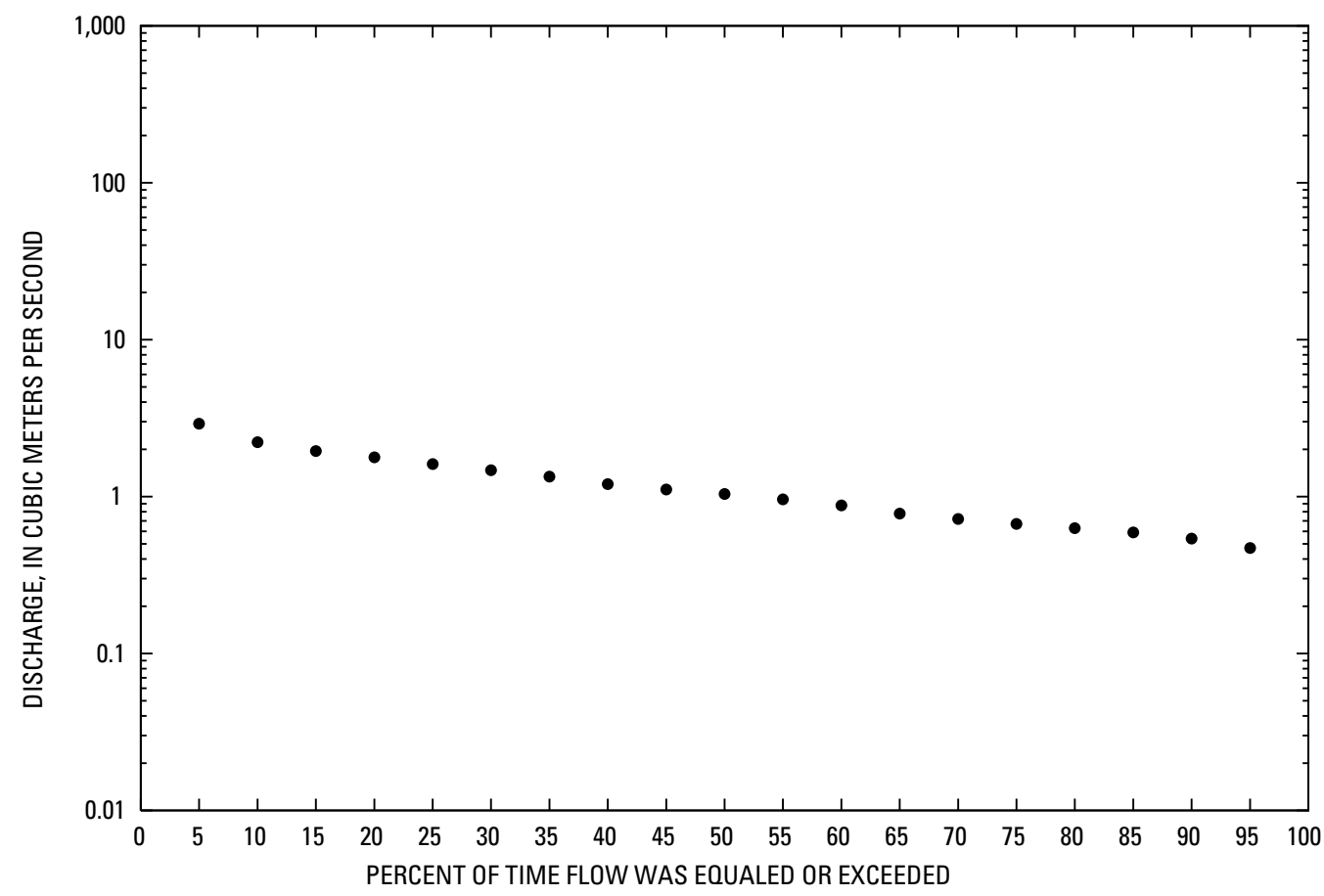




\section{4- 1.21R-7A TARNAK RIVER NEAR SHAHJUY, Continued}

Monthly and annual flow duration, in cubic meters per second

[Data were not rounded in accordance with U.S. Geological Survey publication standards]

\begin{tabular}{|c|c|c|c|c|c|c|c|c|c|c|c|c|c|}
\hline $\begin{array}{l}\text { Percentage } \\
\text { of days } \\
\text { discharge } \\
\text { equaled or } \\
\text { exceeded }\end{array}$ & January & February & March & April & May & June & July & August & September & October & November & December & Annual \\
\hline 95 & 0.4 & 0.38 & 0.92 & 0.76 & 0.56 & 0.49 & 0.45 & 0.43 & 0.46 & 0.51 & 0.49 & 0.41 & 0.47 \\
\hline 90 & 0.42 & 0.41 & 1.12 & 0.85 & 0.6 & 0.51 & 0.48 & 0.49 & 0.5 & 0.51 & 0.71 & 0.59 & 0.54 \\
\hline 80 & 0.98 & 1.05 & 1.3 & 0.96 & 0.66 & 0.56 & 0.56 & 0.56 & 0.52 & 0.6 & 0.83 & 0.94 & 0.63 \\
\hline 75 & 1.02 & 1.13 & 1.35 & 1 & 0.69 & 0.59 & 0.59 & 0.57 & 0.56 & 0.62 & 0.89 & 0.98 & 0.67 \\
\hline 70 & 1.24 & 1.28 & 1.42 & 1.03 & 0.71 & 0.6 & 0.6 & 0.59 & 0.59 & 0.68 & 0.94 & 1.03 & 0.72 \\
\hline 65 & 1.38 & 1.43 & 1.51 & 1.06 & 0.75 & 0.62 & 0.62 & 0.61 & 0.6 & 0.7 & 0.97 & 1.16 & 0.78 \\
\hline 45 & 1.63 & 1.89 & 1.84 & 1.49 & 0.92 & 0.69 & 0.7 & 0.68 & 0.71 & 0.8 & 1.08 & 1.45 & 1.11 \\
\hline 40 & 1.68 & 1.96 & 1.92 & 1.71 & 1.03 & 0.71 & 0.73 & 0.7 & 0.76 & 0.82 & 1.13 & 1.49 & 1.2 \\
\hline 35 & 1.72 & 2.06 & 2.01 & 1.87 & 1.12 & 0.75 & 0.76 & 0.75 & 0.8 & 0.84 & 1.17 & 1.53 & 1.34 \\
\hline 30 & 1.77 & 2.19 & 2.14 & 1.96 & 1.29 & 0.81 & 0.82 & 0.96 & 0.87 & 0.87 & 1.21 & 1.58 & 1.47 \\
\hline 25 & 1.83 & 2.38 & 2.36 & 2.13 & 1.52 & 0.91 & 0.89 & 1.02 & 0.96 & 0.94 & 1.24 & 1.65 & 1.61 \\
\hline 20 & 1.93 & 2.61 & 2.64 & 2.55 & 1.7 & 1.11 & 1.02 & 1.1 & 1.02 & 0.96 & 1.31 & 1.75 & 1.78 \\
\hline 15 & 2.06 & 2.73 & 2.84 & 2.8 & 1.88 & 1.32 & 1.13 & 1.22 & 1.09 & 1.01 & 1.51 & 1.9 & 1.95 \\
\hline 10 & 2.25 & 2.86 & 3.14 & 2.93 & 2.04 & 1.36 & 1.41 & 1.54 & 1.12 & 1.07 & 1.72 & 2.03 & 2.22 \\
\hline 5 & 2.77 & 3.43 & 3.86 & 3.48 & 2.97 & 0 & 1.77 & 1.85 & 1.22 & 0 & 2.06 & 2.21 & 2.91 \\
\hline
\end{tabular}




\section{4- 1.21R-7A TARNAK RIVER NEAR SHAHJUY, Continued}

Probability of occurrence of annual high discharges

[ $\mathrm{m}^{3} / \mathrm{s}$, meters per second; $\mathrm{ng}$, statistic not given]

\begin{tabular}{|c|c|c|c|c|c|c|}
\hline \multirow{2}{*}{$\begin{array}{c}\text { Exceedance } \\
\text { probability }\end{array}$} & \multirow{2}{*}{$\begin{array}{c}\text { Recurrence } \\
\text { interval } \\
\text { (years) }\end{array}$} & \multirow{2}{*}{$\begin{array}{c}\text { Maximum } \\
\text { instantaneous } \\
\left(\mathrm{m}^{3} / \mathrm{s}\right)\end{array}$} & \multicolumn{4}{|c|}{ Maximum mean discharge } \\
\hline & & & 3-day period & 7-day period & 15-day period & 30-day period \\
\hline 0.99 & 1.01 & 2.10 & 1.47 & 1.25 & 1.30 & ${ }^{1} 1.31$ \\
\hline 0.95 & 1.05 & 3.90 & 2.43 & 1.93 & 1.75 & 1.60 \\
\hline 0.90 & 1.11 & 5.40 & 3.20 & 2.45 & 2.06 & 1.81 \\
\hline 0.80 & 1.25 & 8.10 & 4.50 & 3.31 & 2.54 & 2.11 \\
\hline 0.50 & 2 & 17.6 & 8.86 & 5.99 & 3.92 & 2.91 \\
\hline 0.20 & 5 & 38.6 & 18.0 & 11.2 & 6.30 & 4.20 \\
\hline 0.10 & 10 & 58.4 & 26.3 & 15.7 & 8.21 & 5.16 \\
\hline 0.04 & 25 & 90.9 & 39.9 & 22.8 & 11.0 & 6.53 \\
\hline 0.02 & 50 & 121 & 52.5 & 29.2 & 13.4 & 7.66 \\
\hline 0.01 & 100 & 157 & 67.5 & 36.6 & 16.1 & 8.88 \\
\hline 0.005 & 200 & 199 & 85.1 & 45.0 & 19.2 & 10.2 \\
\hline 0.002 & 500 & 266 & $\mathrm{ng}$ & ng & $\mathrm{ng}$ & $\mathrm{ng}$ \\
\hline
\end{tabular}

'Data does not fit log-Pearson Type III curve, use with caution.

\section{4- 1.21R-7A TARNAK RIVER NEAR SHAHJUY, Continued}

Probability of occurrence of annual low discharges

$\left[\mathrm{m}^{3} / \mathrm{s}\right.$, meters per second]

\begin{tabular}{|c|c|c|c|c|c|c|c|c|c|c|}
\hline \multirow{3}{*}{$\begin{array}{c}\text { Nonexceedance } \\
\text { probability }\end{array}$} & \multirow{3}{*}{$\begin{array}{c}\text { Recurrence } \\
\text { interval } \\
\text { (years) }\end{array}$} & \multicolumn{9}{|c|}{ Minimum mean discharge $\left(\mathrm{m}^{3} / \mathrm{s}\right)$} \\
\hline & & \multicolumn{9}{|c|}{ Number of consecutive days } \\
\hline & & 1 & 3 & 7 & 14 & 30 & 60 & 90 & 120 & 183 \\
\hline 0.05 & 20 & 0.254 & 0.273 & 0.300 & 0.332 & 0.358 & 0.423 & 0.432 & 0.438 & 0.458 \\
\hline 0.10 & 10 & 0.276 & 0.297 & 0.322 & 0.353 & 0.380 & 0.451 & 0.467 & 0.485 & 0.516 \\
\hline 0.20 & 5 & 0.309 & 0.333 & 0.356 & 0.385 & 0.413 & 0.493 & 0.517 & 0.549 & 0.597 \\
\hline 0.50 & 2 & 0.404 & 0.431 & 0.451 & 0.475 & 0.506 & 0.600 & 0.644 & 0.707 & 0.794 \\
\hline
\end{tabular}




\section{4- 1.21R-7A TARNAK RIVER NEAR SHAHJUY, Continued}

\section{Probability of occurrence of seasonal low discharges}

$\left[\mathrm{m}^{3} / \mathrm{s}\right.$, meters per second]

\begin{tabular}{|c|c|c|c|c|c|c|c|c|c|}
\hline \multirow{3}{*}{$\begin{array}{c}\text { Nonexceedance } \\
\text { probability }\end{array}$} & \multirow{3}{*}{$\begin{array}{c}\text { Recurrence } \\
\text { interval } \\
\text { (years) }\end{array}$} & \multicolumn{8}{|c|}{ Minimum mean discharge $\left(\mathrm{m}^{3} / \mathrm{s}\right)$} \\
\hline & & \multicolumn{8}{|c|}{ Number of consecutive days } \\
\hline & & 1 & 7 & 14 & 30 & 1 & 7 & 14 & 30 \\
\hline & & \multicolumn{4}{|c|}{ December-January-February } & \multicolumn{4}{|c|}{ March-April-May } \\
\hline 0.05 & 20 & 0.287 & 0.340 & 0.356 & 0.368 & 0.388 & 0.450 & 0.515 & 0.549 \\
\hline 0.10 & 10 & 0.406 & 0.466 & 0.492 & 0.525 & 0.419 & 0.482 & 0.549 & 0.607 \\
\hline 0.20 & 5 & 0.587 & 0.653 & 0.693 & 0.759 & 0.471 & 0.535 & 0.603 & 0.700 \\
\hline \multirow[t]{2}{*}{0.50} & 2 & 1.02 & 1.08 & 1.16 & 1.29 & 0.637 & 0.702 & 0.776 & 0.980 \\
\hline & & \multicolumn{4}{|c|}{ June-July-August } & \multicolumn{4}{|c|}{ September-October-November } \\
\hline 0.05 & 20 & 0.296 & 0.339 & 0.381 & 0.447 & 0.380 & 0.397 & 0.437 & 0.444 \\
\hline 0.10 & 10 & 0.320 & 0.365 & 0.402 & 0.460 & 0.407 & 0.428 & 0.465 & 0.476 \\
\hline 0.20 & 5 & 0.361 & 0.407 & 0.438 & 0.485 & 0.448 & 0.474 & 0.508 & 0.522 \\
\hline 0.50 & 2 & 0.491 & 0.538 & 0.557 & 0.584 & 0.560 & 0.595 & 0.620 & 0.641 \\
\hline
\end{tabular}

4- 1.L00-9A ARGHANDAB RIVER AT SANG-I-MASHA, Continued

Annual peak discharge and corresponding gage height

[ $\mathrm{m}^{3} / \mathrm{s}$, meters per second; --, no data]

\begin{tabular}{|c|c|c|c|c|c|c|c|}
\hline $\begin{array}{c}\text { Water } \\
\text { year }\end{array}$ & Date & $\begin{array}{c}\text { Gage } \\
\text { height } \\
\text { (meters) }\end{array}$ & $\begin{array}{c}\text { Peak } \\
\text { discharge } \\
\left(\mathrm{m}^{3} / \mathrm{s}\right)\end{array}$ & $\begin{array}{c}\text { Water } \\
\text { year }\end{array}$ & Date & $\begin{array}{c}\text { Gage } \\
\text { height } \\
\text { (meters) }\end{array}$ & $\begin{array}{c}\text { Peak } \\
\text { discharge } \\
\left(\mathrm{m}^{3} / \mathrm{s}\right)\end{array}$ \\
\hline
\end{tabular}

Annual peak discharge, by year

\begin{tabular}{lccrcccr}
1970 & 15-Apr & -- & 23.0 & 1976 & 23-Jul & -- & 216 \\
1971 & 15-Apr & -- & 7.98 & 1977 & 30-Jun & -- & 46.1 \\
1972 & 27-Apr & -- & 46.5 & 1978 & 5-Jul & -- & 220 \\
1973 & 20-Jul & -- & 111 & 1979 & 6-Aug & -- & 83.0 \\
1974 & 28-Mar & -- & 26.9 & 1980 & 19-Apr & -- & 81.0 \\
1975 & 3-Apr & -- & 57.6 & & & \\
\multicolumn{2}{c}{ Annual peak discharge, from highest to lowest } & & \\
1978 & 5-Jul & -- & 220 & 1972 & 27-Apr & -- & 46.5 \\
1976 & 23-Jul & -- & 216 & 1977 & 30-Jun & -- & 46.1 \\
1973 & 20-Jul & -- & 111 & 1974 & 28-Mar & -- & 26.9 \\
1979 & 6-Aug & -- & 83.0 & 1970 & 15-Apr & -- & 23.0 \\
1980 & 19-Apr & -- & 81.0 & 1971 & 15-Apr & -- & 7.98 \\
1975 & 3-Apr & - & 57.6 & & & & \\
\hline
\end{tabular}




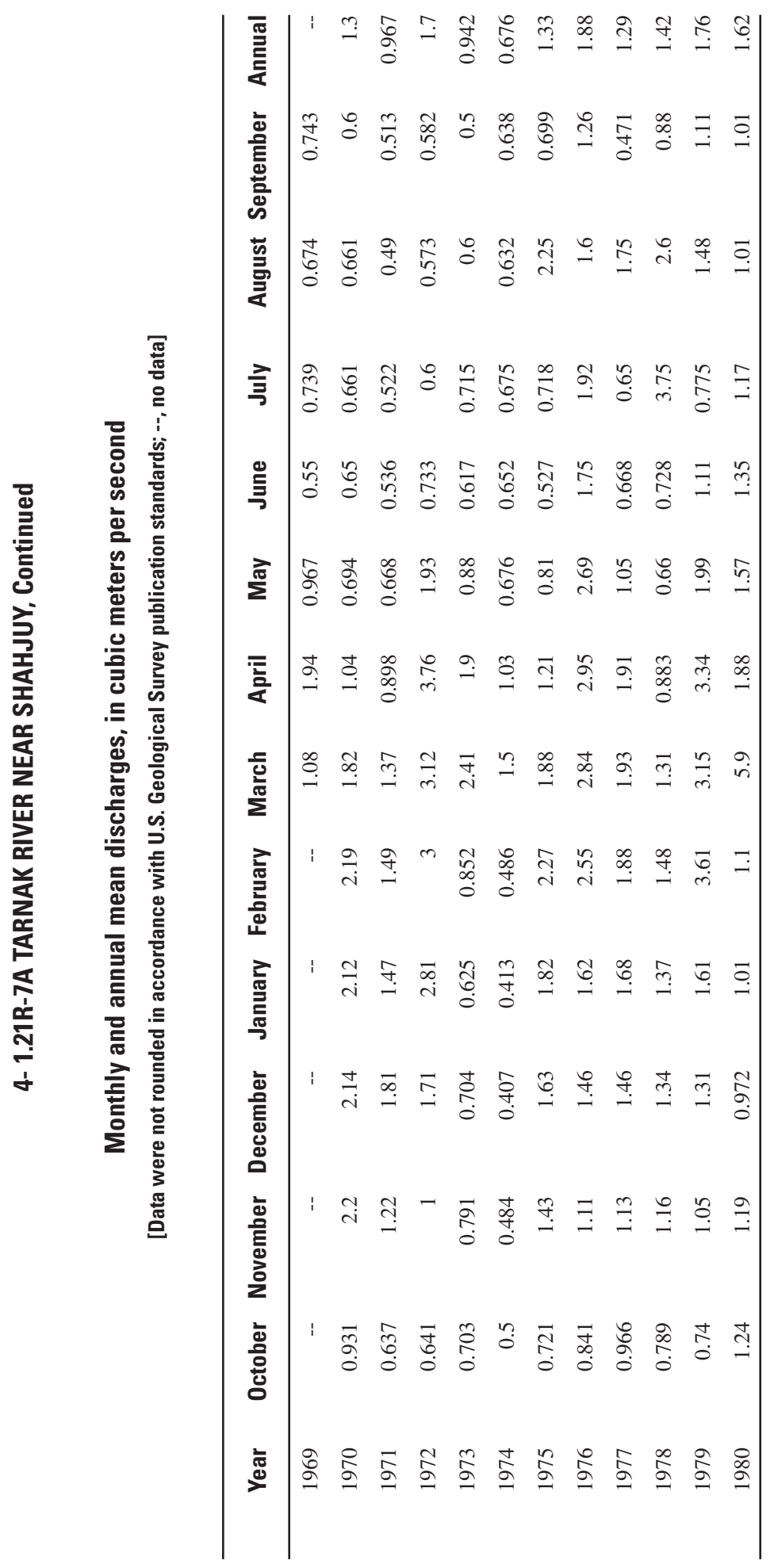




\section{4- 1.222R-6A LORA RIVER NEAR SHINKAY}

\section{(U.S. Geological Survey identification number: 320000067180000)}

LOCATION: Lat $32^{\circ} 00^{\prime} \mathrm{N}$., long $67^{\circ} 18^{\prime} \mathrm{E}$.

DRAINAGE AREA: $2,930 \mathrm{~km}^{2}$.

ALTITUDE: 1,882 meters plus mean sea level.

PERIOD OF RECORD: October 1, 1977 to September 30, 1979.

GAGE: --

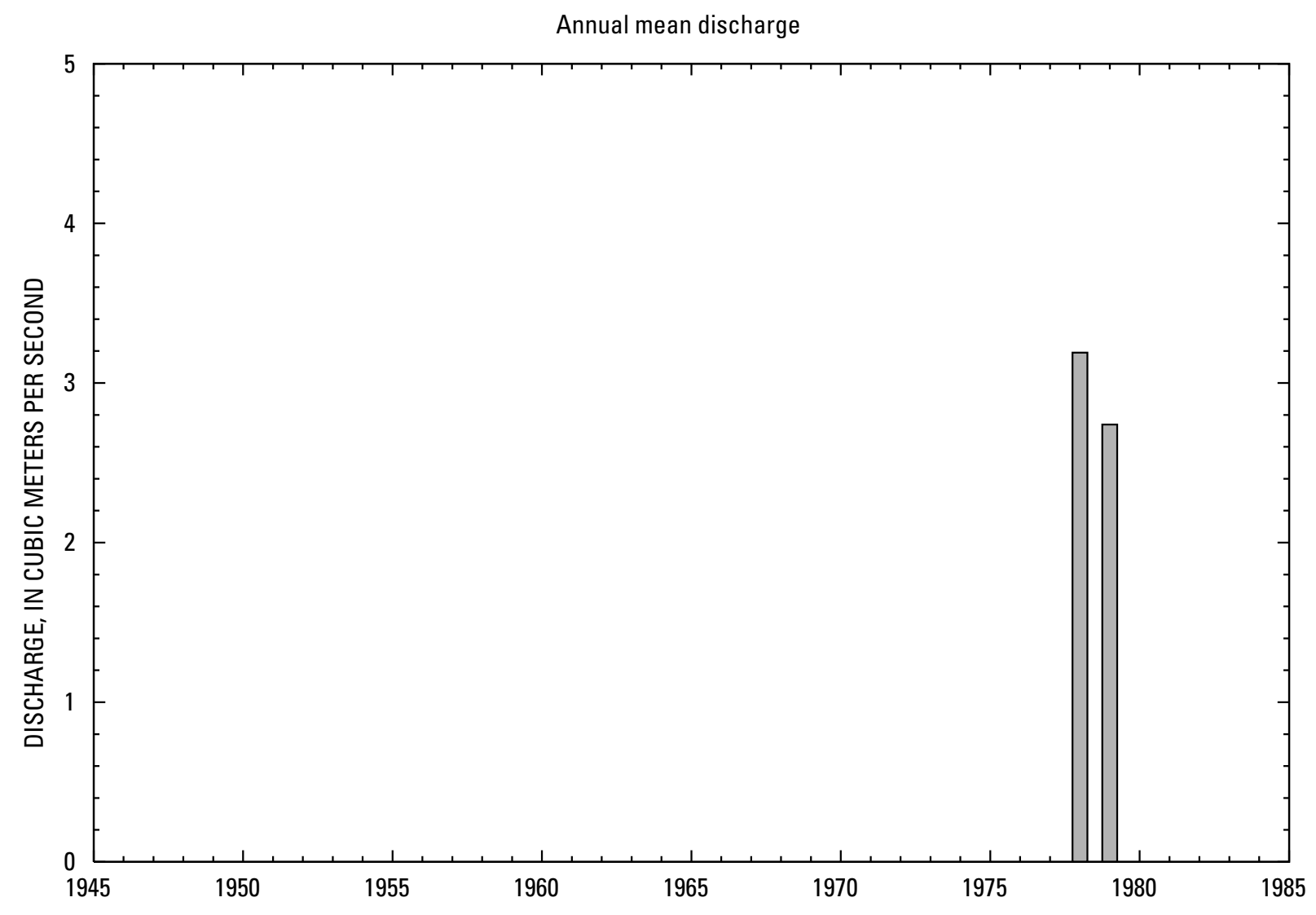




\section{4- 1.222R-6A LORA RIVER NEAR SHINKAY, Continued}

\section{Statistics of monthly and annual mean discharges}

$\left[\mathrm{m}^{3} / \mathrm{s}\right.$, meters per second]

\begin{tabular}{|c|c|c|c|c|c|c|c|c|}
\hline \multirow[b]{2}{*}{ Month } & \multicolumn{2}{|c|}{ Maximum } & \multicolumn{2}{|c|}{ Minimum } & \multicolumn{4}{|c|}{ Mean } \\
\hline & $\begin{array}{c}\text { Discharge } \\
\left(\mathrm{m}^{3} / \mathrm{s}\right)\end{array}$ & $\begin{array}{c}\text { Water year } \\
\text { of } \\
\text { occurrence }\end{array}$ & $\begin{array}{c}\text { Discharge } \\
\left(\mathrm{m}^{3} / \mathrm{s}\right)\end{array}$ & $\begin{array}{c}\text { Water year } \\
\text { of } \\
\text { occurrence }\end{array}$ & $\begin{array}{c}\text { Discharge } \\
\left(\mathrm{m}^{3} / \mathrm{s}\right)\end{array}$ & $\begin{array}{c}\text { Standard } \\
\text { deviation } \\
\left(\mathrm{m}^{3} / \mathrm{s}\right)\end{array}$ & $\begin{array}{c}\text { Coefficient } \\
\text { of } \\
\text { variation }\end{array}$ & $\begin{array}{c}\text { Percentage } \\
\text { of annual } \\
\text { discharge }\end{array}$ \\
\hline October & 1.28 & 1979 & 0.064 & 1978 & 0.67 & 0.86 & 1.28 & 1.87 \\
\hline November & 3.11 & 1979 & 0.232 & 1978 & 1.67 & 2.04 & 1.22 & 4.66 \\
\hline December & 7.67 & 1978 & 0.615 & 1979 & 4.14 & 4.99 & 1.20 & 11.6 \\
\hline January & 4.16 & 1978 & 0.242 & 1979 & 2.20 & 2.77 & 1.26 & 6.14 \\
\hline February & 9.04 & 1979 & 5.68 & 1978 & 7.36 & 2.38 & 0.32 & 20.5 \\
\hline March & 4.91 & 1978 & 2.90 & 1979 & 3.90 & 1.42 & 0.36 & 10.9 \\
\hline April & 7.96 & 1979 & 1.16 & 1978 & 4.56 & 4.81 & 1.05 & 12.7 \\
\hline May & 2.95 & 1979 & 0.955 & 1978 & 1.95 & 1.41 & 0.72 & 5.45 \\
\hline June & 1.06 & 1979 & 0.445 & 1978 & 0.75 & 0.43 & 0.58 & 2.10 \\
\hline July & 7.16 & 1978 & 0.897 & 1979 & 4.03 & 4.43 & 1.10 & 11.2 \\
\hline August & 5.13 & 1978 & 2.34 & 1979 & 3.74 & 1.97 & 0.53 & 10.4 \\
\hline September & 1.11 & 1979 & 0.626 & 1978 & 0.87 & 0.34 & 0.39 & 2.42 \\
\hline Annual & 3.19 & 1978 & 2.74 & 1979 & 2.97 & 0.32 & 0.11 & 100 \\
\hline
\end{tabular}

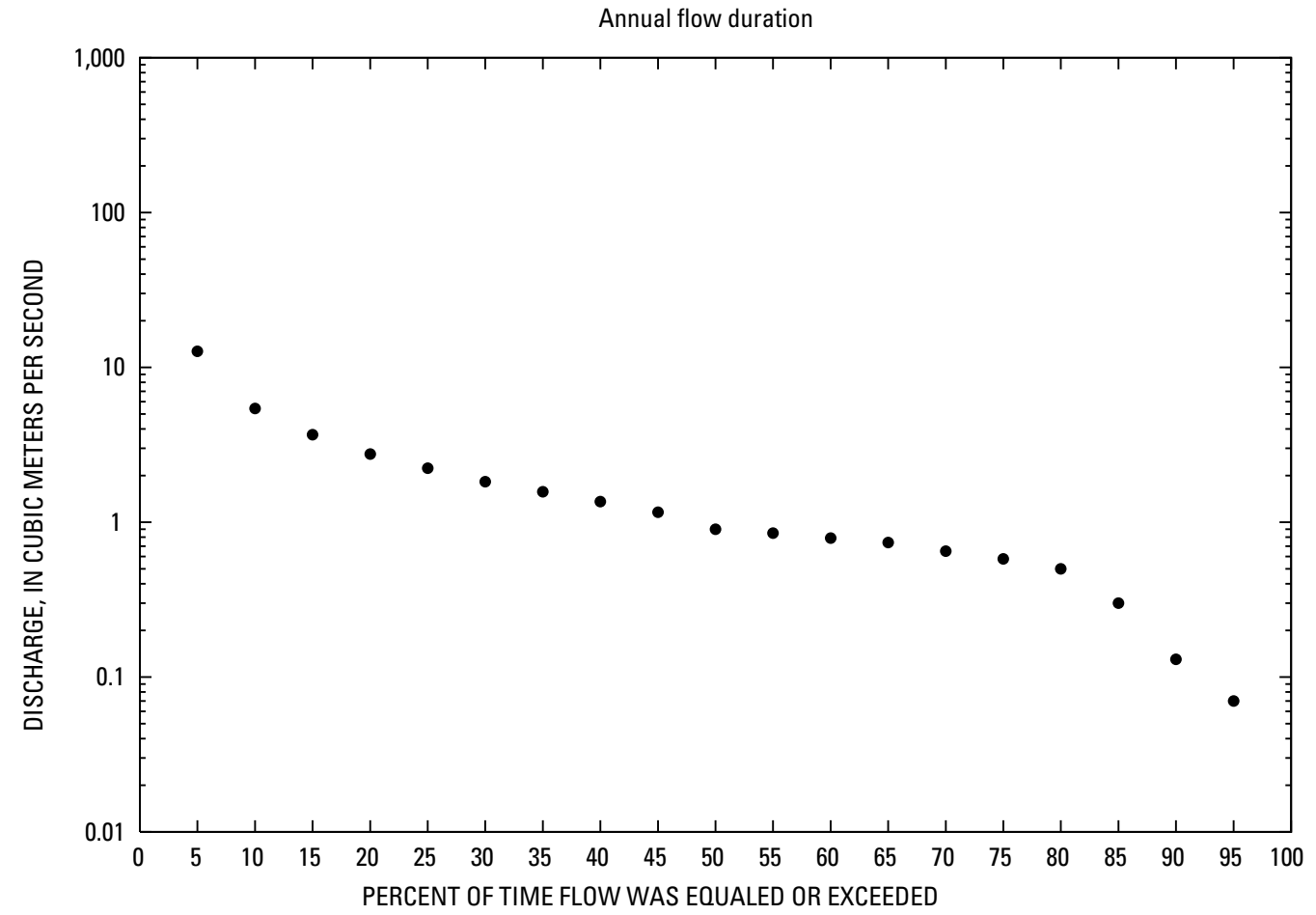




\section{4- 1.222R-6A LORA RIVER NEAR SHINKAY, Continued}

Monthly and annual flow duration, in cubic meters per second

[Data were not rounded in accordance with U.S. Geological Survey publication standards]

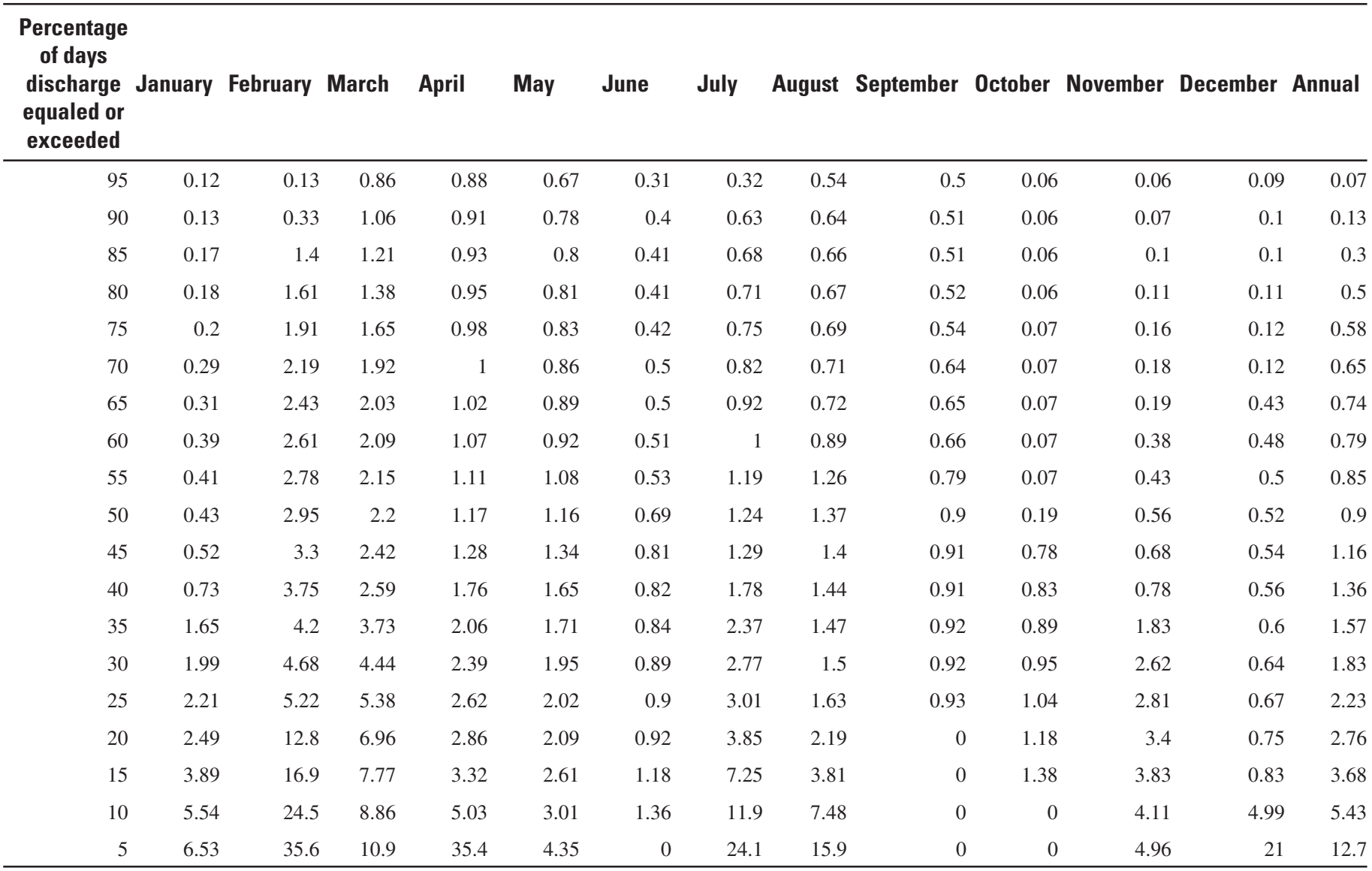




\section{4- 1.222R-6A LORA RIVER NEAR SHINKAY, Continued}

Probability of occurrence of annual high discharges

[ $\mathrm{m}^{3} / \mathrm{s}$, meters per second; $\mathrm{ng}$, statistic not given]

\begin{tabular}{|c|c|c|c|c|c|c|}
\hline \multirow{2}{*}{$\begin{array}{c}\text { Exceedance } \\
\text { probability }\end{array}$} & \multirow{2}{*}{$\begin{array}{c}\text { Recurrence } \\
\text { interval } \\
\text { (years) }\end{array}$} & \multirow{2}{*}{$\begin{array}{c}\text { Maximum } \\
\text { instantaneous } \\
\left(\mathrm{m}^{3} / \mathrm{s}\right)\end{array}$} & \multicolumn{4}{|c|}{ Maximum mean discharge } \\
\hline & & & 3-day period & 7-day period & 15-day period & 30-day period \\
\hline 0.99 & 1.01 & ng & ng & ng & ng & ng \\
\hline 0.95 & 1.05 & ng & ng & ng & ng & ng \\
\hline 0.90 & 1.11 & ng & ng & ng & ng & ng \\
\hline 0.80 & 1.25 & ng & ng & $\mathrm{ng}$ & ng & ng \\
\hline 0.50 & 2 & ng & ng & ng & ng & ng \\
\hline 0.20 & 5 & ng & ng & ng & ng & ng \\
\hline 0.10 & 10 & ng & ng & ng & ng & ng \\
\hline 0.04 & 25 & ng & ng & ng & ng & ng \\
\hline 0.02 & 50 & ng & ng & ng & ng & ng \\
\hline 0.01 & 100 & ng & ng & ng & ng & ng \\
\hline 0.005 & 200 & ng & ng & $\mathrm{ng}$ & ng & ng \\
\hline 0.002 & 500 & $\mathrm{ng}$ & ng & ng & $\mathrm{ng}$ & ng \\
\hline
\end{tabular}

\section{4- 1.222R-6A LORA RIVER NEAR SHINKAY, Continued}

Probability of occurrence of annual low discharges

[m³/s, meters per second; ng, statistic not given]

\begin{tabular}{|c|c|c|c|c|c|c|c|c|c|c|}
\hline \multirow{3}{*}{$\begin{array}{c}\text { Nonexceedance } \\
\text { probability }\end{array}$} & \multirow{3}{*}{$\begin{array}{c}\text { Recurrence } \\
\text { interval } \\
\text { (years) }\end{array}$} & \multicolumn{9}{|c|}{ Minimum mean discharge $\left(\mathrm{m}^{3} / \mathrm{s}\right)$} \\
\hline & & \multicolumn{9}{|c|}{ Number of consecutive days } \\
\hline & & 1 & 3 & 7 & 14 & 30 & 60 & 90 & 120 & 183 \\
\hline 0.05 & 20 & ng & ng & ng & ng & ng & ng & ng & ng & ng \\
\hline 0.10 & 10 & ng & ng & ng & ng & ng & ng & ng & ng & ng \\
\hline 0.20 & 5 & ng & ng & ng & ng & ng & ng & ng & ng & ng \\
\hline 0.50 & 2 & ng & ng & ng & ng & ng & ng & ng & $\mathrm{ng}$ & ng \\
\hline
\end{tabular}




\section{4- 1.222R-6A LORA RIVER NEAR SHINKAY, Continued}

Probability of occurrence of seasonal low discharges

[ $\mathrm{m}^{3} / \mathrm{s}$, meters per second; $\mathbf{n g}$, statistic not given]

\begin{tabular}{|c|c|c|c|c|c|c|c|c|c|}
\hline \multirow{3}{*}{$\begin{array}{c}\text { Nonexceedance } \\
\text { probability }\end{array}$} & \multirow{3}{*}{$\begin{array}{c}\text { Recurrence } \\
\text { interval } \\
\text { (years) }\end{array}$} & \multicolumn{8}{|c|}{ Minimum mean discharge $\left(\mathrm{m}^{3} / \mathrm{s}\right)$} \\
\hline & & \multicolumn{8}{|c|}{ Number of consecutive days } \\
\hline & & 1 & 7 & 14 & 30 & 1 & 7 & 14 & 30 \\
\hline & & \multicolumn{4}{|c|}{ December-January-February } & \multicolumn{4}{|c|}{ March-April-May } \\
\hline 0.05 & 20 & ng & ng & ng & $\mathrm{ng}$ & ng & ng & ng & ng \\
\hline 0.10 & 10 & ng & ng & ng & $\mathrm{ng}$ & ng & ng & ng & ng \\
\hline 0.20 & 5 & ng & ng & $\mathrm{ng}$ & ng & ng & ng & ng & ng \\
\hline \multirow[t]{2}{*}{0.50} & 2 & ng & ng & $\mathrm{ng}$ & $\mathrm{ng}$ & ng & ng & ng & ng \\
\hline & & \multicolumn{4}{|c|}{ June-July-August } & \multicolumn{4}{|c|}{ September-October-November } \\
\hline 0.05 & 20 & ng & ng & $\mathrm{ng}$ & ng & ng & ng & ng & ng \\
\hline 0.10 & 10 & ng & $\mathrm{ng}$ & $\mathrm{ng}$ & $\mathrm{ng}$ & $\mathrm{ng}$ & ng & ng & ng \\
\hline 0.20 & 5 & ng & ng & ng & $\mathrm{ng}$ & $\mathrm{ng}$ & ng & ng & ng \\
\hline 0.50 & 2 & ng & $\mathrm{ng}$ & $\mathrm{ng}$ & $\mathrm{ng}$ & $\mathrm{ng}$ & $\mathrm{ng}$ & $\mathrm{ng}$ & $\mathrm{ng}$ \\
\hline
\end{tabular}

\section{4- 1.222R-6A LORA RIVER NEAR SHINKAY, Continued}

Annual peak discharge and corresponding gage height

[ $\mathrm{m}^{3} / \mathrm{s}$, meters per second; --, no data]

\begin{tabular}{|c|c|c|c|c|c|c|c|}
\hline $\begin{array}{c}\text { Water } \\
\text { year }\end{array}$ & Date & $\begin{array}{c}\text { Gage } \\
\text { height } \\
\text { (meters) }\end{array}$ & $\begin{array}{c}\text { Peak } \\
\text { discharge } \\
\left(\mathrm{m}^{3} / \mathbf{s}\right)\end{array}$ & $\begin{array}{l}\text { Water } \\
\text { year }\end{array}$ & Date & $\begin{array}{c}\text { Gage } \\
\text { height } \\
\text { (meters) }\end{array}$ & $\begin{array}{c}\text { Peak } \\
\text { discharge } \\
\left(\mathrm{m}^{3} / \mathrm{s}\right)\end{array}$ \\
\hline
\end{tabular}

Annual peak discharge, by year

\begin{tabular}{lccc}
1978 & $25-\mathrm{Dec}$ & -- & 160 \\
1979 & $2-\mathrm{Apr}$ & -- & 66.6 \\
& \multicolumn{3}{r}{ Annual peak discharge, from highest to lowest } \\
1978 & $25-\mathrm{Dec}$ & -- & 160 \\
1979 & $2-\mathrm{Apr}$ & -- & 66.6 \\
\hline
\end{tabular}




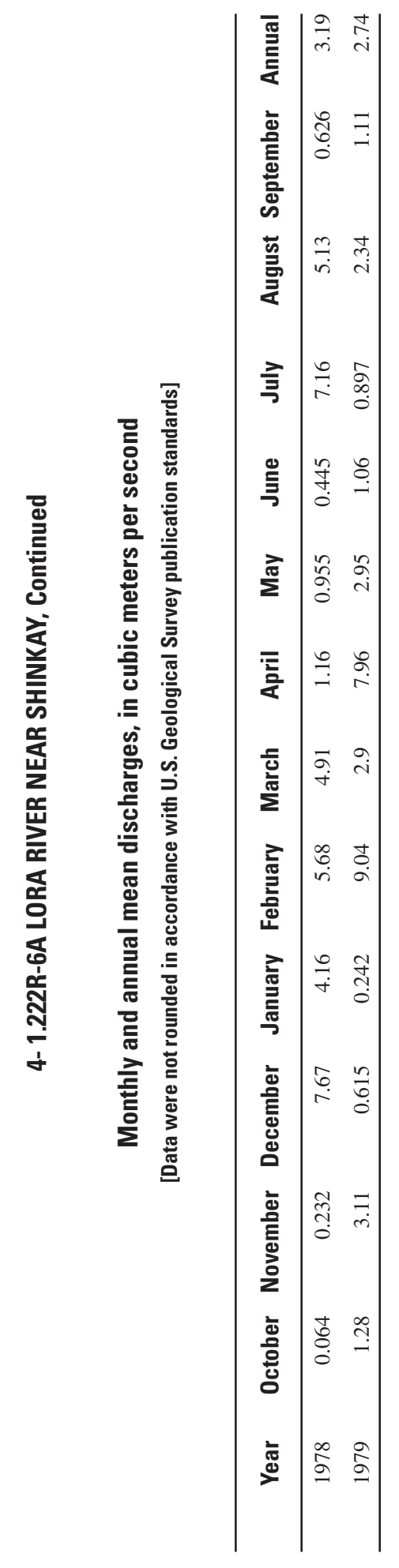




\section{4- 1.22R-1A ARGHASTAN RIVER NEAR KANDAHAR}

\section{(U.S. Geological Survey identification number: 312600065550000)}

LOCATION: Lat $31^{\circ} 26^{\prime}$ N., long $65^{\circ} 55^{\prime}$ E., on upstream side of bridge pier on Kandahar-Chaman Highway, 22 km upstream from Dori River, and $28 \mathrm{~km}$ southeast of Kandahar.

DRAINAGE AREA: $15,245 \mathrm{~km}^{2}$.

ALTITUDE: 1,031 meters plus mean sea level.

PERIOD OF RECORD: October 1, 1952 to September 30, 1979.

GAGE: Water-stage recorder.

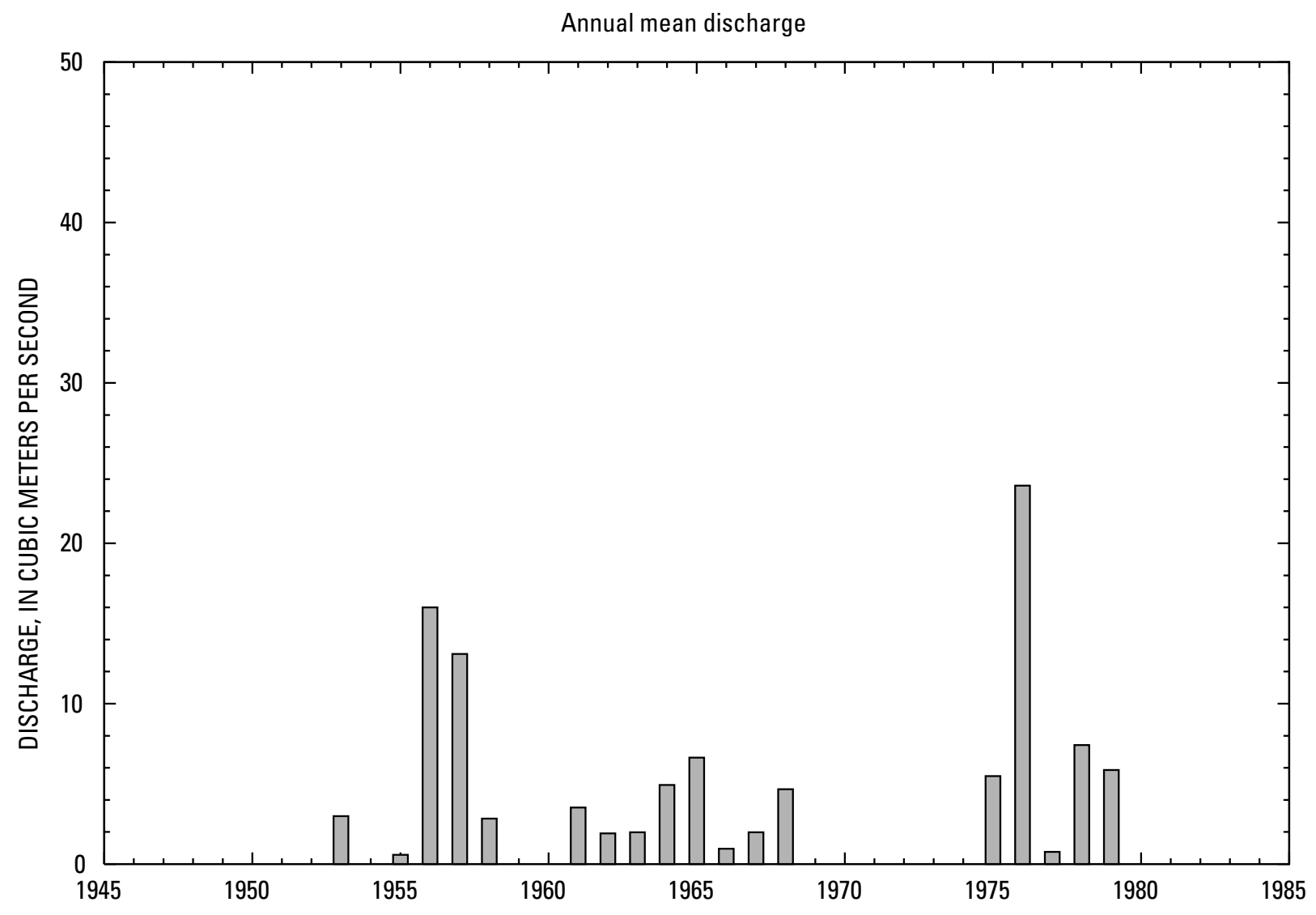




\section{4- 1.22R-1A ARGHASTAN RIVER NEAR KANDAHAR, Continued}

\section{Statistics of monthly and annual mean discharges}

[m3/s, meters per second; $\mathbf{m}$, more than 2 years of occurrence; ng, statistic not given]

\begin{tabular}{|c|c|c|c|c|c|c|c|c|}
\hline \multirow[b]{2}{*}{ Month } & \multicolumn{2}{|c|}{ Maximum } & \multicolumn{2}{|c|}{ Minimum } & \multicolumn{4}{|c|}{ Mean } \\
\hline & $\begin{array}{c}\text { Discharge } \\
\left(\mathrm{m}^{3} / \mathrm{s}\right)\end{array}$ & $\begin{array}{c}\text { Water year } \\
\text { of } \\
\text { occurrence }\end{array}$ & $\begin{array}{c}\text { Discharge } \\
\left(\mathrm{m}^{3} / \mathrm{s}\right)\end{array}$ & $\begin{array}{c}\text { Water year } \\
\text { of } \\
\text { occurrence }\end{array}$ & $\begin{array}{c}\text { Discharge } \\
\left(\mathrm{m}^{3} / \mathrm{s}\right)\end{array}$ & $\begin{array}{c}\text { Standard } \\
\text { deviation } \\
\left(\mathrm{m}^{3} / \mathrm{s}\right)\end{array}$ & $\begin{array}{c}\text { Coefficient } \\
\text { of } \\
\text { variation }\end{array}$ & $\begin{array}{c}\text { Percentage } \\
\text { of annual } \\
\text { discharge }\end{array}$ \\
\hline October & 0 & $\mathrm{~m}$ & 0 & $\mathrm{~m}$ & 0 & 0 & ng & 0 \\
\hline November & 0.78 & 1979 & 0 & $\mathrm{~m}$ & 0.04 & 0.18 & 4.17 & 0.06 \\
\hline December & 16.1 & 1978 & 0 & $\mathrm{~m}$ & 2.25 & 5.16 & 2.29 & 3.18 \\
\hline January & 15.7 & 1957 & 0 & $\mathrm{~m}$ & 3.21 & 4.72 & 1.47 & 4.53 \\
\hline February & 50.1 & 1957 & 0 & 1961,1962 & 15.3 & 15.2 & 0.99 & 21.6 \\
\hline March & 123 & 1976 & 0.201 & 1965 & 21.3 & 29.8 & 1.40 & 30.1 \\
\hline April & 111 & 1976 & 0 & $\mathrm{~m}$ & 16.8 & 29.2 & 1.73 & 23.8 \\
\hline May & 13.6 & 1957 & 0 & $\mathrm{~m}$ & 2.25 & 4.37 & 1.95 & 3.17 \\
\hline June & 0.779 & 1957 & 0 & $\mathrm{~m}$ & 0.07 & 0.2 & 2.96 & 0.09 \\
\hline July & 97.5 & 1956 & 0 & $\mathrm{~m}$ & 7.52 & 23.1 & 3.07 & 10.6 \\
\hline August & 15.3 & 1978 & 0 & $\mathrm{~m}$ & 2.04 & 4.49 & 2.20 & 2.88 \\
\hline September & 0.007 & 1956 & 0 & $\mathrm{~m}$ & 0 & 0 & 3.38 & 0 \\
\hline Annual & 23.6 & 1976 & 0.573 & 1955 & 5.84 & 6.05 & 1.04 & 100 \\
\hline
\end{tabular}

Annual flow duration

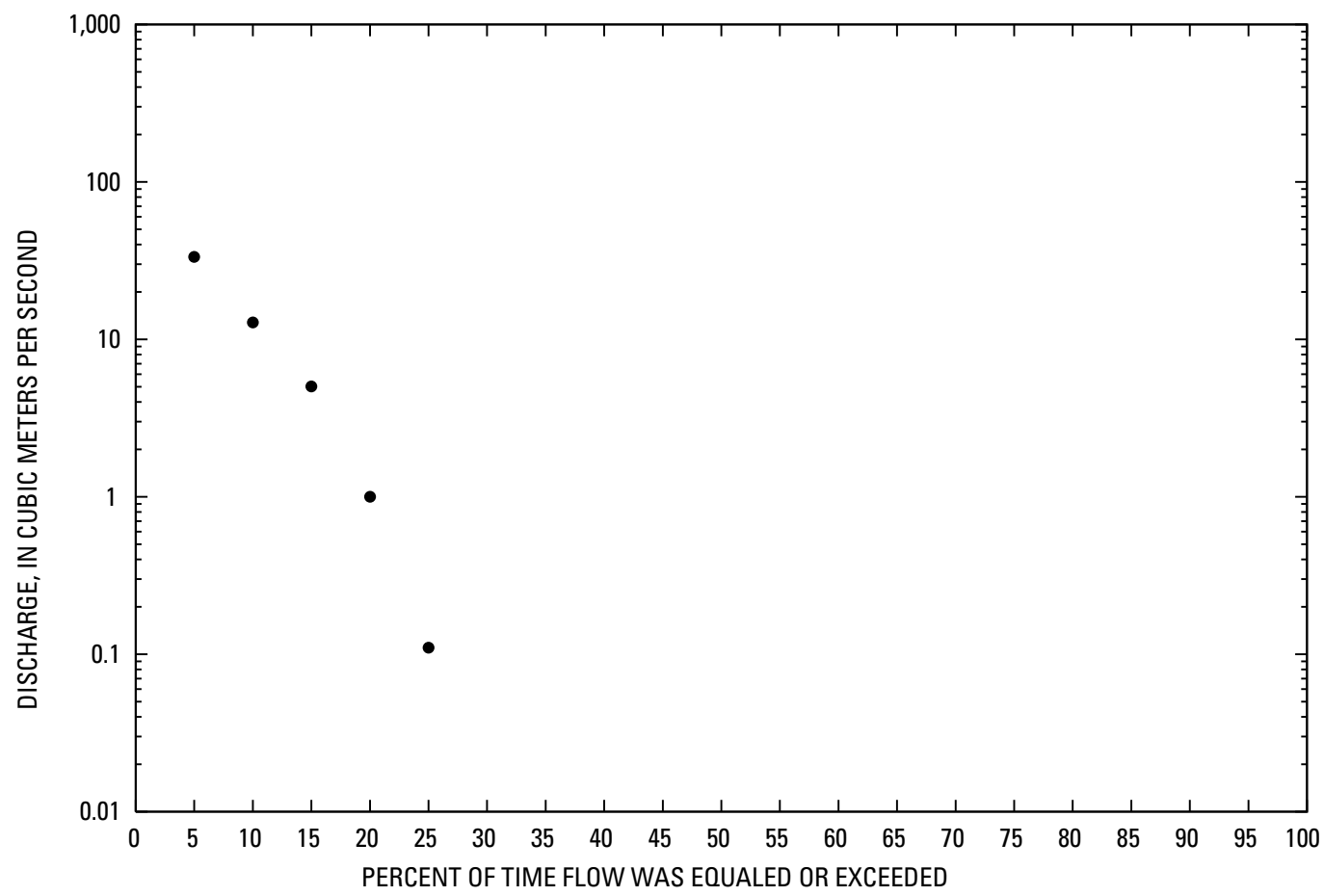




\begin{tabular}{|c|c|c|c|c|c|c|c|c|c|c|c|c|c|}
\hline $\begin{array}{l}\text { Percentage } \\
\text { of days } \\
\text { discharge } \\
\text { equaled or } \\
\text { exceeded }\end{array}$ & January & February & March & April & May & June & July & August & September & October & November & December & Annual \\
\hline 95 & 0 & 0 & 0 & 0 & 0 & 0 & 0 & 0 & 0 & 0 & 0 & 0 & 0 \\
\hline 90 & 0 & 0 & 0 & 0 & 0 & 0 & 0.01 & 0 & 0 & 0 & 0 & 0 & 0 \\
\hline 85 & 0 & 0 & 0 & 0 & 0 & 0 & 0.02 & 0 & 0 & 0 & 0 & 0 & 0 \\
\hline 80 & 0 & 0 & 0 & 0 & 0 & 0 & 0.02 & 0 & 0 & 0 & 0 & 0 & 0 \\
\hline 75 & 0 & 0 & 0 & 0 & 0 & 0 & 0.03 & 0 & 0 & 0 & 0 & 0 & 0 \\
\hline 70 & 0 & 0 & 0.08 & 0 & 0 & 0 & 0.04 & 0 & 0 & 0 & 0 & 0 & 0 \\
\hline 65 & 0 & 0 & 0.55 & 0 & 0 & 0 & 0.04 & 0 & 0 & 0 & 0 & 0 & 0 \\
\hline 60 & 0 & 0.37 & 1.55 & 0 & 0 & 0 & 0.05 & 0 & 0 & 0 & 0 & 0 & 0 \\
\hline 55 & 0 & 1.79 & 4.57 & 0 & 0 & 0 & 0.05 & 0 & 0 & 0 & 0 & 0 & 0 \\
\hline 50 & 0 & 3.44 & 7.26 & 0.09 & 0 & 0 & 0.06 & 0 & 0 & 0 & 0 & 0 & 0 \\
\hline 45 & 0 & 5.28 & 10.4 & 0.37 & 0 & 0 & 0.06 & 0 & 0 & 0 & 0 & 0 & 0 \\
\hline 40 & 0.02 & 6.64 & 12.8 & 1.02 & 0 & 0 & 0.07 & 0 & 0 & 0 & 0 & 0 & 0 \\
\hline 35 & 0.42 & 8.4 & 15.5 & 4.1 & 0 & 0 & 0.08 & 0 & 0 & 0 & 0 & 0 & 0 \\
\hline 30 & 0.92 & 11.6 & 18.5 & 8.16 & 0 & 0 & 0.08 & 0 & 0 & 0 & 0 & 0 & 0 \\
\hline 25 & 1.84 & 18.1 & 24.2 & 13.5 & 0.1 & 0 & 0.09 & 0 & 0 & 0 & 0 & 0 & 0.11 \\
\hline 20 & 3.12 & 27.4 & 30.2 & 20.4 & 0.35 & 0 & 0.09 & 0 & 0 & 0 & 0 & 0.01 & 1 \\
\hline 15 & 6.88 & 37.5 & 38.1 & 32.2 & 0.76 & 0 & 0.1 & 0.29 & 0 & 0 & 0 & 0.1 & 5.02 \\
\hline 10 & 9.87 & 48.9 & 53.6 & 53.4 & 4.52 & 0.02 & 3 & 2.03 & 0 & 0 & 0 & 2 & 12.8 \\
\hline 5 & 15.7 & 66.5 & 86.9 & 89 & 11.7 & 0.31 & 23.3 & 7.8 & 0 & 0 & 0 & 11.5 & 33.4 \\
\hline
\end{tabular}




\section{4- 1.22R-1A ARGHASTAN RIVER NEAR KANDAHAR, Continued}

Probability of occurrence of annual high discharges

[ $\mathrm{m}^{3} / \mathrm{s}$, meters per second; $\mathrm{ng}$, statistic not given]

\begin{tabular}{|c|c|c|c|c|c|c|}
\hline \multirow{2}{*}{$\begin{array}{l}\text { Exceedance } \\
\text { probability }\end{array}$} & \multirow{2}{*}{$\begin{array}{c}\text { Recurrence } \\
\text { interval } \\
\text { (years) }\end{array}$} & \multirow{2}{*}{$\begin{array}{c}\text { Maximum } \\
\text { instantaneous } \\
\left(\mathrm{m}^{3} / \mathrm{s}\right)\end{array}$} & \multicolumn{4}{|c|}{ Maximum mean discharge } \\
\hline & & & 3-day period & 7-day period & 15-day period & 30-day period \\
\hline 0.99 & 1.01 & 22.6 & 18.6 & 11.4 & 6.24 & 2.61 \\
\hline 0.95 & 1.05 & 44.0 & 30.5 & 19.2 & 10.5 & 5.46 \\
\hline 0.90 & 1.11 & 62.9 & 39.8 & 25.4 & 14.0 & 8.00 \\
\hline 0.80 & 1.25 & 97.0 & 54.9 & 35.4 & 20.0 & 12.6 \\
\hline 0.50 & 2 & 222 & 102 & 66.7 & 40.6 & 29.1 \\
\hline 0.20 & 5 & 512 & 191 & 125 & 85.3 & 64.9 \\
\hline 0.10 & 10 & 793 & 266 & 173 & 128 & 97.1 \\
\hline 0.04 & 25 & 1,260 & 378 & 244 & 198 & 148 \\
\hline 0.02 & 50 & 1,710 & 476 & 304 & 265 & 192 \\
\hline 0.01 & 100 & 2,240 & 585 & 371 & 345 & 243 \\
\hline 0.005 & 200 & 2,880 & 708 & 445 & 442 & 300 \\
\hline 0.002 & 500 & 3,900 & ng & ng & ng & ng \\
\hline
\end{tabular}

\section{4- 1.22R-1A ARGHASTAN RIVER NEAR KANDAHAR, Continued}

Probability of occurrence of annual low discharges

[m3/s, meters per second; ng, statistic not given]

\begin{tabular}{|c|c|c|c|c|c|c|c|c|c|c|}
\hline \multirow{3}{*}{$\begin{array}{c}\text { Nonexceedance } \\
\text { probability }\end{array}$} & \multirow{3}{*}{$\begin{array}{c}\text { Recurrence } \\
\text { interval } \\
\text { (years) }\end{array}$} & \multicolumn{9}{|c|}{ Minimum mean discharge $\left(\mathrm{m}^{3} / \mathrm{s}\right)$} \\
\hline & & \multicolumn{9}{|c|}{ Number of consecutive days } \\
\hline & & 1 & 3 & 7 & 14 & 30 & 60 & 90 & 120 & 183 \\
\hline 0.05 & 20 & ng & ng & ng & ng & ng & $\mathrm{ng}$ & ng & 0 & 0 \\
\hline 0.10 & 10 & $\mathrm{ng}$ & $\mathrm{ng}$ & ng & ng & ng & $\mathrm{ng}$ & ng & 0 & 0 \\
\hline 0.20 & 5 & ng & ng & ng & ng & ng & ng & ng & 0 & 0 \\
\hline 0.50 & 2 & ng & $\mathrm{ng}$ & ng & ng & ng & ng & ng & 0 & 0 \\
\hline
\end{tabular}




\section{4- 1.22R-1A ARGHASTAN RIVER NEAR KANDAHAR, Continued}

\section{Probability of occurrence of seasonal low discharges}

[m3/s, meters per second; ng, statistic not given]

\begin{tabular}{|c|c|c|c|c|c|c|c|c|c|}
\hline \multirow{3}{*}{$\begin{array}{c}\text { Nonexceedance } \\
\text { probability }\end{array}$} & \multirow{3}{*}{$\begin{array}{c}\text { Recurrence } \\
\text { interval } \\
\text { (years) }\end{array}$} & \multicolumn{8}{|c|}{ Minimum mean discharge $\left(\mathrm{m}^{3} / \mathrm{s}\right)$} \\
\hline & & \multicolumn{8}{|c|}{ Number of consecutive days } \\
\hline & & 1 & 7 & 14 & 30 & 1 & 7 & 14 & 30 \\
\hline & & \multicolumn{4}{|c|}{ December-January-February } & \multicolumn{4}{|c|}{ March-April-May } \\
\hline 0.05 & 20 & $\mathrm{ng}$ & ng & 0 & 0 & ng & ng & 0 & 0 \\
\hline 0.10 & 10 & $\mathrm{ng}$ & ng & 0 & 0 & ng & $\mathrm{ng}$ & 0 & 0 \\
\hline 0.20 & 5 & ng & ng & 0 & 0 & ng & $\mathrm{ng}$ & 0 & 0 \\
\hline \multirow[t]{2}{*}{0.50} & 2 & ng & ng & 0 & 0 & ng & ng & 0 & 0 \\
\hline & & \multicolumn{4}{|c|}{ June-July-August } & \multicolumn{4}{|c|}{ September-October-November } \\
\hline 0.05 & 20 & ng & ng & ng & ng & ng & ng & ng & ng \\
\hline 0.10 & 10 & ng & ng & ng & ng & ng & ng & ng & ng \\
\hline 0.20 & 5 & ng & ng & ng & ng & ng & ng & ng & $\mathrm{ng}$ \\
\hline 0.50 & 2 & $\mathrm{ng}$ & ng & ng & ng & ng & ng & $\mathrm{ng}$ & $\mathrm{ng}$ \\
\hline
\end{tabular}




\section{4- 1.22R-1A ARGHASTAN RIVER NEAR KANDAHAR, Continued}

\section{Annual peak discharge and corresponding gage height}

[m³/s, meters per second; --, no data]

\begin{tabular}{|c|c|c|c|c|c|c|c|}
\hline $\begin{array}{l}\text { Water } \\
\text { year }\end{array}$ & Date & $\begin{array}{c}\text { Gage } \\
\text { height } \\
\text { (meters) }\end{array}$ & $\begin{array}{c}\text { Peak } \\
\text { discharge } \\
\left(\mathrm{m}^{3} / \mathrm{s}\right)\end{array}$ & $\begin{array}{c}\text { Water } \\
\text { year }\end{array}$ & Date & $\begin{array}{c}\text { Gage } \\
\text { height } \\
\text { (meters) }\end{array}$ & $\begin{array}{c}\text { Peak } \\
\text { discharge } \\
\left(\mathrm{m}^{3} / \mathrm{s}\right)\end{array}$ \\
\hline \multicolumn{8}{|c|}{ Annual peak discharge, by year } \\
\hline 1953 & 14-Feb & $1,020.00$ & 314 & 1964 & 6-Jan & -- & 219 \\
\hline 1954 & 13-Feb & $1,021.09$ & 1,190 & 1965 & 23-Apr & -- & 800 \\
\hline 1955 & 16-Mar & $1,019.98$ & 81.2 & 1966 & 26-Feb & -- & 113 \\
\hline 1956 & 20-Jul & $1,020.80$ & 620 & 1967 & 19-Feb & -- & 160 \\
\hline 1957 & 8-Apr & $1,020.01$ & 147 & 1968 & 26-Feb & -- & 131 \\
\hline 1958 & 14-Dec & $1,020.40$ & 169 & 1975 & 13-Feb & -- & 329 \\
\hline 1959 & 2-Mar & $1,019.98$ & 122 & 1976 & 15-Mar & -- & 980 \\
\hline 1961 & 9-Apr & -- & 228 & 1977 & 13-Aug & -- & 26.6 \\
\hline 1962 & 31-Mar & -- & 78 & 1978 & 25-Dec & -- & 731 \\
\hline 1963 & 27-Apr & -- & 157 & & & & \\
\hline \multicolumn{8}{|c|}{ Annual peak discharge, from highest to lowest } \\
\hline 1954 & 13-Feb & $1,021.09$ & 1,190 & 1967 & 19-Feb & -- & 160 \\
\hline 1976 & 15-Mar & -- & 980 & 1963 & 27-Apr & -- & 157 \\
\hline 1965 & 23-Apr & -- & 800 & 1957 & 8-Apr & $1,020.01$ & 147 \\
\hline 1978 & 25-Dec & -- & 731 & 1968 & 26-Feb & -- & 131 \\
\hline 1956 & 20-Jul & $1,020.80$ & 620 & 1959 & 2-Mar & $1,019.98$ & 122 \\
\hline 1975 & 13-Feb & -- & 329 & 1966 & 26-Feb & -- & 113 \\
\hline 1953 & 14-Feb & $1,020.00$ & 314 & 1955 & 16-Mar & $1,019.98$ & 81.2 \\
\hline 1961 & 9-Apr & -- & 228 & 1962 & 31-Mar & -- & 78 \\
\hline 1964 & 6-Jan & -- & 219 & 1977 & 13-Aug & -- & 26.6 \\
\hline 1958 & 14-Dec & $1,020.40$ & 169 & & & & \\
\hline
\end{tabular}




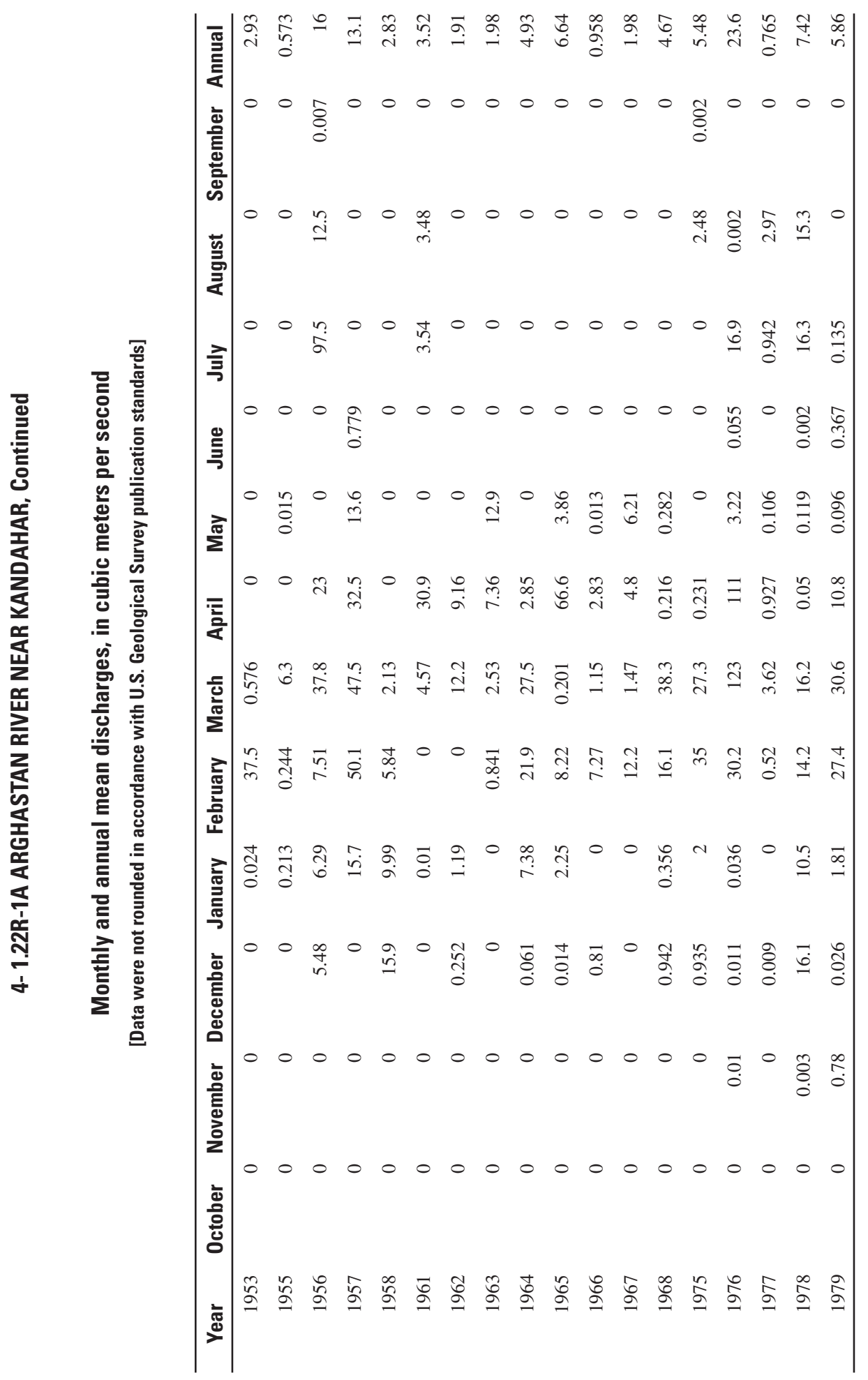




\section{4- 1.2L0-5A DORI RIVER NEAR TAKHTAPUL}

\section{(U.S. Geological Survey identification number: 311300065570000)}

LOCATION: Lat $31^{\circ} 13^{\prime} \mathrm{N}$., long $65^{\circ} 57^{\prime} \mathrm{E}$.

DRAINAGE AREA: $4,185 \mathrm{~km}^{2}$, of which approximately $960 \mathrm{~km}^{2}$ is considered non-contributing.

ALTITUDE: 1,050 meters plus mean sea level.

PERIOD OF RECORD: October 1, 1976 to March 2, 1980.

GAGE: --

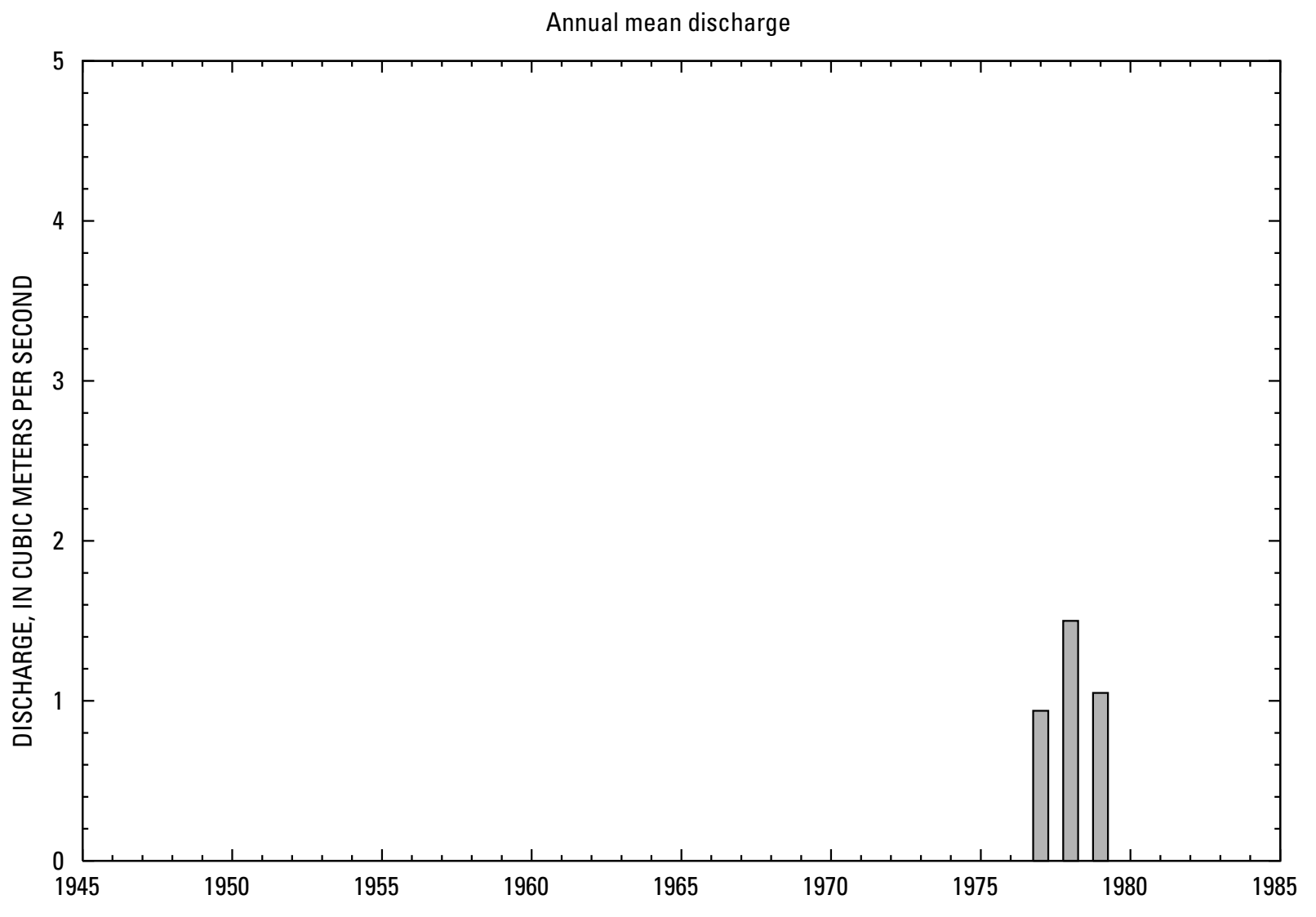




\section{4- 1.2L0-5A DORI RIVER NEAR TAKHTAPUL, Continued}

\section{Statistics of monthly and annual mean discharges}

[ $\mathrm{m}^{3} / \mathrm{s}$, meters per second]

\begin{tabular}{|c|c|c|c|c|c|c|c|c|}
\hline \multirow[b]{2}{*}{ Month } & \multicolumn{2}{|c|}{ Maximum } & \multicolumn{2}{|c|}{ Minimum } & \multicolumn{4}{|c|}{ Mean } \\
\hline & $\begin{array}{c}\text { Discharge } \\
\left(\mathrm{m}^{3} / \mathrm{s}\right)\end{array}$ & $\begin{array}{c}\text { Water year } \\
\text { of } \\
\text { occurrence }\end{array}$ & $\begin{array}{c}\text { Discharge } \\
\left(\mathrm{m}^{3} / \mathrm{s}\right)\end{array}$ & $\begin{array}{c}\text { Water year } \\
\text { of } \\
\text { occurrence }\end{array}$ & $\begin{array}{c}\text { Discharge } \\
\left(\mathrm{m}^{3} / \mathrm{s}\right)\end{array}$ & $\begin{array}{c}\text { Standard } \\
\text { deviation } \\
\left(\mathrm{m}^{3} / \mathrm{s}\right)\end{array}$ & $\begin{array}{c}\text { Coefficient } \\
\text { of } \\
\text { variation }\end{array}$ & $\begin{array}{c}\text { Percentage } \\
\text { of annual } \\
\text { discharge }\end{array}$ \\
\hline October & 0.655 & 1977 & 0.010 & 1979,1980 & 0.17 & 0.32 & 1.88 & 1.14 \\
\hline November & 0.537 & 1979 & 0.010 & 1977 & 0.17 & 0.24 & 1.41 & 1.15 \\
\hline December & 0.901 & 1978 & 0.339 & 1979 & 0.68 & 0.27 & 0.40 & 4.51 \\
\hline January & 5.14 & 1980 & 0.652 & 1977 & 2.70 & 2.08 & 0.77 & 17.9 \\
\hline February & 9.12 & 1979 & 0.742 & 1978 & 3.85 & 3.94 & 1.02 & 25.5 \\
\hline March & 0.658 & 1979 & 0.048 & 1978 & 0.28 & 0.33 & 1.20 & 1.84 \\
\hline April & 0.192 & 1979 & 0.053 & 1978 & 0.11 & 0.07 & 0.68 & 0.72 \\
\hline May & 0.512 & 1979 & 0.010 & 1978 & 0.24 & 0.25 & 1.06 & 1.59 \\
\hline June & 0.229 & 1979 & 0.010 & 1978 & 0.11 & 0.11 & 0.96 & 0.76 \\
\hline July & 6.47 & 1978 & 0.110 & 1979 & 2.66 & 3.36 & 1.27 & 17.6 \\
\hline August & 6.36 & 1977 & 0.088 & 1979 & 3.96 & 3.38 & 0.86 & 26.2 \\
\hline September & 0.301 & 1978 & 0.044 & 1977 & 0.14 & 0.14 & 0.97 & 0.95 \\
\hline Annual & 1.50 & 1978 & 0.938 & 1977 & 1.16 & 0.30 & 0.26 & 100 \\
\hline
\end{tabular}

Annual flow duration

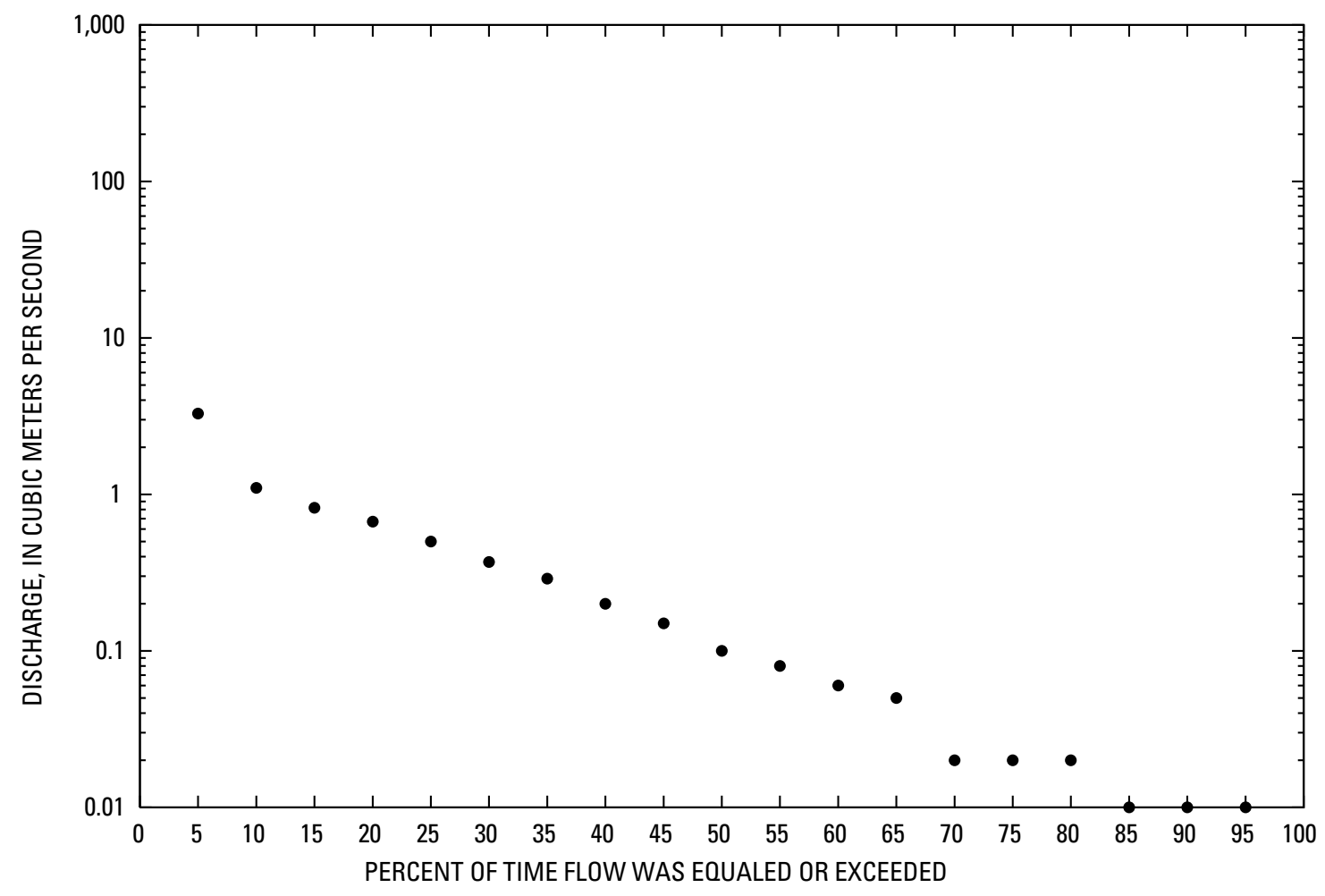




\section{4- 1.2L0-5A DORI RIVER NEAR TAKHTAPUL, Continued}

\section{Monthly and annual flow duration, in cubic meters per second}

[Data were not rounded in accordance with U.S. Geological Survey publication standards]

\begin{tabular}{|c|c|c|c|c|c|c|c|c|c|c|c|c|c|}
\hline $\begin{array}{l}\text { Percentage } \\
\text { of days } \\
\text { discharge } \\
\text { equaled or } \\
\text { exceeded }\end{array}$ & January & February & March & April & May & June & July & August & September & October & November & December & Annual \\
\hline 95 & 0.17 & 0.01 & 0.01 & 0.01 & 0.01 & 0.01 & 0.01 & 0.01 & 0.01 & 0.01 & 0.01 & 0.09 & 0.01 \\
\hline 90 & 0.3 & 0.02 & 0.01 & 0.01 & 0.01 & 0.01 & 0.02 & 0.02 & 0.01 & 0.01 & 0.01 & 0.12 & 0.01 \\
\hline 85 & 0.57 & 0.06 & 0.02 & 0.02 & 0.01 & 0.01 & 0.02 & 0.02 & 0.01 & 0.01 & 0.01 & 0.21 & 0.01 \\
\hline 80 & 0.61 & 0.2 & 0.02 & 0.02 & 0.02 & 0.02 & 0.05 & 0.04 & 0.01 & 0.01 & 0.01 & 0.24 & 0.02 \\
\hline 75 & 0.64 & 0.33 & 0.02 & 0.04 & 0.02 & 0.02 & 0.06 & 0.05 & 0.02 & 0.01 & 0.01 & 0.26 & 0.02 \\
\hline 70 & 0.67 & 0.43 & 0.05 & 0.04 & 0.02 & 0.02 & 0.09 & 0.05 & 0.02 & 0.01 & 0.01 & 0.28 & 0.02 \\
\hline 65 & 0.71 & 0.55 & 0.06 & 0.04 & 0.05 & 0.04 & 0.1 & 0.06 & 0.02 & 0.01 & 0.02 & 0.3 & 0.05 \\
\hline 60 & 0.75 & 0.75 & 0.08 & 0.05 & 0.06 & 0.05 & 0.1 & 0.06 & 0.02 & 0.01 & 0.02 & 0.32 & 0.06 \\
\hline 55 & 0.79 & 0.8 & 0.09 & 0.05 & 0.08 & 0.05 & 0.11 & 0.09 & 0.04 & 0.01 & 0.02 & 0.34 & 0.08 \\
\hline 50 & 0.84 & 0.84 & 0.09 & 0.05 & 0.1 & 0.06 & 0.12 & 0.12 & 0.05 & 0.02 & 0.02 & 0.38 & 0.1 \\
\hline 45 & 0.92 & 0.88 & 0.1 & 0.05 & 0.14 & 0.06 & 0.13 & 0.18 & 0.06 & 0.02 & 0.02 & 0.46 & 0.15 \\
\hline 40 & 1.03 & 0.93 & 0.1 & 0.06 & 0.21 & 0.1 & 0.17 & 0.2 & 0.09 & 0.02 & 0.02 & 0.55 & 0.2 \\
\hline 35 & 1.13 & 0.99 & 0.12 & 0.08 & 0.32 & 0.11 & 0.22 & 0.22 & 0.11 & 0.02 & 0.05 & 0.63 & 0.29 \\
\hline 30 & 1.22 & 1.18 & 0.23 & 0.08 & 0.41 & 0.13 & 0.41 & 0.26 & 0.13 & 0.02 & 0.09 & 0.71 & 0.37 \\
\hline 25 & 1.38 & 1.74 & 0.42 & 0.09 & 0.48 & 0.2 & 0.63 & 0.72 & 0.2 & 0.02 & 0.11 & 0.78 & 0.5 \\
\hline 20 & 1.64 & 2.24 & 0.62 & 0.09 & 0.51 & 0.21 & 1.68 & 2.05 & 0.22 & 0.02 & 0.24 & 0.85 & 0.67 \\
\hline 15 & 2.06 & 3.45 & 0.75 & 0.11 & 0.55 & 0.26 & 3.56 & 5.26 & 0.24 & 0.02 & 0.32 & 0.93 & 0.82 \\
\hline 10 & 3.05 & 7.11 & 0.89 & 0.22 & 0.61 & 0.29 & 6.44 & 13.7 & 0.38 & 0.02 & 0.5 & 1.09 & 1.1 \\
\hline 5 & 7.78 & 14.6 & 1.16 & 0.3 & 0.78 & 0 & 15 & 26 & 0.62 & 0.02 & 0.54 & 2.33 & 3.29 \\
\hline
\end{tabular}




\section{4- 1.2L0-5A DORI RIVER NEAR TAKHTAPUL, Continued}

\section{Probability of occurrence of annual high discharges}

[ $\mathrm{m}^{3} / \mathrm{s}$, meters per second; $\mathbf{n g}$, statistic not given]

\begin{tabular}{|c|c|c|c|c|c|c|}
\hline \multirow{2}{*}{$\begin{array}{c}\text { Exceedance } \\
\text { probability }\end{array}$} & \multirow{2}{*}{$\begin{array}{c}\text { Recurrence } \\
\text { interval } \\
\text { (years) }\end{array}$} & \multirow{2}{*}{$\begin{array}{c}\text { Maximum } \\
\text { instantaneous } \\
\left(\mathrm{m}^{3} / \mathrm{s}\right)\end{array}$} & \multicolumn{4}{|c|}{ Maximum mean discharge } \\
\hline & & & 3-day period & 7-day period & 15-day period & 30-day period \\
\hline 0.99 & 1.01 & 32.8 & ng & ng & ng & $\mathrm{ng}$ \\
\hline 0.95 & 1.05 & 47.0 & ng & ng & ng & ng \\
\hline 0.90 & 1.11 & 57.2 & $\mathrm{ng}$ & ng & ng & ng \\
\hline 0.80 & 1.25 & 73.1 & $\mathrm{ng}$ & ng & ng & $\mathrm{ng}$ \\
\hline 0.50 & 2 & 119 & ng & ng & ng & $\mathrm{ng}$ \\
\hline 0.20 & 5 & 197 & ng & ng & ng & ng \\
\hline 0.10 & 10 & 258 & $\mathrm{ng}$ & ng & ng & ng \\
\hline 0.04 & 25 & 348 & $\mathrm{ng}$ & ng & ng & ng \\
\hline 0.02 & 50 & 423 & ng & ng & ng & ng \\
\hline 0.01 & 100 & 506 & $\mathrm{ng}$ & ng & ng & $\mathrm{ng}$ \\
\hline 0.005 & 200 & 597 & ng & ng & ng & ng \\
\hline 0.002 & 500 & 732 & ng & $\mathrm{ng}$ & ng & ng \\
\hline
\end{tabular}

'Less than 10 years of data used.

\section{4- 1.2L0-5A DORI RIVER NEAR TAKHTAPUL, Continued}

\section{Probability of occurrence of annual low discharges}

$\left[\mathrm{m}^{3} / \mathrm{s}\right.$, meters per second]

\begin{tabular}{|c|c|c|c|c|c|c|c|c|c|c|}
\hline \multirow{3}{*}{$\begin{array}{c}\text { Nonexceedance } \\
\text { probability }\end{array}$} & \multirow{3}{*}{$\begin{array}{c}\text { Recurrence } \\
\text { interval } \\
\text { (years) }\end{array}$} & \multicolumn{9}{|c|}{ Minimum mean discharge $\left(\mathrm{m}^{3} / \mathrm{s}\right)$} \\
\hline & & \multicolumn{9}{|c|}{ Number of consecutive days } \\
\hline & & 1 & 3 & 7 & 14 & 30 & 60 & 90 & 120 & 183 \\
\hline 0.05 & 20 & ng & ng & ng & ng & ng & ng & ng & ng & ng \\
\hline 0.10 & 10 & ng & ng & ng & ng & ng & ng & ng & ng & ng \\
\hline 0.20 & 5 & ng & ng & $\mathrm{ng}$ & ng & ng & ng & ng & ng & ng \\
\hline 0.50 & 2 & ng & $\mathrm{ng}$ & ng & ng & ng & ng & ng & ng & ng \\
\hline
\end{tabular}




\section{4- 1.2L0-5A DORI RIVER NEAR TAKHTAPUL, Continued}

Probability of occurrence of seasonal low discharges

$\left[\mathrm{m}^{3} / \mathrm{s}\right.$, meters per second]

\begin{tabular}{|c|c|c|c|c|c|c|c|c|c|}
\hline \multirow{3}{*}{$\begin{array}{c}\text { Nonexceedance } \\
\text { probability }\end{array}$} & \multirow{3}{*}{$\begin{array}{c}\text { Recurrence } \\
\text { interval } \\
\text { (years) }\end{array}$} & \multicolumn{8}{|c|}{ Minimum mean discharge $\left(\mathrm{m}^{3} / \mathrm{s}\right)$} \\
\hline & & \multicolumn{8}{|c|}{ Number of consecutive days } \\
\hline & & 1 & 7 & 14 & 30 & 1 & 7 & 14 & 30 \\
\hline & & \multicolumn{4}{|c|}{ December-January-February } & \multicolumn{4}{|c|}{ March-April-May } \\
\hline 0.05 & 20 & ng & ng & ng & ng & ng & ng & ng & ng \\
\hline 0.10 & 10 & ng & ng & ng & ng & ng & ng & ng & ng \\
\hline 0.20 & 5 & ng & ng & ng & ng & ng & ng & ng & ng \\
\hline \multirow[t]{2}{*}{0.50} & 2 & ng & ng & $\mathrm{ng}$ & ng & ng & ng & ng & ng \\
\hline & & \multicolumn{4}{|c|}{ June-July-August } & \multicolumn{4}{|c|}{ September-October-November } \\
\hline 0.05 & 20 & ng & ng & ng & ng & ng & $\mathrm{ng}$ & ng & ng \\
\hline 0.10 & 10 & ng & $\mathrm{ng}$ & $\mathrm{ng}$ & $\mathrm{ng}$ & $\mathrm{ng}$ & $\mathrm{ng}$ & ng & ng \\
\hline 0.20 & 5 & ng & ng & ng & ng & ng & ng & ng & ng \\
\hline 0.50 & 2 & $\mathrm{ng}$ & $\mathrm{ng}$ & ng & $\mathrm{ng}$ & $\mathrm{ng}$ & $\mathrm{ng}$ & $\mathrm{ng}$ & ng \\
\hline
\end{tabular}

\section{4- 1.2L0-5A DORI RIVER NEAR TAKHTAPUL, Continued}

Annual peak discharge and corresponding gage height

[m³/s, meters per second; --, no data]

\begin{tabular}{llccccc}
\hline $\begin{array}{l}\text { Water } \\
\text { year }\end{array}$ Date & $\begin{array}{c}\text { Gage } \\
\text { height } \\
(\text { meters })\end{array}$ & $\begin{array}{c}\text { Peak } \\
\text { discharge } \\
\left(\mathrm{m}^{3} / \mathrm{s}\right)\end{array}$ & $\begin{array}{c}\text { Water } \\
\text { year }\end{array}$ & Date & $\begin{array}{c}\text { Gage } \\
\text { height } \\
(\text { meters })\end{array}$ & $\begin{array}{c}\text { Peak } \\
\text { discharge } \\
\left(\mathrm{m}^{3} / \mathrm{s}\right)\end{array}$ \\
\hline
\end{tabular}

Annual peak discharge, by year

$\begin{array}{rrrr}1976 & 15-\mathrm{Feb} & -- & 90.3 \\ 1977 & 10-\text { Aug } & -- & 63 \\ 1978 & 21-\text { Aug } & -- & 154 \\ 1979 & 12-\mathrm{Feb} & -- & 240\end{array}$

Annual peak discharge, from highest to lowest

$\begin{array}{rrrr}1979 & 12-\text { Feb } & -- & 240 \\ 1978 & 21-\text { Aug } & -- & 154 \\ 1976 & 15-\text { Feb } & -- & 90.3 \\ 1977 & 10-\text { Aug } & -- & 63\end{array}$




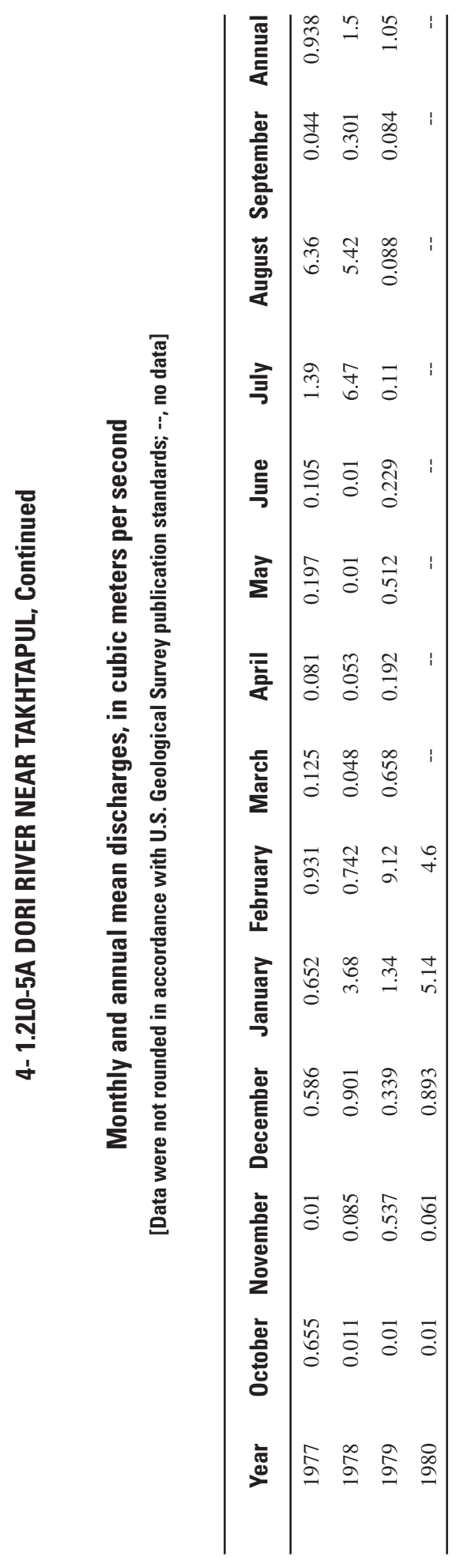




\section{4- 1.L00-1A ARGHANDAB RIVER AT OALA-I-BUST}

\section{(U.S. Geological Survey identification number: 313000064230000 )}

LOCATION: Lat $31^{\circ} 30^{\prime} \mathrm{N}$., long 64²3'E., on right bank $3 \mathrm{~km}$ northeast of ruins of Qala-i-Bust, $6 \mathrm{~km}$ upstream from Helmand River, 8 km southeast of Lashkargah, and $179 \mathrm{~km}$ downstream from Arghandab Dam.

DRAINAGE AREA: $52,920 \mathrm{~km}^{2}$, of which approximately $3,940 \mathrm{~km}^{2}$ is considered noncontributing.

ALTITUDE: 756 meters plus mean sea level.

PERIOD OF RECORD: October 1, 1947 to September 30, 1980.

GAGE: Water-stage recorder.

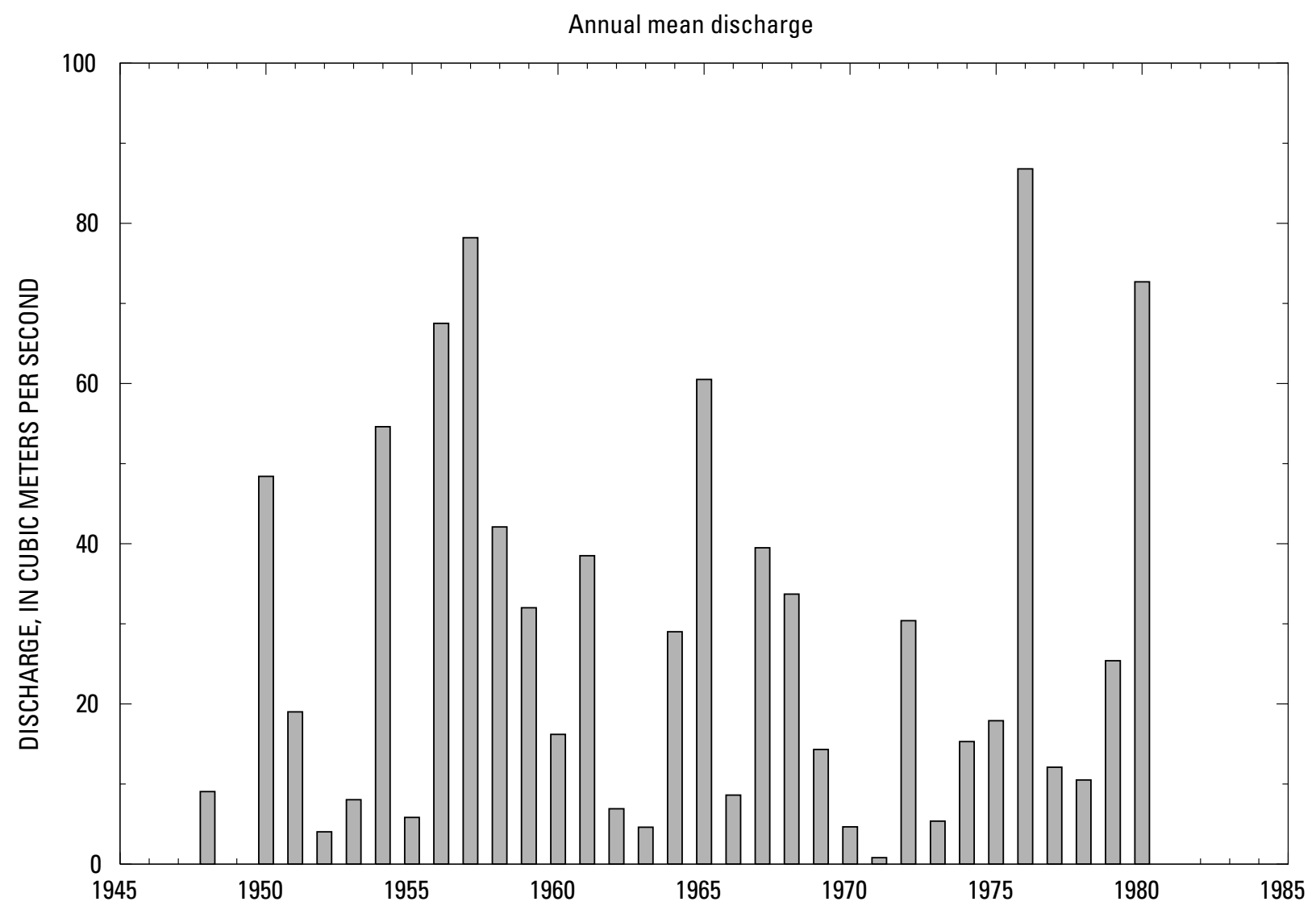




\section{4- 1.L00-1A ARGHANDAB RIVER AT QALA-I-BUST, Continued}

\section{Statistics of monthly and annual mean discharges}

[ $\mathrm{m}^{3} / \mathrm{s}$, meters per second; $\mathrm{m}$, more than 2 years of occurrence]

\begin{tabular}{|c|c|c|c|c|c|c|c|c|}
\hline \multirow[b]{2}{*}{ Month } & \multicolumn{2}{|c|}{ Maximum } & \multicolumn{2}{|c|}{ Minimum } & \multicolumn{4}{|c|}{ Mean } \\
\hline & $\begin{array}{c}\text { Discharge } \\
\left(\mathrm{m}^{3} / \mathrm{s}\right)\end{array}$ & $\begin{array}{c}\text { Water year } \\
\text { of } \\
\text { occurrence }\end{array}$ & $\begin{array}{c}\text { Discharge } \\
\left(\mathrm{m}^{3} / \mathrm{s}\right)\end{array}$ & $\begin{array}{c}\text { Water year } \\
\text { of } \\
\text { occurrence }\end{array}$ & $\begin{array}{c}\text { Discharge } \\
\left(\mathrm{m}^{3} / \mathrm{s}\right)\end{array}$ & $\begin{array}{c}\text { Standard } \\
\text { deviation } \\
\left(\mathrm{m}^{3} / \mathrm{s}\right)\end{array}$ & $\begin{array}{c}\text { Coefficient } \\
\text { of } \\
\text { variation }\end{array}$ & $\begin{array}{c}\text { Percentage } \\
\text { of annual } \\
\text { discharge }\end{array}$ \\
\hline October & 12.1 & 1957 & 0 & $\mathrm{~m}$ & 1.77 & 2.86 & 1.62 & 0.52 \\
\hline November & 19.2 & 1958 & 0 & $\mathrm{~m}$ & 3.28 & 4.51 & 1.38 & 0.97 \\
\hline December & 199 & 1958 & 0 & $\mathrm{~m}$ & 12.5 & 34.3 & 2.74 & 3.71 \\
\hline January & 69.5 & 1957 & 0 & $\mathrm{~m}$ & 16.9 & 18.1 & 1.07 & 4.99 \\
\hline February & 202 & 1954 & 0.312 & 1948 & 36.8 & 40.2 & 1.09 & 10.9 \\
\hline March & 395 & 1980 & 1.26 & 1953 & 69.8 & 86.8 & 1.24 & 20.7 \\
\hline April & 312 & 1965 & 0.177 & 1971 & 110 & 112 & 1.01 & 32.6 \\
\hline May & 211 & 1965 & 0.018 & 1971 & 55.5 & 62.1 & 1.12 & 16.4 \\
\hline June & 70.5 & 1957 & 0 & $\mathrm{~m}$ & 12.7 & 18.8 & 1.49 & 3.75 \\
\hline July & 255 & 1956 & 0 & $\mathrm{~m}$ & 12.6 & 44.5 & 3.52 & 3.74 \\
\hline August & 44.4 & 1956 & 0 & $\mathrm{~m}$ & 3.76 & 8.59 & 2.29 & 1.11 \\
\hline September & 13.9 & 1976 & 0 & $\mathrm{~m}$ & 1.94 & 3.49 & 1.80 & 0.57 \\
\hline Annual & 86.8 & 1976 & 0.812 & 1971 & 28.2 & 24.4 & 0.86 & 100 \\
\hline
\end{tabular}

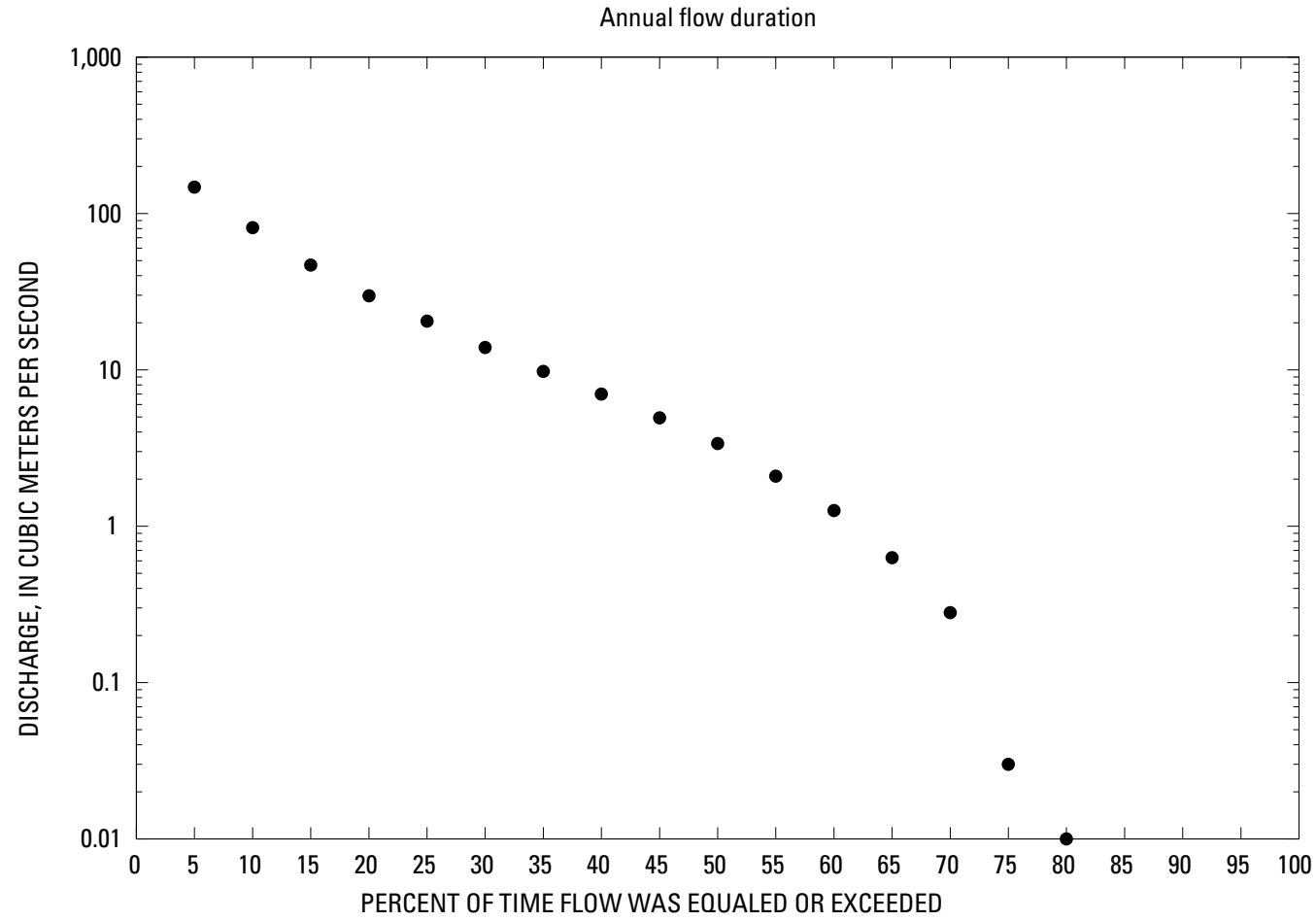




\section{4- 1.L00-1A ARGHANDAB RIVER AT QALA-I-BUST, Continued}

Monthly and annual flow duration, in cubic meters per second

[Data were not rounded in accordance with U.S. Geological Survey publication standards]

\begin{tabular}{|c|c|c|c|c|c|c|c|c|c|c|c|c|c|}
\hline $\begin{array}{l}\text { Percentage } \\
\text { of days } \\
\text { discharge } \\
\text { equaled or } \\
\text { exceeded }\end{array}$ & January & February & March & April & May & June & July & August & September & October & November & December & Annual \\
\hline 95 & 0 & 0.07 & 0.3 & 0.16 & 0.01 & 0 & 0 & 0 & 0 & 0 & 0 & 0 & 0 \\
\hline 90 & 0 & 0.82 & 1.37 & 0.35 & 0.13 & 0 & 0 & 0 & 0 & 0 & 0 & 0 & 0 \\
\hline 85 & 0 & 1.67 & 3.24 & 3.58 & 0.63 & 0 & 0 & 0 & 0 & 0 & 0 & 0 & 0 \\
\hline 80 & 0.24 & 2.86 & 5.12 & 6.95 & 1.61 & 0 & 0 & 0 & 0 & 0 & 0 & 0 & 0.01 \\
\hline 75 & 0.54 & 4.26 & 7.83 & 13.5 & 3.49 & 0.01 & 0 & 0 & 0 & 0 & 0 & 0.02 & 0.03 \\
\hline 70 & 1.07 & 5.59 & 10.6 & 21.2 & 6.71 & 0.03 & 0 & 0 & 0 & 0 & 0 & 0.37 & 0.28 \\
\hline 65 & 1.86 & 7.61 & 16.1 & 29.8 & 9.73 & 0.17 & 0.01 & 0.01 & 0.01 & 0 & 0.01 & 0.67 & 0.63 \\
\hline 60 & 3.35 & 9.88 & 19.6 & 44 & 16.2 & 0.43 & 0.02 & 0.01 & 0.02 & 0.01 & 0.16 & 1.72 & 1.26 \\
\hline 55 & 5.2 & 12.1 & 23.3 & 58 & 21.4 & 0.69 & 0.02 & 0.02 & 0.02 & 0.02 & 0.32 & 2.42 & 2.09 \\
\hline 50 & 6.92 & 14.4 & 28.7 & 70.8 & 26.2 & 1.59 & 0.14 & 0.13 & 0.47 & 0.28 & 0.68 & 3.11 & 3.38 \\
\hline 45 & 8.68 & 18.9 & 34.7 & 83.1 & 32.7 & 3.46 & 0.27 & 0.42 & 0.64 & 0.37 & 1.42 & 4.23 & 4.93 \\
\hline 40 & 10.5 & 24.1 & 41.5 & 99 & 40.8 & 5.98 & 0.53 & 0.68 & 0.9 & 0.53 & 2.27 & 5.26 & 7 \\
\hline 35 & 13 & 30.4 & 49.7 & 115.9 & 50.8 & 8.98 & 1.37 & 1.05 & 1.16 & 0.77 & 3 & 6.13 & 9.79 \\
\hline 30 & 15.9 & 37.4 & 60.1 & 133.1 & 66.3 & 13.9 & 2.22 & 1.51 & 1.35 & 1.84 & 4.3 & 7.81 & 13.9 \\
\hline 25 & 18.5 & 44.6 & 78.4 & 150.4 & 83.9 & 18.9 & 3.12 & 2.42 & 1.53 & 2.47 & 5.46 & 9.97 & 20.5 \\
\hline 20 & 21.1 & 54.3 & 103 & 180.9 & 107.5 & 24 & 5.63 & 3.27 & 3.28 & 3.51 & 6.53 & 11.6 & 29.8 \\
\hline 15 & 25.9 & 66.4 & 126.8 & 243.9 & 136.2 & 29.1 & 10.7 & 3.95 & 4.66 & 5.47 & 8.29 & 13.5 & 46.8 \\
\hline 10 & 35.5 & 82.9 & 202.6 & 300.6 & 164.8 & 36.6 & 21.8 & 7.97 & 6.03 & 6.3 & 10.2 & 20.2 & 81.3 \\
\hline 5 & 68.8 & 111.8 & 320.4 & 388.7 & 210.9 & 57 & 34 & 14.7 & 8.94 & 7.71 & 12.1 & 35.5 & 147.7 \\
\hline
\end{tabular}




\section{4- 1.LO0-1A ARGHANDAB RIVER AT OALA-I-BUST, Continued}

\section{Probability of occurrence of annual high discharges}

[ $\mathrm{m}^{3} / \mathrm{s}$, meters per second; $\mathbf{n g}$, statistic not given]

\begin{tabular}{|c|c|c|c|c|c|c|}
\hline \multirow{2}{*}{$\begin{array}{c}\text { Exceedance } \\
\text { probability }\end{array}$} & \multirow{2}{*}{$\begin{array}{c}\text { Recurrence } \\
\text { interval } \\
\text { (years) }\end{array}$} & \multirow{2}{*}{$\begin{array}{c}\text { Maximum } \\
\text { instantaneous } \\
\left(\mathrm{m}^{3} / \mathrm{s}\right)\end{array}$} & \multicolumn{4}{|c|}{ Maximum mean discharge } \\
\hline & & & 3-day period & 7-day period & 15-day period & 30-day period \\
\hline 0.99 & 1.01 & ng & 7.48 & 6.13 & 4.60 & 3.66 \\
\hline 0.95 & 1.05 & 45.8 & 25.5 & 19.5 & 14.4 & 11.4 \\
\hline 0.90 & 1.11 & 76.5 & 45.7 & 34.0 & 25.0 & 19.7 \\
\hline 0.80 & 1.25 & 137 & 86.6 & 63.2 & 46.3 & 36.3 \\
\hline 0.50 & 2 & 372 & 244 & 176 & 129 & 101 \\
\hline 0.20 & 5 & 877 & 547 & 404 & 300 & 234 \\
\hline 0.10 & 10 & 1,300 & 767 & 579 & 434 & 339 \\
\hline 0.04 & 25 & 1,900 & 1,040 & 808 & 614 & 480 \\
\hline 0.02 & 50 & 2,380 & 1,230 & 977 & 748 & 586 \\
\hline 0.01 & 100 & 2,880 & 1,400 & 1,140 & 879 & 690 \\
\hline 0.005 & 200 & 3,390 & 1,560 & 1,290 & 1,010 & 791 \\
\hline 0.002 & 500 & 4,080 & $\mathrm{ng}$ & $\mathrm{ng}$ & ng & ng \\
\hline
\end{tabular}

\section{4- 1.LO0-1A ARGHANDAB RIVER AT OALA-I-BUST, Continued}

Probability of occurrence of annual low discharges

$\left[\mathrm{m}^{3} / \mathrm{s}\right.$, meters per second]

\begin{tabular}{|c|c|c|c|c|c|c|c|c|c|c|}
\hline \multirow{3}{*}{$\begin{array}{c}\text { Nonexceedance } \\
\text { probability }\end{array}$} & \multirow{3}{*}{$\begin{array}{c}\text { Recurrence } \\
\text { interval } \\
\text { (years) }\end{array}$} & \multicolumn{9}{|c|}{ Minimum mean discharge $\left(\mathrm{m}^{3} / \mathrm{s}\right)$} \\
\hline & & \multicolumn{9}{|c|}{ Number of consecutive days } \\
\hline & & 1 & 3 & 7 & 14 & 30 & 60 & 90 & 120 & 183 \\
\hline 0.05 & 20 & 0 & 0 & 0 & 0 & 0 & 0 & 0 & 0 & 0 \\
\hline 0.10 & 10 & 0 & 0 & 0 & 0 & 0 & 0 & 0 & 0 & 0 \\
\hline 0.20 & 5 & 0 & 0 & 0 & 0 & 0 & 0 & 0 & 0 & 0.021 \\
\hline 0.50 & 2 & 0.010 & 0.010 & 0.010 & 0.010 & 0.010 & 0.029 & 0.123 & 0.465 & 1.16 \\
\hline
\end{tabular}




\section{4- 1.L00-1A ARGHANDAB RIVER AT OALA-I-BUST, Continued}

\section{Probability of occurrence of seasonal low discharges}

$\left[\mathrm{m}^{3} / \mathrm{s}\right.$, meters per second]

\begin{tabular}{|c|c|c|c|c|c|c|c|c|c|}
\hline \multirow{3}{*}{$\begin{array}{l}\text { Nonexceedance } \\
\text { probability }\end{array}$} & \multirow{3}{*}{$\begin{array}{c}\text { Recurrence } \\
\text { interval } \\
\text { (years) }\end{array}$} & \multicolumn{8}{|c|}{ Minimum mean discharge $\left(\mathrm{m}^{3} / \mathrm{s}\right)$} \\
\hline & & \multicolumn{8}{|c|}{ Number of consecutive days } \\
\hline & & 1 & 7 & 14 & 30 & 1 & 7 & 14 & 30 \\
\hline & & \multicolumn{4}{|c|}{ December-January-February } & \multicolumn{4}{|c|}{ March-April-May } \\
\hline 0.05 & 20 & 0 & 0 & 0 & 0 & 0 & 0 & 0.001 & 0.124 \\
\hline 0.10 & 10 & 0 & 0 & 0 & 0 & 0 & 0 & 0.023 & 0.434 \\
\hline 0.20 & 5 & 0 & 0 & 0.010 & 0.117 & 0.002 & 0.046 & 0.214 & 1.70 \\
\hline \multirow[t]{2}{*}{0.50} & 2 & 0.678 & 0.814 & 1.32 & 3.38 & 2.17 & 3.27 & 4.65 & 14.5 \\
\hline & & \multicolumn{4}{|c|}{ June-July-August } & \multicolumn{4}{|c|}{ September-October-November } \\
\hline 0.05 & 20 & 0 & 0 & 0 & 0 & 0 & 0 & 0 & 0 \\
\hline 0.10 & 10 & 0 & 0 & 0 & 0 & 0 & 0 & 0 & 0 \\
\hline 0.20 & 5 & 0 & 0 & 0 & 0 & 0 & 0 & 0 & 0 \\
\hline 0.50 & 2 & 0.015 & 0.017 & 0.019 & 0.024 & 0.040 & 0.082 & 0.093 & 0.115 \\
\hline
\end{tabular}


4- 1.LO0-1A ARGHANDAB RIVER AT QALA-I-BUST, Continued

Annual peak discharge and corresponding gage height

$\left[\mathrm{m}^{3} / \mathrm{s}\right.$, meters per second; --, no data]

\begin{tabular}{|c|c|c|c|c|c|c|c|}
\hline $\begin{array}{l}\text { Water } \\
\text { year }\end{array}$ & Date & $\begin{array}{c}\text { Gage } \\
\text { height } \\
\text { (meters) }\end{array}$ & $\begin{array}{c}\text { Peak } \\
\text { discharge } \\
\left(\mathrm{m}^{3} / \mathrm{s}\right)\end{array}$ & $\begin{array}{c}\text { Water } \\
\text { year }\end{array}$ & Date & $\begin{array}{c}\text { Gage } \\
\text { height } \\
\text { (meters) }\end{array}$ & $\begin{array}{c}\text { Peak } \\
\text { discharge } \\
\left(\mathrm{m}^{3} / \mathrm{s}\right)\end{array}$ \\
\hline \multicolumn{8}{|c|}{ Annual peak discharge, by year } \\
\hline 1948 & 9-Mar & -- & 127 & 1965 & 23-Apr & -- & 955 \\
\hline 1950 & 30-Jan & 3.80 & 1,698 & 1966 & 26-Feb & -- & 100 \\
\hline 1951 & 28-Mar & 1.54 & 273 & 1967 & 1-May & -- & 740 \\
\hline 1952 & $16-\mathrm{Feb}$ & 0.97 & 56.9 & 1968 & 28-Feb & -- & 323 \\
\hline 1953 & $16-\mathrm{Feb}$ & 2.11 & 529 & 1969 & 11-Dec & -- & 120 \\
\hline 1954 & 14-Feb & 3.30 & 1,256 & 1970 & 16-Apr & -- & 60 \\
\hline 1955 & 31-Jan & 0.63 & 28 & 1971 & $28-\mathrm{Feb}$ & -- & 11.6 \\
\hline 1956 & 27-Jul & 3.13 & 990 & 1972 & 14-Mar & -- & 344 \\
\hline 1957 & 6-Apr & 2.90 & 925 & 1973 & 27-Jul & -- & 100 \\
\hline 1958 & 12-Dec & 3.02 & 988 & 1974 & 26-Jan & -- & 203 \\
\hline 1959 & 2-Mar & 1.64 & 256 & 1975 & 29-Apr & -- & 133 \\
\hline 1960 & 18-Apr & 1.85 & 356 & 1976 & 9-Apr & -- & 750 \\
\hline 1961 & 10-Apr & 3.80 & 1,160 & 1977 & 11-Jan & -- & 900 \\
\hline 1962 & 2-Apr & -- & 156 & 1978 & 22-Aug & -- & 300 \\
\hline 1963 & 16-May & -- & 328 & 1979 & $13-\mathrm{Feb}$ & -- & 960 \\
\hline 1964 & 8-Jan & -- & 949 & 1980 & 5-Mar & -- & 1,200 \\
\hline \multicolumn{8}{|c|}{ Annual peak discharge, from highest to lowest } \\
\hline 1950 & 30-Jan & 3.80 & 1,698 & 1963 & 16-May & -- & 328 \\
\hline 1954 & $14-\mathrm{Feb}$ & 3.30 & 1,256 & 1968 & $28-\mathrm{Feb}$ & -- & 323 \\
\hline 1980 & 5-Mar & -- & 1,200 & 1978 & 22-Aug & -- & 300 \\
\hline 1961 & 10-Apr & 3.80 & 1,160 & 1951 & 28-Mar & 1.54 & 273 \\
\hline 1956 & 27-Jul & 3.13 & 990 & 1959 & 2-Mar & 1.64 & 256 \\
\hline 1958 & 12-Dec & 3.02 & 988 & 1974 & 26-Jan & -- & 203 \\
\hline 1979 & $13-\mathrm{Feb}$ & -- & 960 & 1962 & 2-Apr & -- & 156 \\
\hline 1965 & 23-Apr & -- & 955 & 1975 & 29-Apr & -- & 133 \\
\hline 1964 & 8-Jan & -- & 949 & 1948 & 9-Mar & -- & 127 \\
\hline 1957 & 6-Apr & 2.90 & 925 & 1969 & 11-Dec & -- & 120 \\
\hline 1977 & 11-Jan & -- & 900 & 1973 & 27-Jul & -- & 100 \\
\hline 1976 & 9-Apr & -- & 750 & 1966 & 26-Feb & -- & 100 \\
\hline 1967 & 1-May & -- & 740 & 1970 & 16-Apr & -- & 60 \\
\hline 1953 & $16-\mathrm{Feb}$ & 2.11 & 529 & 1952 & $16-\mathrm{Feb}$ & 0.97 & 56.9 \\
\hline 1960 & 18-Apr & 1.85 & 356 & 1955 & 31-Jan & 0.63 & 28 \\
\hline 1972 & 14-Mar & -- & 344 & 1971 & $28-\mathrm{Feb}$ & -- & 11.6 \\
\hline
\end{tabular}




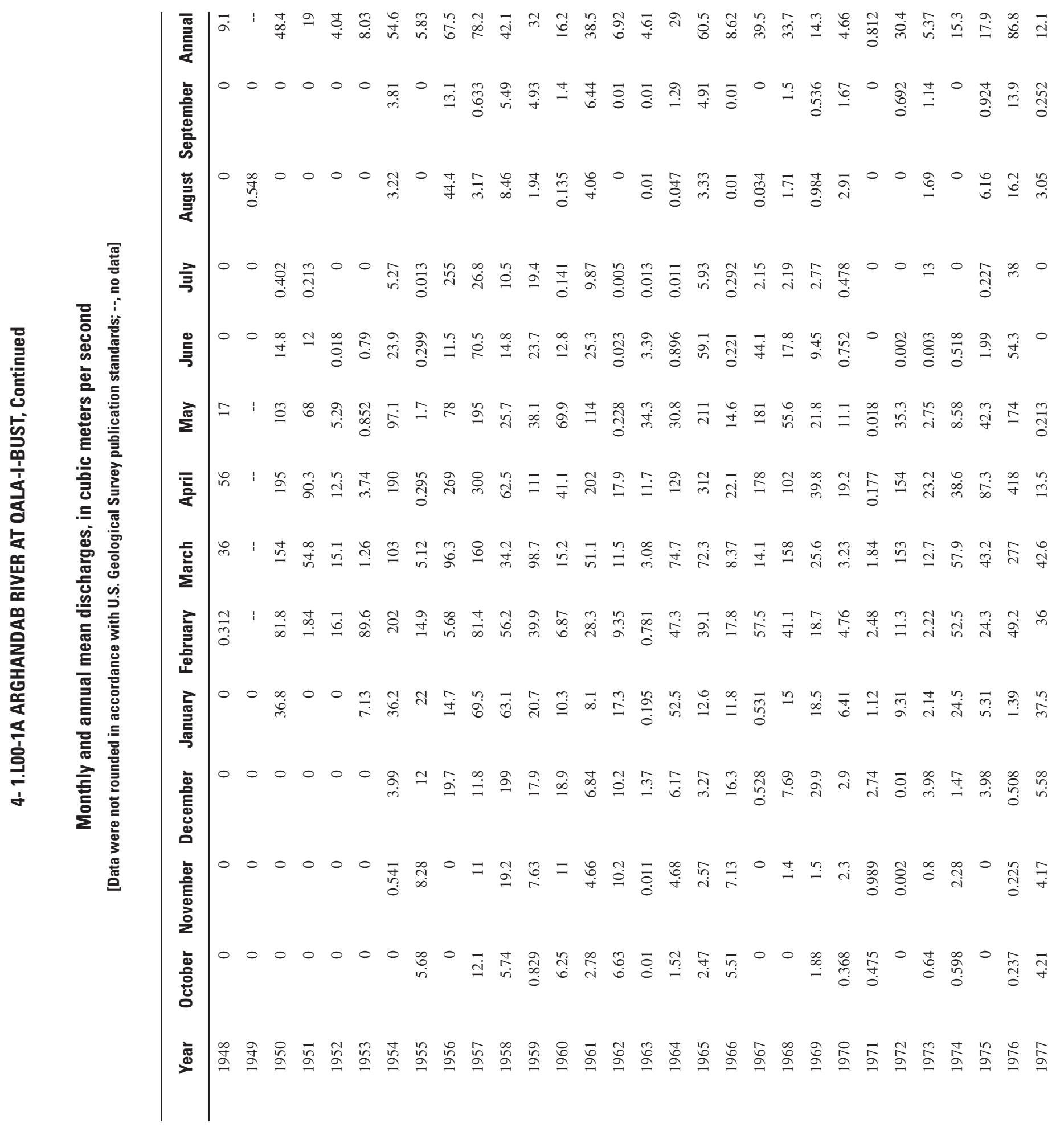




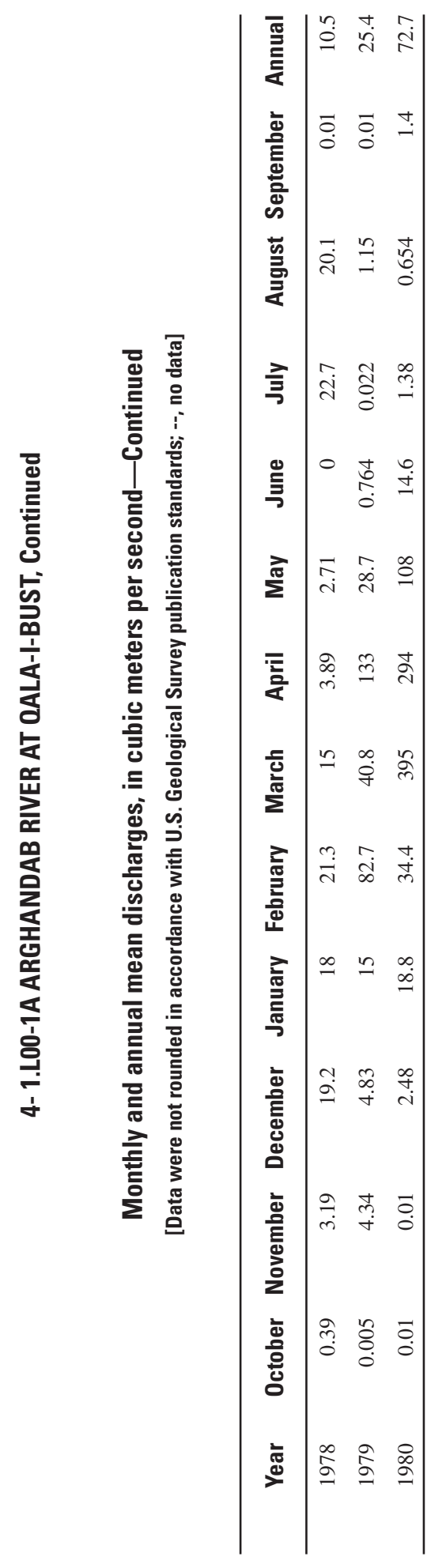




\section{4- 1.LO0-3A ARGHANDAB RIVER NEAR KANDAHAR \\ (U.S. Geological Survey identification number: 313700065340000)}

LOCATION: Lat $31^{\circ} 37^{\prime} N$., long 65³'E.

DRAINAGE AREA: $14,245 \mathrm{~km}^{2}$.

ALTITUDE: 973 meters plus mean sea level.

PERIOD OF RECORD: December 8, 1969 to September 30, 1980.

GAGE: --

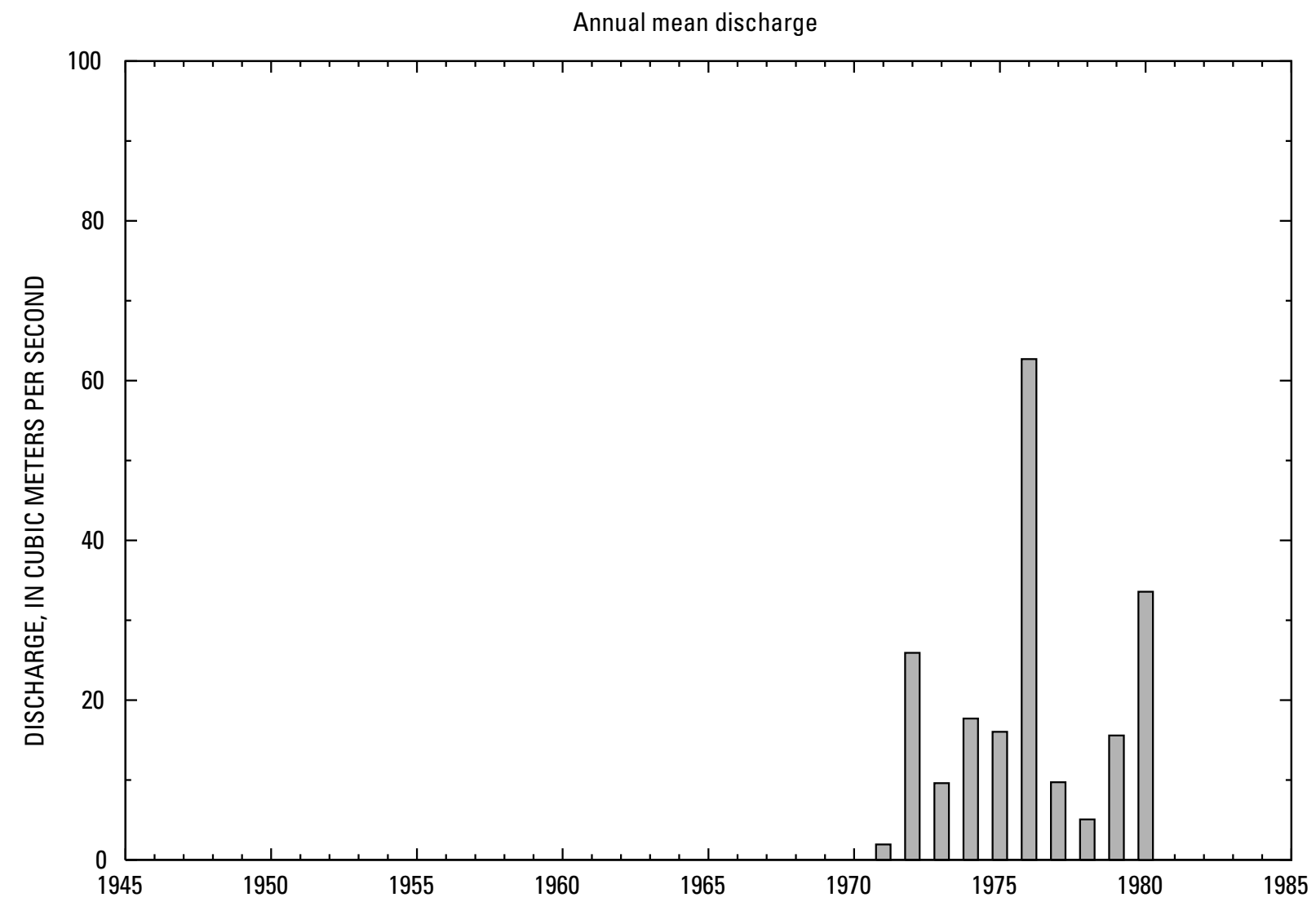




\section{4- 1.L00-3A ARGHANDAB RIVER NEAR KANDAHAR, Continued}

\section{Statistics of monthly and annual mean discharges}

$\left[\mathrm{m}^{3} / \mathrm{s}\right.$, meters per second]

\begin{tabular}{|c|c|c|c|c|c|c|c|c|}
\hline \multirow[b]{2}{*}{ Month } & \multicolumn{2}{|c|}{ Maximum } & \multicolumn{2}{|c|}{ Minimum } & \multicolumn{4}{|c|}{ Mean } \\
\hline & $\begin{array}{c}\text { Discharge } \\
\left(\mathrm{m}^{3} / \mathrm{s}\right)\end{array}$ & $\begin{array}{c}\text { Water year } \\
\text { of } \\
\text { occurrence }\end{array}$ & $\begin{array}{c}\text { Discharge } \\
\left(\mathrm{m}^{3} / \mathrm{s}\right)\end{array}$ & $\begin{array}{c}\text { Water year } \\
\text { of } \\
\text { occurrence }\end{array}$ & $\begin{array}{c}\text { Discharge } \\
\left(\mathrm{m}^{3} / \mathrm{s}\right)\end{array}$ & $\begin{array}{c}\text { Standard } \\
\text { deviation } \\
\left(\mathrm{m}^{3} / \mathrm{s}\right)\end{array}$ & $\begin{array}{c}\text { Coefficient } \\
\text { of } \\
\text { variation }\end{array}$ & $\begin{array}{c}\text { Percentage } \\
\text { of annual } \\
\text { discharge }\end{array}$ \\
\hline October & 12.7 & 1977 & 0 & 1972 & 3.11 & 3.55 & 1.14 & 1.39 \\
\hline November & 8.47 & 1977 & 0 & 1972 & 3.12 & 2.44 & 0.78 & 1.40 \\
\hline December & 5.58 & 1977 & 0 & 1972 & 2.66 & 1.90 & 0.71 & 1.19 \\
\hline January & 4.42 & 1977 & 0.005 & 1973 & 1.91 & 1.25 & 0.66 & 0.86 \\
\hline February & 19.1 & 1977 & 0.265 & 1973 & 3.86 & 5.41 & 1.40 & 1.73 \\
\hline March & 171 & 1976 & 0.971 & 1971 & 48.4 & 56.8 & 1.17 & 21.7 \\
\hline April & 334 & 1976 & 2.16 & 1971 & 101 & 97.5 & 0.97 & 45.3 \\
\hline May & 168 & 1976 & 0.811 & 1971 & 38.7 & 48.3 & 1.25 & 17.3 \\
\hline June & 29.6 & 1976 & 1.77 & 1971 & 6.37 & 7.87 & 1.24 & 2.85 \\
\hline July & 11.9 & 1976 & 0.925 & 1971 & 5.26 & 2.83 & 0.54 & 2.36 \\
\hline August & 11.5 & 1976 & 0.013 & 1971 & 4.75 & 2.82 & 0.59 & 2.13 \\
\hline September & 10.9 & 1976 & 0 & 1971 & 3.87 & 2.70 & 0.70 & 1.73 \\
\hline Annual & 62.7 & 1976 & 1.93 & 1971 & 19.8 & 17.8 & 0.90 & 100 \\
\hline
\end{tabular}

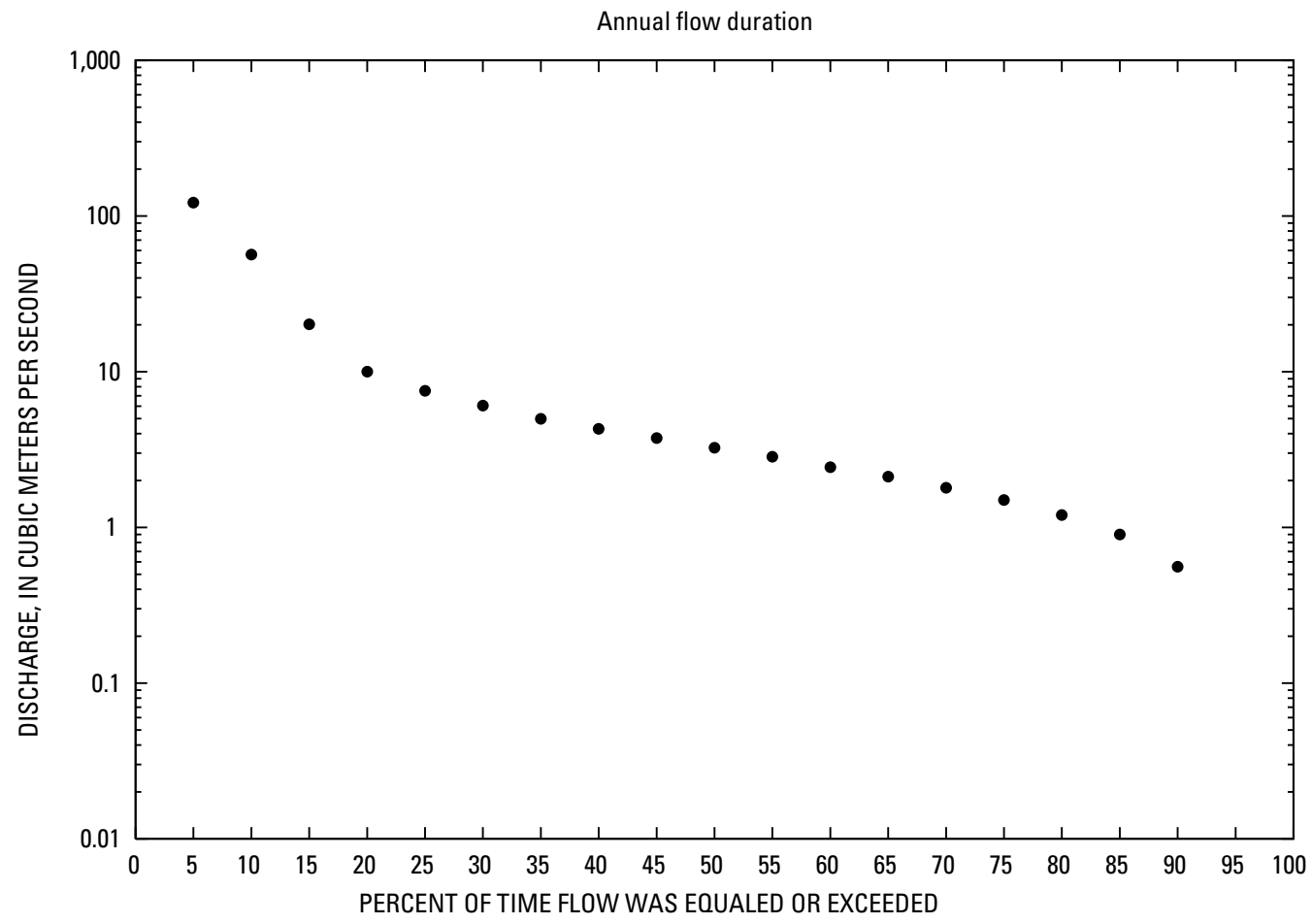




\section{4- 1.L00-3A ARGHANDAB RIVER NEAR KANDAHAR, Continued}

Monthly and annual flow duration, in cubic meters per second

[Data were not rounded in accordance with U.S. Geological Survey publication standards]

\begin{tabular}{|c|c|c|c|c|c|c|c|c|c|c|c|c|c|}
\hline $\begin{array}{l}\text { Percentage } \\
\text { of days } \\
\text { discharge } \\
\text { equaled or } \\
\text { exceeded }\end{array}$ & January & February & March & April & May & June & July & August & September & October & November & December & Annual \\
\hline 95 & 0 & 0.14 & 0.37 & 1.75 & 0.16 & 0.49 & 0.98 & 0 & 0.82 & 0.41 & 0.4 & 0.04 & 0 \\
\hline 90 & 0 & 0.52 & 0.7 & 3.24 & 1.94 & 1.43 & 1.65 & 1.9 & 1.78 & 0.83 & 0.8 & 0.09 & 0.56 \\
\hline 85 & 0.02 & 0.6 & 0.98 & 5.49 & 2.39 & 1.71 & 2.46 & 2.38 & 1.98 & 1.05 & 0.99 & 0.4 & 0.9 \\
\hline 80 & 0.09 & 0.67 & 1.38 & 10.6 & 3.18 & 1.97 & 2.99 & 2.8 & 2.17 & 1.14 & 1.4 & 0.88 & 1.2 \\
\hline 75 & 0.58 & 0.73 & 1.67 & 25.4 & 5.8 & 2.26 & 3.24 & 3.12 & 2.33 & 1.22 & 1.54 & 1 & 1.5 \\
\hline 70 & 0.69 & 0.79 & 1.89 & 37.1 & 6.56 & 2.63 & 3.44 & 3.38 & 2.57 & 1.3 & 1.63 & 1.12 & 1.8 \\
\hline 65 & 0.8 & 0.89 & 2.22 & 44.7 & 7.66 & 3.02 & 3.64 & 3.65 & 2.78 & 1.39 & 1.72 & 1.39 & 2.12 \\
\hline 60 & 0.91 & 1.01 & 2.62 & 57.2 & 10.4 & 3.43 & 3.96 & 3.86 & 2.88 & 1.56 & 1.82 & 1.71 & 2.44 \\
\hline 55 & 1.07 & 1.2 & 3.13 & 66.6 & 13.8 & 3.75 & 4.33 & 4.04 & 3.01 & 2.07 & 1.9 & 1.86 & 2.84 \\
\hline 50 & 1.22 & 1.31 & 4.02 & 76.4 & 18.4 & 3.97 & 4.73 & 4.23 & 3.21 & 2.29 & 1.98 & 1.99 & 3.25 \\
\hline 45 & 1.32 & 1.47 & 5.89 & 87.4 & 22.4 & 4.18 & 5.36 & 4.41 & 3.43 & 2.52 & 2.16 & 2.23 & 3.75 \\
\hline 40 & 1.42 & 1.72 & 12.5 & 99.8 & 26.8 & 4.4 & 5.75 & 4.59 & 3.78 & 2.73 & 2.91 & 2.62 & 4.3 \\
\hline 35 & 1.52 & 2 & 20.4 & 115.7 & 33.1 & 4.62 & 6.06 & 4.8 & 4.05 & 2.9 & 3.56 & 3 & 4.99 \\
\hline 30 & 1.69 & 2.19 & 24.8 & 131 & 42 & 5.03 & 6.37 & 5.18 & 4.26 & 3.07 & 3.77 & 3.71 & 6.06 \\
\hline 25 & 1.87 & 2.38 & 56.1 & 145.9 & 51.3 & 6.08 & 6.68 & 5.56 & 4.8 & 3.23 & 3.95 & 3.98 & 7.54 \\
\hline 20 & 2.12 & 2.81 & 79.2 & 163.3 & 63.4 & 7.2 & 6.99 & 5.94 & 5.24 & 3.38 & 4.48 & 4.36 & 9.99 \\
\hline 15 & 2.47 & 4.51 & 104.3 & 192.5 & 79.1 & 8.48 & 7.35 & 6.89 & 5.61 & 3.78 & 6.13 & 4.92 & 20.2 \\
\hline 10 & 3.47 & 7.95 & 181.8 & 238.1 & 102.2 & 10.6 & 7.84 & 8.73 & 6.48 & 5.82 & 7.61 & 5.56 & 56.6 \\
\hline 5 & 4.16 & 18.1 & 275.1 & 317.5 & 165.4 & 18.8 & 8.6 & 11.2 & 11.2 & 13.6 & 8.02 & 6.19 & 121.9 \\
\hline
\end{tabular}




\section{4- 1.L00-3A ARGHANDAB RIVER NEAR KANDAHAR, Continued}

\section{Probability of occurrence of annual high discharges}

[ $\mathrm{m}^{3} / \mathrm{s}$, meters per second; $\mathrm{ng}$, statistic not given]

\begin{tabular}{|c|c|c|c|c|c|c|}
\hline \multirow{2}{*}{$\begin{array}{l}\text { Exceedance } \\
\text { probability }\end{array}$} & \multirow{2}{*}{$\begin{array}{c}\text { Recurrence } \\
\text { interval } \\
\text { (years) }\end{array}$} & \multirow{2}{*}{$\begin{array}{c}\text { Maximum } \\
\text { instantaneous } \\
\left(\mathrm{m}^{3} / \mathrm{s}\right)\end{array}$} & \multicolumn{4}{|c|}{ Maximum mean discharge } \\
\hline & & & 3-day period & 7-day period & 15-day period & 30-day period \\
\hline 0.99 & 1.01 & ng & ${ }^{1} 2.14$ & 2.17 & 2.11 & 1.92 \\
\hline 0.95 & 1.05 & ng & 10.3 & 9.74 & 9.01 & 7.64 \\
\hline 0.90 & 1.11 & 75.1 & 21.1 & 19.4 & 17.6 & 14.6 \\
\hline 0.80 & 1.25 & 102 & 44.6 & 40.3 & 36.2 & 29.5 \\
\hline 0.50 & 2 & 177 & 136 & 122 & 110 & 90.6 \\
\hline 0.20 & 5 & 290 & 283 & 264 & 242 & 211 \\
\hline 0.10 & 10 & 368 & 367 & 351 & 326 & 297 \\
\hline 0.04 & 25 & 468 & 447 & 440 & 418 & 400 \\
\hline 0.02 & 50 & 541 & 489 & ${ }^{1} 492$ & 474 & 469 \\
\hline 0.01 & 100 & 614 & ${ }^{1} 521$ & ${ }^{1} 533$ & ${ }^{1} 520$ & ${ }^{1} 531$ \\
\hline 0.005 & 200 & 686 & ${ }^{1} 544$ & ${ }^{1} 565$ & ${ }^{1} 557$ & ${ }^{1} 585$ \\
\hline 0.002 & 500 & 780 & ng & ng & ng & ng \\
\hline
\end{tabular}

'Data does not fit log-Pearson Type III curve, use with caution.

\section{4- 1.L00-3A ARGHANDAB RIVER NEAR KANDAHAR, Continued}

Probability of occurrence of annual low discharges

$\left[\mathrm{m}^{3} / \mathrm{s}\right.$, meters per second]

\begin{tabular}{|c|c|c|c|c|c|c|c|c|c|c|}
\hline \multirow{3}{*}{$\begin{array}{c}\text { Nonexceedance } \\
\text { probability }\end{array}$} & \multirow{3}{*}{$\begin{array}{c}\text { Recurrence } \\
\text { interval } \\
\text { (years) }\end{array}$} & \multicolumn{9}{|c|}{ Minimum mean discharge $\left(\mathrm{m}^{3} / \mathrm{s}\right)$} \\
\hline & & \multicolumn{9}{|c|}{ Number of consecutive days } \\
\hline & & 1 & 3 & 7 & 14 & 30 & 60 & 90 & 120 & 183 \\
\hline 0.05 & 20 & 0 & 0 & 0 & 0 & 0 & 0 & 0 & 0 & 0.127 \\
\hline 0.10 & 10 & 0 & 0 & 0 & 0 & 0 & 0 & 0 & 0 & 0.313 \\
\hline 0.20 & 5 & 0 & 0 & 0 & 0 & 0 & 0.276 & 0.495 & 0.834 & 0.771 \\
\hline 0.50 & 2 & 0.260 & 0.306 & 0.344 & 0.401 & 0.881 & 1.36 & 1.45 & 1.77 & 2.51 \\
\hline
\end{tabular}




\section{4- 1.L00-3A ARGHANDAB RIVER NEAR KANDAHAR, Continued}

Probability of occurrence of seasonal low discharges

[m³ $/ \mathrm{s}$, meters per second]

\begin{tabular}{|c|c|c|c|c|c|c|c|c|c|}
\hline \multirow{3}{*}{$\begin{array}{c}\text { Nonexceedance } \\
\text { probability }\end{array}$} & \multirow{3}{*}{$\begin{array}{c}\text { Recurrence } \\
\text { interval } \\
\text { (years) }\end{array}$} & \multicolumn{8}{|c|}{ Minimum mean discharge $\left(\mathrm{m}^{3} / \mathrm{s}\right)$} \\
\hline & & \multicolumn{8}{|c|}{ Number of consecutive days } \\
\hline & & 1 & 7 & 14 & 30 & 1 & 7 & 14 & 30 \\
\hline & & \multicolumn{4}{|c|}{ December-January-February } & \multicolumn{4}{|c|}{ March-April-May } \\
\hline 0.05 & 20 & 0 & 0 & 0 & 0 & 0.091 & 0.096 & 0.120 & 0.725 \\
\hline 0.10 & 10 & 0 & 0 & 0 & 0 & 0.152 & 0.191 & 0.233 & 1.26 \\
\hline 0.20 & 5 & 0 & 0 & 0 & 0 & 0.281 & 0.418 & 0.507 & 2.50 \\
\hline \multirow[t]{2}{*}{0.50} & 2 & 0.329 & 0.389 & 0.471 & 0.881 & 0.875 & 1.62 & 2.10 & 9.78 \\
\hline & & \multicolumn{4}{|c|}{ June-July-August } & \multicolumn{4}{|c|}{ September-October-November } \\
\hline 0.05 & 20 & 0 & 0 & 0 & 0.023 & 0 & 0 & 0 & 0 \\
\hline 0.10 & 10 & 0.001 & 0.001 & 0.012 & 0.139 & 0 & 0 & 0 & 0 \\
\hline 0.20 & 5 & 0.182 & 0.231 & 0.532 & 0.736 & 0.838 & 0.953 & 1.04 & 1.16 \\
\hline 0.50 & 2 & 2.04 & 2.69 & 3.17 & 4.44 & 1.27 & 1.35 & 1.49 & 1.70 \\
\hline
\end{tabular}

\section{4- 1.L00-3A ARGHANDAB RIVER NEAR KANDAHAR, Continued}

Annual peak discharge and corresponding gage height

[m³/s, meters per second; --, no data]

\begin{tabular}{ccccccc}
\hline $\begin{array}{c}\text { Water } \\
\text { year }\end{array}$ Date & $\begin{array}{c}\text { Gage } \\
\text { height } \\
(\text { meters })\end{array}$ & $\begin{array}{c}\text { Peak } \\
\text { discharge } \\
\left(\mathrm{m}^{3} / \mathrm{s}\right)\end{array}$ & $\begin{array}{c}\text { Water } \\
\text { year }\end{array}$ & Date & $\begin{array}{c}\text { Gage } \\
\text { height } \\
(\text { meters })\end{array}$ & $\begin{array}{c}\text { Peak } \\
\text { discharge } \\
\left(\mathrm{m}^{3} / \mathrm{s}\right)\end{array}$
\end{tabular}

Annual peak discharge, by year

\begin{tabular}{|c|c|c|c|c|c|c|c|}
\hline 1970 & 17-Apr & -- & 72.9 & 1976 & 25-Apr & -- & 456 \\
\hline 1971 & $27-\mathrm{Nov}$ & -- & 6.81 & 1977 & 11-Aug & -- & 283 \\
\hline 1972 & 26-Mar & -- & 295 & 1978 & 25-Dec & -- & 168 \\
\hline 1973 & 6-Apr & -- & 82.4 & 1979 & 2-Apr & -- & 195 \\
\hline 1974 & 4-Apr & -- & 168 & 1980 & 21-Mar & -- & 272 \\
\hline 1975 & 5-Apr & -- & 138 & & & & \\
\hline \multicolumn{8}{|c|}{ Annual peak discharge, from highest to lowest } \\
\hline 1976 & 25-Apr & -- & 456 & 1974 & 4-Apr & -- & 168 \\
\hline 1972 & 26-Mar & -- & 295 & 1975 & 5-Apr & -- & 138 \\
\hline 1977 & 11-Aug & -- & 283 & 1973 & 6-Apr & -- & 82.4 \\
\hline 1980 & 21-Mar & -- & 272 & 1970 & 17-Apr & -- & 72.9 \\
\hline 1979 & 2-Apr & -- & 195 & 1971 & 27-Nov & -- & 6.81 \\
\hline 1978 & 25-Dec & -- & 168 & & & & \\
\hline
\end{tabular}




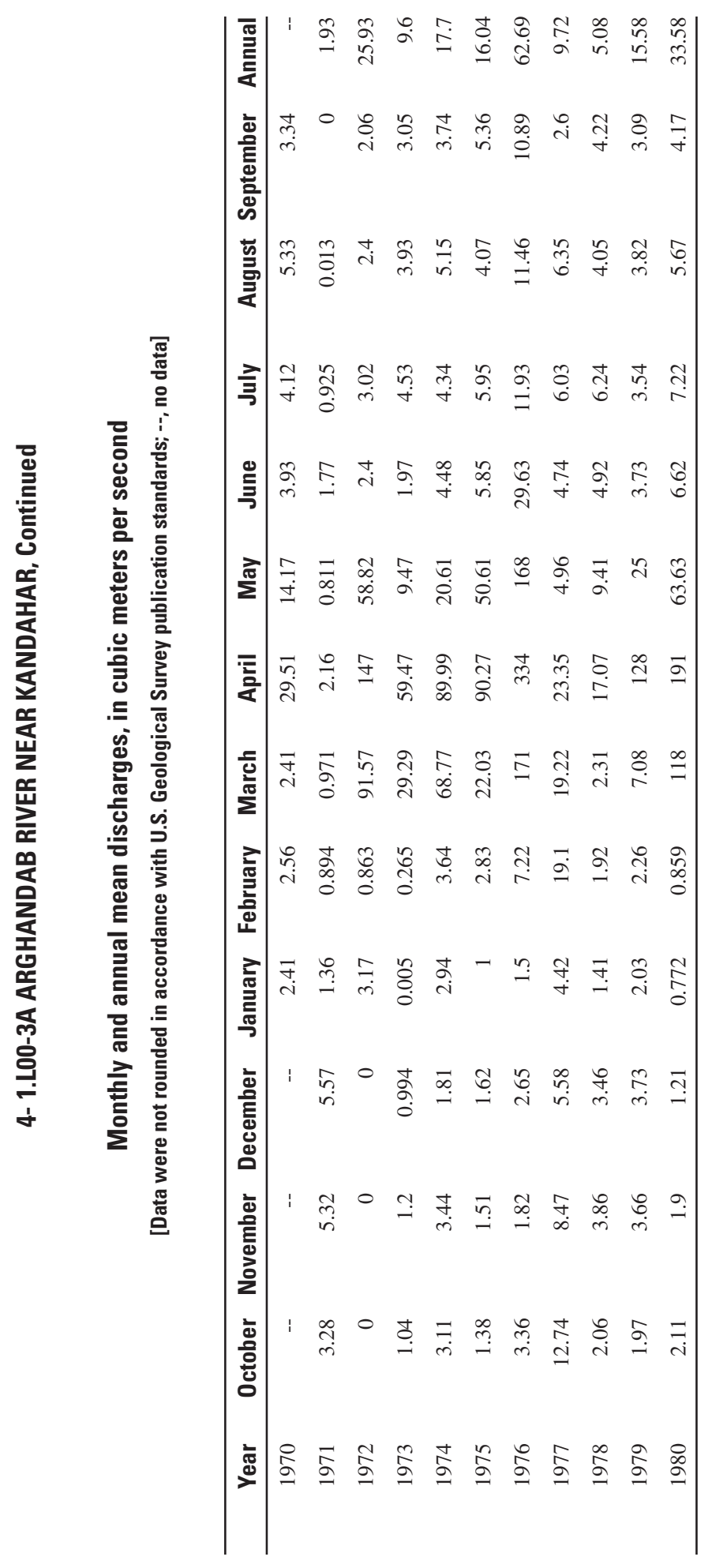




\section{4- 1.L00-4A ARGHANDAB RIVER BELOW ARGHANDAB RESERVOIR}

\section{(U.S. Geological Survey identification number: 315000065520000)}

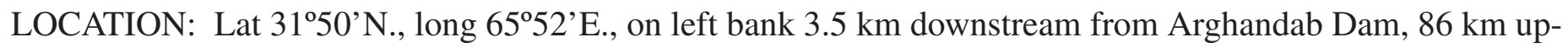
stream from Dori River, and $181 \mathrm{~km}$ upstream from Helmand River.

DRAINAGE AREA: $12,975 \mathrm{~km}^{2}$.

ALTITUDE: 1,100 meters plus mean sea level.

PERIOD OF RECORD: October 1, 1947 to September 30, 1979.

GAGE: Water-stage recorder. December 30, 1947 to December 22, 1951, water-stage recorder $2.5 \mathrm{~km}$ upstream at different datum. December 23, 1951 to October 16, 1952, staff gage and concrete control $3 \mathrm{~km}$ upstream at different datum.

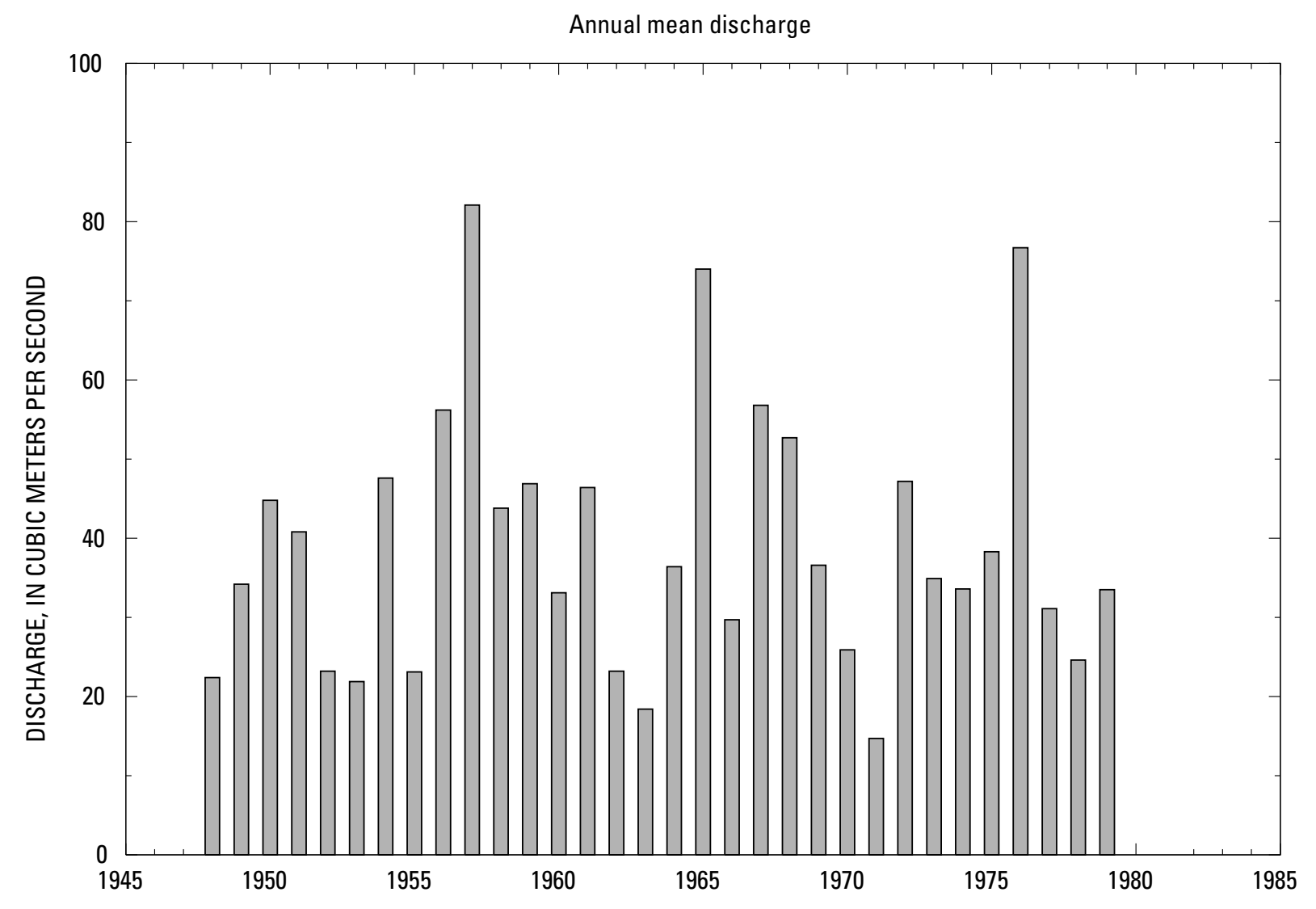




\section{Statistics of monthly and annual mean discharges}

$\left[\mathrm{m}^{3} / \mathrm{s}\right.$, meters per second]

\begin{tabular}{|c|c|c|c|c|c|c|c|c|}
\hline \multirow[b]{2}{*}{ Month } & \multicolumn{2}{|c|}{ Maximum } & \multicolumn{2}{|c|}{ Minimum } & \multicolumn{4}{|c|}{ Mean } \\
\hline & $\begin{array}{c}\text { Discharge } \\
\left(\mathrm{m}^{3} / \mathrm{s}\right)\end{array}$ & $\begin{array}{c}\text { Water year } \\
\text { of } \\
\text { occurrence }\end{array}$ & $\begin{array}{c}\text { Discharge } \\
\left(\mathrm{m}^{3} / \mathrm{s}\right)\end{array}$ & $\begin{array}{c}\text { Water year } \\
\text { of } \\
\text { occurrence }\end{array}$ & $\begin{array}{c}\text { Discharge } \\
\left(\mathrm{m}^{3} / \mathrm{s}\right)\end{array}$ & $\begin{array}{c}\text { Standard } \\
\text { deviation } \\
\left(\mathrm{m}^{3} / \mathrm{s}\right)\end{array}$ & $\begin{array}{c}\text { Coefficient } \\
\text { of } \\
\text { variation }\end{array}$ & $\begin{array}{c}\text { Percentage } \\
\text { of annual } \\
\text { discharge }\end{array}$ \\
\hline October & 31.2 & 1966 & 0.859 & 1972 & 20.5 & 8.11 & 0.40 & 4.35 \\
\hline November & 31.6 & 1966 & 4.22 & 1972 & 20.8 & 6.52 & 0.31 & 4.42 \\
\hline December & 50.6 & 1958 & 5.29 & 1972 & 20.1 & 7.73 & 0.38 & 4.28 \\
\hline January & 53.1 & 1958 & 0.285 & 1979 & 13.3 & 13.8 & 1.04 & 2.83 \\
\hline February & 53.3 & 1958 & 0 & 1972 & 17.3 & 13.1 & 0.76 & 3.67 \\
\hline March & 177 & 1968 & 10.1 & 1963 & 60.2 & 45.8 & 0.76 & 12.8 \\
\hline April & 322 & 1957 & 20.7 & 1962 & 122 & 83.8 & 0.69 & 26.0 \\
\hline May & 235 & 1967 & 14.9 & 1971 & 80.9 & 61.1 & 0.76 & 17.2 \\
\hline June & 91.3 & 1965 & 12.6 & 1948 & 36.1 & 17.6 & 0.49 & 7.68 \\
\hline July & 96.1 & 1956 & 6.76 & 1948 & 30.4 & 15.5 & 0.51 & 6.46 \\
\hline August & 42.7 & 1956 & 4.60 & 1948 & 26.0 & 9.72 & 0.37 & 5.54 \\
\hline September & 35.1 & 1956 & 1.87 & 1971 & 22.7 & 9.33 & 0.41 & 4.83 \\
\hline Annual & 82.1 & 1957 & 14.7 & 1971 & 39.2 & 16.7 & 0.43 & 100 \\
\hline
\end{tabular}

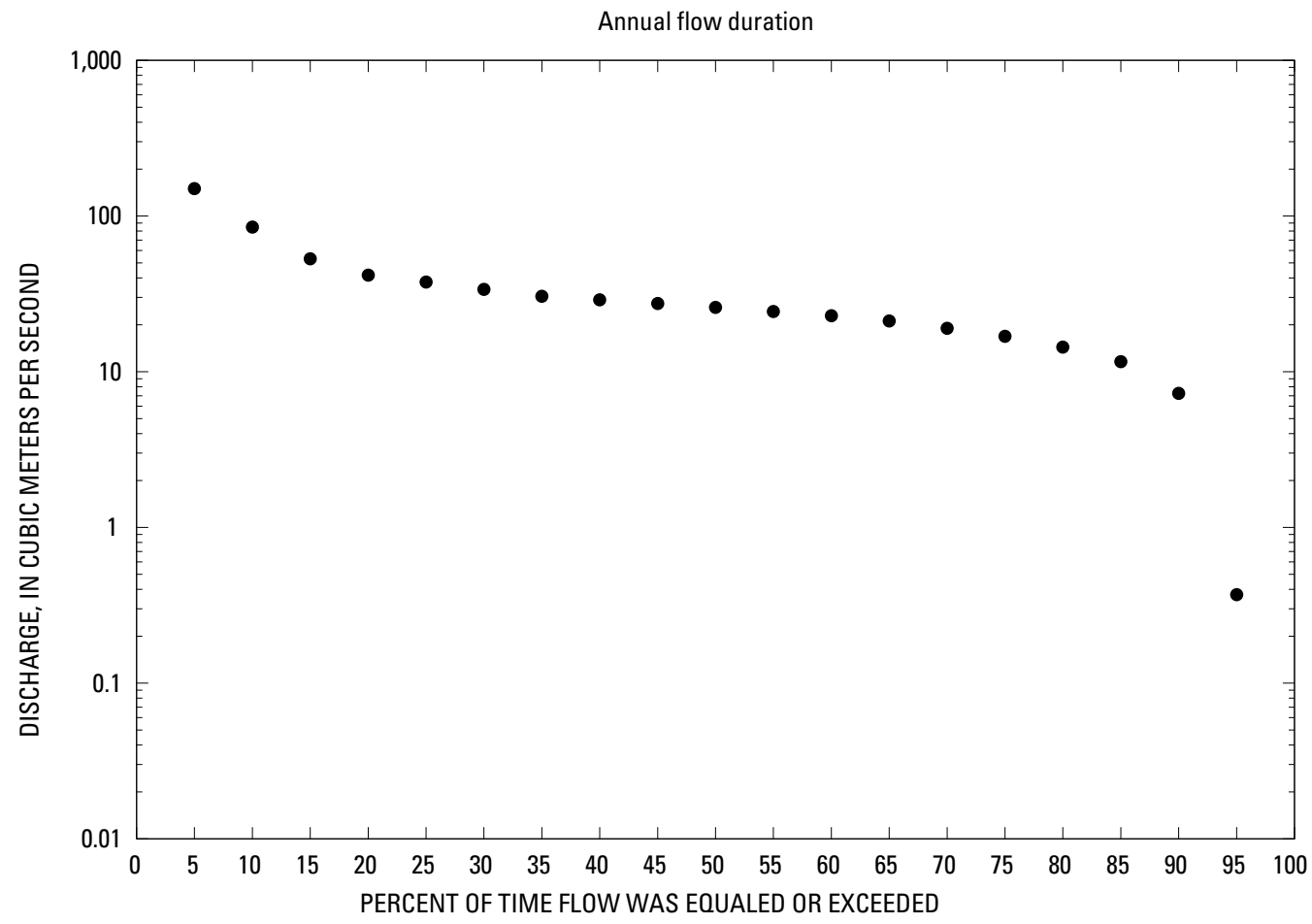




\section{4- 1.L00-4A ARGHANDAB RIVER BELOW ARGHANDAB RESERVOIR, Continued}

Monthly and annual flow duration, in cubic meters per second

[Data were not rounded in accordance with U.S. Geological Survey publication standards]

\begin{tabular}{|c|c|c|c|c|c|c|c|c|c|c|c|c|c|}
\hline $\begin{array}{l}\text { Percentage } \\
\text { of days } \\
\text { discharge } \\
\text { equaled or } \\
\text { exceeded }\end{array}$ & January & February & March & April & May & June & July & August & September & October & November & December & Annual \\
\hline 95 & 0.21 & 0.02 & 8.96 & 20.1 & 20.5 & 15.6 & 9.17 & 4.57 & 3.94 & 4.33 & 8.93 & 1.45 & 0.37 \\
\hline 90 & 0.26 & 0.28 & 12.7 & 26 & 24.4 & 18.8 & 14.4 & 9.08 & 8.15 & 7.8 & 12.3 & 11.9 & 7.26 \\
\hline 85 & 0.29 & 0.36 & 15.2 & 31 & 27.8 & 22.4 & 18.1 & 13.2 & 9.42 & 10.8 & 13.7 & 13.3 & 11.6 \\
\hline 80 & 0.31 & 0.41 & 17.4 & 38.9 & 32.6 & 23.8 & 20.5 & 18.9 & 13 & 12.4 & 15.2 & 14.6 & 14.4 \\
\hline 75 & 0.34 & 0.46 & 19.1 & 45 & 35.2 & 25.3 & 22.3 & 22 & 16.9 & 14.9 & 16.1 & 15.8 & 16.9 \\
\hline 70 & 0.36 & 6.65 & 20.9 & 60.1 & 37.6 & 27 & 23.8 & 24.8 & 20.7 & 17.1 & 19.1 & 17 & 19 \\
\hline 65 & 0.41 & 9.14 & 22.6 & 75.4 & 39.9 & 28.3 & 25.5 & 26 & 23.1 & 19.2 & 20 & 17.7 & 21.2 \\
\hline 60 & 0.51 & 11.4 & 24.8 & 85.6 & 45.8 & 29.2 & 27.7 & 27.2 & 24.3 & 20.7 & 20.8 & 18.5 & 22.9 \\
\hline 55 & 9.3 & 12.9 & 27.3 & 94.1 & 50.4 & 30 & 28.5 & 28.4 & 25.1 & 21.6 & 21.3 & 19.2 & 24.4 \\
\hline 50 & 11.6 & 14.2 & 29.8 & 107 & 55.9 & 31 & 29.3 & 29.5 & 25.8 & 22.5 & 21.8 & 20 & 25.9 \\
\hline 45 & 14.8 & 15.6 & 32.9 & 117.5 & 60.9 & 31.9 & 30.1 & 30.2 & 26.5 & 23.4 & 22.4 & 21 & 27.4 \\
\hline 40 & 15.9 & 17.5 & 41.7 & 127.5 & 67.6 & 32.9 & 30.9 & 30.8 & 27.2 & 24.3 & 23.2 & 22.1 & 29 \\
\hline 35 & 17 & 19.5 & 49.1 & 136.9 & 77.3 & 34.5 & 31.8 & 31.5 & 27.9 & 25.2 & 24 & 23.2 & 30.5 \\
\hline 30 & 18.5 & 21.7 & 55.9 & 148.3 & 91.8 & 36.9 & 32.7 & 32.2 & 28.5 & 26.1 & 24.8 & 24.2 & 33.8 \\
\hline 25 & 21.1 & 24.5 & 66.4 & 161.5 & 107.7 & 40.4 & 33.6 & 32.8 & 29.3 & 27.1 & 25.5 & 25.2 & 37.7 \\
\hline 20 & 23.4 & 27.2 & 91.8 & 183.6 & 128.3 & 43.2 & 34.5 & 33.5 & 30.2 & 28 & 26.1 & 26.1 & 41.7 \\
\hline 15 & 25.4 & 31.8 & 123.9 & 208.4 & 152.6 & 51.1 & 35.7 & 34.2 & 31.1 & 29.3 & 26.8 & 27.1 & 53.1 \\
\hline 10 & 27.5 & 38 & 160.9 & 240.6 & 183.6 & 56.7 & 38.5 & 34.8 & 32 & 30.6 & 27.8 & 28 & 84.9 \\
\hline 5 & 33.2 & 54.5 & 224 & 301.1 & 233.4 & 77.1 & 44.9 & 37.9 & 33.8 & 31.9 & 30.5 & 32 & 150.2 \\
\hline
\end{tabular}


Probability of occurrence of annual high discharges

[m³/s, meters per second; $n g$, statistic not given]

\begin{tabular}{rrrrrrr}
\hline & & & \multicolumn{5}{c}{ Maximum mean discharge } \\
\cline { 6 - 7 } $\begin{array}{c}\text { Exceedance } \\
\text { probability }\end{array}$ & $\begin{array}{c}\text { Recurrence } \\
\text { interval } \\
\text { (years) }\end{array}$ & $\begin{array}{c}\text { Maximum } \\
\text { instantaneous } \\
\left(\mathbf{m}^{3} / \mathbf{s}\right)\end{array}$ & 3-day period & 7-day period & 15-day period & 30-day period \\
\cline { 5 - 7 } 0.99 & 1.01 & 19.5 & 18.8 & 18.1 & 17.5 & 15.9 \\
0.95 & 1.05 & 39.5 & 36.9 & 34.9 & 33.1 & 30.1 \\
0.90 & 1.11 & 56.6 & 52.0 & 48.6 & 45.7 & 41.5 \\
0.80 & 1.25 & 86.3 & 77.3 & 71.4 & 66.4 & 60.0 \\
0.50 & 2 & 185 & 157 & 142 & 129 & 115 \\
0.20 & 5 & 375 & 299 & 266 & 236 & 207 \\
0.10 & 10 & 530 & 409 & 361 & 315 & 275 \\
0.04 & 25 & 755 & 560 & 491 & 421 & 363 \\
0.02 & 50 & 941 & 679 & 593 & 504 & 431 \\
0.01 & 100 & 1,140 & 803 & 699 & 588 & 499 \\
0.005 & 200 & 1,350 & 931 & 808 & 673 & 567 \\
0.002 & 500 & 1,660 & ng & ng & ng & ng \\
\hline
\end{tabular}

4- 1.L00-4A ARGHANDAB RIVER BELOW ARGHANDAB RESERVOIR, Continued

Probability of occurrence of annual low discharges

$\left[\mathrm{m}^{3} / \mathrm{s}\right.$, meters per second]

\begin{tabular}{|c|c|c|c|c|c|c|c|c|c|c|}
\hline \multirow{3}{*}{$\begin{array}{c}\text { Nonexceedance } \\
\text { probability }\end{array}$} & \multirow{3}{*}{$\begin{array}{c}\text { Recurrence } \\
\text { interval } \\
\text { (years) }\end{array}$} & \multicolumn{9}{|c|}{ Minimum mean discharge $\left(\mathrm{m}^{3} / \mathrm{s}\right)$} \\
\hline & & \multicolumn{9}{|c|}{ Number of consecutive days } \\
\hline & & 1 & 3 & 7 & 14 & 30 & 60 & 90 & 120 & 183 \\
\hline 0.05 & 20 & 0 & ${ }^{1} 0.015$ & 0.014 & 0.015 & 0.055 & 0.554 & 1.82 & 4.28 & 5.83 \\
\hline 0.10 & 10 & 0.025 & ${ }^{1} 0.054$ & 0.053 & 0.057 & 0.160 & 1.11 & 3.38 & 6.11 & 8.41 \\
\hline 0.20 & 5 & 0.097 & 0.165 & 0.173 & 0.188 & 0.420 & 2.33 & 6.18 & 8.82 & 12.1 \\
\hline 0.50 & 2 & 0.706 & 1.06 & 1.23 & 1.34 & 2.14 & 7.32 & 13.1 & 14.8 & 19.0 \\
\hline
\end{tabular}

'Data does not fit log-Pearson Type III curve, use with caution. 
4- 1.LO0-4A ARGHANDAB RIVER BELOW ARGHANDAB RESERVOIR, Continued

Probability of occurrence of seasonal low discharges

[m³/s, meters per second; $\mathrm{ng}$, statistic not given]

\begin{tabular}{|c|c|c|c|c|c|c|c|c|c|}
\hline \multirow{3}{*}{$\begin{array}{c}\text { Nonexceedance } \\
\text { probability }\end{array}$} & \multirow{3}{*}{$\begin{array}{c}\text { Recurrence } \\
\text { interval } \\
\text { (years) }\end{array}$} & \multicolumn{8}{|c|}{ Minimum mean discharge $\left(\mathrm{m}^{3} / \mathrm{s}\right)$} \\
\hline & & \multicolumn{8}{|c|}{ Number of consecutive days } \\
\hline & & 1 & 7 & 14 & 30 & 1 & 7 & 14 & 30 \\
\hline & & \multicolumn{4}{|c|}{ December-January-February } & \multicolumn{4}{|c|}{ March-April-May } \\
\hline 0.05 & 20 & 0.011 & 0.012 & 0.013 & 0.047 & 0.047 & 0.926 & 2.93 & 10.8 \\
\hline 0.10 & 10 & 0.043 & 0.052 & 0.057 & 0.158 & 0.615 & 3.53 & 6.08 & 14.1 \\
\hline 0.20 & 5 & 0.147 & 0.196 & 0.212 & 0.477 & 3.55 & 8.98 & 12.2 & 19.4 \\
\hline 0.50 & 2 & 1.19 & 1.70 & 1.82 & 2.91 & 19.6 & 23.6 & 27.5 & 36.2 \\
\hline & & \multicolumn{4}{|c|}{ June-July-August } & \multicolumn{4}{|c|}{ September-October-November } \\
\hline 0.05 & 20 & 0.743 & ng & ng & 5.84 & 1.94 & 2.25 & 2.61 & ng \\
\hline 0.10 & 10 & 3.56 & $\mathrm{ng}$ & $\mathrm{ng}$ & 9.47 & 5.17 & 5.77 & 6.16 & ng \\
\hline 0.20 & 5 & 9.92 & $\mathrm{ng}$ & ng & 15.1 & 10.1 & 10.9 & 11.1 & ng \\
\hline 0.50 & 2 & 24.7 & ng & ng & 26.7 & 20.4 & 21.0 & 21.0 & ng \\
\hline
\end{tabular}


Annual peak discharge and corresponding gage height

$\left[\mathrm{m}^{3} / \mathrm{s}\right.$, meters per second; --, no data]

\begin{tabular}{|c|c|c|c|c|c|c|c|}
\hline $\begin{array}{l}\text { Water } \\
\text { year }\end{array}$ & Date & $\begin{array}{c}\text { Gage } \\
\text { height } \\
\text { (meters) }\end{array}$ & $\begin{array}{c}\text { Peak } \\
\text { discharge } \\
\left(\mathrm{m}^{3} / \mathrm{s}\right)\end{array}$ & $\begin{array}{l}\text { Water } \\
\text { year }\end{array}$ & Date & $\begin{array}{c}\text { Gage } \\
\text { height } \\
\text { (meters) }\end{array}$ & $\begin{array}{c}\text { Peak } \\
\text { discharge } \\
\left(\mathrm{m}^{3} / \mathrm{s}\right)\end{array}$ \\
\hline \multicolumn{8}{|c|}{ Annual peak discharge, by year } \\
\hline 1948 & 8-Mar & $1,042.71$ & 359 & 1964 & 22-Apr & -- & 140 \\
\hline 1949 & 11-Mar & $1,043.98$ & 792 & 1965 & 23-Apr & -- & 671 \\
\hline 1950 & 28-Jan & $1,042.87$ & 416 & 1966 & 15-Apr & -- & 104 \\
\hline 1951 & 7-May & $1,042.92$ & 433 & 1967 & 29-Apr & -- & 361 \\
\hline 1952 & 14-Feb & -- & 144 & 1968 & 13-Mar & -- & 250 \\
\hline 1953 & 16-Apr & $1,055.58$ & 44.4 & 1969 & 16-Apr & -- & 153 \\
\hline 1954 & 30-Mar & $1,057.35$ & 391 & 1970 & 16-Apr & -- & 113 \\
\hline 1955 & 6-Apr & $1,055.92$ & 45.3 & 1971 & 4-Apr & -- & 28.1 \\
\hline 1956 & 3-Apr & $1,057.52$ & 286 & 1972 & 26-Mar & -- & 324 \\
\hline 1957 & 6-Apr & $1,058.54$ & 637 & 1973 & 1-Apr & -- & 120 \\
\hline 1958 & 19-Apr & $1,056.44$ & 99.9 & 1974 & 23-Mar & -- & 175 \\
\hline 1959 & 3-Apr & $1,057.02$ & 207 & 1975 & 5-Apr & -- & 170 \\
\hline 1960 & 4-May & $1,056.73$ & 143 & 1976 & 23-Mar & -- & 526 \\
\hline 1961 & 20-Apr & $1,057.18$ & 258 & 1977 & 14-Apr & -- & 95 \\
\hline 1962 & 22-Dec & -- & 47 & 1978 & 23-Apr & -- & 85.6 \\
\hline 1963 & 31-May & $1,055.89$ & 47.5 & 1979 & 3-Apr & -- & 258 \\
\hline \multicolumn{8}{|c|}{ Annual peak discharge, from highest to lowest } \\
\hline 1949 & 11-Mar & $1,043.98$ & 792 & 1975 & 5-Apr & -- & 170 \\
\hline 1965 & 23-Apr & -- & 671 & 1969 & 16-Apr & -- & 153 \\
\hline 1957 & 6-Apr & $1,058.54$ & 637 & 1952 & $14-\mathrm{Feb}$ & -- & 144 \\
\hline 1976 & 23-Mar & -- & 526 & 1960 & 4-May & $1,056.73$ & 143 \\
\hline 1951 & 7-May & $1,042.92$ & 433 & 1964 & 22-Apr & -- & 140 \\
\hline 1950 & 28-Jan & $1,042.87$ & 416 & 1973 & 1-Apr & -- & 120 \\
\hline 1954 & 30-Mar & $1,057.35$ & 391 & 1970 & 16-Apr & -- & 113 \\
\hline 1967 & 29-Apr & -- & 361 & 1966 & 15-Apr & -- & 104 \\
\hline 1948 & 8-Mar & $1,042.71$ & 359 & 1958 & 19-Apr & $1,056.44$ & 99.9 \\
\hline 1972 & 26-Mar & -- & 324 & 1977 & 14-Apr & -- & 95 \\
\hline 1956 & 3-Apr & $1,057.52$ & 286 & 1978 & 23-Apr & -- & 85.6 \\
\hline 1979 & 3-Apr & -- & 258 & 1963 & 31-May & $1,055.89$ & 47.5 \\
\hline 1961 & 20-Apr & $1,057.18$ & 258 & 1962 & 22-Dec & -- & 47 \\
\hline 1968 & 13-Mar & -- & 250 & 1955 & 6-Apr & $1,055.92$ & 45.3 \\
\hline 1959 & 3-Apr & $1,057.02$ & 207 & 1953 & 16-Apr & $1,055.58$ & 44.4 \\
\hline 1974 & 23-Mar & -- & 175 & 1971 & 4-Apr & -- & 28.1 \\
\hline
\end{tabular}




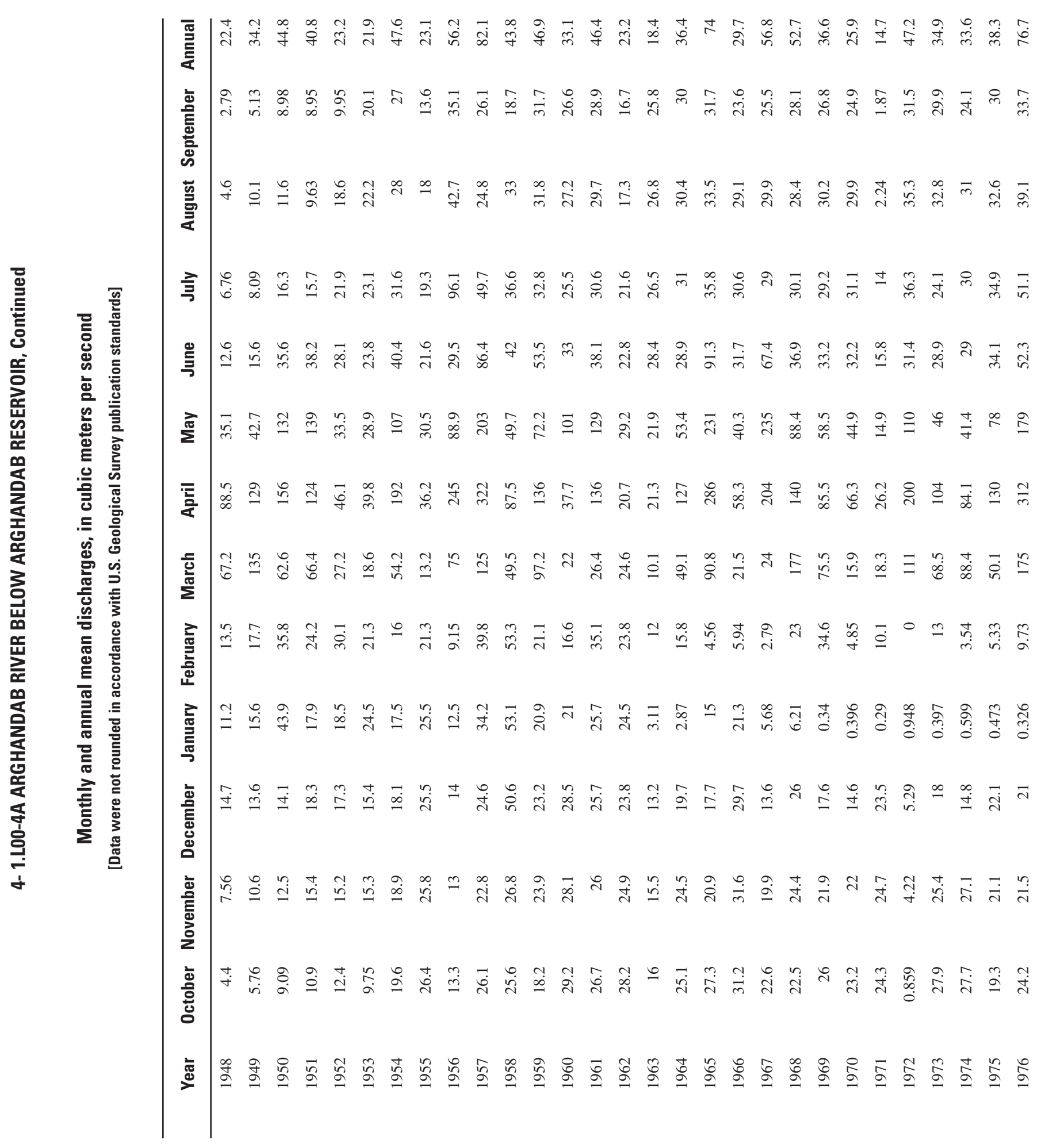




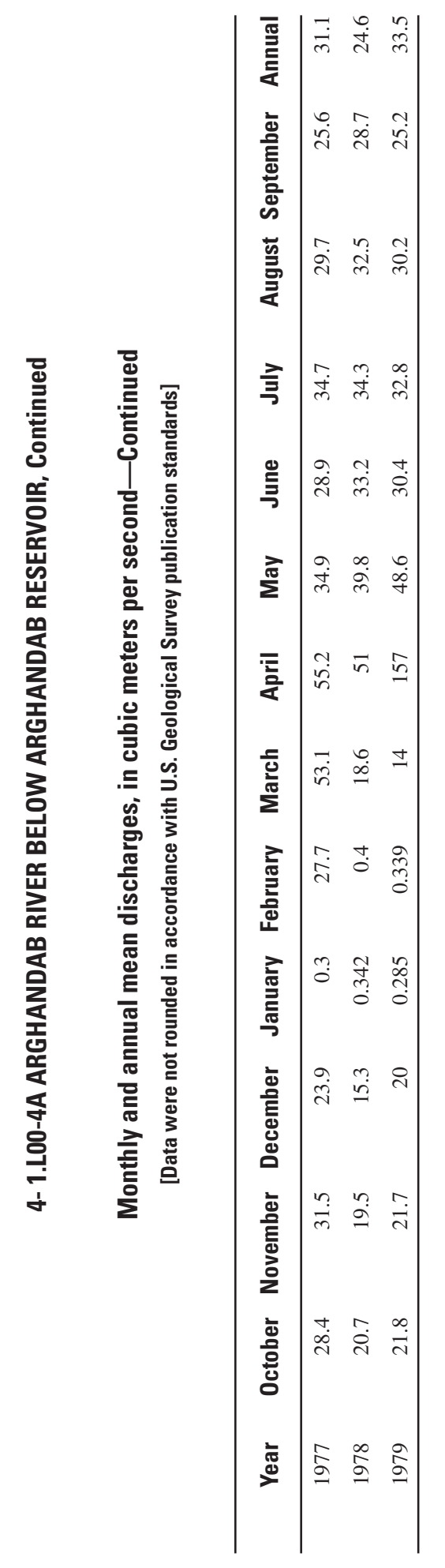




\section{4- 1.LO0-5A ARGHANDAB RIVER ABOVE ARGHANDAB RESERVOIR}

\section{(U.S. Geological Survey identification number: 315700066020000)}

LOCATION: Lat $31^{\circ} 57^{\prime} N$., long 66을 $\mathrm{km}$ northeast of Kandahar.

DRAINAGE AREA: $11,525 \mathrm{~km}^{2}$ (from Survey of India maps).

ALTITUDE: 1,154 meters plus mean sea level.

PERIOD OF RECORD: October 1, 1951 to March 31, 1979.

GAGE: Water-stage recorder. Prior to January 13, 1952, staff gage at same site and datum; January 13,1952 to August 7, 1958, water-stage recorder at site about $0.8 \mathrm{~km}$ downstream at datum $14 \mathrm{~m}$ lower.

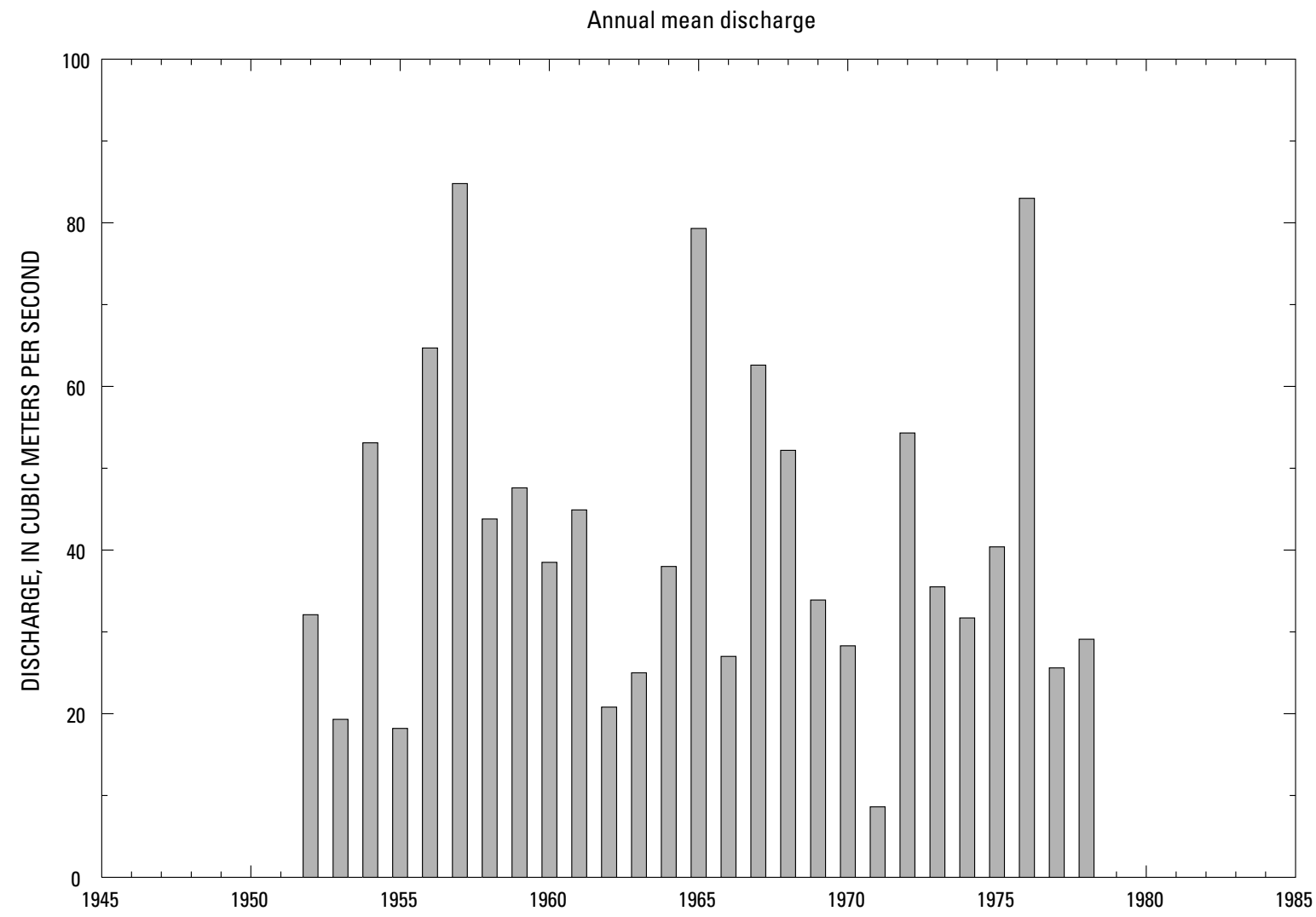


Statistics of monthly and annual mean discharges

$\left[\mathrm{m}^{3} / \mathrm{s}\right.$, meters per second]

\begin{tabular}{|c|c|c|c|c|c|c|c|c|}
\hline \multirow[b]{2}{*}{ Month } & \multicolumn{2}{|c|}{ Maximum } & \multicolumn{2}{|c|}{ Minimum } & \multicolumn{4}{|c|}{ Mean } \\
\hline & $\begin{array}{c}\text { Discharge } \\
\left(\mathrm{m}^{3} / \mathrm{s}\right)\end{array}$ & $\begin{array}{c}\text { Water year } \\
\text { of } \\
\text { occurrence }\end{array}$ & $\begin{array}{c}\text { Discharge } \\
\left(\mathrm{m}^{3} / \mathrm{s}\right)\end{array}$ & $\begin{array}{c}\text { Water year } \\
\text { of } \\
\text { occurrence }\end{array}$ & $\begin{array}{c}\text { Discharge } \\
\left(\mathrm{m}^{3} / \mathrm{s}\right)\end{array}$ & $\begin{array}{c}\text { Standard } \\
\text { deviation } \\
\left(\mathrm{m}^{3} / \mathrm{s}\right)\end{array}$ & $\begin{array}{c}\text { Coefficient } \\
\text { of } \\
\text { variation }\end{array}$ & $\begin{array}{c}\text { Percentage } \\
\text { of annual } \\
\text { discharge }\end{array}$ \\
\hline October & 18.7 & 1966 & 1.08 & 1972 & 11.1 & 4.10 & 0.37 & 2.24 \\
\hline November & 41.8 & 1958 & 6.87 & 1972 & 17.1 & 6.61 & 0.39 & 3.43 \\
\hline December & 59.1 & 1969 & 7.85 & 1972 & 21.4 & 10.9 & 0.51 & 4.30 \\
\hline January & 57.3 & 1958 & 13.0 & 1963 & 23.4 & 9.39 & 0.40 & 4.71 \\
\hline February & 82.8 & 1954 & 12.7 & 1963 & 39.9 & 18.1 & 0.45 & 8.02 \\
\hline March & 216 & 1972 & 20.5 & 1963 & 95.1 & 57.9 & 0.61 & 19.1 \\
\hline April & 333 & 1976 & 13.7 & 1971 & 138 & 89.4 & 0.65 & 27.8 \\
\hline May & 230 & 1965 & 3.03 & 1971 & 81.8 & 60.6 & 0.74 & 16.4 \\
\hline June & 90.2 & 1965 & 0.505 & 1971 & 29.2 & 22.4 & 0.77 & 5.87 \\
\hline July & 104 & 1956 & 0.001 & 1971 & 21.5 & 21.3 & 0.99 & 4.31 \\
\hline August & 28.7 & 1956 & 0.123 & 1971 & 11.0 & 6.95 & 0.63 & 2.21 \\
\hline September & 18.2 & 1957 & 0.460 & 1971 & 8.01 & 4.39 & 0.55 & 1.61 \\
\hline Annual & 84.8 & 1957 & 8.61 & 1971 & 41.6 & 20.0 & 0.48 & 100 \\
\hline
\end{tabular}

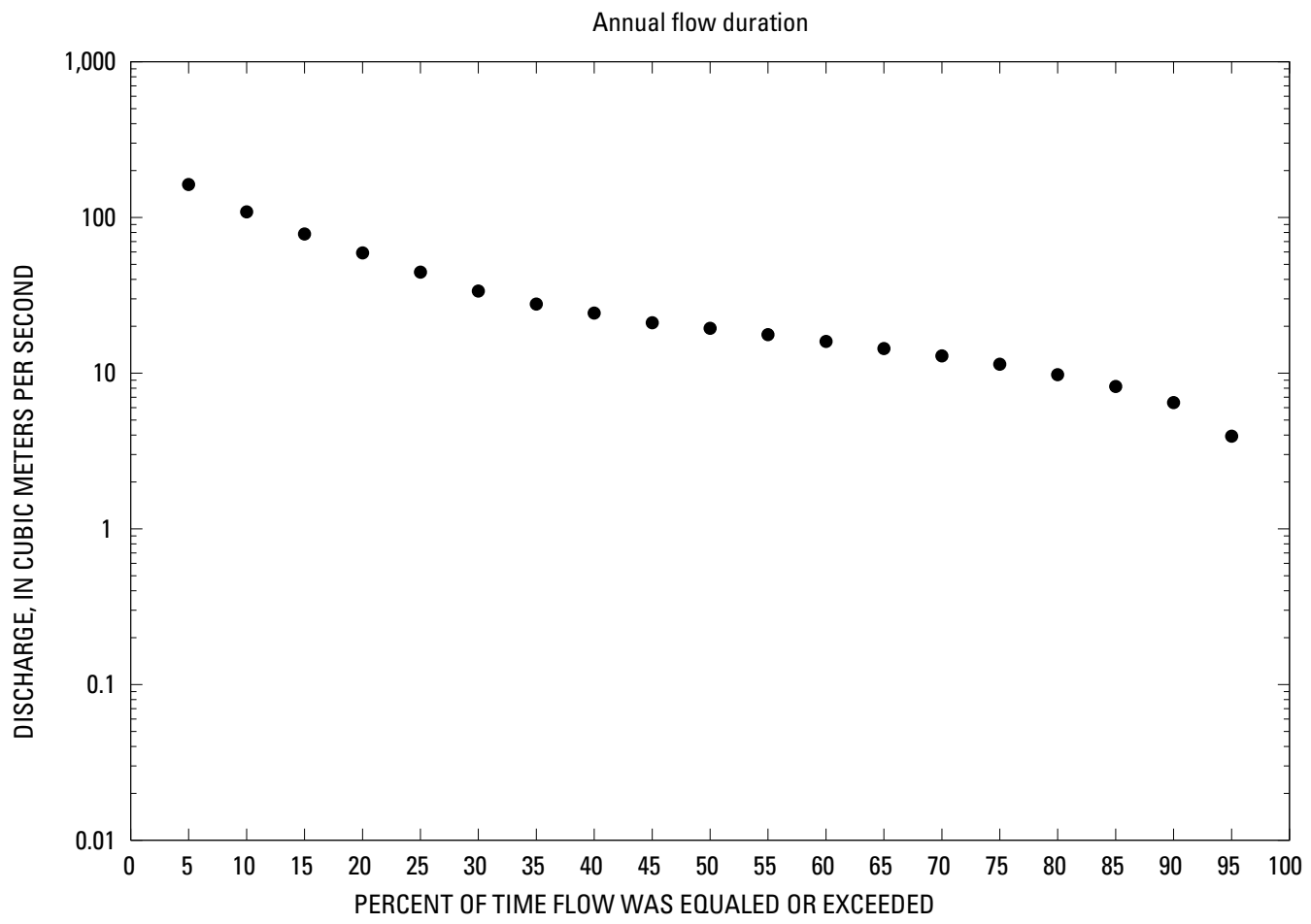




\section{4- 1.L00-5A ARGHANDAB RIVER ABOVE ARGHANDAB RESERVOIR, Continued}

\section{Monthly and annual flow duration, in cubic meters per second}

[Data were not rounded in accordance with U.S. Geological Survey publication standards]

\begin{tabular}{|c|c|c|c|c|c|c|c|c|c|c|c|c|c|}
\hline $\begin{array}{l}\text { Percentage } \\
\text { of days } \\
\text { discharge } \\
\text { equaled or } \\
\text { exceeded }\end{array}$ & January & February & March & April & May & June & July & August & September & October & November & December & Annual \\
\hline 95 & 13 & 15 & 19.7 & 26.5 & 14.7 & 6.09 & 2.69 & 2.01 & 2.15 & 4.18 & 8.37 & 11.5 & 3.94 \\
\hline 90 & 13.7 & 16.4 & 23.4 & 39.7 & 19 & 7.91 & 4.22 & 2.97 & 2.87 & 6.12 & 9.88 & 13.5 & 6.46 \\
\hline 85 & 14.6 & 17.4 & 35.2 & 53.2 & 23.9 & 9.43 & 5.35 & 3.54 & 3.42 & 6.98 & 11.2 & 14.1 & 8.21 \\
\hline 80 & 15.5 & 18.6 & 41.6 & 63.1 & 28.8 & 11 & 6.26 & 3.91 & 4.05 & 7.71 & 12.1 & 14.7 & 9.79 \\
\hline 75 & 16.5 & 20.2 & 46.2 & 70 & 33.9 & 12.7 & 7.21 & 5.31 & 5.15 & 8.29 & 12.7 & 15.2 & 11.4 \\
\hline 70 & 17.2 & 21.7 & 50.7 & 78.4 & 39.1 & 14.5 & 8.25 & 5.91 & 5.56 & 8.87 & 13.2 & 15.7 & 12.9 \\
\hline 65 & 17.9 & 22.9 & 56.5 & 86.2 & 44.4 & 16.4 & 9.3 & 6.49 & 5.96 & 9.44 & 13.6 & 16.3 & 14.4 \\
\hline 60 & 18.6 & 24.2 & 62 & 93.7 & 51.3 & 18.4 & 10.7 & 7.28 & 6.33 & 9.93 & 14 & 16.8 & 16 \\
\hline 55 & 19.3 & 25.7 & 68.7 & 102.3 & 56.4 & 21 & 12.1 & 8.2 & 6.69 & 10.4 & 14.6 & 17.5 & 17.7 \\
\hline 50 & 20 & 29.1 & 75.8 & 113.5 & 61.6 & 23.6 & 13.6 & 9.06 & 7.08 & 10.9 & 15.3 & 18.2 & 19.4 \\
\hline 45 & 20.6 & 33.8 & 82.4 & 125.5 & 67 & 25.9 & 15 & 9.83 & 7.67 & 11.4 & 16.3 & 19 & 21.1 \\
\hline 40 & 21.4 & 37.7 & 90.1 & 135.9 & 73.2 & 28.1 & 16.5 & 10.5 & 8.27 & 11.9 & 17.3 & 19.6 & 24.3 \\
\hline 35 & 22.2 & 40.3 & 98.1 & 148.2 & 80.8 & 30.2 & 18.4 & 11.2 & 9.12 & 12.5 & 18.2 & 20.2 & 27.8 \\
\hline 30 & 23.1 & 45.2 & 106.6 & 162.2 & 92.5 & 32.4 & 20.9 & 12 & 9.92 & 13.2 & 19.1 & 20.9 & 33.7 \\
\hline 25 & 24.3 & 53.2 & 118.2 & 181.3 & 109.4 & 36.7 & 23.4 & 12.9 & 10.6 & 13.9 & 20.1 & 22.3 & 44.5 \\
\hline 20 & 26.2 & 58.1 & 135.1 & 201.9 & 129 & 41.2 & 27.3 & 17.4 & 11.3 & 14.5 & 21.1 & 23.8 & 59.2 \\
\hline 15 & 30.3 & 63 & 157.3 & 230.2 & 150.9 & 48.7 & 31.4 & 18.9 & 12.9 & 15.4 & 22.8 & 25.3 & 78.3 \\
\hline 10 & 35.5 & 73.6 & 196 & 270.3 & 181.7 & 61.1 & 39.3 & 21.2 & 14.8 & 17.2 & 24.9 & 28.7 & 108.5 \\
\hline 5 & 53.3 & 96 & 255.2 & 333.2 & 233.6 & 84.9 & 55.3 & 25.4 & 17.5 & 19 & 28.2 & 38.6 & 163.1 \\
\hline
\end{tabular}




\section{4- 1.L00-5A ARGHANDAB RIVER ABOVE ARGHANDAB RESERVOIR, Continued}

Probability of occurrence of annual high discharges

[ $\mathrm{m}^{3} / \mathrm{s}$, meters per second; $\mathrm{ng}$, statistic not given]

\begin{tabular}{|c|c|c|c|c|c|c|}
\hline \multirow{2}{*}{$\begin{array}{l}\text { Exceedance } \\
\text { probability }\end{array}$} & \multirow{2}{*}{$\begin{array}{c}\text { Recurrence } \\
\text { interval } \\
\text { (years) }\end{array}$} & \multirow{2}{*}{$\begin{array}{c}\text { Maximum } \\
\text { instantaneous } \\
\left(\mathrm{m}^{3} / \mathbf{s}\right)\end{array}$} & \multicolumn{4}{|c|}{ Maximum mean discharge } \\
\hline & & & 3-day period & 7-day period & 15-day period & 30-day period \\
\hline 0.99 & 1.01 & ng & 24.9 & 23.9 & 23.4 & 21.1 \\
\hline 0.95 & 1.05 & 120 & 53.7 & 47.7 & 42.7 & 39.4 \\
\hline 0.90 & 1.11 & 150 & 77.3 & 66.6 & 58.6 & 53.5 \\
\hline 0.80 & 1.25 & 195 & 115 & 96.8 & 83.9 & 75.8 \\
\hline 0.50 & 2 & 320 & 219 & 181 & 154 & 137 \\
\hline 0.20 & 5 & 520 & 359 & 302 & 259 & 228 \\
\hline 0.10 & 10 & 668 & 441 & 379 & 328 & 288 \\
\hline 0.04 & 25 & 871 & 529 & 470 & 411 & 360 \\
\hline 0.02 & 50 & 1,030 & 584 & 531 & 470 & 410 \\
\hline 0.01 & 100 & 1,200 & 631 & 587 & 525 & 458 \\
\hline 0.005 & 200 & 1,380 & 672 & 639 & 577 & 504 \\
\hline 0.002 & 500 & 1,630 & $\mathrm{ng}$ & ng & $\mathrm{ng}$ & ng \\
\hline
\end{tabular}

'Historic peak of 1939 not used.

\section{4- 1.L00-5A ARGHANDAB RIVER ABOVE ARGHANDAB RESERVOIR, Continued}

Probability of occurrence of annual low discharges

[ $\mathbf{m}^{3} / \mathbf{s}$, meters per second; $\mathbf{n g}$, statistic not given]

\begin{tabular}{|c|c|c|c|c|c|c|c|c|c|c|}
\hline \multirow{3}{*}{$\begin{array}{c}\text { Nonexceedance } \\
\text { probability }\end{array}$} & \multirow{3}{*}{$\begin{array}{c}\text { Recurrence } \\
\text { interval } \\
\text { (years) }\end{array}$} & \multicolumn{9}{|c|}{ Minimum mean discharge $\left(\mathrm{m}^{3} / \mathrm{s}\right)$} \\
\hline & & \multicolumn{9}{|c|}{ Number of consecutive days } \\
\hline & & 1 & 3 & 7 & 14 & 30 & 60 & 90 & ${ }^{1} 120$ & 183 \\
\hline 0.05 & 20 & 0.805 & 0.845 & 0.919 & 1.08 & 1.56 & 1.91 & ${ }^{1} 1.15$ & ng & 2.79 \\
\hline 0.10 & 10 & 1.96 & 2.02 & 2.12 & 2.32 & 2.86 & 3.44 & 12.53 & ng & 4.78 \\
\hline 0.20 & 5 & 3.19 & 3.24 & 3.36 & 3.57 & 4.08 & 4.84 & 5.23 & ng & 8.01 \\
\hline 0.50 & 2 & 6.22 & 6.28 & 6.40 & 6.56 & 6.96 & 8.04 & 11.1 & ng & 14.9 \\
\hline
\end{tabular}

'Data does not fit log-Pearson Type III curve, use with caution. 


\section{4- 1.L00-5A ARGHANDAB RIVER ABOVE ARGHANDAB RESERVOIR, Continued}

\section{Probability of occurrence of seasonal low discharges}

$\left[\mathrm{m}^{3} / \mathrm{s}\right.$, meters per second]

\begin{tabular}{|c|c|c|c|c|c|c|c|c|c|}
\hline \multirow{3}{*}{$\begin{array}{c}\text { Nonexceedance } \\
\text { probability }\end{array}$} & \multirow{3}{*}{$\begin{array}{c}\text { Recurrence } \\
\text { interval } \\
\text { (years) }\end{array}$} & \multicolumn{8}{|c|}{ Minimum mean discharge $\left(\mathrm{m}^{3} / \mathrm{s}\right)$} \\
\hline & & \multicolumn{8}{|c|}{ Number of consecutive days } \\
\hline & & 1 & 7 & 14 & 30 & 1 & 7 & 14 & 30 \\
\hline & & \multicolumn{4}{|c|}{ December-January-February } & \multicolumn{4}{|c|}{ March-April-May } \\
\hline 0.05 & 20 & 9.49 & 9.94 & 10.3 & 11.0 & 5.54 & 6.36 & 7.32 & 9.93 \\
\hline 0.10 & 10 & 10.7 & 11.1 & 11.5 & 12.1 & 8.88 & 10.1 & 11.8 & 16.1 \\
\hline 0.20 & 5 & 12.2 & 12.6 & 13.2 & 13.8 & 14.5 & 16.4 & 19.4 & 27.0 \\
\hline \multirow[t]{2}{*}{0.50} & 2 & 15.7 & 16.3 & 17.1 & 17.9 & 29.9 & 33.8 & 40.7 & 57.8 \\
\hline & & \multicolumn{4}{|c|}{ June-July-August } & \multicolumn{4}{|c|}{ September-October-November } \\
\hline 0.05 & 20 & 1.13 & 1.22 & 1.40 & 1.83 & 1.50 & 1.63 & 1.73 & 2.01 \\
\hline 0.10 & 10 & 2.13 & 2.28 & 2.50 & 2.99 & 2.51 & 2.65 & 2.78 & 3.12 \\
\hline 0.20 & 5 & 3.39 & 3.59 & 3.84 & 4.38 & 3.65 & 3.80 & 3.95 & 4.33 \\
\hline 0.50 & 2 & 6.84 & 7.15 & 7.46 & 8.15 & 6.42 & 6.56 & 6.75 & 7.19 \\
\hline
\end{tabular}




\section{Annual peak discharge and corresponding gage height}

$\left[\mathrm{m}^{3} / \mathrm{s}\right.$, meters per second; --, no data]

\begin{tabular}{|c|c|c|c|c|c|c|c|}
\hline $\begin{array}{c}\text { Water } \\
\text { year }\end{array}$ & Date & $\begin{array}{c}\text { Gage } \\
\text { height } \\
\text { (meters) }\end{array}$ & $\begin{array}{c}\text { Peak } \\
\text { discharge } \\
\left(\mathrm{m}^{3} / \mathrm{s}\right)\end{array}$ & $\begin{array}{c}\text { Water } \\
\text { year }\end{array}$ & Date & $\begin{array}{c}\text { Gage } \\
\text { height } \\
\text { (meters) }\end{array}$ & $\begin{array}{c}\text { Peak } \\
\text { discharge } \\
\left(\mathrm{m}^{3} / \mathrm{s}\right)\end{array}$ \\
\hline
\end{tabular}

Annual peak discharge, by year

\begin{tabular}{|c|c|c|c|c|c|c|c|}
\hline 1939 & -- & $1,118.00$ & 1,190 & 1965 & 23-Apr & -- & 603 \\
\hline 1952 & $14-\mathrm{Feb}$ & $1,115.41$ & 203 & 1966 & 14-Apr & -- & 181 \\
\hline 1953 & $12-\mathrm{Feb}$ & $1,116.00$ & 354 & 1967 & 27-Apr & -- & 561 \\
\hline 1954 & 30-Mar & $1,116.10$ & 399 & 1968 & $25-\mathrm{Feb}$ & -- & 356 \\
\hline 1955 & 15-Mar & $1,115.76$ & 279 & 1969 & 10-Dec & -- & 250 \\
\hline 1956 & 28-Jul & $1,117.23$ & 841 & 1970 & 26-Mar & -- & 135 \\
\hline 1957 & 17-Mar & $1,117.54$ & 1,010 & 1971 & 27-Mar & -- & 24.7 \\
\hline 1958 & 19-Nov & -- & 255 & 1972 & 12-Mar & -- & 500 \\
\hline 1959 & 2-Mar & -- & 337 & 1973 & 20-Jul & -- & 175 \\
\hline 1960 & 17-Apr & $1,119.71$ & 323 & 1974 & 14-Jan & -- & 190 \\
\hline 1961 & 9-Apr & -- & 620 & 1975 & 27-Dec & -- & 248 \\
\hline 1962 & 21-Apr & -- & 97 & 1976 & 23-Mar & -- & 581 \\
\hline 1963 & 13-May & -- & 495 & 1977 & 9-Jan & -- & 195 \\
\hline 1964 & 17-Feb & -- & 298 & 1978 & 21-Aug & -- & 436 \\
\hline \multicolumn{8}{|c|}{ Annual peak discharge, from highest to lowest } \\
\hline 1939 & -- & $1,118.00$ & 1,190 & 1960 & 17-Apr & $1,119.71$ & 323 \\
\hline 1957 & 17-Mar & $1,117.54$ & 1,010 & 1964 & 17-Feb & -- & 298 \\
\hline 1956 & 28-Jul & $1,117.23$ & 841 & 1955 & 15-Mar & $1,115.76$ & 279 \\
\hline 1961 & 9-Apr & -- & 620 & 1958 & 19-Nov & -- & 255 \\
\hline 1965 & 23-Apr & -- & 603 & 1969 & 10-Dec & -- & 250 \\
\hline 1976 & 23-Mar & -- & 581 & 1975 & 27-Dec & -- & 248 \\
\hline 1967 & 27-Apr & -- & 561 & 1952 & 14-Feb & $1,115.41$ & 203 \\
\hline 1972 & 12-Mar & -- & 500 & 1977 & 9-Jan & -- & 195 \\
\hline 1963 & 13-May & -- & 495 & 1974 & 14-Jan & -- & 190 \\
\hline 1978 & 21-Aug & -- & 436 & 1966 & 14-Apr & -- & 181 \\
\hline 1954 & 30-Mar & $1,116.10$ & 399 & 1973 & 20-Jul & -- & 175 \\
\hline 1968 & $25-\mathrm{Feb}$ & -- & 356 & 1970 & 26-Mar & -- & 135 \\
\hline 1953 & $12-\mathrm{Feb}$ & $1,116.00$ & 354 & 1962 & 21-Apr & -- & 97 \\
\hline 1959 & 2-Mar & -- & 337 & 1971 & 27-Mar & -- & 24.7 \\
\hline
\end{tabular}




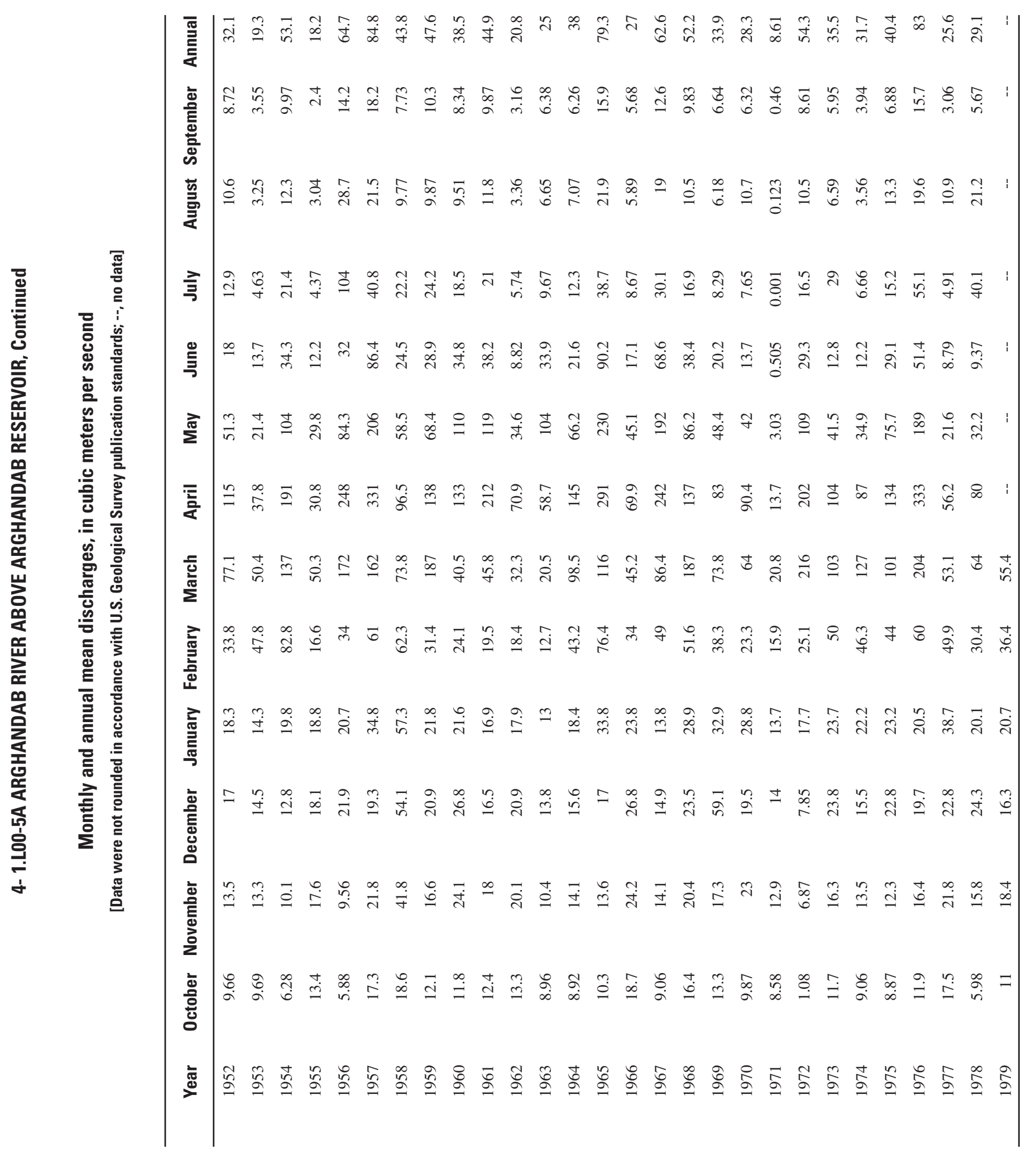




\section{4- 1.L00-6A ARGHANDAB RIVER AT MIZAN}

\section{(U.S. Geological Survey identification number: 321000066270000 )}

LOCATION: Lat $32^{\circ} 10^{\prime} \mathrm{N}$., long $66^{\circ} 27^{\prime} \mathrm{E}$.

DRAINAGE AREA: $9,310 \mathrm{~km}^{2}$.

ALTITUDE: 1,312 meters plus mean sea level.

PERIOD OF RECORD: February 12, 1972 to September 30, 1980.

GAGE: --

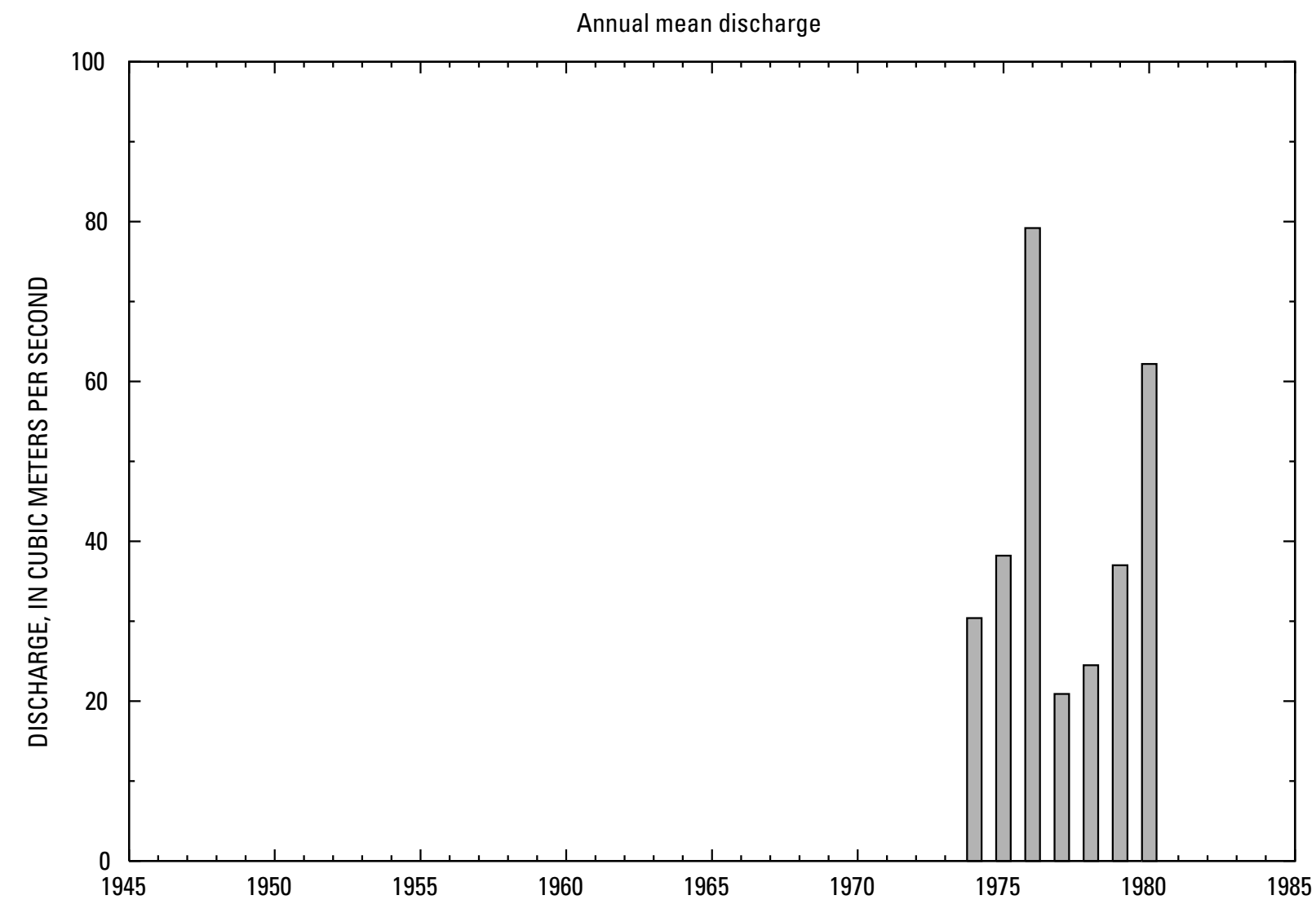




\section{4- 1.L00-6A ARGHANDAB RIVER AT MIZAN, Continued}

\section{Statistics of monthly and annual mean discharges}

$\left[\mathrm{m}^{3} / \mathrm{s}\right.$, meters per second]

\begin{tabular}{|c|c|c|c|c|c|c|c|c|}
\hline \multirow[b]{2}{*}{ Month } & \multicolumn{2}{|c|}{ Maximum } & \multicolumn{2}{|c|}{ Minimum } & \multicolumn{4}{|c|}{ Mean } \\
\hline & $\begin{array}{c}\text { Discharge } \\
\left(\mathrm{m}^{3} / \mathrm{s}\right)\end{array}$ & $\begin{array}{c}\text { Water year } \\
\text { of } \\
\text { occurrence }\end{array}$ & $\begin{array}{c}\text { Discharge } \\
\left(\mathrm{m}^{3} / \mathrm{s}\right)\end{array}$ & $\begin{array}{c}\text { Water year } \\
\text { of } \\
\text { occurrence }\end{array}$ & $\begin{array}{c}\text { Discharge } \\
\left(\mathrm{m}^{3} / \mathrm{s}\right)\end{array}$ & $\begin{array}{c}\text { Standard } \\
\text { deviation } \\
\left(\mathrm{m}^{3} / \mathrm{s}\right)\end{array}$ & $\begin{array}{c}\text { Coefficient } \\
\text { of } \\
\text { variation }\end{array}$ & $\begin{array}{c}\text { Percentage } \\
\text { of annual } \\
\text { discharge }\end{array}$ \\
\hline October & 16.3 & 1980 & 4.35 & 1978 & 10.6 & 4.39 & 0.41 & 2.17 \\
\hline November & 24.2 & 1980 & 9.86 & 1978 & 15.3 & 5.03 & 0.33 & 3.13 \\
\hline December & 28.8 & 1980 & 12.8 & 1978 & 18.3 & 5.18 & 0.28 & 3.73 \\
\hline January & 28.9 & 1980 & 14.8 & 1979 & 21.0 & 5.17 & 0.25 & 4.28 \\
\hline February & 57.8 & 1976 & 26.2 & 1978 & 39.7 & 11.5 & 0.29 & 8.11 \\
\hline March & 195 & 1976 & 46.0 & 1977 & 96.9 & 48.7 & 0.50 & 19.8 \\
\hline April & 323 & 1976 & 50.0 & 1977 & 152 & 98.9 & 0.65 & 31.1 \\
\hline May & 182 & 1976 & 17.1 & 1977 & 70.8 & 56.4 & 0.80 & 14.5 \\
\hline June & 47.8 & 1976 & 6.18 & 1978 & 22.4 & 16.8 & 0.75 & 4.57 \\
\hline July & 51.0 & 1976 & 4.19 & 1977 & 22.7 & 15.5 & 0.68 & 4.63 \\
\hline August & 18.4 & 1976 & 4.21 & 1974 & 12.0 & 5.91 & 0.49 & 2.46 \\
\hline September & 14.8 & 1976 & 2.67 & 1977 & 8.01 & 4.28 & 0.53 & 1.64 \\
\hline Annual & 79.2 & 1976 & 20.9 & 1977 & 41.8 & 21.3 & 0.51 & 100 \\
\hline
\end{tabular}

Annual flow duration

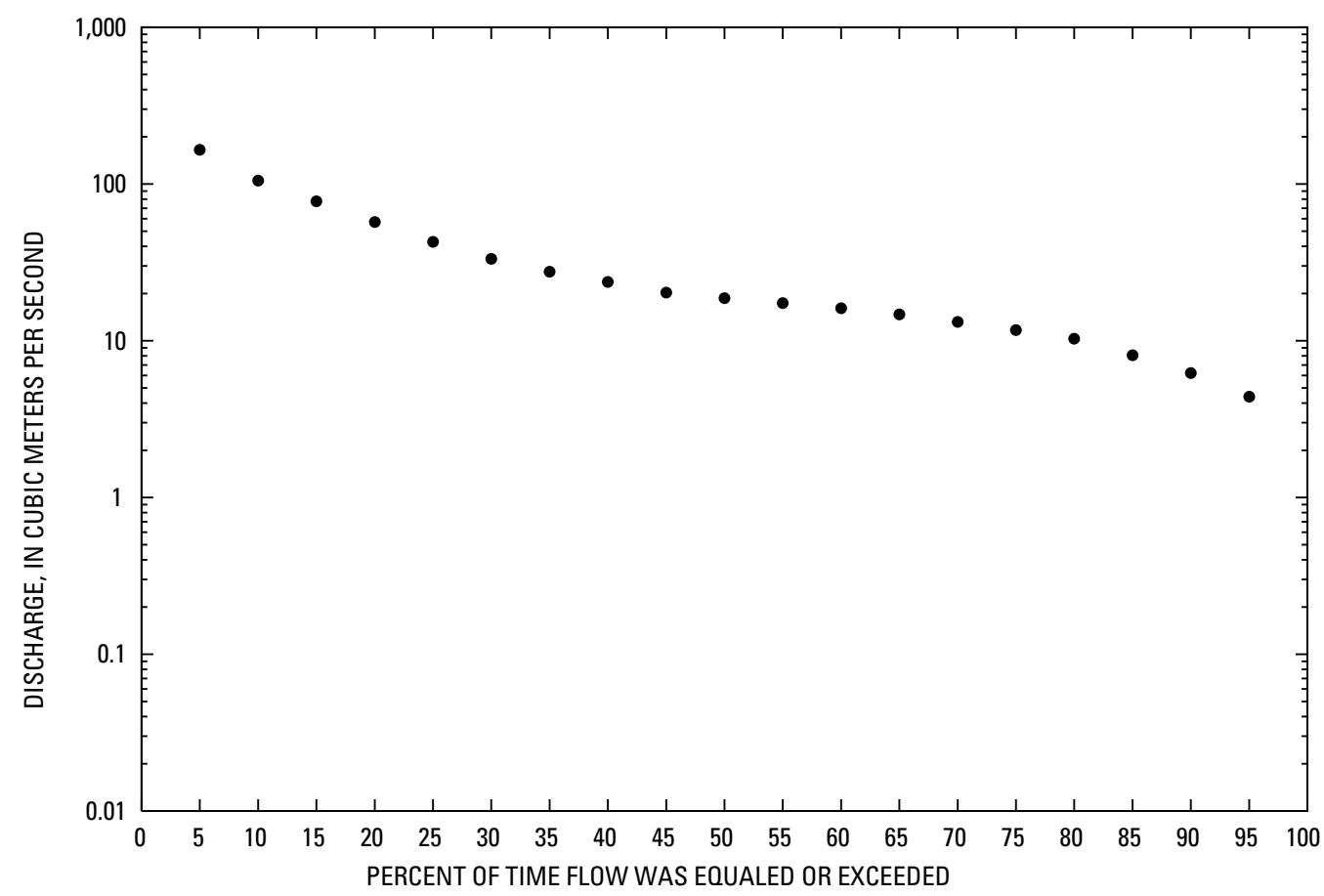




\section{4- 1.L00-6A ARGHANDAB RIVER AT MIZAN, Continued}

\section{Monthly and annual flow duration, in cubic meters per second}

[Data were not rounded in accordance with U.S. Geological Survey publication standards]

\begin{tabular}{|c|c|c|c|c|c|c|c|c|c|c|c|c|c|}
\hline $\begin{array}{l}\text { Percentage } \\
\text { of days } \\
\text { discharge } \\
\text { equaled or } \\
\text { exceeded }\end{array}$ & January & February & March & April & May & June & July & August & September & October & November & December & Annual \\
\hline 95 & 13.4 & 16.3 & 36.3 & 46.7 & 12.1 & 5.46 & 2.72 & 2.64 & 2.55 & 4.01 & 8.3 & 7.87 & 4.39 \\
\hline 90 & 14.2 & 19.2 & 42.5 & 57.4 & 15.5 & 6.06 & 4.17 & 3.94 & 3.05 & 4.46 & 9.96 & 9.02 & 6.23 \\
\hline 85 & 14.8 & 20.6 & 45.2 & 64 & 18 & 6.63 & 5.44 & 4.18 & 4.29 & 5.61 & 10.2 & 13.4 & 8.08 \\
\hline 80 & 15.2 & 22.8 & 48.1 & 69.1 & 22.5 & 7.28 & 5.9 & 4.57 & 4.53 & 6.45 & 10.8 & 14.3 & 10.3 \\
\hline 75 & 15.6 & 23.7 & 52.5 & 75 & 28 & 8.06 & 6.53 & 5.75 & 5.05 & 7.51 & 11.3 & 15 & 11.7 \\
\hline 70 & 16 & 26.1 & 58.2 & 83.4 & 32 & 8.75 & 7.76 & 6.34 & 5.54 & 8.07 & 12.1 & 15.5 & 13.2 \\
\hline 65 & 16.4 & 27.5 & 65.8 & 88.4 & 38.3 & 10.3 & 11.6 & 7.48 & 5.67 & 8.51 & 12.6 & 15.9 & 14.7 \\
\hline 60 & 17 & 30.6 & 76.5 & 99 & 41.3 & 11.7 & 13.9 & 8.47 & 5.79 & 9 & 13.1 & 16.3 & 16.1 \\
\hline 55 & 17.9 & 32.7 & 83.9 & 110.9 & 44.8 & 12.5 & 15.7 & 9.66 & 6.1 & 9.81 & 13.8 & 17 & 17.4 \\
\hline 50 & 18.9 & 34.4 & 87.7 & 119.4 & 49.6 & 14.9 & 17 & 11.3 & 6.55 & 10.1 & 14.8 & 17.5 & 18.7 \\
\hline 45 & 19.5 & 36 & 90.6 & 126.8 & 57.1 & 21.2 & 17.7 & 12.5 & 6.93 & 10.9 & 15.4 & 18 & 20.3 \\
\hline 40 & 20.3 & 40.1 & 94.8 & 134.2 & 67.5 & 23.9 & 18.5 & 13 & 7.3 & 11.5 & 16 & 18.4 & 23.7 \\
\hline 35 & 22.4 & 42.4 & 100.8 & 153.5 & 77.1 & 26 & 19.2 & 13.6 & 9.78 & 11.9 & 17 & 18.7 & 27.6 \\
\hline 30 & 23.5 & 44.5 & 106 & 181.5 & 87.3 & 28.7 & 20.6 & 14.9 & 10.3 & 12.5 & 18.4 & 19.2 & 33.3 \\
\hline 25 & 25.8 & 48 & 115 & 203.4 & 95.6 & 31.1 & 22.2 & 16 & 10.7 & 13.6 & 19 & 19.8 & 42.7 \\
\hline 20 & 26.7 & 54.1 & 120.9 & 230.1 & 103.9 & 33.2 & 24.4 & 17.1 & 11.1 & 15 & 19.3 & 20.9 & 57.2 \\
\hline 15 & 28.8 & 57.8 & 130.4 & 292 & 117.7 & 37.6 & 27.4 & 17.9 & 12.6 & 16.1 & 20.7 & 26.3 & 77.4 \\
\hline 10 & 30.3 & 67.1 & 152.5 & 326 & 162.2 & 48.8 & 32.9 & 18.7 & 14 & 17.6 & 23.4 & 28.3 & 105.2 \\
\hline 5 & 31.5 & 87.8 & 234.2 & 358.2 & 210.6 & 64.4 & 83.3 & 21 & 16.7 & 18.3 & 25.2 & 29.9 & 165.3 \\
\hline
\end{tabular}




\section{4- 1.L00-6A ARGHANDAB RIVER AT MIZAN, Continued}

Probability of occurrence of annual high discharges

[ $\mathrm{m}^{3} / \mathrm{s}$, meters per second; $\mathbf{n g}$, statistic not given]

\begin{tabular}{|c|c|c|c|c|c|c|}
\hline \multirow{2}{*}{$\begin{array}{c}\text { Exceedance } \\
\text { probability }\end{array}$} & \multirow{2}{*}{$\begin{array}{c}\text { Recurrence } \\
\text { interval } \\
\text { (years) }\end{array}$} & \multirow{2}{*}{$\begin{array}{c}\text { Maximum } \\
\text { instantaneous } \\
\left(\mathrm{m}^{3} / \mathrm{s}\right)\end{array}$} & \multicolumn{4}{|c|}{ Maximum mean discharge } \\
\hline & & & 3-day period & 7-day period & 15-day period & 30-day period \\
\hline 0.99 & 1.01 & 74.1 & 46.1 & 37.2 & 31.3 & 28.3 \\
\hline 0.95 & 1.05 & 104 & 74.4 & 59.5 & 51.0 & 46.2 \\
\hline 0.90 & 1.11 & 125 & 94.9 & 76.2 & 66.0 & 59.6 \\
\hline 0.80 & 1.25 & 158 & 126 & 102 & 89.7 & 80.7 \\
\hline 0.50 & 2 & 252 & 211 & 179 & 160 & 142 \\
\hline 0.20 & 5 & 413 & 340 & 308 & 281 & 244 \\
\hline 0.10 & 10 & 541 & 430 & 408 & 376 & 322 \\
\hline 0.04 & 25 & 727 & ${ }^{2} 545$ & 548 & 510 & 429 \\
\hline 0.02 & 50 & 884 & ${ }^{2} 631$ & 662 & 619 & 515 \\
\hline 0.01 & 100 & 1,060 & 2718 & 783 & 737 & 606 \\
\hline 0.005 & 200 & 1,250 & ${ }^{2} 805$ & 912 & 863 & 702 \\
\hline 0.002 & 500 & 1,530 & ng & ng & ng & ng \\
\hline
\end{tabular}

'Less than 10 years of data used.

${ }^{2}$ Data does not fit log-Pearson Type III curve, use with caution.

\section{4- 1.L00-6A ARGHANDAB RIVER AT MIZAN, Continued}

Probability of occurrence of annual low discharges

[m³ $/ \mathrm{s}$, meters per second]

\begin{tabular}{|c|c|c|c|c|c|c|c|c|c|c|}
\hline \multirow{3}{*}{$\begin{array}{c}\text { Nonexceedance } \\
\text { probability }\end{array}$} & \multirow{3}{*}{$\begin{array}{c}\text { Recurrence } \\
\text { interval } \\
\text { (years) }\end{array}$} & \multicolumn{9}{|c|}{ Minimum mean discharge $\left(\mathrm{m}^{3} / \mathrm{s}\right)$} \\
\hline & & \multicolumn{9}{|c|}{ Number of consecutive days } \\
\hline & & 1 & 3 & 7 & 14 & 30 & 60 & 90 & 120 & 183 \\
\hline 0.05 & 20 & 1.40 & 1.41 & 1.45 & 1.64 & 2.16 & 2.72 & 3.15 & 3.53 & 5.05 \\
\hline 0.10 & 10 & 2.02 & 2.06 & 2.13 & 2.32 & 2.79 & 3.41 & 4.00 & 4.54 & 6.26 \\
\hline 0.20 & 5 & 3.01 & 3.10 & 3.20 & 3.39 & 3.74 & 4.47 & 5.26 & 6.03 & 7.97 \\
\hline 0.50 & 2 & 5.63 & 5.82 & 5.99 & 6.16 & 6.32 & 7.38 & 8.64 & 9.84 & 12.1 \\
\hline
\end{tabular}




\section{4- 1.L00-6A ARGHANDAB RIVER AT MIZAN, Continued}

\section{Probability of occurrence of seasonal low discharges}

$\left[\mathrm{m}^{3} / \mathrm{s}\right.$, meters per second]

\begin{tabular}{|c|c|c|c|c|c|c|c|c|c|}
\hline \multirow{3}{*}{$\begin{array}{c}\text { Nonexceedance } \\
\text { probability }\end{array}$} & \multirow{3}{*}{$\begin{array}{c}\text { Recurrence } \\
\text { interval } \\
\text { (years) }\end{array}$} & \multicolumn{8}{|c|}{ Minimum mean discharge $\left(\mathrm{m}^{3} / \mathrm{s}\right)$} \\
\hline & & \multicolumn{8}{|c|}{ Number of consecutive days } \\
\hline & & 1 & 7 & 14 & 30 & 1 & 7 & 14 & 30 \\
\hline & & \multicolumn{4}{|c|}{ December-January-February } & \multicolumn{4}{|c|}{ March-April-May } \\
\hline 0.05 & 20 & 7.09 & 7.30 & 7.59 & 11.8 & 7.50 & 8.34 & 9.64 & 15.1 \\
\hline 0.10 & 10 & 8.51 & 8.74 & 9.06 & 12.5 & 10.0 & 11.0 & 12.7 & 19.0 \\
\hline 0.20 & 5 & 10.5 & 10.7 & 11.1 & 13.5 & 14.2 & 15.3 & 17.8 & 25.5 \\
\hline \multirow[t]{2}{*}{0.50} & 2 & 14.8 & 15.2 & 15.7 & 16.4 & 27.2 & 29.5 & 34.0 & 47.4 \\
\hline & & \multicolumn{4}{|c|}{ June-July-August } & \multicolumn{4}{|c|}{ September-October-November } \\
\hline 0.05 & 20 & 1.45 & 1.52 & 1.70 & 2.15 & 1.97 & 1.97 & 2.09 & 2.53 \\
\hline 0.10 & 10 & 2.14 & 2.27 & 2.49 & 3.00 & 2.62 & 2.63 & 2.75 & 3.12 \\
\hline 0.20 & 5 & 3.30 & 3.54 & 3.82 & 4.39 & 3.59 & 3.62 & 3.73 & 4.01 \\
\hline 0.50 & 2 & 6.81 & 7.25 & 7.70 & 8.48 & 5.98 & 6.10 & 6.20 & 6.39 \\
\hline
\end{tabular}

\section{4- 1.L00-6A ARGHANDAB RIVER AT MIZAN, Continued}

\section{Annual peak discharge and corresponding gage height}

$\left[\mathrm{m}^{3} / \mathrm{s}\right.$, meters per second; --, no data]

\begin{tabular}{ccccccc}
\hline $\begin{array}{c}\text { Water } \\
\text { year }\end{array}$ Date & $\begin{array}{c}\text { Gage } \\
\text { height } \\
(\text { meters })\end{array}$ & $\begin{array}{c}\text { Peak } \\
\text { discharge } \\
\left(\mathrm{m}^{3} / \mathrm{s}\right)\end{array}$ & $\begin{array}{c}\text { Water } \\
\text { year }\end{array}$ & Date & $\begin{array}{c}\text { Gage } \\
\text { height } \\
(\text { meters })\end{array}$ & $\begin{array}{c}\text { Peak } \\
\text { discharge } \\
\left(\mathrm{m}^{3} / \mathrm{s}\right)\end{array}$ \\
\hline
\end{tabular}

Annual peak discharge, by year

\begin{tabular}{|c|c|c|c|c|c|c|c|}
\hline 1973 & 5-Apr & -- & 166 & 1977 & 12-Aug & -- & 117 \\
\hline 1974 & 24-Mar & -- & 175 & 1978 & $5-J u l$ & -- & 500 \\
\hline 1975 & 4-Apr & -- & 193 & 1979 & 14-Apr & -- & 261 \\
\hline 1976 & 23-Mar & -- & 560 & 1980 & 20-Apr & -- & 400 \\
\hline \multicolumn{8}{|c|}{ Annual peak discharge, from highest to lowest } \\
\hline 1976 & 23-Mar & -- & 560 & 1975 & 4-Apr & -- & 193 \\
\hline 1978 & $5-J u l$ & -- & 500 & 1974 & 24-Mar & -- & 175 \\
\hline 1980 & 20-Apr & -- & 400 & 1973 & 5-Apr & -- & 166 \\
\hline 1979 & 14-Apr & -- & 261 & 1977 & 12-Aug & -- & 117 \\
\hline
\end{tabular}




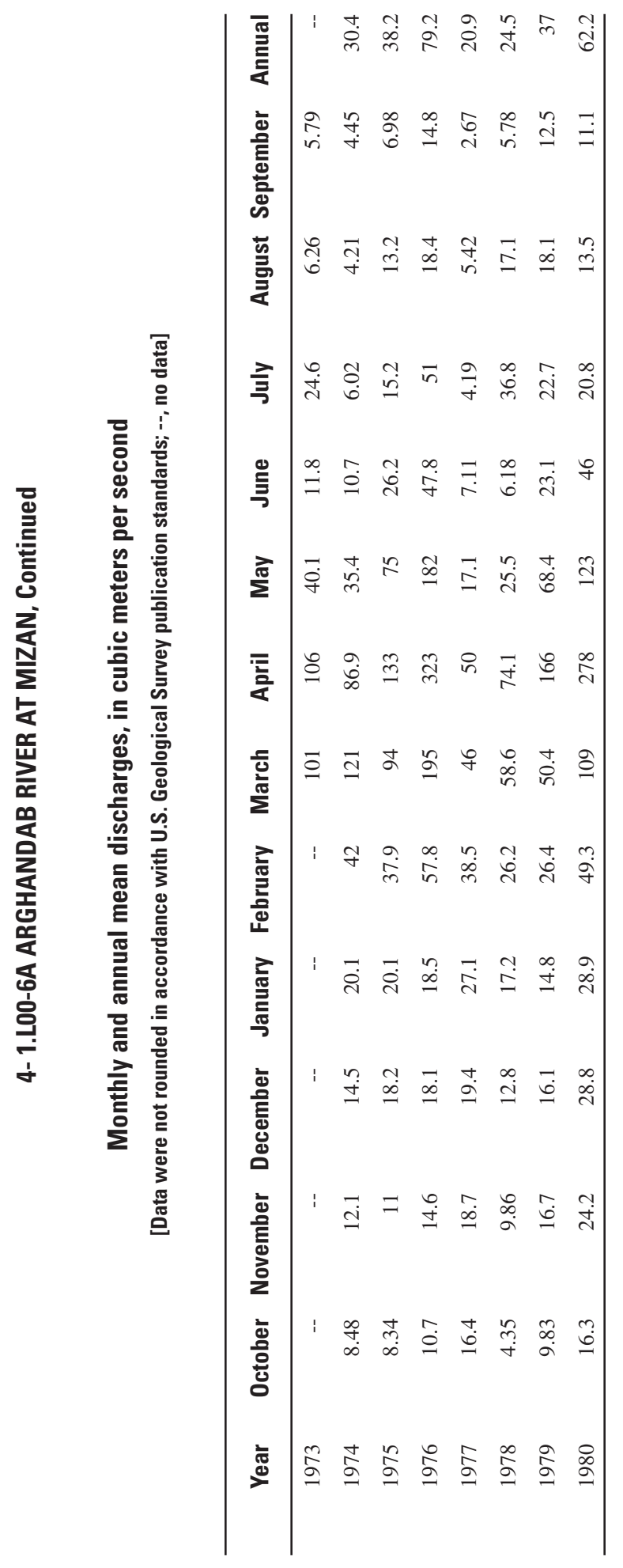




\section{4- 1.L00-9A ARGHANDAB RIVER AT SANG-I-MASHA}

\section{(U.S. Geological Survey identification number: 330800067280000 )}

LOCATION: Lat $33^{\circ} 08^{\prime} \mathrm{N}$., long $67^{\circ} 28^{\prime} \mathrm{E}$.

DRAINAGE AREA: $2,155 \mathrm{~km}^{2}$.

ALTITUDE: 2,302 meters plus mean sea level.

PERIOD OF RECORD: June 22, 1969 to September 30, 1980.

GAGE: --

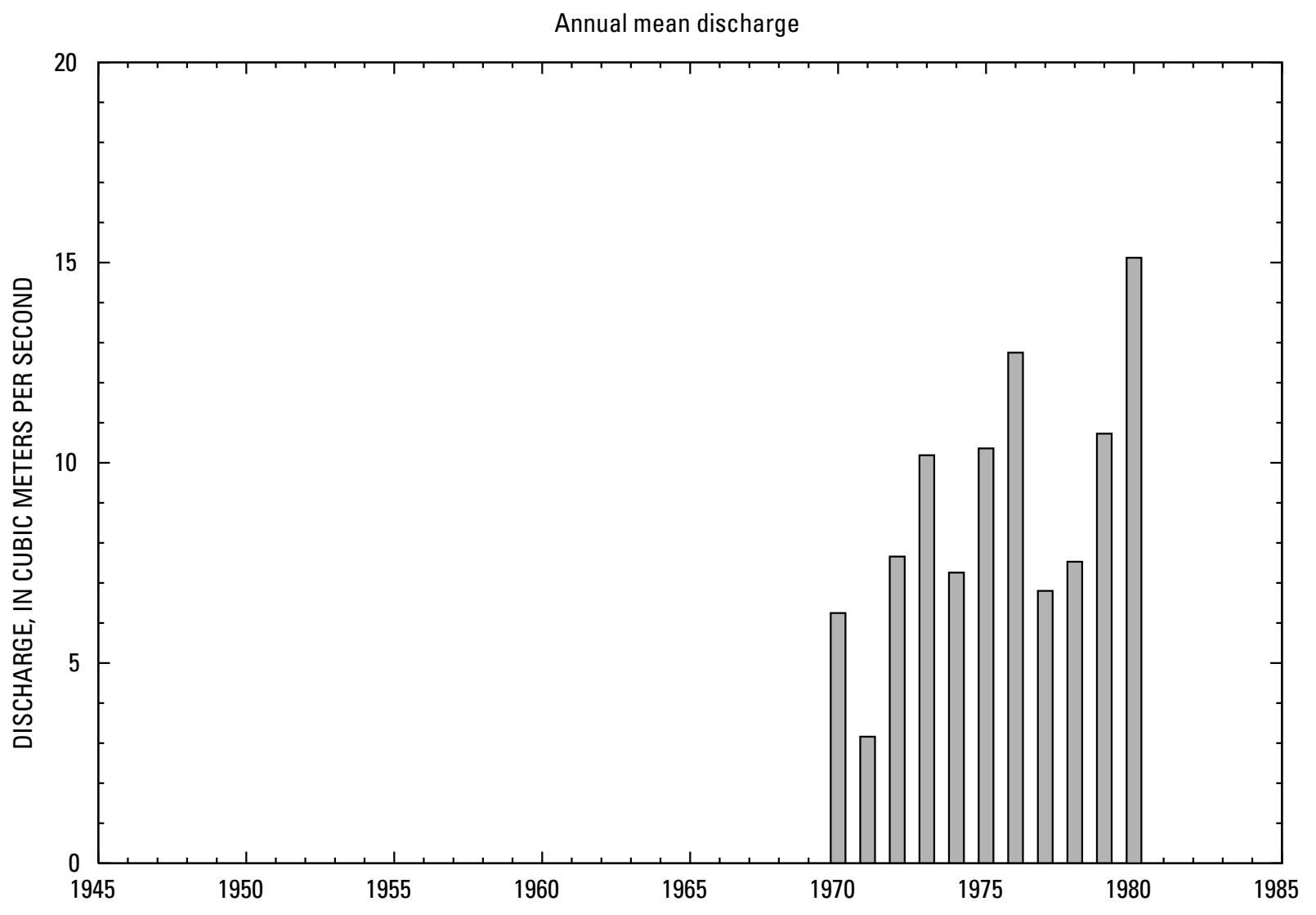




\section{4- 1.L00-9A ARGHANDAB RIVER AT SANG-I-MASHA, Continued}

\section{Statistics of monthly and annual mean discharges}

$\left[\mathrm{m}^{3} / \mathrm{s}\right.$, meters per second]

\begin{tabular}{|c|c|c|c|c|c|c|c|c|}
\hline \multirow[b]{2}{*}{ Month } & \multicolumn{2}{|c|}{ Maximum } & \multicolumn{2}{|c|}{ Minimum } & \multicolumn{4}{|c|}{ Mean } \\
\hline & $\begin{array}{c}\text { Discharge } \\
\left(\mathrm{m}^{3} / \mathrm{s}\right)\end{array}$ & $\begin{array}{c}\text { Water year } \\
\text { of } \\
\text { occurrence }\end{array}$ & $\begin{array}{c}\text { Discharge } \\
\left(\mathrm{m}^{3} / \mathrm{s}\right)\end{array}$ & $\begin{array}{c}\text { Water year } \\
\text { of } \\
\text { occurrence }\end{array}$ & $\begin{array}{c}\text { Discharge } \\
\left(\mathrm{m}^{3} / \mathrm{s}\right)\end{array}$ & $\begin{array}{c}\text { Standard } \\
\text { deviation } \\
\left(\mathrm{m}^{3} / \mathrm{s}\right)\end{array}$ & $\begin{array}{c}\text { Coefficient } \\
\text { of } \\
\text { variation }\end{array}$ & $\begin{array}{c}\text { Percentage } \\
\text { of annual } \\
\text { discharge }\end{array}$ \\
\hline October & 8.92 & 1980 & 2.59 & 1972 & 4.90 & 1.80 & 0.37 & 4.60 \\
\hline November & 10.4 & 1980 & 3.77 & 1972 & 6.12 & 2.19 & 0.36 & 5.74 \\
\hline December & 10.3 & 1980 & 4.25 & 1971 & 6.10 & 1.84 & 0.30 & 5.72 \\
\hline January & 9.54 & 1980 & 3.78 & 1971 & 5.87 & 1.80 & 0.31 & 5.51 \\
\hline February & 10.2 & 1980 & 3.84 & 1971 & 6.15 & 1.89 & 0.31 & 5.77 \\
\hline March & 20.2 & 1973 & 5.54 & 1971 & 12.0 & 4.41 & 0.37 & 11.2 \\
\hline April & 59.4 & 1980 & 5.97 & 1971 & 29.3 & 15.4 & 0.53 & 27.5 \\
\hline May & 58.9 & 1976 & 2.11 & 1971 & 15.3 & 9.30 & 0.61 & 14.3 \\
\hline June & 10.1 & 1980 & 0.775 & 1971 & 5.57 & 3.09 & 0.56 & 5.23 \\
\hline July & 17.0 & 1976 & 0.632 & 1971 & 6.33 & 5.34 & 0.84 & 5.95 \\
\hline August & 11.6 & 1976 & 1.80 & 1971 & 4.95 & 2.99 & 0.60 & 4.65 \\
\hline September & 7.50 & 1976 & 1.15 & 1971 & 3.98 & 1.93 & 0.48 & 3.73 \\
\hline Annual & 15.1 & 1980 & 3.16 & 1971 & 8.89 & 3.33 & 0.37 & 100 \\
\hline
\end{tabular}

Annual flow duration

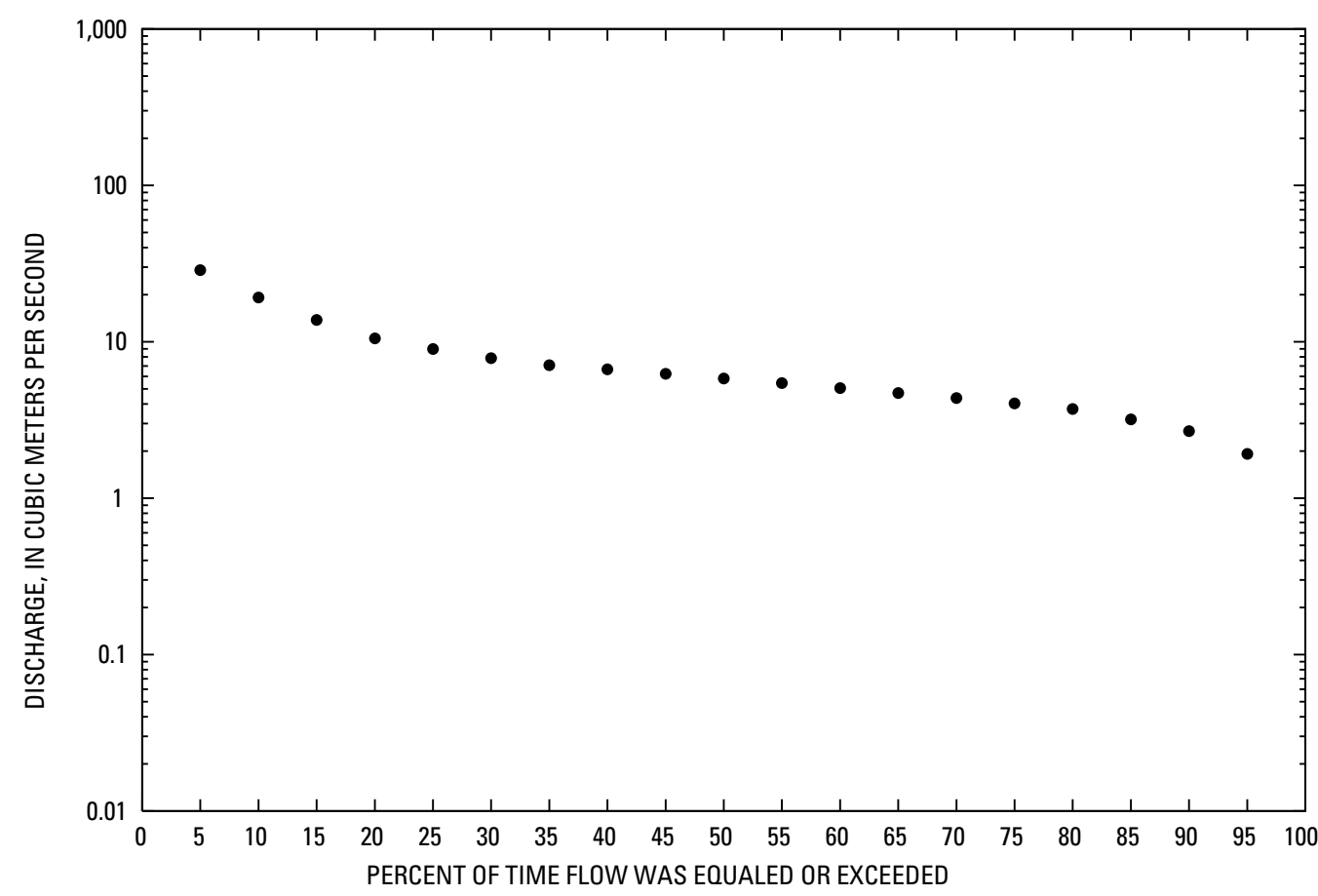




\section{4- 1.L00-9A ARGHANDAB RIVER AT SANG-I-MASHA, Continued}

Monthly and annual flow duration, in cubic meters per second

[Data were not rounded in accordance with U.S. Geological Survey publication standards]

\begin{tabular}{|c|c|c|c|c|c|c|c|c|c|c|c|c|c|}
\hline $\begin{array}{l}\text { Percentage } \\
\text { of days } \\
\text { discharge } \\
\text { equaled or } \\
\text { exceeded }\end{array}$ & January & February & March & April & May & June & July & August & September & October & November & December & Annual \\
\hline 95 & 3.74 & 3.71 & 4.57 & 6.53 & 2.42 & 0.71 & 0.62 & 1.26 & 1.29 & 2.77 & 3.49 & 4.13 & 1.92 \\
\hline 90 & 3.86 & 3.83 & 5.7 & 12.9 & 3.72 & 1.63 & 1.06 & 1.76 & 1.71 & 2.89 & 3.92 & 4.31 & 2.68 \\
\hline 85 & 4.05 & 4.11 & 6.51 & 15 & 5.19 & 2.44 & 1.83 & 1.96 & 2.15 & 3.29 & 4.05 & 4.35 & 3.19 \\
\hline 80 & 4.14 & 4.29 & 6.74 & 16.2 & 5.82 & 2.79 & 2.06 & 2.44 & 2.47 & 3.52 & 4.21 & 4.39 & 3.72 \\
\hline 75 & 4.21 & 4.6 & 6.96 & 17.2 & 6.47 & 3.03 & 2.39 & 2.55 & 2.68 & 3.73 & 4.38 & 4.54 & 4.04 \\
\hline 70 & 4.44 & 4.9 & 7.66 & 18.7 & 7.35 & 3.2 & 2.83 & 2.65 & 2.83 & 3.86 & 4.45 & 4.6 & 4.37 \\
\hline 65 & 4.9 & 5.27 & 8.01 & 19.9 & 9.13 & 3.74 & 3.29 & 2.99 & 3.01 & 3.96 & 4.53 & 4.66 & 4.71 \\
\hline 60 & 5.22 & 5.58 & 8.45 & 20.9 & 10.4 & 4.1 & 3.67 & 3.27 & 3.18 & 4.09 & 4.96 & 4.84 & 5.05 \\
\hline 55 & 5.29 & 5.76 & 9.1 & 22.3 & 11.6 & 4.52 & 3.87 & 3.56 & 3.3 & 4.23 & 5.6 & 5.63 & 5.44 \\
\hline 50 & 5.57 & 5.98 & 9.83 & 24.1 & 13.4 & 4.99 & 4.07 & 3.91 & 3.42 & 4.38 & 5.81 & 6.13 & 5.83 \\
\hline 45 & 5.95 & 6.22 & 10.6 & 26.5 & 14.2 & 5.41 & 4.26 & 4.39 & 3.9 & 4.61 & 6.05 & 6.28 & 6.24 \\
\hline 40 & 6.14 & 6.56 & 11.3 & 28.6 & 15.4 & 5.96 & 4.66 & 4.61 & 4.1 & 4.82 & 6.19 & 6.36 & 6.66 \\
\hline 35 & 6.39 & 6.67 & 12.1 & 31.7 & 17.2 & 6.55 & 5.13 & 4.84 & 4.42 & 5.3 & 6.39 & 6.5 & 7.08 \\
\hline 30 & 6.63 & 6.9 & 14.3 & 36.1 & 19.2 & 7.09 & 5.51 & 5.41 & 4.87 & 5.73 & 6.98 & 6.81 & 7.85 \\
\hline 25 & 7.21 & 7.09 & 15.4 & 39.1 & 22.1 & 7.73 & 5.89 & 5.83 & 5.17 & 5.91 & 7.4 & 7.21 & 8.98 \\
\hline 20 & 7.46 & 7.21 & 17.2 & 42 & 24.5 & 8.34 & 6.33 & 6.22 & 5.47 & 6.14 & 8.4 & 7.34 & 10.5 \\
\hline 15 & 7.79 & 7.83 & 19.5 & 46.5 & 27.1 & 8.99 & 6.87 & 6.78 & 5.85 & 6.59 & 8.72 & 7.82 & 13.8 \\
\hline 10 & 8.17 & 8.96 & 21.2 & 55.5 & 31.3 & 9.79 & 8.1 & 9.64 & 6.45 & 7.48 & 9.57 & 8.39 & 19.2 \\
\hline 5 & 0 & 9.82 & 24.7 & 66.3 & 36.9 & 11.8 & 19.6 & 12.9 & 6.9 & 9.18 & 0 & 0 & 28.7 \\
\hline
\end{tabular}




\section{4- 1.L00-9A ARGHANDAB RIVER AT SANG-I-MASHA, Continued}

Probability of occurrence of annual high discharges

[ $\mathrm{m}^{3} / \mathrm{s}$, meters per second; ng, statistic not given]

\begin{tabular}{|c|c|c|c|c|c|c|}
\hline \multirow{2}{*}{$\begin{array}{l}\text { Exceedance } \\
\text { probability }\end{array}$} & \multirow{2}{*}{$\begin{array}{c}\text { Recurrence } \\
\text { interval } \\
\text { (years) }\end{array}$} & \multirow{2}{*}{$\begin{array}{c}\text { Maximum } \\
\text { instantaneous } \\
\left(\mathrm{m}^{3} / \mathrm{s}\right)\end{array}$} & \multicolumn{4}{|c|}{ Maximum mean discharge } \\
\hline & & & 3-day period & 7-day period & 15-day period & 30-day period \\
\hline 0.99 & 1.01 & 4.20 & 14.23 & 14.13 & ${ }^{14} 4.27$ & ${ }^{14} 4.28$ \\
\hline 0.95 & 1.05 & 10.1 & 9.58 & 9.24 & 8.87 & 8.39 \\
\hline 0.90 & 1.11 & 15.6 & 14.1 & 13.4 & 12.5 & 11.5 \\
\hline 0.80 & 1.25 & 25.8 & 21.5 & 20.1 & 18.2 & 16.4 \\
\hline 0.50 & 2 & 61.9 & 42.0 & 37.9 & 33.2 & 29.0 \\
\hline 0.20 & 5 & 133 & 69.8 & 60.1 & 52.3 & 45.0 \\
\hline 0.10 & 10 & 190 & 85.9 & 72.0 & 63.0 & 54.2 \\
\hline 0.04 & 25 & 269 & 103 & 84.0 & 74.2 & 64.0 \\
\hline 0.02 & 50 & 332 & 114 & 90.9 & 81.0 & 70.1 \\
\hline 0.01 & 100 & 397 & 123 & 96.5 & 86.7 & 75.3 \\
\hline 0.005 & 200 & 464 & 130 & 101 & 91.6 & 79.9 \\
\hline 0.002 & 500 & 554 & $\mathrm{ng}$ & ng & $\mathrm{ng}$ & ng \\
\hline
\end{tabular}

'Data does not fit log-Pearson Type III curve, use with caution.

\section{4- 1.L00-9A ARGHANDAB RIVER AT SANG-I-MASHA, Continued}

Probability of occurrence of annual low discharges

$\left[\mathrm{m}^{3} / \mathrm{s}\right.$, meters per second]

\begin{tabular}{|c|c|c|c|c|c|c|c|c|c|c|}
\hline \multirow{3}{*}{$\begin{array}{c}\text { Nonexceedance } \\
\text { probability }\end{array}$} & \multirow{3}{*}{$\begin{array}{c}\text { Recurrence } \\
\text { interval } \\
\text { (years) }\end{array}$} & \multicolumn{9}{|c|}{ Minimum mean discharge $\left(\mathrm{m}^{3} / \mathrm{s}\right)$} \\
\hline & & \multicolumn{9}{|c|}{ Number of consecutive days } \\
\hline & & 1 & 3 & 7 & 14 & 30 & 60 & 90 & 120 & 183 \\
\hline 0.05 & 20 & 0.456 & 0.457 & 0.484 & 0.510 & 0.603 & 0.843 & 1.04 & 1.22 & 1.66 \\
\hline 0.10 & 10 & 0.692 & 0.697 & 0.732 & 0.769 & 0.901 & 1.20 & 1.43 & 1.64 & 2.12 \\
\hline 0.20 & 5 & 1.10 & 1.11 & 1.15 & 1.20 & 1.39 & 1.78 & 2.04 & 2.27 & 2.79 \\
\hline 0.50 & 2 & 2.30 & 2.33 & 2.38 & 2.47 & 2.75 & 3.34 & 3.65 & 3.89 & 4.44 \\
\hline
\end{tabular}




\section{4- 1.L00-9A ARGHANDAB RIVER AT SANG-I-MASHA, Continued}

Probability of occurrence of seasonal low discharges

$\left[\mathrm{m}^{3} / \mathrm{s}\right.$, meters per second]

\begin{tabular}{|c|c|c|c|c|c|c|c|c|c|}
\hline \multirow{3}{*}{$\begin{array}{c}\text { Nonexceedance } \\
\text { probability }\end{array}$} & \multirow{3}{*}{$\begin{array}{c}\text { Recurrence } \\
\text { interval } \\
\text { (years) }\end{array}$} & \multicolumn{8}{|c|}{ Minimum mean discharge $\left(\mathrm{m}^{3} / \mathrm{s}\right)$} \\
\hline & & \multicolumn{8}{|c|}{ Number of consecutive days } \\
\hline & & 1 & 7 & 14 & 30 & 1 & 7 & 14 & 30 \\
\hline & & \multicolumn{4}{|c|}{ December-January-February } & \multicolumn{4}{|c|}{ March-April-May } \\
\hline 0.05 & 20 & 3.29 & 3.37 & 3.42 & 3.49 & 1.59 & 1.72 & 2.03 & 2.90 \\
\hline 0.10 & 10 & 3.56 & 3.65 & 3.71 & 3.79 & 2.19 & 2.41 & 2.76 & 4.14 \\
\hline 0.20 & 5 & 3.95 & 4.05 & 4.12 & 4.22 & 3.10 & 3.46 & 3.86 & 5.98 \\
\hline \multirow[t]{2}{*}{0.50} & 2 & 4.91 & 5.04 & 5.13 & 5.27 & 5.35 & 6.09 & 6.56 & 10.0 \\
\hline & & \multicolumn{4}{|c|}{ June-July-August } & \multicolumn{4}{|c|}{ September-October-November } \\
\hline 0.05 & 20 & 0.487 & 0.518 & 0.547 & 0.643 & 0.731 & 0.804 & 0.925 & 1.33 \\
\hline 0.10 & 10 & 0.762 & 0.809 & 0.855 & 0.991 & 1.10 & 1.17 & 1.29 & 1.69 \\
\hline 0.20 & 5 & 1.24 & 1.31 & 1.38 & 1.57 & 1.68 & 1.76 & 1.85 & 2.21 \\
\hline 0.50 & 2 & 2.65 & 2.77 & 2.90 & 3.18 & 3.17 & 3.24 & 3.26 & 3.48 \\
\hline
\end{tabular}

4- 1.L00-9A ARGHANDAB RIVER AT SANG-I-MASHA, Continued

Annual peak discharge and corresponding gage height

[m³/s, meters per second; --, no data]

\begin{tabular}{ccccccc}
\hline $\begin{array}{c}\text { Water } \\
\text { year }\end{array}$ Date & $\begin{array}{c}\text { Gage } \\
\text { height } \\
(\text { meters })\end{array}$ & $\begin{array}{c}\text { Peak } \\
\text { discharge } \\
\left(\mathrm{m}^{3} / \mathrm{s}\right)\end{array}$ & $\begin{array}{c}\text { Water } \\
\text { year }\end{array}$ & Date & $\begin{array}{c}\text { Gage } \\
\text { height } \\
(\text { meters })\end{array}$ & $\begin{array}{c}\text { Peak } \\
\text { discharge } \\
\left(\mathrm{m}^{3} / \mathrm{s}\right)\end{array}$ \\
\hline
\end{tabular}

Annual peak discharge, by year

\begin{tabular}{|c|c|c|c|c|c|c|c|}
\hline 1970 & 15-Apr & -- & 23.0 & 1976 & 23-Jul & -- & 216 \\
\hline 1971 & 15-Apr & -- & 7.98 & 1977 & 30-Jun & -- & 46.1 \\
\hline 1972 & 27-Apr & -- & 46.5 & 1978 & 5-Jul & -- & 220 \\
\hline 1973 & 20-Jul & -- & 111 & 1979 & 6-Aug & -- & 83.0 \\
\hline 1974 & 28-Mar & -- & 26.9 & 1980 & 19-Apr & -- & 81.0 \\
\hline 1975 & 3-Apr & -- & 57.6 & & & & \\
\hline \multicolumn{8}{|c|}{ Annual peak discharge, from highest to lowest } \\
\hline 1978 & 5-Jul & -- & 220 & 1972 & 27-Apr & -- & 46.5 \\
\hline 1976 & 23-Jul & -- & 216 & 1977 & 30-Jun & -- & 46.1 \\
\hline 1973 & 20-Jul & -- & 111 & 1974 & 28-Mar & -- & 26.9 \\
\hline 1979 & 6-Aug & -- & 83.0 & 1970 & 15-Apr & -- & 23.0 \\
\hline 1980 & 19-Apr & -- & 81.0 & 1971 & 15-Apr & -- & 7.98 \\
\hline 1975 & 3-Apr & -- & 57.6 & & & & \\
\hline
\end{tabular}




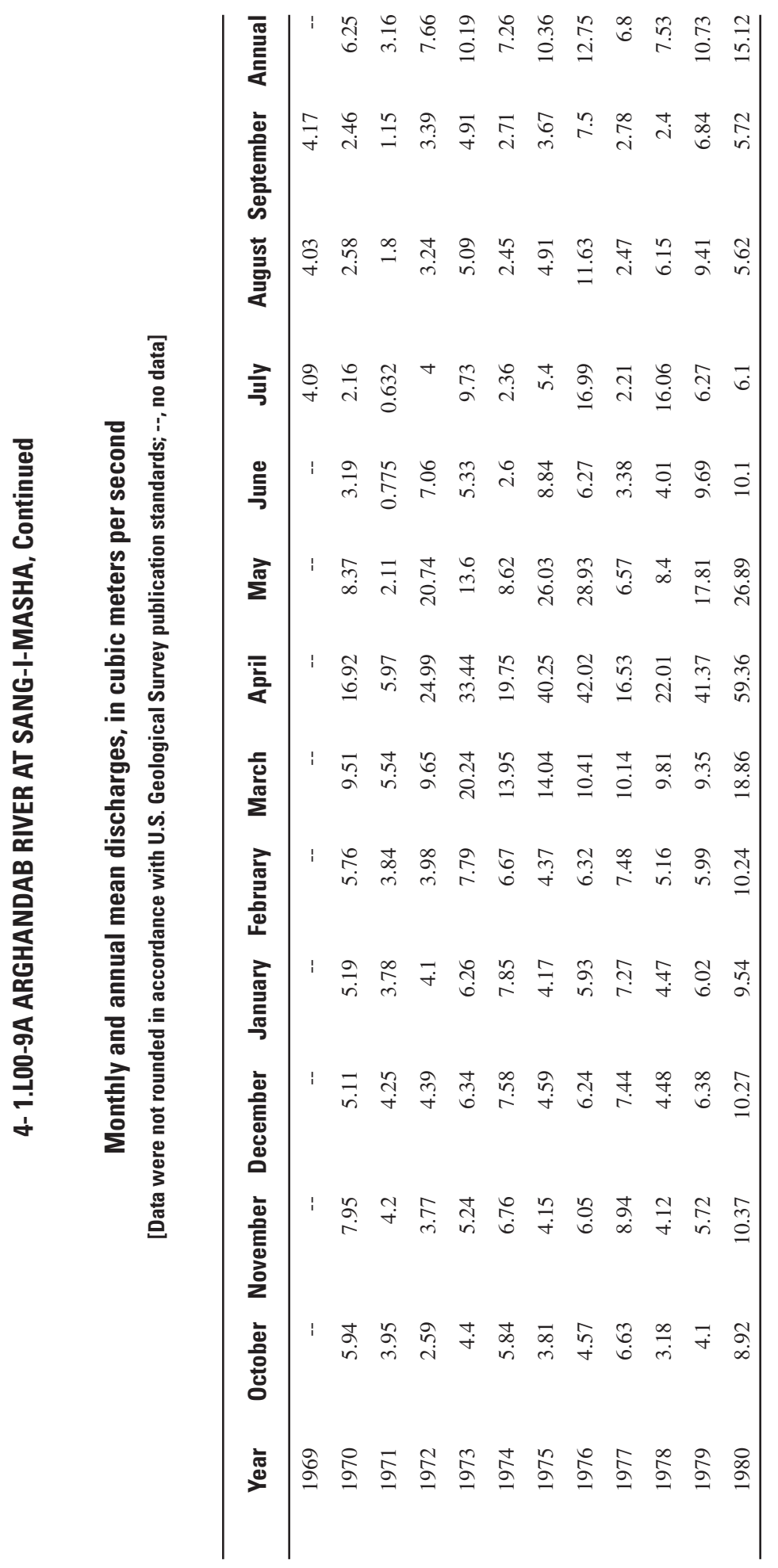




\section{4-13.R00-4A PUNJAB RIVER AT WARAS}

\section{(U.S. Geological Survey identification number: 341400066550000 )}

LOCATION: Lat $34^{\circ} 14^{\prime} \mathrm{N}$., long $66^{\circ} 55^{\prime} \mathrm{E}$.

DRAINAGE AREA: $1,710 \mathrm{~km}^{2}$.

ALTITUDE: 2,480 meters plus mean sea level.

PERIOD OF RECORD: October 1, 1969 to January 20, 1979.

GAGE: --

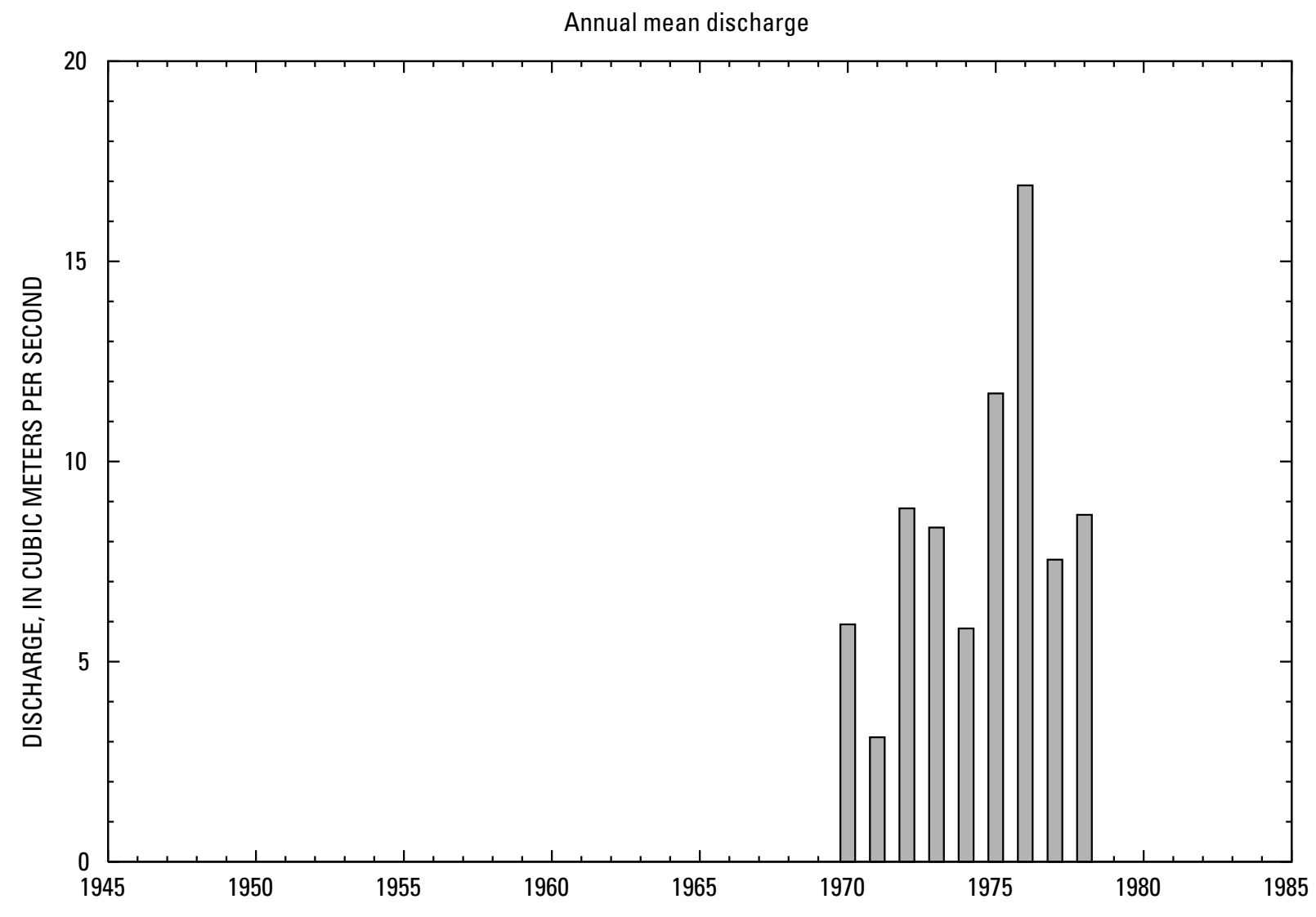




\section{4- 13.RO0-4A PUNJAB RIVER AT WARAS, Continued}

\section{Statistics of monthly and annual mean discharges}

$\left[\mathrm{m}^{3} / \mathrm{s}\right.$, meters per second]

\begin{tabular}{|c|c|c|c|c|c|c|c|c|}
\hline \multirow[b]{2}{*}{ Month } & \multicolumn{2}{|c|}{ Maximum } & \multicolumn{2}{|c|}{ Minimum } & \multicolumn{4}{|c|}{ Mean } \\
\hline & $\begin{array}{c}\text { Discharge } \\
\left(\mathrm{m}^{3} / \mathrm{s}\right)\end{array}$ & $\begin{array}{c}\text { Water year } \\
\text { of } \\
\text { occurrence }\end{array}$ & $\begin{array}{c}\text { Discharge } \\
\left(\mathrm{m}^{3} / \mathrm{s}\right)\end{array}$ & $\begin{array}{c}\text { Water year } \\
\text { of } \\
\text { occurrence }\end{array}$ & $\begin{array}{c}\text { Discharge } \\
\left(\mathrm{m}^{3} / \mathrm{s}\right)\end{array}$ & $\begin{array}{c}\text { Standard } \\
\text { deviation } \\
\left(\mathrm{m}^{3} / \mathrm{s}\right)\end{array}$ & $\begin{array}{c}\text { Coefficient } \\
\text { of } \\
\text { variation }\end{array}$ & $\begin{array}{c}\text { Percentage } \\
\text { of annual } \\
\text { discharge }\end{array}$ \\
\hline October & 5.20 & 1977 & 1.65 & 1972 & 3.26 & 1.11 & 0.34 & 3.18 \\
\hline November & 5.60 & 1970 & 1.85 & 1972 & 3.21 & 1.25 & 0.39 & 3.13 \\
\hline December & 4.19 & 1977 & 1.56 & 1973 & 2.97 & 0.92 & 0.31 & 2.89 \\
\hline January & 6.25 & 1977 & 1.33 & 1973 & 3.38 & 1.34 & 0.40 & 3.30 \\
\hline February & 7.95 & 1977 & 1.78 & 1973 & 4.17 & 1.80 & 0.43 & 4.07 \\
\hline March & 10.7 & 1977 & 3.24 & 1972 & 5.76 & 2.63 & 0.46 & 5.61 \\
\hline April & 53.9 & 1976 & 7.73 & 1971 & 30.3 & 13.5 & 0.45 & 29.6 \\
\hline May & 93.6 & 1976 & 3.45 & 1971 & 33.9 & 27.6 & 0.81 & 33.0 \\
\hline June & 20.5 & 1976 & 0.997 & 1971 & 7.47 & 6.27 & 0.84 & 7.28 \\
\hline July & 5.03 & 1976 & 0.753 & 1971 & 2.73 & 1.43 & 0.52 & 2.66 \\
\hline August & 4.84 & 1978 & 1.06 & 1971 & 2.54 & 1.13 & 0.45 & 2.47 \\
\hline September & 4.50 & 1976 & 1.44 & 1971 & 2.88 & 0.88 & 0.30 & 2.81 \\
\hline Annual & 16.9 & 1976 & 3.11 & 1971 & 8.54 & 3.95 & 0.46 & 100 \\
\hline
\end{tabular}

Annual flow duration

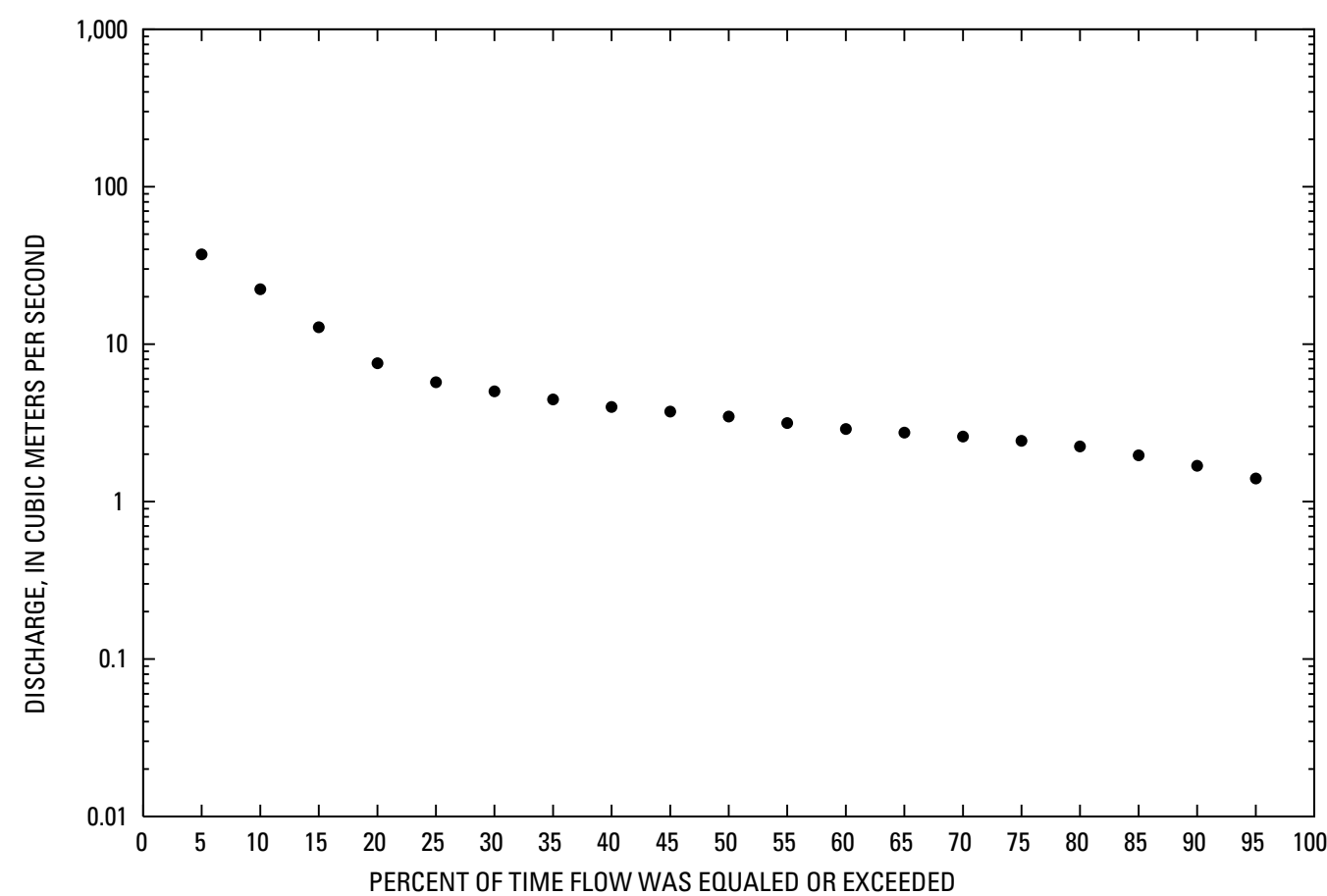




\section{4- 13.R00-4A PUNJAB RIVER AT WARAS, Continued}

Monthly and annual flow duration, in cubic meters per second

[Data were not rounded in accordance with U.S. Geological Survey publication standards]

\begin{tabular}{|c|c|c|c|c|c|c|c|c|c|c|c|c|c|}
\hline $\begin{array}{l}\text { Percentage } \\
\text { of days } \\
\text { discharge } \\
\text { equaled or } \\
\text { exceeded }\end{array}$ & January & February & March & April & May & June & July & August & September & October & November & December & Annual \\
\hline 95 & 1.24 & 1.5 & 2.52 & 7.02 & 2.86 & 0.94 & 0.77 & 0.97 & 1.49 & 1.64 & 1.8 & 1.52 & 1.4 \\
\hline 90 & 1.73 & 2.29 & 2.66 & 7.48 & 5.7 & 1.34 & 0.81 & 1.29 & 1.57 & 1.85 & 1.9 & 1.77 & 1.69 \\
\hline 80 & 2.27 & 2.48 & 2.88 & 12.7 & 12.1 & 2.3 & 1.59 & 1.57 & 2.31 & 2.54 & 2.27 & 1.96 & 2.24 \\
\hline 75 & 2.34 & 2.64 & 3.05 & 16.2 & 13.8 & 2.67 & 1.66 & 1.67 & 2.47 & 2.59 & 2.37 & 2.03 & 2.43 \\
\hline 70 & 2.48 & 2.91 & 3.37 & 18.6 & 15.9 & 3.13 & 1.72 & 1.8 & 2.52 & 2.63 & 2.44 & 2.22 & 2.59 \\
\hline 65 & 2.74 & 3.07 & 3.46 & 20.3 & 17.8 & 3.54 & 1.77 & 1.92 & 2.57 & 2.66 & 2.51 & 2.37 & 2.74 \\
\hline 60 & 2.87 & 3.22 & 3.59 & 23 & 19.5 & 3.88 & 1.84 & 2.02 & 2.63 & 2.69 & 2.57 & 2.84 & 2.89 \\
\hline 45 & 3.46 & 4.3 & 4.53 & 27.1 & 28.3 & 5.04 & 2.83 & 2.36 & 2.81 & 3.09 & 3.05 & 3.07 & 3.73 \\
\hline 40 & 3.55 & 4.46 & 5.08 & 28 & 32.5 & 5.8 & 3.01 & 2.54 & 2.84 & 3.35 & 3.26 & 3.28 & 3.99 \\
\hline 35 & 3.67 & 4.7 & 6.02 & 29.4 & 37.2 & 6.51 & 3.32 & 2.65 & 2.88 & 3.45 & 3.55 & 3.36 & 4.45 \\
\hline 30 & 3.8 & 4.85 & 6.62 & 32 & 42.9 & 7.41 & 3.5 & 2.73 & 3.27 & 3.5 & 3.63 & 3.45 & 5.01 \\
\hline 25 & 3.93 & 4.98 & 7.58 & 36.6 & 49 & 8.69 & 3.62 & 2.8 & 3.52 & 3.56 & 3.72 & 3.73 & 5.74 \\
\hline 20 & 4.06 & 5.25 & 8.69 & 42.2 & 54.6 & 10.1 & 4.01 & 2.87 & 3.62 & 3.73 & 3.99 & 3.91 & 7.57 \\
\hline 15 & 4.25 & 5.85 & 9.83 & 49.4 & 59.1 & 12.4 & 4.6 & 3.05 & 3.75 & 4.87 & 4.52 & 4.05 & 12.8 \\
\hline 10 & 4.77 & 6.98 & 11.7 & 58.4 & 71.6 & 17.7 & 4.82 & 3.34 & 4.17 & 4.98 & 4.94 & 4.21 & 22.3 \\
\hline 5 & 5.81 & 7.96 & 13.4 & 64.5 & 106.1 & 24.7 & 5.47 & 3.56 & 4.51 & 5.34 & 5.7 & 4.44 & 37.2 \\
\hline
\end{tabular}




\section{4- 13.RO0-4A PUNJAB RIVER AT WARAS, Continued}

\section{Probability of occurrence of annual high discharges}

[ $\mathrm{m}^{3} / \mathrm{s}$, meters per second; $\mathbf{n g}$, statistic not given]

\begin{tabular}{|c|c|c|c|c|c|c|}
\hline \multirow{2}{*}{$\begin{array}{c}\text { Exceedance } \\
\text { probability }\end{array}$} & \multirow{2}{*}{$\begin{array}{c}\text { Recurrence } \\
\text { interval } \\
\text { (years) }\end{array}$} & \multirow{2}{*}{$\begin{array}{c}\text { Maximum } \\
\text { instantaneous } \\
\left(\mathrm{m}^{3} / \mathrm{s}\right)\end{array}$} & \multicolumn{4}{|c|}{ Maximum mean discharge } \\
\hline & & & 3-day period & 7-day period & 15-day period & 30-day period \\
\hline 0.99 & 1.01 & ng & 5.24 & 4.80 & 4.33 & 4.23 \\
\hline 0.95 & 1.05 & $\mathrm{ng}$ & 11.2 & 10.5 & 9.58 & 8.94 \\
\hline 0.90 & 1.11 & ng & 16.1 & 15.3 & 14.0 & 12.8 \\
\hline 0.80 & 1.25 & 38.8 & 24.4 & 23.2 & 21.4 & 19.3 \\
\hline 0.50 & 2 & 66.7 & 49.0 & 46.4 & 42.7 & 38.0 \\
\hline 0.20 & 5 & 108 & 87.5 & 81.5 & 74.2 & 66.5 \\
\hline 0.10 & 10 & 137 & 113 & 104 & 94.0 & 85.1 \\
\hline 0.04 & 25 & 173 & 145 & 131 & 117 & 107 \\
\hline 0.02 & 50 & 199 & 167 & 149 & 132 & 123 \\
\hline 0.01 & 100 & 224 & 188 & 165 & 145 & 137 \\
\hline 0.005 & 200 & 250 & 208 & 180 & 158 & 150 \\
\hline 0.002 & 500 & 283 & $\mathrm{ng}$ & ng & ng & $\mathrm{ng}$ \\
\hline
\end{tabular}

'Less than 10 years of data used.

\section{4- 13.RO0-4A PUNJAB RIVER AT WARAS, Continued}

\section{Probability of occurrence of annual low discharges}

$\left[\mathrm{m}^{3} / \mathrm{s}\right.$, meters per second]

\begin{tabular}{|c|c|c|c|c|c|c|c|c|c|c|}
\hline \multirow{3}{*}{$\begin{array}{c}\text { Nonexceedance } \\
\text { probability }\end{array}$} & \multirow{3}{*}{$\begin{array}{c}\text { Recurrence } \\
\text { interval } \\
\text { (years) }\end{array}$} & \multicolumn{9}{|c|}{ Minimum mean discharge $\left(\mathrm{m}^{3} / \mathrm{s}\right)$} \\
\hline & & \multicolumn{9}{|c|}{ Number of consecutive days } \\
\hline & & 1 & 3 & 7 & 14 & 30 & 60 & 90 & 120 & 183 \\
\hline 0.05 & 20 & 0.725 & 0.725 & 0.719 & 0.730 & 0.745 & 0.822 & 0.943 & 1.08 & 1.33 \\
\hline 0.10 & 10 & 0.848 & 0.856 & 0.862 & 0.880 & 0.912 & 0.996 & 1.13 & 1.27 & 1.53 \\
\hline 0.20 & 5 & 1.03 & 1.05 & 1.07 & 1.10 & 1.16 & 1.25 & 1.40 & 1.55 & 1.80 \\
\hline 0.50 & 2 & 1.50 & 1.54 & 1.60 & 1.67 & 1.77 & 1.90 & 2.08 & 2.26 & 2.45 \\
\hline
\end{tabular}




\section{4- 13.RO0-4A PUNJAB RIVER AT WARAS, Continued}

\section{Probability of occurrence of seasonal low discharges}

$\left[\mathrm{m}^{3} / \mathrm{s}\right.$, meters per second]

\begin{tabular}{|c|c|c|c|c|c|c|c|c|c|}
\hline \multirow{3}{*}{$\begin{array}{c}\text { Nonexceedance } \\
\text { probability }\end{array}$} & \multirow{3}{*}{$\begin{array}{c}\text { Recurrence } \\
\text { interval } \\
\text { (years) }\end{array}$} & \multicolumn{8}{|c|}{ Minimum mean discharge $\left(\mathrm{m}^{3} / \mathrm{s}\right)$} \\
\hline & & \multicolumn{8}{|c|}{ Number of consecutive days } \\
\hline & & 1 & 7 & 14 & 30 & 1 & 7 & 14 & 30 \\
\hline & & \multicolumn{4}{|c|}{ December-January-February } & \multicolumn{4}{|c|}{ March-April-May } \\
\hline 0.05 & 20 & 1.15 & 1.24 & 1.24 & 1.28 & 1.46 & 1.68 & 1.92 & 2.69 \\
\hline 0.10 & 10 & 1.34 & 1.45 & 1.47 & 1.55 & 1.65 & 1.86 & 2.07 & 2.96 \\
\hline 0.20 & 5 & 1.60 & 1.73 & 1.79 & 1.93 & 1.94 & 2.14 & 2.32 & 3.39 \\
\hline \multirow[t]{2}{*}{0.50} & 2 & 2.16 & 2.35 & 2.48 & 2.76 & 2.87 & 3.06 & 3.20 & 4.66 \\
\hline & & \multicolumn{4}{|c|}{ June-July-August } & \multicolumn{4}{|c|}{ September-October-November } \\
\hline 0.05 & 20 & 0.758 & 0.766 & 0.783 & 0.807 & 1.31 & 1.34 & 1.40 & 1.52 \\
\hline 0.10 & 10 & 0.937 & 0.963 & 1.01 & 1.05 & 1.44 & 1.49 & 1.56 & 1.70 \\
\hline 0.20 & 5 & 1.19 & 1.24 & 1.33 & 1.40 & 1.62 & 1.70 & 1.78 & 1.93 \\
\hline 0.50 & 2 & 1.80 & 1.89 & 2.06 & 2.18 & 2.12 & 2.23 & 2.33 & 2.51 \\
\hline
\end{tabular}

\section{4- 13.R00-4A PUNJAB RIVER AT WARAS, Continued}

\section{Annual peak discharge and corresponding gage height}

[ $\mathrm{m}^{3} / \mathrm{s}$, meters per second; --, no data]

\begin{tabular}{|c|c|c|c|c|c|c|c|}
\hline $\begin{array}{c}\text { Water } \\
\text { year }\end{array}$ & Date & $\begin{array}{c}\text { Gage } \\
\text { height } \\
\text { (meters) }\end{array}$ & $\begin{array}{c}\text { Peak } \\
\text { discharge } \\
\left(\mathrm{m}^{3} / \mathrm{s}\right)\end{array}$ & $\begin{array}{c}\text { Water } \\
\text { year }\end{array}$ & Date & $\begin{array}{c}\text { Gage } \\
\text { height } \\
\text { (meters) }\end{array}$ & $\begin{array}{c}\text { Peak } \\
\text { discharge } \\
\left(\mathrm{m}^{3} / \mathrm{s}\right)\end{array}$ \\
\hline \multicolumn{8}{|c|}{ Annual peak discharge, by year } \\
\hline 1970 & 26-Apr & - & 30.8 & 1975 & 15-May & & 138 \\
\hline 1971 & 13-Apr & - & 10.8 & 1976 & 24-Apr & & 144 \\
\hline 1972 & 11-May & - & 77.6 & 1977 & 1-May & & 69.9 \\
\hline 1973 & 25-Apr & - & 65.5 & 1978 & 17-Apr & & 83.2 \\
\hline 1974 & 12-May & - & 35.3 & & & & \\
\hline \multicolumn{8}{|c|}{ Annual peak discharge, from highest to lowest } \\
\hline 1976 & 24-Apr & - & 144 & 1973 & 25-Apr & & 65.5 \\
\hline 1975 & 15-May & - & 138 & 1974 & 12-Маy & & 35.3 \\
\hline 1978 & 17-Apr & - & 83.2 & 1970 & 26-Apr & & 30.8 \\
\hline 1972 & 11-May & - & 77.6 & 1971 & 13-Apr & & 10.8 \\
\hline 1977 & 1-May & - & 69.9 & & & & \\
\hline
\end{tabular}




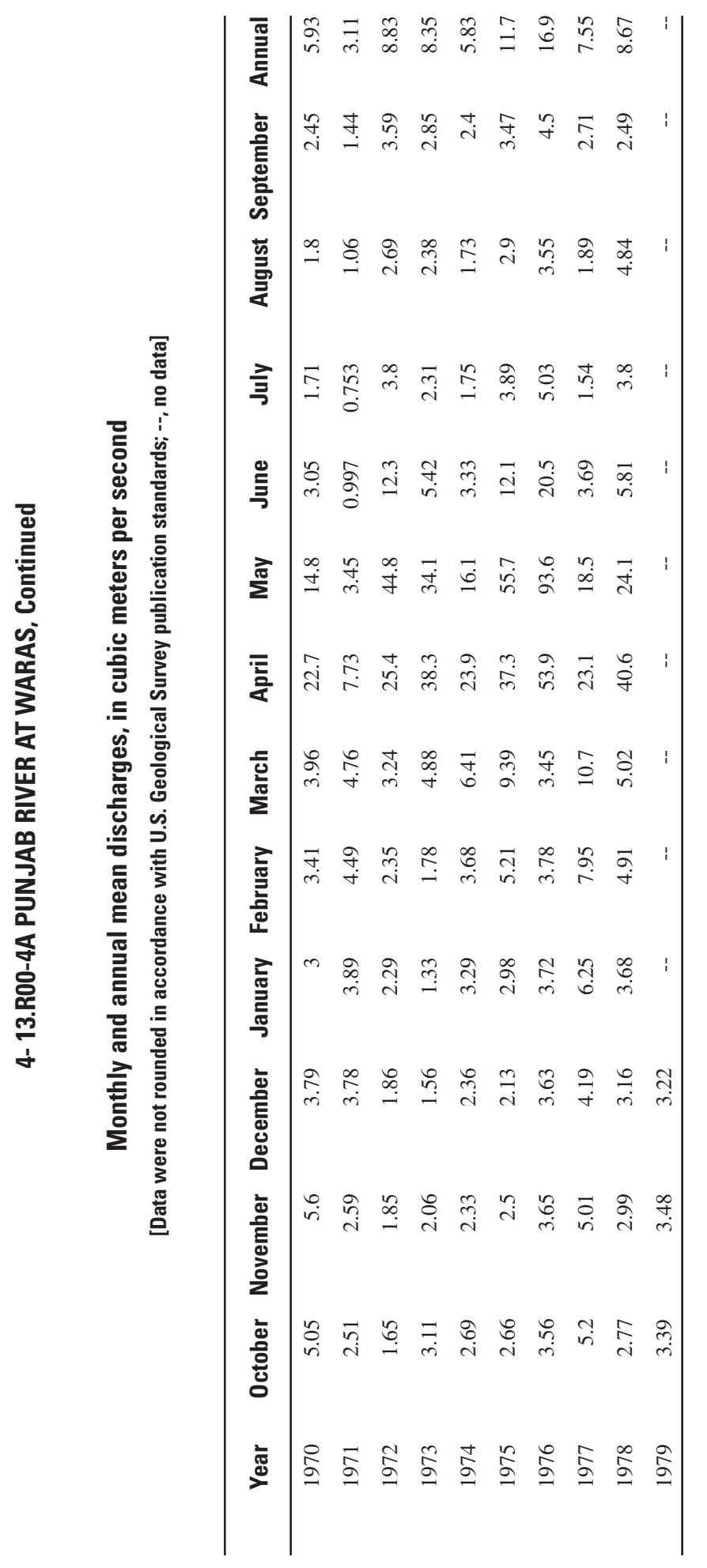




\section{4-14.RO0-1A MARKHANA RIVER AT DAHANE RISHOA}

\section{(U.S. Geological Survey identification number: 342100067290000 )}

LOCATION: Lat $34^{\circ} 21^{\prime} \mathrm{N}$., long $67^{\circ} 29^{\prime} \mathrm{E}$.

DRAINAGE AREA: $1,080 \mathrm{~km}^{2}$.

ALTITUDE: 2,580 meters plus mean sea level.

PERIOD OF RECORD: August 9, 1969 to September 30, 1978.

GAGE: --

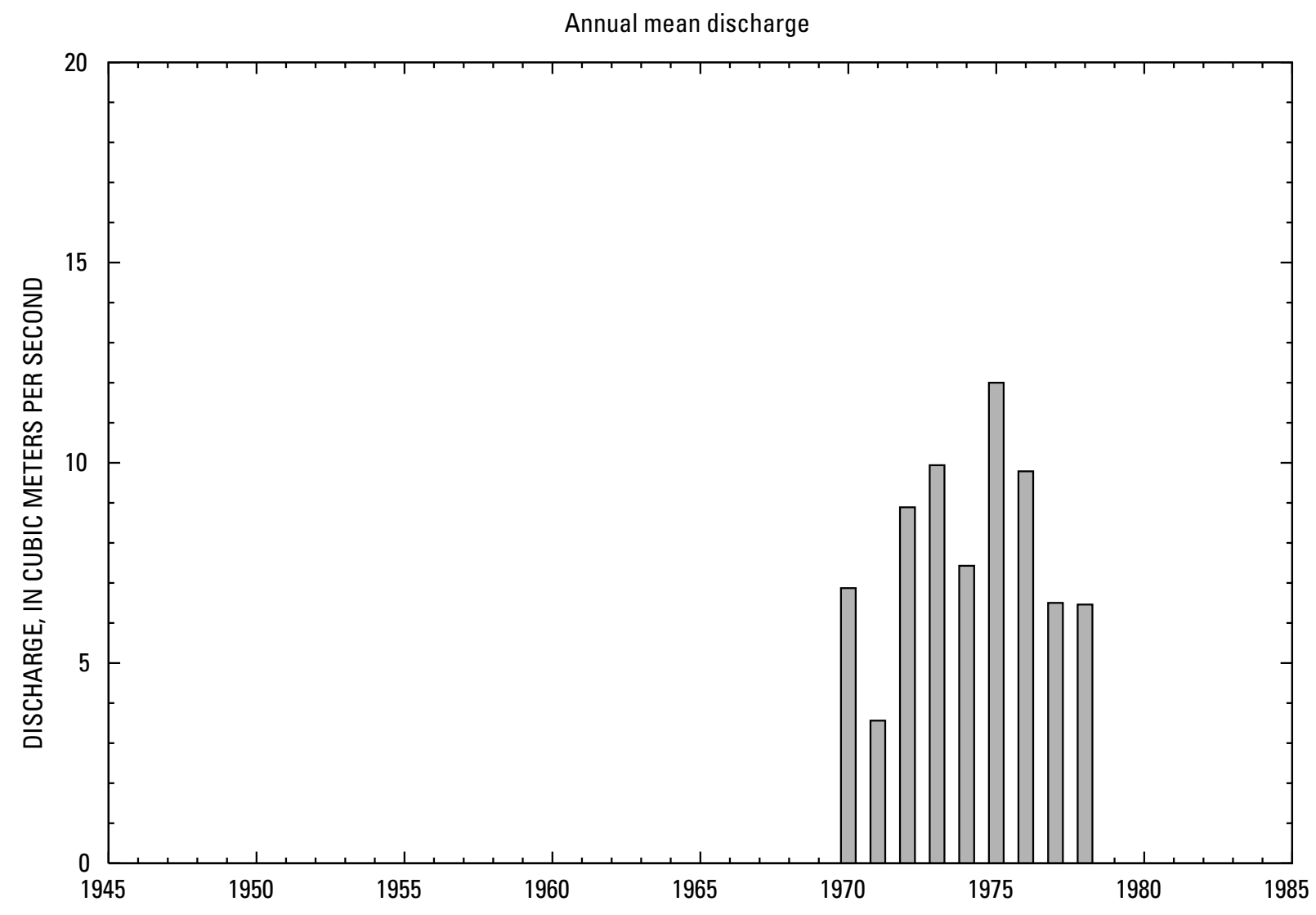




\section{4- 14.RO0-1A MARKHANA RIVER AT DAHANE RISHOA, Continued}

\section{Statistics of monthly and annual mean discharges}

$\left[\mathrm{m}^{3} / \mathrm{s}\right.$, meters per second]

\begin{tabular}{|c|c|c|c|c|c|c|c|c|}
\hline \multirow[b]{2}{*}{ Month } & \multicolumn{2}{|c|}{ Maximum } & \multicolumn{2}{|c|}{ Minimum } & \multicolumn{4}{|c|}{ Mean } \\
\hline & $\begin{array}{c}\text { Discharge } \\
\left(\mathrm{m}^{3} / \mathrm{s}\right)\end{array}$ & $\begin{array}{c}\text { Water year } \\
\text { of } \\
\text { occurrence }\end{array}$ & $\begin{array}{c}\text { Discharge } \\
\left(\mathrm{m}^{3} / \mathrm{s}\right)\end{array}$ & $\begin{array}{c}\text { Water year } \\
\text { of } \\
\text { occurrence }\end{array}$ & $\begin{array}{l}\text { Discharge } \\
\left(\mathrm{m}^{3} / \mathrm{s}\right)\end{array}$ & $\begin{array}{c}\text { Standard } \\
\text { deviation } \\
\left(\mathrm{m}^{3} / \mathrm{s}\right)\end{array}$ & $\begin{array}{c}\text { Coefficient } \\
\text { of } \\
\text { variation }\end{array}$ & $\begin{array}{c}\text { Percentage } \\
\text { of annual } \\
\text { discharge }\end{array}$ \\
\hline October & 5.13 & 1970 & 2.31 & 1972 & 3.47 & 0.85 & 0.24 & 3.64 \\
\hline November & 5.35 & 1970 & 2.19 & 1972 & 3.25 & 0.92 & 0.28 & 3.41 \\
\hline December & 4.64 & 1970 & 2.27 & 1972 & 3.04 & 0.73 & 0.24 & 3.19 \\
\hline January & 3.62 & 1970 & 2.38 & 1978 & 2.86 & 0.44 & 0.15 & 3.00 \\
\hline February & 5.56 & 1975 & 2.31 & 1978 & 3.19 & 1.01 & 0.32 & 3.35 \\
\hline March & 9.89 & 1974 & 2.55 & 1976 & 5.91 & 3.03 & 0.51 & 6.21 \\
\hline April & 27.6 & 1975 & 7.63 & 1971 & 18.8 & 6.08 & 0.32 & 19.7 \\
\hline May & 44.7 & 1976 & 7.83 & 1971 & 27.2 & 13.2 & 0.48 & 28.5 \\
\hline June & 25.2 & 1975 & 3.18 & 1971 & 13.6 & 6.65 & 0.49 & 14.3 \\
\hline July & 11.0 & 1975 & 2.24 & 1971 & 6.23 & 2.72 & 0.44 & 6.54 \\
\hline August & 5.39 & 1976 & 2.23 & 1971 & 3.88 & 1.12 & 0.29 & 4.08 \\
\hline September & 5.36 & 1969 & 2.58 & 1971 & 3.77 & 0.82 & 0.22 & 3.96 \\
\hline Annual & 12.0 & 1975 & 3.56 & 1971 & 7.94 & 2.49 & 0.31 & 100 \\
\hline
\end{tabular}

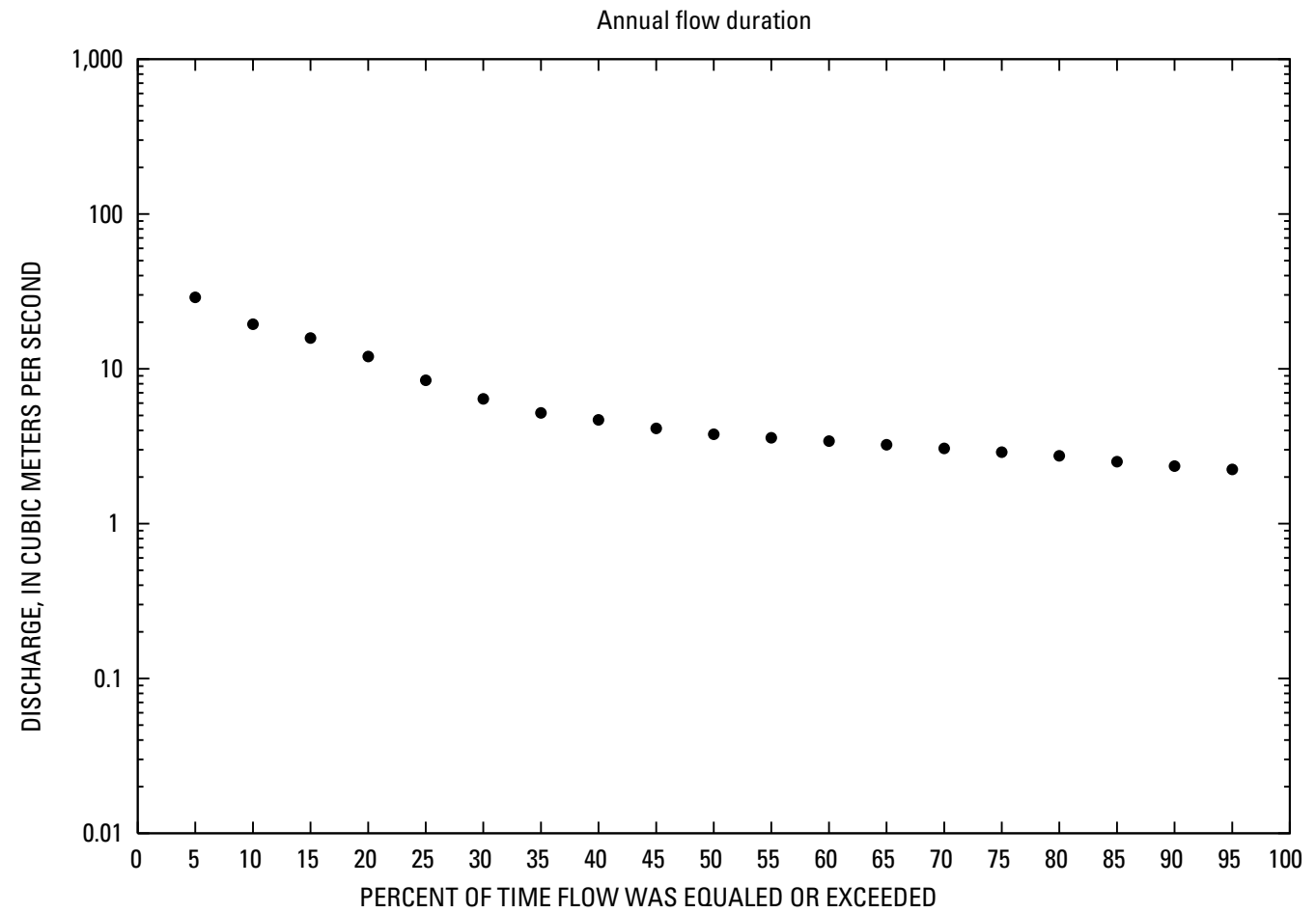




\section{4- 14.RO0-1A MARKHANA RIVER AT DAHANE RISHOA, Continued}

Monthly and annual flow duration, in cubic meters per second

[Data were not rounded in accordance with U.S. Geological Survey publication standards]

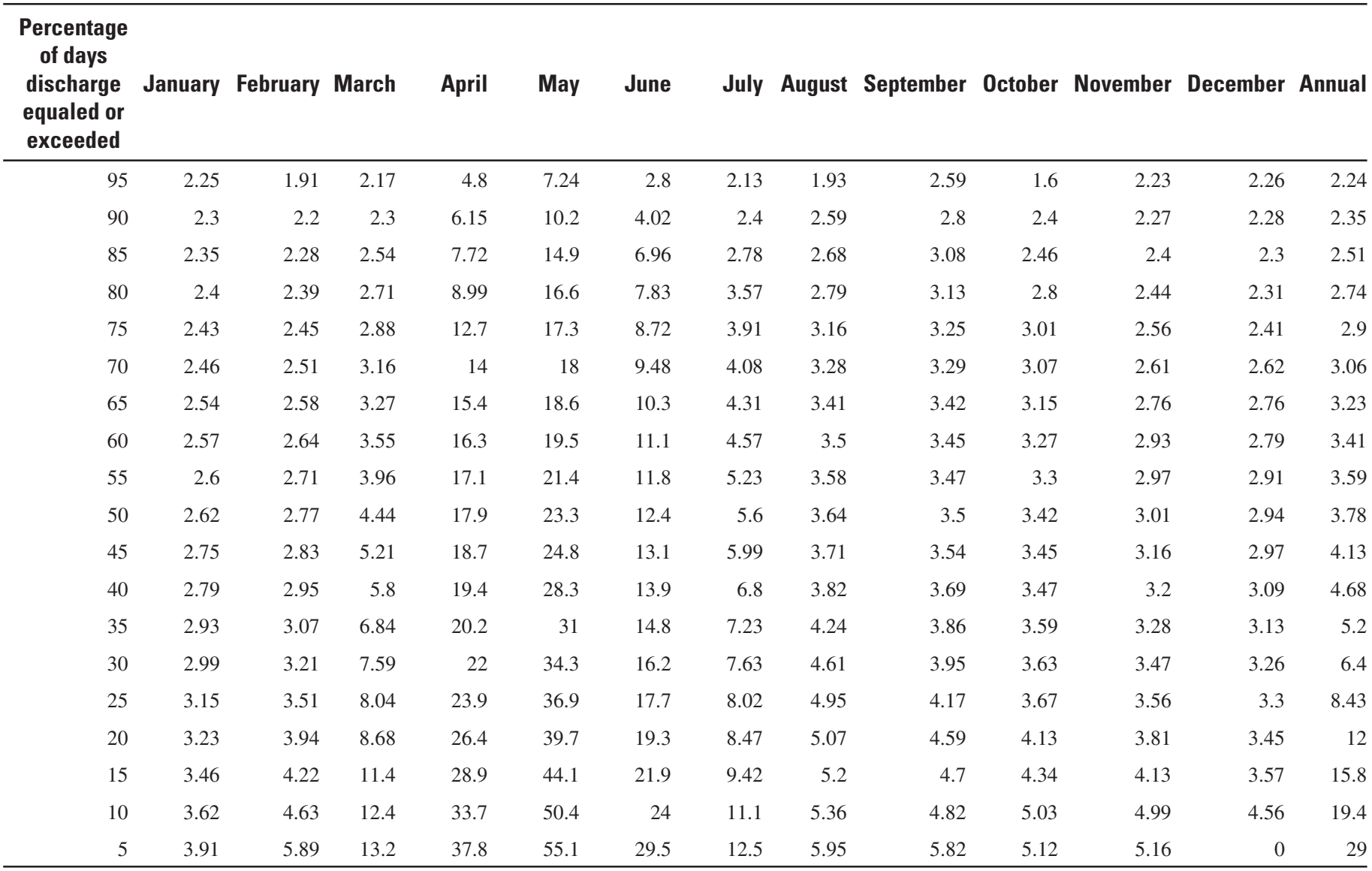


Probability of occurrence of annual high discharges

[m³/s, meters per second; $n g$, statistic not given]

\begin{tabular}{|c|c|c|c|c|c|c|}
\hline \multirow{2}{*}{$\begin{array}{c}\text { Exceedance } \\
\text { probability }\end{array}$} & \multirow{2}{*}{$\begin{array}{c}\text { Recurrence } \\
\text { interval } \\
\text { (years) }\end{array}$} & \multirow{2}{*}{$\begin{array}{c}\text { Maximum } \\
\text { instantaneous } \\
\left(\mathrm{m}^{3} / \mathrm{s}\right)\end{array}$} & \multicolumn{4}{|c|}{ Maximum mean discharge ${ }^{2}$} \\
\hline & & & 3-day period & 7-day period & 15-day period & 30-day period \\
\hline 0.99 & 1.01 & ng & 6.01 & 6.16 & 5.98 & 5.61 \\
\hline 0.95 & 1.05 & ng & 10.8 & 10.6 & 10.2 & 9.72 \\
\hline 0.90 & 1.11 & ng & 14.4 & 13.9 & 13.2 & 12.7 \\
\hline 0.80 & 1.25 & 31.5 & 19.8 & 18.8 & 17.8 & 17.0 \\
\hline 0.50 & 2 & 45.0 & 33.6 & 31.6 & 29.7 & 27.6 \\
\hline 0.20 & 5 & 60.1 & 51.8 & 49.2 & 46.0 & 40.9 \\
\hline 0.10 & 10 & 68.2 & 62.5 & 60.3 & 56.3 & 48.5 \\
\hline 0.04 & 25 & 76.7 & 74.6 & 73.4 & 68.5 & 56.7 \\
\hline 0.02 & 50 & 82.0 & 82.5 & 82.4 & 76.9 & 62.0 \\
\hline 0.01 & 100 & 86.5 & 89.6 & 90.8 & 84.8 & 66.6 \\
\hline 0.005 & 200 & 90.5 & 96.0 & 98.8 & 92.2 & 70.7 \\
\hline 0.002 & 500 & 95.0 & $\mathrm{ng}$ & ng & $\mathrm{ng}$ & ng \\
\hline
\end{tabular}

'Less than 10 years of data used.

${ }^{2}$ Data does not fit log-Pearson Type III curve, use with caution.

\section{4- 14.RO0-1A MARKHANA RIVER AT DAHANE RISHQA, Continued}

Probability of occurrence of annual low discharges

$\left[\mathrm{m}^{3} / \mathrm{s}\right.$, meters per second]

\begin{tabular}{|c|c|c|c|c|c|c|c|c|c|c|}
\hline \multirow{3}{*}{$\begin{array}{l}\text { Nonexceedance } \\
\text { probability }\end{array}$} & \multirow{3}{*}{$\begin{array}{c}\text { Recurrence } \\
\text { interval } \\
\text { (years) }\end{array}$} & \multicolumn{9}{|c|}{ Minimum mean discharge $\left(\mathrm{m}^{3} / \mathrm{s}\right)$} \\
\hline & & \multicolumn{9}{|c|}{ Number of consecutive days } \\
\hline & & 1 & 3 & 7 & 14 & 30 & 60 & 90 & 120 & 183 \\
\hline 0.05 & 20 & 1.40 & 1.50 & 1.58 & 1.76 & 1.91 & 2.12 & 2.15 & 2.18 & 2.23 \\
\hline 0.10 & 10 & 1.54 & 1.62 & 1.71 & 1.86 & 2.05 & 2.23 & 2.26 & 2.30 & 2.39 \\
\hline 0.20 & 5 & 1.71 & 1.77 & 1.87 & 1.99 & 2.22 & 2.36 & 2.40 & 2.45 & 2.58 \\
\hline 0.50 & 2 & 2.02 & 2.06 & 2.17 & 2.23 & 2.50 & 2.63 & 2.68 & 2.76 & 2.96 \\
\hline
\end{tabular}


Probability of occurrence of seasonal low discharges

$\left[\mathrm{m}^{3} / \mathrm{s}\right.$, meters per second]

\begin{tabular}{|c|c|c|c|c|c|c|c|c|c|}
\hline \multirow{3}{*}{$\begin{array}{l}\text { Nonexceedance } \\
\text { probability }\end{array}$} & \multirow{3}{*}{$\begin{array}{c}\text { Recurrence } \\
\text { interval } \\
\text { (years) }\end{array}$} & \multicolumn{8}{|c|}{ Minimum mean discharge $\left(\mathrm{m}^{3} / \mathrm{s}\right)$} \\
\hline & & \multicolumn{8}{|c|}{ Number of consecutive days } \\
\hline & & 1 & 7 & 14 & 30 & 1 & 7 & 14 & 30 \\
\hline & & \multicolumn{4}{|c|}{ December-January-February } & \multicolumn{4}{|c|}{ March-April-May } \\
\hline 0.05 & 20 & 1.45 & 1.67 & 1.89 & 2.21 & 1.92 & 1.93 & 1.84 & 1.96 \\
\hline 0.10 & 10 & 1.61 & 1.80 & 1.97 & 2.27 & 2.15 & 2.18 & 2.22 & 2.44 \\
\hline 0.20 & 5 & 1.82 & 1.98 & 2.09 & 2.36 & 2.48 & 2.54 & 2.77 & 3.18 \\
\hline \multirow[t]{2}{*}{0.50} & 2 & 2.19 & 2.33 & 2.37 & 2.58 & 3.30 & 3.50 & 4.15 & 5.16 \\
\hline & & \multicolumn{4}{|c|}{ June-July-August } & \multicolumn{4}{|c|}{ September-October-November } \\
\hline 0.05 & 20 & 1.72 & 1.74 & 1.83 & 1.94 & 1.95 & 2.07 & 2.15 & 2.21 \\
\hline 0.10 & 10 & 1.98 & 2.02 & 2.12 & 2.28 & 2.06 & 2.17 & 2.24 & 2.32 \\
\hline 0.20 & 5 & 2.34 & 2.40 & 2.53 & 2.72 & 2.22 & 2.32 & 2.37 & 2.49 \\
\hline 0.50 & 2 & 3.19 & 3.27 & 3.43 & 3.70 & 2.62 & 2.71 & 2.75 & 2.96 \\
\hline
\end{tabular}

4- 14.RO0-1A MARKHANA RIVER AT DAHANE RISHQA, Continued

Annual peak discharge and corresponding gage height

$\left[\mathrm{m}^{3} / \mathrm{s}\right.$, meters per second; --, no data]

\begin{tabular}{|c|c|c|c|c|c|c|c|}
\hline $\begin{array}{c}\text { Water } \\
\text { year }\end{array}$ & Date & $\begin{array}{c}\text { Gage } \\
\text { height } \\
\text { (meters) }\end{array}$ & $\begin{array}{c}\text { Peak } \\
\text { discharge } \\
\left(\mathrm{m}^{3} / \mathrm{s}\right)\end{array}$ & $\begin{array}{c}\text { Water } \\
\text { year }\end{array}$ & Date & $\begin{array}{c}\text { Gage } \\
\text { height } \\
\text { (meters) }\end{array}$ & $\begin{array}{c}\text { Peak } \\
\text { discharge } \\
\left(\mathrm{m}^{3} / \mathrm{s}\right)\end{array}$ \\
\hline
\end{tabular}

Annual peak discharge, by year

\begin{tabular}{|c|c|c|c|c|c|c|c|}
\hline 1970 & 26-Apr & -- & 24.5 & 1975 & 18-May & -- & 60.6 \\
\hline 1971 & 4-May & -- & 11.2 & 1976 & 18-May & -- & 72.0 \\
\hline 1972 & 20-May & -- & 57.0 & 1977 & 26-May & -- & 35.4 \\
\hline 1973 & 2-May & -- & 52.1 & 1978 & 6-May & -- & 47.2 \\
\hline 1974 & 10-May & -- & 36.5 & & & & \\
\hline \multicolumn{8}{|c|}{ Annual peak discharge, from highest to lowest } \\
\hline 1976 & 18-May & -- & 72.0 & 1974 & 10-May & -- & 36.5 \\
\hline 1975 & 18-May & -- & 60.6 & 1977 & 26-May & -- & 35.4 \\
\hline 1972 & 20-May & -- & 57.0 & 1970 & 26-Apr & -- & 24.5 \\
\hline 1973 & 2-May & -- & 52.1 & 1971 & 4-May & -- & 11.2 \\
\hline 1978 & 6-May & -- & 47.2 & & & & \\
\hline
\end{tabular}




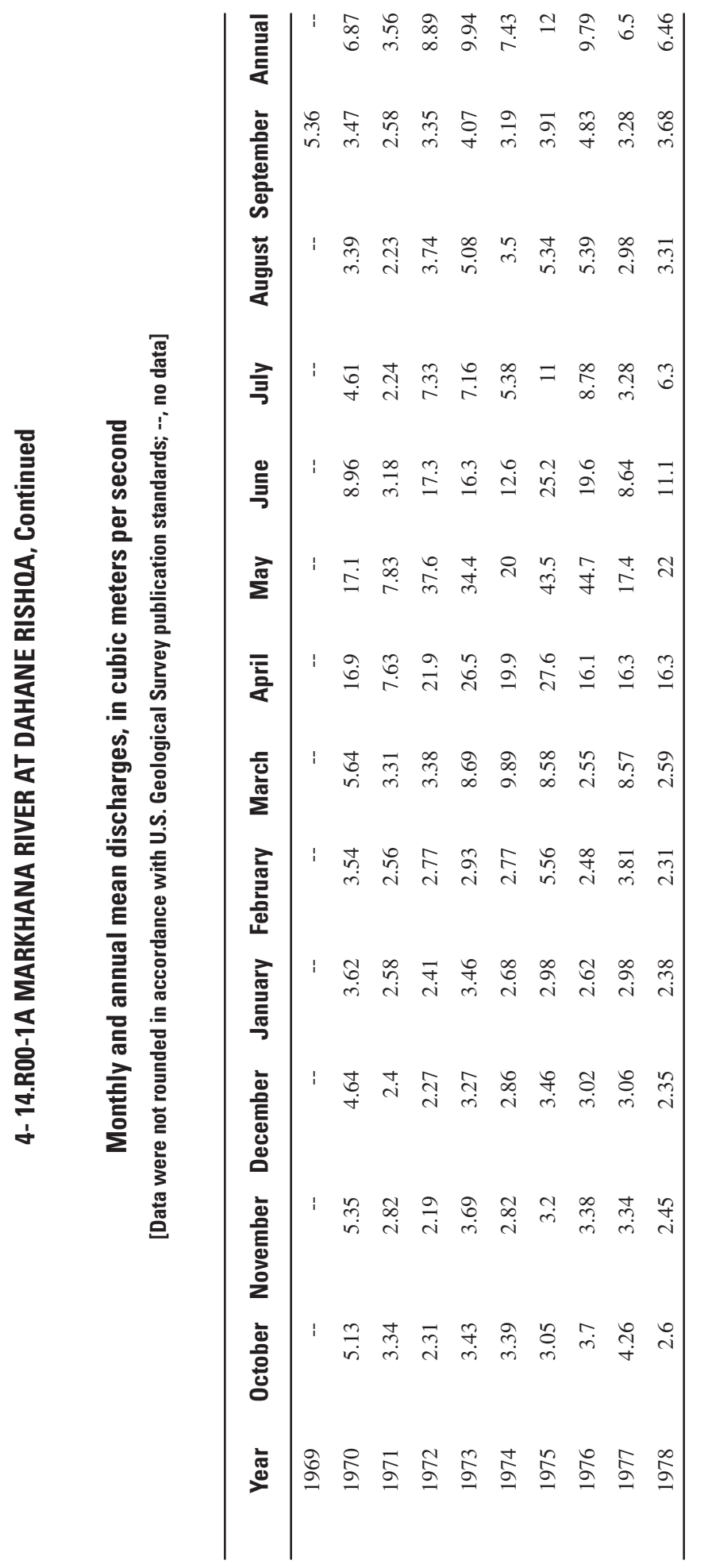




\section{4-16.R00-3A SYAHSANG RIVER NEAR GARDANDEWAL}

\section{(U.S. Geological Survey identification number: 343400068110000 )}

LOCATION: Lat $34^{\circ} 34^{\prime} N$., long $68^{\circ} 11^{\prime} \mathrm{E}$.

DRAINAGE AREA: $160 \mathrm{~km}^{2}$.

ALTITUDE: 3,010 meters plus mean sea level.

PERIOD OF RECORD: May 9, 1970 to September 30, 1980.

GAGE: --

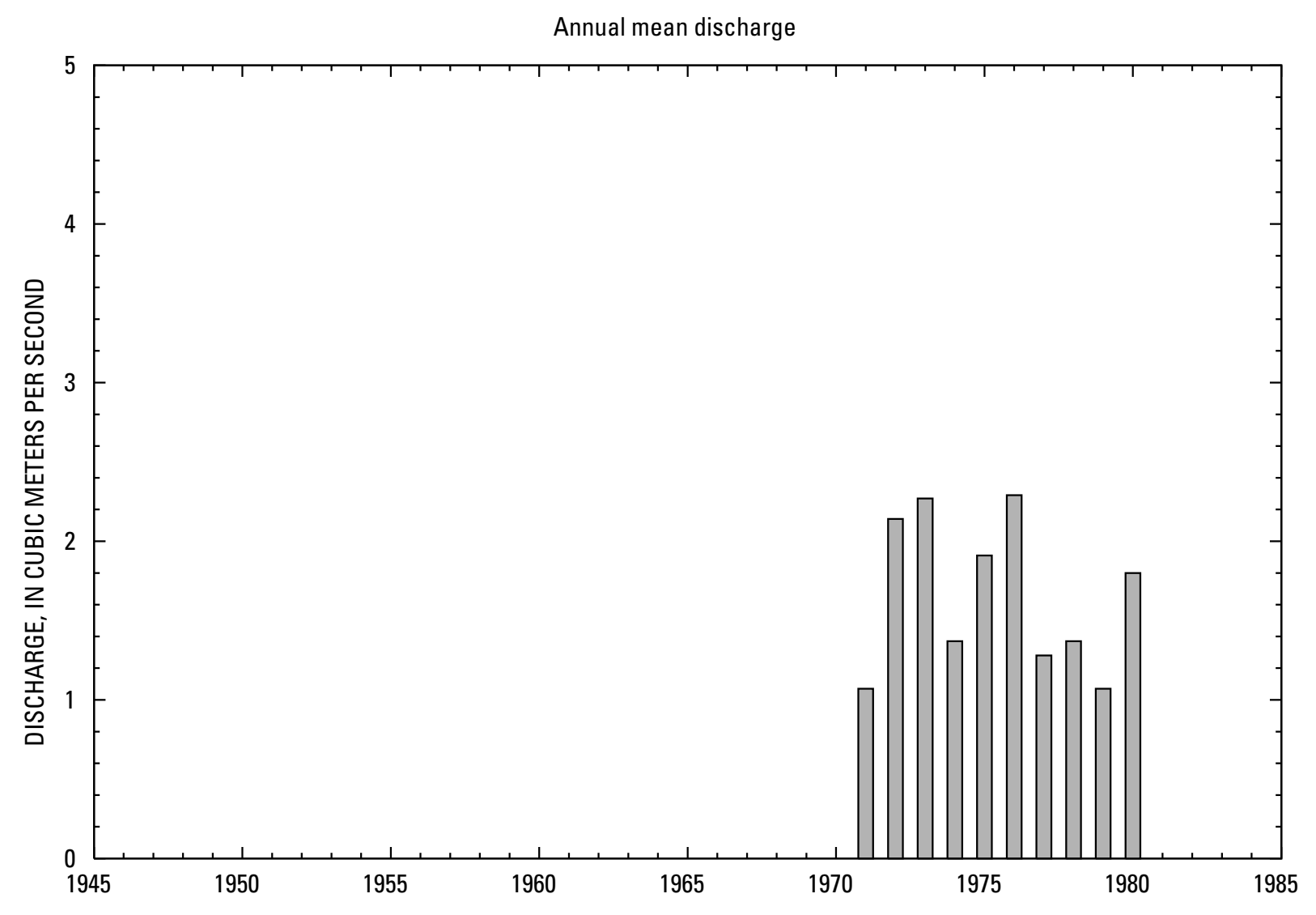




\section{4- 16.RO0-3A SYAHSANG RIVER NEAR GARDANDEWAL, Continued}

\section{Statistics of monthly and annual mean discharges}

[ $\mathrm{m}^{3} / \mathrm{s}$, meters per second]

\begin{tabular}{|c|c|c|c|c|c|c|c|c|}
\hline \multirow[b]{2}{*}{ Month } & \multicolumn{2}{|c|}{ Maximum } & \multicolumn{2}{|c|}{ Minimum } & \multicolumn{4}{|c|}{ Mean } \\
\hline & $\begin{array}{c}\text { Discharge } \\
\left(\mathrm{m}^{3} / \mathrm{s}\right)\end{array}$ & $\begin{array}{c}\text { Water year } \\
\text { of } \\
\text { occurrence }\end{array}$ & $\begin{array}{c}\text { Discharge } \\
\left(\mathrm{m}^{3} / \mathrm{s}\right)\end{array}$ & $\begin{array}{c}\text { Water year } \\
\text { of } \\
\text { occurrence }\end{array}$ & $\begin{array}{c}\text { Discharge } \\
\left(\mathrm{m}^{3} / \mathrm{s}\right)\end{array}$ & $\begin{array}{c}\text { Standard } \\
\text { deviation } \\
\left(\mathrm{m}^{3} / \mathrm{s}\right)\end{array}$ & $\begin{array}{c}\text { Coefficient } \\
\text { of } \\
\text { variation }\end{array}$ & $\begin{array}{c}\text { Percentage } \\
\text { of annual } \\
\text { discharge }\end{array}$ \\
\hline October & 1.12 & 1973 & 0.554 & 1972 & 0.75 & 0.18 & 0.24 & 3.81 \\
\hline November & 1.06 & 1977 & 0.513 & 1972 & 0.72 & 0.18 & 0.25 & 3.66 \\
\hline December & 0.916 & 1973 & 0.386 & 1972 & 0.64 & 0.16 & 0.25 & 3.27 \\
\hline January & 0.820 & 1973 & 0.451 & 1972 & 0.63 & 0.13 & 0.21 & 3.18 \\
\hline February & 0.820 & 1973 & 0.470 & 1972 & 0.63 & 0.11 & 0.18 & 3.22 \\
\hline March & 1.31 & 1977 & 0.588 & 1972 & 0.91 & 0.24 & 0.26 & 4.63 \\
\hline April & 6.79 & 1973 & 2.32 & 1980 & 3.97 & 1.37 & 0.34 & 20.2 \\
\hline May & 12.5 & 1976 & 2.80 & 1979 & 6.60 & 3.62 & 0.55 & 33.6 \\
\hline June & 4.89 & 1972 & 1.12 & 1971 & 2.46 & 1.43 & 0.58 & 12.5 \\
\hline July & 2.72 & 1980 & 0.181 & 1971 & 0.99 & 0.67 & 0.67 & 5.04 \\
\hline August & 0.925 & 1978 & 0.125 & 1971 & 0.62 & 0.24 & 0.39 & 3.15 \\
\hline September & 1.00 & 1972 & 0.486 & 1971 & 0.74 & 0.18 & 0.24 & 3.77 \\
\hline Annual & 2.29 & 1976 & 1.07 & 1971, 1979 & 1.66 & 0.48 & 0.29 & 100 \\
\hline
\end{tabular}

Annual flow duration

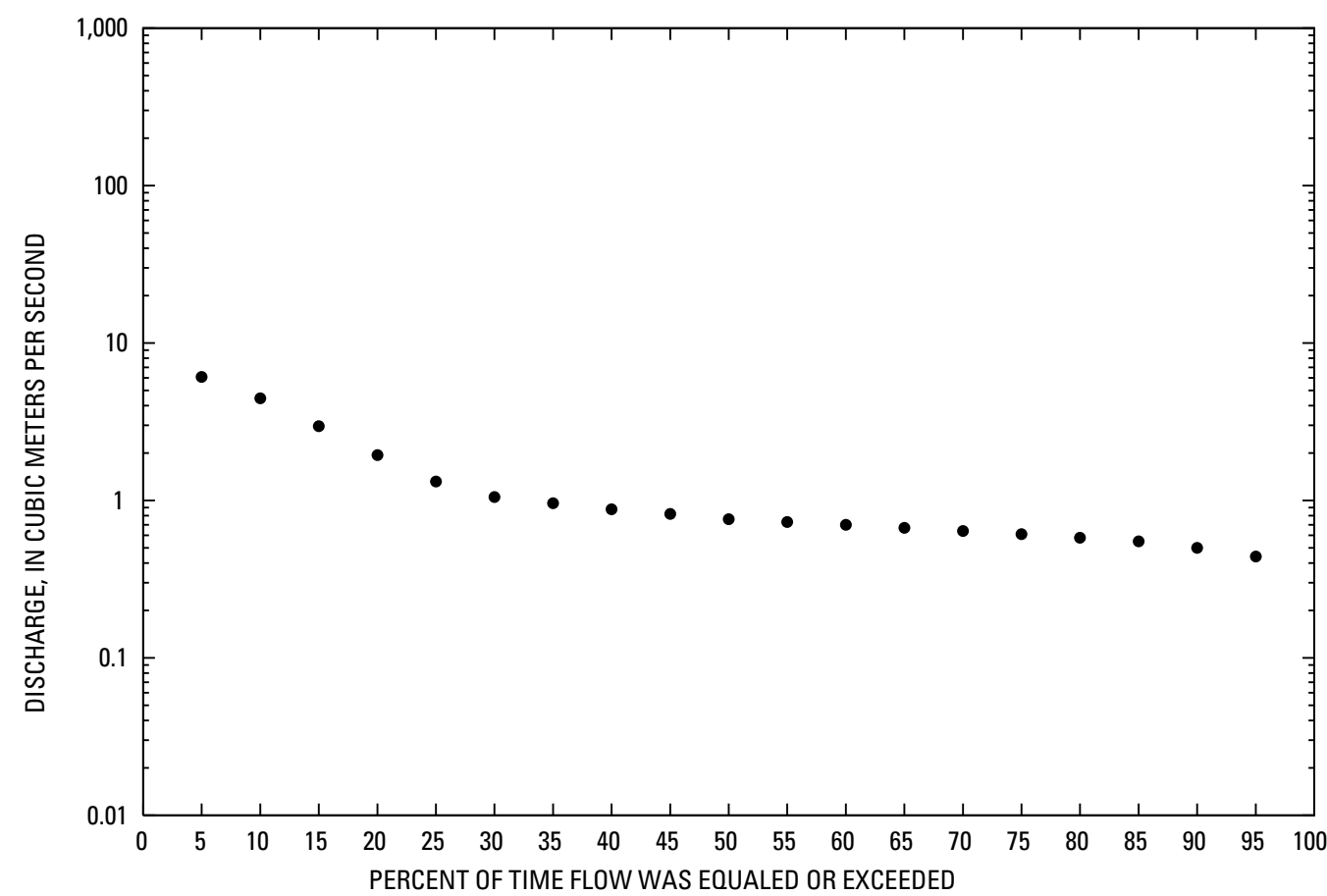




\section{4- 16.R00-3A SYAHSANG RIVER NEAR GARDANDEWAL, Continued}

\section{Monthly and annual flow duration, in cubic meters per second}

[Data were not rounded in accordance with U.S. Geological Survey publication standards]

\begin{tabular}{|c|c|c|c|c|c|c|c|c|c|c|c|c|c|}
\hline $\begin{array}{l}\text { Percentage } \\
\text { of days } \\
\text { discharge } \\
\text { equaled or } \\
\text { exceeded }\end{array}$ & January & February & March & April & May & June & July & August & September & October & November & December & Annual \\
\hline 95 & 0.43 & 0.47 & 0.52 & 1.19 & 2.1 & 0.53 & 0.18 & 0.14 & 0.5 & 0.56 & 0.5 & 0.34 & 0.44 \\
\hline 90 & 0.45 & 0.48 & 0.57 & 1.59 & 2.51 & 0.94 & 0.34 & 0.28 & 0.54 & 0.57 & 0.52 & 0.49 & 0.5 \\
\hline 85 & 0.47 & 0.5 & 0.6 & 1.86 & 2.86 & 1.02 & 0.43 & 0.36 & 0.56 & 0.59 & 0.57 & 0.5 & 0.55 \\
\hline 80 & 0.48 & 0.51 & 0.63 & 2.16 & 3.29 & 1.09 & 0.5 & 0.43 & 0.58 & 0.6 & 0.6 & 0.5 & 0.58 \\
\hline 75 & 0.5 & 0.55 & 0.67 & 2.36 & 3.55 & 1.16 & 0.53 & 0.48 & 0.59 & 0.61 & 0.61 & 0.51 & 0.61 \\
\hline 70 & 0.51 & 0.59 & 0.7 & 2.61 & 3.78 & 1.29 & 0.56 & 0.5 & 0.6 & 0.61 & 0.61 & 0.58 & 0.64 \\
\hline 65 & 0.57 & 0.6 & 0.72 & 2.75 & 4.14 & 1.39 & 0.71 & 0.52 & 0.64 & 0.66 & 0.61 & 0.59 & 0.67 \\
\hline 60 & 0.59 & 0.61 & 0.74 & 2.93 & 4.51 & 1.48 & 0.75 & 0.55 & 0.67 & 0.67 & 0.62 & 0.6 & 0.7 \\
\hline 55 & 0.6 & 0.62 & 0.76 & 3.16 & 4.77 & 1.58 & 0.79 & 0.58 & 0.69 & 0.67 & 0.67 & 0.61 & 0.73 \\
\hline 50 & 0.62 & 0.67 & 0.82 & 3.36 & 5.04 & 1.79 & 0.87 & 0.6 & 0.72 & 0.68 & 0.68 & 0.62 & 0.76 \\
\hline 45 & 0.68 & 0.67 & 0.84 & 3.59 & 5.47 & 1.96 & 0.92 & 0.68 & 0.76 & 0.71 & 0.68 & 0.62 & 0.82 \\
\hline 40 & 0.69 & 0.68 & 0.85 & 4.01 & 5.81 & 2.18 & 0.97 & 0.72 & 0.78 & 0.74 & 0.69 & 0.63 & 0.88 \\
\hline 35 & 0.71 & 0.7 & 0.91 & 4.36 & 7.22 & 2.64 & 1.04 & 0.73 & 0.81 & 0.75 & 0.74 & 0.68 & 0.96 \\
\hline 30 & 0.72 & 0.71 & 0.94 & 4.74 & 8.16 & 3.23 & 1.11 & 0.75 & 0.83 & 0.82 & 0.76 & 0.74 & 1.05 \\
\hline 25 & 0.72 & 0.71 & 1.03 & 5.25 & 8.78 & 3.8 & 1.17 & 0.78 & 0.9 & 0.83 & 0.83 & 0.81 & 1.32 \\
\hline 20 & 0 & 0.72 & 1.12 & 5.8 & 9.74 & 4.47 & 1.24 & 0.81 & 0.93 & 0.94 & 0.87 & 0.83 & 1.94 \\
\hline 15 & 0 & 0 & 1.27 & 6.34 & 11.3 & 4.72 & 1.34 & 0.85 & 0.95 & 1 & 0.93 & 0.84 & 2.96 \\
\hline 10 & 0 & 0 & 1.49 & 6.92 & 13.8 & 4.99 & 1.67 & 0.89 & 0.99 & 1.06 & 1.02 & 0.85 & 4.45 \\
\hline 5 & 0 & 0 & 1.73 & 8.41 & 15.5 & 5.48 & 2.31 & 0.98 & 1.06 & 0 & 1.06 & 0 & 6.09 \\
\hline
\end{tabular}




\section{4- 16.R00-3A SYAHSANG RIVER NEAR GARDANDEWAL, Continued}

Probability of occurrence of annual high discharges

[ $\mathrm{m}^{3} / \mathrm{s}$, meters per second; $\mathrm{ng}$, statistic not given]

\begin{tabular}{|c|c|c|c|c|c|c|}
\hline \multirow{2}{*}{$\begin{array}{c}\text { Exceedance } \\
\text { probability }\end{array}$} & \multirow{2}{*}{$\begin{array}{c}\text { Recurrence } \\
\text { interval } \\
\text { (years) }\end{array}$} & \multirow{2}{*}{$\begin{array}{c}\text { Maximum } \\
\text { instantaneous } \\
\left(\mathrm{m}^{3} / \mathrm{s}\right)\end{array}$} & \multicolumn{4}{|c|}{ Maximum mean discharge } \\
\hline & & & 3-day period & 7-day period & 15-day period & 30-day period \\
\hline 0.99 & 1.01 & 2.70 & 12.72 & 12.68 & 12.71 & 12.55 \\
\hline 0.95 & 1.05 & 4.20 & 3.74 & 3.64 & 3.56 & 3.30 \\
\hline 0.90 & 1.11 & 5.20 & 4.45 & 4.32 & 4.16 & 3.81 \\
\hline 0.80 & 1.25 & 6.70 & 5.54 & 5.35 & 5.05 & 4.57 \\
\hline 0.50 & 2 & 10.8 & 8.57 & 8.20 & 7.54 & 6.63 \\
\hline 0.20 & 5 & 16.7 & 13.6 & 12.9 & 11.7 & 9.93 \\
\hline 0.10 & 10 & 20.6 & 17.4 & 16.6 & 14.9 & 12.4 \\
\hline 0.04 & 25 & 25.6 & 23.0 & 21.8 & 19.5 & 15.9 \\
\hline 0.02 & 50 & 29.3 & 27.5 & 26.1 & 23.3 & 18.8 \\
\hline 0.01 & 100 & 33.0 & 32.5 & 30.9 & 27.6 & 21.9 \\
\hline 0.005 & 200 & 36.7 & 138.0 & 36.1 & 32.2 & 25.3 \\
\hline 0.002 & 500 & 41.5 & $\mathrm{ng}$ & ng & ng & ng \\
\hline
\end{tabular}

'Data does not fit log-Pearson Type III curve, use with caution.

\section{4- 16.R00-3A SYAHSANG RIVER NEAR GARDANDEWAL, Continued}

Probability of occurrence of annual low discharges

$\left[\mathrm{m}^{3} / \mathrm{s}\right.$, meters per second]

\begin{tabular}{|c|c|c|c|c|c|c|c|c|c|c|}
\hline \multirow{3}{*}{$\begin{array}{c}\text { Nonexceedance } \\
\text { probability }\end{array}$} & \multirow{3}{*}{$\begin{array}{c}\text { Recurrence } \\
\text { interval } \\
\text { (years) }\end{array}$} & \multicolumn{9}{|c|}{ Minimum mean discharge $\left(\mathrm{m}^{3} / \mathrm{s}\right)$} \\
\hline & & \multicolumn{9}{|c|}{ Number of consecutive days } \\
\hline & & 1 & 3 & 7 & 14 & 30 & 60 & 90 & 120 & 183 \\
\hline 0.05 & 20 & 0.129 & 0.129 & 0.131 & 0.132 & 0.153 & 0.186 & 0.252 & 0.322 & 0.384 \\
\hline 0.10 & 10 & 0.194 & 0.196 & 0.201 & 0.205 & 0.227 & 0.264 & 0.325 & 0.384 & 0.436 \\
\hline 0.20 & 5 & 0.291 & 0.298 & 0.306 & 0.315 & 0.336 & 0.375 & 0.423 & 0.464 & 0.504 \\
\hline 0.50 & 2 & 0.492 & 0.512 & 0.528 & 0.545 & 0.562 & 0.595 & 0.612 & 0.624 & 0.650 \\
\hline
\end{tabular}




\section{4- 16.RO0-3A SYAHSANG RIVER NEAR GARDANDEWAL, Continued}

\section{Probability of occurrence of seasonal low discharges}

$\left[\mathrm{m}^{3} / \mathrm{s}\right.$, meters per second]

\begin{tabular}{|c|c|c|c|c|c|c|c|c|c|}
\hline \multirow{3}{*}{$\begin{array}{c}\text { Nonexceedance } \\
\text { probability }\end{array}$} & \multirow{3}{*}{$\begin{array}{c}\text { Recurrence } \\
\text { interval } \\
\text { (years) }\end{array}$} & \multicolumn{8}{|c|}{ Minimum mean discharge $\left(\mathrm{m}^{3} / \mathrm{s}\right)$} \\
\hline & & \multicolumn{8}{|c|}{ Number of consecutive days } \\
\hline & & 1 & 7 & 14 & 30 & 1 & 7 & 14 & 30 \\
\hline & & \multicolumn{4}{|c|}{ December-January-February } & \multicolumn{4}{|c|}{ March-April-May } \\
\hline 0.05 & 20 & 0.341 & 0.341 & 0.345 & 0.377 & 0.477 & ${ }^{1} 0.476$ & 0.477 & 0.550 \\
\hline 0.10 & 10 & 0.389 & 0.389 & 0.393 & 0.420 & 0.515 & 0.520 & 0.524 & 0.611 \\
\hline 0.20 & 5 & 0.450 & 0.450 & 0.454 & 0.475 & 0.562 & 0.575 & 0.583 & 0.691 \\
\hline \multirow[t]{2}{*}{0.50} & 2 & 0.575 & 0.575 & 0.578 & 0.588 & 0.655 & 0.684 & 0.703 & 0.868 \\
\hline & & \multicolumn{4}{|c|}{ June-July-August } & \multicolumn{4}{|c|}{ September-October-November } \\
\hline 0.05 & 20 & 0.138 & 0.142 & 0.144 & 0.166 & 0.213 & 0.349 & 0.409 & 0.476 \\
\hline 0.10 & 10 & 0.203 & 0.214 & 0.220 & 0.246 & 0.275 & 0.401 & 0.451 & 0.507 \\
\hline 0.20 & 5 & 0.299 & 0.323 & 0.336 & 0.365 & 0.361 & 0.469 & 0.506 & 0.550 \\
\hline 0.50 & 2 & 0.511 & 0.557 & 0.588 & 0.623 & 0.547 & 0.611 & 0.629 & 0.659 \\
\hline
\end{tabular}

'Data does not fit log-Pearson Type III curve, use with caution.

\section{4- 16.R00-3A SYAHSANG RIVER NEAR GARDANDEWAL, Continued}

\section{Annual peak discharge and corresponding gage height}

$\left[\mathrm{m}^{3} / \mathrm{s}\right.$, meters per second; --, no data]

\begin{tabular}{|c|c|c|c|c|c|c|c|}
\hline $\begin{array}{c}\text { Water } \\
\text { year }\end{array}$ & Date & $\begin{array}{c}\text { Gage } \\
\text { height } \\
\text { (meters) }\end{array}$ & $\begin{array}{c}\text { Peak } \\
\text { discharge } \\
\left(\mathrm{m}^{3} / \mathrm{s}\right)\end{array}$ & $\begin{array}{c}\text { Water } \\
\text { year }\end{array}$ & Date & $\begin{array}{c}\text { Gage } \\
\text { height } \\
\text { (meters) }\end{array}$ & $\begin{array}{c}\text { Peak } \\
\text { discharge } \\
\left(\mathrm{m}^{3} / \mathrm{s}\right)\end{array}$ \\
\hline \multicolumn{8}{|c|}{ Annual peak discharge, by year } \\
\hline 1971 & 18-Apr & & 4.66 & 1976 & 14-May & & 21.5 \\
\hline 1972 & 20-May & & 16.7 & 1977 & $15-\mathrm{Nov}$ & & 14.6 \\
\hline 1973 & 5-May & & 17.4 & 1978 & 19-Apr & & 8.84 \\
\hline 1974 & 31-May & & 6.00 & 1979 & 18-Apr & & 5.65 \\
\hline 1975 & 22-May & & 9.00 & 1980 & 21-May & & 14.6 \\
\hline \multicolumn{8}{|c|}{ Annual peak discharge, from highest to lowest } \\
\hline 1976 & 14-May & & 21.5 & 1975 & 22-May & & 9.00 \\
\hline 1973 & 5-May & & 17.4 & 1978 & 19-Apr & & 8.84 \\
\hline 1972 & 20-May & & 16.7 & 1974 & 31-May & & 6.00 \\
\hline 1980 & 21-May & & 14.6 & 1979 & 18-Apr & & 5.65 \\
\hline 1977 & $15-\mathrm{Nov}$ & & 14.6 & 1971 & 18-Apr & & 4.66 \\
\hline
\end{tabular}




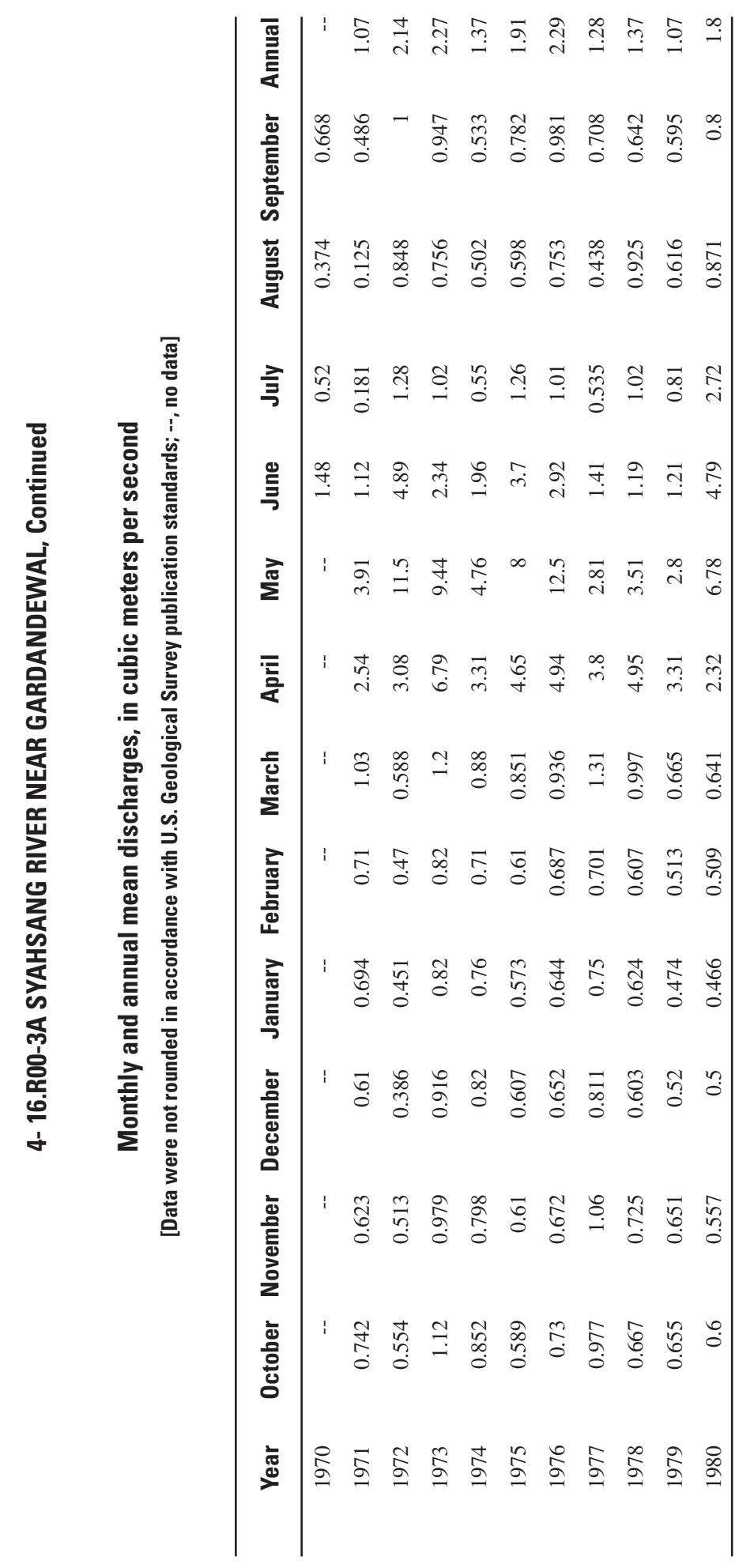




\section{4- 3.L00-2T SANGIN WASH (SERAJ CANAL) AT SANGIN}

\section{(U.S. Geological Survey identification number: 320300064500000 )}

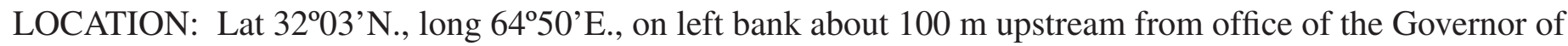
Sangin district in village of Sangin, about $500 \mathrm{~m}$ downstream from canal inlet and control gate structure on Helmand River, $35 \mathrm{~km}$ northeast of Girishk, and about $40 \mathrm{~km}$ downstream from Kajakai Dam.

DRAINAGE AREA: --

\section{ALTITUDE: --}

PERIOD OF RECORD: October 1, 1952 to September 30, 1960.

GAGE: Staff gage read twice daily.

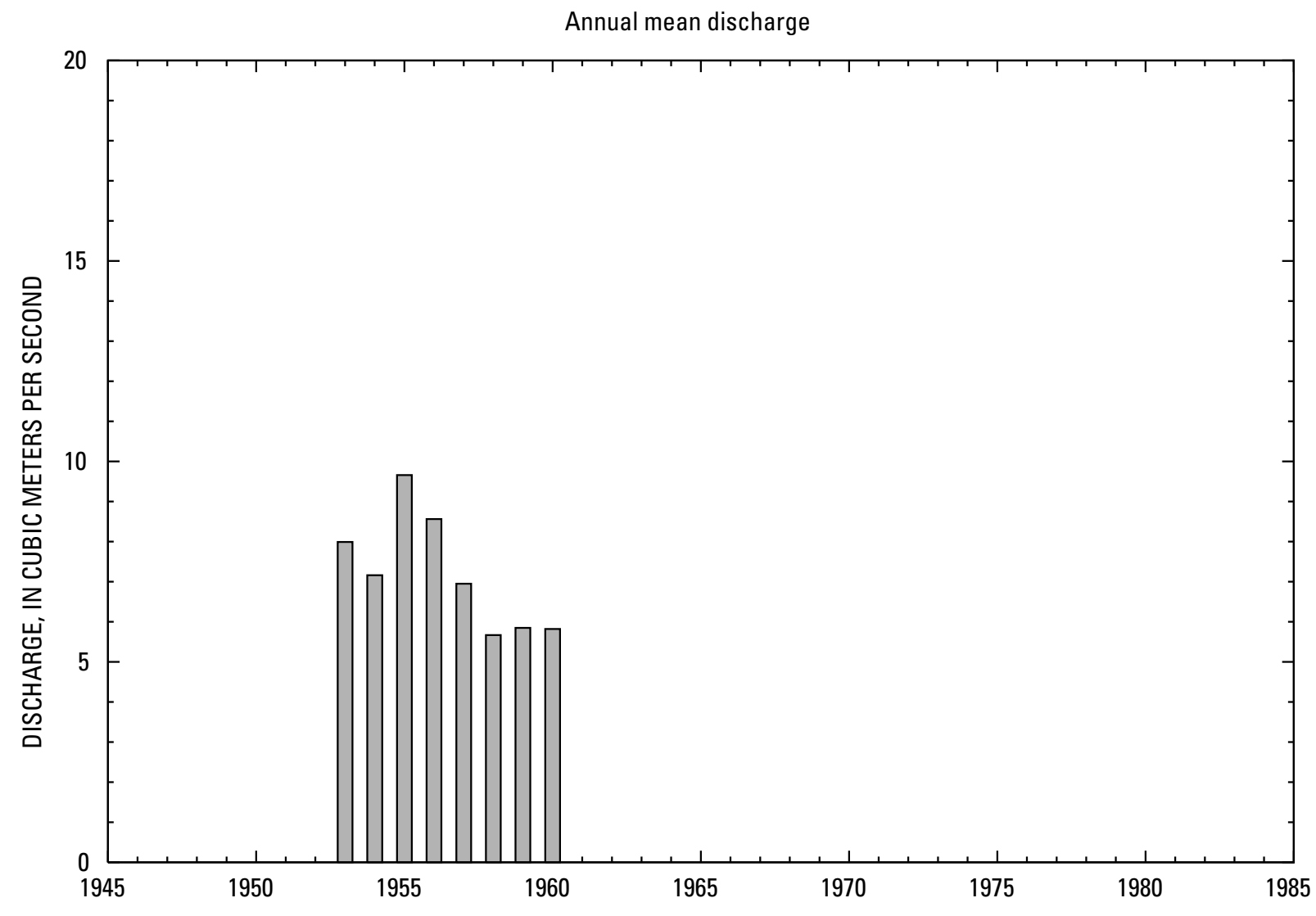




\section{4- 3.LOO-2T SANGIN WASH (SERAJ CANAL) AT SANGIN, Continued}

\section{Statistics of monthly and annual mean discharges}

[ $\mathrm{m}^{3} / \mathrm{s}$, meters per second]

\begin{tabular}{|c|c|c|c|c|c|c|c|c|}
\hline \multirow[b]{2}{*}{ Month } & \multicolumn{2}{|c|}{ Maximum } & \multicolumn{2}{|c|}{ Minimum } & \multicolumn{4}{|c|}{ Mean } \\
\hline & $\begin{array}{c}\text { Discharge } \\
\left(\mathrm{m}^{3} / \mathrm{s}\right)\end{array}$ & $\begin{array}{c}\text { Water year } \\
\text { of } \\
\text { occurrence }\end{array}$ & $\begin{array}{c}\text { Discharge } \\
\left(\mathrm{m}^{3} / \mathrm{s}\right)\end{array}$ & $\begin{array}{c}\text { Water year } \\
\text { of } \\
\text { occurrence }\end{array}$ & $\begin{array}{c}\text { Discharge } \\
\left(\mathrm{m}^{3} / \mathrm{s}\right)\end{array}$ & $\begin{array}{c}\text { Standard } \\
\text { deviation } \\
\left(\mathrm{m}^{3} / \mathrm{s}\right)\end{array}$ & $\begin{array}{c}\text { Coefficient } \\
\text { of } \\
\text { variation }\end{array}$ & $\begin{array}{c}\text { Percentage } \\
\text { of annual } \\
\text { discharge }\end{array}$ \\
\hline October & 12.2 & 1956 & 5.18 & 1960 & 7.90 & 2.24 & 0.28 & 9.15 \\
\hline November & 11.4 & 1956 & 2.51 & 1958 & 6.69 & 3.20 & 0.48 & 7.76 \\
\hline December & 9.01 & 1957 & 2.33 & 1960 & 5.90 & 2.50 & 0.42 & 6.84 \\
\hline January & 6.98 & 1955 & 1.41 & 1958 & 4.72 & 1.80 & 0.38 & 5.48 \\
\hline February & 6.31 & 1960 & 0.359 & 1957 & 4.80 & 2.01 & 0.42 & 5.56 \\
\hline March & 11.2 & 1956 & 4.52 & 1959 & 7.45 & 2.17 & 0.29 & 8.64 \\
\hline April & 13.5 & 1955,1956 & 4.02 & 1960 & 9.13 & 3.23 & 0.35 & 10.6 \\
\hline May & 13.5 & 1955 & 5.65 & 1956 & 8.94 & 2.69 & 0.30 & 10.4 \\
\hline June & 11.2 & 1955 & 3.86 & 1957 & 6.80 & 2.89 & 0.42 & 7.88 \\
\hline July & 9.63 & 1953 & 4.69 & 1960 & 7.70 & 1.69 & 0.22 & 8.92 \\
\hline August & 10.4 & 1956 & 6.66 & 1958 & 8.35 & 1.18 & 0.14 & 9.67 \\
\hline September & 10.6 & 1955 & 6.46 & 1958 & 7.92 & 1.34 & 0.17 & 9.17 \\
\hline Annual & 9.66 & 1955 & 5.67 & 1958 & 7.21 & 1.45 & 0.20 & 100 \\
\hline
\end{tabular}

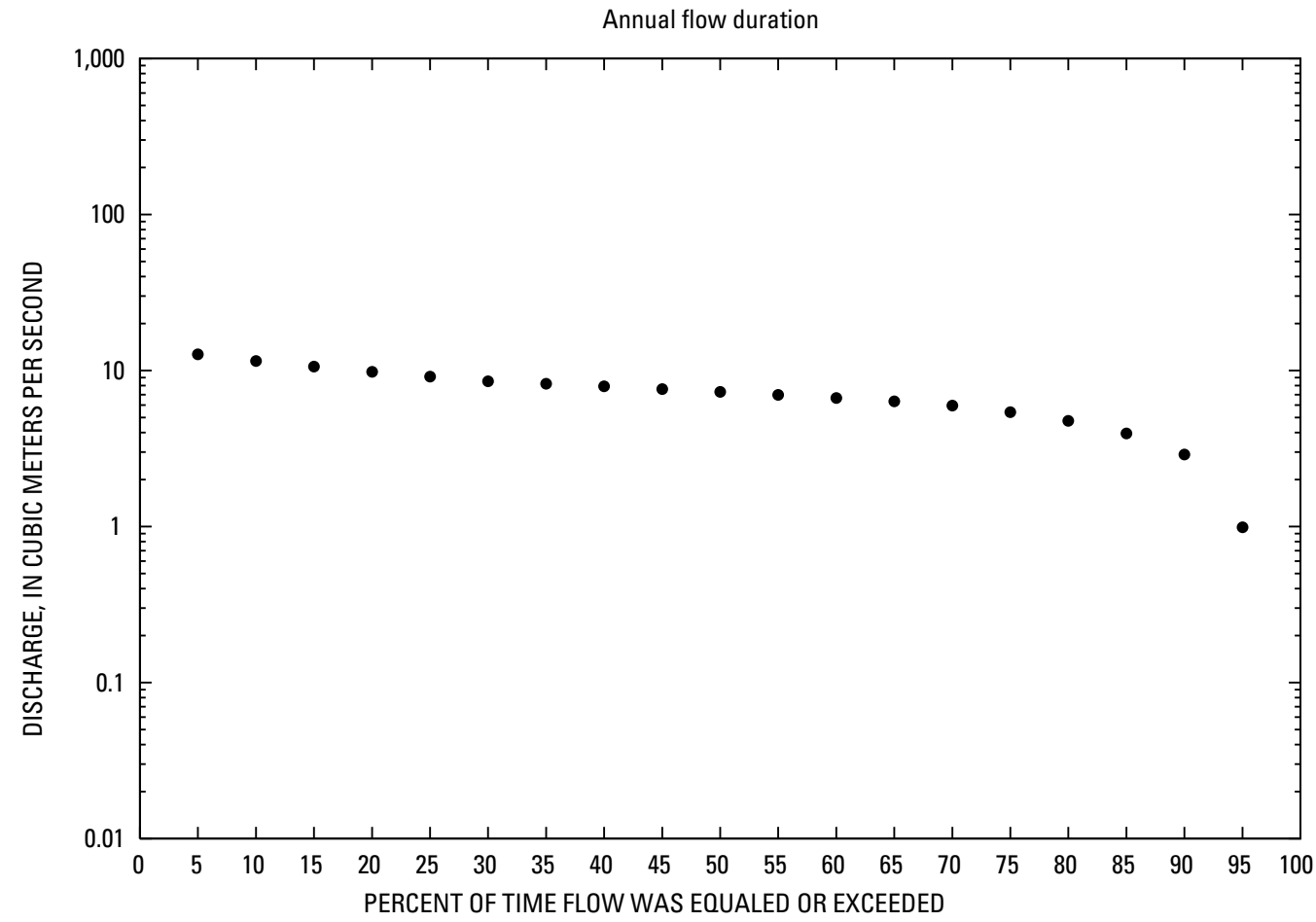




\section{4- 3.LO0-2T SANGIN WASH (SERAJ CANAL) AT SANGIN, Continued}

\section{Monthly and annual flow duration, in cubic meters per second}

[Data were not rounded in accordance with U.S. Geological Survey publication standards]

\begin{tabular}{|c|c|c|c|c|c|c|c|c|c|c|c|c|c|}
\hline $\begin{array}{l}\text { Percentage } \\
\text { of days } \\
\text { discharge } \\
\text { equaled or } \\
\text { exceeded }\end{array}$ & January & February & March & April & May & June & July & August & September & October & November & December & Annual \\
\hline 95 & 0.91 & 0.24 & 3.62 & 0.81 & 2.34 & 0.78 & 2.12 & 5.99 & 4.32 & 5.29 & 0.15 & 0.52 & 0.99 \\
\hline 90 & 1.63 & 0.26 & 4.5 & 4.65 & 4.4 & 1.73 & 4.37 & 6.61 & 5.49 & 5.77 & 0.57 & 1.37 & 2.9 \\
\hline 85 & 2.89 & 1.07 & 4.78 & 6.08 & 5.97 & 2.52 & 5.61 & 6.97 & 6.48 & 6.04 & 1.35 & 2.18 & 3.95 \\
\hline 80 & 3.05 & 3.42 & 5.04 & 6.59 & 6.53 & 3.67 & 5.88 & 7.33 & 6.9 & 6.37 & 3.08 & 2.48 & 4.76 \\
\hline 75 & 3.38 & 4.06 & 5.57 & 7.04 & 6.92 & 4.88 & 6.16 & 7.51 & 7.18 & 6.55 & 3.85 & 2.87 & 5.41 \\
\hline 70 & 3.87 & 4.24 & 5.86 & 7.37 & 7.29 & 5.61 & 6.5 & 7.69 & 7.46 & 6.71 & 6.12 & 3.14 & 5.96 \\
\hline 65 & 4.12 & 4.41 & 6.13 & 7.63 & 7.66 & 6.04 & 6.92 & 7.86 & 7.73 & 6.87 & 6.57 & 3.49 & 6.34 \\
\hline 60 & 4.31 & 4.62 & 6.38 & 7.9 & 8.01 & 6.34 & 7.3 & 8.04 & 7.83 & 7.02 & 6.91 & 4.45 & 6.66 \\
\hline 55 & 4.52 & 5.13 & 6.61 & 8.16 & 8.37 & 6.68 & 7.53 & 8.22 & 7.93 & 7.17 & 7.25 & 5.03 & 6.98 \\
\hline 50 & 4.76 & 5.3 & 6.85 & 8.51 & 8.72 & 7.17 & 7.77 & 8.37 & 8.03 & 7.31 & 7.57 & 6.27 & 7.3 \\
\hline 45 & 5.02 & 5.46 & 7.1 & 8.95 & 9.07 & 7.5 & 8 & 8.53 & 8.13 & 7.48 & 7.88 & 6.56 & 7.61 \\
\hline 40 & 5.32 & 5.61 & 7.36 & 9.4 & 9.72 & 7.73 & 8.24 & 8.68 & 8.23 & 7.85 & 8.18 & 6.86 & 7.92 \\
\hline 35 & 5.64 & 5.77 & 7.61 & 10.4 & 10.7 & 7.96 & 8.49 & 8.84 & 8.33 & 8.19 & 8.44 & 7.69 & 8.23 \\
\hline 30 & 6.02 & 5.97 & 8.05 & 11.5 & 11.4 & 8.19 & 8.79 & 9 & 8.64 & 8.5 & 8.7 & 8.23 & 8.54 \\
\hline 25 & 6.31 & 6.18 & 8.7 & 12.1 & 11.9 & 9.52 & 9.1 & 9.15 & 8.97 & 9.14 & 8.97 & 8.63 & 9.14 \\
\hline 20 & 6.53 & 6.38 & 10.5 & 12.7 & 12.5 & 10.1 & 9.4 & 10.1 & 9.79 & 9.72 & 9.41 & 9.07 & 9.81 \\
\hline 15 & 6.75 & 6.65 & 11.2 & 13.5 & 13 & 10.7 & 9.77 & 10.6 & 10 & 10.4 & 10.2 & 9.58 & 10.6 \\
\hline 10 & 7.03 & 7.07 & 12.1 & 14.4 & 13.8 & 11.3 & 11.2 & 10.9 & 10.2 & 11.8 & 10.9 & 10.5 & 11.5 \\
\hline 5 & 7.48 & 7.94 & 13.2 & 15.9 & 14.7 & 12.2 & 12.5 & 11.2 & 10.4 & 12.2 & 11.5 & 12.1 & 12.7 \\
\hline
\end{tabular}




\section{4- 3.LOO-2T SANGIN WASH (SERAJ CANAL) AT SANGIN, Continued}

Probability of occurrence of annual high discharges

[ $\mathrm{m}^{3} / \mathrm{s}$, meters per second; $\mathbf{n g}$, statistic not given]

\begin{tabular}{|c|c|c|c|c|c|c|}
\hline \multirow{2}{*}{$\begin{array}{c}\text { Exceedance } \\
\text { probability }\end{array}$} & \multirow{2}{*}{$\begin{array}{c}\text { Recurrence } \\
\text { interval } \\
\text { (years) }\end{array}$} & \multirow{2}{*}{$\begin{array}{c}\text { Maximum } \\
\text { instantaneous } \\
\left(\mathrm{m}^{3} / \mathrm{s}\right)\end{array}$} & \multicolumn{4}{|c|}{ Maximum mean discharge } \\
\hline & & & 3-day period & 7-day period & 15-day period & 30-day period \\
\hline 0.99 & 1.01 & 7.00 & ${ }^{2} 6.23$ & 6.30 & 5.99 & 6.10 \\
\hline 0.95 & 1.05 & 9.10 & 7.78 & 7.70 & 7.30 & 7.18 \\
\hline 0.90 & 1.11 & 10.3 & 8.71 & 8.52 & 8.08 & 7.82 \\
\hline 0.80 & 1.25 & 11.8 & 9.96 & 9.60 & 9.11 & 8.67 \\
\hline 0.50 & 2 & 14.7 & 12.7 & 11.9 & 11.3 & 10.5 \\
\hline 0.20 & 5 & 17.3 & 15.9 & 14.5 & 13.8 & 12.7 \\
\hline 0.10 & 10 & 18.5 & 17.8 & 16.0 & 15.2 & 14.0 \\
\hline 0.04 & 25 & 19.7 & 220.0 & 17.7 & 16.8 & 15.5 \\
\hline 0.02 & 50 & 20.3 & 221.5 & 18.8 & 17.9 & 16.6 \\
\hline 0.01 & 100 & 20.8 & 222.9 & 19.9 & 18.9 & 17.6 \\
\hline 0.005 & 200 & 21.2 & 224.2 & 20.8 & 19.8 & 18.5 \\
\hline 0.002 & 500 & 21.7 & ng & ng & ng & ng \\
\hline
\end{tabular}

'Less than 10 years of data used.

${ }^{2}$ Data does not fit log-Pearson Type III curve, use with caution.

\section{4- 3.LO0-2T SANGIN WASH (SERAJ CANAL) AT SANGIN, Continued}

Probability of occurrence of annual low discharges

$\left[\mathrm{m}^{3} / \mathrm{s}\right.$, meters per second]

\begin{tabular}{|c|c|c|c|c|c|c|c|c|c|c|}
\hline \multirow{3}{*}{$\begin{array}{c}\text { Nonexceedance } \\
\text { probability }\end{array}$} & \multirow{3}{*}{$\begin{array}{c}\text { Recurrence } \\
\text { interval } \\
\text { (years) }\end{array}$} & \multicolumn{9}{|c|}{ Minimum mean discharge $\left(\mathrm{m}^{3} / \mathrm{s}\right)$} \\
\hline & & \multicolumn{9}{|c|}{ Number of consecutive days } \\
\hline & & 1 & 3 & 7 & 14 & 30 & 60 & 90 & 120 & 183 \\
\hline 0.05 & 20 & 0 & 0 & 0 & 0.014 & 0.624 & 2.29 & ${ }^{12} 2.20$ & 2.85 & 3.78 \\
\hline 0.10 & 10 & 0 & 0 & 0 & 0.044 & 0.836 & 2.45 & 2.57 & 3.22 & 4.15 \\
\hline 0.20 & 5 & 0 & 0 & 0.113 & 0.146 & 1.17 & 2.69 & 3.09 & 3.71 & 4.65 \\
\hline 0.50 & 2 & 0 & 0.165 & 0.424 & 0.890 & 2.11 & 3.34 & 4.27 & 4.89 & 5.84 \\
\hline
\end{tabular}

'Data does not fit log-Pearson Type III curve, use with caution. 
Probability of occurrence of seasonal low discharges

$\left[\mathrm{m}^{3} / \mathrm{s}\right.$, meters per second]

\begin{tabular}{|c|c|c|c|c|c|c|c|c|c|}
\hline \multirow{3}{*}{$\begin{array}{c}\text { Nonexceedance } \\
\text { probability }\end{array}$} & \multirow{3}{*}{$\begin{array}{c}\text { Recurrence } \\
\text { interval } \\
\text { (years) }\end{array}$} & \multicolumn{8}{|c|}{ Minimum mean discharge $\left(\mathrm{m}^{3} / \mathrm{s}\right)$} \\
\hline & & \multicolumn{8}{|c|}{ Number of consecutive days } \\
\hline & & 1 & 7 & 14 & 30 & 1 & 7 & 14 & 30 \\
\hline & & \multicolumn{4}{|c|}{ December-January-February } & \multicolumn{4}{|c|}{ March-April-May } \\
\hline 0.05 & 20 & 0 & 0.194 & 0.231 & 0.668 & 0 & 0.233 & 0.690 & 3.69 \\
\hline 0.10 & 10 & 0 & 0.296 & 0.391 & 0.949 & 0 & 0.482 & 1.18 & 4.06 \\
\hline 0.20 & 5 & 0 & 0.490 & 0.701 & 1.41 & 0.215 & 1.02 & 2.04 & 4.57 \\
\hline \multirow[t]{2}{*}{0.50} & 2 & 0.477 & 1.26 & 1.85 & 2.72 & 1.79 & 3.00 & 4.33 & 5.78 \\
\hline & & \multicolumn{4}{|c|}{ June-July-August } & \multicolumn{4}{|c|}{ September-October-November } \\
\hline 0.05 & 20 & 0 & 0.476 & 1.57 & 3.09 & 0 & 0 & 0.019 & 2.11 \\
\hline 0.10 & 10 & 0 & 0.674 & 1.91 & 3.45 & 0 & 0 & 0.096 & 2.65 \\
\hline 0.20 & 5 & 0 & 1.03 & 2.45 & 3.98 & 0 & 0.345 & 0.483 & 3.46 \\
\hline 0.50 & 2 & 1.52 & 2.40 & 4.00 & 5.34 & 1.13 & 3.02 & 3.97 & 5.56 \\
\hline
\end{tabular}

4- 3.LOO-2T SANGIN WASH (SERAJ CANAL) AT SANGIN, Continued

Annual peak discharge and corresponding gage height

[m³/s, meters per second; --, no data]

\begin{tabular}{|c|c|c|c|c|c|c|c|}
\hline $\begin{array}{c}\text { Water } \\
\text { year }\end{array}$ & Date & $\begin{array}{c}\text { Gage } \\
\text { height } \\
\text { (meters) }\end{array}$ & $\begin{array}{c}\text { Peak } \\
\text { discharge } \\
\left(\mathrm{m}^{3} / \mathrm{s}\right)\end{array}$ & $\begin{array}{c}\text { Water } \\
\text { year }\end{array}$ & Date & $\begin{array}{c}\text { Gage } \\
\text { height } \\
\text { (meters) }\end{array}$ & $\begin{array}{c}\text { Peak } \\
\text { discharge } \\
\left(\mathrm{m}^{3} / \mathrm{s}\right)\end{array}$ \\
\hline \multicolumn{8}{|c|}{ Annual peak discharge, by year } \\
\hline 1953 & 1-May & 1.68 & 12.6 & 1957 & 19-Mar & 2.00 & 17.3 \\
\hline 1954 & 21-Apr & -- & 16.8 & 1958 & 15-Dec & 1.34 & 11.2 \\
\hline 1955 & 26-Dec & 1.80 & 16.9 & 1959 & 3-Mar & 1.74 & 14.7 \\
\hline 1956 & 13-Apr & 2.00 & 17.3 & 1960 & 15-Apr & 1.44 & 9.29 \\
\hline \multicolumn{8}{|c|}{ Annual peak discharge, from highest to lowest } \\
\hline 1957 & 19-Mar & 2.00 & 17.3 & 1959 & 3-Mar & 1.74 & 14.7 \\
\hline 1956 & 13-Apr & 2.00 & 17.3 & 1953 & 1-May & 1.68 & 12.6 \\
\hline 1955 & 26-Dec & 1.80 & 16.9 & 1958 & 15-Dec & 1.34 & 11.2 \\
\hline 1954 & 21-Apr & & 16.8 & 1960 & 15-Apr & 1.44 & 9.29 \\
\hline
\end{tabular}




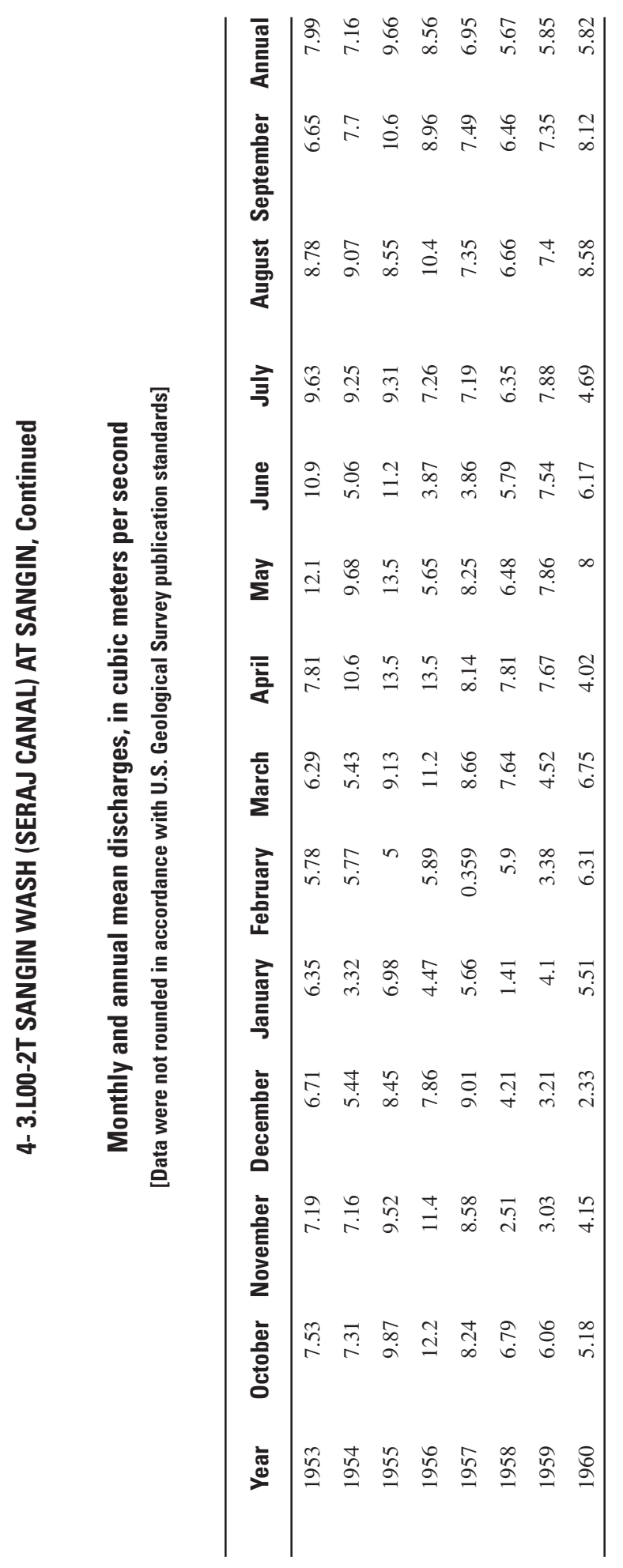




\section{4-4.R00-2A MUSA QALA RIVER NEAR MUSA OALA}

\section{(U.S. Geological Survey identification number: 321700064460000)}

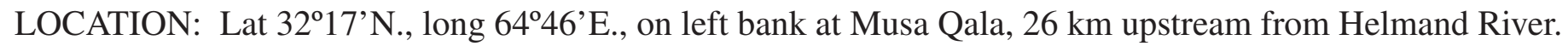

DRAINAGE AREA: $3,215 \mathrm{~km}^{2}$.

ALTITUDE: 1,000 meters plus mean sea level.

PERIOD OF RECORD: April 21, 1952 to September 30, 1979.

GAGE: Water-stage recorder.

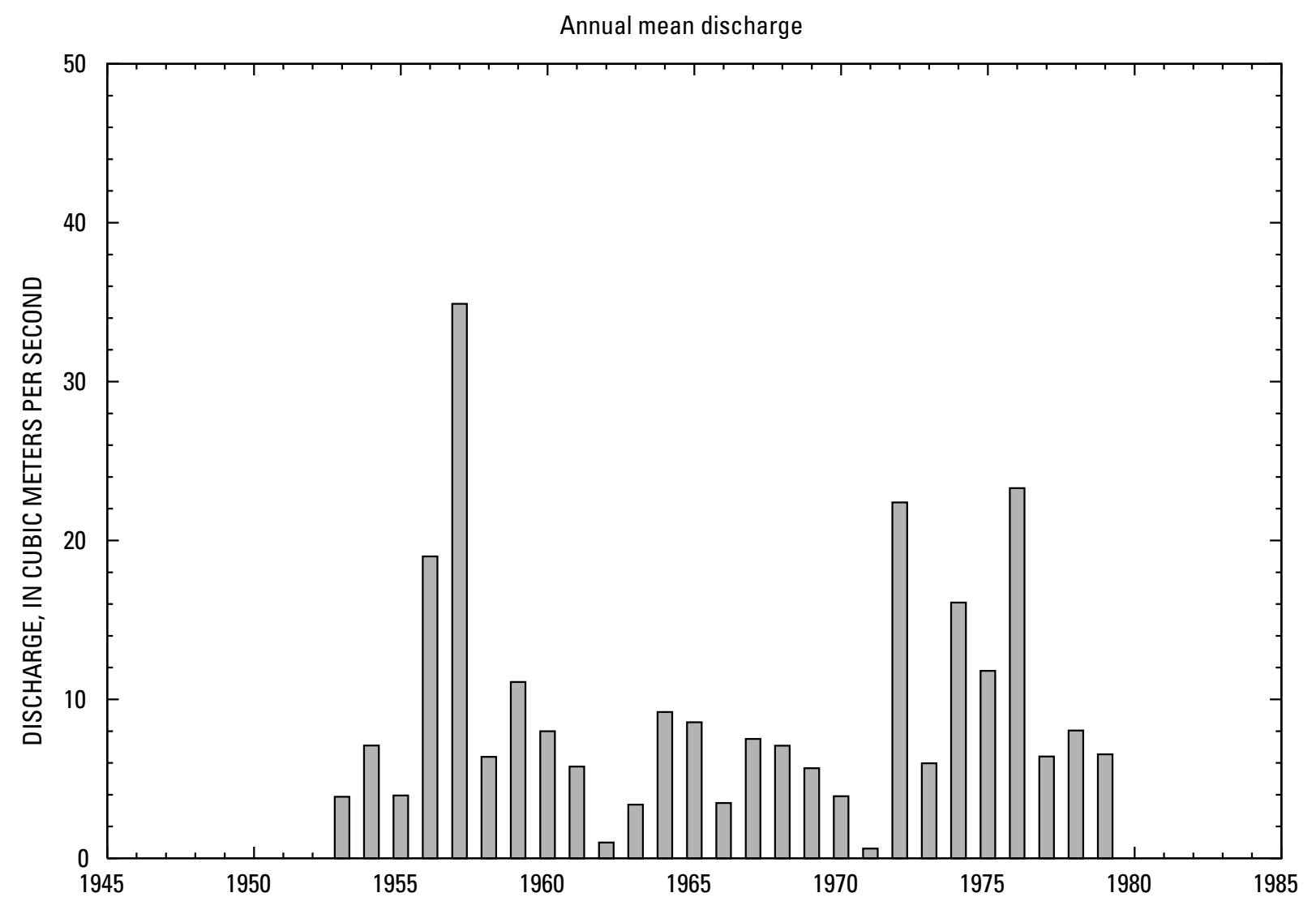




\section{4- 4.RO0-2A MUSA OALA RIVER NEAR MUSA OALA, Continued}

\section{Statistics of monthly and annual mean discharges}

$\left[\mathrm{m}^{3} / \mathrm{s}\right.$, meters per second]

\begin{tabular}{|c|c|c|c|c|c|c|c|c|}
\hline \multirow[b]{2}{*}{ Month } & \multicolumn{2}{|c|}{ Maximum } & \multicolumn{2}{|c|}{ Minimum } & \multicolumn{4}{|c|}{ Mean } \\
\hline & $\begin{array}{c}\text { Discharge } \\
\left(\mathrm{m}^{3} / \mathrm{s}\right)\end{array}$ & $\begin{array}{c}\text { Water year } \\
\text { of } \\
\text { occurrence }\end{array}$ & $\begin{array}{c}\text { Discharge } \\
\left(\mathrm{m}^{3} / \mathrm{s}\right)\end{array}$ & $\begin{array}{c}\text { Water year } \\
\text { of } \\
\text { occurrence }\end{array}$ & $\begin{array}{c}\text { Discharge } \\
\left(\mathrm{m}^{3} / \mathrm{s}\right)\end{array}$ & $\begin{array}{c}\text { Standard } \\
\text { deviation } \\
\left(\mathrm{m}^{3} / \mathrm{s}\right)\end{array}$ & $\begin{array}{c}\text { Coefficient } \\
\text { of } \\
\text { variation }\end{array}$ & $\begin{array}{c}\text { Percentage } \\
\text { of annual } \\
\text { discharge }\end{array}$ \\
\hline October & 1.47 & 1961 & 0.077 & 1953 & 0.32 & 0.33 & 1.04 & 0.29 \\
\hline November & 10.6 & 1958 & 0.054 & 1963 & 0.99 & 2.05 & 2.07 & 0.89 \\
\hline December & 15.4 & 1958 & 0.109 & 1955 & 2.66 & 3.80 & 1.43 & 2.39 \\
\hline January & 15.9 & 1974 & 0.073 & 1967 & 4.58 & 4.45 & 0.97 & 4.11 \\
\hline February & 40.3 & 1974 & 0.172 & 1955 & 15.0 & 10.4 & 0.70 & 13.4 \\
\hline March & 154 & 1957 & 1.52 & 1971 & 41.4 & 45.8 & 1.11 & 37.1 \\
\hline April & 141 & 1957 & 0.885 & 1971 & 31.9 & 30.3 & 0.95 & 28.6 \\
\hline May & 77.4 & 1957 & 0.750 & 1971 & 10.9 & 14.8 & 1.36 & 9.74 \\
\hline June & 10.8 & 1957 & 0.210 & 1962 & 2.19 & 2.16 & 0.99 & 1.96 \\
\hline July & 8.71 & 1976 & 0.111 & 1966 & 1.05 & 1.69 & 1.60 & 0.94 \\
\hline August & 1.34 & 1960 & 0.031 & 1963 & 0.37 & 0.30 & 0.81 & 0.33 \\
\hline September & 1.36 & 1960 & 0.050 & 1952 & 0.28 & 0.30 & 1.08 & 0.25 \\
\hline Annual & 34.9 & 1957 & 0.612 & 1971 & 9.30 & 7.73 & 0.83 & 100 \\
\hline
\end{tabular}

Annual flow duration

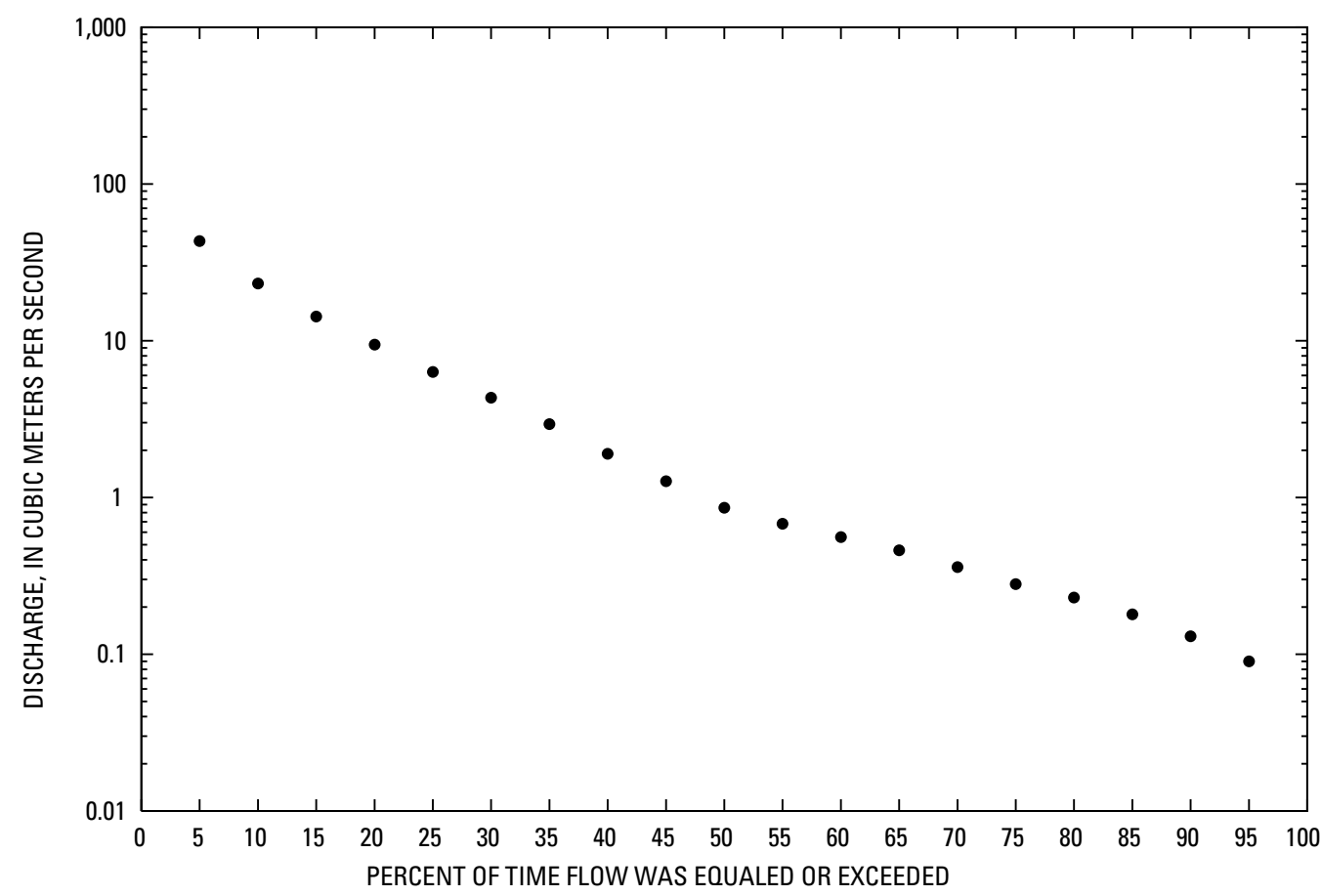




\section{4- 4.RO0-2A MUSA QALA RIVER NEAR MUSA QALA, Continued}

Monthly and annual flow duration, in cubic meters per second

[Data were not rounded in accordance with U.S. Geological Survey publication standards]

\begin{tabular}{|c|c|c|c|c|c|c|c|c|c|c|c|c|c|}
\hline $\begin{array}{l}\text { Percentage } \\
\text { of days } \\
\text { discharge } \\
\text { equaled or } \\
\text { exceeded }\end{array}$ & January & February & March & April & May & June & July & August & September & October & November & December & Annual \\
\hline 95 & 0.18 & 0.19 & 1.26 & 2.39 & 0.58 & 0.24 & 0.1 & 0.04 & 0.06 & 0.07 & 0.07 & 0.13 & 0.09 \\
\hline 90 & 0.22 & 0.43 & 2.94 & 4.54 & 0.75 & 0.33 & 0.14 & 0.07 & 0.07 & 0.08 & 0.08 & 0.19 & 0.13 \\
\hline 85 & 0.41 & 1.01 & 4.66 & 5.92 & 1.12 & 0.41 & 0.2 & 0.09 & 0.08 & 0.09 & 0.12 & 0.25 & 0.18 \\
\hline 80 & 0.51 & 2.28 & 6.9 & 7.06 & 1.75 & 0.51 & 0.23 & 0.12 & 0.09 & 0.11 & 0.15 & 0.33 & 0.23 \\
\hline 75 & 0.64 & 2.78 & 9.59 & 8.54 & 2.47 & 0.57 & 0.27 & 0.15 & 0.1 & 0.12 & 0.18 & 0.38 & 0.28 \\
\hline 70 & 0.79 & 3.57 & 11.7 & 9.88 & 2.97 & 0.67 & 0.3 & 0.18 & 0.12 & 0.13 & 0.22 & 0.47 & 0.36 \\
\hline 65 & 1.06 & 4.37 & 13.4 & 11.5 & 3.57 & 0.82 & 0.36 & 0.2 & 0.14 & 0.14 & 0.26 & 0.56 & 0.46 \\
\hline 60 & 1.44 & 4.95 & 15.3 & 13.6 & 4.2 & 1 & 0.41 & 0.22 & 0.15 & 0.16 & 0.29 & 0.64 & 0.56 \\
\hline 55 & 1.63 & 5.77 & 17.7 & 15.7 & 5.11 & 1.18 & 0.45 & 0.25 & 0.17 & 0.19 & 0.32 & 0.71 & 0.68 \\
\hline 50 & 1.83 & 6.71 & 20.2 & 18.1 & 6.1 & 1.4 & 0.49 & 0.27 & 0.19 & 0.21 & 0.41 & 0.78 & 0.86 \\
\hline 45 & 2.09 & 8.23 & 23 & 21.2 & 7.08 & 1.64 & 0.54 & 0.3 & 0.2 & 0.23 & 0.47 & 0.85 & 1.27 \\
\hline 40 & 2.36 & 9.85 & 26 & 23.8 & 8.07 & 1.88 & 0.58 & 0.33 & 0.21 & 0.26 & 0.52 & 0.92 & 1.9 \\
\hline 35 & 3.15 & 12 & 29.6 & 28.5 & 9.07 & 2.15 & 0.65 & 0.38 & 0.24 & 0.28 & 0.55 & 1.02 & 2.94 \\
\hline 30 & 3.78 & 14.8 & 33.7 & 33.6 & 10.1 & 2.54 & 0.74 & 0.43 & 0.28 & 0.32 & 0.59 & 1.13 & 4.33 \\
\hline 25 & 4.44 & 16.7 & 41.8 & 38.4 & 11.3 & 2.88 & 0.87 & 0.47 & 0.35 & 0.38 & 0.7 & 1.41 & 6.33 \\
\hline 20 & 5.23 & 19.3 & 54.6 & 44.5 & 12.7 & 3.26 & 1.09 & 0.51 & 0.39 & 0.4 & 0.83 & 2.61 & 9.45 \\
\hline 15 & 7.05 & 26.1 & 69.2 & 57.5 & 16.1 & 3.74 & 1.56 & 0.57 & 0.45 & 0.49 & 0.91 & 3.99 & 14.3 \\
\hline 10 & 9.6 & 35.2 & 104 & 79.1 & 22.7 & 4.61 & 2.11 & 0.66 & 0.59 & 0.74 & 1.29 & 5.44 & 23.2 \\
\hline 5 & 15.1 & 52.5 & 167.4 & 120.4 & 36 & 6.23 & 2.71 & 1.21 & 1.09 & 1.14 & 2.86 & 9.64 & 43.2 \\
\hline
\end{tabular}




\section{4- 4.ROO-2A MUSA QALA RIVER NEAR MUSA OALA, Continued}

\section{Probability of occurrence of annual high discharges}

[ $\mathrm{m}^{3} / \mathrm{s}$, meters per second; $\mathrm{ng}$, statistic not given]

\begin{tabular}{rrrrrrr}
\hline & & & \multicolumn{5}{c}{ Maximum mean discharge } \\
\cline { 6 - 7 } $\begin{array}{c}\text { Exceedance } \\
\text { probability }\end{array}$ & $\begin{array}{c}\text { Recurrence } \\
\text { interval } \\
\text { (years) }\end{array}$ & $\begin{array}{c}\text { Maximum } \\
\text { instantaneous } \\
\left(\mathbf{m}^{3} / \mathbf{s}\right)\end{array}$ & 3-day period & 7-day period & 15-day period & 30-day period \\
\cline { 5 - 7 } & 1.01 & 8.20 & 3.40 & 3.01 & 2.56 & 2.06 \\
0.99 & 1.05 & 20.7 & 11.3 & 9.64 & 7.89 & 6.03 \\
0.95 & 1.11 & 33.2 & 19.8 & 16.6 & 13.4 & 10.0 \\
0.90 & 1.25 & 58.1 & 36.9 & 30.2 & 23.7 & 17.5 \\
0.50 & 2 & 162 & 99.8 & 77.8 & 58.8 & 43.2 \\
0.20 & 5 & 427 & 214 & 159 & 116 & 86.9 \\
0.10 & 10 & 694 & 294 & 212 & 152 & 116 \\
0.04 & 25 & 1,140 & 389 & 272 & 193 & 151 \\
0.02 & 50 & 1,570 & 453 & 311 & 219 & 174 \\
0.01 & 100 & 2,070 & 511 & 345 & 241 & 195 \\
0.005 & 200 & 2,650 & 562 & 374 & 260 & 213 \\
0.002 & 500 & 3,560 & ng & ng & ng & ng \\
\hline
\end{tabular}

\section{4- 4.R00-2A MUSA OALA RIVER NEAR MUSA QALA, Continued}

Probability of occurrence of annual low discharges

$\left[\mathrm{m}^{3} / \mathrm{s}\right.$, meters per second]

\begin{tabular}{|c|c|c|c|c|c|c|c|c|c|c|}
\hline \multirow{3}{*}{$\begin{array}{c}\text { Nonexceedance } \\
\text { probability }\end{array}$} & \multirow{3}{*}{$\begin{array}{c}\text { Recurrence } \\
\text { interval } \\
\text { (years) }\end{array}$} & \multicolumn{9}{|c|}{ Minimum mean discharge $\left(\mathrm{m}^{3} / \mathrm{s}\right)$} \\
\hline & & \multicolumn{9}{|c|}{ Number of consecutive days } \\
\hline & & 1 & 3 & 7 & 14 & 30 & 60 & 90 & 120 & 183 \\
\hline 0.05 & 20 & 0.009 & 0.016 & 0.017 & 0.024 & 0.035 & 0.051 & 0.060 & 0.076 & 0.119 \\
\hline 0.10 & 10 & 0.022 & 0.031 & 0.033 & 0.036 & 0.048 & 0.065 & 0.077 & 0.097 & 0.153 \\
\hline 0.20 & 5 & 0.044 & 0.053 & 0.056 & 0.058 & 0.070 & 0.089 & 0.106 & 0.131 & 0.211 \\
\hline 0.50 & 2 & 0.122 & 0.124 & 0.131 & 0.137 & 0.147 & 0.171 & 0.203 & 0.248 & 0.413 \\
\hline
\end{tabular}




\section{4- 4.ROO-2A MUSA QALA RIVER NEAR MUSA OALA, Continued}

\section{Probability of occurrence of seasonal low discharges}

$\left[\mathrm{m}^{3} / \mathrm{s}\right.$, meters per second]

\begin{tabular}{|c|c|c|c|c|c|c|c|c|c|}
\hline \multirow{3}{*}{$\begin{array}{c}\text { Nonexceedance } \\
\text { probability }\end{array}$} & \multirow{3}{*}{$\begin{array}{c}\text { Recurrence } \\
\text { interval } \\
\text { (years) }\end{array}$} & \multicolumn{8}{|c|}{ Minimum mean discharge $\left(\mathrm{m}^{3} / \mathrm{s}\right)$} \\
\hline & & \multicolumn{8}{|c|}{ Number of consecutive days } \\
\hline & & 1 & 7 & 14 & 30 & 1 & 7 & 14 & 30 \\
\hline & & \multicolumn{4}{|c|}{ December-January-February } & \multicolumn{4}{|c|}{ March-April-May } \\
\hline 0.05 & 20 & 0.070 & 0.082 & 0.084 & 0.096 & 0.175 & 0.236 & 0.409 & 0.832 \\
\hline 0.10 & 10 & 0.111 & 0.127 & 0.137 & 0.164 & 0.335 & 0.445 & 0.712 & 1.30 \\
\hline 0.20 & 5 & 0.186 & 0.214 & 0.243 & 0.304 & 0.692 & 0.898 & 1.32 & 2.21 \\
\hline \multirow[t]{2}{*}{0.50} & 2 & 0.455 & 0.556 & 0.679 & 0.933 & 2.30 & 2.84 & 3.73 & 5.81 \\
\hline & & \multicolumn{4}{|c|}{ June-July-August } & \multicolumn{4}{|c|}{ September-October-November } \\
\hline 0.05 & 20 & 0 & 0 & 0.028 & 0.050 & 0.019 & 0.028 & 0.036 & 0.048 \\
\hline 0.10 & 10 & 0.018 & 0.035 & 0.047 & 0.074 & 0.034 & 0.039 & 0.047 & 0.060 \\
\hline 0.20 & 5 & 0.059 & 0.081 & 0.083 & 0.116 & 0.054 & 0.059 & 0.067 & 0.079 \\
\hline 0.50 & 2 & 0.189 & 0.206 & 0.210 & 0.258 & 0.123 & 0.130 & 0.139 & 0.149 \\
\hline
\end{tabular}




\section{4- 4.R00-2A MUSA OALA RIVER NEAR MUSA OALA, Continued}

\section{Annual peak discharge and corresponding gage height}

$\left[\mathrm{m}^{3} / \mathrm{s}\right.$, meters per second; --, no data]

\begin{tabular}{|c|c|c|c|c|c|c|c|}
\hline $\begin{array}{c}\text { Water } \\
\text { year }\end{array}$ & Date & $\begin{array}{c}\text { Gage } \\
\text { height } \\
\text { (meters) }\end{array}$ & $\begin{array}{c}\text { Peak } \\
\text { discharge } \\
\left(\mathrm{m}^{3} / \mathrm{s}\right)\end{array}$ & $\begin{array}{c}\text { Water } \\
\text { year }\end{array}$ & Date & $\begin{array}{c}\text { Gage } \\
\text { height } \\
\text { (meters) }\end{array}$ & $\begin{array}{c}\text { Peak } \\
\text { discharge } \\
\left(\mathrm{m}^{3} / \mathrm{s}\right)\end{array}$ \\
\hline
\end{tabular}

Annual peak discharge, by year

\begin{tabular}{|c|c|c|c|c|c|c|c|}
\hline 1953 & $14-\mathrm{Feb}$ & & 187 & 1967 & 27-Apr & -- & 220 \\
\hline 1954 & 5-Apr & 1.56 & 215 & 1968 & 9-Mar & -- & 681 \\
\hline 1955 & 14-Mar & 2.53 & 1,075 & 1969 & 16-Apr & -- & 85.7 \\
\hline 1956 & 13-Dec & 1.78 & 458 & 1970 & 9-Mar & -- & 30.0 \\
\hline 1957 & 17-Mar & 3.60 & 2,009 & 1971 & $25-\mathrm{Feb}$ & -- & 10.8 \\
\hline 1958 & 11-Dec & -- & 69.3 & 1972 & 12-Mar & -- & 496 \\
\hline 1959 & 3-Mar & -- & 99.0 & 1973 & $25-\mathrm{Feb}$ & -- & 142 \\
\hline 1960 & 18-Apr & 2.93 & 127 & 1974 & 21-Feb & -- & 199 \\
\hline 1961 & 10-Apr & -- & 248 & 1975 & 15-May & -- & 222 \\
\hline 1962 & 14-Nov & -- & 16.4 & 1976 & 27-Mar & -- & 400 \\
\hline 1963 & 12-May & -- & 114 & 1977 & 10-Mar & -- & 42.0 \\
\hline 1964 & 1-Apr & -- & 283 & 1978 & 9-Mar & -- & 158 \\
\hline 1965 & 21-Apr & -- & 246 & 1979 & $18-\mathrm{Feb}$ & -- & 56.0 \\
\hline 1966 & 27-Apr & -- & 72.6 & & & & \\
\hline \multicolumn{8}{|c|}{ Annual peak discharge, from highest to lowest } \\
\hline 1957 & 17-Mar & 3.60 & 2,009 & 1978 & 9-Mar & -- & 158 \\
\hline 1955 & 14-Mar & 2.53 & 1,075 & 1973 & $25-\mathrm{Feb}$ & -- & 142 \\
\hline 1968 & 9-Mar & -- & 681 & 1960 & 18-Apr & 2.93 & 127 \\
\hline 1972 & 12-Mar & -- & 496 & 1963 & 12-May & -- & 114 \\
\hline 1956 & 13-Dec & 1.78 & 458 & 1959 & 3-Mar & -- & 99.0 \\
\hline 1976 & 27-Mar & -- & 400 & 1969 & 16-Apr & -- & 85.7 \\
\hline 1964 & 1-Apr & -- & 283 & 1966 & 27-Apr & -- & 72.6 \\
\hline 1961 & 10-Apr & -- & 248 & 1958 & 11-Dec & -- & 69.3 \\
\hline 1965 & 21-Apr & -- & 246 & 1979 & 18 -Feb & -- & 56.0 \\
\hline 1975 & 15-May & -- & 222 & 1977 & 10-Mar & -- & 42.0 \\
\hline 1967 & 27-Apr & -- & 220 & 1970 & 9-Mar & -- & 30.0 \\
\hline 1954 & 5-Apr & 1.56 & 215 & 1962 & 14-Nov & -- & 16.4 \\
\hline 1974 & $21-\mathrm{Feb}$ & -- & 199 & 1971 & $25-\mathrm{Feb}$ & -- & 10.8 \\
\hline 1953 & $14-\mathrm{Feb}$ & -- & 187 & & & & \\
\hline
\end{tabular}




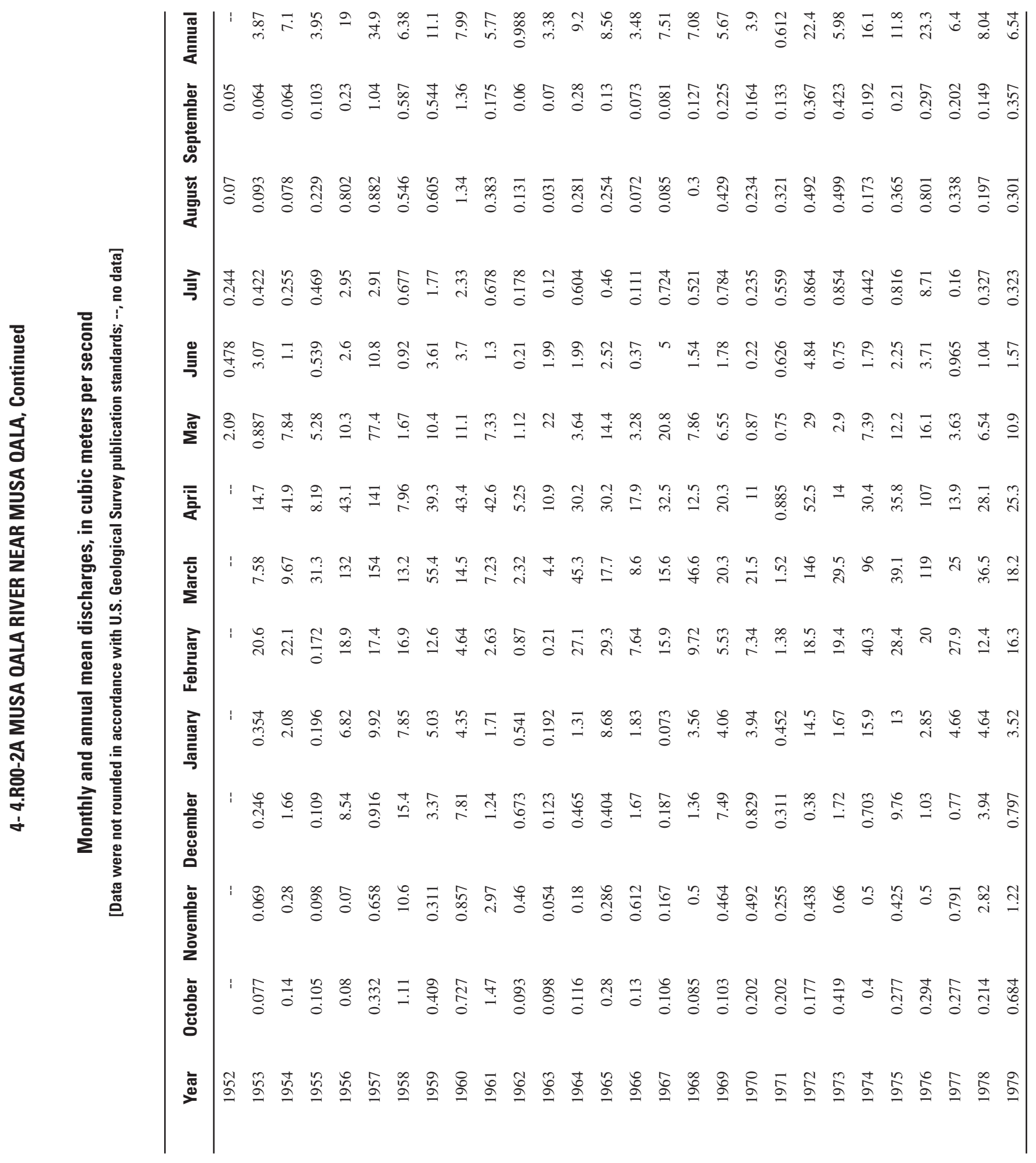




\section{4- 5.LOO-1A TIRIN RIVER AT ANARJUY/DEHRAOUT}

\section{(U.S. Geological Survey identification number: 323800065340000)}

LOCATION: Lat $32^{\circ} 38^{\prime}$ N., long 65'34'E., on left bank at Dehraout, $6 \mathrm{~km}$ upstream from Helmand River, and $65 \mathrm{~km}$ upstream from Kajakai Dam.

DRAINAGE AREA: $8,055 \mathrm{~km}^{2}$ (from Survey of India maps).

ALTITUDE: 1,115 meters plus mean sea level.

PERIOD OF RECORD: December 13, 1951 to September 30, 1979.

GAGE: Water-stage recorder.

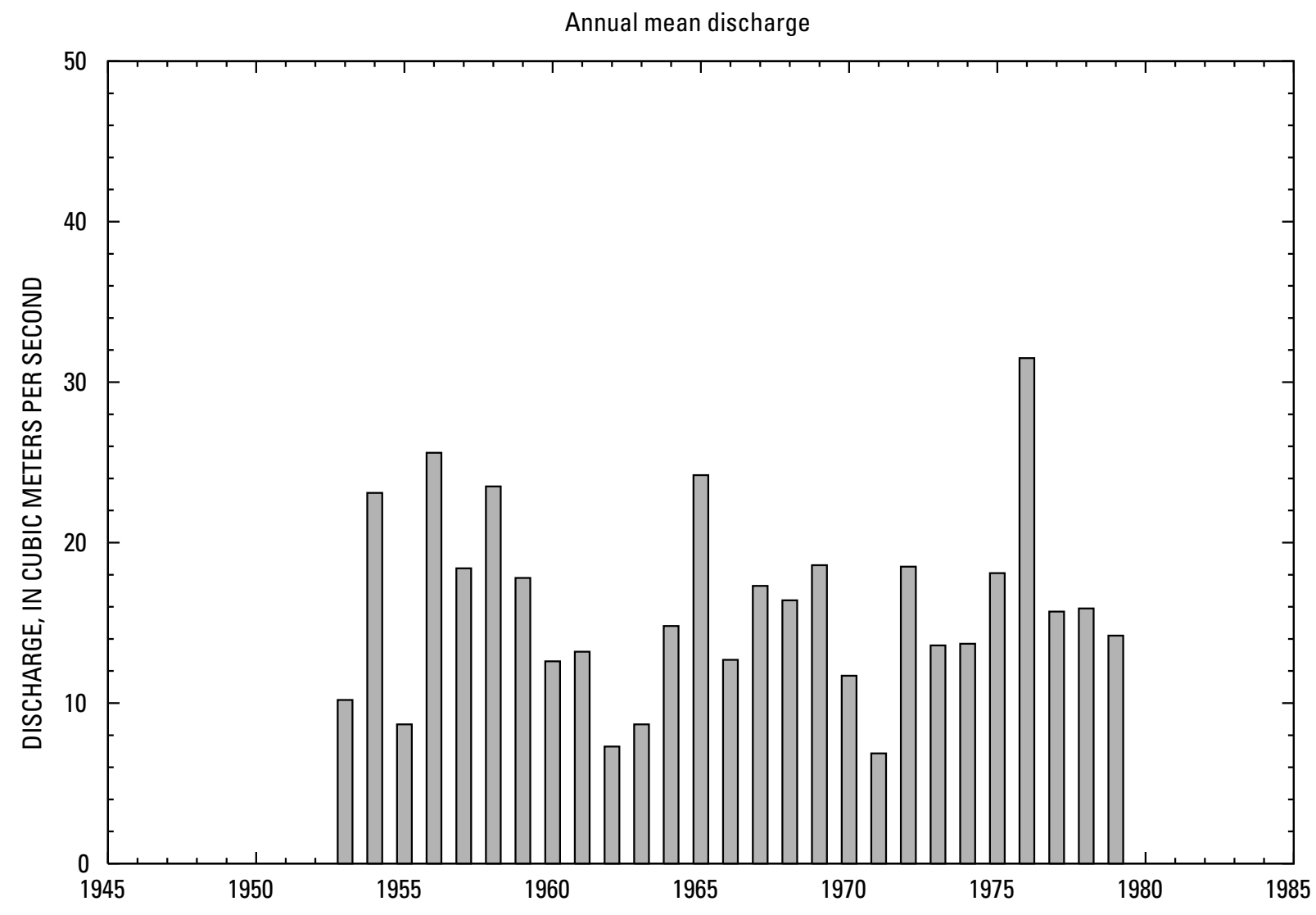




\section{4-5.LO0-1A TIRIN RIVER AT ANARJUY/DEHRAOUT, Continued}

\section{Statistics of monthly and annual mean discharges}

$\left[\mathrm{m}^{3} / \mathrm{s}\right.$, meters per second]

\begin{tabular}{|c|c|c|c|c|c|c|c|c|}
\hline \multirow[b]{2}{*}{ Month } & \multicolumn{2}{|c|}{ Maximum } & \multicolumn{2}{|c|}{ Minimum } & \multicolumn{4}{|c|}{ Mean } \\
\hline & $\begin{array}{c}\text { Discharge } \\
\left(\mathrm{m}^{3} / \mathrm{s}\right)\end{array}$ & $\begin{array}{c}\text { Water year } \\
\text { of } \\
\text { occurrence }\end{array}$ & $\begin{array}{c}\text { Discharge } \\
\left(\mathrm{m}^{3} / \mathrm{s}\right)\end{array}$ & $\begin{array}{c}\text { Water year } \\
\text { of } \\
\text { occurrence }\end{array}$ & $\begin{array}{c}\text { Discharge } \\
\left(\mathrm{m}^{3} / \mathrm{s}\right)\end{array}$ & $\begin{array}{c}\text { Standard } \\
\text { deviation } \\
\left(\mathrm{m}^{3} / \mathrm{s}\right)\end{array}$ & $\begin{array}{c}\text { Coefficient } \\
\text { of } \\
\text { variation }\end{array}$ & $\begin{array}{c}\text { Percentage } \\
\text { of annual } \\
\text { discharge }\end{array}$ \\
\hline October & 13.1 & 1966 & 3.26 & 1964 & 6.62 & 2.55 & 0.39 & 3.44 \\
\hline November & 19.0 & 1958 & 4.90 & 1972 & 9.78 & 3.57 & 0.37 & 5.09 \\
\hline December & 28.6 & 1958 & 6.06 & 1972 & 12.9 & 5.29 & 0.41 & 6.72 \\
\hline January & 29.9 & 1958 & 7.25 & 1963 & 14.4 & 5.57 & 0.39 & 7.51 \\
\hline February & 46.0 & 1958 & 7.56 & 1963 & 20.0 & 9.09 & 0.45 & 10.4 \\
\hline March & 70.3 & 1956 & 11.2 & 1963 & 33.2 & 14.8 & 0.45 & 17.3 \\
\hline April & 115 & 1976 & 10.3 & 1971 & 44.0 & 25.0 & 0.57 & 22.9 \\
\hline May & 71.0 & 1976 & 1.77 & 1971 & 22.5 & 16.2 & 0.72 & 11.7 \\
\hline June & 26.6 & 1965 & 1.53 & 1971 & 9.21 & 6.74 & 0.73 & 4.79 \\
\hline July & 28.4 & 1976 & 1.49 & 1971 & 8.41 & 7.07 & 0.84 & 4.37 \\
\hline August & 18.4 & 1957 & 1.41 & 1971 & 6.05 & 3.95 & 0.65 & 3.15 \\
\hline September & 14.2 & 1957 & 2.19 & 1971 & 5.15 & 2.99 & 0.58 & 2.68 \\
\hline Annual & 31.5 & 1976 & 6.87 & 1971 & 16.0 & 5.88 & 0.37 & 100 \\
\hline
\end{tabular}

Annual flow duration

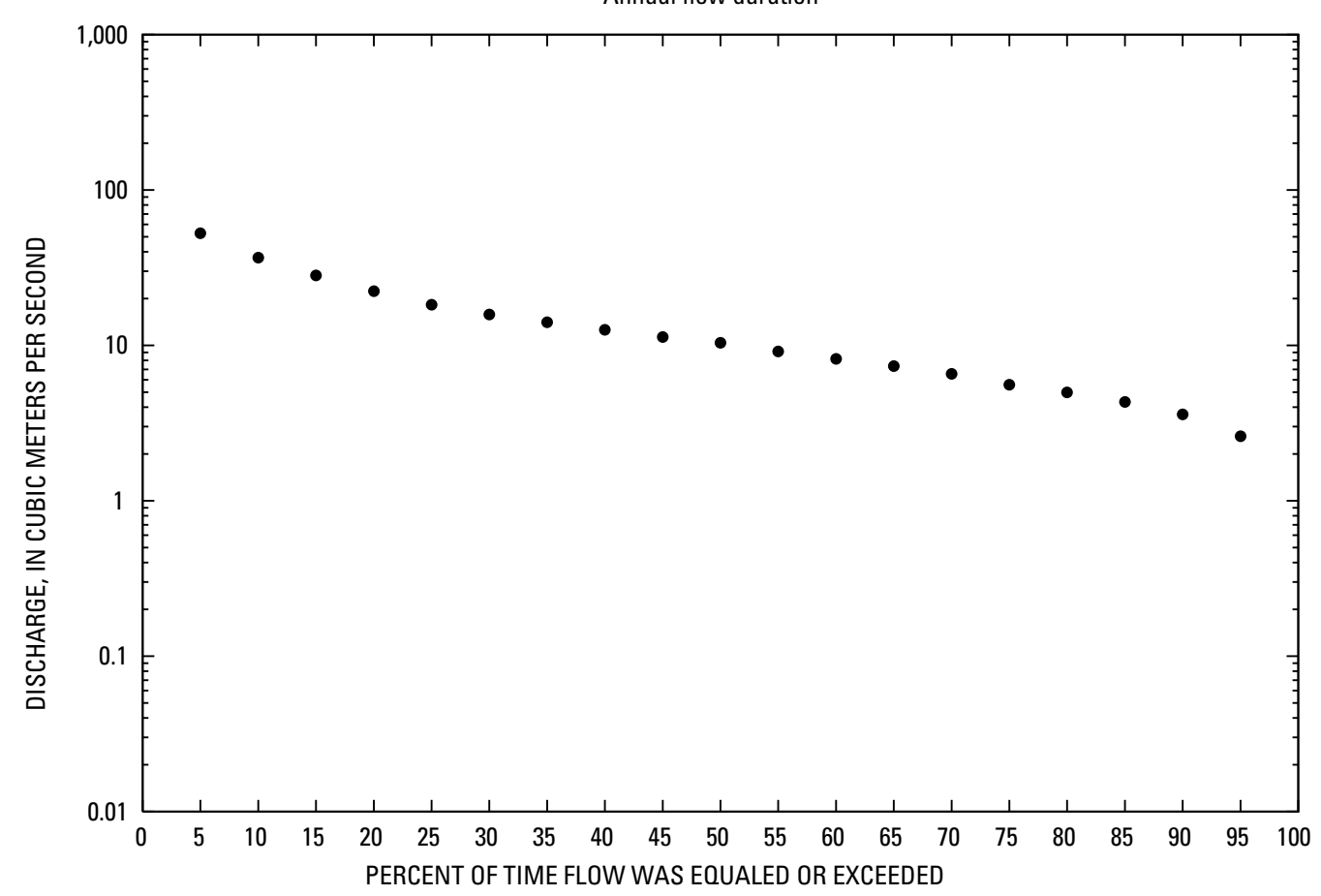




\section{4- 5.LO0-1A TIRIN RIVER AT ANARJUY/DEHRAOUT, Continued}

Monthly and annual flow duration, in cubic meters per second

[Data were not rounded in accordance with U.S. Geological Survey publication standards]

\begin{tabular}{|c|c|c|c|c|c|c|c|c|c|c|c|c|c|}
\hline $\begin{array}{l}\text { Percentage } \\
\text { of days } \\
\text { discharge } \\
\text { equaled or } \\
\text { exceeded }\end{array}$ & January & February & March & April & May & June & July & August & September & October & November & December & Annual \\
\hline 95 & 7.4 & 8.03 & 10.1 & 11.6 & 3.27 & 2.21 & 2.03 & 1.82 & 2.21 & 3.1 & 4.72 & 5.93 & 2.6 \\
\hline 90 & 8.29 & 8.7 & 12 & 14.6 & 5.62 & 2.85 & 2.39 & 2.13 & 2.35 & 3.6 & 5.33 & 7.19 & 3.6 \\
\hline 85 & 8.9 & 10.1 & 14.5 & 17.5 & 6.83 & 3.32 & 2.72 & 2.51 & 2.62 & 3.96 & 5.65 & 7.73 & 4.32 \\
\hline 80 & 9.57 & 11.1 & 16.2 & 19.9 & 8.19 & 4 & 3.19 & 2.77 & 2.91 & 4.26 & 6.34 & 8.25 & 4.98 \\
\hline 75 & 10.2 & 11.8 & 18.9 & 22.6 & 9.18 & 4.43 & 3.67 & 3.15 & 3.34 & 4.56 & 6.9 & 8.99 & 5.57 \\
\hline 70 & 10.7 & 12.5 & 20.6 & 25 & 10.3 & 4.93 & 4.09 & 3.53 & 3.73 & 4.88 & 7.22 & 9.58 & 6.55 \\
\hline 65 & 11.1 & 13.5 & 22.9 & 28.6 & 11.6 & 5.34 & 4.47 & 3.83 & 3.92 & 5.14 & 7.75 & 10.1 & 7.35 \\
\hline 60 & 11.5 & 14.7 & 25.2 & 32.1 & 13 & 5.98 & 4.87 & 4.14 & 4.05 & 5.45 & 8.29 & 10.4 & 8.18 \\
\hline 55 & 12 & 15.7 & 27.5 & 34.8 & 14.7 & 6.38 & 5.22 & 4.46 & 4.25 & 5.92 & 8.69 & 10.8 & 9.14 \\
\hline 50 & 12.4 & 16.7 & 29.3 & 38.2 & 16.5 & 6.81 & 5.54 & 4.78 & 4.51 & 6.35 & 9.07 & 11.3 & 10.4 \\
\hline 45 & 13.1 & 17.8 & 31.3 & 41.5 & 18.8 & 7.45 & 6.01 & 5 & 4.69 & 6.69 & 9.48 & 11.9 & 11.3 \\
\hline 40 & 13.8 & 18.8 & 33.5 & 44.8 & 21.6 & 8.12 & 6.63 & 5.22 & 4.98 & 6.97 & 10.1 & 12.6 & 12.6 \\
\hline 35 & 14.5 & 20 & 36.1 & 49.2 & 23.7 & 9.38 & 7.1 & 5.53 & 5.18 & 7.24 & 10.5 & 13.2 & 14.1 \\
\hline 30 & 15.4 & 21.4 & 39.1 & 53.6 & 25.4 & 10.5 & 7.59 & 5.95 & 5.35 & 7.48 & 10.9 & 13.7 & 15.8 \\
\hline 25 & 16.6 & 23.6 & 41.6 & 58.1 & 29.9 & 11.6 & 8.29 & 6.77 & 5.59 & 7.77 & 11.3 & 14.2 & 18.3 \\
\hline 20 & 17.6 & 26.8 & 46.7 & 63.3 & 35.1 & 12.9 & 9.22 & 7.35 & 6.12 & 8.38 & 12.3 & 14.9 & 22.3 \\
\hline 15 & 19.1 & 29.8 & 51.4 & 70.1 & 42.2 & 15.2 & 12.7 & 8.77 & 6.89 & 8.98 & 13.9 & 16 & 28.2 \\
\hline 10 & 23.1 & 36.7 & 58.7 & 78.8 & 50.4 & 20.5 & 18.9 & 12.3 & 9.85 & 10.9 & 14.9 & 18.4 & 36.7 \\
\hline 5 & 28.8 & 43.4 & 72.6 & 103.3 & 61.2 & 23.8 & 22.9 & 16.6 & 12.9 & 12.6 & 16.5 & 26.8 & 52.7 \\
\hline
\end{tabular}




\section{4- 5.LO0-1A TIRIN RIVER AT ANARJUY/DEHRAOUT, Continued}

Probability of occurrence of annual high discharges

$\left[\mathrm{m}^{3} / \mathrm{s}\right.$, meters per second; $\mathrm{ng}$, statistic not given]

\begin{tabular}{|c|c|c|c|c|c|c|}
\hline \multirow{2}{*}{$\begin{array}{l}\text { Exceedance } \\
\text { probability }\end{array}$} & \multirow{2}{*}{$\begin{array}{c}\text { Recurrence } \\
\text { interval } \\
\text { (years) }\end{array}$} & \multirow{2}{*}{$\begin{array}{c}\text { Maximum } \\
\text { instantaneous } \\
\left(\mathrm{m}^{3} / \mathrm{s}\right)\end{array}$} & \multicolumn{4}{|c|}{ Maximum mean discharge } \\
\hline & & & 3-day period & 7-day period & 15-day period & 30-day period \\
\hline 0.99 & 1.01 & ng & 16.6 & 15.0 & 13.8 & 13.1 \\
\hline 0.95 & 1.05 & 54.8 & 28.2 & 24.0 & 21.1 & 19.4 \\
\hline 0.90 & 1.11 & 68.0 & 36.3 & 30.2 & 26.1 & 23.7 \\
\hline 0.80 & 1.25 & 87.2 & 47.8 & 39.2 & 33.4 & 30.0 \\
\hline 0.50 & 2 & 135 & 74.7 & 61.2 & 51.9 & 45.9 \\
\hline 0.20 & 5 & 199 & 106 & 89.0 & 77.0 & 68.3 \\
\hline 0.10 & 10 & 240 & 122 & 105 & 93.1 & 83.1 \\
\hline 0.04 & 25 & 288 & 139 & 124 & 112 & 102 \\
\hline 0.02 & 50 & 322 & 149 & 137 & 126 & 115 \\
\hline 0.01 & 100 & 354 & 157 & 148 & 140 & 128 \\
\hline 0.005 & 200 & 385 & 165 & 158 & 152 & 142 \\
\hline 0.002 & 500 & 424 & ng & ng & ng & ng \\
\hline
\end{tabular}

4- 5.L00-1A TIRIN RIVER AT ANARJUY/DEHRAOUT, Continued

Probability of occurrence of annual low discharges

[ $\mathrm{m}^{3} / \mathrm{s}$, meters per second]

\begin{tabular}{|c|c|c|c|c|c|c|c|c|c|c|}
\hline \multirow{3}{*}{$\begin{array}{c}\text { Nonexceedance } \\
\text { probability }\end{array}$} & \multirow{3}{*}{$\begin{array}{c}\text { Recurrence } \\
\text { interval } \\
\text { (years) }\end{array}$} & \multicolumn{9}{|c|}{ Minimum mean discharge $\left(\mathrm{m}^{3} / \mathrm{s}\right)$} \\
\hline & & \multicolumn{9}{|c|}{ Number of consecutive days } \\
\hline & & 1 & 3 & 7 & 14 & 30 & 60 & 90 & 120 & 183 \\
\hline 0.05 & 20 & 1.45 & 1.48 & 1.55 & 1.60 & 1.72 & 1.88 & 1.98 & 2.10 & 2.70 \\
\hline 0.10 & 10 & 1.73 & 1.77 & 1.84 & 1.91 & 2.04 & 2.26 & 2.40 & 2.58 & 3.29 \\
\hline 0.20 & 5 & 2.16 & 2.20 & 2.30 & 2.38 & 2.54 & 2.82 & 3.04 & 3.31 & 4.18 \\
\hline 0.50 & 2 & 3.38 & 3.45 & 3.60 & 3.72 & 3.96 & 4.38 & 4.78 & 5.26 & 6.48 \\
\hline
\end{tabular}




\section{4- 5.LO0-1A TIRIN RIVER AT ANARJUY/DEHRAOUT, Continued}

\section{Probability of occurrence of seasonal low discharges}

$\left[\mathrm{m}^{3} / \mathrm{s}\right.$, meters per second]

\begin{tabular}{|c|c|c|c|c|c|c|c|c|c|}
\hline \multirow{3}{*}{$\begin{array}{c}\text { Nonexceedance } \\
\text { probability }\end{array}$} & \multirow{3}{*}{$\begin{array}{c}\text { Recurrence } \\
\text { interval } \\
\text { (years) }\end{array}$} & \multicolumn{8}{|c|}{ Minimum mean discharge $\left(\mathrm{m}^{3} / \mathrm{s}\right)$} \\
\hline & & \multicolumn{8}{|c|}{ Number of consecutive days } \\
\hline & & 1 & 7 & 14 & 30 & 1 & 7 & 14 & 30 \\
\hline & & \multicolumn{4}{|c|}{ December-January-February } & \multicolumn{4}{|c|}{ March-April-May } \\
\hline 0.05 & 20 & 4.89 & 5.46 & 5.84 & 6.41 & 2.58 & 2.83 & 2.98 & 3.85 \\
\hline 0.10 & 10 & 5.53 & 6.06 & 6.42 & 7.07 & 3.44 & 3.84 & 4.23 & 5.61 \\
\hline 0.20 & 5 & 6.44 & 6.92 & 7.27 & 8.03 & 4.79 & 5.43 & 6.23 & 8.48 \\
\hline \multirow[t]{2}{*}{0.50} & 2 & 8.68 & 9.14 & 9.58 & 10.6 & 8.62 & 9.83 & 11.9 & 16.7 \\
\hline & & \multicolumn{4}{|c|}{ June-July-August } & \multicolumn{4}{|c|}{ September-October-November } \\
\hline 0.05 & 20 & 1.47 & 1.58 & 1.65 & 1.78 & 1.86 & 1.97 & 2.08 & 2.24 \\
\hline 0.10 & 10 & 1.74 & 1.88 & 1.96 & 2.12 & 2.12 & 2.26 & 2.37 & 2.54 \\
\hline 0.20 & 5 & 2.17 & 2.35 & 2.46 & 2.65 & 2.52 & 2.69 & 2.80 & 3.00 \\
\hline 0.50 & 2 & 3.50 & 3.80 & 3.97 & 4.27 & 3.68 & 3.91 & 4.06 & 4.31 \\
\hline
\end{tabular}




\section{4- 5.LO0-1A TIRIN RIVER AT ANARJUY/DEHRAOUT, Continued}

Annual peak discharge and corresponding gage height

[m³/s, meters per second; --, no data]

\begin{tabular}{|c|c|c|c|c|c|c|c|}
\hline $\begin{array}{l}\text { Water } \\
\text { year }\end{array}$ & Date & $\begin{array}{c}\text { Gage } \\
\text { height } \\
\text { (meters) }\end{array}$ & $\begin{array}{c}\text { Peak } \\
\text { discharge } \\
\left(\mathrm{m}^{3} / \mathrm{s}\right)\end{array}$ & $\begin{array}{c}\text { Water } \\
\text { year }\end{array}$ & Date & $\begin{array}{c}\text { Gage } \\
\text { height } \\
\text { (meters) }\end{array}$ & $\begin{array}{c}\text { Peak } \\
\text { discharge } \\
\left(\mathrm{m}^{3} / \mathrm{s}\right)\end{array}$ \\
\hline \multicolumn{8}{|c|}{ Annual peak discharge, by year } \\
\hline 1952 & 27-Mar & 2.00 & 102 & 1966 & 15-Apr & -- & 205 \\
\hline 1953 & 26-May & 1.64 & 70.2 & 1967 & 31-Mar & -- & 220 \\
\hline 1954 & 15-Mar & -- & 142 & 1968 & 22-Dec & -- & 142 \\
\hline 1955 & 14-Mar & 1.19 & 47.8 & 1969 & 15-Apr & -- & 166 \\
\hline 1956 & 20-Mar & 2.10 & 172 & 1970 & 23-Aug & -- & 88.4 \\
\hline 1957 & 17-Mar & 2.65 & 249 & 1971 & $25-\mathrm{Feb}$ & -- & 50.0 \\
\hline 1958 & 2-Feb & 1.70 & 125 & 1972 & 28-Apr & -- & 191 \\
\hline 1959 & 2-Mar & 1.70 & 122 & 1973 & 19-Jul & -- & 256 \\
\hline 1960 & 16-Apr & 1.71 & 95.9 & 1974 & 26-Jan & -- & 122 \\
\hline 1961 & 3-Apr & -- & 75.0 & 1975 & 15-May & -- & 148 \\
\hline 1962 & 24-Dec & -- & 24.3 & 1976 & 24-Apr & -- & 236 \\
\hline 1963 & 12-May & -- & 112 & 1977 & 9-Jan & -- & 169 \\
\hline 1964 & 30-Mar & -- & 98.3 & 1978 & 25-Dec & -- & 315 \\
\hline 1965 & $3-\mathrm{Feb}$ & -- & 109 & 1979 & 29-Mar & -- & 182 \\
\hline \multicolumn{8}{|c|}{ Annual peak discharge, from highest to lowest } \\
\hline 1978 & 25-Dec & -- & 315 & 1958 & 2-Feb & 1.70 & 125 \\
\hline 1973 & 19-Jul & -- & 256 & 1974 & 26-Jan & -- & 122 \\
\hline 1957 & 17-Mar & 2.65 & 249 & 1959 & 2-Mar & 1.70 & 122 \\
\hline 1976 & 24-Apr & -- & 236 & 1963 & 12-May & -- & 112 \\
\hline 1967 & 31-Mar & -- & 220 & 1965 & $3-\mathrm{Feb}$ & -- & 109 \\
\hline 1966 & 15-Apr & -- & 205 & 1952 & 27-Mar & 2.00 & 102 \\
\hline 1972 & 28-Apr & -- & 191 & 1964 & 30-Mar & -- & 98.3 \\
\hline 1979 & 29-Mar & -- & 182 & 1960 & 16-Apr & 1.71 & 95.9 \\
\hline 1956 & 20-Mar & 2.10 & 172 & 1970 & 23-Aug & -- & 88.4 \\
\hline 1977 & 9-Jan & -- & 169 & 1961 & 3-Apr & -- & 75.0 \\
\hline 1969 & 15-Apr & -- & 166 & 1953 & 26-May & 1.64 & 70.2 \\
\hline 1975 & 15-May & -- & 148 & 1971 & 25-Feb & -- & 50.0 \\
\hline 1968 & 22-Dec & -- & 142 & 1955 & 14-Mar & 1.19 & 47.8 \\
\hline 1954 & 15-Mar & -- & 142 & 1962 & 24-Dec & -- & 24.3 \\
\hline
\end{tabular}




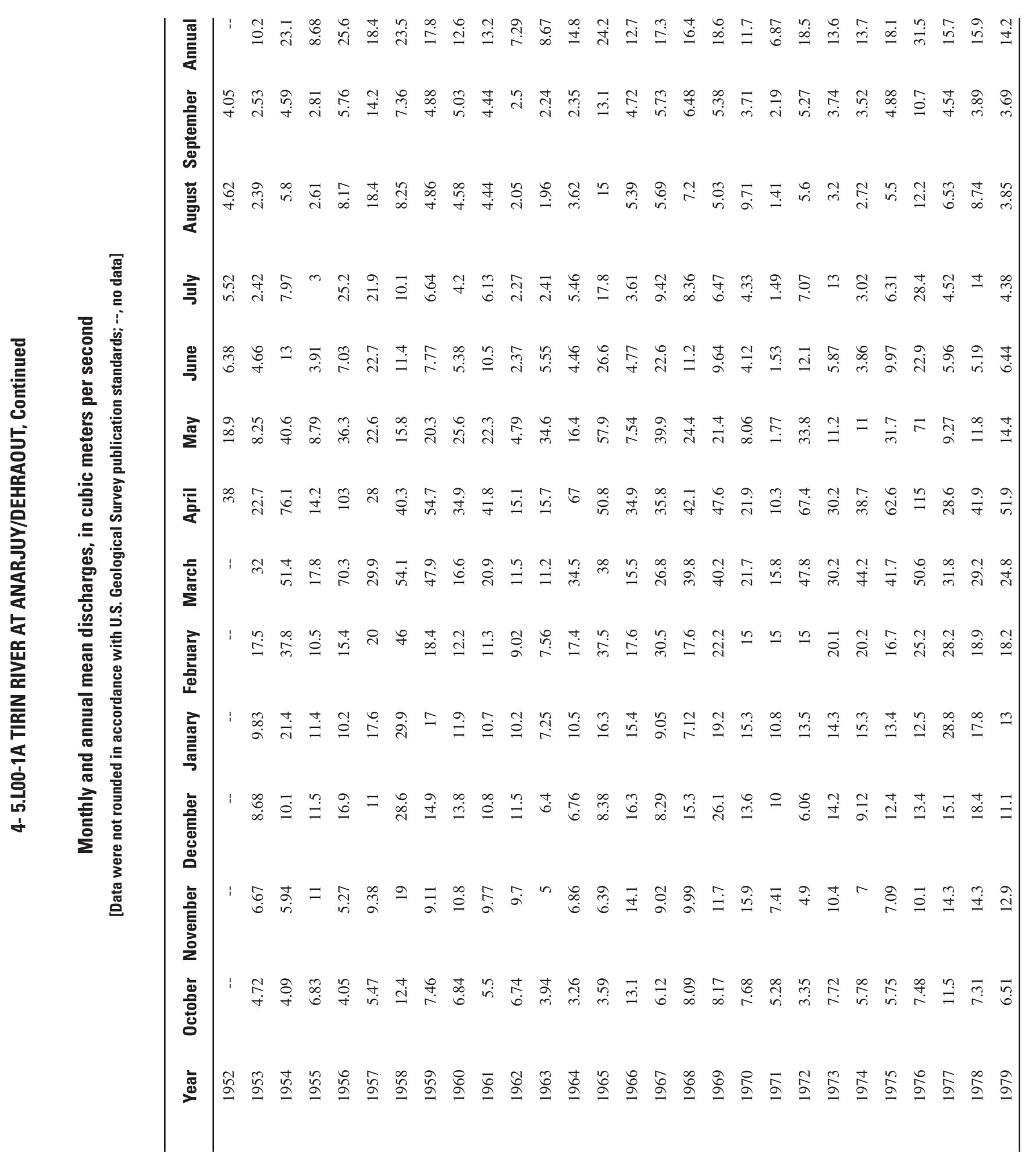




\section{4- 5.L00-4A TIRIN RIVER AT TIRIN}

\section{(U.S. Geological Survey identification number: 323800065560000 )}

LOCATION: Lat $32^{\circ} 38^{\prime} \mathrm{N}$., long $65^{\circ} 56^{\prime} \mathrm{E}$.

DRAINAGE AREA: $3,680 \mathrm{~km}^{2}$.

ALTITUDE: 1,358 meters plus mean sea level.

PERIOD OF RECORD: December 21, 1969 to March 19, 1980.

GAGE: --

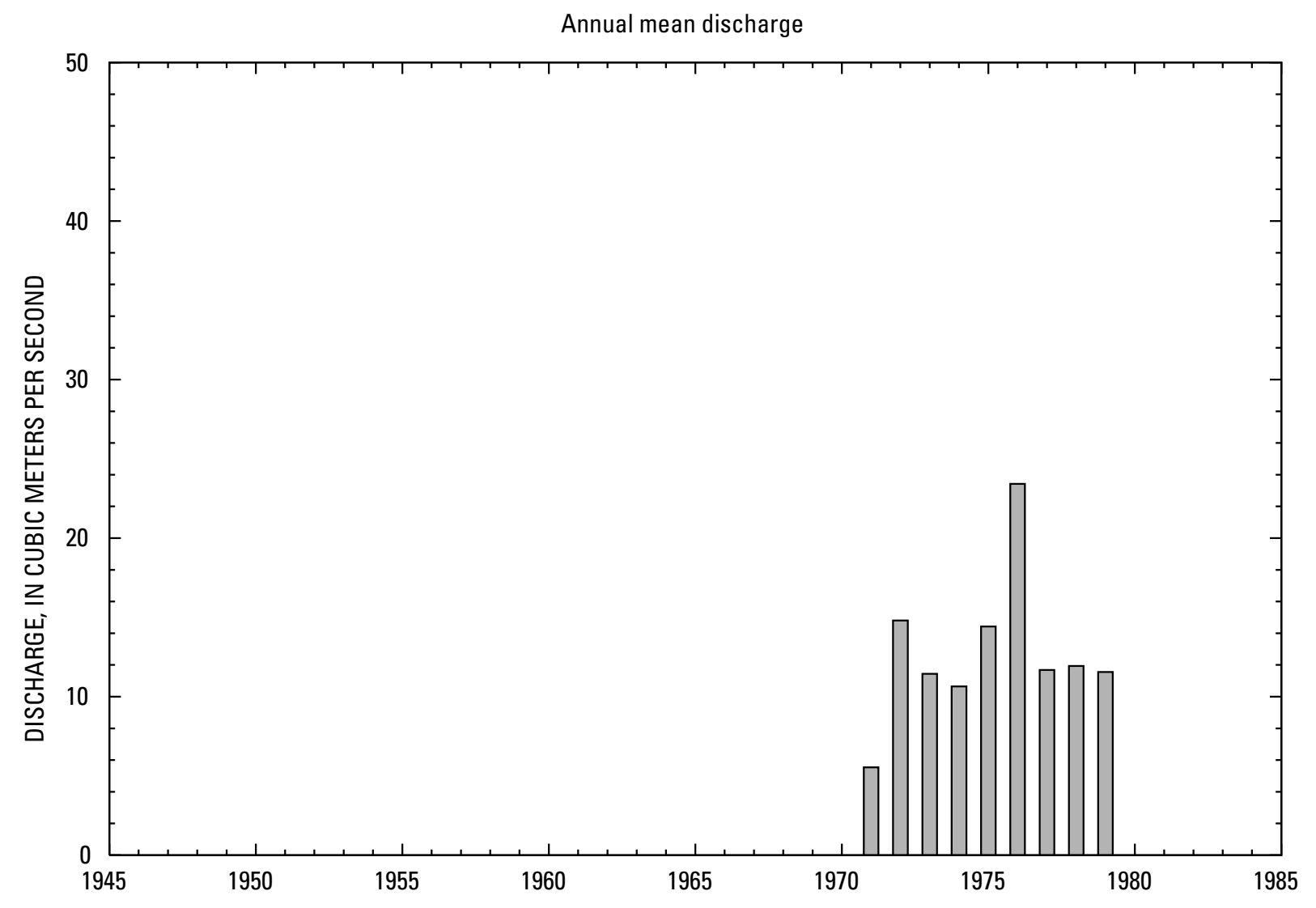




\section{4- 5.LO0-4A TIRIN RIVER AT TIRIN, Continued}

\section{Statistics of monthly and annual mean discharges}

$\left[\mathrm{m}^{3} / \mathrm{s}\right.$, meters per second]

\begin{tabular}{|c|c|c|c|c|c|c|c|c|}
\hline \multirow[b]{2}{*}{ Month } & \multicolumn{2}{|c|}{ Maximum } & \multicolumn{2}{|c|}{ Minimum } & \multicolumn{4}{|c|}{ Mean } \\
\hline & $\begin{array}{c}\text { Discharge } \\
\left(\mathrm{m}^{3} / \mathrm{s}\right)\end{array}$ & $\begin{array}{c}\text { Water year } \\
\text { of } \\
\text { occurrence }\end{array}$ & $\begin{array}{c}\text { Discharge } \\
\left(\mathrm{m}^{3} / \mathrm{s}\right)\end{array}$ & $\begin{array}{c}\text { Water year } \\
\text { of } \\
\text { occurrence }\end{array}$ & $\begin{array}{c}\text { Discharge } \\
\left(\mathrm{m}^{3} / \mathrm{s}\right)\end{array}$ & $\begin{array}{c}\text { Standard } \\
\text { deviation } \\
\left(\mathrm{m}^{3} / \mathrm{s}\right)\end{array}$ & $\begin{array}{c}\text { Coefficient } \\
\text { of } \\
\text { variation }\end{array}$ & $\begin{array}{c}\text { Percentage } \\
\text { of annual } \\
\text { discharge }\end{array}$ \\
\hline October & 8.66 & 1977 & 2.86 & 1972 & 5.14 & 1.49 & 0.29 & 3.44 \\
\hline November & 10.1 & 1977 & 4.10 & 1972 & 6.89 & 1.77 & 0.26 & 4.61 \\
\hline December & 10.7 & 1977 & 4.37 & 1972 & 7.96 & 1.66 & 0.21 & 5.32 \\
\hline January & 15.3 & 1977 & 7.20 & 1978 & 9.25 & 2.26 & 0.24 & 6.18 \\
\hline February & 18.7 & 1977 & 9.18 & 1978 & 12.4 & 3.16 & 0.26 & 8.27 \\
\hline March & 36.9 & 1972 & 11.6 & 1971 & 25.0 & 7.39 & 0.30 & 16.7 \\
\hline April & 91.3 & 1976 & 8.67 & 1971 & 39.8 & 23.9 & 0.60 & 26.6 \\
\hline May & 57.3 & 1976 & 2.26 & 1971 & 17.4 & 15.9 & 0.91 & 11.6 \\
\hline June & 18.3 & 1976 & 1.91 & 1971 & 7.23 & 4.44 & 0.61 & 4.83 \\
\hline July & 22.7 & 1976 & 2.08 & 1971 & 8.24 & 6.55 & 0.79 & 5.51 \\
\hline August & 10.4 & 1976 & 1.74 & 1971 & 5.73 & 2.56 & 0.45 & 3.83 \\
\hline September & 9.04 & 1976 & 2.25 & 1971 & 4.57 & 1.81 & 0.40 & 3.06 \\
\hline Annual & 23.4 & 1976 & 5.54 & 1971 & 12.8 & 4.77 & 0.37 & 100 \\
\hline
\end{tabular}

Annual flow duration

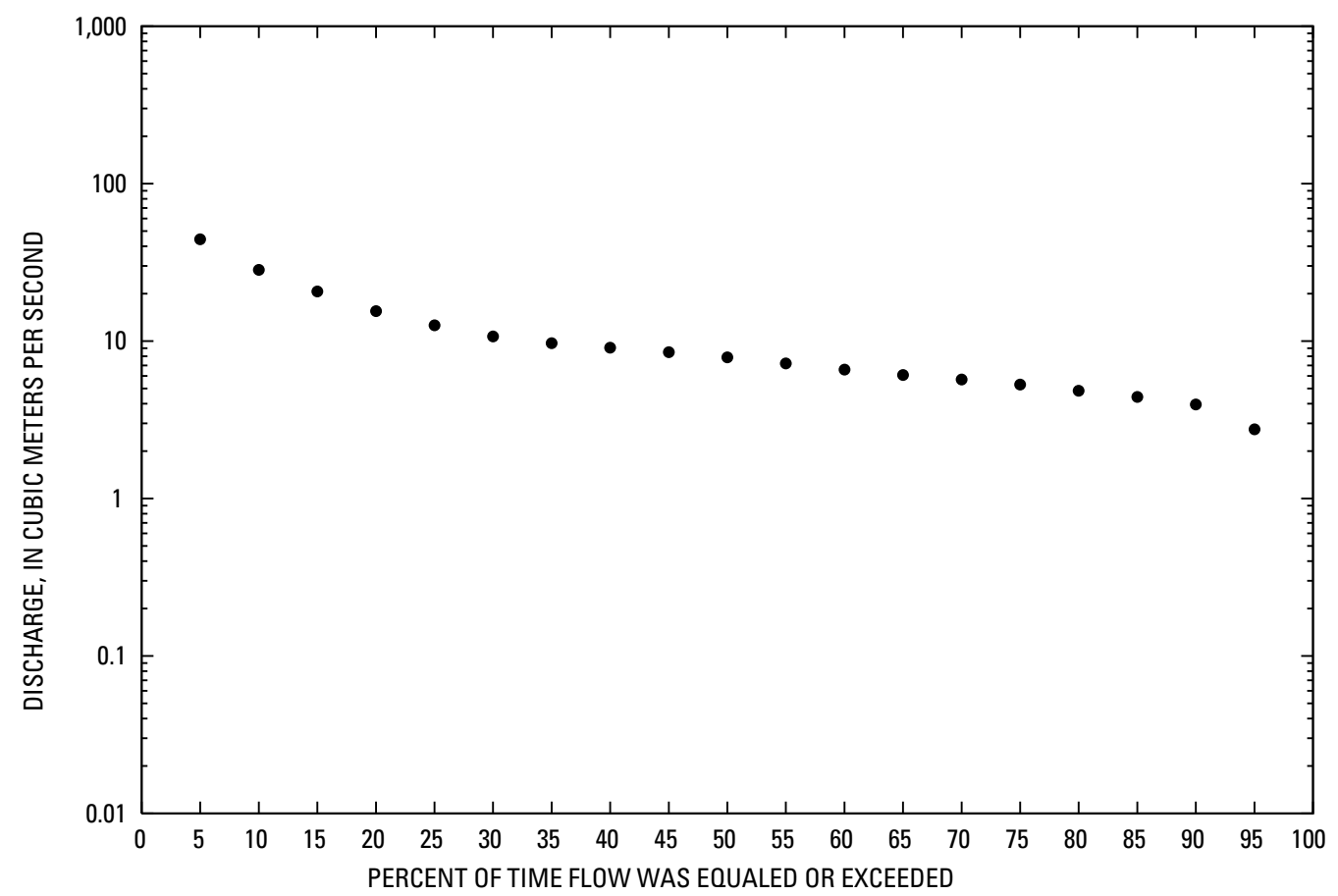




\section{4- 5.L00-4A TIRIN RIVER AT TIRIN, Continued}

Monthly and annual flow duration, in cubic meters per second

[Data were not rounded in accordance with U.S. Geological Survey publication standards]

\begin{tabular}{|c|c|c|c|c|c|c|c|c|c|c|c|c|c|}
\hline $\begin{array}{l}\text { Percentage } \\
\text { of days } \\
\text { discharge } \\
\text { equaled or } \\
\text { exceeded }\end{array}$ & January & February & March & April & May & June & July & August & September & October & November & December & Annual \\
\hline 95 & 6.06 & 7.84 & 10.2 & 9.58 & 2.25 & 2 & 2.19 & 1.76 & 2.24 & 2.78 & 4.17 & 4.4 & 2.75 \\
\hline 90 & 6.82 & 8.2 & 12.2 & 12.1 & 3.04 & 2.16 & 2.84 & 2.16 & 2.64 & 3.23 & 4.71 & 5.69 & 3.96 \\
\hline 80 & 7.61 & 8.59 & 13.7 & 19.9 & 7.38 & 4.56 & 4.1 & 3.78 & 3.42 & 4.11 & 5.46 & 6.62 & 4.83 \\
\hline 75 & 7.81 & 8.79 & 15.3 & 22.1 & 7.96 & 4.88 & 4.32 & 3.99 & 3.95 & 4.27 & 5.69 & 6.97 & 5.29 \\
\hline 70 & 8 & 9.09 & 16.5 & 23.7 & 8.38 & 5.29 & 4.53 & 4.13 & 4.01 & 4.38 & 5.99 & 7.18 & 5.7 \\
\hline 65 & 8.19 & 9.89 & 17.7 & 25 & 8.94 & 5.5 & 4.74 & 4.28 & 4.07 & 4.53 & 6.2 & 7.36 & 6.09 \\
\hline 60 & 8.38 & 10.3 & 19 & 26.8 & 9.77 & 5.74 & 5.12 & 4.46 & 4.13 & 4.79 & 6.33 & 7.54 & 6.59 \\
\hline 45 & 8.81 & 11.5 & 23.6 & 38.7 & 12.2 & 6.43 & 5.72 & 5.26 & 4.31 & 5.16 & 6.83 & 8.31 & 8.51 \\
\hline 40 & 8.93 & 11.9 & 24.8 & 42.1 & 13.4 & 6.61 & 5.87 & 5.46 & 4.36 & 5.27 & 6.95 & 8.5 & 9.09 \\
\hline 35 & 9.09 & 12.4 & 25.9 & 44.9 & 14.7 & 6.83 & 6.01 & 5.63 & 4.49 & 5.4 & 7.13 & 8.59 & 9.71 \\
\hline 30 & 9.48 & 13.2 & 27.6 & 47.8 & 16.2 & 7.06 & 6.16 & 5.79 & 4.63 & 5.56 & 7.35 & 8.69 & 10.7 \\
\hline 25 & 9.88 & 13.9 & 30.9 & 51 & 19.1 & 7.77 & 6.54 & 5.95 & 4.83 & 5.76 & 7.63 & 8.78 & 12.6 \\
\hline 20 & 10.3 & 15.1 & 34.7 & 54.3 & 24.6 & 9.17 & 6.92 & 6.34 & 5.2 & 5.94 & 7.94 & 8.94 & 15.5 \\
\hline 15 & 10.8 & 15.8 & 37.2 & 57.7 & 30.8 & 10.3 & 11 & 8.95 & 5.47 & 6.2 & 9.36 & 9.39 & 20.7 \\
\hline 10 & 11.5 & 18 & 41.6 & 69.7 & 40.3 & 12.6 & 13.6 & 9.85 & 6.23 & 7.07 & 10.2 & 10.4 & 28.3 \\
\hline 5 & 15.2 & 21.4 & 52.8 & 93 & 61.5 & 17.9 & 21.5 & 11.1 & 8.77 & 8.7 & 10.5 & 10.9 & 44.3 \\
\hline
\end{tabular}




\section{4- 5.L00-4A TIRIN RIVER AT TIRIN, Continued}

Probability of occurrence of annual high discharges

$\left[\mathrm{m}^{3} / \mathrm{s}\right.$, meters per second; $\mathrm{ng}$, statistic not given]

\begin{tabular}{|c|c|c|c|c|c|c|}
\hline \multirow{2}{*}{$\begin{array}{c}\text { Exceedance } \\
\text { probability }\end{array}$} & \multirow{2}{*}{$\begin{array}{c}\text { Recurrence } \\
\text { interval } \\
\text { (years) }\end{array}$} & \multirow{2}{*}{$\begin{array}{c}\text { Maximum } \\
\text { instantaneous } \\
\left(\mathrm{m}^{3} / \mathrm{s}\right)\end{array}$} & \multicolumn{4}{|c|}{ Maximum mean discharge } \\
\hline & & & 3-day period & 7-day period & 15-day period & 30-day period \\
\hline 0.99 & 1.01 & 16.8 & ${ }^{17} 7.56$ & 8.67 & 8.32 & 7.58 \\
\hline 0.95 & 1.05 & 34.8 & 15.8 & 15.5 & 14.7 & 13.6 \\
\hline 0.90 & 1.11 & 49.6 & 22.5 & 20.6 & 19.5 & 18.1 \\
\hline 0.80 & 1.25 & 74.0 & 33.1 & 28.7 & 26.9 & 24.9 \\
\hline 0.50 & 2 & 145 & 62.1 & 51.0 & 46.8 & 42.6 \\
\hline 0.20 & 5 & 255 & 102 & 84.6 & 75.8 & 66.2 \\
\hline 0.10 & 10 & 329 & 125 & 107 & 94.8 & 80.5 \\
\hline 0.04 & 25 & 418 & 150 & 136 & 118 & 96.8 \\
\hline 0.02 & 50 & 481 & 167 & 156 & 134 & 108 \\
\hline 0.01 & 100 & 540 & 181 & 176 & 150 & 118 \\
\hline 0.005 & 200 & 596 & ${ }^{1} 193$ & 195 & 165 & 127 \\
\hline 0.002 & 500 & 665 & ng & ng & ng & ng \\
\hline
\end{tabular}

'Data does not fit log-Pearson Type III curve, use with caution.

\section{4- 5.L00-4A TIRIN RIVER AT TIRIN, Continued}

Probability of occurrence of annual low discharges

$\left[\mathrm{m}^{3} / \mathrm{s}\right.$, meters per second]

\begin{tabular}{|c|c|c|c|c|c|c|c|c|c|c|}
\hline \multirow{3}{*}{$\begin{array}{c}\text { Nonexceedance } \\
\text { probability }\end{array}$} & \multirow{3}{*}{$\begin{array}{l}\text { Recurrence } \\
\text { interval } \\
\text { (years) }\end{array}$} & \multicolumn{9}{|c|}{ Minimum mean discharge $\left(\mathrm{m}^{3} / \mathrm{s}\right)$} \\
\hline & & \multicolumn{9}{|c|}{ Number of consecutive days } \\
\hline & & 1 & 3 & 7 & 14 & 30 & 60 & 90 & 120 & 183 \\
\hline 0.05 & 20 & 1.70 & 1.78 & 1.82 & 1.84 & 1.90 & 2.06 & 2.13 & 2.18 & 2.44 \\
\hline 0.10 & 10 & 2.04 & 2.13 & 2.17 & 2.22 & 2.28 & 2.46 & 2.62 & 2.70 & 3.05 \\
\hline 0.20 & 5 & 2.53 & 2.62 & 2.67 & 2.75 & 2.82 & 3.02 & 3.30 & 3.42 & 3.89 \\
\hline 0.50 & 2 & 3.73 & 3.82 & 3.89 & 4.02 & 4.10 & 4.36 & 4.86 & 5.07 & 5.78 \\
\hline
\end{tabular}




\section{4- 5.L00-4A TIRIN RIVER AT TIRIN, Continued}

\section{Probability of occurrence of seasonal low discharges}

[ $\mathrm{m}^{3} / \mathrm{s}$, meters per second]

\begin{tabular}{|c|c|c|c|c|c|c|c|c|c|}
\hline \multirow{3}{*}{$\begin{array}{c}\text { Nonexceedance } \\
\text { probability }\end{array}$} & \multirow{3}{*}{$\begin{array}{c}\text { Recurrence } \\
\text { interval } \\
\text { (years) }\end{array}$} & \multicolumn{8}{|c|}{ Minimum mean discharge $\left(\mathrm{m}^{3} / \mathrm{s}\right)$} \\
\hline & & \multicolumn{8}{|c|}{ Number of consecutive days } \\
\hline & & 1 & 7 & 14 & 30 & 1 & 7 & 14 & 30 \\
\hline & & \multicolumn{4}{|c|}{ December-January-February } & \multicolumn{4}{|c|}{ March-April-May } \\
\hline 0.05 & 20 & 4.52 & 4.59 & 4.66 & 4.78 & 2.22 & 2.38 & 2.48 & 2.86 \\
\hline 0.10 & 10 & 5.05 & 5.16 & 5.30 & 5.52 & 3.18 & 3.40 & 3.57 & 4.24 \\
\hline 0.20 & 5 & 5.71 & 5.87 & 6.09 & 6.43 & 4.62 & 4.93 & 5.28 & 6.53 \\
\hline \multirow[t]{2}{*}{0.50} & 2 & 7.04 & 7.28 & 7.61 & 8.04 & 7.94 & 8.54 & 9.63 & 13.0 \\
\hline & & \multicolumn{4}{|c|}{ June-July-August } & \multicolumn{4}{|c|}{ September-October-November } \\
\hline 0.05 & 20 & 1.79 & 1.94 & 1.97 & 2.04 & 2.08 & 2.20 & 2.32 & 2.44 \\
\hline 0.10 & 10 & 2.20 & 2.38 & 2.44 & 2.53 & 2.39 & 2.50 & 2.61 & 2.73 \\
\hline 0.20 & 5 & 2.79 & 3.01 & 3.11 & 3.23 & 2.84 & 2.94 & 3.04 & 3.14 \\
\hline 0.50 & 2 & 4.24 & 4.50 & 4.64 & 4.86 & 3.91 & 3.99 & 4.07 & 4.16 \\
\hline
\end{tabular}




\section{4- 5.L00-4A TIRIN RIVER AT TIRIN, Continued}

\section{Annual peak discharge and corresponding gage height}

[m³/s, meters per second; --, no data]

\begin{tabular}{|c|c|c|c|c|c|c|c|}
\hline $\begin{array}{l}\text { Water } \\
\text { year }\end{array}$ & Date & $\begin{array}{c}\text { Gage } \\
\text { height } \\
\text { (meters) }\end{array}$ & $\begin{array}{c}\text { Peak } \\
\text { discharge } \\
\left(\mathrm{m}^{3} / \mathrm{s}\right)\end{array}$ & $\begin{array}{c}\text { Water } \\
\text { year }\end{array}$ & Date & $\begin{array}{c}\text { Gage } \\
\text { height } \\
\text { (meters) }\end{array}$ & $\begin{array}{c}\text { Peak } \\
\text { discharge } \\
\left(\mathrm{m}^{3} / \mathrm{s}\right)\end{array}$ \\
\hline
\end{tabular}

Annual peak discharge, by year

\begin{tabular}{|c|c|c|c|c|c|c|c|}
\hline 1970 & 23-Aug & -- & 94.2 & 1975 & 17-May & -- & 127 \\
\hline 1971 & 25-Feb & -- & 30.7 & 1976 & 22-Jul & -- & 325 \\
\hline 1972 & 27-Apr & -- & 160 & 1977 & 4-Apr & -- & 66.2 \\
\hline 1973 & 19-Jul & -- & 280 & 1978 & 4-Jul & -- & 318 \\
\hline 1974 & 7-Apr & -- & 100 & 1979 & 30-Mar & -- & 171 \\
\hline
\end{tabular}

\begin{tabular}{rrrrrrrr}
1976 & 22-Jul & -- & 325 & 1975 & 17-May & -- & 127 \\
1978 & 4-Jul & -- & 318 & 1974 & $7-A p r$ & -- & 100 \\
1973 & 19-Jul & -- & 280 & 1970 & $23-A u g$ & -- & 94.2 \\
1979 & 30-Mar & -- & 171 & 1977 & $4-A p r$ & -- & 66.2 \\
1972 & 27-Apr & -- & 160 & 1971 & $25-F e b$ & -- & 30.7 \\
\hline
\end{tabular}




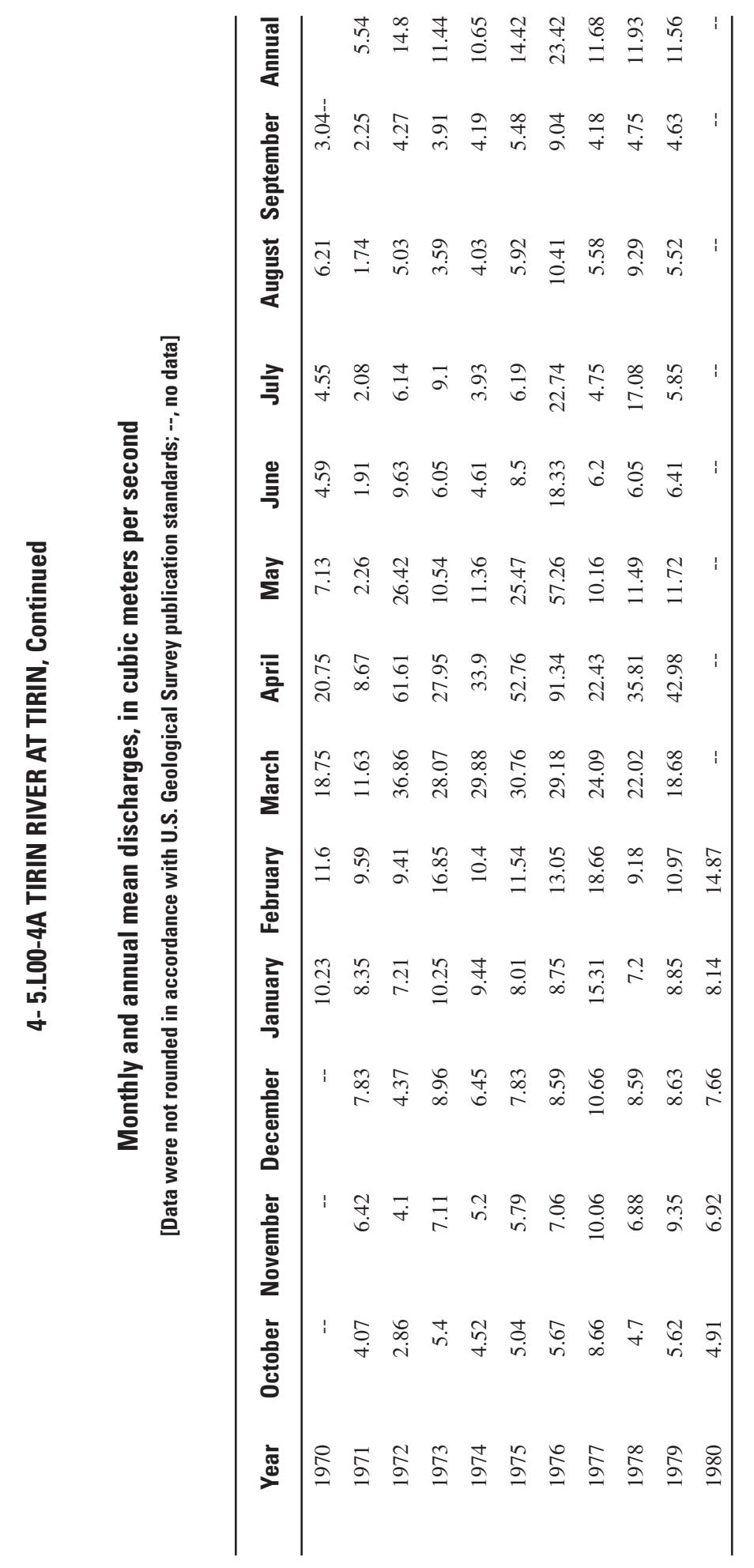




\section{4- 5.LO0-8A TIRIN RIVER AT UROSGAN}

\section{(U.S. Geological Survey identification number: 325800066390000)}

LOCATION: Lat $32^{\circ} 58^{\prime} N$., long 66³9'E.

DRAINAGE AREA: $1,060 \mathrm{~km}^{2}$.

ALTITUDE: 2,028 meters plus mean sea level.

PERIOD OF RECORD: April 1, 1970 to February 19, 1980.

GAGE: --

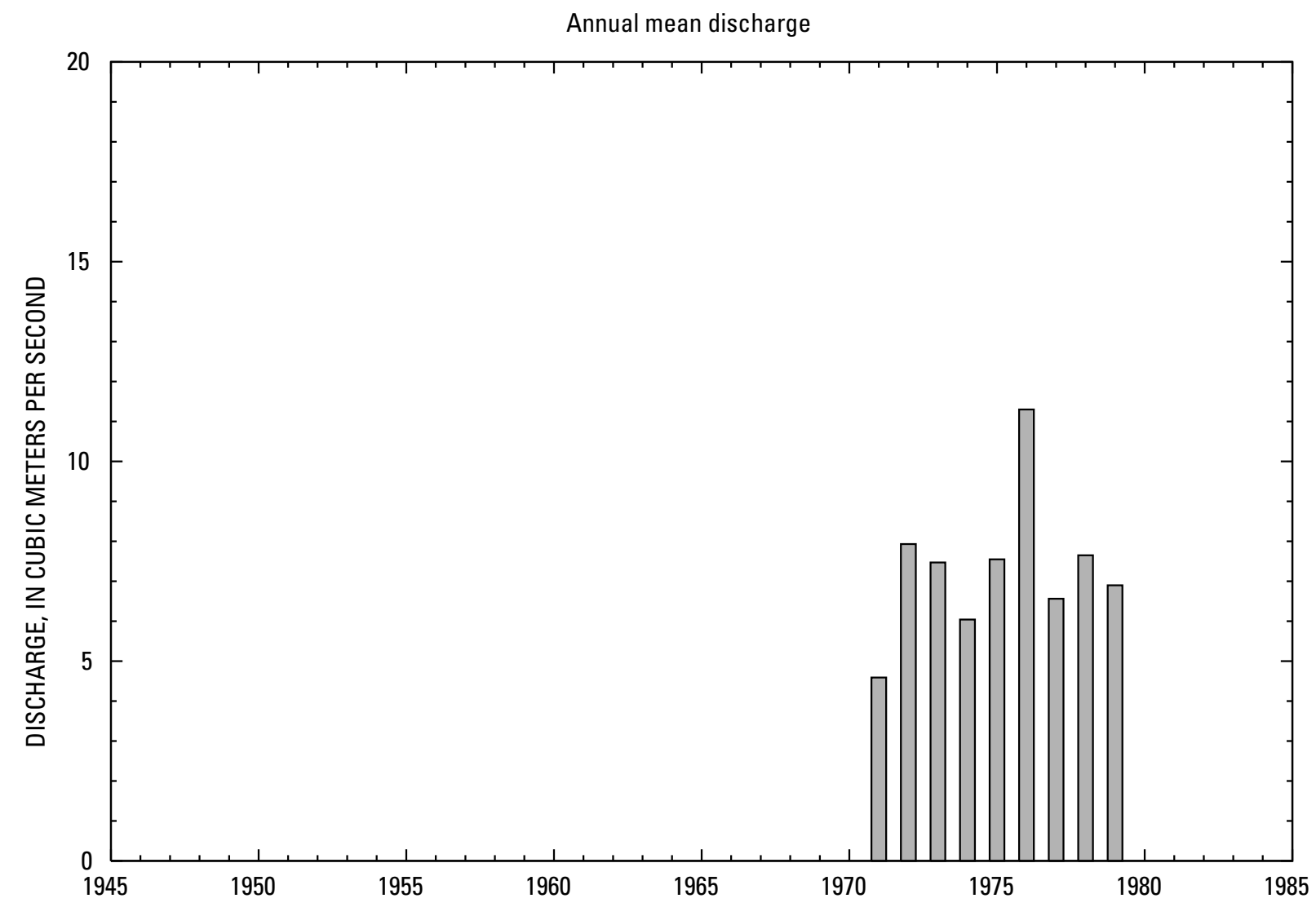




\section{4- 5.L00-8A TIRIN RIVER AT UROSGAN, Continued}

\section{Statistics of monthly and annual mean discharges}

$\left[\mathrm{m}^{3} / \mathrm{s}\right.$, meters per second]

\begin{tabular}{|c|c|c|c|c|c|c|c|c|}
\hline \multirow[b]{2}{*}{ Month } & \multicolumn{2}{|c|}{ Maximum } & \multicolumn{2}{|c|}{ Minimum } & \multicolumn{4}{|c|}{ Mean } \\
\hline & $\begin{array}{c}\text { Discharge } \\
\left(\mathrm{m}^{3} / \mathrm{s}\right)\end{array}$ & $\begin{array}{c}\text { Water year } \\
\text { of } \\
\text { occurrence }\end{array}$ & $\begin{array}{c}\text { Discharge } \\
\left(\mathrm{m}^{3} / \mathrm{s}\right)\end{array}$ & $\begin{array}{c}\text { Water year } \\
\text { of } \\
\text { occurrence }\end{array}$ & $\begin{array}{c}\text { Discharge } \\
\left(\mathrm{m}^{3} / \mathrm{s}\right)\end{array}$ & $\begin{array}{c}\text { Standard } \\
\text { deviation } \\
\left(\mathrm{m}^{3} / \mathrm{s}\right)\end{array}$ & $\begin{array}{c}\text { Coefficient } \\
\text { of } \\
\text { variation }\end{array}$ & $\begin{array}{c}\text { Percentage } \\
\text { of annual } \\
\text { discharge }\end{array}$ \\
\hline October & 7.03 & 1977 & 3.08 & 1975 & 4.38 & 1.27 & 0.29 & 5.03 \\
\hline November & 6.83 & 1977 & 3.19 & 1975 & 5.29 & 1.10 & 0.21 & 6.08 \\
\hline December & 6.61 & 1973 & 3.75 & 1975 & 5.45 & 0.99 & 0.18 & 6.26 \\
\hline January & 7.29 & 1973 & 4.13 & 1975 & 5.53 & 0.96 & 0.17 & 6.36 \\
\hline February & 7.96 & 1973 & 4.55 & 1975 & 6.12 & 1.06 & 0.17 & 7.04 \\
\hline March & 14.8 & 1972 & 6.83 & 1979 & 10.6 & 2.65 & 0.25 & 12.2 \\
\hline April & 29.7 & 1976 & 6.49 & 1971 & 16.8 & 7.59 & 0.45 & 19.3 \\
\hline May & 22.5 & 1976 & 2.62 & 1971 & 9.71 & 5.38 & 0.55 & 11.2 \\
\hline June & 13.2 & 1976 & 2.54 & 1971 & 6.31 & 2.77 & 0.44 & 7.25 \\
\hline July & 15.2 & 1976 & 2.71 & 1971 & 6.60 & 3.98 & 0.60 & 7.58 \\
\hline August & 9.33 & 1976 & 2.63 & 1971 & 5.74 & 2.22 & 0.39 & 6.60 \\
\hline September & 7.93 & 1976 & 2.52 & 1971 & 4.44 & 1.57 & 0.35 & 5.11 \\
\hline Annual & 11.3 & 1976 & 4.59 & 1971 & 7.33 & 1.81 & 0.25 & 100 \\
\hline
\end{tabular}

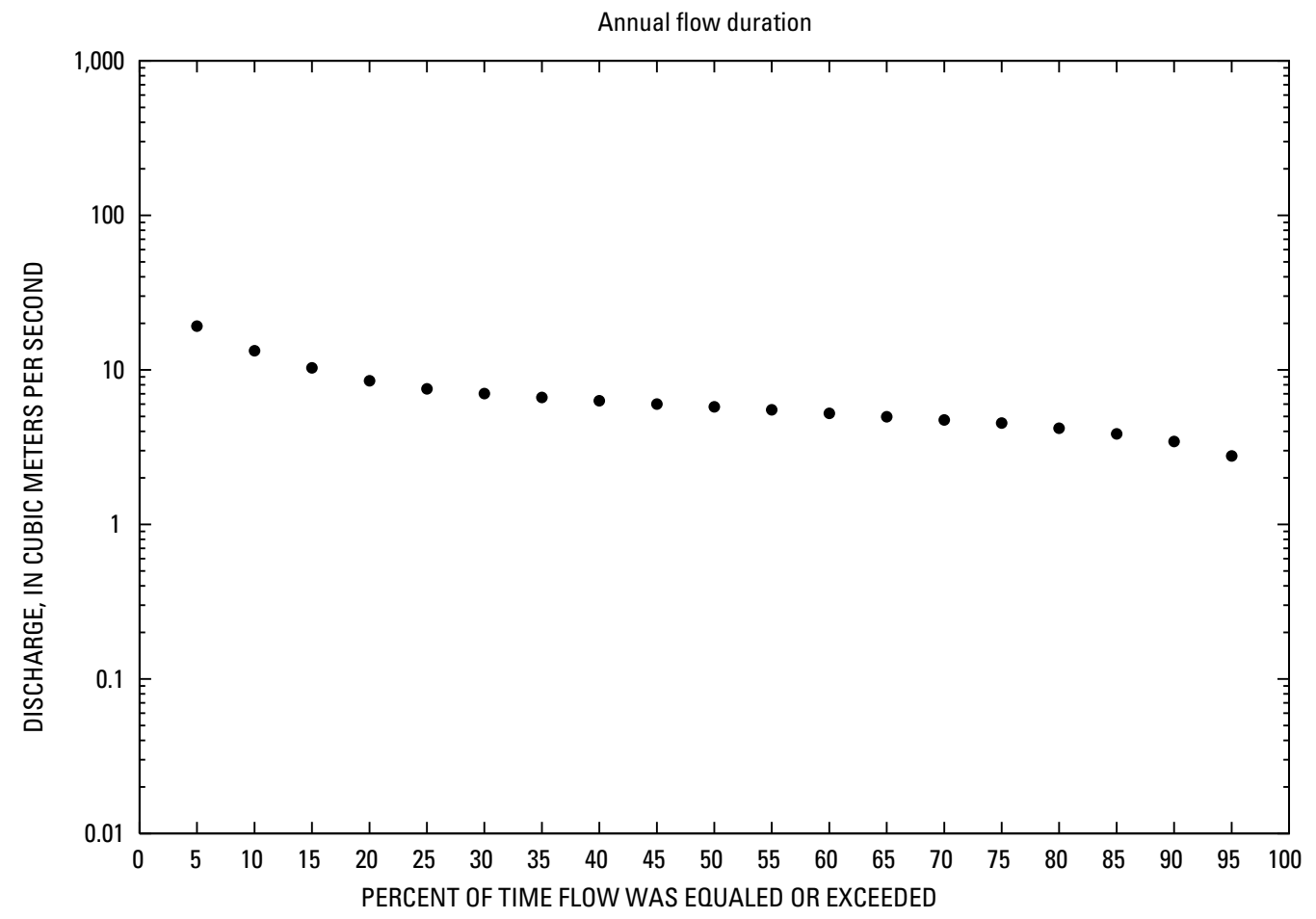




\section{4- 5.LO0-8A TIRIN RIVER AT UROSGAN, Continued}

\section{Monthly and annual flow duration, in cubic meters per second}

[Data were not rounded in accordance with U.S. Geological Survey publication standards]

\begin{tabular}{|c|c|c|c|c|c|c|c|c|c|c|c|c|c|}
\hline $\begin{array}{l}\text { Percentage } \\
\text { of days } \\
\text { discharge } \\
\text { equaled or } \\
\text { exceeded }\end{array}$ & January & February & March & April & May & June & July & August & September & October & November & December & Annual \\
\hline 95 & 4.12 & 4.4 & 5.16 & 6.83 & 2.59 & 2.42 & 2.64 & 2.71 & 2.53 & 2.78 & 3.19 & 3.44 & 2.77 \\
\hline 90 & 4.29 & 4.67 & 6.44 & 8.18 & 3.18 & 3.02 & 2.79 & 2.95 & 2.61 & 2.85 & 3.75 & 4.07 & 3.44 \\
\hline 85 & 4.38 & 4.91 & 6.8 & 8.59 & 6.09 & 4.38 & 3.52 & 3.53 & 2.98 & 3.02 & 4.15 & 4.12 & 3.85 \\
\hline 80 & 4.55 & 5.1 & 7.46 & 8.96 & 6.48 & 4.69 & 3.68 & 3.7 & 3.05 & 3.22 & 4.35 & 4.37 & 4.19 \\
\hline 75 & 4.89 & 5.26 & 7.8 & 9.77 & 6.71 & 4.89 & 4.3 & 3.87 & 3.13 & 3.39 & 4.41 & 4.56 & 4.53 \\
\hline 70 & 5.03 & 5.48 & 8.2 & 10.5 & 6.94 & 5.06 & 4.55 & 4.28 & 3.3 & 3.49 & 4.67 & 4.85 & 4.75 \\
\hline 65 & 5.11 & 5.54 & 8.4 & 11.5 & 7.17 & 5.21 & 4.69 & 4.51 & 3.68 & 3.74 & 4.79 & 5.16 & 4.97 \\
\hline 60 & 5.17 & 5.73 & 8.6 & 12.5 & 7.39 & 5.37 & 4.83 & 4.7 & 3.85 & 3.94 & 4.97 & 5.45 & 5.24 \\
\hline 55 & 5.24 & 5.81 & 8.95 & 14.1 & 7.67 & 5.73 & 4.97 & 4.86 & 3.99 & 4.07 & 5.19 & 5.55 & 5.52 \\
\hline 50 & 5.44 & 6.19 & 9.36 & 16 & 8.15 & 5.98 & 5.11 & 5.05 & 4.11 & 4.32 & 5.28 & 5.73 & 5.76 \\
\hline 45 & 5.52 & 6.41 & 9.78 & 17.2 & 8.4 & 6.14 & 5.25 & 5.29 & 4.32 & 4.41 & 5.5 & 5.78 & 6.01 \\
\hline 40 & 5.73 & 6.45 & 10.2 & 18.3 & 8.63 & 6.31 & 5.4 & 5.57 & 4.4 & 4.5 & 5.78 & 5.84 & 6.31 \\
\hline 35 & 5.81 & 6.49 & 10.7 & 19.5 & 8.89 & 6.57 & 5.55 & 5.82 & 4.48 & 4.59 & 5.87 & 6.11 & 6.63 \\
\hline 30 & 6.04 & 6.52 & 11.3 & 20.6 & 10.2 & 6.83 & 5.7 & 5.98 & 4.87 & 4.76 & 6.04 & 6.16 & 7.03 \\
\hline 25 & 6.3 & 6.71 & 12.2 & 21.7 & 11.2 & 7.05 & 5.85 & 6.14 & 5.41 & 4.91 & 6.16 & 6.22 & 7.55 \\
\hline 20 & 6.51 & 7.02 & 13.1 & 22.7 & 12.7 & 7.24 & 7.12 & 6.69 & 5.86 & 5.29 & 6.26 & 6.41 & 8.51 \\
\hline 15 & 6.81 & 7.57 & 14.3 & 23.7 & 14.6 & 7.5 & 8.72 & 8.07 & 6.33 & 5.8 & 6.56 & 6.49 & 10.3 \\
\hline 10 & 6.92 & 7.72 & 17.5 & 25.9 & 17.7 & 9.07 & 12.7 & 9.35 & 6.77 & 6.76 & 6.88 & 6.59 & 13.3 \\
\hline 5 & 7.27 & 7.91 & 21.2 & 35.6 & 23.2 & 0 & 13.5 & 9.86 & 7.67 & 6.96 & 7.02 & 6.74 & 19.2 \\
\hline
\end{tabular}




\section{4- 5.L00-8A TIRIN RIVER AT UROSGAN, Continued}

Probability of occurrence of annual high discharges

$\left[\mathrm{m}^{3} / \mathrm{s}\right.$, meters per second; $\mathrm{ng}$, statistic not given]

\begin{tabular}{|c|c|c|c|c|c|c|}
\hline \multirow{2}{*}{$\begin{array}{l}\text { Exceedance } \\
\text { probability }\end{array}$} & \multirow{2}{*}{$\begin{array}{c}\text { Recurrence } \\
\text { interval } \\
\text { (years) }\end{array}$} & \multirow{2}{*}{$\begin{array}{c}\text { Maximum } \\
\text { instantaneous } \\
\left(\mathrm{m}^{3} / \mathrm{s}\right)\end{array}$} & \multicolumn{4}{|c|}{ Maximum mean discharge } \\
\hline & & & 3-day period & 7-day period & 15-day period & 30-day period \\
\hline 0.99 & 1.01 & 7.10 & ${ }^{1} 5.81$ & ${ }^{1} 6.18$ & ${ }^{1} 6.53$ & ${ }^{1} 6.32$ \\
\hline 0.95 & 1.05 & 10.4 & 9.54 & 19.6 & 9.37 & 8.87 \\
\hline 0.90 & 1.11 & 13.0 & 12.2 & 11.9 & 11.2 & 10.5 \\
\hline 0.80 & 1.25 & 17.3 & 16.1 & 15.3 & 13.9 & 12.9 \\
\hline 0.50 & 2 & 31.4 & 26.2 & 23.4 & 20.5 & 18.4 \\
\hline 0.20 & 5 & 61.8 & 39.9 & 33.7 & 29.1 & 25.7 \\
\hline 0.10 & 10 & 90.8 & 48.5 & 39.9 & 34.6 & 30.2 \\
\hline 0.04 & 25 & 140 & 58.7 & 47.0 & 41.2 & 35.5 \\
\hline 0.02 & 50 & 188 & 65.8 & 51.8 & 45.9 & 39.3 \\
\hline 0.01 & 100 & 248 & 72.5 & 56.2 & 50.4 & 42.9 \\
\hline 0.005 & 200 & 321 & 78.8 & 60.3 & 54.8 & 46.4 \\
\hline 0.002 & 500 & 444 & ng & ng & ng & ng \\
\hline
\end{tabular}

'Data does not fit log-Pearson Type III curve, use with caution.

\section{4- 5.LO0-8A TIRIN RIVER AT UROSGAN, Continued}

Probability of occurrence of annual low discharges

$\left[\mathrm{m}^{3} / \mathrm{s}\right.$, meters per second]

\begin{tabular}{|c|c|c|c|c|c|c|c|c|c|c|}
\hline \multirow{3}{*}{$\begin{array}{c}\text { Nonexceedance } \\
\text { probability }\end{array}$} & \multirow{3}{*}{$\begin{array}{c}\text { Recurrence } \\
\text { interval } \\
\text { (years) }\end{array}$} & \multicolumn{9}{|c|}{ Minimum mean discharge $\left(\mathrm{m}^{3} / \mathrm{s}\right)$} \\
\hline & & \multicolumn{9}{|c|}{ Number of consecutive days } \\
\hline & & 1 & 3 & 7 & 14 & 30 & 60 & 90 & 120 & 183 \\
\hline 0.05 & 20 & ${ }^{1} 2.11$ & 2.09 & 2.10 & 2.20 & 2.34 & 2.50 & 2.59 & 2.60 & 2.74 \\
\hline 0.10 & 10 & 2.29 & 2.30 & 2.32 & 2.41 & 2.56 & 2.77 & 2.92 & 2.97 & 3.16 \\
\hline 0.20 & 5 & 2.56 & 2.60 & 2.62 & 2.71 & 2.88 & 3.13 & 3.35 & 3.47 & 3.71 \\
\hline 0.50 & 2 & 3.28 & 3.37 & 3.42 & 3.50 & 3.69 & 4.00 & 4.30 & 4.51 & 4.80 \\
\hline
\end{tabular}

'Data does not fit log-Pearson Type III curve, use with caution. 


\section{4- 5.L00-8A TIRIN RIVER AT UROSGAN, Continued}

\section{Probability of occurrence of seasonal low discharges}

$\left[\mathrm{m}^{3} / \mathrm{s}\right.$, meters per second]

\begin{tabular}{|c|c|c|c|c|c|c|c|c|c|}
\hline \multirow{3}{*}{$\begin{array}{c}\text { Nonexceedance } \\
\text { probability }\end{array}$} & \multirow{3}{*}{$\begin{array}{c}\text { Recurrence } \\
\text { interval } \\
\text { (years) }\end{array}$} & \multicolumn{8}{|c|}{ Minimum mean discharge $\left(\mathrm{m}^{3} / \mathrm{s}\right)$} \\
\hline & & \multicolumn{8}{|c|}{ Number of consecutive days } \\
\hline & & 1 & 7 & 14 & 30 & 1 & 7 & 14 & 30 \\
\hline & & \multicolumn{4}{|c|}{ December-January-February } & \multicolumn{4}{|c|}{ March-April-May } \\
\hline 0.05 & 20 & 3.26 & 13.25 & 3.36 & 3.64 & 2.75 & 2.75 & 2.78 & 3.01 \\
\hline 0.10 & 10 & 3.60 & 3.62 & 3.70 & 3.94 & 3.52 & 3.56 & 3.60 & 3.94 \\
\hline 0.20 & 5 & 4.04 & 4.08 & 4.15 & 4.32 & 4.45 & 4.60 & 4.70 & 5.24 \\
\hline \multirow[t]{2}{*}{0.50} & 2 & 4.92 & 4.99 & 5.04 & 5.12 & 5.91 & 6.38 & 6.80 & 7.99 \\
\hline & & \multicolumn{4}{|c|}{ June-July-August } & \multicolumn{4}{|c|}{ September-October-November } \\
\hline 0.05 & 20 & 2.52 & 2.55 & 2.56 & 2.68 & 2.14 & 2.14 & 2.28 & 2.38 \\
\hline 0.10 & 10 & 2.83 & 2.87 & 2.89 & 3.04 & 2.31 & 2.34 & 2.46 & 2.58 \\
\hline 0.20 & 5 & 3.26 & 3.32 & 3.36 & 3.55 & 2.57 & 2.62 & 2.73 & 2.88 \\
\hline 0.50 & 2 & 4.35 & 4.42 & 4.52 & 4.75 & 3.29 & 3.39 & 3.47 & 3.66 \\
\hline
\end{tabular}

'Data does not fit log-Pearson Type III curve, use with caution.

\section{4- 5.L00-8A TIRIN RIVER AT UROSGAN, Continued}

\section{Annual peak discharge and corresponding gage height}

$\left[\mathrm{m}^{3} / \mathrm{s}\right.$, meters per second; --, no data]

\begin{tabular}{|c|c|c|c|c|c|c|c|}
\hline $\begin{array}{l}\text { Water } \\
\text { year }\end{array}$ & Date & $\begin{array}{c}\text { Gage } \\
\text { height } \\
\text { (meters) }\end{array}$ & $\begin{array}{c}\text { Peak } \\
\text { discharge } \\
\left(\mathrm{m}^{3} / \mathrm{s}\right)\end{array}$ & $\begin{array}{c}\text { Water } \\
\text { year }\end{array}$ & Date & $\begin{array}{c}\text { Gage } \\
\text { height } \\
\text { (meters) }\end{array}$ & $\begin{array}{c}\text { Peak } \\
\text { discharge } \\
\left(\mathrm{m}^{3} / \mathrm{s}\right)\end{array}$ \\
\hline \multicolumn{8}{|c|}{ Annual peak discharge, by year } \\
\hline 1970 & 22-Aug & & 20.0 & 1975 & 4-Apr & & 37.2 \\
\hline 1971 & 19-Mar & & 10.2 & 1976 & 23-Jul & & 82.6 \\
\hline 1972 & 26-Apr & & 40.0 & 1977 & 29-Mar & & 15.2 \\
\hline 1973 & 18-Jul & & 40.2 & 1978 & 5 -Jul & & 137 \\
\hline 1974 & 23-Mar & & 28.1 & 1979 & 8-Apr & & 27.5 \\
\hline \multicolumn{8}{|c|}{ Annual peak discharge, from highest to lowest } \\
\hline 1978 & 5-Jul & - & 137 & 1974 & 23-Mar & & 28.1 \\
\hline 1976 & 23-Jul & - & 82.6 & 1979 & 8-Apr & & 27.5 \\
\hline 1973 & 18-Jul & - & 40.2 & 1970 & 22-Aug & & 20.0 \\
\hline 1972 & 26-Apr & - & 40.0 & 1977 & 29-Mar & & 15.2 \\
\hline 1975 & 4-Apr & . & 37.2 & 1971 & 19-Mar & & 10.2 \\
\hline
\end{tabular}




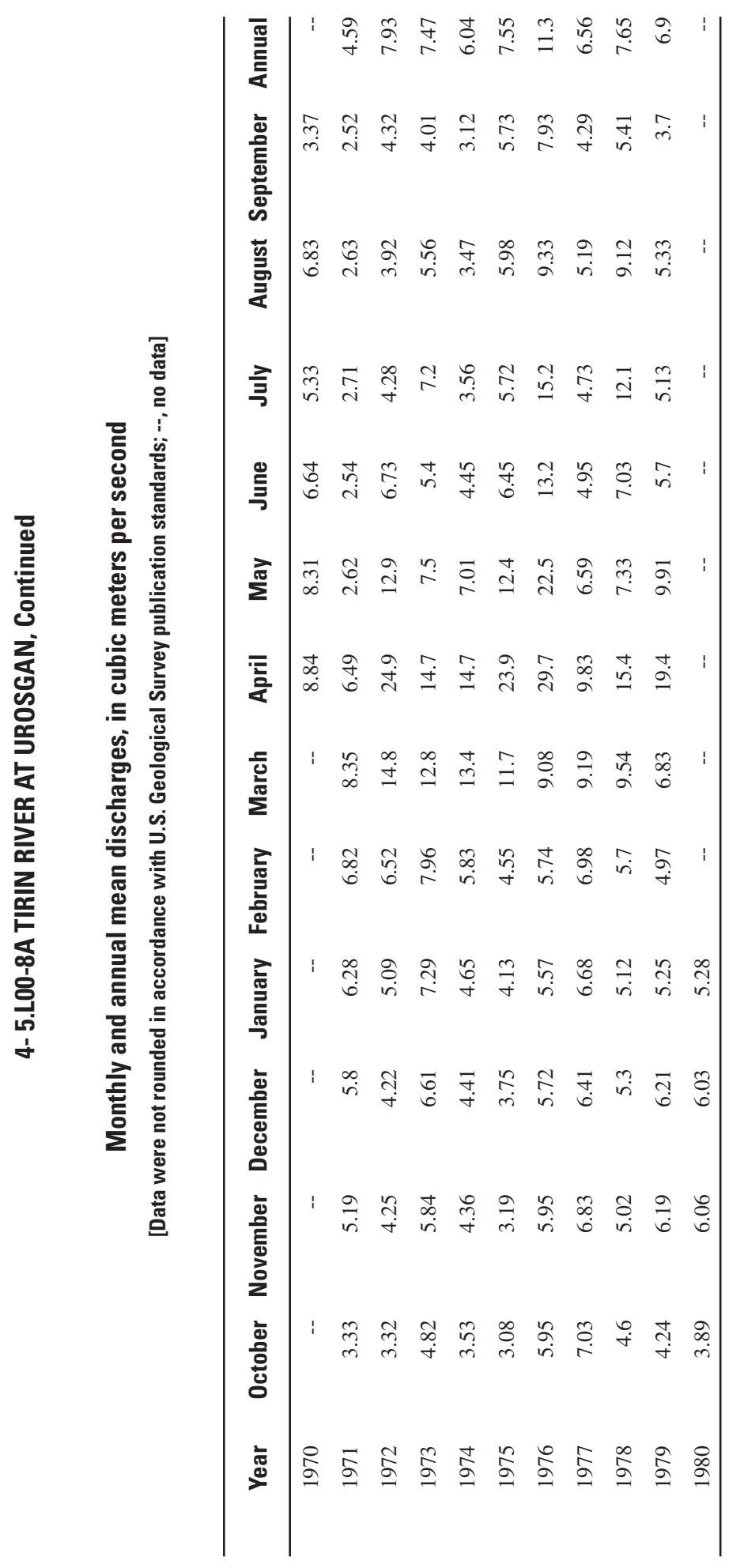




\section{4- 6.R00-1A KAJ RIVER NEAR YAKHDAN}

\section{(U.S. Geological Survey identification number: 325800065300000 )}

LOCATION: Lat $32^{\circ} 58^{\prime} N$., long 65³0'E.

DRAINAGE AREA: $11,590 \mathrm{~km}^{2}$.

ALTITUDE: 1,125 meters plus mean sea level.

PERIOD OF RECORD: October 1, 1973 to March 18, 1980.

GAGE: --

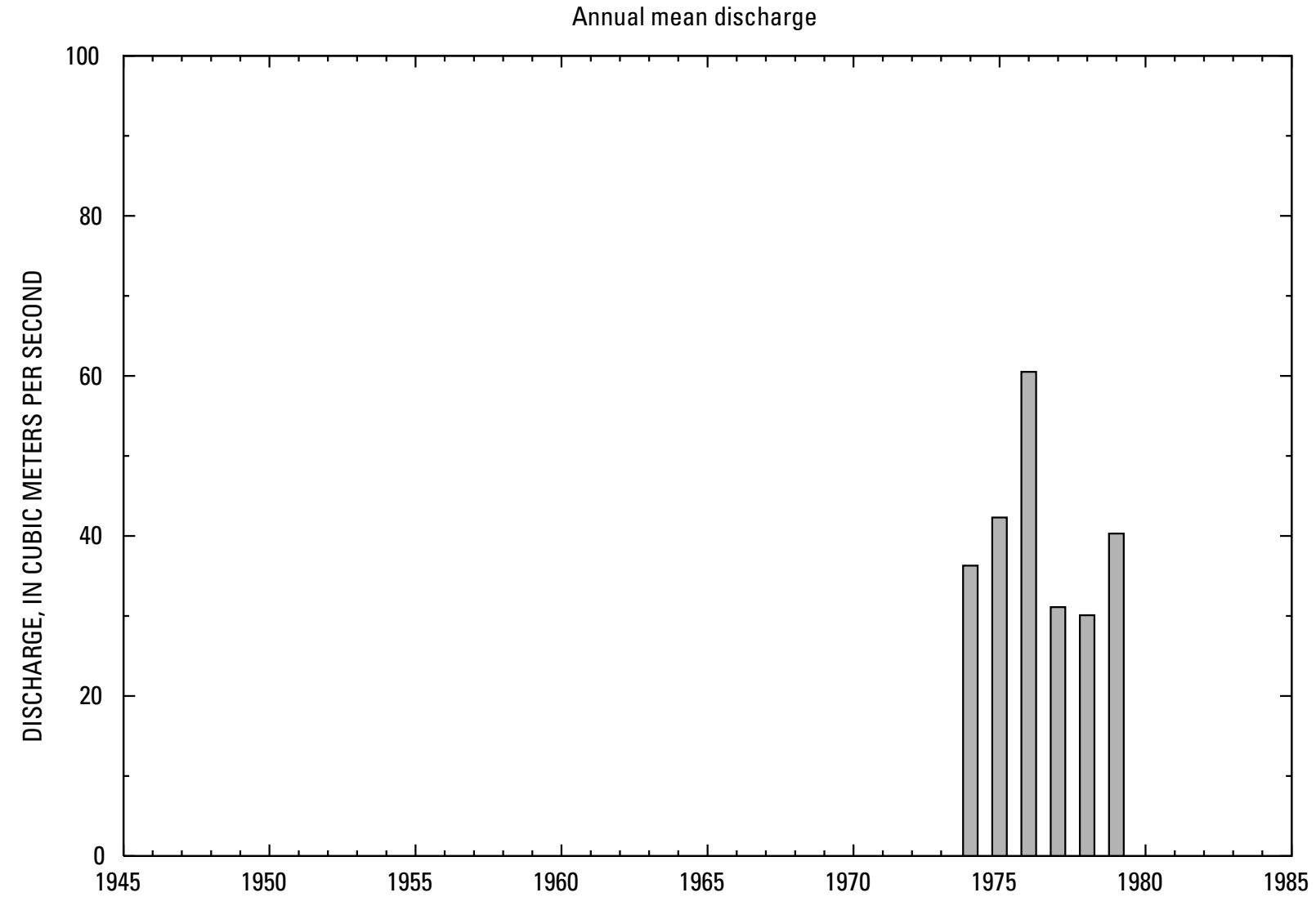




\section{4- 6.R00-1A KAJ RIVER NEAR YAKHDAN, Continued}

\section{Statistics of monthly and annual mean discharges}

$\left[\mathrm{m}^{3} / \mathrm{s}\right.$, meters per second]

\begin{tabular}{|c|c|c|c|c|c|c|c|c|}
\hline \multirow[b]{2}{*}{ Month } & \multicolumn{2}{|c|}{ Maximum } & \multicolumn{2}{|c|}{ Minimum } & \multicolumn{4}{|c|}{ Mean } \\
\hline & $\begin{array}{c}\text { Discharge } \\
\left(\mathrm{m}^{3} / \mathrm{s}\right)\end{array}$ & $\begin{array}{c}\text { Water year } \\
\text { of } \\
\text { occurrence }\end{array}$ & $\begin{array}{c}\text { Discharge } \\
\left(\mathrm{m}^{3} / \mathrm{s}\right)\end{array}$ & $\begin{array}{c}\text { Water year } \\
\text { of } \\
\text { occurrence }\end{array}$ & $\begin{array}{c}\text { Discharge } \\
\left(\mathrm{m}^{3} / \mathrm{s}\right)\end{array}$ & $\begin{array}{c}\text { Standard } \\
\text { deviation } \\
\left(\mathrm{m}^{3} / \mathrm{s}\right)\end{array}$ & $\begin{array}{c}\text { Coefficient } \\
\text { of } \\
\text { variation }\end{array}$ & $\begin{array}{c}\text { Percentage } \\
\text { of annual } \\
\text { discharge }\end{array}$ \\
\hline October & 17.4 & 1980 & 8.36 & 1979 & 10.7 & 3.19 & 0.30 & 2.19 \\
\hline November & 18.6 & 1978 & 10.3 & 1974 & 14.2 & 3.46 & 0.24 & 2.91 \\
\hline December & 17.8 & 1980 & 11.4 & 1974 & 13.7 & 2.11 & 0.15 & 2.80 \\
\hline January & 18.0 & 1977,1980 & 11.0 & 1974 & 14.5 & 2.88 & 0.20 & 2.98 \\
\hline February & 41.4 & 1980 & 17.1 & 1975 & 26.2 & 8.61 & 0.33 & 5.38 \\
\hline March & 116 & 1974 & 60.7 & 1979 & 86.3 & 24.7 & 0.29 & 17.7 \\
\hline April & 287 & 1976 & 115 & 1978 & 171 & 62.3 & 0.36 & 35.1 \\
\hline May & 171 & 1976 & 44.1 & 1978 & 93.1 & 50.3 & 0.54 & 19.1 \\
\hline June & 43.9 & 1976 & 13.4 & 1978 & 26.8 & 11.7 & 0.44 & 5.50 \\
\hline July & 14.9 & 1979 & 7.97 & 1978 & 12.8 & 4.83 & 0.38 & 2.63 \\
\hline August & 14.2 & 1979 & 7.17 & 1974 & 8.96 & 2.62 & 0.29 & 1.84 \\
\hline September & 15.1 & 1979 & 6.53 & 1978 & 9.00 & 3.14 & 0.35 & 1.85 \\
\hline Annual & 60.5 & 1976 & 30.1 & 1978 & 40.1 & 11.1 & 0.28 & 100 \\
\hline
\end{tabular}

Annual flow duration

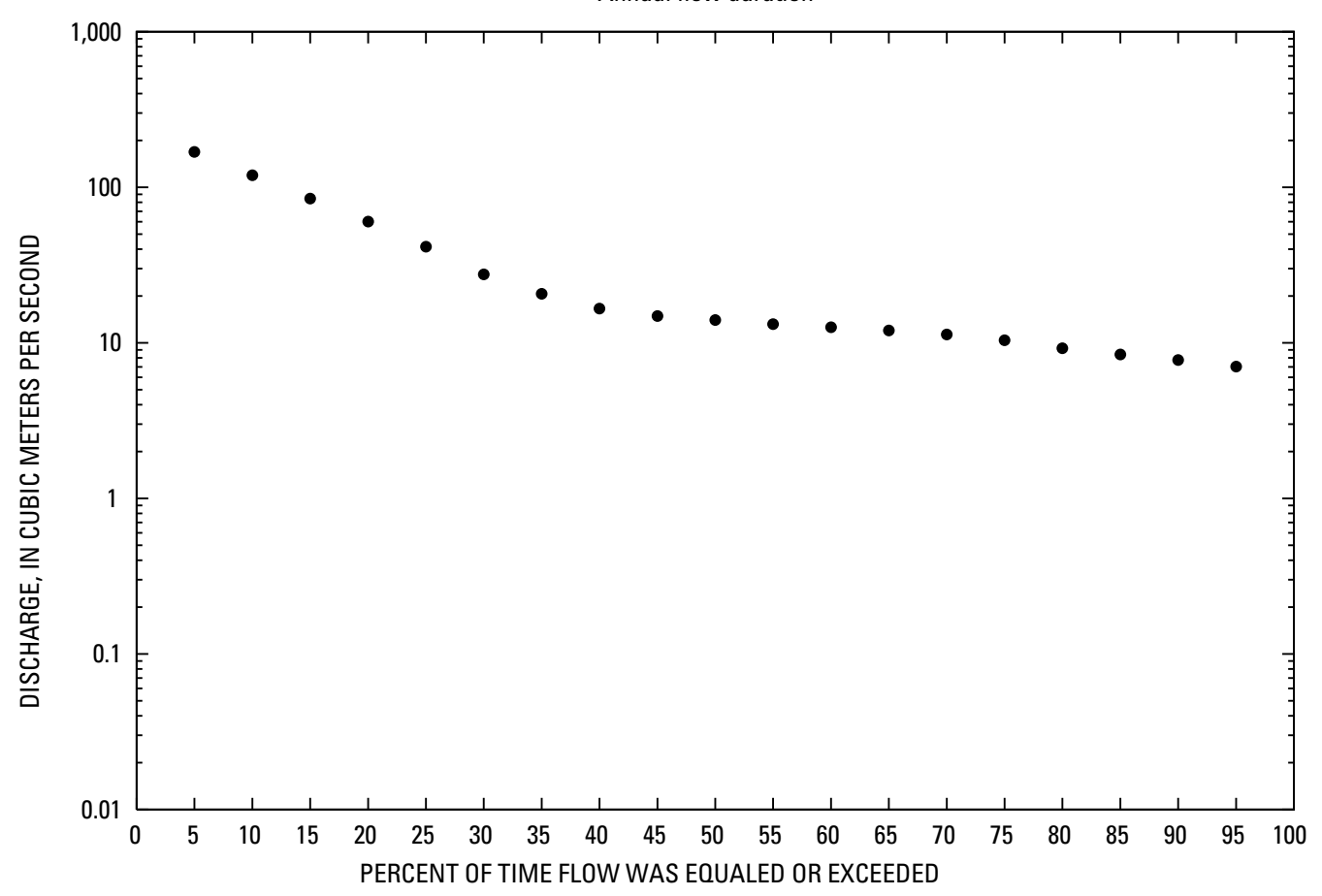




\section{4- 6.RO0-1A KAJ RIVER NEAR YAKHDAN, Continued}

Monthly and annual flow duration, in cubic meters per second

[Data were not rounded in accordance with U.S. Geological Survey publication standards]

\begin{tabular}{|c|c|c|c|c|c|c|c|c|c|c|c|c|c|}
\hline $\begin{array}{l}\text { Percentage } \\
\text { of days } \\
\text { discharge } \\
\text { equaled or } \\
\text { exceeded }\end{array}$ & January & February & March & April & May & June & July & August & September & October & November & December & Annual \\
\hline 95 & 10.2 & 13.3 & 28.5 & 90.6 & 30.3 & 11.4 & 6.7 & 6.41 & 5.76 & 7.48 & 9.61 & 11 & 7.05 \\
\hline 90 & 10.9 & 14 & 31.6 & 98.2 & 37.2 & 12 & 7.74 & 6.67 & 6.25 & 7.91 & 10.1 & 11.2 & 7.74 \\
\hline 80 & 11.3 & 14.8 & 45.9 & 110 & 44.5 & 13.6 & 8.69 & 7.31 & 6.94 & 8.49 & 10.9 & 11.6 & 9.24 \\
\hline 75 & 11.9 & 15.3 & 53.2 & 118 & 49.4 & 15.6 & 9.07 & 7.47 & 7.14 & 8.59 & 11.2 & 11.9 & 10.4 \\
\hline 70 & 12.4 & 16.1 & 58.5 & 127 & 51.9 & 17.5 & 9.4 & 7.8 & 7.54 & 8.68 & 11.4 & 12 & 11.3 \\
\hline 65 & 12.7 & 17.2 & 62 & 136 & 58.5 & 19.2 & 9.72 & 7.85 & 7.67 & 8.8 & 11.6 & 12.2 & 12 \\
\hline 60 & 13 & 18 & 65.8 & 142.2 & 63.4 & 20 & 10 & 7.9 & 7.75 & 8.95 & 11.9 & 12.3 & 12.6 \\
\hline 45 & 13.8 & 21 & 79.9 & 166 & 79.4 & 23.8 & 12.3 & 8.07 & 8.05 & 9.69 & 12.7 & 13 & 14.9 \\
\hline 40 & 14 & 22.9 & 83.1 & 170.2 & 91 & 25.1 & 13 & 8.19 & 8.19 & 10.2 & 13.3 & 13.3 & 16.6 \\
\hline 35 & 14.2 & 24.9 & 86.8 & 174.5 & 101 & 28.3 & 13.5 & 8.31 & 8.35 & 10.7 & 13.7 & 13.5 & 20.7 \\
\hline 30 & 14.8 & 26.8 & 91 & 182.3 & 119.3 & 30.4 & 14.1 & 8.47 & 8.81 & 11.2 & 14.1 & 13.8 & 27.6 \\
\hline 25 & 15.8 & 29 & 105.2 & 198.4 & 129.8 & 33.7 & 14.6 & 9.11 & 9.01 & 11.4 & 14.8 & 14.4 & 41.5 \\
\hline 20 & 16.5 & 34 & 112.2 & 215.5 & 144 & 38.3 & 15.2 & 10.3 & 10.5 & 12.1 & 16 & 15.2 & 60.2 \\
\hline 15 & 17.1 & 38.1 & 126.7 & 229.8 & 160.8 & 44.4 & 15.8 & 12.4 & 14.1 & 14.2 & 17.5 & 16.4 & 84.6 \\
\hline 10 & 18.7 & 45.7 & 170.2 & 273.2 & 184.9 & 49.4 & 16.8 & 13.8 & 14.5 & 16.9 & 19 & 17 & 119.4 \\
\hline 5 & 22 & 51.5 & 192.9 & 330 & 211.4 & 57.6 & 20.2 & 0 & 15.7 & 17.5 & 21.5 & 18.5 & 169 \\
\hline
\end{tabular}




\section{4- 6.RO0-1A KAJ RIVER NEAR YAKHDAN, Continued}

Probability of occurrence of annual high discharges

[ $\mathrm{m}^{3} / \mathrm{s}$, meters per second; $\mathbf{n g}$, statistic not given]

\begin{tabular}{|c|c|c|c|c|c|c|}
\hline \multirow{2}{*}{$\begin{array}{c}\text { Exceedance } \\
\text { probability }\end{array}$} & \multirow{2}{*}{$\begin{array}{c}\text { Recurrence } \\
\text { interval } \\
\text { (years) }\end{array}$} & \multirow{2}{*}{$\begin{array}{c}\text { Maximum } \\
\text { instantaneous } \\
\left(\mathrm{m}^{3} / \mathbf{s}\right)\end{array}$} & \multicolumn{4}{|c|}{ Maximum mean discharge } \\
\hline & & & 3-day period & 7-day period & 15-day period & 30-day period \\
\hline 0.99 & 1.01 & 146 & ${ }^{2} 126$ & 129 & 115 & 90.2 \\
\hline 0.95 & 1.05 & 168 & 142 & 140 & 124 & 104 \\
\hline 0.90 & 1.11 & 183 & 154 & 148 & 132 & 113 \\
\hline 0.80 & 1.25 & 206 & 172 & 162 & 145 & 127 \\
\hline 0.50 & 2 & 268 & 224 & 205 & 183 & 164 \\
\hline 0.20 & 5 & 369 & 314 & 285 & 253 & 223 \\
\hline 0.10 & 10 & 447 & 387 & 354 & 312 & 269 \\
\hline 0.04 & 25 & 560 & 496 & 460 & 402 & 333 \\
\hline 0.02 & 50 & 654 & 590 & 555 & 482 & 385 \\
\hline 0.01 & 100 & 757 & 670 & 667 & 576 & 443 \\
\hline 0.005 & 200 & 871 & 818 & 797 & 684 & 506 \\
\hline 0.002 & 500 & 1,040 & ng & ng & ng & ng \\
\hline
\end{tabular}

'Less than 10 years of data used.

${ }^{2}$ Data does not fit log-Pearson Type III curve, use with caution.

\section{4- 6.R00-1A KAJ RIVER NEAR YAKHDAN, Continued}

Probability of occurrence of annual low discharges

$\left[\mathrm{m}^{3} / \mathrm{s}\right.$, meters per second]

\begin{tabular}{|c|c|c|c|c|c|c|c|c|c|c|}
\hline \multirow{3}{*}{$\begin{array}{c}\text { Nonexceedance } \\
\text { probability }\end{array}$} & \multirow{3}{*}{$\begin{array}{c}\text { Recurrence } \\
\text { interval } \\
\text { (years) }\end{array}$} & \multicolumn{9}{|c|}{ Minimum mean discharge $\left(\mathrm{m}^{3} / \mathbf{s}\right)$} \\
\hline & & \multicolumn{9}{|c|}{ Number of consecutive days } \\
\hline & & 1 & 3 & 7 & 14 & 30 & 60 & 90 & 120 & 183 \\
\hline 0.05 & 20 & 4.91 & 5.05 & 5.12 & 5.40 & 5.91 & 6.44 & 6.69 & 7.24 & 8.85 \\
\hline 0.10 & 10 & 5.31 & 5.43 & 5.54 & 5.80 & 6.22 & 6.72 & 6.96 & 7.46 & 9.10 \\
\hline 0.20 & 5 & 5.80 & 5.88 & 6.04 & 6.26 & 6.60 & 7.06 & 7.32 & 7.79 & 9.47 \\
\hline 0.50 & 2 & 6.71 & 6.75 & 6.94 & 7.09 & 7.30 & 7.75 & 8.09 & 8.67 & 10.4 \\
\hline
\end{tabular}




\section{4- 6.R00-1A KAJ RIVER NEAR YAKHDAN, Continued}

\section{Probability of occurrence of seasonal low discharges}

$\left[\mathrm{m}^{3} / \mathrm{s}\right.$, meters per second]

\begin{tabular}{|c|c|c|c|c|c|c|c|c|c|}
\hline \multirow{3}{*}{$\begin{array}{c}\text { Nonexceedance } \\
\text { probability }\end{array}$} & \multirow{3}{*}{$\begin{array}{c}\text { Recurrence } \\
\text { interval } \\
\text { (years) }\end{array}$} & \multicolumn{8}{|c|}{ Minimum mean discharge $\left(\mathrm{m}^{3} / \mathrm{s}\right)$} \\
\hline & & \multicolumn{8}{|c|}{ Number of consecutive days } \\
\hline & & 1 & 7 & 14 & 30 & 1 & 7 & 14 & 30 \\
\hline & & \multicolumn{4}{|c|}{ December-January-February } & \multicolumn{4}{|c|}{ March-April-May } \\
\hline 0.05 & 20 & 8.17 & 9.45 & 10.6 & 10.9 & 21.4 & 21.6 & 24.0 & 42.0 \\
\hline 0.10 & 10 & 8.60 & 9.74 & 10.7 & 11.1 & 22.6 & 24.7 & 27.3 & 44.0 \\
\hline 0.20 & 5 & 9.19 & 10.2 & 11.0 & 11.4 & 24.2 & 28.8 & 31.9 & 47.3 \\
\hline \multirow[t]{2}{*}{0.50} & 2 & 10.6 & 11.4 & 11.9 & 12.4 & 27.7 & 38.3 & 42.1 & 58.0 \\
\hline & & \multicolumn{4}{|c|}{ June-July-August } & \multicolumn{4}{|c|}{ September-October-November } \\
\hline 0.05 & 20 & 5.41 & 5.92 & 6.13 & 6.53 & 5.08 & 5.30 & 5.55 & 6.26 \\
\hline 0.10 & 10 & 5.62 & 6.07 & 6.26 & 6.67 & 5.28 & 5.55 & 5.78 & 6.43 \\
\hline 0.20 & 5 & 5.97 & 6.35 & 6.50 & 6.95 & 5.64 & 5.96 & 6.17 & 6.75 \\
\hline 0.50 & 2 & 7.04 & 7.28 & 7.41 & 7.94 & 6.89 & 7.32 & 7.48 & 7.93 \\
\hline
\end{tabular}

\section{4- 6.RO0-1A KAJ RIVER NEAR YAKHDAN, Continued}

\section{Annual peak discharge and corresponding gage height}

[m³/s, meters per second; --, no data]

\begin{tabular}{|c|c|c|c|c|c|c|c|}
\hline $\begin{array}{c}\text { Water } \\
\text { year }\end{array}$ & Date & $\begin{array}{c}\text { Gage } \\
\text { height } \\
\text { (meters) }\end{array}$ & $\begin{array}{c}\text { Peak } \\
\text { discharge } \\
\left(\mathrm{m}^{3} / \mathrm{s}\right)\end{array}$ & $\begin{array}{c}\text { Water } \\
\text { year }\end{array}$ & Date & $\begin{array}{c}\text { Gage } \\
\text { height } \\
\text { (meters) }\end{array}$ & $\begin{array}{c}\text { Peak } \\
\text { discharge } \\
\left(\mathrm{m}^{3} / \mathrm{s}\right)\end{array}$ \\
\hline
\end{tabular}

Annual peak discharge, by year

\begin{tabular}{|c|c|c|c|c|c|c|c|}
\hline 1974 & 22-Mar & -- & 200 & 1977 & 14-Apr & -- & 218 \\
\hline 1975 & 15-May & -- & 348 & 1978 & 18-Nov & -- & 206 \\
\hline 1976 & 24-Apr & -- & 485 & 1979 & 29-Mar & -- & 310 \\
\hline \multicolumn{8}{|c|}{ Annual peak discharge, from highest to lowest } \\
\hline 1976 & 24-Apr & -- & 485 & 1977 & 14-Apr & -- & 218 \\
\hline 1975 & 15-May & -- & 348 & 1978 & 18-Nov & -- & 206 \\
\hline 1979 & 29-Mar & -- & 310 & 1974 & 22-Mar & -- & 200 \\
\hline
\end{tabular}




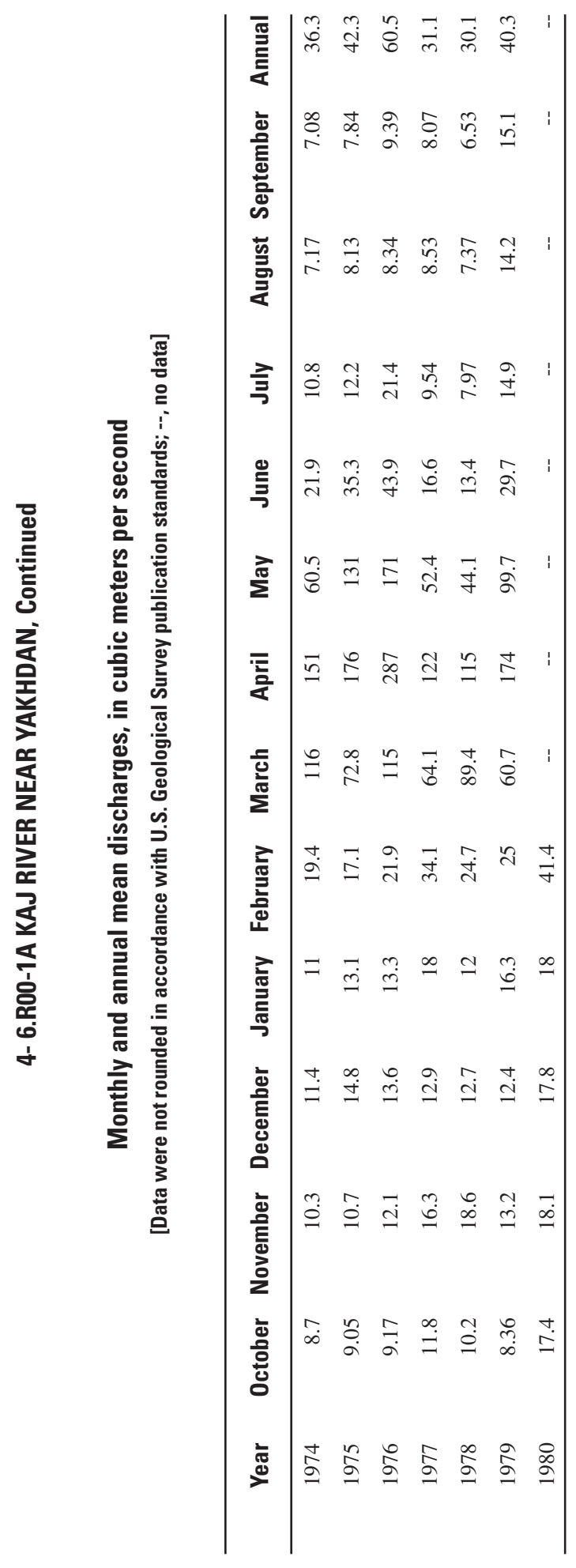




\section{5- 0.000-4W KHASH RIVER AT DEHMAZANG}

\section{(U.S. Geological Survey identification number: 315500063070000$)$}

LOCATION: Lat $31^{\circ} 55^{\prime} \mathrm{N}$., long $63^{\circ} 07^{\prime} \mathrm{E}$.

DRAINAGE AREA: $5,925 \mathrm{~km}^{2}$.

ALTITUDE: 700 meters plus mean sea level.

PERIOD OF RECORD: April 1, 1976 to September 30, 1978.

GAGE: --

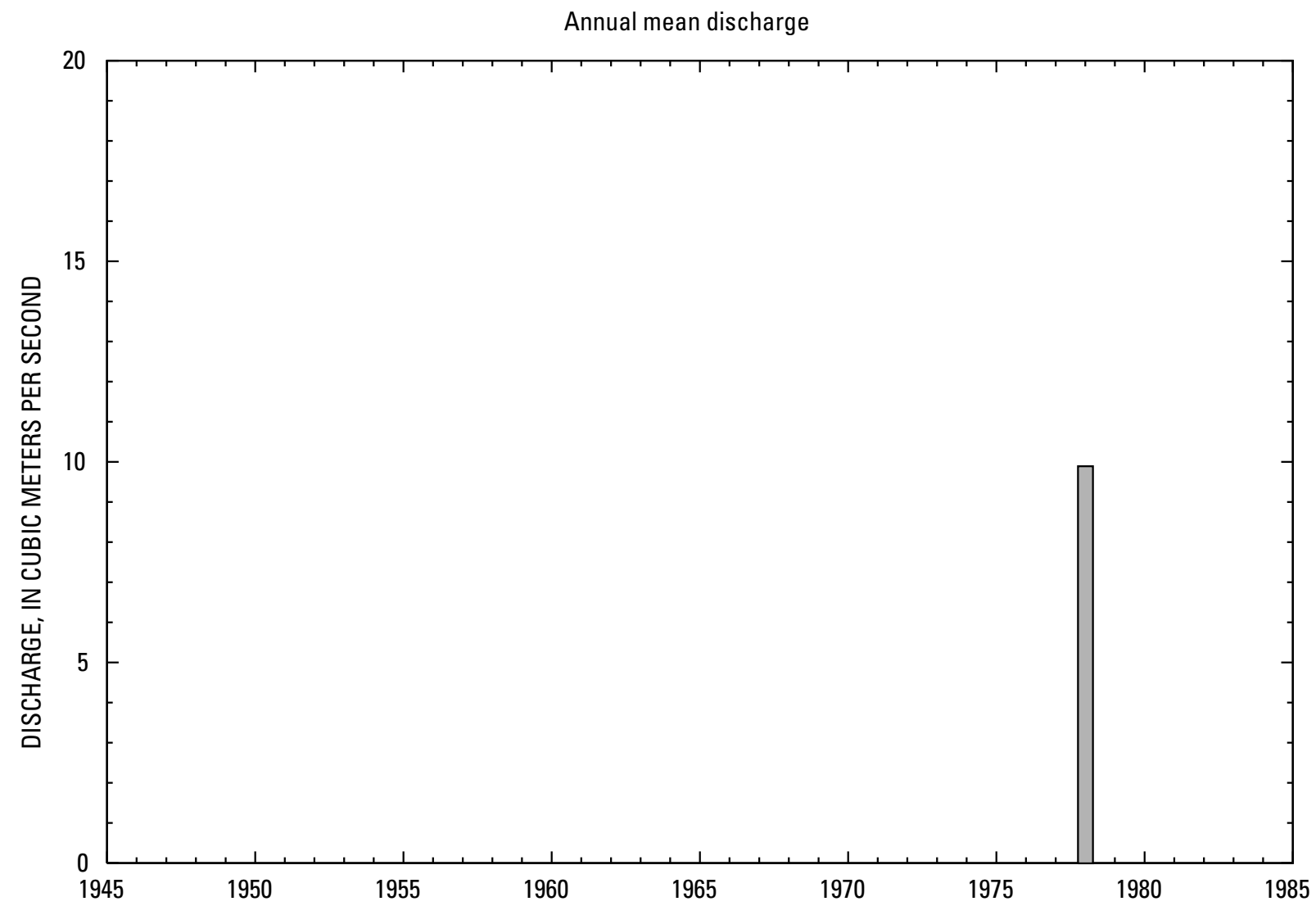




\section{5- 0.000-4W KHASH RIVER AT DEHMAZANG, Continued}

\section{Statistics of monthly and annual mean discharges}

[ $\mathrm{m}^{3} / \mathrm{s}$, meters per second; $\mathrm{ng}$, statistic not given]

\begin{tabular}{|c|c|c|c|c|c|c|c|c|}
\hline \multirow[b]{2}{*}{ Month } & \multicolumn{2}{|c|}{ Maximum } & \multicolumn{2}{|c|}{ Minimum } & \multicolumn{4}{|c|}{ Mean } \\
\hline & $\begin{array}{c}\text { Discharge } \\
\left(\mathrm{m}^{3} / \mathrm{s}\right)\end{array}$ & $\begin{array}{c}\text { Water year } \\
\text { of } \\
\text { occurrence }\end{array}$ & $\begin{array}{c}\text { Discharge } \\
\left(\mathrm{m}^{3} / \mathrm{s}\right)\end{array}$ & $\begin{array}{c}\text { Water year } \\
\text { of } \\
\text { occurrence }\end{array}$ & $\begin{array}{c}\text { Discharge } \\
\left(\mathrm{m}^{3} / \mathrm{s}\right)\end{array}$ & $\begin{array}{c}\text { Standard } \\
\text { deviation } \\
\left(\mathrm{m}^{3} / \mathrm{s}\right)\end{array}$ & $\begin{array}{c}\text { Coefficient } \\
\text { of } \\
\text { variation }\end{array}$ & $\begin{array}{c}\text { Percentage } \\
\text { of annual } \\
\text { discharge }\end{array}$ \\
\hline October & 0.010 & 1978 & ng & $\mathrm{ng}$ & 0.01 & ng & ng & 0.01 \\
\hline November & 3.83 & 1978 & ng & ng & 3.83 & ng & $\mathrm{ng}$ & 3.56 \\
\hline December & 4.50 & 1978 & ng & ng & 4.50 & ng & ng & 4.19 \\
\hline January & 5.81 & 1978 & ng & ng & 5.81 & ng & ng & 0.541 \\
\hline February & 14.6 & 1978 & ng & ng & 14.6 & ng & ng & 13.6 \\
\hline March & 45.8 & 1978 & ng & ng & 45.8 & ng & ng & 42.6 \\
\hline April & 32.6 & 1978 & 16.0 & 1977 & 24.3 & 11.7 & 0.48 & 22.6 \\
\hline May & 9.96 & 1978 & 4.64 & 1977 & 7.30 & 3.76 & 0.52 & 6.79 \\
\hline June & 1.70 & 1978 & 0.698 & 1977 & 1.20 & 0.71 & 0.59 & 1.12 \\
\hline July & 0.070 & 1978 & 0.020 & 1977 & 0.05 & 0.04 & 0.79 & 0.04 \\
\hline August & 0.167 & 1977 & 0.015 & 1978 & 0.09 & 0.11 & 1.18 & 0.08 \\
\hline September & 0.010 & 1977 & 0.003 & 1978 & 0.01 & 0 & 0.76 & 0.01 \\
\hline Annual & 9.87 & 1978 & ng & ng & 9.87 & ng & ng & 100 \\
\hline
\end{tabular}

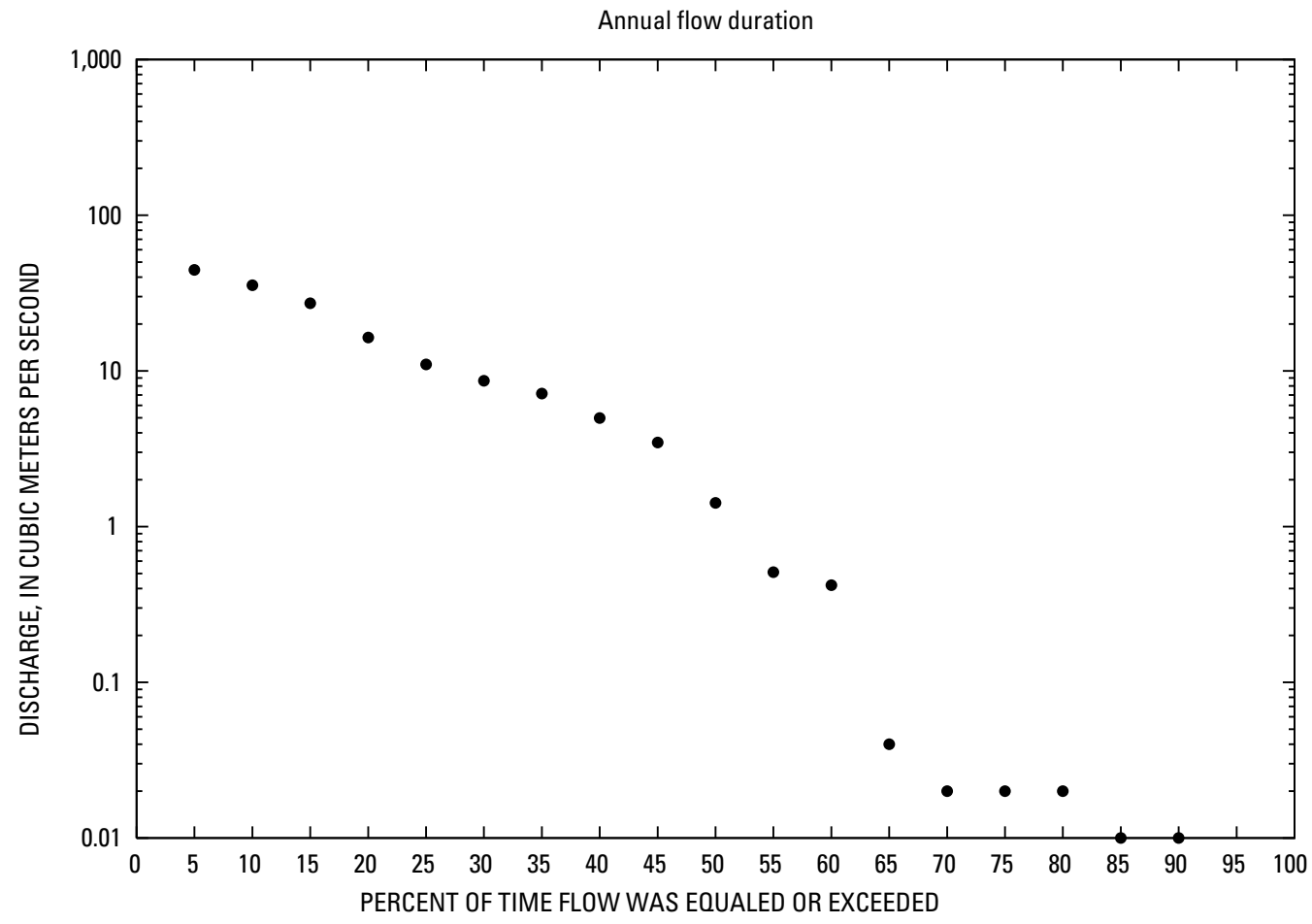




\section{5- 0.000-4W KHASH RIVER AT DEHMAZANG, Continued}

Monthly and annual flow duration, in cubic meters per second

[Data were not rounded in accordance with U.S. Geological Survey publication standards; --, no data]

\begin{tabular}{|c|c|c|c|c|c|c|c|c|c|c|c|c|c|}
\hline $\begin{array}{l}\text { Percentage } \\
\text { of days } \\
\text { discharge } \\
\text { equaled or } \\
\text { exceeded }\end{array}$ & January & February & March & April & May & June & July & August & September & October & November & December & Annual \\
\hline 95 & 3.03 & 7.09 & 26.5 & 9.15 & 2.65 & 0.02 & 0.02 & 0.01 & 0 & 0 & 0.01 & 0.5 & 0 \\
\hline 90 & 3.06 & 7.19 & 26.9 & 10.2 & 3.02 & 0.02 & 0.02 & 0.01 & 0 & 0 & 0.01 & 0.51 & 0.01 \\
\hline 85 & 3.09 & 7.28 & 27.6 & 11.5 & 3.43 & 0.03 & 0.02 & 0.01 & 0 & 0 & 0.01 & 0.51 & 0.01 \\
\hline 80 & 3.12 & 7.38 & 28.8 & 12.3 & 3.93 & 0.03 & 0.02 & 0.01 & 0 & 0 & 0.01 & 0.52 & 0.02 \\
\hline 75 & 3.89 & 7.48 & 30.4 & 14.4 & 4.11 & 0.24 & 0.02 & 0.02 & 0 & 0 & 0.01 & 0.52 & 0.02 \\
\hline 70 & 3.96 & 7.63 & 31.4 & 16.5 & 4.56 & 0.35 & 0.02 & 0.02 & 0 & 0 & 0.02 & 0.53 & 0.02 \\
\hline 65 & 4.02 & 7.79 & 32.2 & 20.6 & 5.03 & 0.41 & 0.02 & 0.02 & 0 & 0 & 0.02 & 0.53 & 0.04 \\
\hline 60 & 5.01 & 9.01 & 33.4 & 21.4 & 5.22 & 0.6 & 0.03 & 0.02 & 0 & 0 & 0.02 & 0.54 & 0.42 \\
\hline 55 & 5.06 & 9.6 & 35.1 & 22 & 5.74 & 0.76 & 0.03 & 0.02 & 0 & 0 & 0.02 & 0.54 & 0.51 \\
\hline 50 & 5.11 & 9.99 & 36.8 & 22.6 & 5.97 & 0.96 & 0.03 & 0.02 & 0 & 0 & 0.02 & 0.55 & 1.42 \\
\hline 45 & 5.15 & 10.4 & 39.6 & 24 & 6.62 & 1.08 & 0.03 & 0.02 & 0 & 0 & 0.02 & 0.55 & 3.47 \\
\hline 40 & 5.95 & 10.8 & 42.5 & 25.3 & 7.04 & 1.25 & 0.03 & 0.02 & 0 & 0 & 0.46 & 0.56 & 4.98 \\
\hline 35 & 6.96 & 11.3 & 43.8 & 26.7 & 7.57 & 1.53 & 0.03 & 0.03 & 0 & 0 & 0.6 & 0.56 & 7.15 \\
\hline 30 & 7.03 & 11.8 & 44.4 & 33.1 & 7.96 & 1.78 & 0.03 & 0.03 & 0 & 0 & 0.77 & 0.57 & 8.65 \\
\hline 25 & 7.11 & 12.2 & 45 & 34.9 & 9.1 & 2 & 0.03 & 0.03 & 0 & 0 & 0.98 & 6.69 & 11 \\
\hline 20 & 7.19 & 15.4 & 50.7 & 36.8 & 10.4 & 2.31 & 0.04 & 0.03 & 0 & 0 & 2.3 & 7.35 & 16.4 \\
\hline 15 & 8 & 26.5 & 53 & 39.3 & 12.5 & 2.63 & 0.04 & 0.08 & 0 & 0 & 5.51 & 9.15 & 27.2 \\
\hline 10 & 9.29 & 36.2 & 65.4 & 40.6 & 14 & 3 & 0.05 & 0.24 & 0 & 0 & 7.92 & 12.1 & 35.5 \\
\hline 5 & 0 & 0 & 0.05 & 0 & 15.7 & 0 & 0.21 & 0.43 & 0 & 0 & 0 & 0 & 44.6 \\
\hline
\end{tabular}




\section{5- 0.000-4W KHASH RIVER AT DEHMAZANG, Continued}

Probability of occurrence of annual high discharges

$\left[\mathrm{m}^{3} / \mathrm{s}\right.$, meters per second; $\mathrm{ng}$, statistic not given]

\begin{tabular}{|c|c|c|c|c|c|c|}
\hline \multirow{2}{*}{$\begin{array}{c}\text { Exceedance } \\
\text { probability }\end{array}$} & \multirow{2}{*}{$\begin{array}{c}\text { Recurrence } \\
\text { interval } \\
\text { (years) }\end{array}$} & \multirow{2}{*}{$\begin{array}{c}\text { Maximum } \\
\text { instantaneous } \\
\left(\mathrm{m}^{3} / \mathrm{s}\right)\end{array}$} & \multicolumn{4}{|c|}{ Maximum mean discharge } \\
\hline & & & 3-day period & 7-day period & 15-day period & 30-day period \\
\hline 0.99 & 1.01 & ng & ng & ng & ng & ng \\
\hline 0.95 & 1.05 & ng & ng & ng & ng & ng \\
\hline 0.90 & 1.11 & ng & ng & ng & ng & ng \\
\hline 0.80 & 1.25 & ng & ng & ng & ng & ng \\
\hline 0.50 & 2 & ng & ng & ng & ng & ng \\
\hline 0.20 & 5 & ng & ng & ng & ng & ng \\
\hline 0.10 & 10 & ng & ng & ng & ng & ng \\
\hline 0.04 & 25 & ng & ng & ng & ng & ng \\
\hline 0.02 & 50 & ng & ng & ng & ng & ng \\
\hline 0.01 & 100 & $\mathrm{ng}$ & ng & ng & ng & $\mathrm{ng}$ \\
\hline 0.005 & 200 & ng & $\mathrm{ng}$ & $\mathrm{ng}$ & ng & ng \\
\hline 0.002 & 500 & $\mathrm{ng}$ & ng & ng & ng & $\mathrm{ng}$ \\
\hline
\end{tabular}

5- 0.000-4W KHASH RIVER AT DEHMAZANG, Continued

Probability of occurrence of annual low discharges

[m3/s, meters per second; ng, statistic not given]

\begin{tabular}{|c|c|c|c|c|c|c|c|c|c|c|}
\hline \multirow{3}{*}{$\begin{array}{c}\text { Nonexceedance } \\
\text { probability }\end{array}$} & \multirow{3}{*}{$\begin{array}{l}\text { Recurrence } \\
\text { interval } \\
\text { (years) }\end{array}$} & \multicolumn{9}{|c|}{ Minimum mean discharge (m3/s) } \\
\hline & & \multicolumn{9}{|c|}{ Number of consecutive days } \\
\hline & & 1 & 3 & 7 & 14 & 30 & 60 & 90 & 120 & 183 \\
\hline 0.05 & 20 & ng & ng & ng & ng & ng & ng & ng & ng & ng \\
\hline 0.10 & 10 & ng & ng & ng & ng & ng & ng & ng & ng & ng \\
\hline 0.20 & 5 & ng & ng & ng & ng & ng & ng & ng & ng & ng \\
\hline 0.50 & 2 & ng & ng & $\mathrm{ng}$ & ng & ng & ng & ng & $\mathrm{ng}$ & ng \\
\hline
\end{tabular}




\section{5- 0.000-4W KHASH RIVER AT DEHMAZANG, Continued}

\section{Probability of occurrence of seasonal low discharges}

[ $\mathrm{m}^{3} / \mathrm{s}$, meters per second; $\mathrm{ng}$, statistic not given]

\begin{tabular}{|c|c|c|c|c|c|c|c|c|c|}
\hline \multirow{3}{*}{$\begin{array}{c}\text { Nonexceedance } \\
\text { probability }\end{array}$} & \multirow{3}{*}{$\begin{array}{c}\text { Recurrence } \\
\text { interval } \\
\text { (years) }\end{array}$} & \multicolumn{8}{|c|}{ Minimum mean discharge $\left(\mathrm{m}^{3} / \mathrm{s}\right)$} \\
\hline & & \multicolumn{8}{|c|}{ Number of consecutive days } \\
\hline & & 1 & 7 & 14 & 30 & 1 & 7 & 14 & 30 \\
\hline & & \multicolumn{4}{|c|}{ December-January-February } & \multicolumn{4}{|c|}{ March-April-May } \\
\hline 0.05 & 20 & ng & ng & ng & ng & ng & ng & ng & ng \\
\hline 0.10 & 10 & ng & ng & ng & ng & ng & ng & ng & ng \\
\hline 0.20 & 5 & ng & ng & ng & ng & ng & ng & ng & ng \\
\hline \multirow[t]{2}{*}{0.50} & 2 & ng & ng & ng & ng & ng & ng & ng & ng \\
\hline & & \multicolumn{4}{|c|}{ June-July-August } & \multicolumn{4}{|c|}{ September-October-November } \\
\hline 0.05 & 20 & ng & ng & ng & ng & ng & ng & ng & $\mathrm{ng}$ \\
\hline 0.10 & 10 & ng & $\mathrm{ng}$ & ng & ng & $\mathrm{ng}$ & $\mathrm{ng}$ & $\mathrm{ng}$ & $\mathrm{ng}$ \\
\hline 0.20 & 5 & ng & ng & ng & ng & ng & ng & ng & $\mathrm{ng}$ \\
\hline 0.50 & 2 & ng & $\mathrm{ng}$ & $\mathrm{ng}$ & ng & $\mathrm{ng}$ & $\mathrm{ng}$ & $\mathrm{ng}$ & $\mathrm{ng}$ \\
\hline
\end{tabular}

\section{5- 0.000-4W KHASH RIVER AT DEHMAZANG, Continued}

Annual peak discharge and corresponding gage height

[m³/s, meters per second; --, no data]

\begin{tabular}{ccccccc}
\hline $\begin{array}{c}\text { Water } \\
\text { year }\end{array} \quad$ Date & $\begin{array}{c}\text { Gage } \\
\text { height } \\
(\text { meters })\end{array}$ & $\begin{array}{c}\text { Peak } \\
\text { discharge } \\
\left(\mathrm{m}^{3} / \mathrm{s}\right)\end{array}$ & $\begin{array}{c}\text { Water } \\
\text { year }\end{array}$ & Date & $\begin{array}{c}\text { Gage } \\
\text { height } \\
(\text { meters })\end{array}$ & $\begin{array}{c}\text { Peak } \\
\text { discharge } \\
\left(\mathrm{m}^{3} / \mathrm{s}\right)\end{array}$ \\
\hline
\end{tabular}

Annual peak discharge, by year

$\begin{array}{llll}1977 & \text { 6-Apr } & -- & 32.2 \\ 1978 & \text { 9-Mar } & -- & 211\end{array}$

Annual peak discharge, from highest to lowest

1978 9-Mar $\quad$-- $\quad 211$

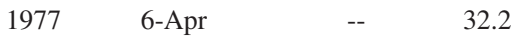




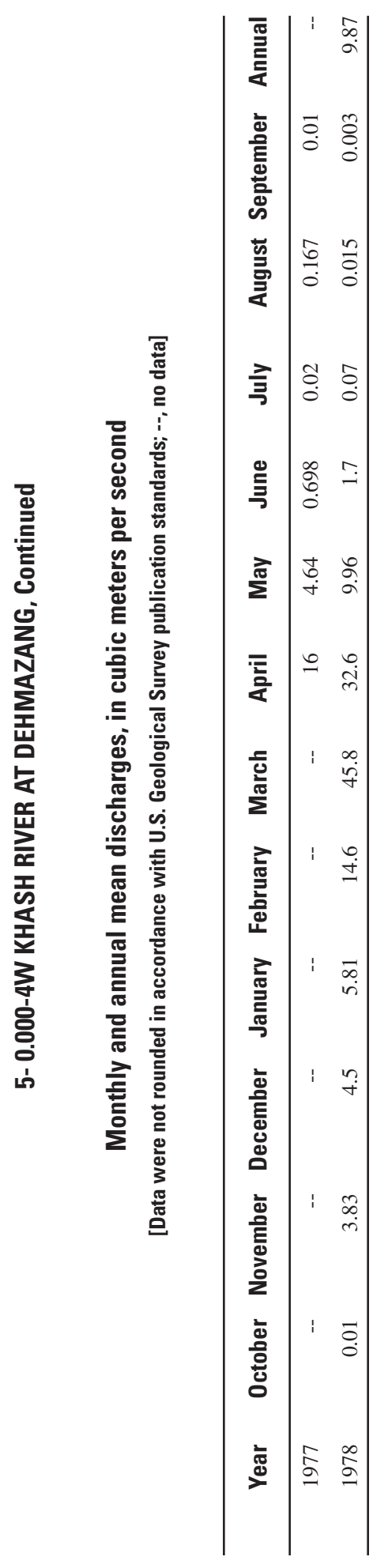




\section{5- 0.000-5M KHASH RIVER AT DILARAM}

\section{(U.S. Geological Survey identification number: 320900063260000)}

LOCATION: Lat $32^{\circ} 09^{\prime} \mathrm{N}$., long 63²6'E., on right bank about $300 \mathrm{~m}$ downstream from highway bridge at Dilaram, about $180 \mathrm{~km}$ upstream from Chakhansur.

DRAINAGE AREA: $5,585 \mathrm{~km}^{2}$.

ALTITUDE: 810 meters plus mean sea level.

PERIOD OF RECORD: October 1, 1952 to September 30, 1978.

GAGE: Water-stage recorder. Prior to August 1, 1958, water-stage recorder on left bank about 3 km downstream at same datum.

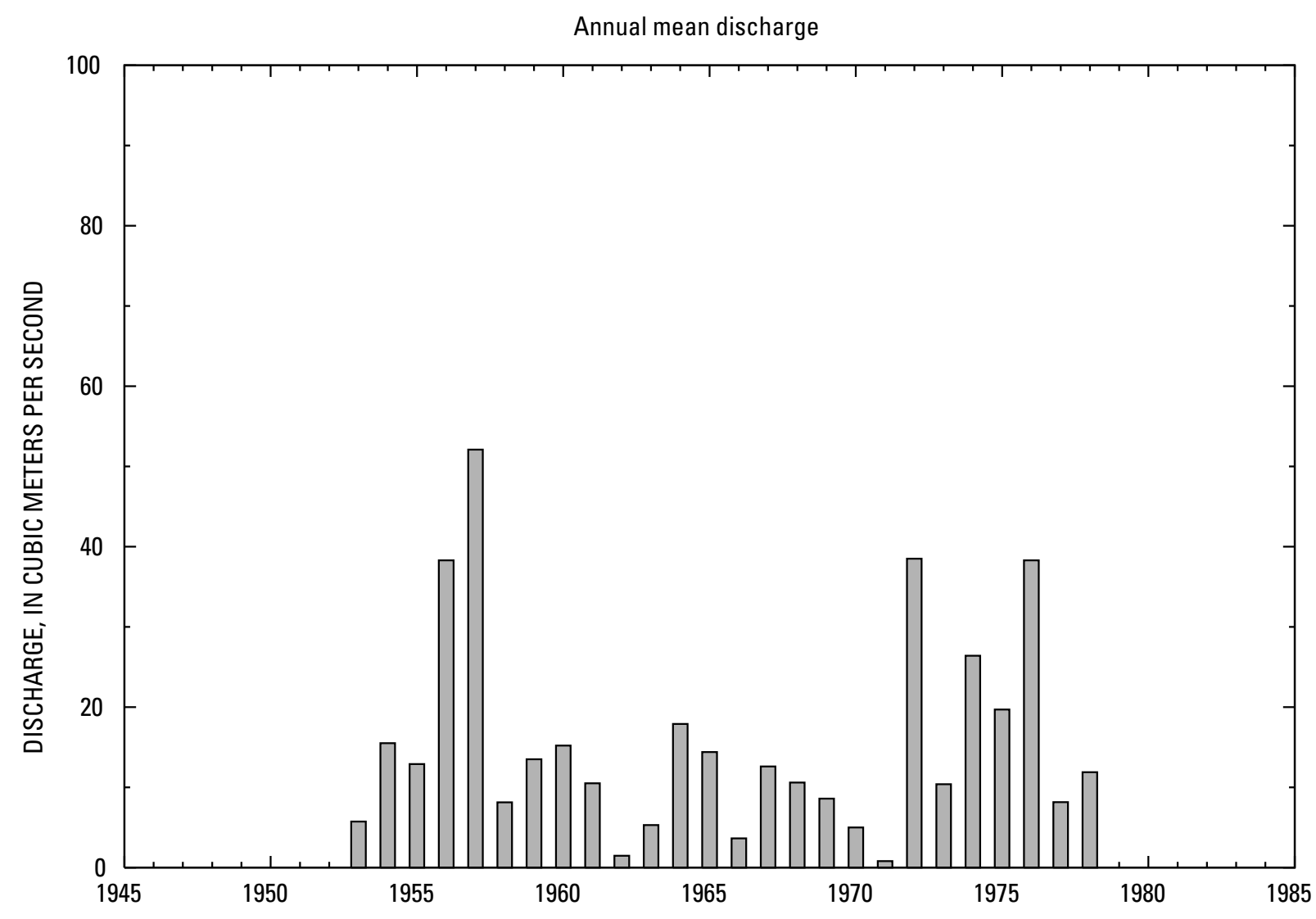




\section{5- 0.000-5M KHASH RIVER AT DILARAM, Continued}

\section{Statistics of monthly and annual mean discharges}

[ $\mathrm{m}^{3} / \mathrm{s}$, meters per second]

\begin{tabular}{|c|c|c|c|c|c|c|c|c|}
\hline \multirow[b]{2}{*}{ Month } & \multicolumn{2}{|c|}{ Maximum } & \multicolumn{2}{|c|}{ Minimum } & \multicolumn{4}{|c|}{ Mean } \\
\hline & $\begin{array}{c}\text { Discharge } \\
\left(\mathrm{m}^{3} / \mathrm{s}\right)\end{array}$ & $\begin{array}{c}\text { Water year } \\
\text { of } \\
\text { occurrence }\end{array}$ & $\begin{array}{c}\text { Discharge } \\
\left(\mathrm{m}^{3} / \mathrm{s}\right)\end{array}$ & $\begin{array}{c}\text { Water year } \\
\text { of } \\
\text { occurrence }\end{array}$ & $\begin{array}{c}\text { Discharge } \\
\left(\mathrm{m}^{3} / \mathrm{s}\right)\end{array}$ & $\begin{array}{c}\text { Standard } \\
\text { deviation } \\
\left(\mathrm{m}^{3} / \mathrm{s}\right)\end{array}$ & $\begin{array}{c}\text { Coefficient } \\
\text { of } \\
\text { variation }\end{array}$ & $\begin{array}{c}\text { Percentage } \\
\text { of annual } \\
\text { discharge }\end{array}$ \\
\hline October & 1.13 & 1958 & 0 & 1953 & 0.18 & 0.25 & 1.38 & 0.1 \\
\hline November & 5.20 & 1958 & 0.009 & 1953 & 0.87 & 1.40 & 1.61 & 0.46 \\
\hline December & 22.5 & 1958 & 0.030 & 1972 & 3.08 & 5.34 & 1.73 & 1.64 \\
\hline January & 34.2 & 1957 & 0.066 & 1967 & 8.07 & 9.74 & 1.21 & 4.30 \\
\hline February & 60.4 & 1974 & 0.087 & 1963 & 22.5 & 18.6 & 0.83 & 12.0 \\
\hline March & 288 & 1956 & 2.35 & 1971 & 69.4 & 78.1 & 1.13 & 37.0 \\
\hline April & 264 & 1957 & 3.28 & 1971 & 57.0 & 56.8 & 1.00 & 30.3 \\
\hline May & 45.3 & 1976 & 0.895 & 1971 & 19.2 & 13.8 & 0.72 & 10.2 \\
\hline June & 16.4 & 1972 & 0.040 & 1971 & 5.01 & 3.59 & 0.72 & 2.67 \\
\hline July & 7.78 & 1976 & 0.030 & 1971 & 1.77 & 2.11 & 1.19 & 0.94 \\
\hline August & 3.86 & 1957 & 0 & 1953 & 0.59 & 0.86 & 1.47 & 0.31 \\
\hline September & 1.73 & 1957 & 0 & 1953 & 0.23 & 0.36 & 1.61 & 0.12 \\
\hline Annual & 52.1 & 1957 & 0.803 & 1971 & 15.6 & 12.9 & 0.83 & 100 \\
\hline
\end{tabular}

Annual flow duration

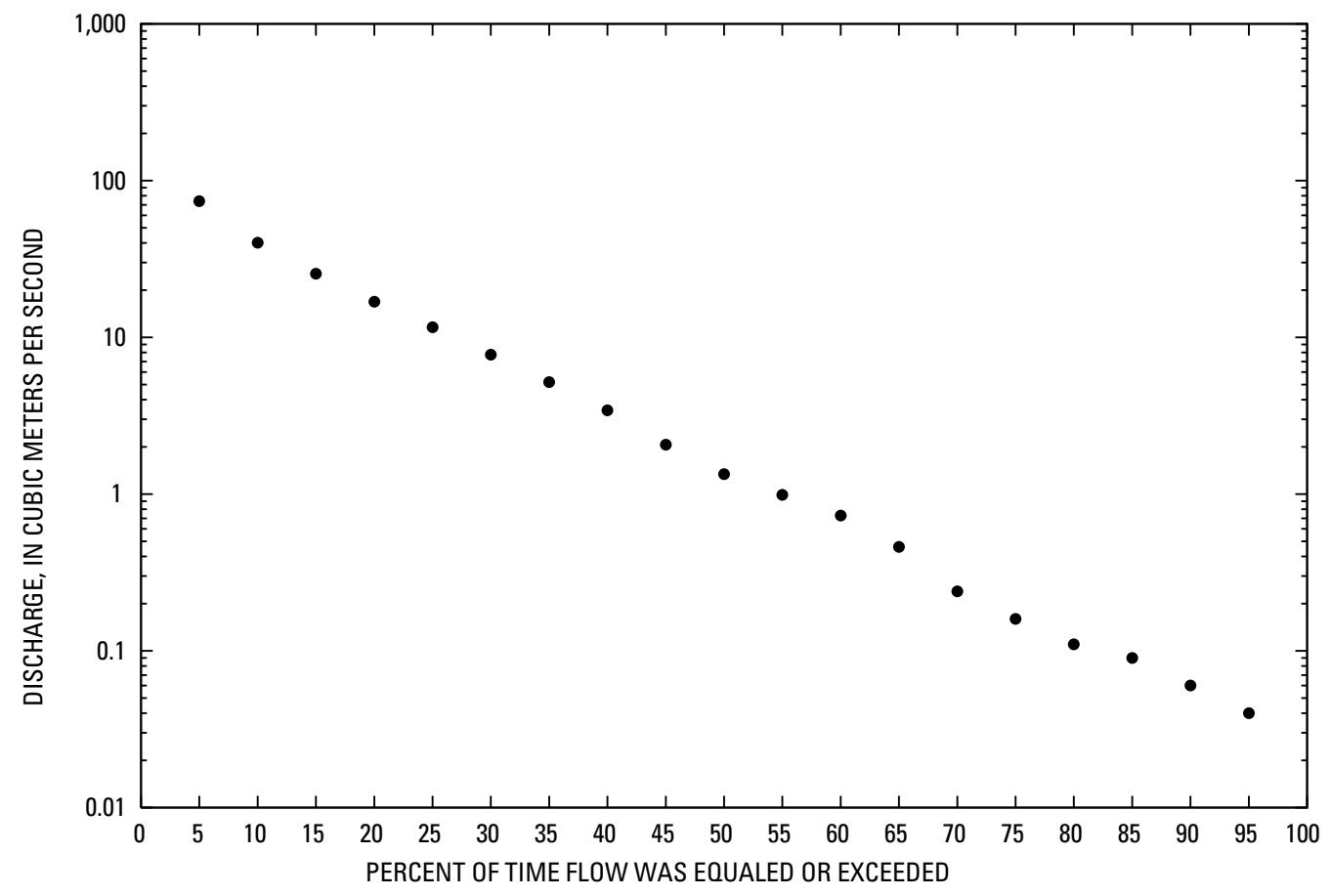




\section{Monthly and annual flow duration, in cubic meter per second}

[Data were not rounded in accordance with U.S. Geological Survey publication standards]

\begin{tabular}{|c|c|c|c|c|c|c|c|c|c|c|c|c|c|}
\hline $\begin{array}{l}\text { Percentage } \\
\text { of days } \\
\text { discharge } \\
\text { equaled or } \\
\text { exceeded }\end{array}$ & January & February & March & April & May & June & July & August & September & October & November & December & Annual \\
\hline 95 & 0.09 & 0.33 & 1.7 & 5.97 & 1.92 & 0.2 & 0.04 & 0.03 & 0.03 & 0 & 0.02 & 0.04 & 0.04 \\
\hline 90 & 0.11 & 0.9 & 3.03 & 8.52 & 3.38 & 0.5 & 0.1 & 0.05 & 0.04 & 0.02 & 0.03 & 0.06 & 0.06 \\
\hline 85 & 0.4 & 1.13 & 6.9 & 10.6 & 4.64 & 0.88 & 0.12 & 0.06 & 0.04 & 0.03 & 0.04 & 0.08 & 0.09 \\
\hline 80 & 0.76 & 1.5 & 10.1 & 13.1 & 5.99 & 1.38 & 0.15 & 0.07 & 0.05 & 0.03 & 0.05 & 0.09 & 0.11 \\
\hline 75 & 0.87 & 2.43 & 12.9 & 15.6 & 7.28 & 1.83 & 0.2 & 0.08 & 0.06 & 0.04 & 0.06 & 0.11 & 0.16 \\
\hline 70 & 0.98 & 3.68 & 17.8 & 18.2 & 8.37 & 2.15 & 0.3 & 0.11 & 0.07 & 0.06 & 0.06 & 0.15 & 0.24 \\
\hline 65 & 1.11 & 4.81 & 21.5 & 21.4 & 10 & 2.55 & 0.4 & 0.13 & 0.07 & 0.06 & 0.08 & 0.34 & 0.46 \\
\hline 60 & 1.25 & 6.52 & 25.7 & 25 & 11.7 & 3.02 & 0.52 & 0.14 & 0.08 & 0.07 & 0.1 & 0.54 & 0.73 \\
\hline 55 & 1.6 & 7.88 & 30 & 29.1 & 13.2 & 3.48 & 0.64 & 0.16 & 0.09 & 0.09 & 0.13 & 0.74 & 0.99 \\
\hline 50 & 1.94 & 10.2 & 33.6 & 34.2 & 14.7 & 3.91 & 0.75 & 0.18 & 0.09 & 0.1 & 0.2 & 0.8 & 1.34 \\
\hline 45 & 2.38 & 11.8 & 37.1 & 39.7 & 16.4 & 4.41 & 0.89 & 0.21 & 0.11 & 0.12 & 0.37 & 0.85 & 2.07 \\
\hline 40 & 3.06 & 13.7 & 41.6 & 45.4 & 18.1 & 4.97 & 1.12 & 0.24 & 0.13 & 0.13 & 0.48 & 0.9 & 3.42 \\
\hline 35 & 3.7 & 16.9 & 48.3 & 52.3 & 19.9 & 5.59 & 1.4 & 0.28 & 0.15 & 0.15 & 0.57 & 1 & 5.18 \\
\hline 30 & 4.52 & 21.9 & 57.8 & 61.6 & 21.8 & 6.23 & 1.7 & 0.32 & 0.15 & 0.17 & 0.7 & 1.13 & 7.76 \\
\hline 25 & 6.37 & 31.2 & 71.9 & 73.4 & 24.1 & 7.07 & 2.08 & 0.5 & 0.17 & 0.19 & 0.84 & 1.37 & 11.6 \\
\hline 20 & 10.4 & 37.9 & 90.2 & 86.8 & 27.1 & 8.1 & 2.97 & 0.77 & 0.22 & 0.24 & 0.97 & 1.96 & 16.9 \\
\hline 15 & 14.2 & 44 & 114.4 & 113.8 & 31.3 & 9.47 & 3.96 & 1.14 & 0.36 & 0.28 & 1.09 & 4.91 & 25.5 \\
\hline 10 & 19.3 & 51.2 & 172.2 & 148.8 & 38 & 11.1 & 4.9 & 1.62 & 0.54 & 0.44 & 1.21 & 7.08 & 40.1 \\
\hline 5 & 30.6 & 82.4 & 270.8 & 183.2 & 55.9 & 13.8 & 6.41 & 3.02 & 1.11 & 0.76 & 1.47 & 12.7 & 74 \\
\hline
\end{tabular}




\section{5- 0.000-5M KHASH RIVER AT DILARAM, Continued}

Probability of occurrence of annual high discharges

[m³/s, meters per second; ng, statistic not given]

\begin{tabular}{rrrrrrr}
\hline & & & & \multicolumn{5}{c}{ Maximum mean discharge } \\
\cline { 6 - 7 } $\begin{array}{c}\text { Exceedance } \\
\text { probability }\end{array}$ & $\begin{array}{c}\text { Recurrence } \\
\text { interval } \\
\text { (years) }\end{array}$ & $\begin{array}{c}\text { Maximum } \\
\text { instantaneous } \\
\left(\mathbf{m}^{3} / \mathbf{s}\right)\end{array}$ & 3-day period & 7-day period & 15-day period & 30-day period \\
\cline { 5 - 7 } & 1.01 & 18.9 & 8.38 & 5.26 & 3.55 & 2.92 \\
0.99 & 1.05 & 47.1 & 19.8 & 14.3 & 10.5 & 8.40 \\
0.95 & 1.11 & 74.2 & 30.9 & 23.5 & 17.9 & 14.0 \\
0.90 & 1.25 & 125 & 52.0 & 41.3 & 32.2 & 25.0 \\
0.50 & 2 & 309 & 135 & 110 & 86.4 & 65.9 \\
0.20 & 5 & 684 & 329 & 255 & 194 & 147 \\
0.10 & 10 & 992 & 513 & 377 & 278 & 211 \\
0.04 & 25 & 1,430 & 811 & 553 & 389 & 296 \\
0.02 & 50 & 1,790 & 1,080 & 695 & 472 & 360 \\
0.01 & 100 & 2,160 & 1,390 & 842 & 553 & 424 \\
0.005 & 200 & 2,540 & 1,740 & 995 & 631 & 486 \\
0.002 & 500 & 3,060 & ng & ng & ng & ng \\
\hline
\end{tabular}

\section{5- 0.000-5M KHASH RIVER AT DILARAM, Continued}

\section{Probability of occurrence of annual low discharges}

$\left[\mathrm{m}^{3} / \mathrm{s}\right.$, meters per second]

\begin{tabular}{|c|c|c|c|c|c|c|c|c|c|c|}
\hline \multirow{3}{*}{$\begin{array}{c}\text { Nonexceedance } \\
\text { probability }\end{array}$} & \multirow{3}{*}{$\begin{array}{c}\text { Recurrence } \\
\text { interval } \\
\text { (years) }\end{array}$} & \multicolumn{9}{|c|}{ Minimum mean discharge $\left(\mathrm{m}^{3} / \mathrm{s}\right)$} \\
\hline & & \multicolumn{9}{|c|}{ Number of consecutive days } \\
\hline & & 1 & 3 & 7 & 14 & 30 & 60 & 90 & 120 & 183 \\
\hline 0.05 & 20 & 0.004 & 0.006 & 0.006 & 0.008 & 0.010 & 0.016 & 0.022 & ${ }^{1} 0.018$ & 0.056 \\
\hline 0.10 & 10 & 0.011 & 0.012 & 0.013 & 0.015 & 0.017 & 0.024 & 0.033 & ${ }^{1} 0.031$ & 0.094 \\
\hline 0.20 & 5 & 0.020 & 0.023 & 0.024 & 0.026 & 0.028 & 0.036 & 0.048 & 0.058 & 0.171 \\
\hline 0.50 & 2 & 0.060 & 0.063 & 0.065 & 0.068 & 0.071 & 0.083 & 0.105 & 0.170 & 0.477 \\
\hline
\end{tabular}

'Data does not fit log-Pearson Type III curve, use with caution. 


\section{5- 0.000-5M KHASH RIVER AT DILARAM, Continued}

\section{Probability of occurrence of seasonal low discharges}

$\left[\mathrm{m}^{3} / \mathrm{s}\right.$, meters per second]

\begin{tabular}{|c|c|c|c|c|c|c|c|c|c|}
\hline \multirow{3}{*}{$\begin{array}{c}\text { Nonexceedance } \\
\text { probability }\end{array}$} & \multirow{3}{*}{$\begin{array}{c}\text { Recurrence } \\
\text { interval } \\
\text { (years) }\end{array}$} & \multicolumn{8}{|c|}{ Minimum mean discharge $\left(\mathrm{m}^{3} / \mathrm{s}\right)$} \\
\hline & & \multicolumn{8}{|c|}{ Number of consecutive days } \\
\hline & & 1 & 7 & 14 & 30 & 1 & 7 & 14 & 30 \\
\hline & & \multicolumn{4}{|c|}{ December-January-February } & \multicolumn{4}{|c|}{ March-April-May } \\
\hline 0.05 & 20 & 0.015 & 0.024 & 0.027 & 0.036 & 0.223 & 0.505 & 0.881 & 2.08 \\
\hline 0.10 & 10 & 0.028 & 0.043 & 0.050 & 0.068 & 0.544 & 1.01 & 1.64 & 3.38 \\
\hline 0.20 & 5 & 0.059 & 0.086 & 0.105 & 0.149 & 1.39 & 2.12 & 3.18 & 5.75 \\
\hline \multirow[t]{2}{*}{0.50} & 2 & 0.246 & 0.331 & 0.425 & 0.646 & 5.54 & 6.67 & 8.86 & 13.5 \\
\hline & & \multicolumn{4}{|c|}{ June-July-August } & \multicolumn{4}{|c|}{ September-October-November } \\
\hline 0.05 & 20 & 0.023 & 0.028 & 0.033 & ${ }^{1} 0.029$ & 0.004 & 0.006 & 0.008 & 0.010 \\
\hline 0.10 & 10 & 0.032 & 0.037 & 0.044 & 0.048 & 0.011 & 0.014 & 0.015 & 0.018 \\
\hline 0.20 & 5 & 0.045 & 0.051 & 0.061 & 0.076 & 0.021 & 0.025 & 0.026 & 0.029 \\
\hline 0.50 & 2 & 0.098 & 0.108 & 0.129 & 0.194 & 0.064 & 0.068 & 0.070 & 0.073 \\
\hline
\end{tabular}

'Data does not fit log-Pearson Type III curve, use with caution. 


\section{5- 0.000-5M KHASH RIVER AT DILARAM, Continued}

\section{Annual peak discharge and corresponding gage height}

[ $\mathrm{m}^{3} / \mathrm{s}$, meters per second; --, no data]

\begin{tabular}{|c|c|c|c|c|c|c|c|}
\hline $\begin{array}{l}\text { Water } \\
\text { year }\end{array}$ & Date & $\begin{array}{c}\text { Gage } \\
\text { height } \\
\text { (meters) }\end{array}$ & $\begin{array}{c}\text { Peak } \\
\text { discharge } \\
\left(\mathrm{m}^{3} / \mathrm{s}\right)\end{array}$ & $\begin{array}{c}\text { Water } \\
\text { year }\end{array}$ & Date & $\begin{array}{c}\text { Gage } \\
\text { height } \\
\text { (meters) }\end{array}$ & $\begin{array}{c}\text { Peak } \\
\text { discharge } \\
\left(\mathrm{m}^{3} / \mathrm{s}\right)\end{array}$ \\
\hline \multicolumn{8}{|c|}{ Annual peak discharge, by year } \\
\hline 1953 & 14-Feb & 2.52 & 756 & 1966 & $25-\mathrm{Feb}$ & -- & 119 \\
\hline 1954 & $13-\mathrm{Feb}$ & 1.83 & 265 & 1967 & 28-Apr & -- & 81.2 \\
\hline 1955 & 15-Mar & 2.97 & 1,387 & 1968 & 10-Mar & -- & 820 \\
\hline 1956 & 4-Mar & 2.56 & 993 & 1969 & 16-Apr & -- & 102 \\
\hline 1957 & 17-Mar & -- & 1,274 & 1970 & 21-Aug & -- & 59 \\
\hline 1958 & 6-Dec & 1.90 & 427 & 1971 & 25-Feb & -- & 138 \\
\hline 1959 & 2-Mar & 6.44 & 229 & 1972 & 12-Mar & -- & 595 \\
\hline 1960 & 16-Apr & 7.52 & 611 & 1973 & $25-\mathrm{Feb}$ & -- & 170 \\
\hline 1961 & 10-Apr & -- & 495 & 1974 & 19-Jan & -- & 424 \\
\hline 1962 & 14-Nov & -- & 30.3 & 1975 & 15-May & -- & 268 \\
\hline 1963 & 12-May & -- & 362 & 1976 & 23-Mar & -- & 765 \\
\hline 1964 & 1-Apr & -- & 317 & 1977 & 5-Apr & -- & 50 \\
\hline 1965 & 22-Apr & -- & 300 & 1978 & 9-Mar & -- & 222 \\
\hline \multicolumn{8}{|c|}{ Annual peak discharge, from highest to lowest } \\
\hline 1955 & 15-Mar & 2.97 & 1,387 & 1965 & 22-Apr & -- & 300 \\
\hline 1957 & 17-Mar & -- & 1,274 & 1975 & 15-Мау & -- & 268 \\
\hline 1956 & 4-Mar & 2.56 & 993 & 1954 & $13-\mathrm{Feb}$ & 1.83 & 265 \\
\hline 1968 & 10-Mar & -- & 820 & 1959 & 2-Mar & 6.44 & 229 \\
\hline 1976 & 23-Mar & -- & 765 & 1978 & 9-Mar & -- & 222 \\
\hline 1953 & $14-\mathrm{Feb}$ & 2.52 & 756 & 1973 & $25-\mathrm{Feb}$ & -- & 170 \\
\hline 1960 & 16-Apr & 7.52 & 611 & 1971 & $25-\mathrm{Feb}$ & -- & 138 \\
\hline 1972 & 12-Mar & -- & 595 & 1966 & $25-\mathrm{Feb}$ & -- & 119 \\
\hline 1961 & 10-Apr & -- & 495 & 1969 & 16-Apr & -- & 102 \\
\hline 1958 & 6-Dec & 1.90 & 427 & 1967 & 28-Apr & -- & 81.2 \\
\hline 1974 & 19-Jan & -- & 424 & 1970 & 21-Aug & -- & 59 \\
\hline 1963 & 12-May & -- & 362 & 1977 & 5-Apr & -- & 50 \\
\hline 1964 & 1-Apr & -- & 317 & 1962 & $14-\mathrm{Nov}$ & -- & 30.3 \\
\hline
\end{tabular}




\section{6- 0.000-3M FARAH RIVER AT FARAH}

\section{(U.S. Geological Survey identification number: 322200062040000)}

LOCATION: Lat $32^{\circ} 22^{\prime} N$., long $62^{\circ} 04^{\prime}$ E., on downstream side of bridge on Farah-Heart Road, 8 km southwest of Farah, and about $130 \mathrm{~km}$ upstream from Hamun-i-Sabari in the Chakhansur-Sistan basin.

DRAINAGE AREA: $26,700 \mathrm{~km}^{2}$.

ALTITUDE: 650 meters plus mean sea level.

PERIOD OF RECORD: April 1, 1953 to September 30, 1978.

GAGE: Water-stage recorder. Prior to July 14, 1958, staff gage $300 \mathrm{~m}$ upstream at datum $0.55 \mathrm{~m}$ higher.

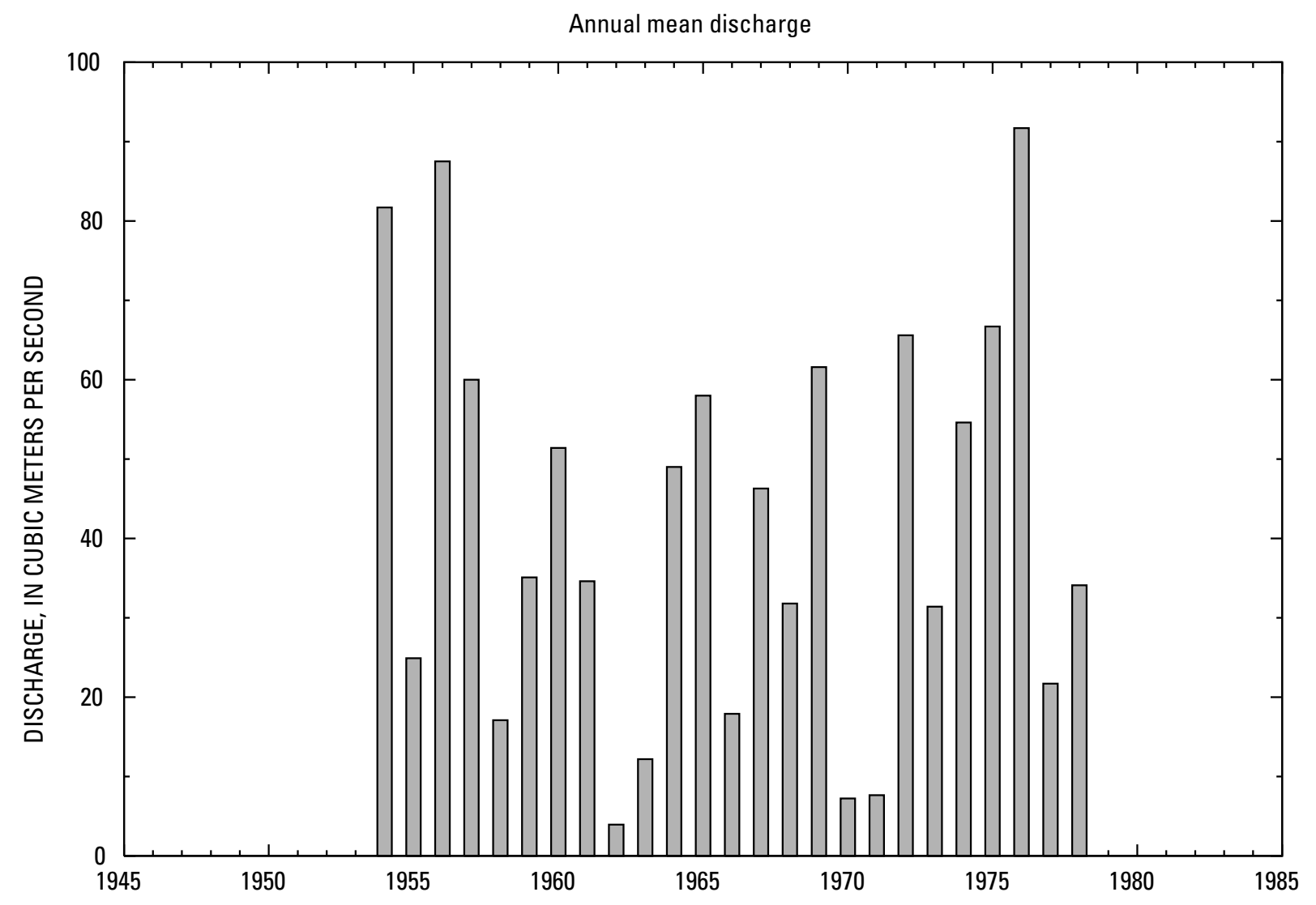




\section{6- 0.000-3M FARAH RIVER AT FARAH, Continued}

\section{Statistics of monthly and annual mean discharges}

[ $\mathrm{m}^{3} / \mathrm{s}$, meters per second; $\mathrm{m}$, more than 2 years of occurrence]

\begin{tabular}{|c|c|c|c|c|c|c|c|c|}
\hline \multirow[b]{2}{*}{ Month } & \multicolumn{2}{|c|}{ Maximum } & \multicolumn{2}{|c|}{ Minimum } & \multicolumn{4}{|c|}{ Mean } \\
\hline & $\begin{array}{c}\text { Discharge } \\
\left(\mathrm{m}^{3} / \mathrm{s}\right)\end{array}$ & $\begin{array}{c}\text { Water year } \\
\text { of } \\
\text { occurrence }\end{array}$ & $\begin{array}{c}\text { Discharge } \\
\left(\mathrm{m}^{3} / \mathrm{s}\right)\end{array}$ & $\begin{array}{c}\text { Water year } \\
\text { of } \\
\text { occurrence }\end{array}$ & $\begin{array}{c}\text { Discharge } \\
\left(\mathrm{m}^{3} / \mathrm{s}\right)\end{array}$ & $\begin{array}{c}\text { Standard } \\
\text { deviation } \\
\left(\mathrm{m}^{3} / \mathrm{s}\right)\end{array}$ & $\begin{array}{c}\text { Coefficient } \\
\text { of } \\
\text { variation }\end{array}$ & $\begin{array}{c}\text { Percentage } \\
\text { of annual } \\
\text { discharge }\end{array}$ \\
\hline October & 1.93 & 1958 & 0 & $\mathrm{~m}$ & 0.15 & 0.38 & 2.51 & 0.03 \\
\hline November & 19.5 & 1964 & 0 & 1971,1972 & 2.41 & 5.41 & 2.25 & 0.47 \\
\hline December & 109 & 1969 & 0.013 & 1971 & 10.9 & 23.4 & 2.14 & 2.15 \\
\hline January & 64.7 & 1965 & 0.044 & 1963 & 20.7 & 24.5 & 1.18 & 4.08 \\
\hline February & 362 & 1954 & 0.059 & 1961 & 64.1 & 80.8 & 1.26 & 12.6 \\
\hline March & 416 & 1976 & 6.79 & 1962 & 144 & 113 & 0.78 & 28.4 \\
\hline April & 508 & 1956 & 16.6 & 1962 & 177 & 130 & 0.74 & 34.8 \\
\hline May & 165 & 1976 & 4.58 & 1962 & 68.7 & 49.0 & 0.71 & 13.5 \\
\hline June & 72.9 & 1954 & 0 & 1971 & 15.2 & 15.8 & 1.04 & 2.99 \\
\hline July & 36.4 & 1956 & 0 & 1970, 1971 & 3.63 & 8.45 & 2.33 & 0.71 \\
\hline August & 10.2 & 1957 & 0 & 1970, 1971 & 0.66 & 2.04 & 3.11 & 0.13 \\
\hline September & 5.89 & 1957 & 0 & 1970, 1971 & 0.28 & 1.15 & 4.08 & 0.06 \\
\hline Annual & 91.7 & 1976 & 3.93 & 1962 & 42.2 & 25.5 & 0.60 & 100 \\
\hline
\end{tabular}

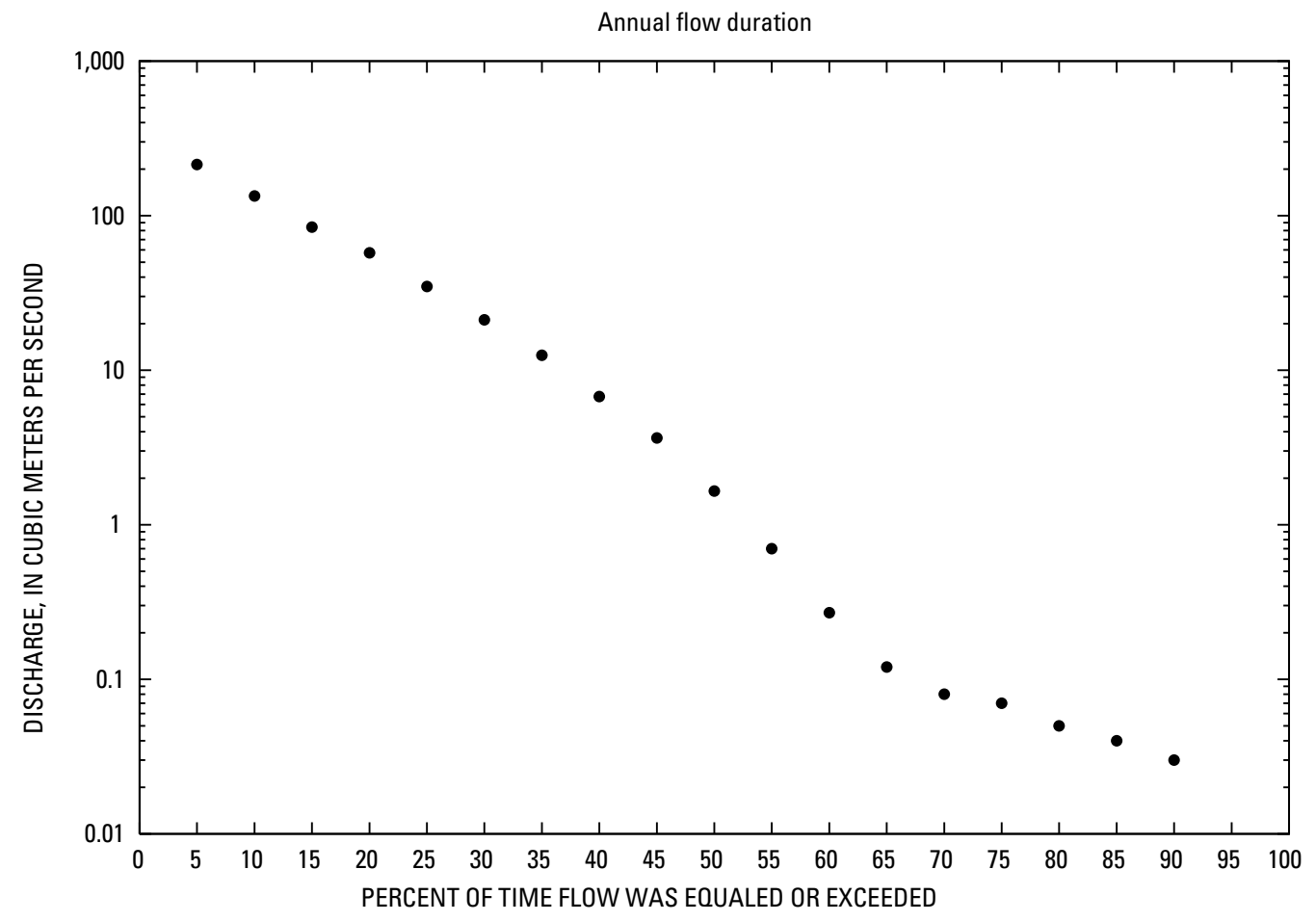




\section{6- 0.000-3M FARAH RIVER AT FARAH, Continued}

\section{Monthly and annual flow duration, in cubic meters per second}

[Data were not rounded in accordance with U.S. Geological Survey publication standards]

\begin{tabular}{|c|c|c|c|c|c|c|c|c|c|c|c|c|c|}
\hline $\begin{array}{l}\text { Percentage } \\
\text { of days } \\
\text { discharge } \\
\text { equaled or } \\
\text { exceeded }\end{array}$ & January & February & March & April & May & June & July & August & September & October & November & December & Annual \\
\hline 95 & 0.06 & 0.07 & 1.15 & 15.2 & 3.83 & 0.04 & 0 & 0 & 0 & 0 & 0 & 0.03 & 0 \\
\hline 90 & 0.11 & 0.19 & 4.56 & 25.1 & 6.25 & 0.21 & 0.02 & 0 & 0 & 0 & 0 & 0.04 & 0.03 \\
\hline 80 & 0.26 & 4.01 & 22.8 & 44.4 & 17.9 & 1.3 & 0.07 & 0.03 & 0.02 & 0.02 & 0.03 & 0.05 & 0.05 \\
\hline 75 & 0.39 & 5.99 & 36.6 & 55.2 & 22.3 & 2.15 & 0.1 & 0.03 & 0.03 & 0.02 & 0.04 & 0.06 & 0.07 \\
\hline 70 & 0.72 & 7.96 & 52.5 & 72.9 & 26.6 & 3.39 & 0.17 & 0.04 & 0.03 & 0.03 & 0.04 & 0.07 & 0.08 \\
\hline 65 & 1.1 & 10.7 & 65.1 & 82.7 & 30.7 & 4.65 & 0.23 & 0.05 & 0.03 & 0.03 & 0.04 & 0.13 & 0.12 \\
\hline 60 & 1.53 & 15.1 & 74.5 & 94.2 & 35.5 & 6.06 & 0.35 & 0.06 & 0.03 & 0.03 & 0.05 & 0.2 & 0.27 \\
\hline 45 & 5.15 & 30.6 & 110.4 & 144 & 56.9 & 10.5 & 0.63 & 0.08 & 0.04 & 0.04 & 0.07 & 0.75 & 3.65 \\
\hline 40 & 6.44 & 36.6 & 125.3 & 163.5 & 65.7 & 11.7 & 0.8 & 0.09 & 0.05 & 0.05 & 0.08 & 1.06 & 6.76 \\
\hline 35 & 7.81 & 45.1 & 140.2 & 180.6 & 75.5 & 13.4 & 1.08 & 0.1 & 0.06 & 0.06 & 0.08 & 1.33 & 12.5 \\
\hline 30 & 12.5 & 55.5 & 156.1 & 195.4 & 85.3 & 15.2 & 1.78 & 0.12 & 0.07 & 0.06 & 0.1 & 2.02 & 21.2 \\
\hline 25 & 18 & 65.8 & 178 & 214.4 & 101.2 & 18 & 2.41 & 0.17 & 0.07 & 0.07 & 0.12 & 2.61 & 34.8 \\
\hline 20 & 25.1 & 77.2 & 199.8 & 244.2 & 117.5 & 21.5 & 3.03 & 0.31 & 0.09 & 0.08 & 0.2 & 5.8 & 57.5 \\
\hline 15 & 33.8 & 102 & 245.2 & 310.7 & 135.7 & 27.1 & 4.42 & 0.45 & 0.1 & 0.11 & 0.51 & 11.1 & 84.4 \\
\hline 10 & 47.1 & 142.1 & 330.9 & 402 & 153.9 & 35.2 & 6.62 & 1.41 & 0.17 & 0.17 & 1.92 & 25.7 & 134.1 \\
\hline 5 & 92.9 & 251.5 & 504 & 559.6 & 200.4 & 53.9 & 17.5 & 3.95 & 0.37 & 1.24 & 8.42 & 58.2 & 214.5 \\
\hline
\end{tabular}




\section{6- 0.000-3M FARAH RIVER AT FARAH, Continued}

\section{Probability of occurrence of annual high discharges}

[m³/s, meters per second; ng, statistic not given]

\begin{tabular}{|c|c|c|c|c|c|c|}
\hline \multirow{2}{*}{$\begin{array}{l}\text { Exceedance } \\
\text { probability }\end{array}$} & \multirow{2}{*}{$\begin{array}{c}\text { Recurrence } \\
\text { interval } \\
\text { (years) }\end{array}$} & \multirow{2}{*}{$\begin{array}{c}\text { Maximum } \\
\text { instantaneous } \\
\left(\mathrm{m}^{3} / \mathrm{s}\right)\end{array}$} & \multicolumn{4}{|c|}{ Maximum mean discharge } \\
\hline & & & 3-day period & 7-day period & 15-day period & 30-day period \\
\hline 0.99 & 1.01 & ng & 27.3 & 23.2 & 17.4 & 14.2 \\
\hline 0.95 & 1.05 & 154 & 80.9 & 61.2 & 47.6 & 38.3 \\
\hline 0.90 & 1.11 & 246 & 134 & 103 & 76.0 & 60.7 \\
\hline 0.80 & 1.25 & 409 & 229 & 172 & 126 & 99.4 \\
\hline 0.50 & 2 & 906 & 528 & 385 & 278 & 213 \\
\hline 0.20 & 5 & 1,620 & 960 & 697 & 497 & 369 \\
\hline 0.10 & 10 & 2,040 & 1,210 & 882 & 625 & 455 \\
\hline 0.04 & 25 & 2,480 & 1,460 & 1,080 & 759 & 542 \\
\hline 0.02 & 50 & 2,750 & 1,610 & 1,200 & 839 & 592 \\
\hline 0.01 & 100 & 2,970 & 1,730 & 1,300 & 905 & 633 \\
\hline 0.005 & 200 & 3,150 & 1,820 & 1,380 & 960 & 665 \\
\hline 0.002 & 500 & 3,340 & ng & ng & ng & $\mathrm{ng}$ \\
\hline
\end{tabular}

\section{6- 0.000-3M FARAH RIVER AT FARAH, Continued}

\section{Probability of occurrence of annual low discharges}

[m³ $/ \mathrm{s}$, meters per second]

\begin{tabular}{|c|c|c|c|c|c|c|c|c|c|c|}
\hline \multirow{3}{*}{$\begin{array}{c}\text { Nonexceedance } \\
\text { probability }\end{array}$} & \multirow{3}{*}{$\begin{array}{c}\text { Recurrence } \\
\text { interval } \\
\text { (years) }\end{array}$} & \multicolumn{9}{|c|}{ Minimum mean discharge $\left(\mathrm{m}^{3} / \mathrm{s}\right)$} \\
\hline & & \multicolumn{9}{|c|}{ Number of consecutive days } \\
\hline & & 1 & 3 & 7 & 14 & 30 & 60 & 90 & 120 & 183 \\
\hline 0.05 & 20 & 0 & 0 & 0 & 0 & 0 & 0 & 0 & 0 & 0.001 \\
\hline 0.10 & 10 & 0 & 0 & 0 & 0 & 0 & 0.008 & 0.015 & 0.019 & 0.014 \\
\hline 0.20 & 5 & 0 & 0 & 0 & 0 & 0 & 0.011 & 0.017 & 0.026 & 0.073 \\
\hline 0.50 & 2 & 0.020 & 0.020 & 0.021 & ${ }^{1} 0.020$ & 0.023 & 0.025 & 0.032 & 0.055 & 0.588 \\
\hline
\end{tabular}

'Data does not fit log-Pearson Type III curve, use with caution. 


\section{6- 0.000-3M FARAH RIVER AT FARAH, Continued}

\section{Probability of occurrence of seasonal low discharges}

$\left[\mathrm{m}^{3} / \mathrm{s}\right.$, meters per second]

\begin{tabular}{|c|c|c|c|c|c|c|c|c|c|}
\hline \multirow{3}{*}{$\begin{array}{c}\text { Nonexceedance } \\
\text { probability }\end{array}$} & \multirow{3}{*}{$\begin{array}{c}\text { Recurrence } \\
\text { interval } \\
\text { (years) }\end{array}$} & \multicolumn{8}{|c|}{ Minimum mean discharge $\left(\mathrm{m}^{3} / \mathrm{s}\right)$} \\
\hline & & \multicolumn{8}{|c|}{ Number of consecutive days } \\
\hline & & 1 & 7 & 14 & 30 & 1 & 7 & 14 & 30 \\
\hline & & \multicolumn{4}{|c|}{ December-January-February } & \multicolumn{4}{|c|}{ March-April-May } \\
\hline 0.05 & 20 & 0.009 & 0.014 & ${ }^{1} 0.008$ & 0.019 & 0.002 & 0.009 & 0.019 & 4.80 \\
\hline 0.10 & 10 & 0.018 & 0.020 & 0.021 & 0.041 & 0.115 & 0.353 & 0.728 & 8.82 \\
\hline 0.20 & 5 & 0.032 & ${ }^{1} 0.031$ & 0.049 & 0.103 & 1.05 & 2.64 & 4.96 & 16.9 \\
\hline \multirow[t]{2}{*}{0.50} & 2 & 0.103 & ${ }^{1} 0.102$ & 0.250 & 0.640 & 13.8 & 22.3 & 33.6 & 46.4 \\
\hline & & \multicolumn{4}{|c|}{ June-July-August } & \multicolumn{4}{|c|}{ September-October-November } \\
\hline 0.05 & 20 & 0 & 0 & 0 & 0 & 0 & 0 & 0 & 0 \\
\hline 0.10 & 10 & 0 & 0 & 0 & 0.003 & 0 & 0 & 0 & 0 \\
\hline 0.20 & 5 & 0.017 & 0.017 & 0.018 & ${ }^{1} 0.013$ & 0 & 0 & 0.003 & 0.011 \\
\hline 0.50 & 2 & 0.042 & 0.044 & 0.049 & 0.081 & 0.020 & 0.021 & ${ }^{1} 0.019$ & 0.023 \\
\hline
\end{tabular}

'Data does not fit log-Pearson Type III curve, use with caution. 


\section{Annual peak discharge and corresponding gage height}

$\left[\mathrm{m}^{3} / \mathrm{s}\right.$, meters per second; --, no data]

\begin{tabular}{|c|c|c|c|c|c|c|c|}
\hline $\begin{array}{c}\text { Water } \\
\text { year }\end{array}$ & Date & $\begin{array}{c}\text { Gage } \\
\text { height } \\
\text { (meters) }\end{array}$ & $\begin{array}{c}\text { Peak } \\
\text { discharge } \\
\left(\mathrm{m}^{3} / \mathrm{s}\right)\end{array}$ & $\begin{array}{c}\text { Water } \\
\text { year }\end{array}$ & Date & $\begin{array}{c}\text { Gage } \\
\text { height } \\
\text { (meters) }\end{array}$ & $\begin{array}{c}\text { Peak } \\
\text { discharge } \\
\left(\mathrm{m}^{3} / \mathrm{s}\right)\end{array}$ \\
\hline \multicolumn{8}{|c|}{ Annual peak discharge, by year } \\
\hline 1953 & 11-Apr & 5.75 & 3,102 & 1966 & 27-Apr & -- & 500 \\
\hline 1954 & $10-\mathrm{Feb}$ & 5.35 & 1,840 & 1967 & 27-Apr & -- & 700 \\
\hline 1955 & 14-Mar & 5.85 & 2,173 & 1968 & 11-Mar & -- & 700 \\
\hline 1956 & 5-Mar & -- & 2,207 & 1969 & 16-Apr & -- & 1,138 \\
\hline 1957 & 17-Mar & -- & 1,132 & 1970 & 28-Mar & -- & 61.6 \\
\hline 1958 & 29-Mar & 2.22 & 170 & 1971 & 26-Mar & -- & 840 \\
\hline 1959 & 29-Mar & 3.10 & 736 & 1972 & 19-Mar & -- & 1,062 \\
\hline 1960 & 17-Apr & 3.51 & 1,087 & 1973 & $25-\mathrm{Feb}$ & -- & 995 \\
\hline 1961 & 3-Apr & -- & 1,104 & 1974 & 20-Feb & -- & 827 \\
\hline 1962 & 24-Dec & -- & 133 & 1975 & 4-Apr & -- & 1,052 \\
\hline 1963 & 13-May & -- & 914 & 1976 & 23-Mar & -- & 1,800 \\
\hline 1964 & 17-Feb & -- & 694 & 1977 & 6-Apr & -- & 118 \\
\hline 1965 & 2-Feb & -- & 1,104 & 1978 & 9-Mar & -- & 375 \\
\hline \multicolumn{8}{|c|}{ Annual peak discharge, from highest to lowest } \\
\hline 1953 & 11-Apr & 5.75 & 3,102 & 1963 & 13-May & -- & 914 \\
\hline 1956 & 5-Mar & -- & 2,207 & 1971 & 26-Mar & -- & 840 \\
\hline 1955 & 14-Mar & 5.85 & 2,173 & 1974 & $20-\mathrm{Feb}$ & -- & 827 \\
\hline 1954 & $10-\mathrm{Feb}$ & 5.35 & 1,840 & 1959 & 29-Mar & 3.10 & 736 \\
\hline 1976 & 23-Mar & -- & 1,800 & 1968 & 11-Mar & -- & 700 \\
\hline 1969 & 16-Apr & -- & 1,138 & 1967 & 27-Apr & -- & 700 \\
\hline 1957 & 17-Mar & -- & 1,132 & 1964 & 17-Feb & -- & 694 \\
\hline 1965 & 2-Feb & -- & 1,104 & 1966 & 27-Apr & -- & 500 \\
\hline 1961 & 3-Apr & -- & 1,104 & 1978 & 9-Mar & -- & 375 \\
\hline 1960 & 17-Apr & 3.51 & 1,087 & 1958 & 29-Mar & 2.22 & 170 \\
\hline 1972 & 19-Mar & -- & 1,062 & 1962 & 24-Dec & -- & 133 \\
\hline 1975 & 4-Apr & -- & 1,052 & 1977 & 6-Apr & -- & 118 \\
\hline 1973 & $25-\mathrm{Feb}$ & -- & 995 & 1970 & 28-Mar & -- & 61.6 \\
\hline
\end{tabular}




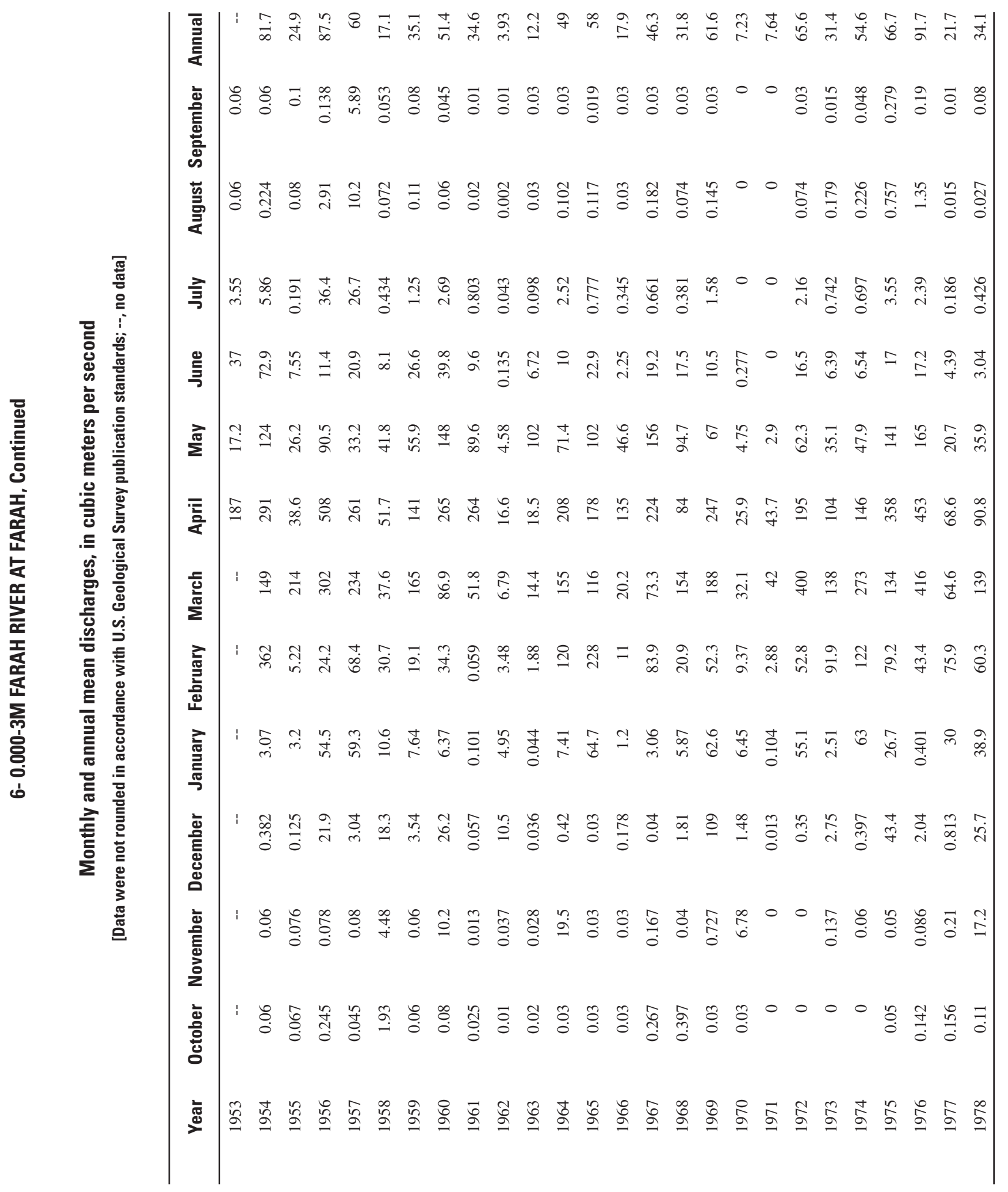




\section{6- 0.000-4S FARAH RIVER NEAR DAULATABAD}

\section{(U.S. Geological Survey identification number: 324500062370000 )}

LOCATION: Lat $32^{\circ} 45^{\prime} \mathrm{N}$., long $62^{\circ} 37^{\prime}$ E., on upstream side of bridge pier on Kandahar-Heart Highway at Daulatabad and about $70 \mathrm{~km}$ upstream from Farah.

DRAINAGE AREA: $21,850 \mathrm{~km}^{2}$.

ALTITUDE: 785 meters plus mean sea level.

PERIOD OF RECORD: March 29, 1963 to September 30, 1971.

GAGE: Staff gage read once or twice daily.

Annual mean discharge

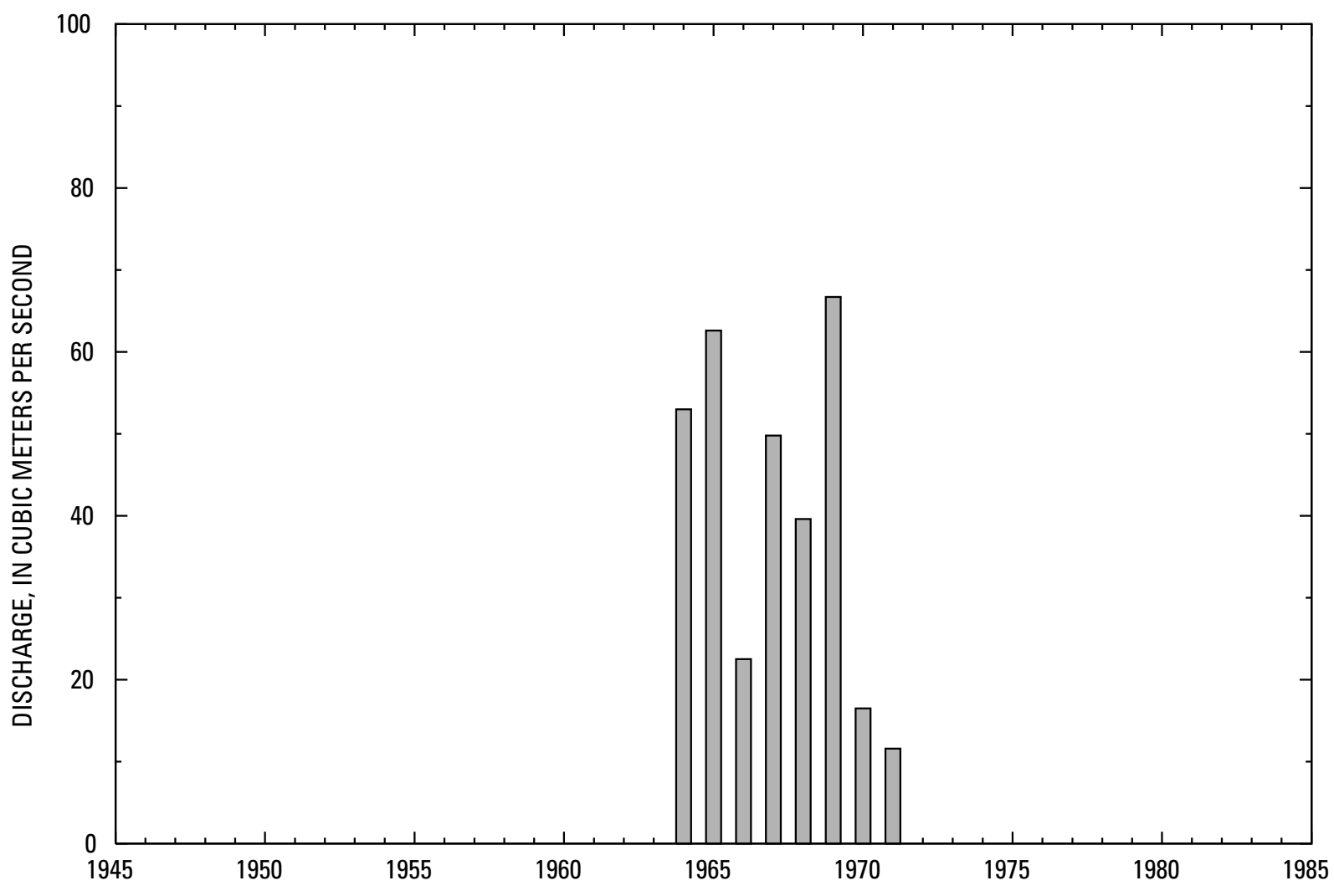




\section{6- 0.000-4S FARAH RIVER NEAR DAULATABAD, Continued}

\section{Statistics of monthly and annual mean discharges}

$\left[\mathrm{m}^{3} / \mathrm{s}\right.$, meters per second]

\begin{tabular}{|c|c|c|c|c|c|c|c|c|}
\hline \multirow[b]{2}{*}{ Month } & \multicolumn{2}{|c|}{ Maximum } & \multicolumn{2}{|c|}{ Minimum } & \multicolumn{4}{|c|}{ Mean } \\
\hline & $\begin{array}{c}\text { Discharge } \\
\left(\mathrm{m}^{3} / \mathrm{s}\right)\end{array}$ & $\begin{array}{c}\text { Water year } \\
\text { of } \\
\text { occurrence }\end{array}$ & $\begin{array}{c}\text { Discharge } \\
\left(\mathrm{m}^{3} / \mathrm{s}\right)\end{array}$ & $\begin{array}{c}\text { Water year } \\
\text { of } \\
\text { occurrence }\end{array}$ & $\begin{array}{c}\text { Discharge } \\
\left(\mathrm{m}^{3} / \mathrm{s}\right)\end{array}$ & $\begin{array}{c}\text { Standard } \\
\text { deviation } \\
\left(\mathrm{m}^{3} / \mathrm{s}\right)\end{array}$ & $\begin{array}{c}\text { Coefficient } \\
\text { of } \\
\text { variation }\end{array}$ & $\begin{array}{c}\text { Percentage } \\
\text { of annual } \\
\text { discharge }\end{array}$ \\
\hline October & 5.79 & 1968 & 0.267 & 1971 & 3.31 & 1.66 & 0.50 & 0.69 \\
\hline November & 25.4 & 1964 & 1.87 & 1971 & 11.1 & 7.58 & 0.68 & 2.32 \\
\hline December & 98.6 & 1969 & 3.56 & 1971 & 19.9 & 32.0 & 1.60 & 4.16 \\
\hline January & 72.7 & 1969 & 3.84 & 1971 & 27.7 & 28.1 & 1.01 & 5.79 \\
\hline February & 204 & 1965 & 9.97 & 1971 & 71.6 & 65.6 & 0.92 & 14.9 \\
\hline March & 194 & 1969 & 25.1 & 1966 & 105 & 60.9 & 0.58 & 22.0 \\
\hline April & 238 & 1969 & 40.3 & 1963 & 134 & 77.2 & 0.58 & 27.9 \\
\hline May & 149 & 1967 & 8.40 & 1971 & 80.1 & 47.2 & 0.59 & 16.7 \\
\hline June & 33.3 & 1965 & 0.700 & 1971 & 17.2 & 11.7 & 0.68 & 3.59 \\
\hline July & 11.5 & 1965 & 0 & 1971 & 4.49 & 3.92 & 0.87 & 0.94 \\
\hline August & 6.29 & 1965 & 0 & 1970. 1971 & 2.28 & 2.32 & 1.02 & 0.48 \\
\hline September & 5.40 & 1965 & 0 & 1970 & 2.11 & 1.94 & 0.92 & 0.44 \\
\hline Annual & 66.7 & 1969 & 11.6 & 1971 & 40.3 & 21.2 & 0.53 & 100 \\
\hline
\end{tabular}

Annual flow duration

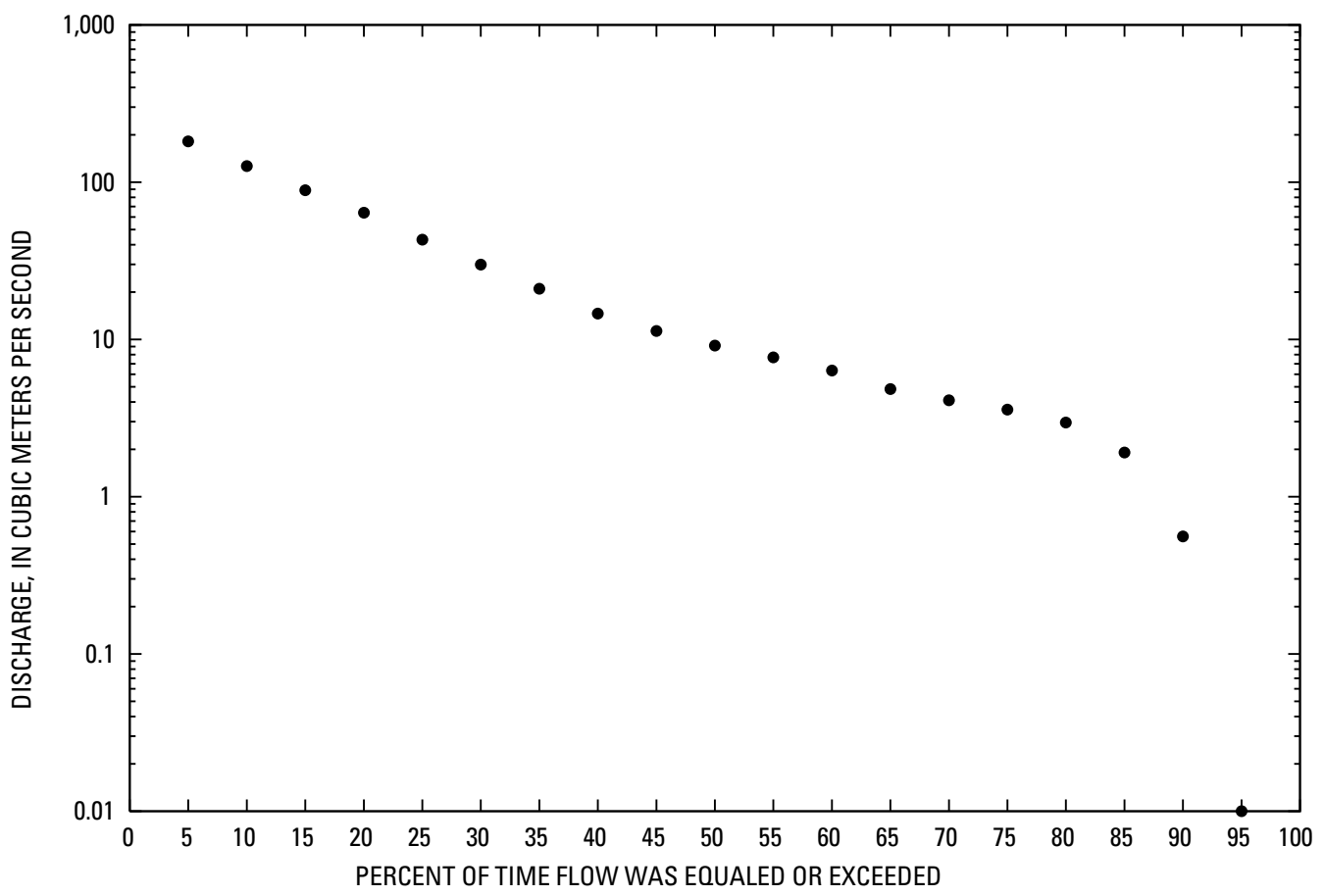




\section{6- 0.000-4S FARAH RIVER NEAR DAULATABAD, Continued}

Monthly and annual flow duration, in cubic meters per second

[Data were not rounded in accordance with U.S. Geological Survey publication standards]

\begin{tabular}{|c|c|c|c|c|c|c|c|c|c|c|c|c|c|}
\hline $\begin{array}{l}\text { Percentage } \\
\text { of days } \\
\text { discharge } \\
\text { equaled or } \\
\text { exceeded }\end{array}$ & January & February & March & April & May & June & July & August & September & October & November & December & Annual \\
\hline 95 & 3.31 & 4.5 & 11.4 & 29 & 6.56 & 0.02 & 0.01 & 0.04 & 0 & 0.23 & 0.69 & 3.64 & 0.01 \\
\hline 90 & 3.59 & 6.89 & 22.5 & 32.5 & 10.1 & 1.73 & 0.03 & 0.09 & 0 & 0.44 & 3.2 & 3.78 & 0.56 \\
\hline 85 & 3.99 & 10.5 & 25.3 & 36.9 & 15.4 & 2.79 & 0.04 & 0.13 & 0 & 1.19 & 3.65 & 3.92 & 1.91 \\
\hline 80 & 4.62 & 13.7 & 27.3 & 45.1 & 21.2 & 3.89 & 0.37 & 0.17 & 0.02 & 1.41 & 4.31 & 4.17 & 2.96 \\
\hline 75 & 6.78 & 16.6 & 42.5 & 51.2 & 27.3 & 5 & 0.79 & 0.2 & 0.27 & 1.56 & 5.18 & 4.6 & 3.58 \\
\hline 70 & 7.79 & 20.7 & 54.7 & 59.4 & 39.2 & 6.85 & 1.25 & 0.25 & 0.34 & 2.03 & 5.93 & 7.97 & 4.1 \\
\hline 65 & 8.37 & 25.1 & 62.2 & 69.7 & 49.9 & 9.02 & 1.89 & 0.55 & 1.02 & 2.24 & 6.48 & 8.29 & 4.84 \\
\hline 60 & 9.03 & 27.9 & 68.5 & 78.4 & 56.2 & 11.1 & 2.38 & 0.83 & 1.22 & 2.49 & 7 & 8.61 & 6.34 \\
\hline 55 & 10.1 & 29.6 & 75 & 87 & 61.2 & 12.3 & 2.78 & 1.33 & 1.39 & 2.74 & 7.51 & 8.93 & 7.69 \\
\hline 50 & 12 & 31.4 & 88.5 & 95.3 & 69 & 13.6 & 3.64 & 1.56 & 1.64 & 2.98 & 8.01 & 9.31 & 9.14 \\
\hline 45 & 13 & 36.6 & 96.3 & 117.6 & 76.3 & 15.5 & 4.38 & 2.1 & 2.05 & 3.23 & 8.51 & 9.76 & 11.3 \\
\hline 40 & 14.3 & 42.6 & 113.8 & 135.2 & 83.5 & 18.2 & 5.3 & 2.42 & 2.79 & 3.61 & 8.99 & 10.2 & 14.6 \\
\hline 35 & 17.4 & 50.2 & 126.2 & 150.1 & 92.1 & 21 & 5.84 & 3.51 & 3.16 & 3.93 & 9.47 & 10.8 & 21 \\
\hline 30 & 21.6 & 73 & 138.1 & 161.1 & 101 & 23.9 & 6.57 & 3.92 & 3.38 & 4.08 & 9.94 & 11.5 & 29.9 \\
\hline 25 & 27 & 81.3 & 150 & 171.5 & 110.2 & 26.9 & 7.4 & 4.25 & 3.6 & 4.23 & 10.7 & 12.2 & 43.1 \\
\hline 20 & 30.7 & 99.2 & 163 & 185.7 & 121.6 & 29.9 & 8.21 & 4.56 & 3.88 & 4.38 & 12.2 & 12.9 & 63.9 \\
\hline 15 & 35.3 & 121 & 180.2 & 211.1 & 136.5 & 33.7 & 9.08 & 4.91 & 4.23 & 4.54 & 13.9 & 13.6 & 88.8 \\
\hline 10 & 55 & 156.9 & 207.5 & 267 & 159.3 & 37.9 & 10.1 & 5.37 & 4.76 & 4.85 & 17.6 & 43.1 & 126.7 \\
\hline 5 & 119.2 & 285.1 & 244.5 & 360.8 & 196.6 & 45.6 & 12.5 & 0 & 0 & 7.1 & 24.1 & 69.6 & 181.7 \\
\hline
\end{tabular}




\section{6- 0.000-4S FARAH RIVER NEAR DAULATABAD, Continued}

Probability of occurrence of annual high discharges

[ $\mathrm{m}^{3} / \mathrm{s}$, meters per second; $\mathrm{ng}$, statistic not given]

\begin{tabular}{rrrrrrr}
\hline & & & \multicolumn{5}{c}{ Maximum mean discharge } \\
$\begin{array}{c}\text { Exceedance } \\
\text { probability }\end{array}$ & $\begin{array}{c}\text { Recurrence } \\
\text { interval } \\
\text { (years) }\end{array}$ & $\begin{array}{c}\text { Maximum } \\
\text { instantaneous } \\
\left(\mathbf{m}^{3} / \mathbf{s}\right)\end{array}$ & 3-day period & 7-day period & 15-day period & 30-day period \\
\cline { 5 - 7 } & 1.01 & ng & 47.6 & 41.0 & 35.8 & 34.6 \\
0.99 & 1.05 & ng & 115 & 87.3 & 68.9 & 61.1 \\
0.95 & 1.11 & ng & 170 & 124 & 93.6 & 79.7 \\
0.80 & 1.25 & 497 & 256 & 179 & 131 & 106 \\
0.50 & 2 & 646 & 459 & 317 & 222 & 168 \\
0.20 & 5 & 863 & 659 & 474 & 329 & 237 \\
0.10 & 10 & 1,020 & 740 & 551 & 386 & 272 \\
0.04 & 25 & 1,220 & 803 & 623 & 443 & 306 \\
0.02 & 50 & 1,370 & 831 & 662 & 477 & 326 \\
0.01 & 100 & 1,540 & 850 & 692 & 505 & 342 \\
0.005 & 200 & 1,710 & 862 & 715 & 527 & 356 \\
0.002 & 500 & 1,950 & ng & ng & ng & ng \\
\hline
\end{tabular}

'Less than 10 years of data used.

\section{6- 0.000-4S FARAH RIVER NEAR DAULATABAD, Continued}

Probability of occurrence of annual low discharges

$\left[\mathrm{m}^{3} / \mathrm{s}\right.$, meters per second]

\begin{tabular}{|c|c|c|c|c|c|c|c|c|c|c|}
\hline \multirow{3}{*}{$\begin{array}{c}\text { Nonexceedance } \\
\text { probability }\end{array}$} & \multirow{3}{*}{$\begin{array}{c}\text { Recurrence } \\
\text { interval } \\
\text { (years) }\end{array}$} & \multicolumn{9}{|c|}{ Minimum mean discharge $\left(\mathrm{m}^{3} / \mathrm{s}\right)$} \\
\hline & & \multicolumn{9}{|c|}{ Number of consecutive days } \\
\hline & & 1 & 3 & 7 & 14 & 30 & 60 & 90 & 120 & 183 \\
\hline 0.05 & 20 & 0 & 0 & 0 & 0 & 0 & 0 & 0.028 & 0.141 & 1.01 \\
\hline 0.10 & 10 & 0 & 0 & 0 & 0 & 0 & 0 & 0.102 & 0.330 & 1.67 \\
\hline 0.20 & 5 & 0.241 & 0.246 & 0.256 & 0.283 & 0.323 & 0.387 & ${ }^{1} 0.378$ & 0.793 & 2.78 \\
\hline 0.50 & 2 & 1.13 & 1.18 & 1.28 & 1.43 & 1.63 & 1.89 & 2.14 & 2.72 & 5.67 \\
\hline
\end{tabular}

'Data does not fit log-Pearson Type III curve, use with caution. 


\section{6- 0.000-4S FARAH RIVER NEAR DAULATABAD, Continued}

Probability of occurrence of seasonal low discharges

$\left[\mathrm{m}^{3} / \mathrm{s}\right.$, meters per second]

\begin{tabular}{|c|c|c|c|c|c|c|c|c|c|}
\hline \multirow{3}{*}{$\begin{array}{c}\text { Nonexceedance } \\
\text { probability }\end{array}$} & \multirow{3}{*}{$\begin{array}{c}\text { Recurrence } \\
\text { interval } \\
\text { (years) }\end{array}$} & \multicolumn{8}{|c|}{ Minimum mean discharge $\left(\mathrm{m}^{3} / \mathrm{s}\right)$} \\
\hline & & \multicolumn{8}{|c|}{ Number of consecutive days } \\
\hline & & 1 & 7 & 14 & 30 & 1 & 7 & 14 & 30 \\
\hline & & \multicolumn{4}{|c|}{ December-January-February } & \multicolumn{4}{|c|}{ March-April-May } \\
\hline 0.05 & 20 & 2.29 & 2.63 & 2.81 & 3.05 & 2.58 & 3.38 & 4.41 & 7.25 \\
\hline 0.10 & 10 & 3.06 & 3.20 & 3.45 & 3.70 & 4.96 & 6.20 & 7.95 & 12.1 \\
\hline 0.20 & 5 & 4.16 & ${ }^{1} 4.12$ & 4.49 & 4.77 & 9.92 & 11.9 & 15.0 & 21.3 \\
\hline \multirow[t]{2}{*}{0.50} & 2 & 6.56 & 7.11 & 7.80 & 8.37 & 28.4 & 32.0 & 40.1 & 52.1 \\
\hline & & \multicolumn{4}{|c|}{ June-July-August } & \multicolumn{4}{|c|}{ September-October-November } \\
\hline 0.05 & 20 & 0 & 0 & 0 & 0 & 0 & 0 & 0 & 0 \\
\hline 0.10 & 10 & 0 & 0 & 0 & 0 & 0 & 0 & 0 & 0 \\
\hline 0.20 & 5 & 0 & 0 & 0 & 0 & 0.317 & 0.352 & 0.389 & 0.535 \\
\hline 0.50 & 2 & 1.19 & 1.22 & 1.30 & 1.51 & 1.29 & 1.48 & 1.63 & 1.94 \\
\hline
\end{tabular}

'Data does not fit log-Pearson Type III curve, use with caution.

6- 0.000-4S FARAH RIVER NEAR DAULATABAD, Continued

Annual peak discharge and corresponding gage height

$\left[\mathrm{m}^{3} / \mathrm{s}\right.$, meters per second; --, no data]

\begin{tabular}{|c|c|c|c|c|c|c|c|}
\hline $\begin{array}{c}\text { Water } \\
\text { year }\end{array}$ & Date & $\begin{array}{c}\text { Gage } \\
\text { height } \\
\text { (meters) }\end{array}$ & $\begin{array}{c}\text { Peak } \\
\text { discharge } \\
\left(\mathrm{m}^{3} / \mathrm{s}\right)\end{array}$ & $\begin{array}{c}\text { Water } \\
\text { year }\end{array}$ & Date & $\begin{array}{c}\text { Gage } \\
\text { height } \\
\text { (meters) }\end{array}$ & $\begin{array}{c}\text { Peak } \\
\text { discharge } \\
\left(\mathrm{m}^{3} / \mathrm{s}\right)\end{array}$ \\
\hline \multicolumn{8}{|c|}{ Annual peak discharge, by year } \\
\hline 1963 & 12-May & & 875 & 1968 & 10-Mar & & 716 \\
\hline 1964 & 17-Feb & & 451 & 1969 & 15-Apr & & 1,200 \\
\hline 1965 & $3-\mathrm{Feb}$ & & 749 & 1970 & 27-Mar & & 111 \\
\hline 1966 & 27-Apr & & 585 & 1971 & 25-Mar & & 520 \\
\hline 1967 & 28-Apr & & 692 & & & & \\
\hline \multicolumn{8}{|c|}{ Annual peak discharge, from highest to lowest } \\
\hline 1969 & 15-Apr & & 1,200 & 1966 & 27-Apr & & 585 \\
\hline 1963 & 12-May & & 875 & 1971 & 25-Mar & & 520 \\
\hline 1965 & $3-\mathrm{Feb}$ & & 749 & 1964 & $17-\mathrm{Feb}$ & & 451 \\
\hline 1968 & 10-Mar & & 716 & 1970 & 27-Mar & & 111 \\
\hline 1967 & 28-Apr & & 692 & & & & \\
\hline
\end{tabular}




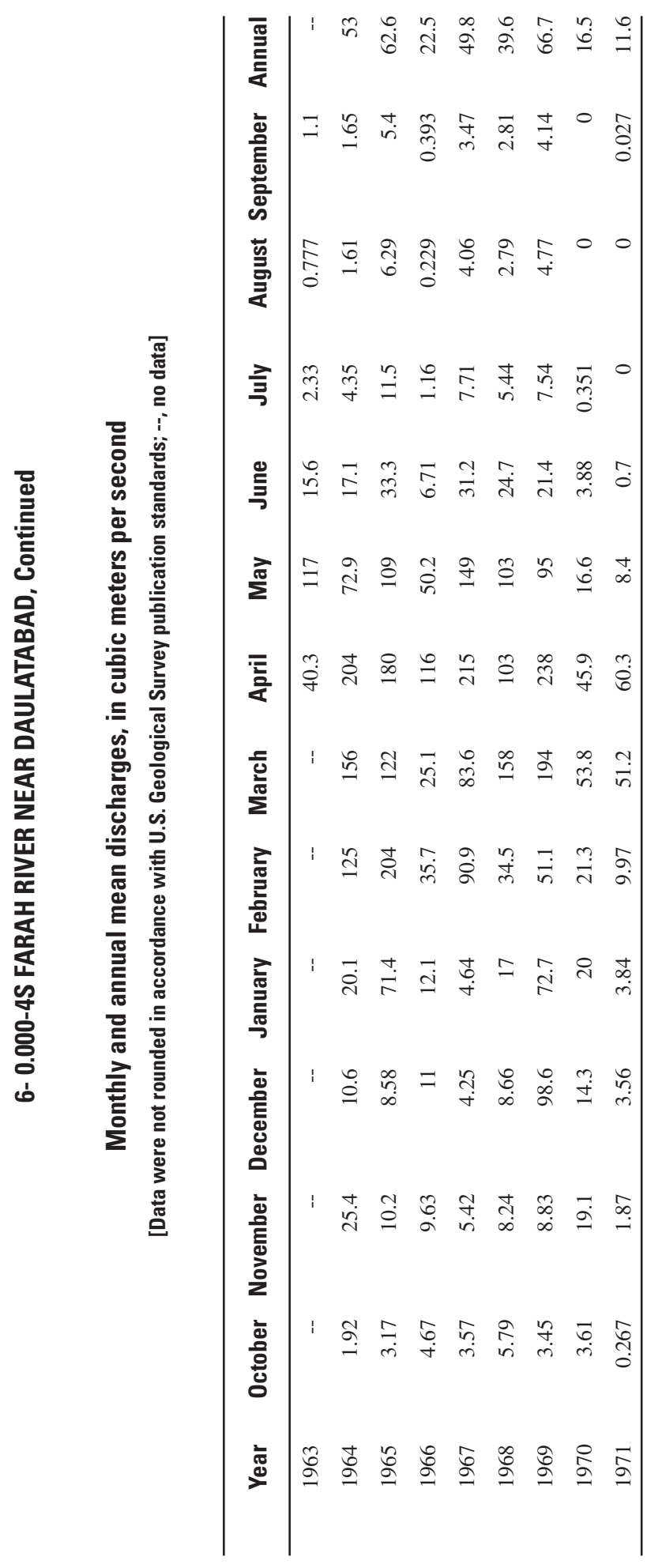




\section{6- 0.000-5M FARAH RIVER NEAR PETCH TANGI}

\section{(U.S. Geological Survey identification number: 325100062520000)}

LOCATION: Lat $32^{\circ} 51^{\prime}$ N., long $62^{\circ} 52^{\prime}$ E., on left bank at Petch-i-Tangi and about $45 \mathrm{~km}$ upstream from Daulatabad.

DRAINAGE AREA: $19,215 \mathrm{~km}^{2}$.

ALTITUDE: 860 meters plus mean sea level.

PERIOD OF RECORD: December 8, 1960 to September 30, 1978.

GAGE: Staff gage read once or twice daily.

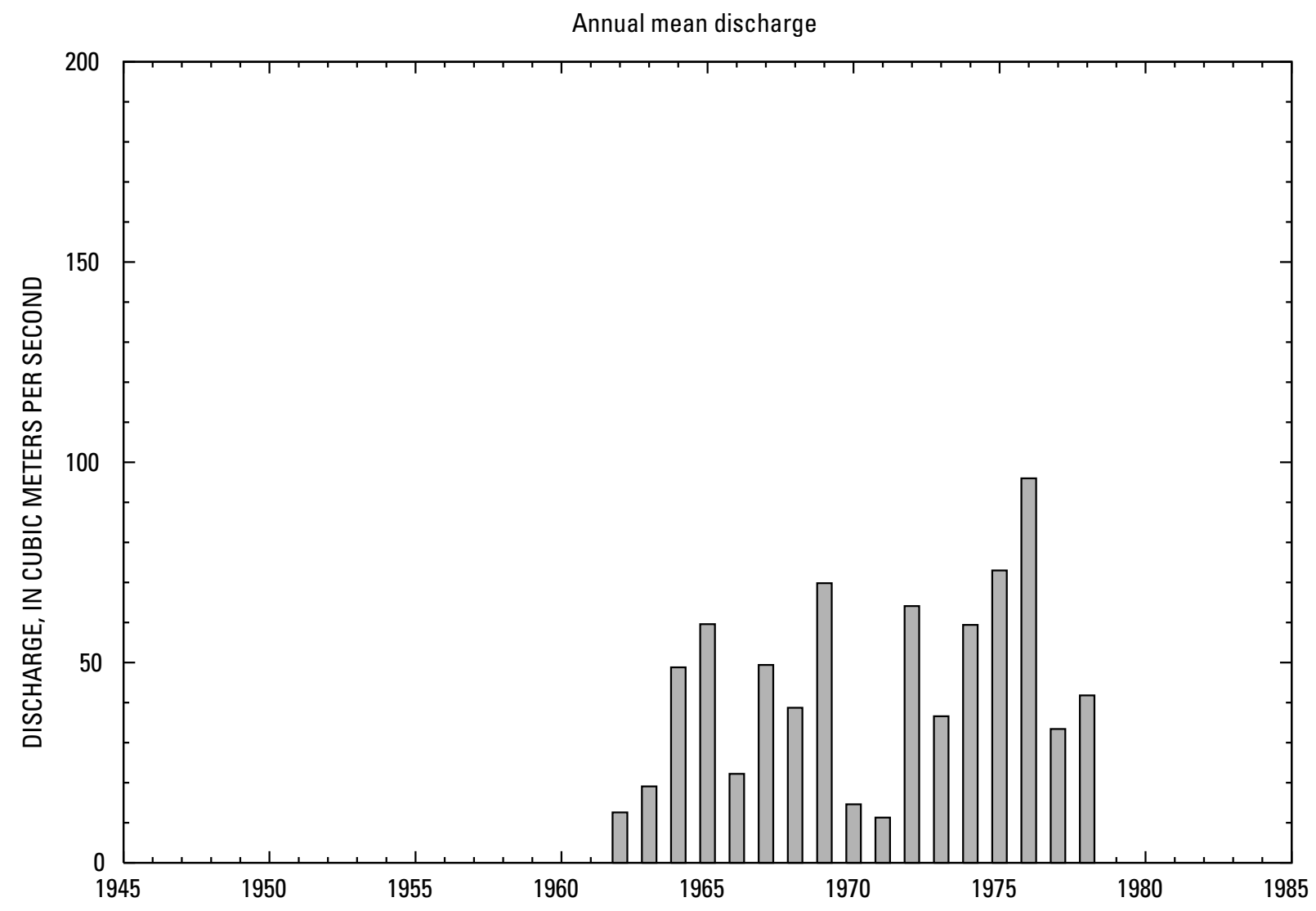




\section{6- 0.000-5M FARAH RIVER NEAR PETCH TANGI, Continued}

\section{Statistics of monthly and annual mean discharges}

$\left[\mathrm{m}^{3} / \mathrm{s}\right.$, meters per second]

\begin{tabular}{|c|c|c|c|c|c|c|c|c|}
\hline \multirow[b]{2}{*}{ Month } & \multicolumn{2}{|c|}{ Maximum } & \multicolumn{2}{|c|}{ Minimum } & \multicolumn{4}{|c|}{ Mean } \\
\hline & $\begin{array}{c}\text { Discharge } \\
\left(\mathrm{m}^{3} / \mathrm{s}\right)\end{array}$ & $\begin{array}{c}\text { Water year } \\
\text { of } \\
\text { occurrence }\end{array}$ & $\begin{array}{c}\text { Discharge } \\
\left(\mathrm{m}^{3} / \mathrm{s}\right)\end{array}$ & $\begin{array}{c}\text { Water year } \\
\text { of } \\
\text { occurrence }\end{array}$ & $\begin{array}{c}\text { Discharge } \\
\left(\mathrm{m}^{3} / \mathrm{s}\right)\end{array}$ & $\begin{array}{c}\text { Standard } \\
\text { deviation } \\
\left(\mathrm{m}^{3} / \mathrm{s}\right)\end{array}$ & $\begin{array}{c}\text { Coefficient } \\
\text { of } \\
\text { variation }\end{array}$ & $\begin{array}{c}\text { Percentage } \\
\text { of annual } \\
\text { discharge }\end{array}$ \\
\hline October & 8.69 & 1977 & 0.519 & 1972 & 4.22 & 2.37 & 0.56 & 0.80 \\
\hline November & 23.6 & 1964 & 2.42 & 1972 & 9.85 & 5.91 & 0.60 & 1.87 \\
\hline December & 97.6 & 1969 & 4.16 & 1972 & 18.2 & 23.0 & 1.26 & 3.45 \\
\hline January & 74.8 & 1965 & 4.33 & 1971 & 27.5 & 23.6 & 0.86 & 5.21 \\
\hline February & 186 & 1965 & 6.73 & 1961 & 65.3 & 47.5 & 0.73 & 12.4 \\
\hline March & 373 & 1976 & 25.5 & 1962 & 133 & 103 & 0.78 & 25.1 \\
\hline April & 435 & 1976 & 39.1 & 1962 & 158 & 106 & 0.67 & 29.9 \\
\hline May & 149 & 1975 & 10.6 & 1971 & 77.2 & 45.9 & 0.59 & 14.6 \\
\hline June & 48.9 & 1975 & 1.69 & 1971 & 20.8 & 14.3 & 0.69 & 3.94 \\
\hline July & 18.6 & 1975 & 0 & 1971 & 6.99 & 5.69 & 0.81 & 1.32 \\
\hline August & 10.7 & 1976 & 0 & 1970,1971 & 3.91 & 3.09 & 0.79 & 0.74 \\
\hline September & 8.77 & 1976 & 0.005 & 1971 & 3.37 & 2.46 & 0.73 & 0.64 \\
\hline Annual & 96 & 1976 & 11.3 & 1971 & 44.1 & 24.2 & 0.55 & 100 \\
\hline
\end{tabular}

Annual flow duration

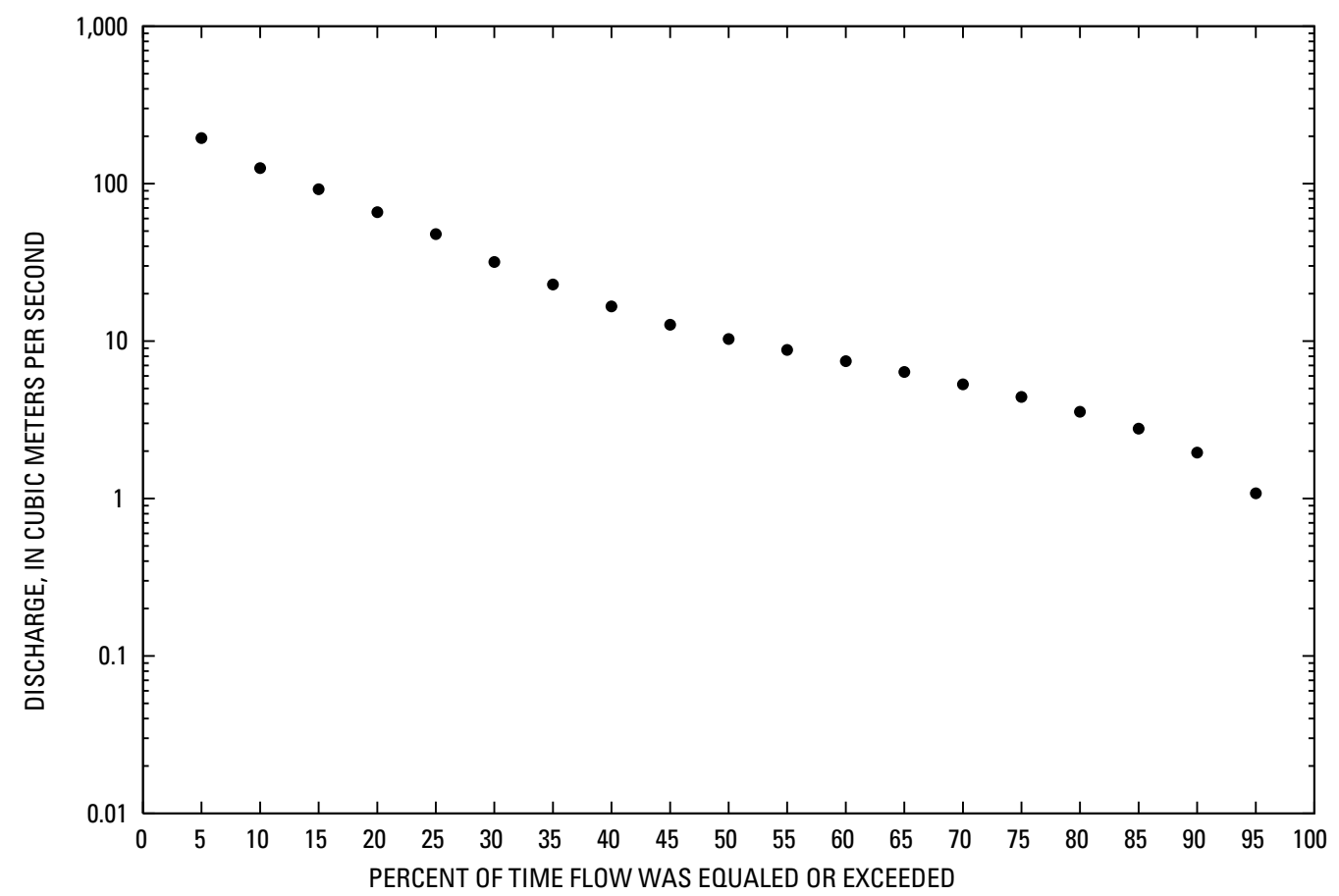




\section{6- 0.000-5M FARAH RIVER NEAR PETCH TANGI, Continued}

Monthly and annual flow duration, in cubic meters per second

[Data were not rounded in accordance with U.S. Geological Survey publication standards]

\begin{tabular}{|c|c|c|c|c|c|c|c|c|c|c|c|c|c|}
\hline $\begin{array}{l}\text { Percentage } \\
\text { of days } \\
\text { discharge } \\
\text { equaled or } \\
\text { exceeded }\end{array}$ & January & February & March & April & May & June & July & August & September & October & November & December & Annual \\
\hline 95 & 3.93 & 5.07 & 11.3 & 30.4 & 8.83 & 2.31 & 0.01 & 0.38 & 0 & 0.52 & 2.57 & 3.41 & 1.08 \\
\hline 90 & 4.37 & 6.17 & 18.8 & 36.4 & 16.8 & 3.62 & 1.13 & 0.77 & 0.03 & 1.28 & 3.19 & 3.97 & 1.96 \\
\hline 85 & 4.94 & 8.3 & 22.5 & 44.6 & 21.5 & 4.69 & 1.42 & 0.95 & 1.07 & 1.76 & 3.59 & 4.32 & 2.78 \\
\hline 80 & 5.73 & 11.9 & 25.4 & 53.4 & 26.3 & 5.95 & 1.76 & 1.09 & 1.29 & 1.99 & 3.96 & 4.96 & 3.56 \\
\hline 75 & 6.66 & 16.3 & 42.1 & 70.2 & 31.6 & 7.37 & 2.13 & 1.19 & 1.48 & 2.21 & 4.57 & 6 & 4.42 \\
\hline 70 & 7.45 & 19.9 & 57 & 82.6 & 37.6 & 8.9 & 2.94 & 1.91 & 1.66 & 2.41 & 5.33 & 6.81 & 5.31 \\
\hline 65 & 8.66 & 23.2 & 70.9 & 93.4 & 45.2 & 10.5 & 3.49 & 2.46 & 2.13 & 2.74 & 6 & 7.49 & 6.36 \\
\hline 60 & 9.66 & 27 & 80.5 & 104.4 & 52.9 & 12.3 & 4.14 & 2.58 & 2.33 & 3.05 & 6.46 & 8.06 & 7.45 \\
\hline 55 & 10.6 & 33.9 & 89.5 & 116 & 58.9 & 14.2 & 4.67 & 2.8 & 2.55 & 3.39 & 6.93 & 8.61 & 8.8 \\
\hline 50 & 11.7 & 42.6 & 101.6 & 123.7 & 64 & 16.5 & 5.21 & 3.32 & 3.05 & 3.72 & 7.41 & 9.11 & 10.3 \\
\hline 45 & 12.9 & 46.9 & 109.5 & 133.1 & 71.5 & 18.9 & 5.83 & 3.5 & 3.31 & 4.04 & 7.91 & 9.62 & 12.7 \\
\hline 40 & 14.5 & 52.5 & 117.8 & 145 & 78.4 & 21.2 & 6.6 & 3.84 & 3.53 & 4.5 & 8.41 & 10.2 & 16.6 \\
\hline 35 & 16.7 & 60 & 130.2 & 158.5 & 85.7 & 23.5 & 8.16 & 4.59 & 3.75 & 4.86 & 9.29 & 10.8 & 22.9 \\
\hline 30 & 20 & 68.3 & 145.9 & 173 & 94.7 & 26.9 & 9.1 & 5.15 & 4.34 & 5.19 & 10.2 & 11.4 & 31.8 \\
\hline 25 & 27.6 & 77.7 & 167.8 & 192.4 & 107.8 & 30.3 & 10.1 & 6.14 & 5.06 & 5.7 & 10.9 & 12.4 & 47.8 \\
\hline 20 & 32 & 91.5 & 193.6 & 214.3 & 120.5 & 35.1 & 11.8 & 6.75 & 5.73 & 6.31 & 11.5 & 13.3 & 65.9 \\
\hline 15 & 41.3 & 109.9 & 222.3 & 250 & 136.8 & 40.1 & 13.7 & 7.36 & 6.41 & 7.04 & 12.5 & 16.6 & 92.2 \\
\hline 10 & 53.7 & 131.4 & 280.5 & 326.8 & 162.9 & 47.3 & 16.1 & 8.25 & 7.11 & 7.92 & 14.7 & 26.3 & 125.5 \\
\hline 5 & 88.8 & 228.2 & 429 & 453 & 190.5 & 56.1 & 19.3 & 10.3 & 8.51 & 9.08 & 21 & 62.8 & 194.8 \\
\hline
\end{tabular}




\section{6- 0.000-5M FARAH RIVER NEAR PETCH TANGI, Continued}

Probability of occurrence of annual high discharges

[ $\mathrm{m}^{3} / \mathrm{s}$, meters per second; $\mathrm{ng}$, statistic not given]

\begin{tabular}{|c|c|c|c|c|c|c|}
\hline \multirow{2}{*}{$\begin{array}{l}\text { Exceedance } \\
\text { probability }\end{array}$} & \multirow{2}{*}{$\begin{array}{c}\text { Recurrence } \\
\text { interval } \\
\text { (years) }\end{array}$} & \multirow{2}{*}{$\begin{array}{c}\text { Maximum } \\
\text { instantaneous } \\
\left(\mathrm{m}^{3} / \mathrm{s}\right)\end{array}$} & \multicolumn{4}{|c|}{ Maximum mean discharge } \\
\hline & & & 3-day period & 7-day period & 15-day period & 30-day period \\
\hline 0.99 & 1.01 & ng & 36.1 & 29.9 & 26.7 & 127.8 \\
\hline 0.95 & 1.05 & ng & 86.3 & 67.1 & 55.1 & 50.5 \\
\hline 0.90 & 1.11 & 271 & 130 & 98.3 & 78.1 & 67.9 \\
\hline 0.80 & 1.25 & 404 & 201 & 150 & 115 & 95.4 \\
\hline 0.50 & 2 & 714 & 398 & 294 & 220 & 173 \\
\hline 0.20 & 5 & 1,000 & 657 & 495 & 370 & 291 \\
\hline 0.10 & 10 & 1,120 & 800 & 615 & 465 & 372 \\
\hline 0.04 & 25 & 1,200 & 945 & 746 & 574 & 474 \\
\hline 0.02 & 50 & 1,240 & 1,030 & 828 & 647 & 549 \\
\hline 0.01 & 100 & 1,260 & 1,100 & 899 & 713 & 621 \\
\hline 0.005 & 200 & 1,270 & 1,160 & 961 & 773 & 692 \\
\hline 0.002 & 500 & 1,280 & ng & ng & ng & ng \\
\hline
\end{tabular}

'Data does not fit log-Pearson Type III curve, use with caution.

\section{6- 0.000-5M FARAH RIVER NEAR PETCH TANGI, Continued}

Probability of occurrence of annual low discharges

[m³/s, meters per second]

\begin{tabular}{|c|c|c|c|c|c|c|c|c|c|c|}
\hline \multirow{3}{*}{$\begin{array}{c}\text { Nonexceedance } \\
\text { probability }\end{array}$} & \multirow{3}{*}{$\begin{array}{c}\text { Recurrence } \\
\text { interval } \\
\text { (years) }\end{array}$} & \multicolumn{9}{|c|}{ Minimum mean discharge $\left(\mathrm{m}^{3} / \mathrm{s}\right)$} \\
\hline & & \multicolumn{9}{|c|}{ Number of consecutive days } \\
\hline & & 1 & 3 & 7 & 14 & 30 & 60 & 90 & 120 & 183 \\
\hline 0.05 & 20 & 0 & 0 & 0 & 0 & 0 & 0 & 0.022 & 0.249 & 1.13 \\
\hline 0.10 & 10 & 0 & 0 & 0 & 0 & 0 & 0 & 0.128 & 0.557 & 1.79 \\
\hline 0.20 & 5 & 0.983 & 0.995 & 1.04 & 1.09 & 1.14 & 1.25 & 0.663 & 1.26 & 2.89 \\
\hline 0.50 & 2 & 2.51 & 2.52 & 2.58 & 2.67 & 2.77 & 2.94 & 4.12 & 3.90 & 5.91 \\
\hline
\end{tabular}




\section{6- 0.000-5M FARAH RIVER NEAR PETCH TANGI, Continued}

\section{Probability of occurrence of seasonal low discharges}

$\left[\mathrm{m}^{3} / \mathrm{s}\right.$, meters per second]

\begin{tabular}{|c|c|c|c|c|c|c|c|c|c|}
\hline \multirow{3}{*}{$\begin{array}{c}\text { Nonexceedance } \\
\text { probability }\end{array}$} & \multirow{3}{*}{$\begin{array}{c}\text { Recurrence } \\
\text { interval } \\
\text { (years) }\end{array}$} & \multicolumn{8}{|c|}{ Minimum mean discharge $\left(\mathrm{m}^{3} / \mathrm{s}\right)$} \\
\hline & & \multicolumn{8}{|c|}{ Number of consecutive days } \\
\hline & & 1 & 7 & 14 & 30 & 1 & 7 & 14 & 30 \\
\hline & & \multicolumn{4}{|c|}{ December-January-February } & \multicolumn{4}{|c|}{ March-April-May } \\
\hline 0.05 & 20 & 2.69 & 2.89 & 3.02 & 3.47 & 4.19 & 5.42 & 7.24 & 12.5 \\
\hline 0.10 & 10 & 3.39 & 3.46 & 3.67 & 4.27 & 6.39 & 8.19 & 10.6 & 17.7 \\
\hline 0.20 & 5 & 4.38 & ${ }^{1} 4.34$ & 4.66 & 5.54 & 10.4 & 13.1 & 16.6 & 26.4 \\
\hline \multirow[t]{2}{*}{0.50} & 2 & 6.63 & 6.87 & 7.53 & 9.40 & 24.3 & 29.5 & 36.0 & 52.7 \\
\hline & & \multicolumn{4}{|c|}{ June-July-August } & \multicolumn{4}{|c|}{ September-October-November } \\
\hline 0.05 & 20 & 0 & 0 & 0 & 0 & 0 & 0 & 0 & 0.034 \\
\hline 0.10 & 10 & 0 & 0 & 0 & 0 & 0 & 0 & 0 & 0.141 \\
\hline 0.20 & 5 & 1.07 & 1.12 & 1.18 & 1.27 & 1.10 & 1.14 & 1.20 & 0.569 \\
\hline 0.50 & 2 & 2.66 & 2.74 & 2.86 & 3.13 & 2.64 & 2.69 & 2.77 & 3.24 \\
\hline
\end{tabular}

'Data does not fit log-Pearson Type III curve, use with caution. 


\section{6- 0.000-5M FARAH RIVER NEAR PETCH TANGI, Continued}

Annual peak discharge and corresponding gage height

[ $\mathrm{m}^{3} / \mathrm{s}$, meters per second; --, no data]

\begin{tabular}{|c|c|c|c|c|c|c|c|}
\hline $\begin{array}{l}\text { Water } \\
\text { year }\end{array}$ & Date & $\begin{array}{c}\text { Gage } \\
\text { height } \\
\text { (meters) }\end{array}$ & $\begin{array}{c}\text { Peak } \\
\text { discharge } \\
\left(\mathrm{m}^{3} / \mathrm{s}\right)\end{array}$ & $\begin{array}{c}\text { Water } \\
\text { year }\end{array}$ & Date & $\begin{array}{c}\text { Gage } \\
\text { height } \\
\text { (meters) }\end{array}$ & $\begin{array}{c}\text { Peak } \\
\text { discharge } \\
\left(\mathrm{m}^{3} / \mathrm{s}\right)\end{array}$ \\
\hline \multicolumn{8}{|c|}{ Annual peak discharge, by year } \\
\hline 1961 & 2-Apr & - & 996 & 1970 & 29-Jan & & 88.2 \\
\hline 1962 & 24-Dec & - & 175 & 1971 & 25-Mar & & 751 \\
\hline 1963 & 12-May & - & 549 & 1972 & 20-Jan & & 1,062 \\
\hline 1964 & 2-Apr & - & 430 & 1973 & 24-Feb & & 836 \\
\hline 1965 & 3-Feb & - & 722 & 1974 & 26-Jan & & 712 \\
\hline 1966 & 26-Apr & - & 498 & 1975 & 3-Apr & & 966 \\
\hline 1967 & 27-Apr & - & 639 & 1976 & 23-Mar & & 1,125 \\
\hline 1968 & 10-Mar & - & 792 & 1977 & 5-Apr & & 170 \\
\hline 1969 & 15-Apr & - & 1,028 & 1978 & 9-Mar & & 801 \\
\hline \multicolumn{8}{|c|}{ Annual peak discharge, from highest to lowest } \\
\hline 1976 & 23-Mar & - & 1,125 & 1965 & $3-\mathrm{Feb}$ & & 722 \\
\hline 1972 & 20-Jan & - & 1,062 & 1974 & 26-Jan & & 712 \\
\hline 1969 & 15-Apr & - & 1,028 & 1967 & 27-Apr & & 639 \\
\hline 1961 & 2-Apr & - & 996 & 1963 & 12-May & & 549 \\
\hline 1975 & 3-Apr & - & 966 & 1966 & 26-Apr & & 498 \\
\hline 1973 & 24-Feb & - & 836 & 1964 & 2-Apr & & 430 \\
\hline 1978 & 9-Mar & - & 801 & 1962 & 24-Dec & & 175 \\
\hline 1968 & 10-Mar & - & 792 & 1977 & 5-Apr & & 170 \\
\hline 1971 & 25-Mar & - & 751 & 1970 & 29-Jan & & 88.2 \\
\hline
\end{tabular}




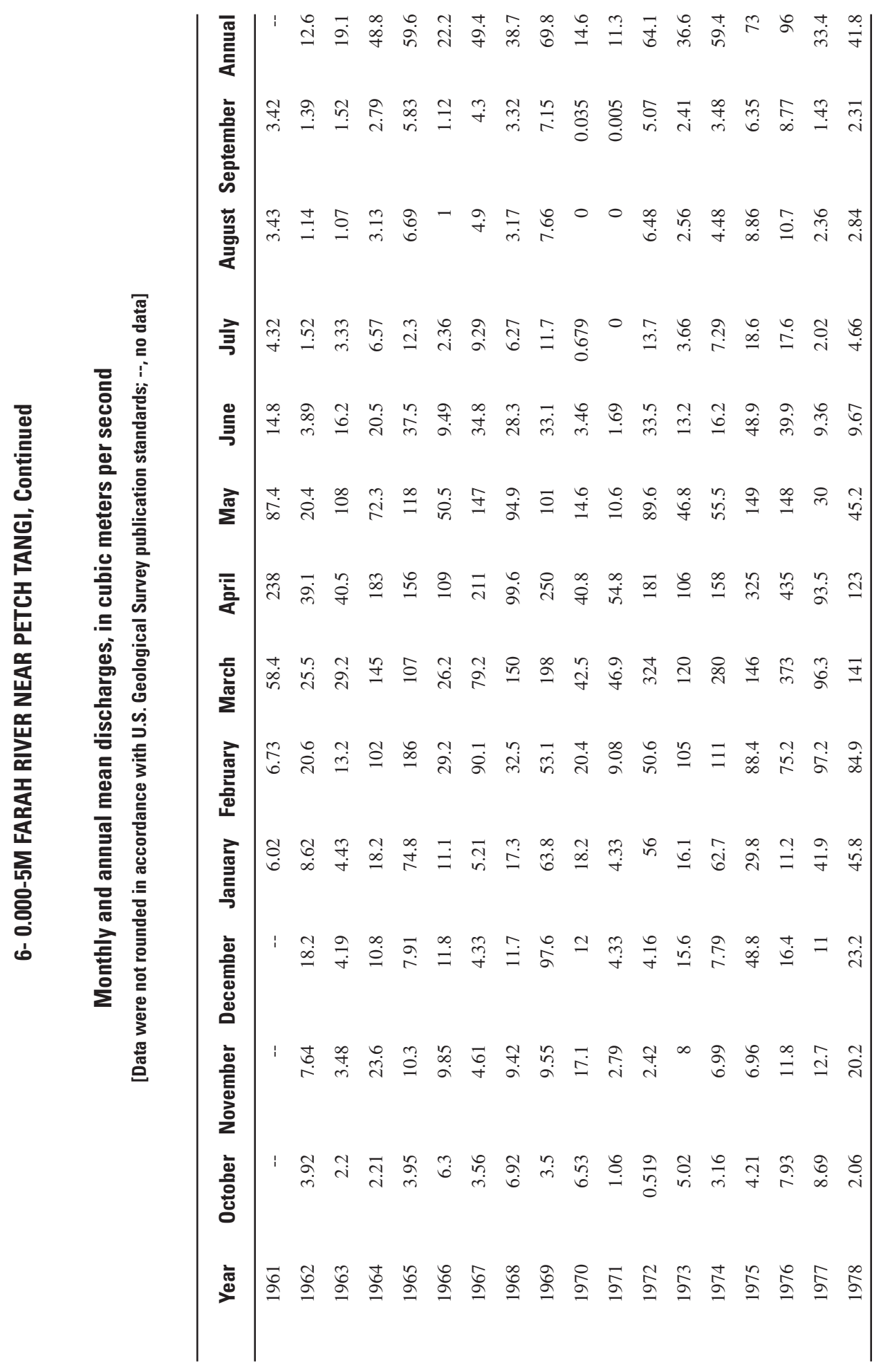




\section{6- 0.L00-1A MALMAND RIVER NEAR SHAWALAT}

\section{(U.S. Geological Survey identification number: 325100063180000)}

LOCATION: Lat $32^{\circ} 51^{\prime}$ N., long $63^{\circ} 18^{\prime} \mathrm{E}$. , on bridge pier, about $0.5 \mathrm{~km}$ upstream from Adraskand River, and about $1 \mathrm{~km}$ north of Adraskand.

DRAINAGE AREA: $1,450 \mathrm{~km}^{2}$.

ALTITUDE: 1,060 meters plus mean sea level.

PERIOD OF RECORD: March 1, 1961 to September 30, 1978.

GAGE: Staff gage read once or twice daily.

Annual mean discharge

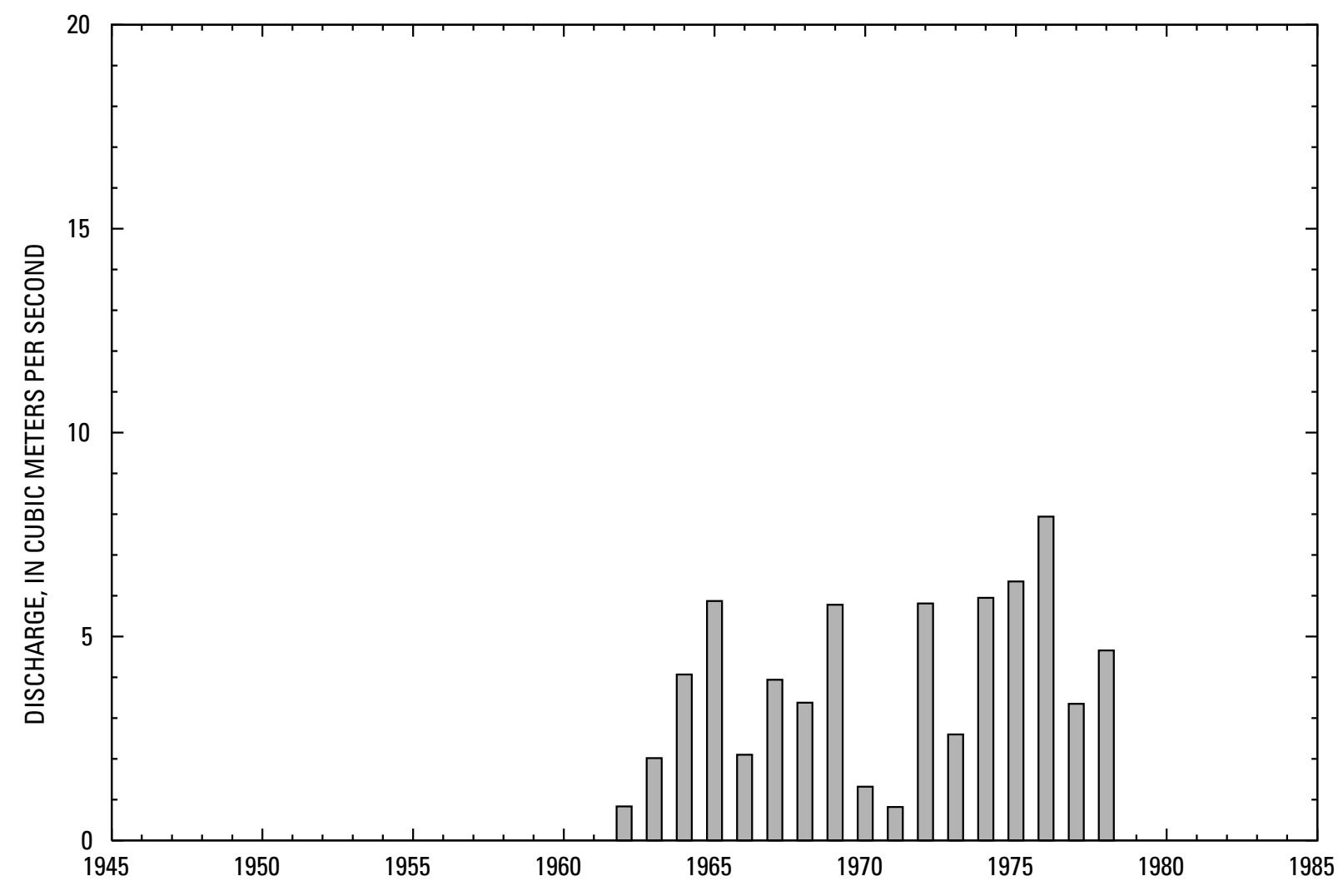




\section{6- 0.L00-1A MALMAND RIVER NEAR SHAWALAT, Continued}

\section{Statistics of monthly and annual mean discharges}

$\left[\mathrm{m}^{3} / \mathrm{s}\right.$, meters per second]

\begin{tabular}{|c|c|c|c|c|c|c|c|c|}
\hline \multirow[b]{2}{*}{ Month } & \multicolumn{2}{|c|}{ Maximum } & \multicolumn{2}{|c|}{ Minimum } & \multicolumn{4}{|c|}{ Mean } \\
\hline & $\begin{array}{c}\text { Discharge } \\
\left(\mathrm{m}^{3} / \mathrm{s}\right)\end{array}$ & $\begin{array}{c}\text { Water year } \\
\text { of } \\
\text { occurrence }\end{array}$ & $\begin{array}{c}\text { Discharge } \\
\left(\mathrm{m}^{3} / \mathrm{s}\right)\end{array}$ & $\begin{array}{c}\text { Water year } \\
\text { of } \\
\text { occurrence }\end{array}$ & $\begin{array}{c}\text { Discharge } \\
\left(\mathrm{m}^{3} / \mathrm{s}\right)\end{array}$ & $\begin{array}{c}\text { Standard } \\
\text { deviation } \\
\left(\mathrm{m}^{3} / \mathrm{s}\right)\end{array}$ & $\begin{array}{c}\text { Coefficient } \\
\text { of } \\
\text { variation }\end{array}$ & $\begin{array}{c}\text { Percentage } \\
\text { of annual } \\
\text { discharge }\end{array}$ \\
\hline October & 1.01 & 1967 & 0.037 & 1972 & 0.330 & 0.220 & 0.65 & 0.70 \\
\hline November & 3.09 & 1978 & 0.147 & 1971 & 0.670 & 0.690 & 1.03 & 1.41 \\
\hline December & 5.52 & 1969 & 0.222 & 1965 & 1.27 & 1.55 & 1.22 & 2.67 \\
\hline January & 9.19 & 1978 & 0.247 & 1971 & 2.76 & 2.64 & 0.95 & 5.81 \\
\hline February & 24.1 & 1965 & 0.764 & 1971 & 5.90 & 5.40 & 0.92 & 12.4 \\
\hline March & 26.5 & 1972 & 1.71 & 1962 & 10.4 & 7.19 & 0.69 & 21.9 \\
\hline April & 44.8 & 1976 & 2.59 & 1962 & 14.4 & 10.2 & 0.70 & 30.3 \\
\hline May & 18.1 & 1976 & 0.920 & 1962 & 7.90 & 4.48 & 0.57 & 16.6 \\
\hline June & 4.65 & 1975 & 0.256 & 1971 & 2.43 & 1.44 & 0.59 & 5.12 \\
\hline July & 1.78 & 1972 & 0.059 & 1971 & 0.840 & 0.550 & 0.66 & 1.77 \\
\hline August & 1.28 & 1977 & 0.010 & 1971 & 0.380 & 0.300 & 0.80 & 0.80 \\
\hline September & 0.490 & 1976 & 0.016 & 1970 & 0.260 & 0.140 & 0.54 & 0.55 \\
\hline Annual & 7.94 & 1976 & 0.821 & 1971 & 3.93 & 2.13 & 0.54 & 100 \\
\hline
\end{tabular}

Annual flow duration

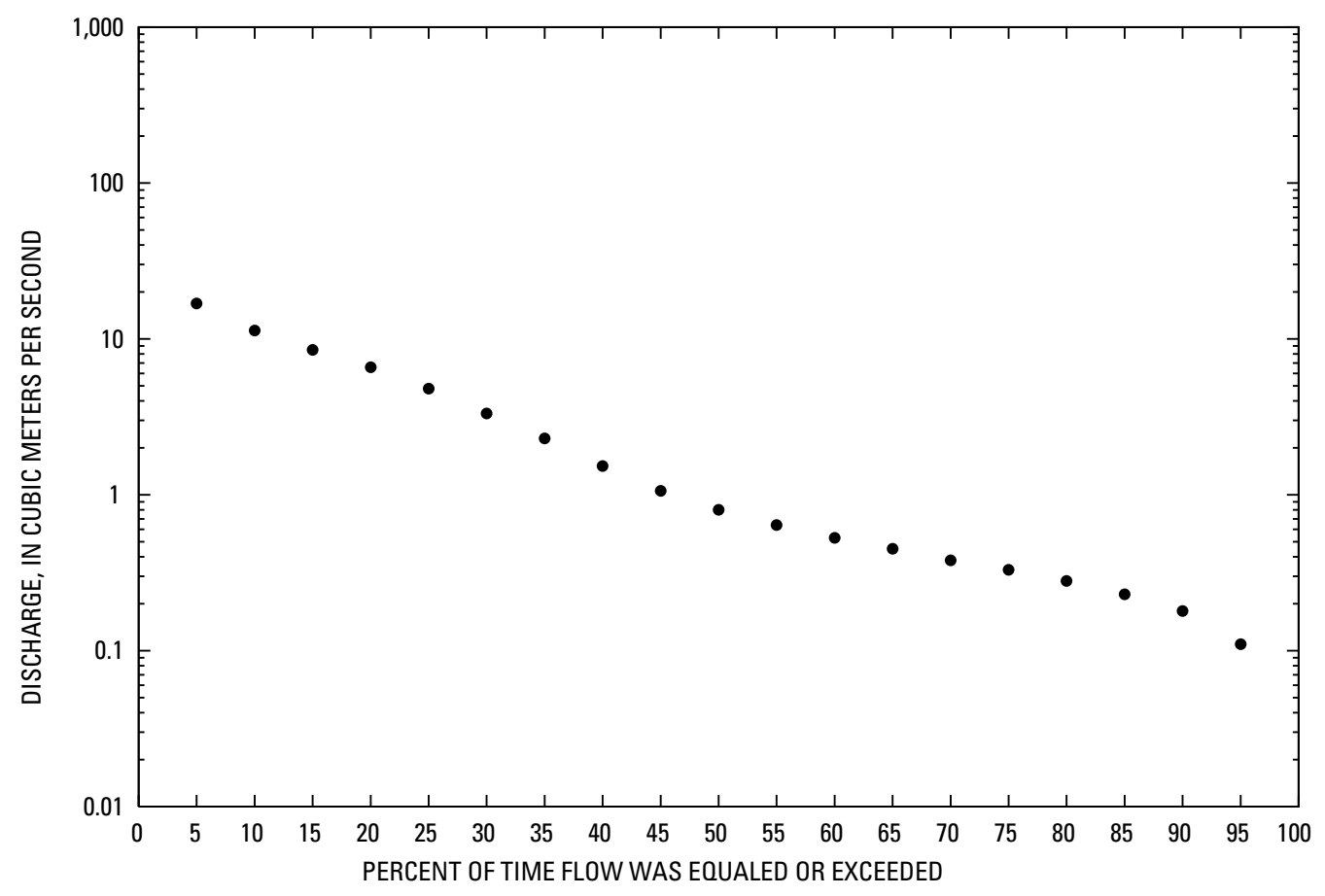




\section{6- 0.L00-1A MALMAND RIVER NEAR SHAWALAT, Continued}

Monthly and annual flow duration, in cubic meters per second

[Data were not rounded in accordance with U.S. Geological Survey publication standards]

\begin{tabular}{|c|c|c|c|c|c|c|c|c|c|c|c|c|c|}
\hline $\begin{array}{l}\text { Percentage } \\
\text { of days } \\
\text { discharge } \\
\text { equaled or } \\
\text { exceeded }\end{array}$ & January & February & March & April & May & June & July & August & September & October & November & December & Annual \\
\hline 95 & 0.24 & 0.4 & 0.86 & 2.57 & 0.68 & 0.27 & 0.07 & 0.01 & 0.02 & 0.05 & 0.13 & 0.2 & 0.11 \\
\hline 90 & 0.32 & 0.65 & 1.4 & 3.39 & 1.26 & 0.31 & 0.15 & 0.02 & 0.04 & 0.08 & 0.18 & 0.24 & 0.18 \\
\hline 85 & 0.37 & 0.74 & 1.98 & 4.25 & 2.27 & 0.41 & 0.17 & 0.1 & 0.14 & 0.17 & 0.21 & 0.28 & 0.23 \\
\hline 80 & 0.42 & 1.13 & 2.94 & 5.88 & 3.06 & 0.86 & 0.25 & 0.12 & 0.15 & 0.19 & 0.22 & 0.31 & 0.28 \\
\hline 75 & 0.58 & 1.64 & 4.29 & 7.09 & 4.25 & 1.06 & 0.31 & 0.14 & 0.16 & 0.21 & 0.24 & 0.35 & 0.33 \\
\hline 70 & 0.7 & 1.87 & 5.05 & 7.74 & 5.17 & 1.2 & 0.45 & 0.18 & 0.17 & 0.22 & 0.26 & 0.38 & 0.38 \\
\hline 65 & 0.76 & 2.26 & 6.15 & 8.39 & 5.73 & 1.45 & 0.5 & 0.25 & 0.2 & 0.23 & 0.28 & 0.4 & 0.45 \\
\hline 60 & 0.81 & 2.68 & 6.76 & 9.12 & 6.15 & 1.67 & 0.6 & 0.27 & 0.23 & 0.25 & 0.32 & 0.44 & 0.53 \\
\hline 55 & 0.88 & 3.19 & 7.25 & 10.1 & 6.65 & 1.81 & 0.64 & 0.3 & 0.25 & 0.28 & 0.35 & 0.49 & 0.64 \\
\hline 50 & 0.97 & 3.76 & 7.68 & 11.4 & 7.25 & 2.11 & 0.67 & 0.33 & 0.26 & 0.29 & 0.38 & 0.56 & 0.8 \\
\hline 45 & 1.1 & 4.53 & 8.18 & 12.4 & 7.95 & 2.32 & 0.72 & 0.36 & 0.26 & 0.31 & 0.4 & 0.61 & 1.06 \\
\hline 40 & 1.23 & 5.44 & 9.15 & 13.3 & 8.66 & 2.73 & 0.8 & 0.39 & 0.28 & 0.33 & 0.44 & 0.67 & 1.53 \\
\hline 35 & 1.33 & 6.1 & 10.2 & 14.4 & 9.33 & 3.11 & 0.89 & 0.42 & 0.31 & 0.35 & 0.47 & 0.73 & 2.3 \\
\hline 30 & 2.31 & 6.76 & 11.4 & 15.9 & 10 & 3.39 & 0.96 & 0.44 & 0.35 & 0.37 & 0.49 & 0.79 & 3.32 \\
\hline 25 & 3.15 & 7.42 & 13.5 & 17.8 & 10.9 & 3.68 & 1.1 & 0.47 & 0.37 & 0.4 & 0.52 & 0.95 & 4.8 \\
\hline 20 & 3.86 & 8.2 & 16.4 & 19.7 & 11.8 & 4.02 & 1.34 & 0.52 & 0.4 & 0.41 & 0.56 & 1.11 & 6.58 \\
\hline 15 & 4.51 & 9.04 & 19 & 21.9 & 13 & 4.42 & 1.57 & 0.56 & 0.44 & 0.43 & 0.79 & 1.43 & 8.51 \\
\hline 10 & 6.14 & 11 & 23 & 26.5 & 14.4 & 4.89 & 1.87 & 0.63 & 0.47 & 0.48 & 0.95 & 1.7 & 11.3 \\
\hline 5 & 9.97 & 17 & 31 & 41.9 & 16.9 & 5.4 & 2.24 & 0.76 & 0.52 & 0.54 & 1.26 & 3.04 & 16.9 \\
\hline
\end{tabular}




\section{6- 0.L00-1A MALMAND RIVER NEAR SHAWALAT, Continued}

Probability of occurrence of annual high discharges

$\left[\mathrm{m}^{3} / \mathrm{s}\right.$, meters per second; $\mathrm{ng}$, statistic not given]

\begin{tabular}{|c|c|c|c|c|c|c|}
\hline \multirow{2}{*}{$\begin{array}{c}\text { Exceedance } \\
\text { probability }\end{array}$} & \multirow{2}{*}{$\begin{array}{c}\text { Recurrence } \\
\text { interval } \\
\text { (years) }\end{array}$} & \multirow{2}{*}{$\begin{array}{c}\text { Maximum } \\
\text { instantaneous } \\
\left(\mathrm{m}^{3} / \mathrm{s}\right)\end{array}$} & \multicolumn{4}{|c|}{ Maximum mean discharge } \\
\hline & & & 3-day period & 7-day period & 15-day period & 30-day period \\
\hline 0.99 & 1.01 & ng & 3.72 & 2.75 & 2.14 & 1.95 \\
\hline 0.95 & 1.05 & ng & 7.57 & 5.48 & 4.30 & 3.76 \\
\hline 0.90 & 1.11 & 63.8 & 10.7 & 7.72 & 6.07 & 5.21 \\
\hline 0.80 & 1.25 & 79.3 & 15.9 & 11.4 & 8.99 & 7.55 \\
\hline 0.50 & 2 & 122 & 30.9 & 22.5 & 17.8 & 14.4 \\
\hline 0.20 & 5 & 193 & 54.3 & 40.8 & 32.1 & 25.3 \\
\hline 0.10 & 10 & 248 & 70.1 & 53.9 & 42.2 & 32.9 \\
\hline 0.04 & 25 & 326 & 89.4 & 70.8 & 55.2 & 42.5 \\
\hline 0.02 & 50 & 390 & 103 & 83.3 & 64.7 & 49.6 \\
\hline 0.01 & 100 & 460 & 116 & 95.8 & 74.1 & 56.5 \\
\hline 0.005 & 200 & 536 & 129 & 108 & 83.3 & 63.3 \\
\hline 0.002 & 500 & 648 & ng & ng & ng & ng \\
\hline
\end{tabular}

\section{6- 0.L00-1A MALMAND RIVER NEAR SHAWALAT, Continued}

\section{Probability of occurrence of annual low discharges}

$\left[\mathrm{m}^{3} / \mathrm{s}\right.$, meters per second]

\begin{tabular}{|c|c|c|c|c|c|c|c|c|c|c|}
\hline \multirow{3}{*}{$\begin{array}{l}\text { Nonexceedance } \\
\text { probability }\end{array}$} & \multirow{3}{*}{$\begin{array}{c}\text { Recurrence } \\
\text { interval } \\
\text { (years) }\end{array}$} & \multicolumn{9}{|c|}{ Minimum mean discharge $\left(\mathrm{m}^{3} / \mathrm{s}\right)$} \\
\hline & & \multicolumn{9}{|c|}{ Number of consecutive days } \\
\hline & & 1 & 3 & 7 & 14 & 30 & 60 & 90 & 120 & 183 \\
\hline 0.05 & 20 & 0 & 0.014 & 0.015 & 0.017 & 0.017 & 0.021 & 0.032 & 0.047 & 0.107 \\
\hline 0.10 & 10 & 0.019 & 0.028 & 0.030 & 0.034 & 0.036 & 0.043 & 0.061 & 0.083 & 0.162 \\
\hline 0.20 & 5 & 0.055 & 0.056 & 0.061 & 0.071 & 0.078 & 0.091 & 0.115 & 0.147 & 0.251 \\
\hline 0.50 & 2 & ${ }^{1} 0.165$ & 0.154 & 0.164 & 0.190 & 0.219 & 0.247 & 0.274 & 0.329 & 0.484 \\
\hline
\end{tabular}

'Data does not fit log-Pearson Type III curve, use with caution. 


\section{6- 0.LO0-1A MALMAND RIVER NEAR SHAWALAT, Continued}

\section{Probability of occurrence of seasonal low discharges}

$\left[\mathrm{m}^{3} / \mathrm{s}\right.$, meters per second]

\begin{tabular}{|c|c|c|c|c|c|c|c|c|c|}
\hline \multirow{3}{*}{$\begin{array}{c}\text { Nonexceedance } \\
\text { probability }\end{array}$} & \multirow{3}{*}{$\begin{array}{c}\text { Recurrence } \\
\text { interval } \\
\text { (years) }\end{array}$} & \multicolumn{8}{|c|}{ Minimum mean discharge $\left(\mathrm{m}^{3} / \mathrm{s}\right)$} \\
\hline & & \multicolumn{8}{|c|}{ Number of consecutive days } \\
\hline & & 1 & 7 & 14 & 30 & 1 & 7 & 14 & 30 \\
\hline & & \multicolumn{4}{|c|}{ December-January-February } & \multicolumn{4}{|c|}{ March-April-May } \\
\hline 0.05 & 20 & 0.159 & 0.170 & ${ }^{1} 0.167$ & 0.193 & 0.340 & 0.396 & 0.582 & 1.12 \\
\hline 0.10 & 10 & 0.192 & 0.207 & 0.209 & 0.240 & 0.579 & 0.681 & 0.967 & 1.71 \\
\hline 0.20 & 5 & 0.240 & 0.264 & 0.276 & 0.320 & 1.04 & 1.24 & 1.68 & 2.72 \\
\hline \multirow[t]{2}{*}{0.50} & 2 & 0.374 & 0.431 & 0.483 & 0.607 & 2.74 & 3.27 & 4.10 & 5.78 \\
\hline & & \multicolumn{4}{|c|}{ June-July-August } & \multicolumn{4}{|c|}{ September-October-November } \\
\hline 0.05 & 20 & 0.015 & 0.016 & 0.018 & 0.020 & 0 & 0.017 & 0.017 & 0.027 \\
\hline 0.10 & 10 & 0.030 & 0.034 & 0.039 & 0.045 & 0.026 & 0.035 & 0.037 & 0.051 \\
\hline 0.20 & 5 & 0.066 & 0.073 & 0.085 & 0.099 & ${ }^{1} 0.077$ & 0.075 & 0.079 & 0.098 \\
\hline 0.50 & 2 & 0.206 & 0.218 & 0.254 & 0.297 & ${ }^{1} 0.209$ & 0.204 & 0.216 & 0.237 \\
\hline
\end{tabular}

'Data does not fit log-Pearson Type III curve, use with caution. 


\section{6- 0.L00-1A MALMAND RIVER NEAR SHAWALAT, Continued}

Annual peak discharge and corresponding gage height

$\left[\mathrm{m}^{3} / \mathrm{s}\right.$, meters per second; --, no data]

\begin{tabular}{|c|c|c|c|c|c|c|c|}
\hline $\begin{array}{c}\text { Water } \\
\text { year }\end{array}$ & Date & $\begin{array}{c}\text { Gage } \\
\text { height } \\
\text { (meters) }\end{array}$ & $\begin{array}{c}\text { Peak } \\
\text { discharge } \\
\left(\mathrm{m}^{3} / \mathrm{s}\right)\end{array}$ & $\begin{array}{c}\text { Water } \\
\text { year }\end{array}$ & Date & $\begin{array}{c}\text { Gage } \\
\text { height } \\
\text { (meters) }\end{array}$ & $\begin{array}{c}\text { Peak } \\
\text { discharge } \\
\left(\mathrm{m}^{3} / \mathrm{s}\right)\end{array}$ \\
\hline \multicolumn{8}{|c|}{ Annual peak discharge, by year } \\
\hline 1961 & 2-Apr & - & 87.0 & 1970 & 9-Mar & & 6.88 \\
\hline 1962 & 13-Apr & - & 115 & 1971 & 25-Mar & & 76.0 \\
\hline 1963 & 14-Mar & - & 145 & 1972 & 24-Apr & & 70.0 \\
\hline 1964 & $13-\mathrm{Nov}$ & - & 99.2 & 1973 & 24-Feb & & 57.0 \\
\hline 1965 & $1-\mathrm{Feb}$ & - & 167 & 1974 & 18-Jan & & 117 \\
\hline 1966 & 12-Apr & - & 96.6 & 1975 & 25-Apr & & 133 \\
\hline 1967 & 21-Apr & - & 199 & 1976 & 23-Jul & & 324 \\
\hline 1968 & 10-Mar & - & 232 & 1977 & 26-May & & 254 \\
\hline 1969 & 15-Apr & - & 80.9 & 1978 & $18-\mathrm{Nov}$ & & 242 \\
\hline \multicolumn{8}{|c|}{ Annual peak discharge, from highest to lowest } \\
\hline 1976 & 23-Jul & - & 324 & 1962 & 13-Apr & & 115 \\
\hline 1977 & 26-May & - & 254 & 1964 & 13-Nov & & 99.2 \\
\hline 1978 & $18-\mathrm{Nov}$ & - & 242 & 1966 & 12-Apr & & 96.6 \\
\hline 1968 & 10-Mar & - & 232 & 1961 & 2-Apr & & 87.0 \\
\hline 1967 & 21-Apr & - & 199 & 1969 & 15-Apr & & 80.9 \\
\hline 1965 & $1-\mathrm{Feb}$ & - & 167 & 1971 & 25-Mar & & 76.0 \\
\hline 1963 & 14-Mar & - & 145 & 1972 & 24-Apr & & 70.0 \\
\hline 1975 & 25-Apr & - & 133 & 1973 & 24-Feb & & 57.0 \\
\hline 1974 & 18-Jan & - & 117 & 1970 & 9-Mar & & 6.88 \\
\hline
\end{tabular}




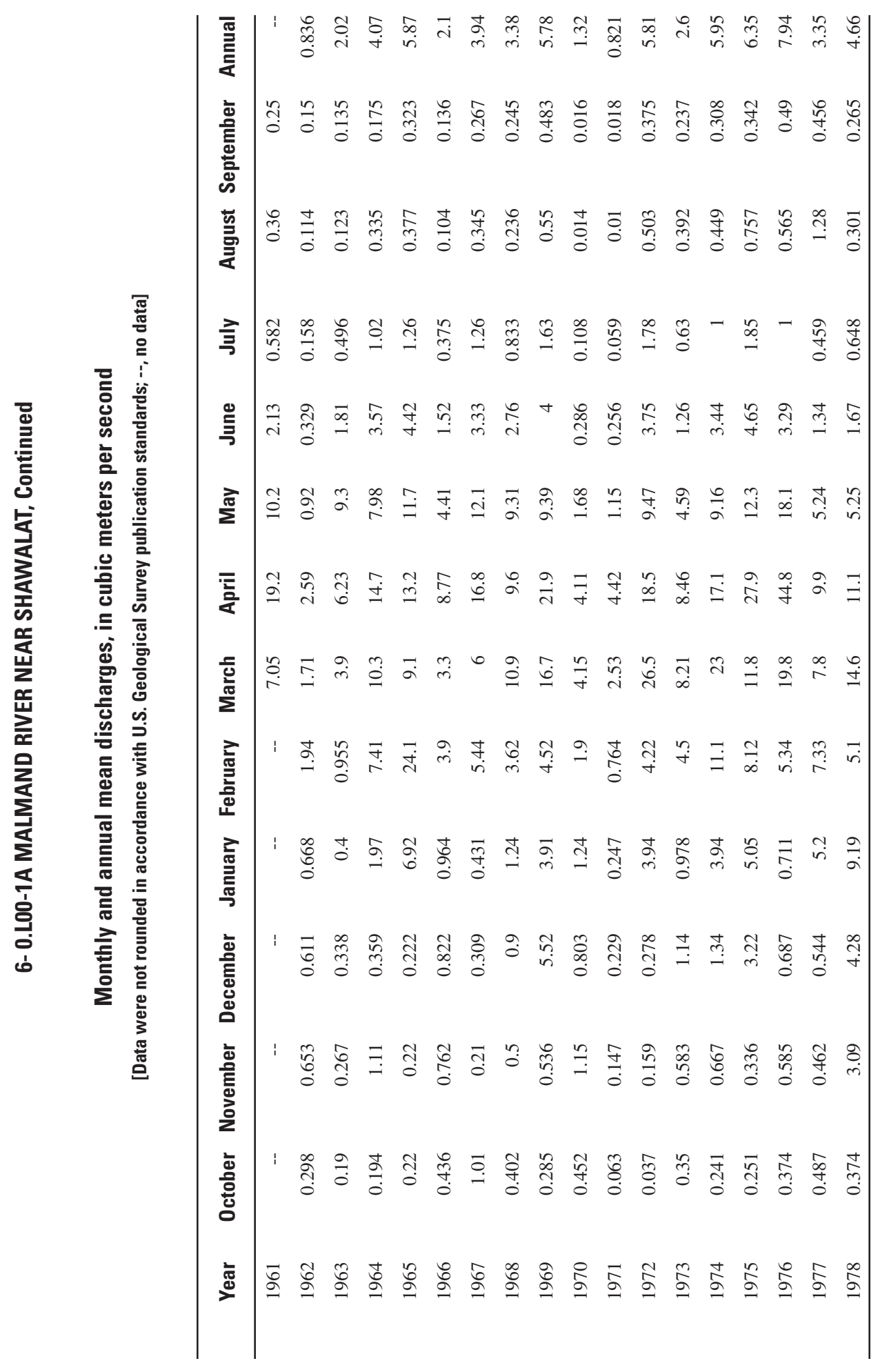




\section{7-0.000-7 M ADRASKAN RIVER AT ADRASKAN}

\section{(U.S. Geological Survey identification number: 333800062160000)}

LOCATION: Lat $33^{\circ} 38^{\prime}$ N., long $62^{\circ} 16^{\prime}$ E., on bridge pier on Kandahar-Heart Highway at Adraskand, about 0.5 km upstream from Gaz River.

DRAINAGE AREA: $1,970 \mathrm{~km}^{2}$.

ALTITUDE: 1,340 meters plus mean sea level.

PERIOD OF RECORD: March 30, 1933 to September 30, 1978.

GAGE: Staff gage read once or twice daily.

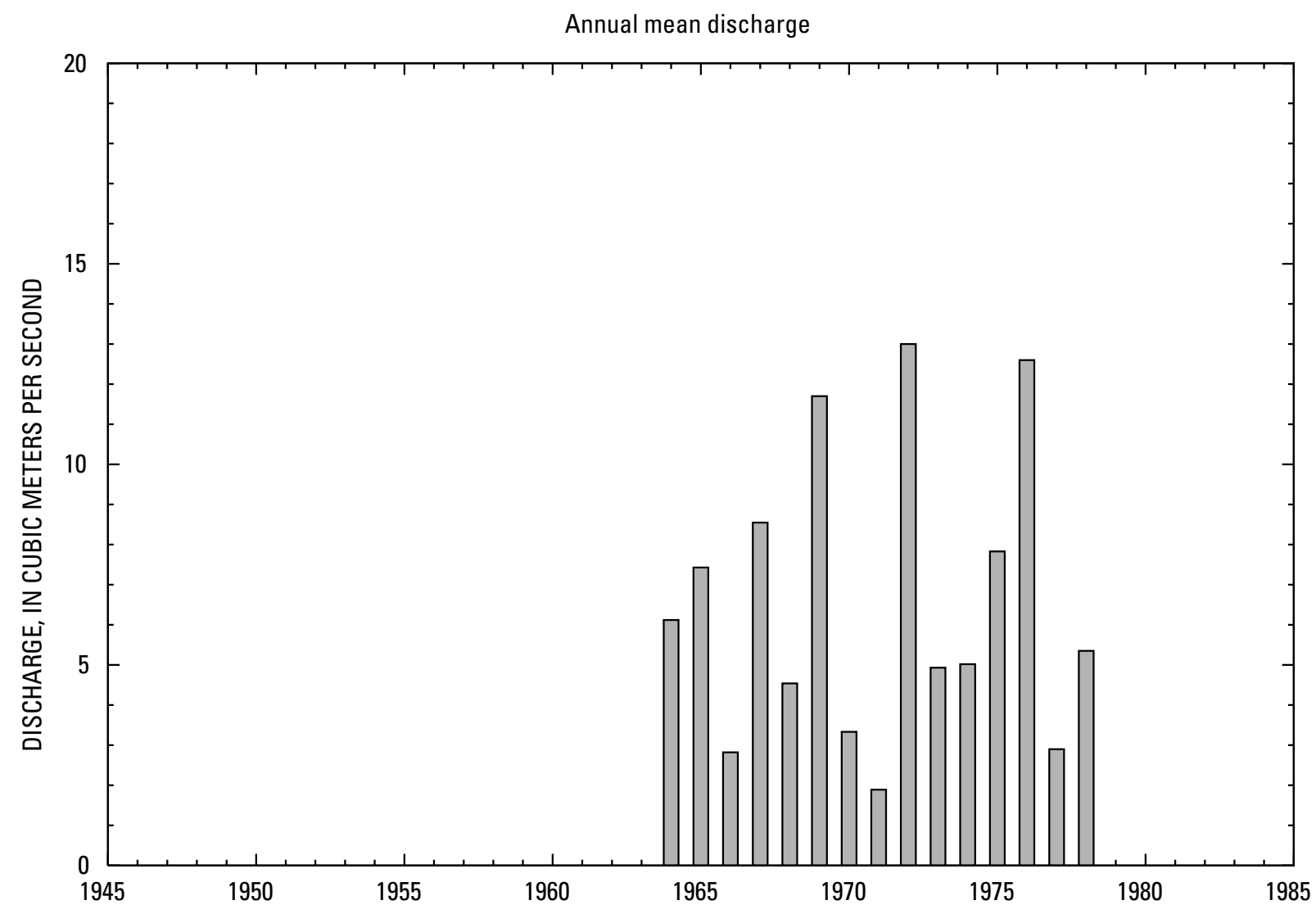




\section{7- 0.000-7M ADRASKAN RIVER AT ADRASKAN, Continued}

\section{Statistics of monthly and annual mean discharges}

$\left[\mathrm{m}^{3} / \mathrm{s}\right.$, meters per second]

\begin{tabular}{|c|c|c|c|c|c|c|c|c|}
\hline \multirow[b]{2}{*}{ Month } & \multicolumn{2}{|c|}{ Maximum } & \multicolumn{2}{|c|}{ Minimum } & \multicolumn{4}{|c|}{ Mean } \\
\hline & $\begin{array}{c}\text { Discharge } \\
\left(\mathrm{m}^{3} / \mathrm{s}\right)\end{array}$ & $\begin{array}{c}\text { Water year } \\
\text { of } \\
\text { occurrence }\end{array}$ & $\begin{array}{c}\text { Discharge } \\
\left(\mathrm{m}^{3} / \mathrm{s}\right)\end{array}$ & $\begin{array}{c}\text { Water year } \\
\text { of } \\
\text { occurrence }\end{array}$ & $\begin{array}{c}\text { Discharge } \\
\left(\mathrm{m}^{3} / \mathrm{s}\right)\end{array}$ & $\begin{array}{c}\text { Standard } \\
\text { deviation } \\
\left(\mathrm{m}^{3} / \mathrm{s}\right)\end{array}$ & $\begin{array}{c}\text { Coefficient } \\
\text { of } \\
\text { variation }\end{array}$ & $\begin{array}{c}\text { Percentage } \\
\text { of annual } \\
\text { discharge }\end{array}$ \\
\hline October & 1.77 & 1970 & 0.149 & 1964 & 0.79 & 0.50 & 0.64 & 1.02 \\
\hline November & 4.50 & 1978 & 0.703 & 1972 & 1.69 & 1.00 & 0.59 & 2.19 \\
\hline December & 19.3 & 1969 & 0.415 & 1964 & 2.79 & 4.62 & 1.66 & 3.61 \\
\hline January & 10.7 & 1969 & 1.02 & 1971 & 3.03 & 2.65 & 0.87 & 3.93 \\
\hline February & 19.5 & 1972 & 1.57 & 1971 & 7.66 & 5.24 & 0.68 & 9.93 \\
\hline March & 40.8 & 1972 & 2.46 & 1971 & 17.9 & 10.4 & 0.58 & 23.2 \\
\hline April & 57.1 & 1976 & 3.85 & 1963 & 23.4 & 17.6 & 0.75 & 30.4 \\
\hline May & 34.4 & 1967 & 2.80 & 1970 & 13.4 & 9.40 & 0.70 & 17.4 \\
\hline June & 10.9 & 1976 & 0.715 & 1970 & 3.98 & 3.00 & 0.75 & 5.17 \\
\hline July & 3.52 & 1972 & 0.306 & 1970 & 1.35 & 0.93 & 0.69 & 1.75 \\
\hline August & 2.04 & 1976 & 0.010 & 1971 & 0.58 & 0.53 & 0.92 & 0.75 \\
\hline September & 1.37 & 1976 & 0.010 & 1971 & 0.56 & 0.42 & 0.75 & 0.72 \\
\hline Annual & 13.0 & 1972 & 1.89 & 1971 & 6.53 & 3.60 & 0.55 & 100 \\
\hline
\end{tabular}

Annual flow duration

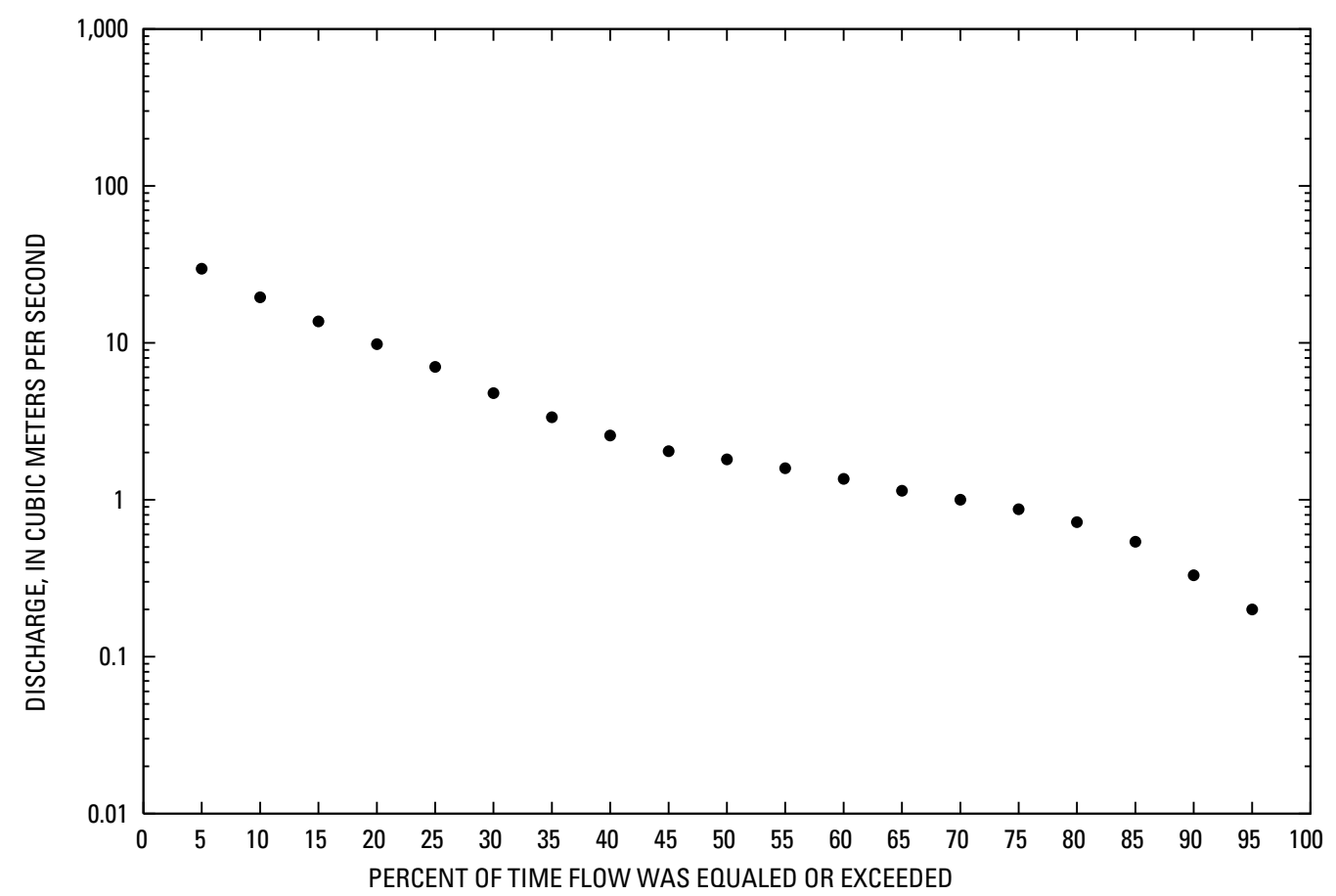




\section{7- 0.000-7M ADRASKAN RIVER AT ADRASKAN, Continued}

Monthly and annual flow duration, in cubic meters per second

[Data were not rounded in accordance with U.S. Geological Survey publication standards]

\begin{tabular}{|c|c|c|c|c|c|c|c|c|c|c|c|c|c|}
\hline $\begin{array}{l}\text { Percentage } \\
\text { of days } \\
\text { discharge } \\
\text { equaled or } \\
\text { exceeded }\end{array}$ & January & February & March & April & May & June & July & August & September & October & November & December & Annual \\
\hline 95 & 0.91 & 1.29 & 2.07 & 3.16 & 2.41 & 0.73 & 0.14 & 0.01 & 0.01 & 0.12 & 0.37 & 0.45 & 0.2 \\
\hline 90 & 0.98 & 1.4 & 2.97 & 4.86 & 3.21 & 0.8 & 0.28 & 0.02 & 0.02 & 0.19 & 0.53 & 0.85 & 0.33 \\
\hline 85 & 1.04 & 1.6 & 3.58 & 6.08 & 3.69 & 1.01 & 0.35 & 0.14 & 0.09 & 0.23 & 0.72 & 0.9 & 0.54 \\
\hline 80 & 1.19 & 1.77 & 5.52 & 7.01 & 4.33 & 1.42 & 0.42 & 0.18 & 0.16 & 0.31 & 0.77 & 0.95 & 0.72 \\
\hline 75 & 1.33 & 1.92 & 6.9 & 8.14 & 5.06 & 1.61 & 0.55 & 0.2 & 0.24 & 0.35 & 0.81 & 1.02 & 0.87 \\
\hline 70 & 1.43 & 2.33 & 9.83 & 9.82 & 5.68 & 1.76 & 0.7 & 0.21 & 0.29 & 0.44 & 0.86 & 1.08 & 1 \\
\hline 65 & 1.54 & 2.72 & 11.7 & 11.8 & 6.31 & 1.94 & 0.78 & 0.22 & 0.32 & 0.51 & 0.91 & 1.15 & 1.14 \\
\hline 60 & 1.64 & 2.98 & 13.3 & 13.5 & 6.96 & 2.3 & 0.86 & 0.27 & 0.34 & 0.59 & 0.97 & 1.25 & 1.36 \\
\hline 55 & 1.74 & 3.64 & 14.5 & 14.9 & 7.61 & 2.76 & 0.93 & 0.31 & 0.42 & 0.63 & 1.13 & 1.54 & 1.59 \\
\hline 50 & 1.82 & 4.92 & 15.6 & 16.6 & 8.36 & 2.99 & 1 & 0.4 & 0.51 & 0.68 & 1.26 & 1.67 & 1.81 \\
\hline 45 & 1.9 & 6 & 16.7 & 19.9 & 9.29 & 3.23 & 1.12 & 0.53 & 0.58 & 0.82 & 1.37 & 1.78 & 2.04 \\
\hline 40 & 2.02 & 7.56 & 17.9 & 23.1 & 10.7 & 3.51 & 1.27 & 0.65 & 0.64 & 0.88 & 1.48 & 1.85 & 2.57 \\
\hline 35 & 2.18 & 8.21 & 19.4 & 26 & 13.1 & 3.94 & 1.51 & 0.73 & 0.71 & 0.93 & 1.59 & 1.92 & 3.36 \\
\hline 30 & 2.63 & 9.67 & 21.2 & 29.1 & 15.3 & 4.59 & 1.67 & 0.77 & 0.77 & 0.97 & 1.71 & 1.98 & 4.79 \\
\hline 25 & 2.89 & 11 & 23.3 & 31.5 & 17.6 & 5.18 & 1.84 & 0.82 & 0.81 & 1.11 & 1.84 & 2.05 & 7.02 \\
\hline 20 & 3.18 & 12.2 & 25.8 & 34.5 & 19.9 & 6.46 & 2.23 & 0.9 & 0.86 & 1.2 & 1.98 & 2.29 & 9.82 \\
\hline 15 & 4.3 & 13.3 & 29.4 & 41.5 & 22.8 & 7.56 & 2.51 & 1.03 & 1.04 & 1.34 & 2.18 & 2.79 & 13.7 \\
\hline 10 & 8.93 & 16.7 & 35.8 & 51.7 & 29.6 & 8.73 & 2.83 & 1.24 & 1.18 & 1.57 & 2.57 & 3.35 & 19.5 \\
\hline 5 & 12.1 & 24.5 & 47.5 & 67.9 & 38.8 & 11.2 & 3.21 & 1.9 & 1.33 & 1.75 & 3.41 & 13.5 & 29.7 \\
\hline
\end{tabular}




\section{7- 0.000-7M ADRASKAN RIVER AT ADRASKAN, Continued}

Probability of occurrence of annual high discharges

$\left[\mathrm{m}^{3} / \mathrm{s}\right.$, meters per second; ng, statistic not given]

\begin{tabular}{|c|c|c|c|c|c|c|}
\hline \multirow{2}{*}{$\begin{array}{c}\text { Exceedance } \\
\text { probability }\end{array}$} & \multirow{2}{*}{$\begin{array}{c}\text { Recurrence } \\
\text { interval } \\
\text { (years) }\end{array}$} & \multirow{2}{*}{$\begin{array}{c}\text { Maximum } \\
\text { instantaneous } \\
\left(\mathrm{m}^{3} / \mathrm{s}\right)\end{array}$} & \multicolumn{4}{|c|}{ Maximum mean discharge } \\
\hline & & & 3-day period & 7-day period & 15-day period & 30-day period \\
\hline 0.99 & 1.01 & 9.60 & ${ }^{1} 8.70$ & 8.90 & 7.09 & 5.08 \\
\hline 0.95 & 1.05 & 19.2 & 15.0 & 13.7 & 10.9 & 8.69 \\
\hline 0.90 & 1.11 & 27.6 & 19.6 & 17.2 & 13.7 & 11.4 \\
\hline 0.80 & 1.25 & 42.4 & 26.8 & 22.4 & 17.9 & 15.5 \\
\hline 0.50 & 2 & 94.4 & 46.0 & 36.6 & 29.8 & 26.6 \\
\hline 0.20 & 5 & 204 & 74.1 & 58.6 & 49.0 & 42.9 \\
\hline 0.10 & 10 & 302 & 92.7 & 74.3 & 63.2 & 53.8 \\
\hline 0.04 & 25 & 456 & 116 & 95.0 & 82.7 & 67.4 \\
\hline 0.02 & 50 & 591 & 132 & 111 & 98.2 & 77.2 \\
\hline 0.01 & 100 & 744 & 148 & 127 & 114 & 86.8 \\
\hline 0.005 & 200 & 917 & 163 & 144 & 132 & 96.1 \\
\hline 0.002 & 500 & 1,180 & ng & ng & $\mathrm{ng}$ & $\mathrm{ng}$ \\
\hline
\end{tabular}

'Data does not fit log-Pearson Type III curve, use with caution.

\section{7- 0.000-7M ADRASKAN RIVER AT ADRASKAN, Continued}

Probability of occurrence of annual low discharges

$\left[\mathrm{m}^{3} / \mathrm{s}\right.$, meters per second]

\begin{tabular}{|c|c|c|c|c|c|c|c|c|c|c|}
\hline \multirow{3}{*}{$\begin{array}{c}\text { Nonexceedance } \\
\text { probability }\end{array}$} & \multirow{3}{*}{$\begin{array}{c}\text { Recurrence } \\
\text { interval } \\
\text { (years) }\end{array}$} & \multicolumn{9}{|c|}{ Minimum mean discharge $\left(\mathrm{m}^{3} / \mathrm{s}\right)$} \\
\hline & & \multicolumn{9}{|c|}{ Number of consecutive days } \\
\hline & & 1 & 3 & 7 & 14 & 30 & 60 & 90 & 120 & 183 \\
\hline 0.05 & 20 & 0 & 0 & 0.007 & 0.010 & 0.013 & 0.017 & 0.053 & 0.136 & 0.374 \\
\hline 0.10 & 10 & 0 & 0.012 & 0.019 & 0.024 & 0.031 & 0.041 & 0.095 & 0.192 & 0.457 \\
\hline 0.20 & 5 & ${ }^{1} 0.052$ & 0.050 & 0.052 & 0.063 & 0.079 & 0.108 & 0.177 & 0.286 & 0.580 \\
\hline 0.50 & 2 & ${ }^{1} 0.256$ & 0.246 & 0.244 & 0.275 & 0.329 & 0.427 & 0.479 & 0.580 & 0.904 \\
\hline
\end{tabular}

'Data does not fit log-Pearson Type III curve, use with caution. 


\section{7- 0.000-7M ADRASKAN RIVER AT ADRASKAN, Continued}

\section{Probability of occurrence of seasonal low discharges}

$\left[\mathrm{m}^{3} / \mathrm{s}\right.$, meters per second]

\begin{tabular}{|c|c|c|c|c|c|c|c|c|c|}
\hline \multirow{3}{*}{$\begin{array}{c}\text { Nonexceedance } \\
\text { probability }\end{array}$} & \multirow{3}{*}{$\begin{array}{c}\text { Recurrence } \\
\text { interval } \\
\text { (years) }\end{array}$} & \multicolumn{8}{|c|}{ Minimum mean discharge $\left(\mathrm{m}^{3} / \mathrm{s}\right)$} \\
\hline & & \multicolumn{8}{|c|}{ Number of consecutive days } \\
\hline & & 1 & 7 & 14 & 30 & 1 & 7 & 14 & 30 \\
\hline & & \multicolumn{4}{|c|}{ December-January-February } & \multicolumn{4}{|c|}{ March-April-May } \\
\hline 0.05 & 20 & 0.401 & 0.451 & 0.539 & 0.587 & 0.963 & 1.24 & 1.45 & 2.14 \\
\hline 0.10 & 10 & ${ }^{1} 0.559$ & 0.537 & 0.624 & 0.683 & 1.31 & 1.65 & 2.00 & 2.89 \\
\hline 0.20 & 5 & ${ }^{1} 0.777$ & 0.680 & 0.764 & 0.843 & 1.88 & 2.33 & 2.91 & 4.12 \\
\hline \multirow[t]{2}{*}{0.50} & 2 & ${ }^{1} 1.19$ & 1.15 & 1.24 & 1.38 & 3.70 & 4.42 & 5.69 & 7.95 \\
\hline & & \multicolumn{4}{|c|}{ June-July-August } & \multicolumn{4}{|c|}{ September-October-November } \\
\hline 0.05 & 20 & 0.014 & 0.014 & 0.015 & 0.016 & 0 & 0.008 & 0.011 & 0.017 \\
\hline 0.10 & 10 & 0.030 & 0.031 & 0.035 & 0.039 & 0 & 0.023 & 0.029 & 0.042 \\
\hline 0.20 & 5 & 0.070 & 0.075 & 0.086 & 0.101 & ${ }^{1} 0.077$ & 0.071 & 0.081 & 0.107 \\
\hline 0.50 & 2 & 0.271 & 0.294 & 0.338 & 0.414 & ${ }^{1} 0.408$ & 0.354 & 0.375 & 0.427 \\
\hline
\end{tabular}

'Data does not fit log-Pearson Type III curve, use with caution. 
7- 0.000-7M ADRASKAN RIVER AT ADRASKAN, Continued

Annual peak discharge and corresponding gage height

[m³/s, meters per second; --, no data]

\begin{tabular}{|c|c|c|c|c|c|c|c|}
\hline $\begin{array}{c}\text { Water } \\
\text { year }\end{array}$ & Date & $\begin{array}{c}\text { Gage } \\
\text { height } \\
\text { (meters) }\end{array}$ & $\begin{array}{c}\text { Peak } \\
\text { discharge } \\
\left(\mathrm{m}^{3} / \mathrm{s}\right)\end{array}$ & $\begin{array}{c}\text { Water } \\
\text { year }\end{array}$ & Date & $\begin{array}{c}\text { Gage } \\
\text { height } \\
\text { (meters) }\end{array}$ & $\begin{array}{c}\text { Peak } \\
\text { discharge } \\
\left(\mathrm{m}^{3} / \mathrm{s}\right)\end{array}$ \\
\hline
\end{tabular}

Annual peak discharge, by year

$\begin{array}{rrrrrrrr}1963 & \text { 12-May } & -- & 390 & 1971 & \text { 16-Apr } & -- & 15.6 \\ 1964 & \text { 13-Nov } & -- & 310 & 1972 & \text { 26-Apr } & -- & 120 \\ 1965 & \text { 6-May } & -- & 37.5 & 1973 & \text { 25-Mar } & -- & 70.0 \\ 1966 & \text { 15-Apr } & -- & 45.5 & 1974 & \text { 3-Mar } & -- & 76.0 \\ 1967 & \text { 26-Apr } & -- & 300 & 1975 & \text { 3-Apr } & -- & 46.6 \\ 1968 & \text { 18-May } & -- & 198 & 1976 & \text { 26-Mar } & -- & 148 \\ 1969 & \text { 8-May } & -- & 200 & 1977 & \text { 28-Mar } & -- & 29.0 \\ 1970 & \text { 9-Mar } & -- & 54.0 & 1978 & \text { 18-Nov } & -- & 109\end{array}$

Annual peak discharge, from highest to lowest

\begin{tabular}{lllllcll}
1963 & 12-May & -- & 390 & 1974 & 3-Mar & -- & 76.0 \\
1964 & 13-Nov & -- & 310 & 1973 & 25-Mar & -- & 70.0 \\
1967 & 26-Apr & -- & 300 & 1970 & 9-Mar & -- & 54.0 \\
1969 & 8-May & -- & 200 & 1975 & 3-Apr & -- & 46.6 \\
1968 & 18-May & -- & 198 & 1966 & 15-Apr & -- & 45.5 \\
1976 & 26-Mar & -- & 148 & 1965 & 6-May & -- & 37.5 \\
1972 & 26-Apr & -- & 120 & 1977 & 28-Mar & -- & 29.0 \\
1978 & 18-Nov & -- & 109 & 1971 & 16-Apr & -- & 15.6 \\
\hline
\end{tabular}




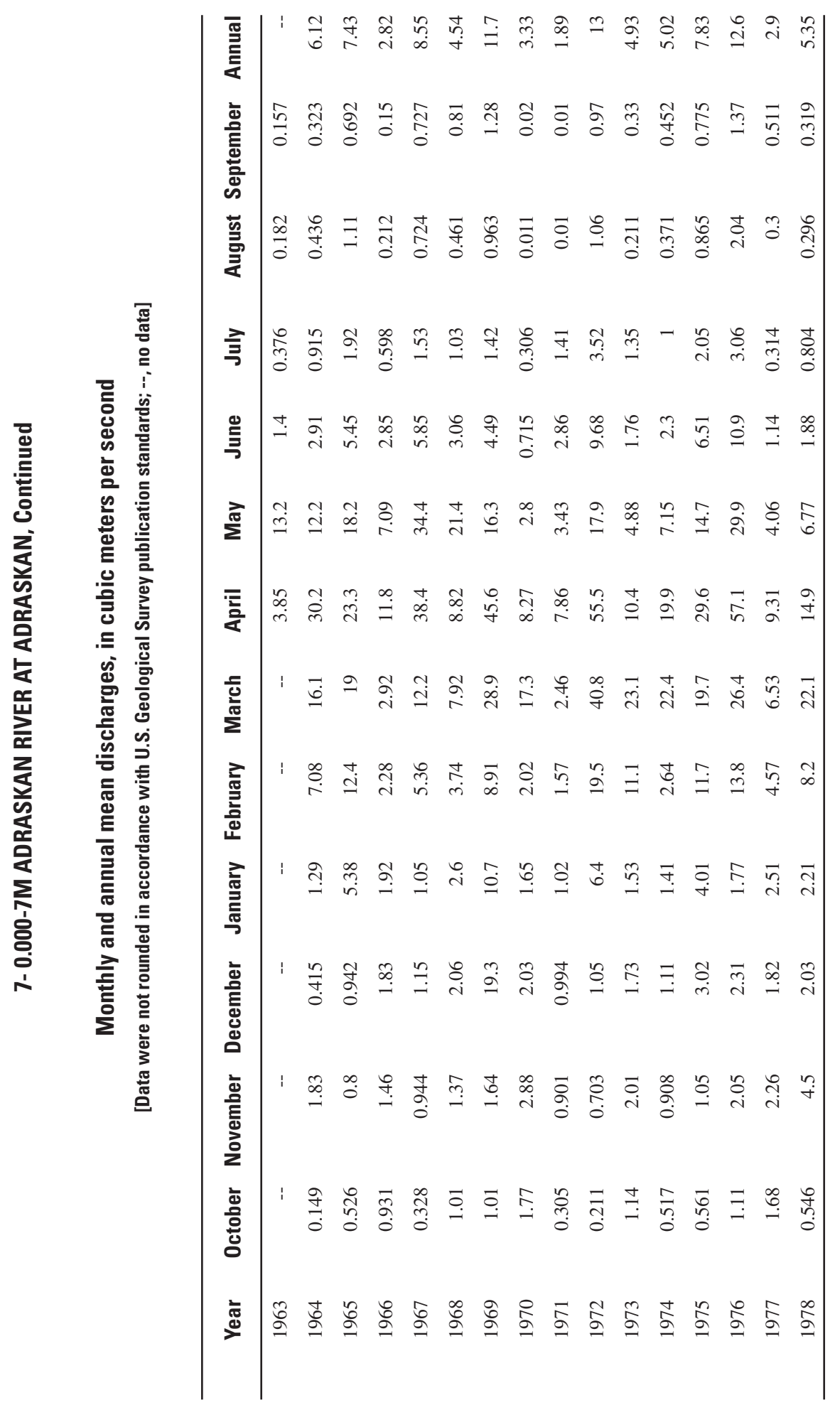




\section{7-5.R00-1A RUD-I-GAZ RIVER NEAR ADRASKAN}

\section{(U.S. Geological Survey identification number: 334200062170000)}

LOCATION: Lat $33^{\circ} 42^{\prime} \mathrm{N}$., long $62^{\circ} 17^{\prime} \mathrm{E}$., on bridge pier, about $0.5 \mathrm{~km}$ upstream from Adraskan River, and about $1 \mathrm{~km}$ north of Adraskan.

DRAINAGE AREA: $2,180 \mathrm{~km}^{2}$.

ALTITUDE: 1,380 meters plus mean sea level.

PERIOD OF RECORD: May 12, 1963 to September 30, 1978.

GAGE: Staff gage read once or twice daily.

Annual mean discharge

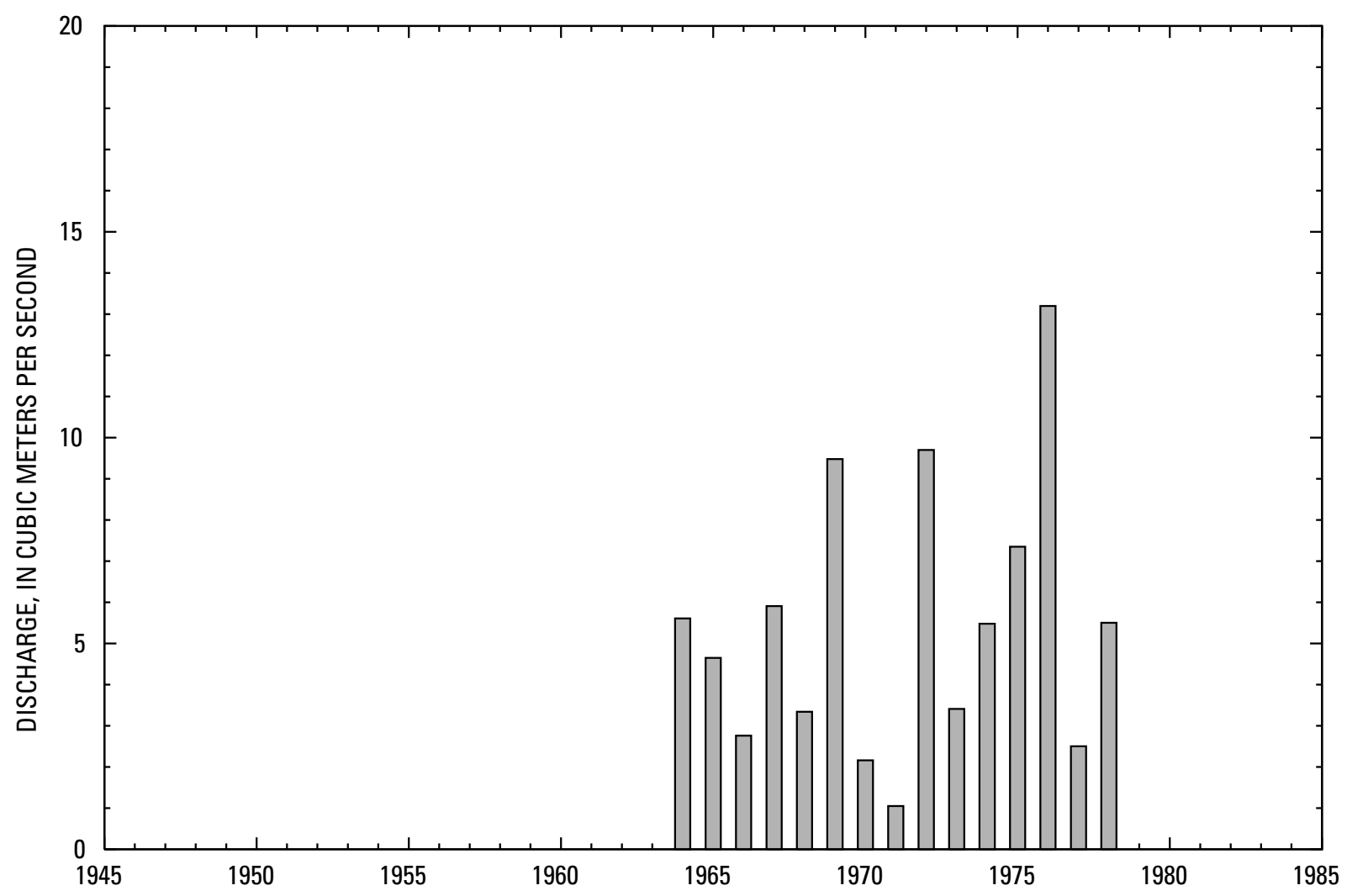




\section{7- 5.R00-1A RUD-I-GAZ RIVER NEAR ADRASKAN, Continued}

\section{Statistics of monthly and annual mean discharges}

$\left[\mathrm{m}^{3} / \mathrm{s}\right.$, meters per second]

\begin{tabular}{|c|c|c|c|c|c|c|c|c|}
\hline \multirow[b]{2}{*}{ Month } & \multicolumn{2}{|c|}{ Maximum } & \multicolumn{2}{|c|}{ Minimum } & \multicolumn{4}{|c|}{ Mean } \\
\hline & $\begin{array}{c}\text { Discharge } \\
\left(\mathrm{m}^{3} / \mathrm{s}\right)\end{array}$ & $\begin{array}{c}\text { Water year } \\
\text { of } \\
\text { occurrence }\end{array}$ & $\begin{array}{c}\text { Discharge } \\
\left(\mathrm{m}^{3} / \mathrm{s}\right)\end{array}$ & $\begin{array}{c}\text { Water year } \\
\text { of } \\
\text { occurrence }\end{array}$ & $\begin{array}{c}\text { Discharge } \\
\left(\mathrm{m}^{3} / \mathrm{s}\right)\end{array}$ & $\begin{array}{c}\text { Standard } \\
\text { deviation } \\
\left(\mathrm{m}^{3} / \mathrm{s}\right)\end{array}$ & $\begin{array}{c}\text { Coefficient } \\
\text { of } \\
\text { variation }\end{array}$ & $\begin{array}{c}\text { Percentage } \\
\text { of annual } \\
\text { discharge }\end{array}$ \\
\hline October & 1.10 & 1977 & 0.062 & 1972 & 0.51 & 0.33 & 0.66 & 0.77 \\
\hline November & 2.48 & 1978 & 0.209 & 1971 & 0.96 & 0.62 & 0.65 & 1.46 \\
\hline December & 11.9 & 1969 & 0.410 & 1965 & 1.74 & 2.84 & 1.63 & 2.65 \\
\hline January & 6.68 & 1969 & 0.602 & 1964 & 2.07 & 1.51 & 0.73 & 3.15 \\
\hline February & 8.14 & 1969 & 1.04 & 1971 & 4.51 & 2.42 & 0.54 & 6.86 \\
\hline March & 39.9 & 1972 & 1.75 & 1967 & 19.3 & 13.4 & 0.70 & 29.3 \\
\hline April & 76.7 & 1976 & 6.45 & 1971 & 24.2 & 18.7 & 0.77 & 36.9 \\
\hline May & 25.6 & 1976 & 1.29 & 1971 & 9.10 & 8.14 & 0.89 & 13.8 \\
\hline June & 4.80 & 1967 & 0.232 & 1971 & 1.78 & 1.53 & 0.86 & 2.70 \\
\hline July & 1.93 & 1976 & 0.075 & 1971 & 0.75 & 0.58 & 0.77 & 1.14 \\
\hline August & 1.16 & 1976 & 0 & 1970,1971 & 0.39 & 0.35 & 0.90 & 0.59 \\
\hline September & 1.05 & 1976 & 0 & 1971 & 0.40 & 0.34 & 0.85 & 0.61 \\
\hline Annual & 13.2 & 1976 & 1.05 & 1971 & 5.47 & 3.31 & 0.61 & 100 \\
\hline
\end{tabular}

Annual flow duration

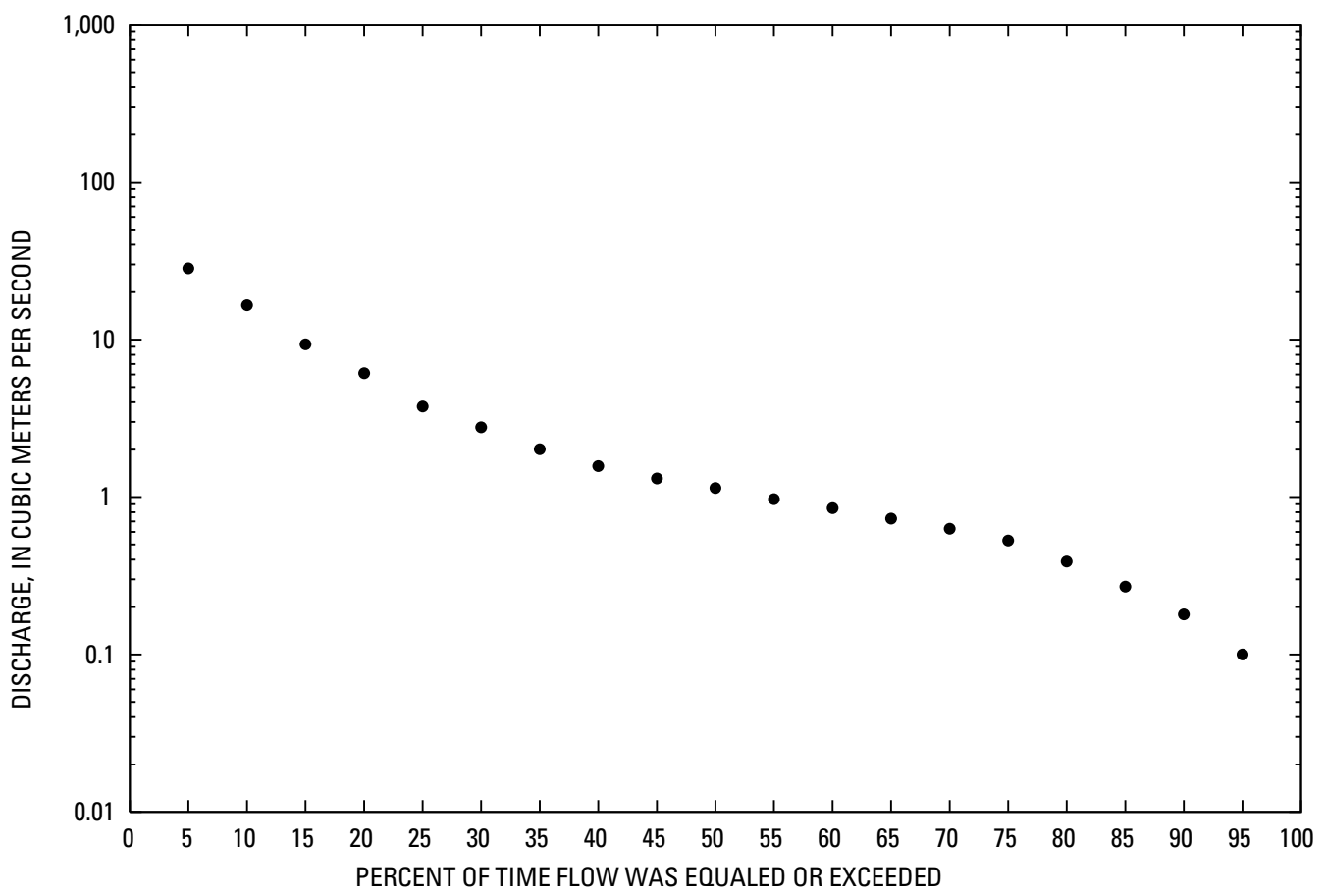




\section{7- 5.R00-1A RUD-I-GAZ RIVER NEAR ADRASKAN, Continued}

Monthly and annual flow duration, in cubic meters per second

[Data were not rounded in accordance with U.S. Geological Survey publication standards]

\begin{tabular}{|c|c|c|c|c|c|c|c|c|c|c|c|c|c|}
\hline $\begin{array}{l}\text { Percentage } \\
\text { of days } \\
\text { discharge } \\
\text { equaled or } \\
\text { exceeded }\end{array}$ & January & February & March & April & May & June & July & August & September & October & November & December & Annual \\
\hline 95 & 0.55 & 0.76 & 1.48 & 3.7 & 0.79 & 0.1 & 0.08 & 0.02 & 0 & 0.09 & 0.17 & 0.37 & 0.1 \\
\hline 90 & 0.63 & 1 & 2.25 & 5.43 & 1.21 & 0.31 & 0.11 & 0.03 & 0.03 & 0.12 & 0.2 & 0.49 & 0.18 \\
\hline 85 & 0.69 & 1.3 & 3.19 & 6.75 & 1.65 & 0.48 & 0.17 & 0.06 & 0.08 & 0.14 & 0.23 & 0.55 & 0.27 \\
\hline 80 & 0.76 & 1.56 & 3.69 & 7.75 & 1.95 & 0.57 & 0.23 & 0.1 & 0.11 & 0.18 & 0.27 & 0.59 & 0.39 \\
\hline 75 & 0.85 & 1.71 & 4.18 & 9.36 & 2.33 & 0.66 & 0.3 & 0.13 & 0.14 & 0.21 & 0.35 & 0.67 & 0.53 \\
\hline 70 & 0.98 & 2.03 & 5.56 & 10.4 & 2.63 & 0.77 & 0.34 & 0.14 & 0.16 & 0.26 & 0.45 & 0.75 & 0.63 \\
\hline 65 & 1.29 & 2.3 & 8.31 & 11.4 & 2.91 & 0.88 & 0.37 & 0.19 & 0.18 & 0.29 & 0.52 & 0.82 & 0.73 \\
\hline 60 & 1.38 & 2.62 & 10.9 & 12.6 & 3.22 & 0.97 & 0.41 & 0.21 & 0.19 & 0.32 & 0.59 & 0.89 & 0.85 \\
\hline 55 & 1.44 & 3.01 & 14 & 14.8 & 3.61 & 1.07 & 0.51 & 0.23 & 0.21 & 0.43 & 0.67 & 0.96 & 0.97 \\
\hline 50 & 1.49 & 3.27 & 15.8 & 17 & 4.67 & 1.23 & 0.58 & 0.28 & 0.29 & 0.46 & 0.74 & 1.1 & 1.14 \\
\hline 45 & 1.55 & 3.55 & 17.9 & 20.4 & 5.28 & 1.34 & 0.64 & 0.31 & 0.38 & 0.54 & 0.81 & 1.16 & 1.31 \\
\hline 40 & 1.6 & 3.88 & 20 & 23.3 & 6.17 & 1.44 & 0.7 & 0.35 & 0.43 & 0.64 & 0.86 & 1.2 & 1.57 \\
\hline 35 & 1.66 & 4.7 & 22.1 & 25.5 & 7.85 & 1.62 & 0.89 & 0.45 & 0.56 & 0.7 & 0.93 & 1.25 & 2.01 \\
\hline 30 & 1.96 & 5.62 & 24.7 & 27.7 & 9.84 & 1.95 & 1.01 & 0.52 & 0.6 & 0.74 & 0.99 & 1.29 & 2.77 \\
\hline 25 & 2.23 & 6.2 & 27.2 & 31.7 & 12.3 & 2.36 & 1.13 & 0.64 & 0.64 & 0.76 & 1.05 & 1.35 & 3.76 \\
\hline 20 & 2.44 & 6.96 & 29.8 & 35.8 & 15.2 & 2.98 & 1.26 & 0.78 & 0.7 & 0.8 & 1.15 & 1.41 & 6.13 \\
\hline 15 & 2.74 & 7.83 & 35.3 & 41.1 & 17.7 & 3.54 & 1.42 & 0.85 & 0.81 & 0.93 & 1.24 & 1.47 & 9.34 \\
\hline 10 & 5.8 & 8.6 & 43.5 & 50.6 & 23 & 4.39 & 1.65 & 0.91 & 0.92 & 1.01 & 1.45 & 1.64 & 16.5 \\
\hline 5 & 6.74 & 10.4 & 54.5 & 77 & 30.2 & 5.36 & 2.06 & 1.05 & 0 & 1.08 & 1.86 & 7.29 & 28.3 \\
\hline
\end{tabular}




\section{7- 5.R00-1A RUD-I-GAZ RIVER NEAR ADRASKAN, Continued}

Probability of occurrence of annual high discharges

[ $\mathrm{m}^{3} / \mathrm{s}$, meters per second; $\mathrm{ng}$, statistic not given]

\begin{tabular}{|c|c|c|c|c|c|c|}
\hline \multirow{2}{*}{$\begin{array}{c}\text { Exceedance } \\
\text { probability }\end{array}$} & \multirow{2}{*}{$\begin{array}{c}\text { Recurrence } \\
\text { interval } \\
\text { (years) }\end{array}$} & \multirow{2}{*}{$\begin{array}{c}\text { Maximum } \\
\text { instantaneous } \\
\left(\mathrm{m}^{3} / \mathrm{s}\right)\end{array}$} & \multicolumn{4}{|c|}{ Maximum mean discharge } \\
\hline & & & 3-day period & 7-day period & 15-day period & 30-day period \\
\hline 0.99 & 1.01 & ng & ${ }^{1} 6.92$ & 6.95 & 6.18 & 4.47 \\
\hline 0.95 & 1.05 & ng & 13.6 & 12.0 & 10.1 & 7.73 \\
\hline 0.90 & 1.11 & 35.3 & 18.9 & 15.8 & 13.1 & 10.2 \\
\hline 0.80 & 1.25 & 49.7 & 27.1 & 21.7 & 17.7 & 14.2 \\
\hline 0.50 & 2 & 89.5 & 49.1 & 38.1 & 30.9 & 25.5 \\
\hline 0.20 & 5 & 148 & 78.8 & 63.4 & 52.4 & 43.9 \\
\hline 0.10 & 10 & 187 & 96.6 & 80.8 & 68.4 & 57.3 \\
\hline 0.04 & 25 & 234 & 116 & 103 & 90.0 & 75.2 \\
\hline 0.02 & 50 & 268 & 129 & 120 & 107 & 88.9 \\
\hline 0.01 & 100 & 299 & 141 & 136 & 125 & 103 \\
\hline 0.005 & 200 & 329 & ${ }^{1} 151$ & 153 & 143 & 117 \\
\hline 0.002 & 500 & 367 & ng & ng & ng & $\mathrm{ng}$ \\
\hline
\end{tabular}

'Data does not fit log-Pearson Type III curve, use with caution.

\section{7- 5.R00-1A RUD-I-GAZ RIVER NEAR ADRASKAN, Continued}

\section{Probability of occurrence of annual low discharges}

$\left[\mathrm{m}^{3} / \mathrm{s}\right.$, meters per second]

\begin{tabular}{|c|c|c|c|c|c|c|c|c|c|c|}
\hline \multirow{3}{*}{$\begin{array}{c}\text { Nonexceedance } \\
\text { probability }\end{array}$} & \multirow{3}{*}{$\begin{array}{c}\text { Recurrence } \\
\text { interval } \\
\text { (years) }\end{array}$} & \multicolumn{9}{|c|}{ Minimum mean discharge $\left(\mathrm{m}^{3} / \mathrm{s}\right)$} \\
\hline & & \multicolumn{9}{|c|}{ Number of consecutive days } \\
\hline & & 1 & 3 & 7 & 14 & 30 & 60 & 90 & 120 & 183 \\
\hline 0.05 & 20 & 0 & 0 & 0 & 0 & 0 & 0 & 0 & 0.042 & 0.141 \\
\hline 0.10 & 10 & 0 & 0 & 0 & 0 & 0 & 0.010 & 0.034 & 0.075 & 0.198 \\
\hline 0.20 & 5 & 0.061 & 0.063 & 0.069 & 0.074 & 0.084 & ${ }^{1} 0.067$ & 0.110 & 0.142 & 0.289 \\
\hline 0.50 & 2 & 0.216 & 0.222 & 0.234 & 0.244 & 0.271 & 0.354 & 0.364 & 0.386 & 0.550 \\
\hline
\end{tabular}

'Data does not fit log-Pearson Type III curve, use with caution. 


\section{7- 5.R00-1A RUD-I-GAZ RIVER NEAR ADRASKAN, Continued}

\section{Probability of occurrence of seasonal low discharges}

$\left[\mathrm{m}^{3} / \mathrm{s}\right.$, meters per second]

\begin{tabular}{|c|c|c|c|c|c|c|c|c|c|}
\hline \multirow{3}{*}{$\begin{array}{c}\text { Nonexceedance } \\
\text { probability }\end{array}$} & \multirow{3}{*}{$\begin{array}{c}\text { Recurrence } \\
\text { interval } \\
\text { (years) }\end{array}$} & \multicolumn{8}{|c|}{ Minimum mean discharge $\left(\mathrm{m}^{3} / \mathrm{s}\right)$} \\
\hline & & \multicolumn{8}{|c|}{ Number of consecutive days } \\
\hline & & 1 & 7 & 14 & 30 & 1 & 7 & 14 & 30 \\
\hline & & \multicolumn{4}{|c|}{ December-January-February } & \multicolumn{4}{|c|}{ March-April-May } \\
\hline 0.05 & 20 & 0.258 & 0.336 & 0.344 & 0.438 & 0.475 & 0.576 & 0.611 & 1.05 \\
\hline 0.10 & 10 & 0.342 & 0.387 & 0.402 & 0.490 & 0.606 & 0.746 & 0.850 & 1.43 \\
\hline 0.20 & 5 & 0.465 & 0.473 & 0.499 & 0.581 & 0.832 & 1.04 & 1.28 & 2.09 \\
\hline \multirow[t]{2}{*}{0.50} & 2 & 0.770 & 0.780 & 0.835 & 0.909 & 1.62 & 2.04 & 2.84 & 4.54 \\
\hline & & \multicolumn{4}{|c|}{ June-July-August } & \multicolumn{4}{|c|}{ September-Ocotber-November } \\
\hline 0.05 & 20 & 0 & 0 & 0 & 0 & 0 & 0 & 0 & 0 \\
\hline 0.10 & 10 & 0 & 0 & 0 & 0 & 0 & 0.007 & 0.015 & 0.027 \\
\hline 0.20 & 5 & 0.064 & 0.074 & 0.083 & 0.102 & ${ }^{1} 0.072$ & 0.048 & 0.066 & 0.091 \\
\hline 0.50 & 2 & 0.207 & 0.229 & 0.239 & 0.286 & 0.246 & 0.302 & 0.297 & 0.331 \\
\hline
\end{tabular}

'Data does not fit log-Pearson Type III curve, use with caution. 


\section{7- 5.R00-1A RUD-I-GAZ RIVER NEAR ADRASKAN, Continued}

Annual peak discharge and corresponding gage height

$\left[\mathrm{m}^{3} / \mathrm{s}\right.$, meters per second; --, no data]

\begin{tabular}{|c|c|c|c|c|c|c|c|c|}
\hline $\begin{array}{l}\text { Water } \\
\text { year }\end{array}$ & Date & $\begin{array}{c}\text { Gage } \\
\text { height } \\
\text { (meter) }\end{array}$ & $\begin{array}{c}\text { Peak } \\
\text { discharge } \\
\left(\mathrm{m}^{3} / \mathrm{s}\right)\end{array}$ & $\begin{array}{c}\text { Water } \\
\text { year }\end{array}$ & Date & $\begin{array}{c}\text { Gage } \\
\text { height } \\
\text { (meter) }\end{array}$ & & $\begin{array}{c}\text { Peak } \\
\text { discharge } \\
\left(\mathrm{m}^{3} / \mathrm{s}\right)\end{array}$ \\
\hline \multicolumn{9}{|c|}{ Annual peak discharge, by year } \\
\hline 1963 & 12-May & & 70.0 & 1971 & 12-Apr & & -- & 13.4 \\
\hline 1964 & 24-Apr & & 40.0 & 1972 & 24-Mar & & -- & 133 \\
\hline 1965 & 24-Apr & & 97.2 & 1973 & 26-Mar & & -- & 75.0 \\
\hline 1966 & 15-Apr & & 34.0 & 1974 & 12-Apr & & -- & 131 \\
\hline 1967 & 28-Apr & & 132 & 1975 & 14-May & & -- & 153 \\
\hline 1968 & 18-May & & 156 & 1976 & 8-Apr & & -- & 156 \\
\hline 1969 & 8-May & & 229 & 1977 & 4-Apr & & -- & 29.7 \\
\hline 1970 & 25-Mar & & 53.1 & 1978 & $18-\mathrm{Nov}$ & & -- & 89.5 \\
\hline \multicolumn{9}{|c|}{ Annual peak discharge, from highest to lowest } \\
\hline 1969 & 8-May & & 229 & 1978 & 18 -Nov & & -- & 89.5 \\
\hline 1976 & 8-Apr & & 156 & 1973 & 26-Mar & & -- & 75.0 \\
\hline 1968 & 18-May & & 156 & 1963 & 12-May & & -- & 70.0 \\
\hline 1975 & 14-May & & 153 & 1970 & 25-Mar & & -- & 53.1 \\
\hline 1972 & 24-Mar & & 133 & 1964 & 24-Apr & & -- & 40.0 \\
\hline 1967 & 28-Apr & & 132 & 1966 & 15-Apr & & -- & 34.0 \\
\hline 1974 & 12-Apr & & 131 & 1977 & 4-Apr & & -- & 29.7 \\
\hline 1965 & 24-Apr & & 97.2 & 1971 & 12-Apr & & -- & 13.4 \\
\hline
\end{tabular}




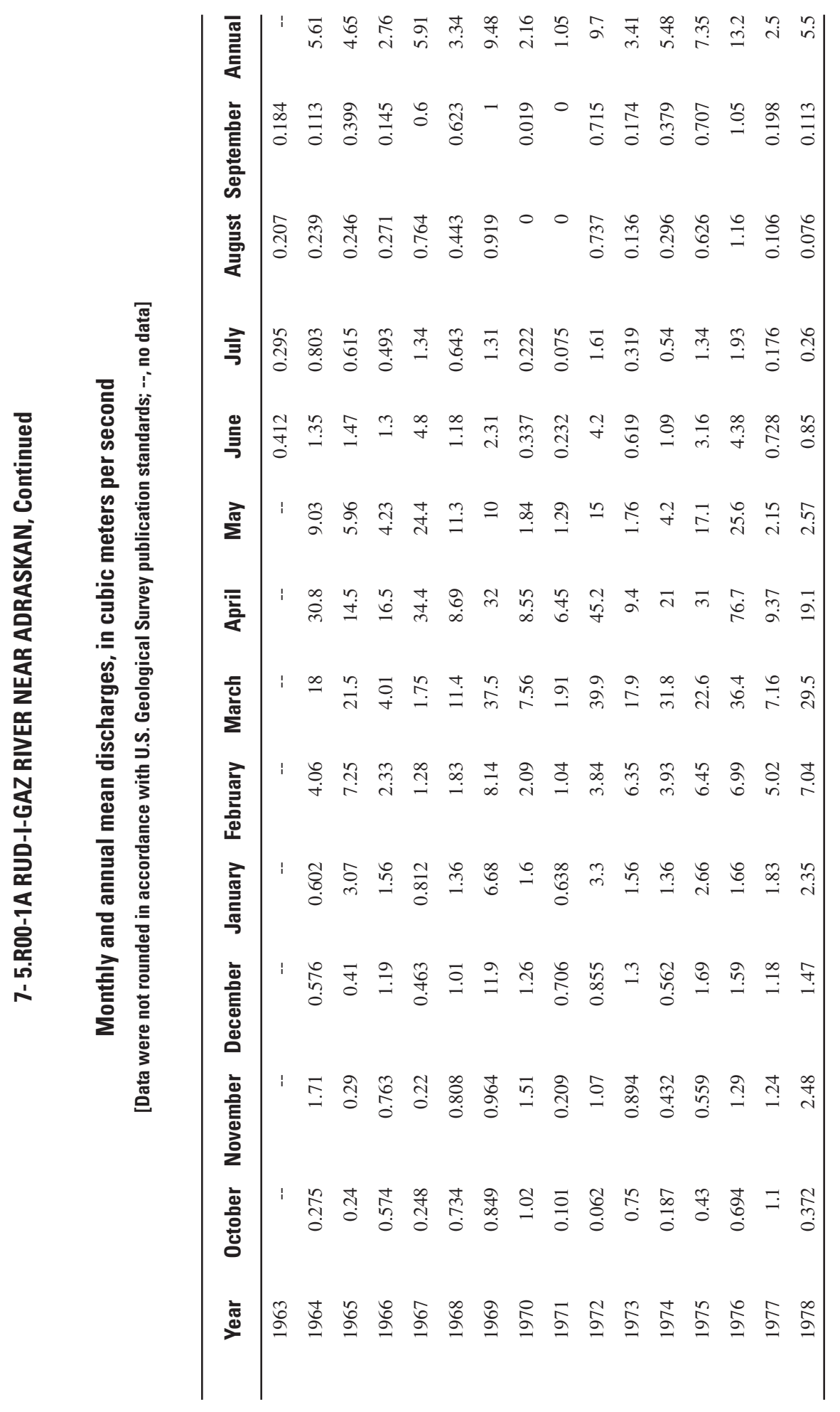




\section{$\frac{\mathbb{3}}{3}$}

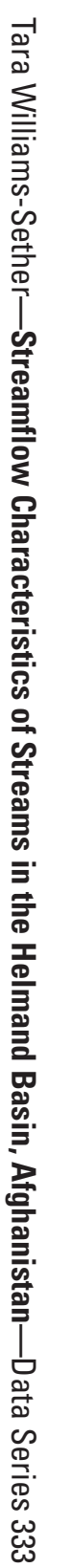

8 Printed on recycled paper 